\title{
Magnetic Properties of Santa Fe Group Sediments in the 98th Street Core Hole, Albuquerque, New Mexico
}

By Mark R. Hudson', Marlo Mikolas², John W. Geissman², and Bruce D. Allen ${ }^{3}$

\section{U.S. Geological Survey Open-File Report 98-592}

Prepared in cooperation with the CITY OF ALBUQUERQUE NEW MEXICO OFFICE OF THE STATE ENGINEER NEW MEXICO BUREAU OF MINES AND MINERAL RESOURCES

NATIONAL COOPERATIVE GEOLOGIC MAPPING PROGRAM

1 - U.S. Geological Survey, Box 25046, MS 913, Denver, Colorado, 80225

2 - Dept. Earth and Planetary Sciences, Univ. of New Mexico, Albuquerque, NM, 87131

${ }^{3}$ - New Mexico Bureau of Mines and Mineral Resources, 2808 Central Ave, Albuquerque, NM, 87106 


\section{U.S. DEPARTMENT OF INTERIOR BRUCE BABBITT, SECRETARY}

U.S. GEOLOGICAL SURVEY

Charles G. Grout, Director

Copies of this report can be purchased from:

U.S. Geological Survey

Branch of Information Services

Box 25286, Building 810

Denver, CO 80225-0286 


\section{CONTENTS}

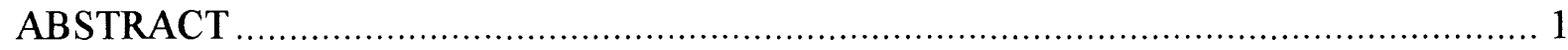

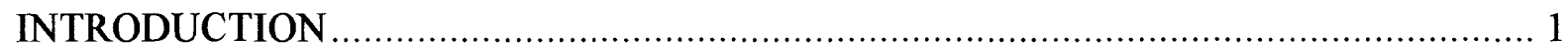

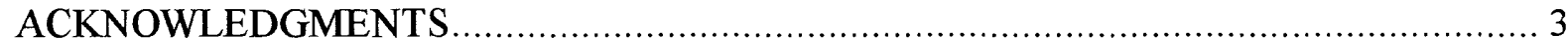

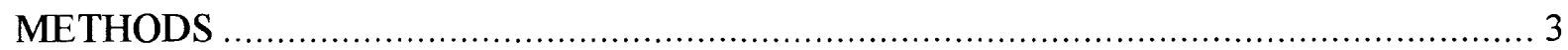

MAGNETIC POLARITY ZONATION .......................................................... 5

VARIATION OF MAGNETIC PROPERTIES WITH DEPTH AND GRAIN SIZE ........... 9

SANTA FE GROUP SEDIMENTS AS MAGNETIC ANOMALY SOURCES ............... 13

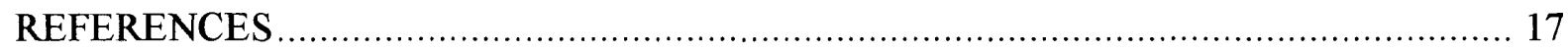

APPENDIX A, PALEOMAGNETIC DATA...................................................... 19

APPENDIX B, WHOLE CORE MAGNETIC SUSCEPTIBШITY.............................57

APPENDIX C, MAGNETIC PROPERTY DATA ..............................................65

CONVERSION FACTORS

Multiply by To obtain

foot $\quad 0.3048 \quad$ meter

\section{SYMBOLS AND TERMS}

$\mathrm{A} / \mathrm{m}=$ Amperes per meter

$\mathrm{DRM}=$ Detrital remanent magnetization

IRM = Isothermal remanent magnetization

NRM = Natural remanent magnetization

MS = Magnetic susceptibility (SI volume units)

$\mathrm{Q}=$ Koenigsburger ratio

$\mathrm{mT}=$ millitesla

$\mu \mathrm{T}=$ microtesla

$\mathrm{nT}=$ nanotesla 


\title{
MAGNETIC PROPERTIES OF SANTA FE GROUP SEDIMENTS IN THE 98TH STREET CORE HOLE, ALBUQUERQUE, NEW MEXICO
}

\author{
By Mark R. Hudson, Marlo Mikolas, John W. Geissman, and Bruce D. Allen
}

\section{Abstract}

Paleomagnetic and rock magnetic studies of upper to middle parts of the rift-filling Santa Fe Group were conducted in the $1500 \mathrm{ft}(457 \mathrm{~m})$ 98th Street core from western Albuquerque to determine the age of the deposition and to assess their possible contribution to magnetic anomalies. A total of 336 samples distributed over the core length were subjected to alternating field demagnetization to identify magnetic components and to determine their polarity. A magnetic mineral having moderate coercivity, probably magnetite, carries the principal component of magnetization. Magnetic polarity is dominantly normal, but samples with reversed polarity cluster over three main depth intervals through the core section. Twenty-one alternating normal and reverse polarity zones are defined by at least two adjacent samples of the same polarity. Comparison of core polarity zonation to the geomagnetic polarity time scale does not yield a unique correlation, yet the dominance of normal polarity suggests that the sampled interval may correlate with one of several potential periods of closely spaced normal subchrons that spanned 1-2 m.y. in middle Miocene or younger time.

Numerous linear anomalies identified in recent high-resolution aeromagnetic surveys of the Albuquerque Basin are probably related to fault offsets of sediments within the Santa Fe Group. Variation of magnetic properties within the core gives insight into potential contrasts of total magnetizations that control the aeromagnetic expression of these sediments. Within the 98th Street core, magnetic susceptibility (MS) varies greatly, both in logs of whole-core MS (8.9E-5 to 6.7E-2 SI) and in individual samples extracted from the core
(6.0E-6 to 2.4E-2 SI). Likewise, natural remanent magnetization (NRM) intensity varies from $8.4 \mathrm{E}-4$ to $2.7 \mathrm{E}-1 \mathrm{~A} / \mathrm{m}$. Both $\mathrm{MS}$ and NRM generally correlate with sediment grain size such that the coarsest grained rocks have highest magnetic property values. Plots of NRM versus MS define into two discrete groups of magnetized sediments that probably reflect contrasting magnetic mineral properties or magnetization acquisition mechanisms. Total magnetizations calculated from the NRM and MS values indicate that Santa Fe Group sediments are capable of producing moderate to weak magnetic anomahes. The data suggest that coarse-grained aquifer rocks have highest total magnetization and thus greatest potential for generation of aeromagnetic anomalies where juxtaposed with fine sediments along faults.

\section{Introduction}

The 98th Street core hole (fig. 1) was drilled on the west side of Albuquerque, New Mexico (3505'32" N, 106 $44^{\circ} 52^{\prime}$ ' W), to provide key stratigraphic and hydrauhic-property information for upper parts of the Santa Fe Group within the Albuquerque basin of the Rio Grande rift (Stone et al., 1998). The core hole was drilled cooperatively by the U.S. Geological Survey and the City of Albuquerque and investigated in collaboration with the New Mexico Bureau of Mines and Mineral Resources and the New Mexico Office of the State Engineer. Clastic sediment recovered in the core provides material for detailed investigation of magnetic properties of the upper and middle Santa Fe Group. Remanent magnetization has been studied in the core sediments to define a magnetic polarity zonation for the stratigraphic 


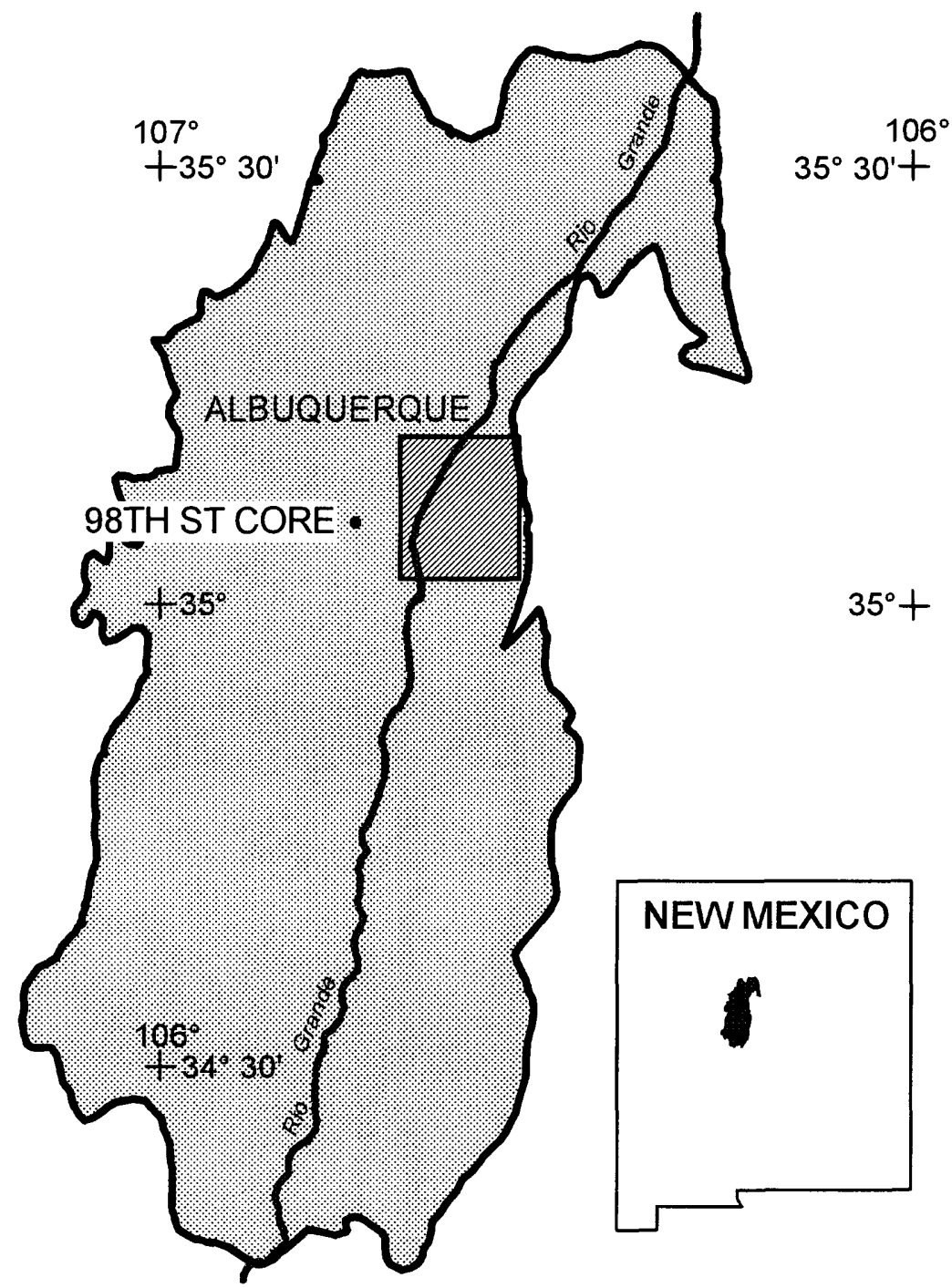

Figure 1. Outline of the middle Rio Grande basin with location of 98th Street core hole, New Mexico. 
interval. Comparison of this polarity zonation to the geomagnetic polarity time scale (GPTS) has potential to provide close chronologic control. Variations among the magnetic properties magnetic susceptibility (MS) and natural remanent magnetization (NRM) give insight into the potential sources within the Santa Fe Group for aeromagnetic anomalies that have been detected in recent high-resolution surveys in the region (e.g., Grauch, 1998). This report tabulates magnetic properties obtained for the core sediments (Appendix A, B, C) and gives some preliminary interpretations of their significance.

A total of $760.6 \mathrm{ft}(232 \mathrm{~m})$ of sediment, or about 50 percent, of the $1500 \mathrm{ft}(457 \mathrm{~m})$ total depth was recovered during drilling of the 98th Street core hole (fig. 2). Stone and others (1998) proposed a fourfold division of the core sequence consisting of: (1) Quaternary eolian sand and valley-border alluvium from $0-19 \mathrm{ft}$ (0$8 \mathrm{~m}$ ) depths, (2) coarse-grained pebbly sandstone of the upper unit of the Santa Fe from 19-97 $\mathrm{ft}(8-30 \mathrm{~m})$ depths, (3) a fine-grained interval tentatively correlated with the middle unit of Santa Fe from 97-787 ft (30-240 m) depths, and (4) a lower part of the middle Santa Fe unit from 787-1500 ft (240-457 m) depths consisting of channel-fill medium sand and underlying sand, silt, and clay overbank deposits. The upper part of the middle unit of the Santa $\mathrm{Fe}$ Group contains thick laminated red and olive brown clay and silt deposits (487-787 ft, 148$240 \mathrm{~m}$ ) within a laterally extensive unit named the Atrisco member (informal) by Connell and others (1998).

Ages for the 98th Street core interval are only broadly constrained. Coarse deposits of the upper Santa Fe Group unit are correlated with an integrated river system that was established within the Rio Grande rift before $4.5 \mathrm{Ma}$ (Stone and others, 1998), suggesting this core unit is probably $4.5 \mathrm{Ma}$ or younger. Stone and others (1998) suggested that the middle unit of the Santa $\mathrm{Fe}$ in the core may correlate with middle
Santa Fe sediments exposed about $22 \mathrm{~km}$ to the north at Loma Colorado containing vertebrate fauna that were considered to be Hemphillian (about 4.6 to $8.9 \mathrm{Ma}$ ) age. Recent reexamination of the Loma Colorado fauna, however, indicates a Blancan (about 2.5 to 4.6 Ma) age (G.S. Morgan, personal communication, 1998).

\section{Acknowledgments}

We appreciate helpful discussions with V.J.S. Grauch on aeromagnetic surveys in area and with J.G. Rosenbaum on sediment magnetic properties. G.S. Morgan kindly shared age information for vertebrate fauna within the Santa Fe Group. We received a valuable review from R.L. Reynolds.

\section{Methods}

Discrete samples were taken from the core in the form of $7 \mathrm{cc}$ nonmagnetic plastic cubes. Fine-grained sediments were preferentially sampled. The samples were oriented vertically within the core segments but lack azimuthal orientation. Natural remanent magnetization (NRM) for the cube samples (336 total) was measured with a three-axis superconducting magnetometer in a magnetically shielded room at the University of New Mexico (Appendix A). To assess the character and stability of the remanent magnetization, samples were subjected to progressive alternating-field (AF) demagnetization in 13-16 steps at peak fields ranging from 3 to $115 \mathrm{mT}$. All demagnetization results for samples were inspected on orthogonal vector diagrams to interpret their magnetic polarity and reliability. Directions of remanent magnetization components isolated by demagnetization were determined using principal component analysis (Kirschvink, 1980). Magnetic susceptibility for the cube samples was measured using a commercial induction coil operating at about $750 \mathrm{~Hz}$ (Appendix C, 282 samples). 


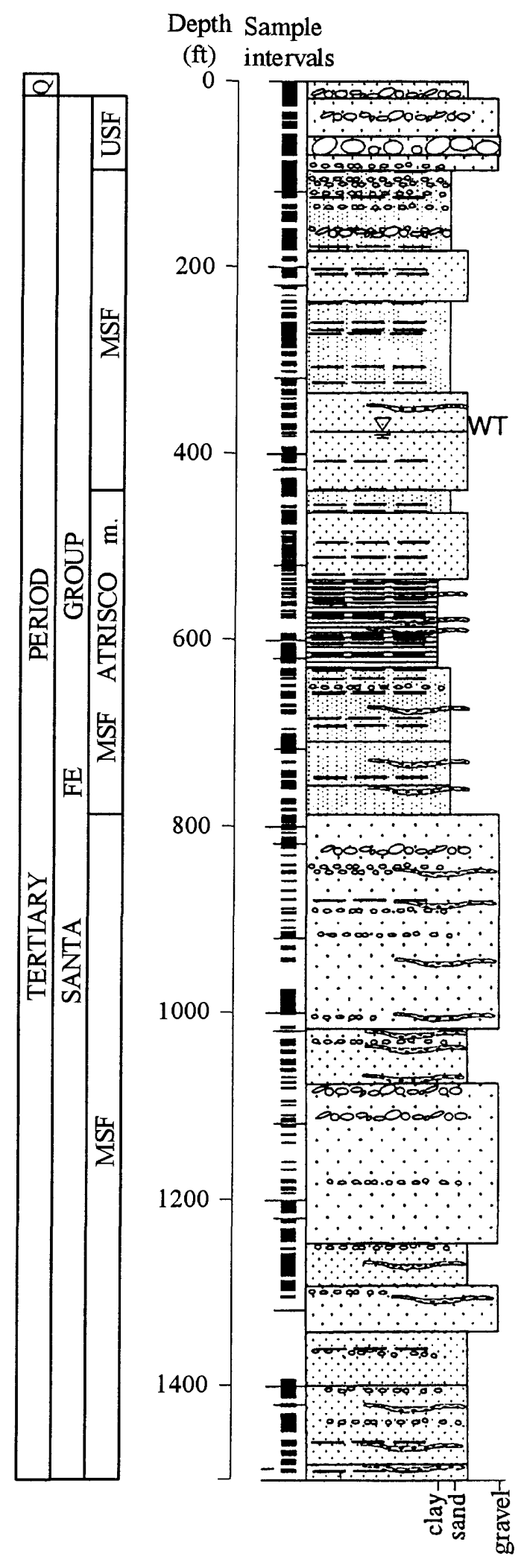

EXPLANATION OF SYMBOLS
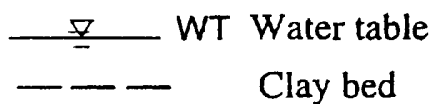

$00000000000 \quad$ Coarse sand bed

\$000000000 Pebble gravel bed

Sandstone

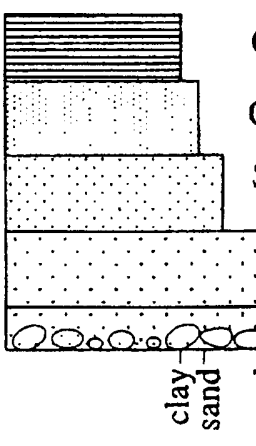

Clay

Clayey, sandy silt

Silty fine sand

Medium sand

Sand and gravel

Increasing

grain size

Figure 2. General stratigraphic column of the 98th Street core hole adapted from Stone and others (1998). Q, Quaternary; USF, upper unit of Santa Fe Group; MSF, middle unit of Santa Fe group; Atrisco m., Atrisco member of Connell and others (1998). 
Magnetic susceptibility measurements were made on whole-core segments employing a commercial pass-through induction coil operated at a $800 \mathrm{~Hz}$ frequency. A total of 1344 measurements were spaced through the $1500 \mathrm{ft}$ $(457 \mathrm{~m})$ core length (Appendix B). The coil sensed an approximate $5 \mathrm{~cm}$ core length centered about the coil midpoint.

\section{Magnetic Polarity Zonation}

The demagnetization character of each sample was examined on orthogonal vector diagrams (fig. 3) to assign a magnetic polarity and well as an estimate of its quality (A, B, or C). Greatest weight in assigning polarity was given to the demagnetization response over the interval between 15 and $115 \mathrm{mT}$. Strongly magnetic cubic spinels such as magnetite, that would most likely carry a detrital remanence, typically unblock over this interval. Many samples also contained a low-coercivity, positive-inclination component of probable viscous origin that was removed by about $15 \mathrm{mT}$ (fig. 3C, E, F). After removal of the magnetite component by $115 \mathrm{mT}$, some samples retained a high-coercivity component that is probably carried by hematite (fig. 3E).

Sample polarities were assigned a highest A quality if they had linear trajectories that trended toward the origin of vector diagrams and if most of their remanence was removed over the 15-115 mT interval (fig. 3). Sixty-three percent of the samples were classified as quality A (fig. 4). Quality B samples (24 percent) also liad clear polarity but yielded either partly curved trajectories on vector diagrams or trajectories that did not trend toward the origin due to the presence of a high coercivity hematite component (fig. 3E). Assigning polarities for quality $\mathrm{C}$ samples ( 12.5 percent) was more subjective, either because they displayed erratic trajectories on vector diagrams (fig. 3C) or because most of their remanence was dominated by a low-coercivity viscous component (fig. 3F).
No polarity was assigned for two samples having remanence with nearly horizontal inclination.

Inclination was determined by principal component analysis (typically between about 18 to $85 \mathrm{mT}$ ) for the moderate-coercivity component in each sample having A or B quality demagnetization behavior (fig. 5). Positive inclinations for these samples cluster most strongly between $50^{\circ}$ and $70^{\circ}$ (fig. 5B), similar to time-averaged inclinations $\left(53-55^{\circ}\right)$ for normal-polarity paleofields from middle Miocene to present at the location. The inclination angles for many normal- and most reversed-polarity samples, however, are less than $35^{\circ}$. These low inclination angles might reflect shallowing of magnetization due to sediment compaction (e.g., Kodama and Sun, 1992) or an incomplete separation of the principal magnetite component from a component of opposite polarity, such as a hard viscous component carried by pigmentary hematite (Dunlop and Stirling, 1977).

The magnetic polarity of samples in the core (fig. 4) is dominantly normal ( 83 percent), but samples with reversed polarity cluster over three main depth intervals (120-170 ft, 37-46 m; 248-377 ft, 76-115 m; 708-784 ft, 216-239 m). A total of 21 polarity zones were defined to include intervals containing at least two adjacent samples of the same polarity (fig. 4). In addition to these polarity zones, several single samples having polarity opposite to those of adjacent samples were identified. Such single-sample polarity changes could reflect short-lived transitional geomagnetic fields, sediment rotation due to core deformation, or perhaps a misorientation of samples. The prevalence of normal-polarity magnetization raises the question of whether the 98th Street core could have been remagnetized in the contemporary magnetic field during drilling. Paleomagnetic studies of other drill cores have detected low coercivity, steeply inclined, isothermal remanent magnetizations (IRM's) that were generated by fields aligned along axes of steel core barrels 
A QUALITYA
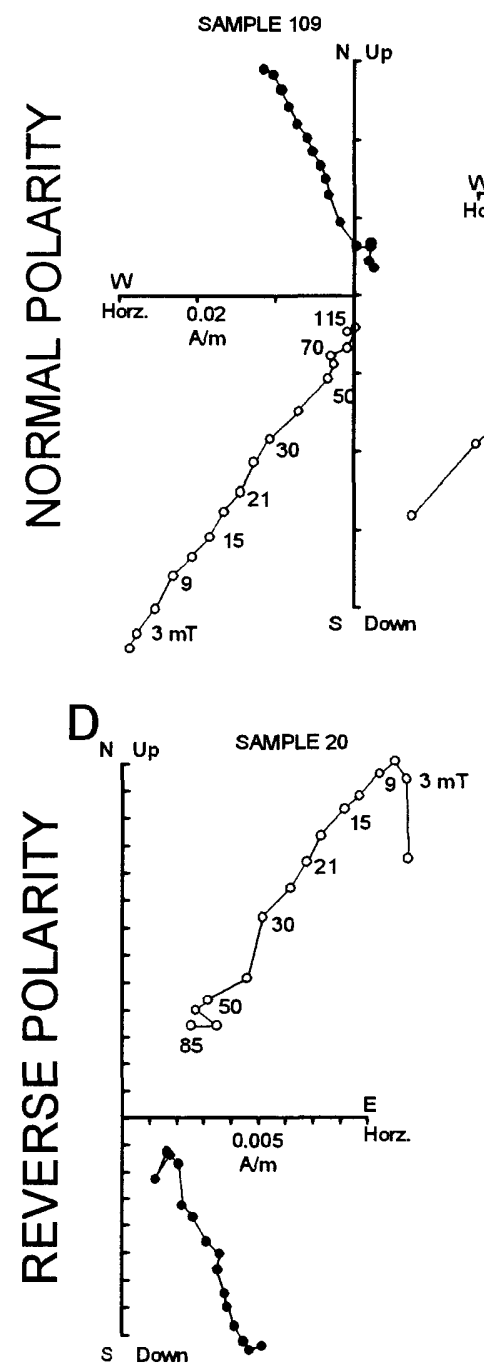

B QUALITY B

SAMPLE 13B

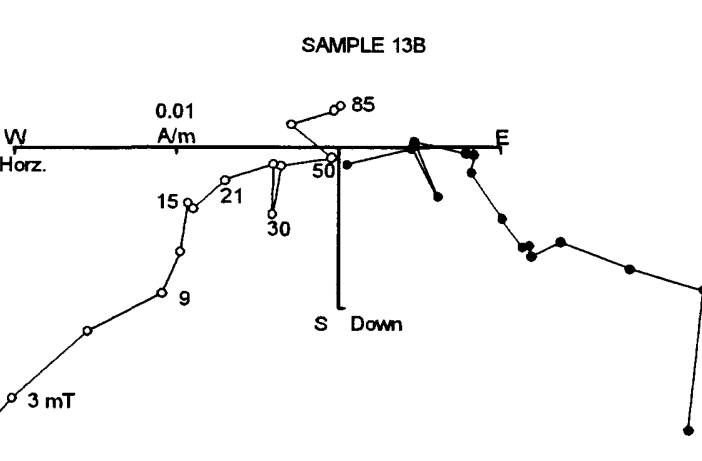

$E$

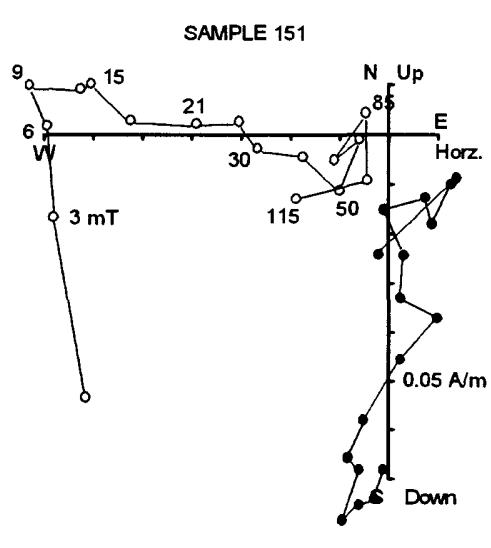

C QUALITY C

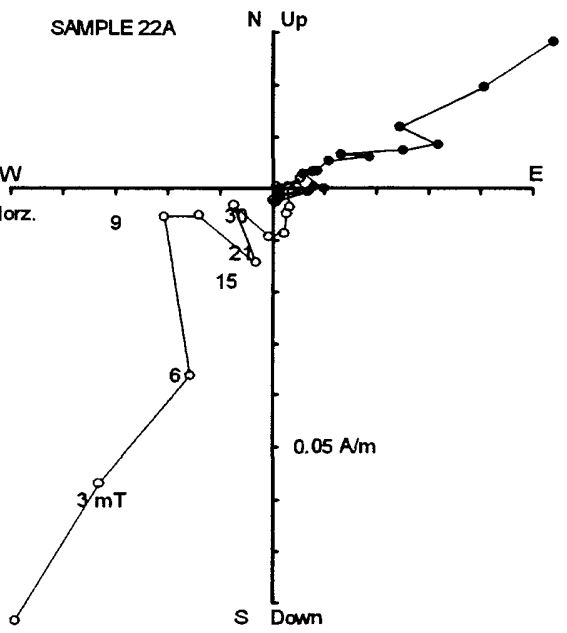

$\mathrm{F}$

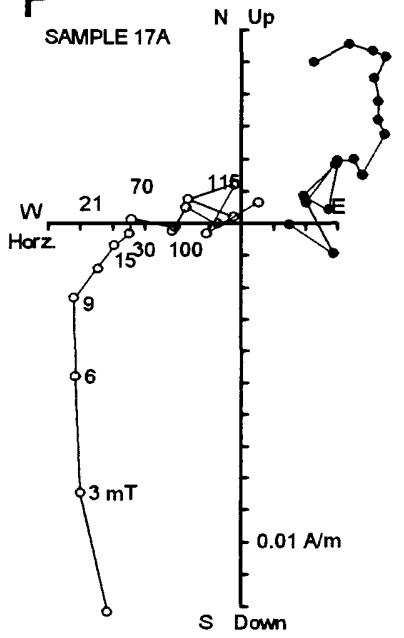

Figure 3. Representative orthogonal demagnetization diagrams depicting quality A, B, and $\mathrm{C}$ alternating field demagnetization behavior for samples assigned normal and reversed polarities. Solid and open circles are projections on horizontal and vertical planes, respectively. 


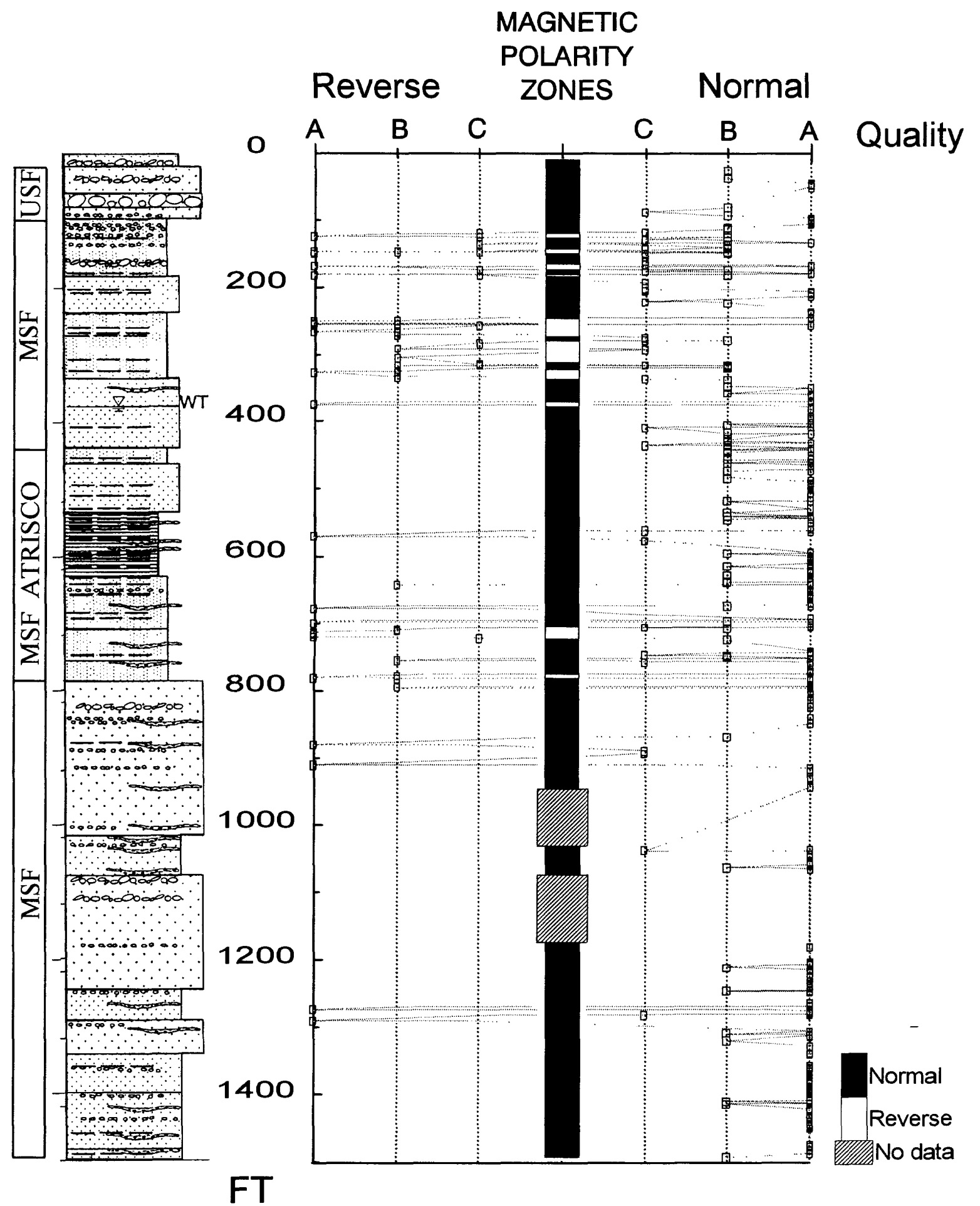

Figure 4. Interpretative magnetic polarity zonation for the 98th St core. Polarity determinations of quality $\mathbf{A}, \mathbf{B}$, and $\mathbf{C}$ for individual samples are shown by squares. 

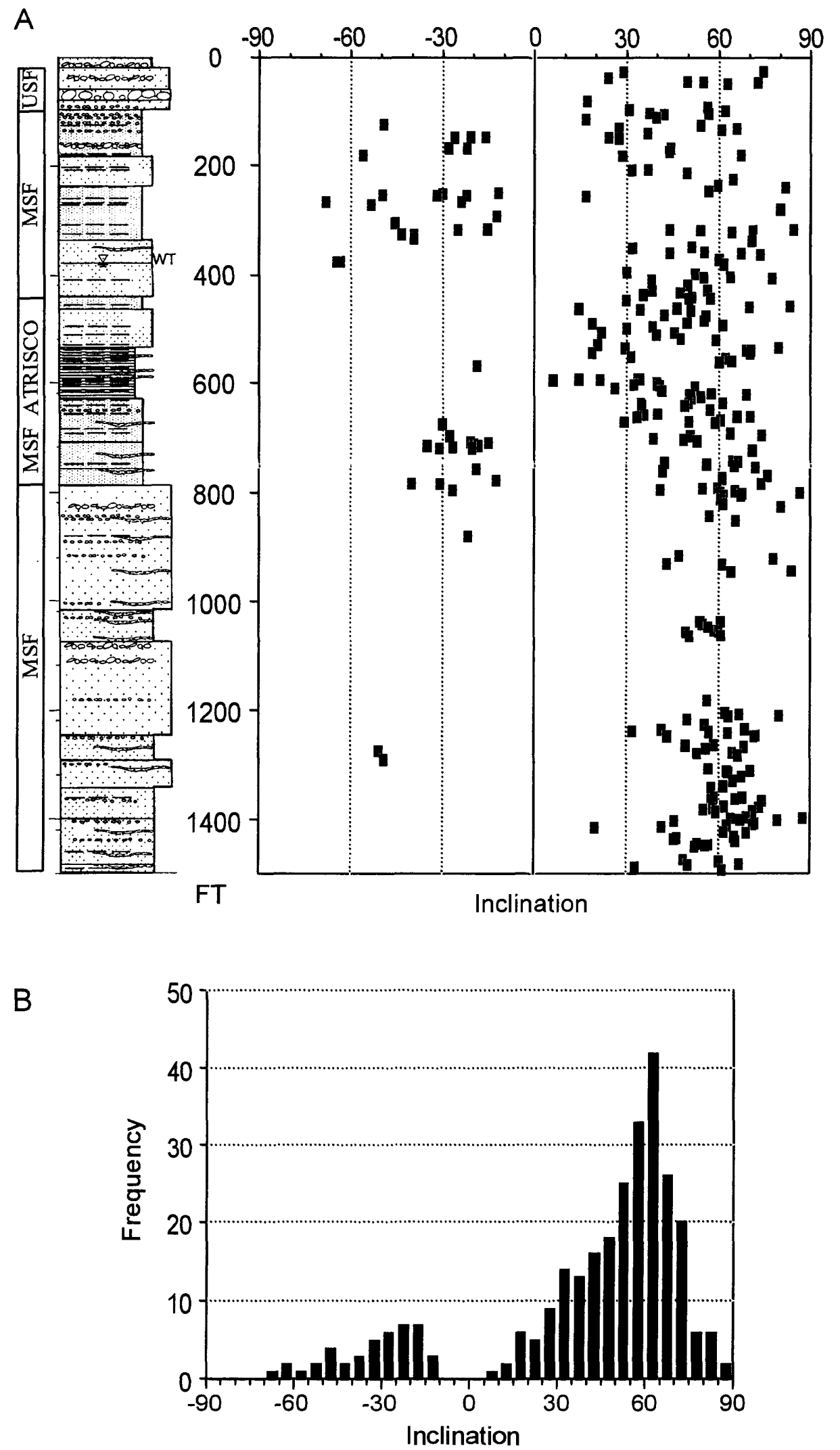

Figure 5. Inclinations of the moderate coercivity magnetization component, derived for samples with A and B quality demagnetization behavior, versus (A) depth, and (B) frequency. 
(e.g., Ade-Hall and Johnson, 1976). For the 98th Street core, however, the moderate inclinations (fig. 5) and coercivities of most magnetization components do not support a secondary IRM origin.

Comparison of the 98th Street core polarity zonation to the geomagnetic polarity time scale for the last $11 \mathrm{Ma}$ (Cande and Kent, 1995 ) does not yield a unique, convincing correlation (fig. 6). As defined by its polarity record, the time span recorded by the core interval should correlate with a one of several possible periods of closely spaced GPTS normal subchrons since middle Miocene. If the correlation by Stone and others (1998) of the middle unit of the Santa $\mathrm{Fe}$ in core to strata at Loma Colorado (bearing Blancan fauna) is accepted, the core polarity zonation may correspond with closely spaced normal polarity subchrons of the Gauss chron at 2.6-3.6 Ma or within the upper Gilbert chron at 4.2-5.2 Ma (fig. 6). Alternatively, the polarity zonation might correlate with older periods of closely spaced normal subchrons such as those at about 6-6.5 Ma, 7-8 Ma, or 9-11 Ma. Any correlation of the core polarity zonation to the GPTS will be complicated if there are hiatuses within the core sequence, as, for instance, are probably present at the base of the upper unit of the Santa Fe (Stone and other, 1988). Whichever correlation is favored, the relative length of the normal polarity zones suggests a rapid accumulation rate for most of the core sediment. For the $427 \mathrm{~m}$ thickness of the middle unit of the Santa Fe in the core, deposition over 1-2 m.y. gives accumulation rates of $43-22 \mathrm{~cm} / \mathrm{ka}$. For comparison, accumulation rates calculated from magnetostratigraphic studies of, for example, the Pho-Pleistocene Camp Rice and Palomas Formations in the southern part of the Rio Grande rift (Mack and others, 1993) are 2-3 $\mathrm{cm} / \mathrm{ka}$ and from the middle Miocene Tesuque Formation of the Española Basin (Barghorn, 1981) are $12-17 \mathrm{~cm} / \mathrm{ka}$, depending on GPTS correlation. If verified by further studies, the high accumulation rate imphed for most of the 98th Street core interval might give evidence for active tectonism and creation of accommodation space within the basin during sedimentation. Plotted at a high $40 \mathrm{~cm} / \mathrm{ka}$ rate, the thin $(<1 \mathrm{~m})$ reversed intervals within the 98th Street core become insignificant (fig. 6), implying that they represent short-lived paleofield events rather than magnetic subchrons shown within the polarity time scale.

\section{Variation of Magnetic Properties with Depth and Grain Size}

Both magnetic susceptibility and natural remanent magnetization vary greatly over the $1500 \mathrm{ft}(457 \mathrm{~m})$ depth of the core sequence (fig. 7, Appendix B, C). Magnetic susceptibility values from the whole core measurements range from a minimum of 8.9E-5 SI to a maximum of 6.7E-2 SI. Magnetic susceptibility values obtained from the cube samples vary from 5.97E-08 to 2.36E-02. Excepting a few highMS values, the whole-core MS measurements vary less over short depth intervals than do the cube sample values from the same interval (fig. 7). We interpret this narrowed range to reflect some averaging of MS variations that are thinner than the $5 \mathrm{~cm}$ length sensed by the whole-core induction coil. Natural remanent magnetization for the cube samples varies from a minimum of $8.37 \mathrm{E}-04$ to a maximum of $2.72 \mathrm{E}-01 \mathrm{~A} / \mathrm{m}$ (fig. 7).

The variation of MS and NRM with stratigraphic depth corresponds, at least partly, to hithologic variations of the sediments (fig. 7). Clay- and silt-rich horizons like those within the Atrisco member generally have lowest values of MS and NRM, whereas fine- to medium-grained sandstones of surrounding intervals typically have higher values. Comparing MS and NRM versus sediment grain size (estimated for samples during core logging) reveals that both MS and NRM are higher for increasing sediment grain size (fig. 8), supporting their lithologic dependence. 


\section{Geomagnetic \\ Polarity \\ Time Scale}

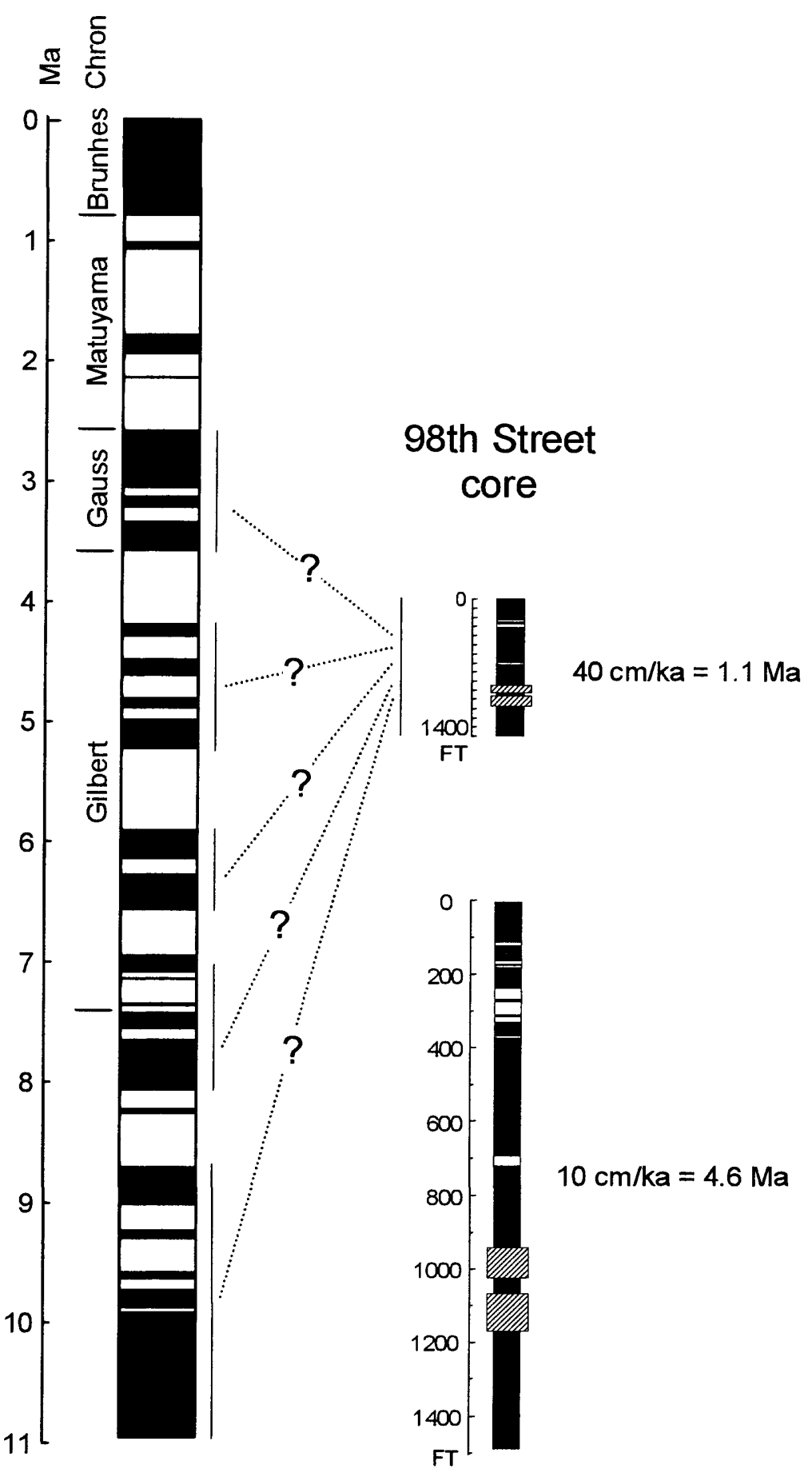

Figure 6. Geomagnetic polarity time scale for the last $11 \mathrm{Ma}$ (Cande and Kent, 1995) compared to the polarity zonation for the $98 \mathrm{th}$ St corehole plotted at scales of $10 \mathrm{~cm} / \mathrm{ka}$ and $40 \mathrm{~cm} / \mathrm{ka}$. 


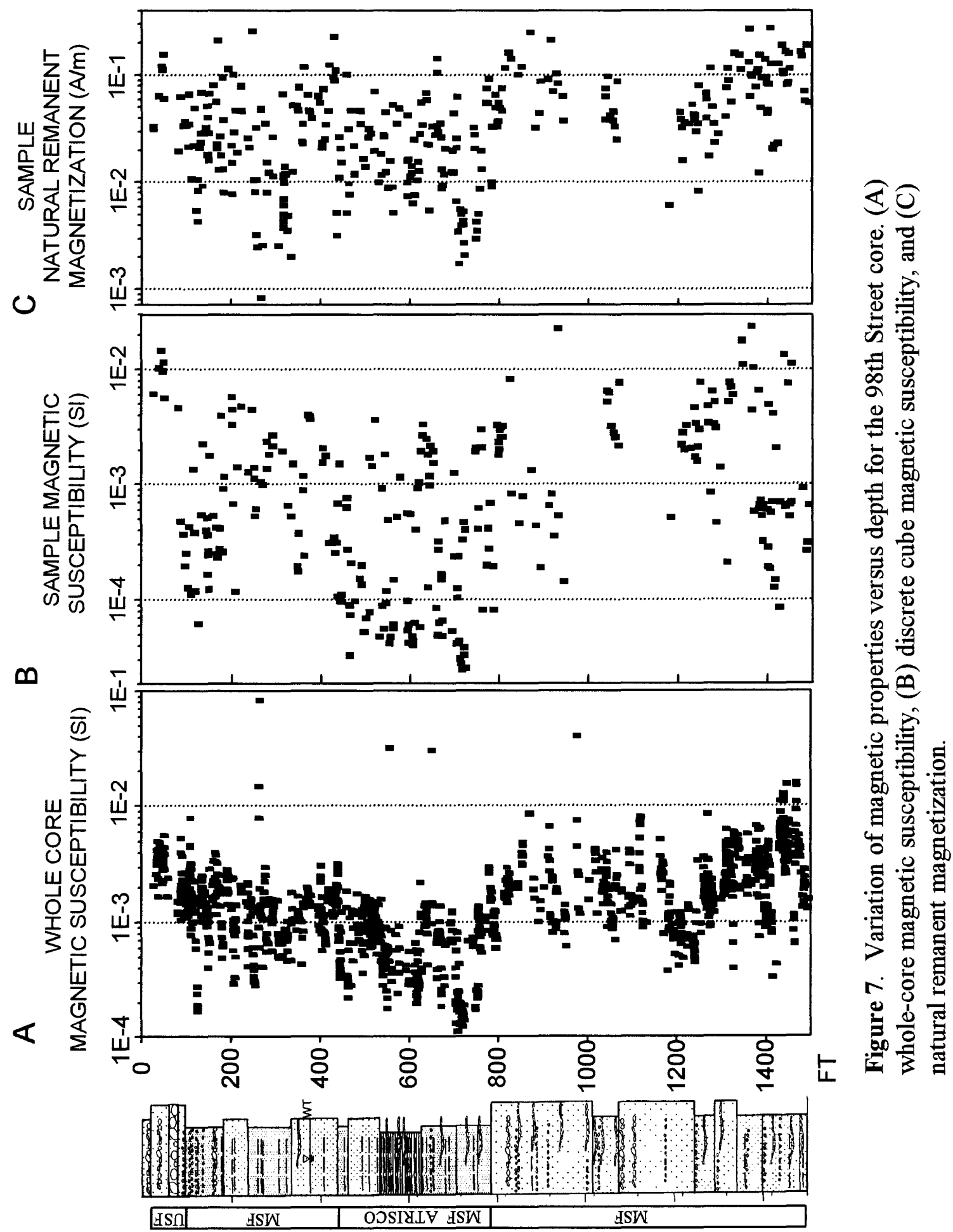



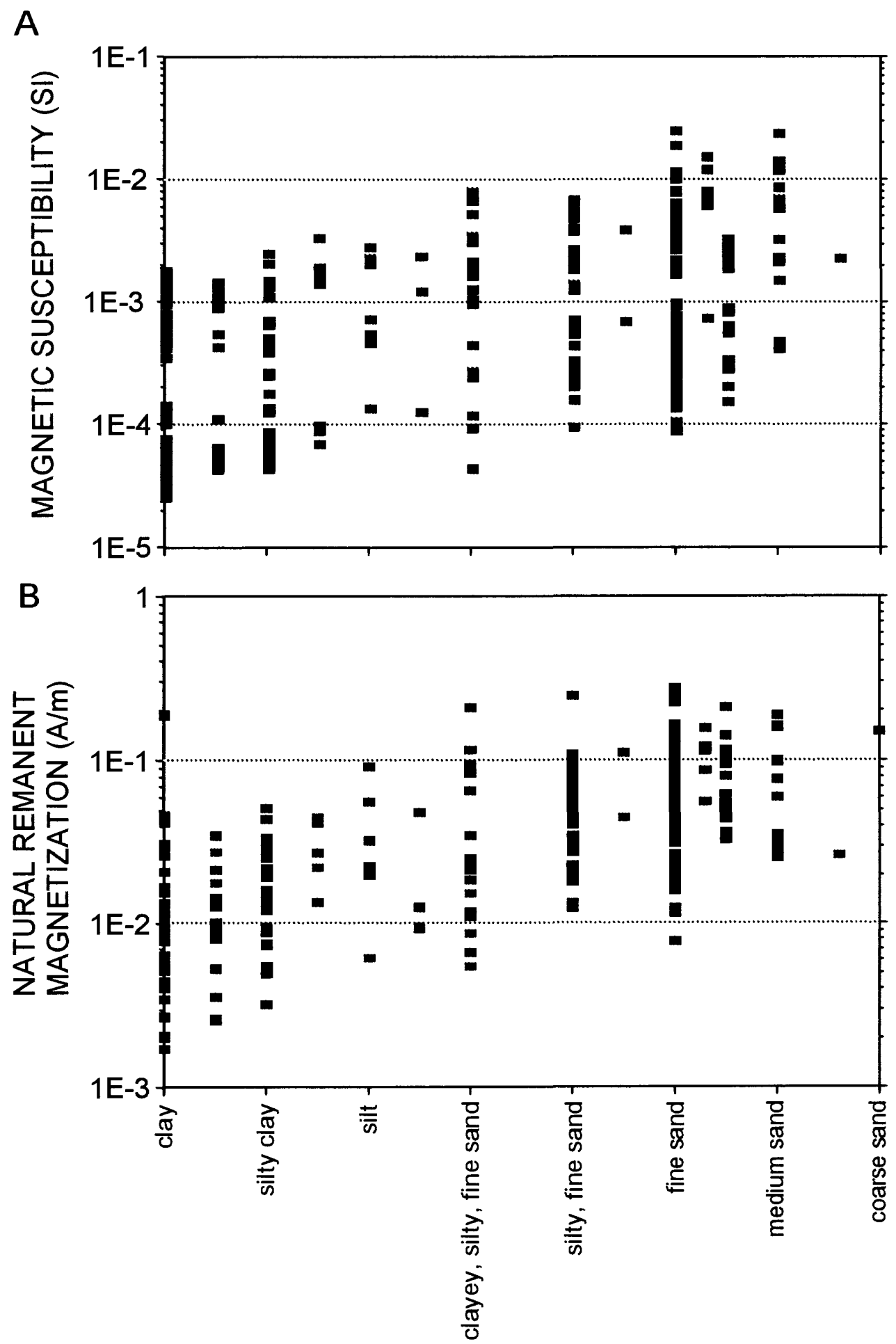

Figure 8. Variation of sediment grain size versus (A) magnetic susceptibility and (B) natural remanent magnetization for samples from the 98th Street core. 
Although magnetic properties and sediment grain size correlate broadly, both NRM and MS have considerable ranges within each size category, suggesting that factors beyond sediment size also influence magnetic property variations. Comparing MS and NRM reveals two distinct populations of magnetized sediments (fig. 9A). Whereas the NRM range from each population is similar, NRM in each population correlates against MS values having different ranges, resulting in a higher NRM/MS ratio for samples in group 1 than in group 2. As endmember models, the two populations (fig. 9A) could be produced either by contrasts in magnetic mineral properties or by a varying efficiency of remanence acquisition due to depositional environment. As an example of the first scenario, the high NRM/MS ratio for group 1 might be produced if remanent magnetization is carried by fine pseudosingle-domain magnetite grains that efficiently acquired a detrital remanent magnetization (DRM) due to their small size and high magnetic moment (Dunlap and Ozdemir, 1997, chapter 15). Group 2 samples could then be produced by addition of a coarse-grained assemblage that increased MS but not NRM because of inefficient DRM acquisition caused by poor alignment of large, low-moment, multidomain magnetite grains. If verified, such contrasts of magnetic grain size might highlight materials derived from different source terranes, such as volcanic versus crystalline. In a second scenario (probably less likely), creation of groups 1 and 2 via efficient versus inefficient DRM acquisition might simply reflect differing ahignment of similar magnetite grains in contrasting fluid flows of different depositional environments, such as quiet-water overbank versus channel-fill settings. These ad hoc explanations are plainly speculative based on current data. With further rock magnetic and petrographic data, however, the distinct magnetic properties of group 1 and 2 sediments may give insight into contrasting source areas or depositional environments recorded within the core.

\section{Santa Fe Group Sediments as Magnetic Anomaly Sources}

Recent high-resolution aeromagnetic surveys within the middle Rio Grande Rift have detected numerous low amplitude (2-10 nT), linear anomahes that have been attributed to fault offsets within Santa Fe sediments (Grauch, 1998). Magnetic anomahes arise from contrasts in total magnetization of the source materials, where total magnetization is the vector sum of remanent and induced magnetization component Total magnetizations can be approximated from MS and NRM values for the 98th Street core samples to assess potential contrasts of total magnetization within Santa Fe Group sediments.

Whereas remanent magnetization is directly measured in the samples, induced magnetization is calculated from the product of MS and the Earth's field strength (about $50 \mu \mathrm{T}$ ). The quotient of NRM and induced magnetization magnitudes, the Koenigsberger ratio $(\mathrm{Q})$, is a common measure of the relative importance of these two components in the total magnetization. The different NRM/MS ratios for groups 1 and 2 (fig. 9) result in two distinct $Q$ populations in the core samples (fig. 9B, 10, 11). Samples from low-MS group 1 have high $Q$ values with a mode of 5 , indicating a dominance of remanent magnetization. Samples from high-MS group 2 have low $Q$ values with a mode of 0.4 , indicating a dominance of induced magnetization.

Lacking azimuthal orientation, declination of NRM for the core samples is unknown and thus it is impossible to calculate a true vector sum of the remanent and induced magnetization components. Normal-polarity late Miocene through Quaternary paleofields, however, typically had directions that were nearly parallel (within $30^{\circ}$ ) to the present-day field. Thus as an approximation, the remanent 

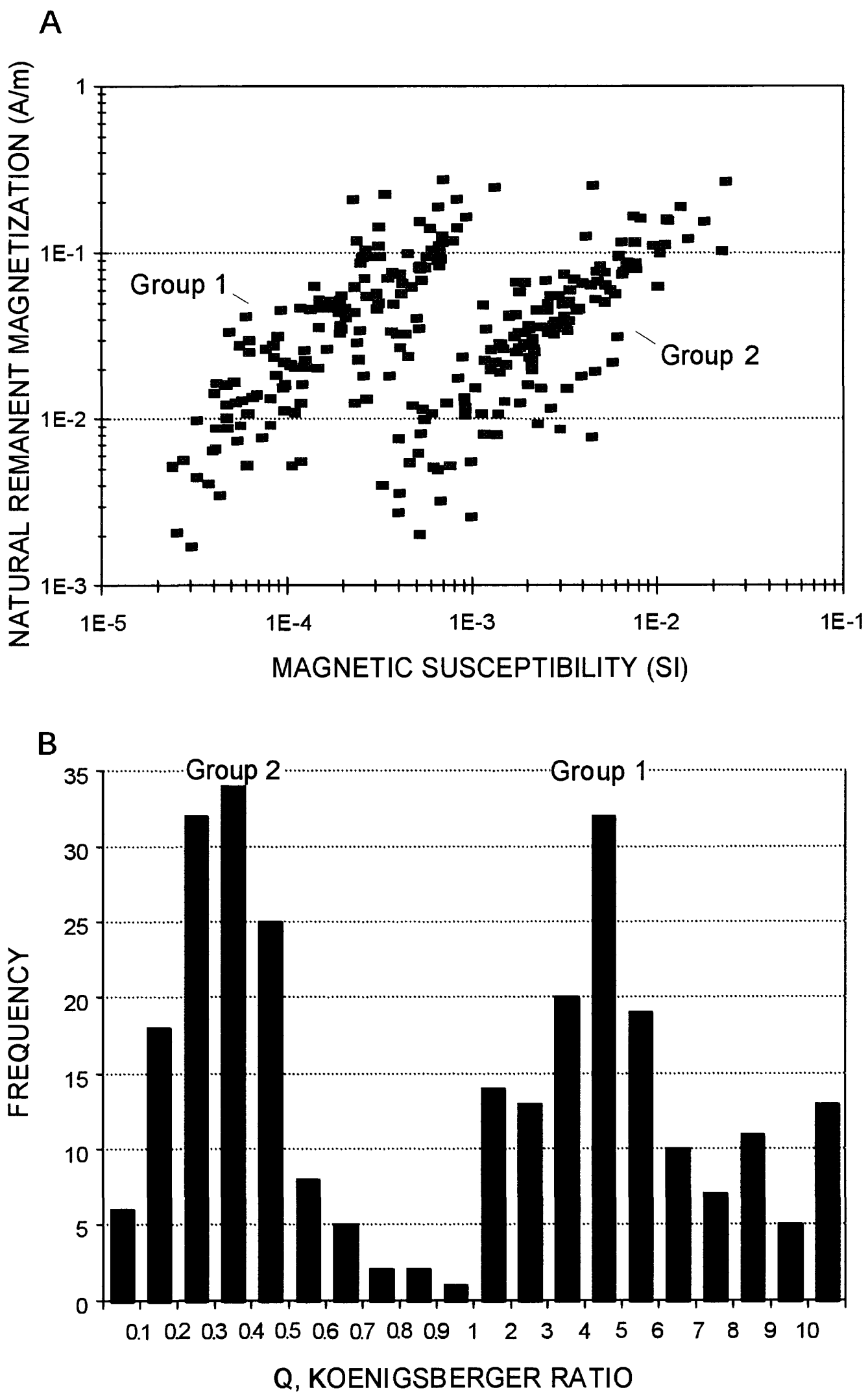

Figure 9. (A) Magnetic susceptibility versus NRM and (B) histogram of Koenigsberger ratio, $Q$, for samples from the 98th Street core. Note dual bin sizes for $Q$ values. 

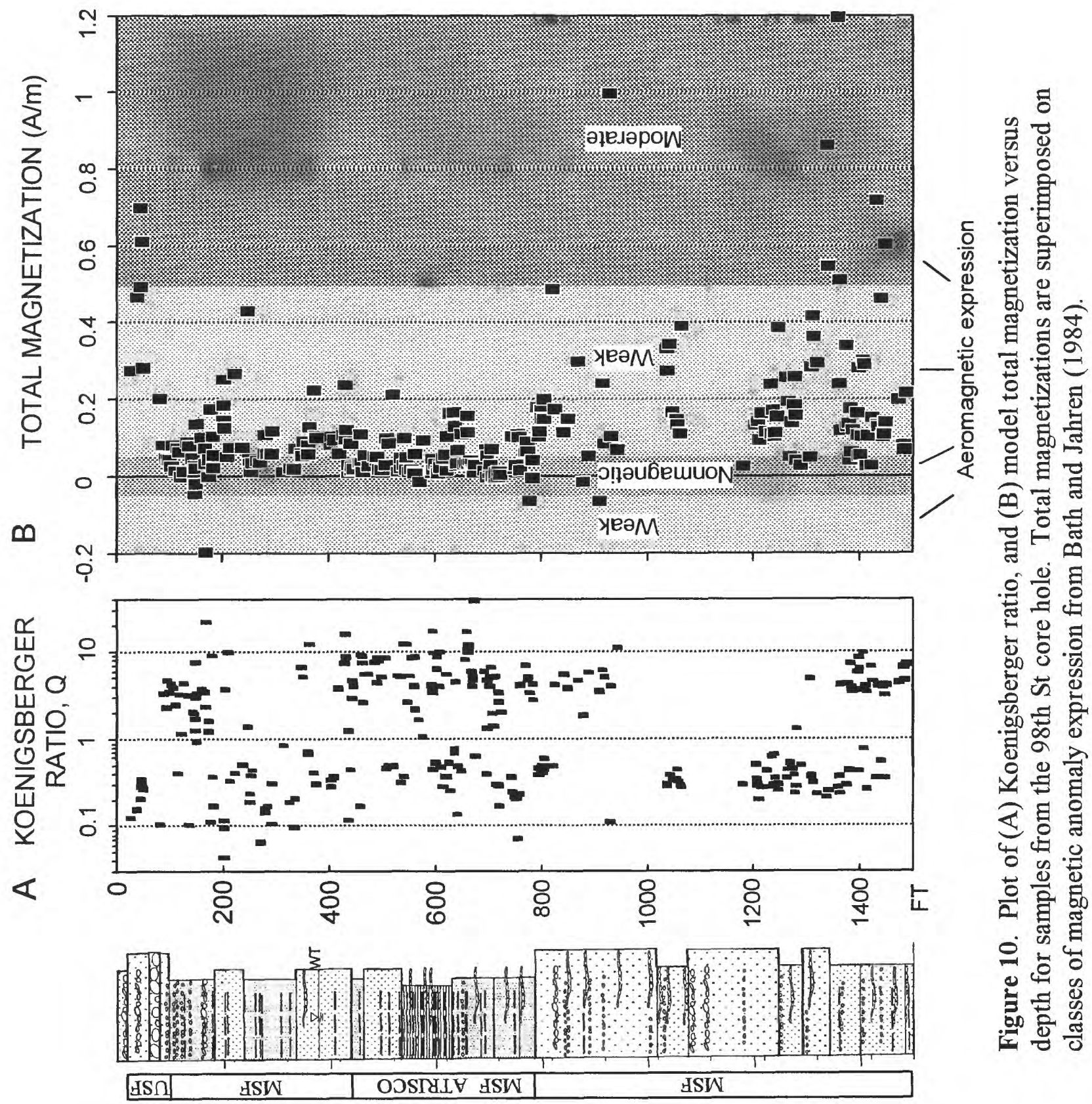

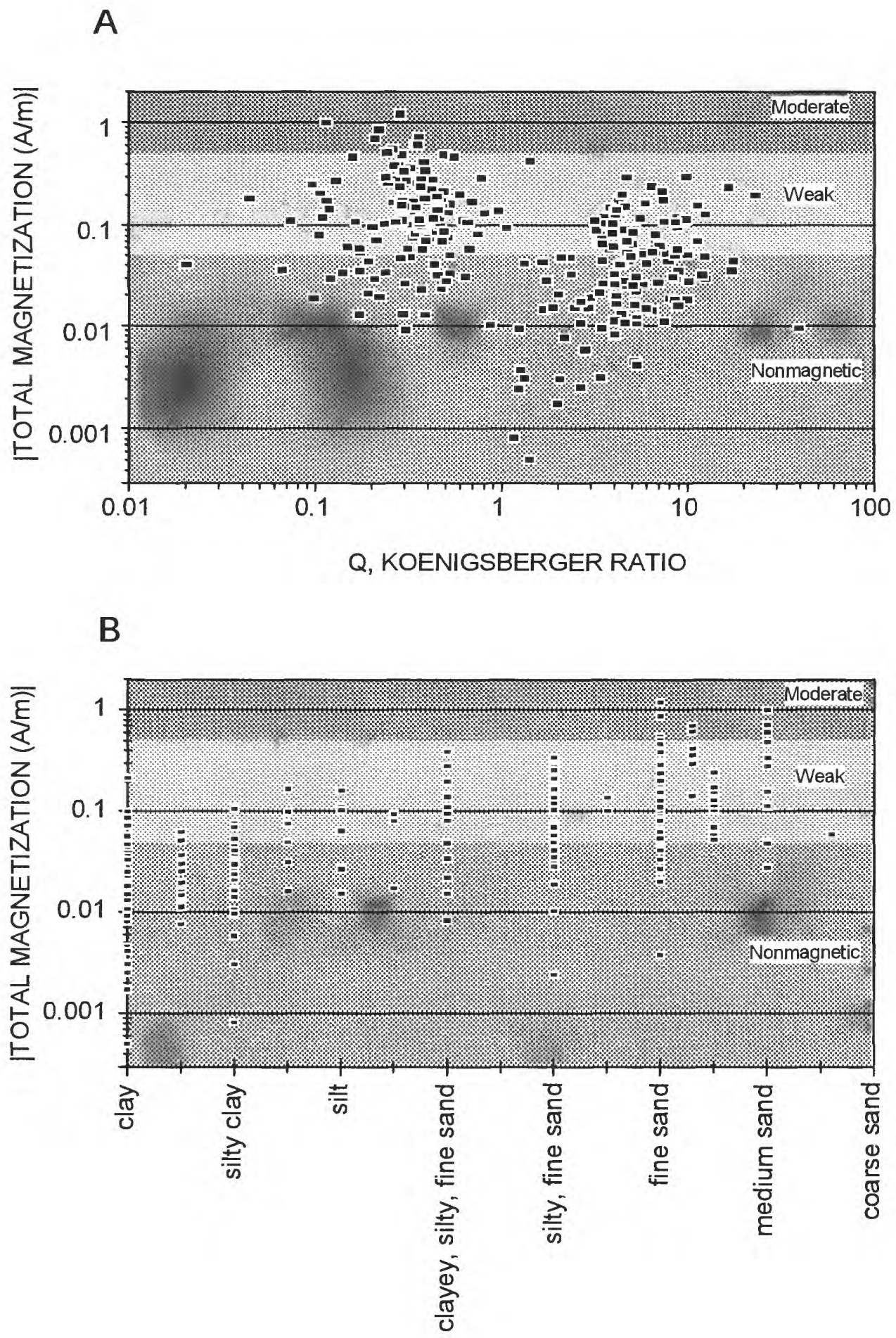

Figure 11. Absolute value of total magnetization versus (A) Koenigsberger ratio, and (B) sediment grain size superimposed on classes of magnetic anomaly expression from Bath and Jahren (1984). 
magnetization for normal polarity samples can be added as a scalar value to the induced magnetization component that is parallel to the present field. Conversely, remanent magnetization for reversed polarity samples can be subtracted from the induced magnetization component. Total magnetizations for core samples calculated in this manner (Appendix C) range from $-1.2 \mathrm{E}-1$ to $1.2 \mathrm{~A} / \mathrm{m}$ (fig. 10). Bath and Jahren (1984) proposed the following classification for expression of magnetic anomalies based on total magnetization: (1) strong, > $1.5 \mathrm{~A} / \mathrm{m},(2)$ moderate, $1.5-0.5 \mathrm{~A} / \mathrm{m}$, (3) weak, 0.5-0.05 A/m, and (4) nonmagnetic, < $0.05 \mathrm{~A} / \mathrm{m}$. Compared to these classes, maximum contrasts in the total magnetizations for the core sediments will generate weak to moderate anomalies (fig. 10), qualitatively compatible with the 2-10 $\mathrm{nT}$ anomalies detected over intrabasin faults in the Albuquerque basin (Grauch, 1998).

Total magnetizations calculated in the manner above probably overestimate contrasts in magnetization if they are in error. The error potential is greatest for high- $Q$, remanencedominant samples. For normal polarity samples, nonparallellism of induced and remanent components will reduce the resultant magnitude of the total magnetization vector from the estimate. High-Q, reversed polarity samples have greatest potential for error because the presence of an overprinting normal polarity viscous component (e.g., fig. 3E) can strongly bias the NRM direction away from the assumed antipode to the field direction. In this case, subtracting the remanent magnetization component will result in a calculated total magnetization that is less (more negative) than the true resultant vector. Despite these potential errors, we think figure 10B illustrates a reasonable range of total magnetizations within the core. Samples with highest total magnetizations have low $Q$ values (fig. 11A) and thus are least affected by potential errors. Negative total magnetizations calculated for most reverse polarity samples are small (fig. 10) and effectively can be considered nonmagnetic.

Within the 98th Street core, stratigraphic intervals that are dominantly sandstone have higher total magnetizations than clay- and siltrich intervals such as the Atrisco member (fig. 10). Predictably, total magnetization has positive correspondence with sediment grain size (fig. 11B) similar to that for NRM and MS (fig. 8). These data suggest that coarse-grained rocks that are the principal aquifers in the basin should have maximum total magnetizations and thus greatest potential for generation of aeromagnetic anomalies where juxtaposed with fine sediments along faults.

\section{References}

Ade-Hall, J.M., and Johnson, H.P., 1976, Paleomagnetism of basalts, Leg 34: Initial Reports of the Deep Sea Drilling Project, v. 34, p. 513-532.

Barghorn, Steven, 1981, Magnetic stratigraphy of the Miocene type Tesuque Formation, Santa Fe Group, in the Española Valley, New Mexico: Geological Society of America Bulletin, v. 92, p. 1027-1041.

Bath, G.D., and Jahren, C.E., 1984, Interpretation of magnetic anomalies at a potential repository site located in the Yucca Mountain areas, Nevada Test Sites: U.S. Geological Survey Open-File Report 84-120, $40 \mathrm{p}$.

Cande, S.C, and Kent, D.V., 1995, Revised calibration for the geomagnetic polarity timescale for the Late Cretaceous and Cenozoic: Journal of Geophysical Research, v. 100, p. 6093-6095.

Connell, S.D., Allen, B.D., and Hawley, J.W., 1998, Subsurface stratigraphy, structure, and hydrogeologic framework of the Santa Fe Group from borehole geophysical logs, northern Albuquerque basin, New Mexico, in Slate, J.L., ed., U.S. Geological Survey Middle Rio 
Grande Basin Study - Second Annual

Workshop Proceedings: U.S. Geological

Survey Open-File Report 98-337, p. 3334.

Dunlop, D.J., and Ozdemir, Ozden, 1997, Rock magnetism: Fundamentals and Frontiers: Cambridge, Cambridge University Press, $573 \mathrm{p}$.

Dunlop, D.J., and Stirling, J.M., 1977, "Hard" viscous remanent magnetization (VRM) in fine-grained hematite: Geophysical Research Letters, v. 4, p. 163-166.

Grauch, V.J.S., 1998, Expression of faults in high-resolution aeromagnetic data near Albuquerque, New Mexico - preliminary results, in Slate, J.L., ed., U.S.

Geological Survey Middle Rio Grande Basin Study - Second Annual Workshop Proceedings: U.S. Geological Survey Open-File Report 98-337, p. 34-35.

Kirschvink, J.L, 1980, The least-square line and plane and analysis of paleomagnetic data: Geophysical Journal of the Royal Astronomical Society, v. 62, p. 699-718. Kodama, K.P., and Sun, W.W., 1992, Magnetic anisotropy as a correction for compaction-caused inclination shallowing: Geophysical Journal International, v. 111, p. 465-469.

Mack, G.H., Salyards, S.L., and James, W.C., 1993, Magnetostratigraphy of the PlioPleistocene Camp Rice and Palomas Formations in the Rio Grande rift of southern New Mexico: American Journal of Science, v. 293, p. 49-77.

Stone, B.D., Allen, B.D., Mikolas, Marlo, Hawley, J.W., Haneberg, W.C., Johnson, P.S., Allred, Barry, and Thorn, C.R., 1998, Preliminary lithostratigraphy, interpreted geophysical logs, and hydrogeologic characteristics of the 98th Street core hole, Albuquerque, New Mexico: U.S. Geological Survey OpenFile Report 98-210, 82 p. 


\section{Appendix A, Paleomagnetic data for 98th Street core samples}

Note: Depth, ft; Samp, sample number; D, declination in degrees clockwise from north; I, inclination in degrees, positive downward; $J$, magnetization $(\mathrm{A} / \mathrm{m}) ; \mathrm{AF}$, alternating-field demagnetization in peak induction (mT)

\begin{tabular}{|c|c|c|c|c|c|}
\hline Depth & Samp & D & I & $\mathbf{J}$ & $\overline{\mathbf{A F}}$ \\
\hline 25.7 & $13 \mathrm{~B}$ & 228 & 37.3 & $3.30 \mathrm{E}-02$ & 0 \\
\hline 25.7 & $13 \mathrm{~B}$ & 246.4 & 35 & $2.72 \mathrm{E}-02$ & 3 \\
\hline 25.7 & $13 \mathrm{~B}$ & 244.5 & 32.9 & $2.10 \mathrm{E}-02$ & 6 \\
\hline 25.7 & $13 \mathrm{~B}$ & 242.8 & 34.9 & $1.59 \mathrm{E}-02$ & 9 \\
\hline 25.7 & $13 \mathrm{~B}$ & 235.2 & 28.6 & $1.36 \mathrm{E}-02$ & 12 \\
\hline 25.7 & $13 \mathrm{~B}$ & 237.4 & 16.9 & $1.20 \mathrm{E}-02$ & 15 \\
\hline 25.7 & $13 \mathrm{~B}$ & 235.8 & 19 & $1.18 \mathrm{E}-02$ & 18 \\
\hline 25.7 & $13 \mathrm{~B}$ & 240.5 & 12.8 & $9.39 \mathrm{E}-03$ & 21 \\
\hline 25.7 & $13 \mathrm{~B}$ & 254.9 & 9.7 & $6.34 \mathrm{E}-03$ & 25 \\
\hline 25.7 & $13 \mathrm{~B}$ & 265 & 33.9 & $7.54 \mathrm{E}-03$ & 30 \\
\hline 25.7 & $13 \mathrm{~B}$ & 265.7 & 11.3 & $5.84 \mathrm{E}-03$ & 40 \\
\hline 25.7 & $13 \mathrm{~B}$ & 276.2 & 15.3 & $2.70 \mathrm{E}-03$ & 50 \\
\hline 25.7 & $13 \mathrm{~B}$ & 231.4 & -15.4 & $5.27 \mathrm{E}-03$ & 60 \\
\hline 25.7 & $13 \mathrm{~B}$ & 267 & 43.5 & $3.27 \mathrm{E}-03$ & 70 \\
\hline 25.7 & $13 \mathrm{~B}$ & 124.9 & -52.3 & $3.22 \mathrm{E}-03$ & 85 \\
\hline 26.8 & $14 \mathrm{~B}$ & 143.7 & 66.5 & $3.14 \mathrm{E}-02$ & 0 \\
\hline 26.8 & $14 \mathrm{~B}$ & 151.2 & 72.6 & $2.76 \mathrm{E}-02$ & 3 \\
\hline 26.8 & $14 \mathrm{~B}$ & 140.3 & 73.9 & $2.36 \mathrm{E}-02$ & 6 \\
\hline 26.8 & $14 \mathrm{~B}$ & 141.9 & 71.2 & $2.03 \mathrm{E}-02$ & 9 \\
\hline 26.8 & $14 \mathrm{~B}$ & 134.5 & 69.7 & $1.77 \mathrm{E}-02$ & 12 \\
\hline 26.8 & $14 \mathrm{~B}$ & 122.8 & 70.7 & $1.61 \mathrm{E}-02$ & 15 \\
\hline 26.8 & $14 \mathrm{~B}$ & 126.3 & 66.7 & $1.43 \mathrm{E}-02$ & 18 \\
\hline 26.8 & $14 \mathrm{~B}$ & 108.9 & 67.9 & $1.38 \mathrm{E}-02$ & 21 \\
\hline 26.8 & $14 \mathrm{~B}$ & 99.7 & 61.5 & $1.23 \mathrm{E}-02$ & 25 \\
\hline 26.8 & $14 \mathrm{~B}$ & 86.9 & 66.1 & $1.10 \mathrm{E}-02$ & 30 \\
\hline 26.8 & $14 \mathrm{~B}$ & 91.4 & 48.1 & $9.44 \mathrm{E}-03$ & 40 \\
\hline 26.8 & $14 \mathrm{~B}$ & 90.7 & 64.8 & $5.88 \mathrm{E}-03$ & 50 \\
\hline 26.8 & $14 \mathrm{~B}$ & 79.4 & 30.8 & $6.40 \mathrm{E}-03$ & 60 \\
\hline 26.8 & $14 \mathrm{~B}$ & 80.4 & 11.7 & $5.91 \mathrm{E}-03$ & 70 \\
\hline 26.8 & $14 \mathrm{~B}$ & 77.4 & 31.1 & $3.07 \mathrm{E}-03$ & 85 \\
\hline 26.8 & $14 \mathrm{~B}$ & 77 & 8.3 & $5.22 \mathrm{E}-03$ & 100 \\
\hline 26.8 & $14 \mathrm{~B}$ & 46.3 & 33.2 & $3.85 \mathrm{E}-03$ & 115 \\
\hline 38.1 & $15 \mathrm{~B}$ & 154.7 & 44.2 & $6.34 \mathrm{E}-02$ & 0 \\
\hline 38.1 & $15 \mathrm{~B}$ & 155.2 & 38.4 & $5.51 \mathrm{E}-02$ & 3 \\
\hline 38.1 & $15 \mathrm{~B}$ & 160.9 & 32.1 & $4.82 \mathrm{E}-02$ & 6 \\
\hline 38.1 & $15 B$ & 163.2 & 27.2 & $4.48 \mathrm{E}-02$ & 9 \\
\hline 38.1 & $15 \mathrm{~B}$ & 164.4 & 23.5 & $4.04 \mathrm{E}-02$ & 12 \\
\hline 38.1 & $15 \mathrm{~B}$ & 163.7 & 19.8 & $3.87 \mathrm{E}-02$ & 15 \\
\hline 38.1 & $15 \mathrm{~B}$ & 166.3 & 19.1 & $3.45 \mathrm{E}-02$ & 18 \\
\hline 38.1 & $15 \mathrm{~B}$ & 167.3 & 23.9 & $3.23 \mathrm{E}-02$ & 21 \\
\hline 38.1 & $15 \mathrm{~B}$ & 169.9 & 21.3 & $2.81 \mathrm{E}-02$ & 25 \\
\hline & $15 B$ & 172.7 & 20.7 & $3 \mathrm{E}-02$ & \\
\hline
\end{tabular}

\begin{tabular}{|c|c|c|c|c|c|}
\hline 38.1 & $15 \mathrm{~B}$ & 183.2 & 21.2 & $1.51 \mathrm{E}-02$ & 40 \\
\hline 38.1 & $15 \mathrm{~B}$ & 184.6 & 3.9 & $9.50 \mathrm{E}-03$ & 50 \\
\hline 38.1 & $15 B$ & 190.5 & 19.5 & $1.06 \mathrm{E}-02$ & 60 \\
\hline 38.1 & $15 \mathrm{~B}$ & 209.1 & -15.9 & $5.47 \mathrm{E}-03$ & 70 \\
\hline 38.1 & $15 B$ & 162.6 & 44.9 & $8.15 \mathrm{E}-03$ & 85 \\
\hline 38.1 & $15 \mathrm{~B}$ & 264.2 & -35.9 & $3.70 \mathrm{E}-03$ & 100 \\
\hline 38.1 & $15 \mathrm{~B}$ & 263.2 & 3.5 & $8.11 \mathrm{E}-03$ & 115 \\
\hline 44.8 & $16 \mathrm{~B}$ & 274.8 & 57.9 & $1.21 \mathrm{E}-01$ & 0 \\
\hline 44.8 & $16 \mathrm{~B}$ & 275 & 57.6 & $1.02 \mathrm{E}-01$ & 3 \\
\hline 44.8 & $16 \mathrm{~B}$ & 274.4 & 54.9 & $8.57 \mathrm{E}-02$ & \\
\hline 44.8 & $16 \mathrm{~B}$ & 273.5 & 52.2 & $7.39 \mathrm{E}-02$ & 0 \\
\hline 44.8 & $16 \mathrm{~B}$ & 272.8 & 50.2 & $6.95 \mathrm{E}-02$ & 12 \\
\hline 44.8 & $16 \mathrm{~B}$ & 272.7 & 52.7 & $6.04 \mathrm{E}-02$ & 15 \\
\hline 44.8 & $16 \mathrm{~B}$ & 270.4 & 46.5 & $5.60 \mathrm{E}-02$ & 18 \\
\hline 44.8 & $16 \mathrm{~B}$ & 272.6 & 49.6 & $5.04 \mathrm{E}-02$ & 21 \\
\hline 44.8 & $16 \mathrm{~B}$ & 275.5 & 47.1 & $4.35 \mathrm{E}-02$ & 25 \\
\hline 44.8 & $16 \mathrm{~B}$ & 268.1 & 47.6 & $3.41 \mathrm{E}-02$ & 30 \\
\hline 44.8 & $16 \mathrm{~B}$ & 288.3 & 44.3 & $2.25 \mathrm{E}-02$ & 40 \\
\hline 44.8 & $16 \mathrm{~B}$ & 282.6 & 31 & $1.31 \mathrm{E}-02$ & 50 \\
\hline 44.8 & $16 \mathrm{~B}$ & 278.8 & 51.5 & $1.37 \mathrm{E}-02$ & 60 \\
\hline 44.8 & $16 \mathrm{~B}$ & 269.9 & 70.3 & $8.98 \mathrm{E}-03$ & 70 \\
\hline 44.8 & $16 \mathrm{~B}$ & 314.6 & 25.7 & $9.30 \mathrm{E}-03$ & 85 \\
\hline 44.8 & $16 \mathrm{~B}$ & 283.1 & 17.7 & $7.39 \mathrm{E}-03$ & 100 \\
\hline 44.8 & $16 \mathrm{~B}$ & 274.6 & 47.2 & $5.62 \mathrm{E}-03$ & 115 \\
\hline 46.5 & $17 \mathrm{~B}$ & 339.5 & 59.1 & $1.11 \mathrm{E}-01$ & 0 \\
\hline 46.5 & $17 \mathrm{~B}$ & 339.2 & 59.2 & $8.88 \mathrm{E}-02$ & 3 \\
\hline 46.5 & $17 \mathrm{~B}$ & 338.3 & 57 & $6.98 \mathrm{E}-02$ & 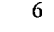 \\
\hline 46.5 & 17B & 335.8 & 56.3 & $5.77 \mathrm{E}-02$ & 9 \\
\hline 46.5 & $17 \mathrm{~B}$ & 335.9 & 54.9 & $5.12 \mathrm{E}-02$ & 12 \\
\hline 46.5 & $17 \mathrm{~B}$ & 338.4 & 56.4 & $4.52 \mathrm{E}-02$ & 15 \\
\hline 46.5 & $17 \mathrm{~B}$ & 338.6 & 56.9 & $4.05 \mathrm{E}-02$ & 18 \\
\hline 46.5 & $17 \mathrm{~B}$ & 339.4 & 55.7 & $3.60 \mathrm{E}-02$ & 21 \\
\hline 46.5 & $17 \mathrm{~B}$ & 350.8 & 61.6 & $3.16 \mathrm{E}-02$ & 25 \\
\hline 46.5 & $17 \mathrm{~B}$ & 336.8 & 58.9 & $2.86 \mathrm{E}-02$ & 30 \\
\hline 46.5 & $17 \mathrm{~B}$ & 341.7 & 55.3 & $1.87 \mathrm{E}-02$ & 40 \\
\hline 46.5 & $17 \mathrm{~B}$ & 3.6 & 63.3 & $1.67 \mathrm{E}-02$ & 50 \\
\hline 46.5 & $17 \mathrm{~B}$ & 4 & 53 & $1.32 \mathrm{E}-02$ & 60 \\
\hline 46.5 & $17 \mathrm{~B}$ & 3.6 & 65 & $8.10 \mathrm{E}-03$ & 70 \\
\hline 46.5 & $17 \mathrm{~B}$ & 3.7 & 43.1 & $6.79 \mathrm{E}-03$ & 85 \\
\hline 46.5 & $17 \mathrm{~B}$ & 60 & 56.9 & $7.52 \mathrm{E}-03$ & 100 \\
\hline 46.5 & $17 \mathrm{~B}$ & 48.3 & 33.4 & 7.57E-03 & 115 \\
\hline 47.8 & $18 \mathrm{~B}$ & 36.2 & 73.4 & $1.56 \mathrm{E}-01$ & 0 \\
\hline 47.8 & $18 \mathrm{~B}$ & 35.1 & 68.8 & $1.19 \mathrm{E}-01$ & 3 \\
\hline
\end{tabular}

\begin{tabular}{|c|c|c|c|c|c|}
\hline & B & 7.4 & 67.6 & $8.86 \mathrm{E}$ & \\
\hline & 18B & 35 & 65.2 & $6.49 \mathrm{E}-02$ & \\
\hline 8 & 8B & 29.8 & 65.2 & $5.04 \mathrm{E}-02$ & \\
\hline 8 & 8B & 25 & 65.9 & $4.11 \mathrm{E}-02$ & \\
\hline .8 & $8 \mathrm{~B}$ & 8.4 & 69.7 & 3.54E-02 & \\
\hline 8 & $8 \mathrm{~B}$ & 7.7 & 64.9 & $2.73 \mathrm{E}-02$ & \\
\hline 8 & 8B & 18.3 & 56.8 & $2.31 \mathrm{E}-02$ & 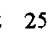 \\
\hline 8 & 8B & 52.9 & 58.3 & $1.87 \mathrm{E}-02$ & 3 \\
\hline .8 & $8 \mathrm{~B}$ & 6.8 & 57.2 & $1.31 \mathrm{E}-02$ & \\
\hline 8 & $8 \mathrm{~B}$ & 338.6 & 56.4 & $1.09 \mathrm{E}-02$ & \\
\hline & 8B & 1.1 & 80.4 & $3.07 \mathrm{E}-\mathrm{C}$ & \\
\hline & $8 \mathrm{~B}$ & 1.4 & 35.7 & & \\
\hline 8.8 & $8 \mathrm{~B}$ & 14.4 & 11.4 & & \\
\hline 7.8 & $8 \mathrm{~B}$ & 67.2 & -11.5 & 6.64 & 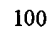 \\
\hline 47.8 & $18 \mathrm{~B}$ & 234 & -12.8 & 3.071 & 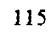 \\
\hline 49.8 & 19B & 18.4 & 66.4 & $6.02 \mathrm{~F}$ & \\
\hline 49.8 & 19B & 17.6 & 69.4 & $5.01 \mathrm{E}-02$ & \\
\hline 19.8 & $19 \mathrm{~B}$ & 11.3 & 68.7 & $3.78 \mathrm{E}-02$ & \\
\hline 19.8 & 19B & 306.3 & 66.1 & $2.79 \mathrm{E}-02$ & \\
\hline 49.8 & 19B & 04.2 & 64 & $2.35 \mathrm{E}-02$ & 1 \\
\hline 19.8 & $9 \mathrm{~B}$ & 08.2 & 66.1 & $1.93 \mathrm{E}-02$ & 1 \\
\hline 49.8 & 19B & 301.9 & 69 & $1.53 \mathrm{E}-02$ & 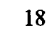 \\
\hline 49.8 & $19 \mathrm{~B}$ & 311.2 & 68.1 & $1.48 \mathrm{E}-02$ & 2 \\
\hline 19.8 & 19B & 306.1 & 62.2 & $1.22 \mathrm{E}-02$ & 2 \\
\hline 19.8 & 19B & 24.5 & 70.2 & $1.00 \mathrm{E}-02$ & 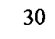 \\
\hline 99.8 & 19B & 34.2 & 62.9 & $6.64 \mathrm{E}-03$ & 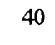 \\
\hline 49.8 & 19B & 308.9 & 81 & $6.07 \mathrm{E}-03$ & 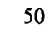 \\
\hline 49.8 & 19B & 17.1 & 71.1 & $5.26 \mathrm{E}-03$ & 6 \\
\hline 49.8 & 19B & 1.7 & 58.1 & $3.78 \mathrm{E}-03$ & 7 \\
\hline 49.8 & 19B & 3.9 & 65.3 & $2.66 \mathrm{E}-03$ & 85 \\
\hline 49.8 & $9 \mathrm{~B}$ & 6.5 & 41.5 & $5.52 \mathrm{E}-03$ & 10 \\
\hline 49.8 & 19B & 9.4 & -30.3 & $7.20 \mathrm{E}-03$ & 11 \\
\hline 81.1 & OB & 33.6 & 83 & $1.94 \mathrm{E}$ & \\
\hline 81.1 & $20 \mathrm{~B}$ & 35.6 & 72.8 & $1.25 \mathrm{E}-02$ & \\
\hline 81.1 & $20 \mathrm{~B}$ & 243.8 & 48.9 & $9.64 \mathrm{E}-03$ & \\
\hline 81.1 & $20 \mathrm{P}$ & 243.4 & 37.8 & $8.73 \mathrm{E}-03$ & \\
\hline 81.1 & $20 \mathrm{~B}$ & & 22.2 & 8777 & 12 \\
\hline 81.1 & $20 \mathrm{~B}$ & & 19.4 & $8.27 \mathrm{E}-03$ & 15 \\
\hline 81.1 & $20 \mathrm{~B}$ & & 7.8 & $7.80 \mathrm{E}-03$ & \\
\hline & 列 & & 17.5 & & \\
\hline & & & 24 & $7.29 \mathrm{E}-03$ & \\
\hline 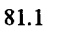 & & & 25.1 & $6.39 \mathrm{E}-03$ & \\
\hline & & & & & \\
\hline
\end{tabular}




\begin{tabular}{|c|c|c|c|c|c|}
\hline 81.1 & OB & 35.7 & -2 & $2.59 \mathrm{E}-03$ & \\
\hline 81.1 & $20 \mathrm{~B}$ & 216.6 & 45.2 & $2.11 \mathrm{E}-03$ & \\
\hline 81.1 & 20B & 218.4 & 7.5 & $2.74 \mathrm{E}-03$ & \\
\hline 87.8 & $1 \mathrm{~A}$ & 102.1 & 79.9 & $6.28 \mathrm{E}-02$ & \\
\hline 87.8 & $1 \mathrm{~A}$ & 87.3 & 74 & $3.92 \mathrm{E}-02$ & \\
\hline 87.8 & IA & 101 & 67.5 & $1.96 \mathrm{E}-02$ & \\
\hline 87.8 & IA & 106.9 & 58.6 & $1.08 \mathrm{E}-02$ & \\
\hline 87.8 & $1 \mathrm{~A}$ & 106.8 & 51.1 & $7.31 \mathrm{E}-03$ & \\
\hline 87.8 & IA & 130 & 37.9 & $5.85 \mathrm{E}-03$ & \\
\hline 87.8 & $1 \mathrm{~A}$ & 121.9 & 38.9 & $4.76 \mathrm{E}$ & \\
\hline 87.8 & $1 \mathrm{~A}$ & 133.3 & 8 & $4.64 \mathrm{E}$ & \\
\hline 87.8 & $1 \mathrm{~A}$ & 149.9 & 10.4 & $3.89 \mathrm{E}$ & \\
\hline 87.8 & $1 \mathrm{~A}$ & 119.8 & 1.3 & $3.75 \mathrm{E}$ & 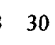 \\
\hline 87.8 & $1 \mathrm{~A}$ & 151.7 & -5.5 & 4.21 & 4 \\
\hline 87.8 & $1 \mathrm{~A}$ & 151.2 & 56.6 & 3.50 & 5 \\
\hline 87.8 & & 9.3 & 47.6 & $2.41 \mathrm{E}-03$ & 6 \\
\hline 87.8 & $1 \mathrm{~A}$ & 96.7 & -24.8 & $9.33 \mathrm{E}-04$ & 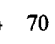 \\
\hline 87.8 & $1 \mathrm{~A}$ & 232.4 & -50.2 & $3.77 \mathrm{E}-03$ & 88 \\
\hline 87.8 & IA & 38.4 & 68.3 & $3.77 \mathrm{E}-03$ & 10 \\
\hline 87.8 & IA & 253.8 & 1.4 & $4.47 \mathrm{E}-03$ & 11 \\
\hline 93.1 & $2 \mathrm{C}$ & 104.3 & 74.9 & $3.37 \mathrm{E}-02$ & \\
\hline 93.1 & $2 \mathrm{C}$ & 105.3 & 74.2 & $2.45 \mathrm{E}-02$ & \\
\hline 93.1 & $2 \mathrm{C}$ & 117.6 & 70.9 & $1.76 \mathrm{E}-02$ & \\
\hline 93.1 & $2 \mathrm{C}$ & 123.2 & 70.8 & $1.29 \mathrm{E}-02$ & \\
\hline 93.1 & $2 \mathrm{C}$ & 125.8 & 74.5 & $1.10 \mathrm{E}-02$ & 12 \\
\hline 93.1 & $2 \mathrm{C}$ & 129.2 & 73.7 & $9.33 \mathrm{E}-03$ & 1 \\
\hline 93.1 & $2 \mathrm{C}$ & 122.7 & 65.8 & $9.06 \mathrm{E}-03$ & 1 \\
\hline 93.1 & $2 \mathrm{C}$ & 146 & 71.1 & $7.58 \mathrm{E}-03$ & 2 \\
\hline 93.1 & $2 \mathrm{C}$ & 168 & 69.9 & $5.51 \mathrm{E}-03$ & 2 \\
\hline 93.1 & $2 \mathrm{C}$ & 124 & 67.1 & $5.15 \mathrm{E}-03$ & 3 \\
\hline 93.1 & $2 \mathrm{C}$ & 45.2 & 58.4 & $3.36 \mathrm{E}-03$ & 4 \\
\hline 93.1 & $2 \mathrm{C}$ & 291.2 & -19.1 & $1.19 \mathrm{E}-03$ & 5 \\
\hline 93.1 & $2 \mathrm{C}$ & 294.4 & 9.7 & $2.49 \mathrm{E}-03$ & 6 \\
\hline 93.1 & $2 \mathrm{C}$ & 314.8 & 13.6 & $2.87 \mathrm{E}-03$ & 70 \\
\hline 93.1 & $2 \mathrm{C}$ & 273.1 & 26.1 & $2.90 \mathrm{E}-03$ & 8. \\
\hline 93.1 & $2 \mathrm{C}$ & 255.4 & -51.1 & $4.02 \mathrm{E}-03$ & 100 \\
\hline 93.1 & $2 \mathrm{C}$ & 288.4 & -6.3 & $4.35 \mathrm{E}-03$ & 115 \\
\hline 96.7 & $2 \mathrm{~A}$ & 25.8 & 49.9 & $3.66 \mathrm{E}-02$ & \\
\hline 96.7 & $2 \mathrm{~A}$ & 24.6 & 41.7 & $2.92 \mathrm{E}-02$ & \\
\hline 96.7 & $2 A$ & 23.5 & 35.8 & 2.45E-02 & \\
\hline 96.7 & 24 & 24.8 & 31.3 & $2.12 \mathrm{E}-02$ & \\
\hline 96.7 & & 23.6 & 30.8 & $1.87 \mathrm{E}-02$ & 12 \\
\hline 96.7 & $2 \mathrm{~A}$ & 22.8 & 29.5 & $1.60 \mathrm{E}-02$ & 15 \\
\hline 96.7 & $2 \mathrm{~A}$ & 24.1 & 28.8 & $1.48 \mathrm{E}-02$ & 18 \\
\hline 96.7 & $2 \mathrm{~A}$ & 23.8 & 29.5 & $1.28 \mathrm{E}-02$ & 21 \\
\hline 96.7 & $2 \mathrm{~A}$ & 23.8 & 27.4 & $1.11 \mathrm{E}-02$ & 25 \\
\hline 96.7 & & 24.4 & 27 & $1.00 \mathrm{E}-02$ & 3 \\
\hline 96.7 & & 23.8 & 24.9 & $5.60 \mathrm{E}-03$ & 40 \\
\hline 96.7 & $2 \mathrm{~A}$ & 15 & 23.8 & $4.35 \mathrm{E}-03$ & \\
\hline 96.7 & . & 28.4 & 39.9 & $3.72 \mathrm{E}-03$ & \\
\hline 96.7 & & & 8.5 & $2.95 \mathrm{E}-03$ & \\
\hline
\end{tabular}

\begin{tabular}{|c|c|c|c|c|c|}
\hline .7 & $2 \mathrm{~A}$ & 11.1 & 33.4 & $4.23 \mathrm{E}-03$ & \\
\hline .7 & $2 \mathrm{~A}$ & 2 & 2.2 & $1.23 \mathrm{E}-03$ & 100 \\
\hline .7 & $2 \mathrm{~A}$ & 18.8 & -3 & $1.60 \mathrm{E}-03$ & 115 \\
\hline .3 & $2 \mathrm{~B}$ & 111.3 & 67.9 & $3.40 \mathrm{E}-02$ & 0 \\
\hline .3 & $2 \mathrm{~B}$ & 93.5 & 64.2 & $2.94 \mathrm{E}-02$ & 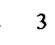 \\
\hline .3 & $2 B$ & 92 & 60.7 & $2.44 \mathrm{E}-02$ & 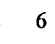 \\
\hline 3 & $2 \mathrm{~B}$ & 91.9 & 58.7 & $2.05 \mathrm{E}-02$ & 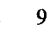 \\
\hline 9.3 & $2 \mathrm{~B}$ & 89.1 & 58.1 & $1.82 \mathrm{E}-02$ & 12 \\
\hline 9.3 & $2 \mathrm{~B}$ & 91.3 & 57.5 & $1.65 \mathrm{E}-02$ & 15 \\
\hline 9.3 & $2 \mathrm{~B}$ & 92.3 & 60.4 & $1.43 \mathrm{E}-02$ & 18 \\
\hline 9.3 & $2 \mathrm{~B}$ & 92 & 61.6 & $1.23 \mathrm{E}-02$ & 21 \\
\hline 9.3 & $2 \mathrm{~B}$ & 89.1 & 60.9 & 1. $12 \mathrm{E}-02$ & 25 \\
\hline 9.3 & $2 \mathrm{~B}$ & 84.4 & 64.4 & $9.73 \mathrm{E}-03$ & 30 \\
\hline 9.3 & $2 \mathrm{~B}$ & 91.6 & 54.7 & $6.48 \mathrm{E}-03$ & 40 \\
\hline 9.3 & $2 \mathrm{~B}$ & 76 & 52.4 & $3.48 \mathrm{E}-03$ & 50 \\
\hline 99.3 & $2 \mathrm{~B}$ & 84 & 28.3 & $3.73 \mathrm{E}-03$ & 60 \\
\hline 9.3 & $2 \mathrm{~B}$ & 85.2 & 49.1 & $3.37 \mathrm{E}-03$ & 70 \\
\hline 9.3 & $2 \mathrm{~B}$ & 25.1 & 55.8 & $3.49 \mathrm{E}-03$ & 85 \\
\hline 9.3 & & 40.9 & 63.3 & $2.85 \mathrm{E}-03$ & 100 \\
\hline 9.3 & $2 B$ & 68 & 38.2 & $2.36 \mathrm{E}-03$ & 115 \\
\hline 103.4 & $3 \mathrm{~A}$ & 0.5 & 41.5 & $2.15 \mathrm{E}-02$ & 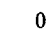 \\
\hline 103.4 & $3 \mathrm{~A}$ & 2.3 & 40 & $1.99 \mathrm{E}-02$ & 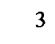 \\
\hline 103.4 & $3 \mathrm{~A}$ & 2.1 & 38.7 & $1.78 \mathrm{E}-02$ & 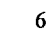 \\
\hline 103.4 & $3 \mathrm{~A}$ & 1.8 & 37.2 & $1.65 \mathrm{E}-02$ & \\
\hline 103.4 & $3 \mathrm{~A}$ & 1.2 & 35.5 & $1.50 \mathrm{E}-02$ & 12 \\
\hline 103.4 & $3 \mathrm{~A}$ & 1.5 & 35.4 & $1.34 \mathrm{E}-02$ & 15 \\
\hline 3.4 & $3 \mathrm{~A}$ & 2.8 & 36.4 & $1.25 \mathrm{E}-02$ & 18 \\
\hline 3.4 & $3 \mathrm{~A}$ & 9.5 & 19 & $1.15 \mathrm{E}-02$ & 21 \\
\hline 3.4 & $3 \mathrm{~A}$ & 2.8 & 34.4 & $1.04 \mathrm{E}-02$ & 25 \\
\hline 3.4 & $3 \mathrm{~A}$ & 0 & 35.9 & $9.02 \mathrm{E}-03$ & 30 \\
\hline 103.4 & $3 \mathrm{~A}$ & 8.5 & 35.5 & $6.86 \mathrm{E}-03$ & 40 \\
\hline 3.4 & $3 \mathrm{~A}$ & 355 & 30.5 & $5.48 \mathrm{E}-03$ & 50 \\
\hline 103.4 & $3 \mathrm{~A}$ & 50.1 & 37 & $3.89 \mathrm{E}-03$ & 60 \\
\hline 103.4 & $3 \mathrm{~A}$ & 59.9 & 22.8 & $3.64 \mathrm{E}-03$ & 70 \\
\hline 103.4 & $3 \mathrm{~A}$ & 3 & 32.3 & 2.94E-03 & 85 \\
\hline 103.4 & $3 \mathrm{~A}$ & 4.2 & 20.6 & $2.37 \mathrm{E}-03$ & 100 \\
\hline 103.4 & $3 \mathrm{~A}$ & 22.5 & 22.5 & $1.87 \mathrm{E}-03$ & 115 \\
\hline 04.6 & $4 \mathrm{~A}$ & 08.7 & 62.4 & $3.49 \mathrm{E}-02$ & 0 \\
\hline 04.6 & $4 \mathrm{~A}$ & 306.9 & 63.5 & $3.08 \mathrm{E}-02$ & 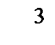 \\
\hline 04.6 & $4 \mathrm{~A}$ & 303.4 & 61.9 & $2.54 \mathrm{E}-02$ & 6 \\
\hline 104.6 & $4 \mathrm{~A}$ & 01.4 & 61.9 & $2.18 \mathrm{E}-02$ & 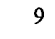 \\
\hline 04.6 & $4 \mathrm{~A}$ & 300.7 & 62.6 & $1.89 \mathrm{E}-02$ & 12 \\
\hline 104.6 & $4 \mathrm{~A}$ & 301.2 & 64.7 & $1.69 \mathrm{E}-02$ & 15 \\
\hline 104.6 & $4 \mathrm{~A}$ & 302.5 & 62.6 & $1.57 \mathrm{E}-02$ & 18 \\
\hline 104.6 & $4 \mathrm{~A}$ & 302.6 & 62.2 & $1.41 \mathrm{E}-02$ & 21 \\
\hline 104.6 & $4 \mathrm{~A}$ & 305.9 & 61.9 & $1.24 \mathrm{E}-02$ & 25 \\
\hline 104.6 & $4 A$ & 294.1 & 68 & $1.12 \mathrm{E}-02$ & 30 \\
\hline 舟 & $4 \mathrm{~A}$ & 300.1 & 70.1 & $8.91 \mathrm{E}-03$ & 40 \\
\hline 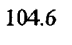 & $4 \mathrm{~A}$ & 305.8 & 59.3 & $5.40 \mathrm{E}-03$ & 50 \\
\hline 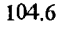 & $4 \mathrm{~A}$ & 49.9 & 64.5 & $4.87 \mathrm{E}-03$ & 60 \\
\hline & & 93 & 86.5 & $2.99 \mathrm{E}-03$ & \\
\hline
\end{tabular}

\begin{tabular}{|c|c|c|c|c|c|}
\hline 4.6 & $4 \mathrm{~A}$ & 119 & 81.7 & $2.34 \mathrm{E}_{-}$ & \\
\hline 4.6 & $4 \mathrm{~A}$ & 66.2 & 24 & 4.72E-03 & \\
\hline 4.6 & $4 \mathrm{~A}$ & 44.6 & 24.2 & $2.41 \mathrm{E}-03$ & 115 \\
\hline 06.2 & $5 \mathrm{~A}$ & 139.3 & 67.7 & $6.59 \mathrm{E}-02$ & 8 \\
\hline 6.2 & $5 \mathrm{~A}$ & 137.2 & 60.3 & $5.54 \mathrm{E}-02$ & \\
\hline 06.2 & $5 \mathrm{~A}$ & 139 & 54.1 & 4. $54 \mathrm{E}-02$ & \\
\hline 6.2 & $5 \mathrm{~A}$ & 138.1 & 49.2 & $3.85 \mathrm{E}-02$ & \\
\hline 6.2 & $5 \mathrm{~A}$ & 137.3 & 46.3 & $3.43 \mathrm{E}-02$ & 12 \\
\hline 06.2 & $5 \mathrm{~A}$ & 137.2 & 45 & $3.05 \mathrm{E}$ & 15 \\
\hline 06.2 & $5 \mathrm{~A}$ & 138.2 & 43.8 & $2.81 \mathrm{E}$ & , \\
\hline 106.2 & $5 \mathrm{~A}$ & 132.2 & 43.7 & $2.58 \mathrm{E}$ & 2 \\
\hline 106.2 & $5 A$ & 129.2 & 42.5 & $2.29 \mathrm{I}$ & 25 \\
\hline 106.2 & $5 \mathrm{~A}$ & 132.3 & 42.1 & 2.09 & 30 \\
\hline 106.2 & $5 \mathrm{~A}$ & 123.1 & 44 & & 40 \\
\hline 106.2 & $5 \mathrm{~A}$ & 131.5 & 45.4 & & 50 \\
\hline 106.2 & $5 \mathrm{~A}$ & 142 & 39 & & 60 \\
\hline 106.2 & $5 A$ & 128.2 & 47.4 & $8.68 \mathrm{E}-03$ & 70 \\
\hline 106.2 & $5 A$ & 152.7 & 44.3 & $7.19 \mathrm{I}$ & 8 \\
\hline 106.2 & $5 \mathrm{~A}$ & 118.9 & 51.7 & 5.391 & 100 \\
\hline 6.2 & $5 \mathrm{~A}$ & 106.2 & 44 & $4.98 \mathrm{I}$ & 115 \\
\hline 11 & $6 \mathrm{~A}$ & 306.5 & 51.8 & $1.09 \mathrm{E}-02$ & \\
\hline 111 & $6 \mathrm{~A}$ & 06.5 & 48.9 & $8.96 \mathrm{E}-03$ & \\
\hline 11 & $6 \mathrm{~A}$ & 05.4 & 42.8 & $7.24 \mathrm{E}-03$ & \\
\hline 11 & $6 \mathrm{~A}$ & 07.6 & 38.5 & $6.12 \mathrm{I}$ & \\
\hline 11 & $6 \mathrm{~A}$ & 07.4 & 37.1 & $5.55 \mathrm{E}-03$ & 12 \\
\hline 11 & $6 \mathrm{~A}$ & 12.9 & 33.8 & $5.12 \mathrm{E}-03$ & 15 \\
\hline 11 & $6 \mathrm{~A}$ & 13.4 & 36.4 & $4.76 \mathrm{E}$ & 18 \\
\hline 11 & $\mathrm{AA}$ & 14.7 & 31.8 & $4.45 \mathrm{E}$ & 21 \\
\hline 11 & $6 \mathrm{~A}$ & 16.9 & 33.9 & $3.90 \mathrm{E}$ & 25 \\
\hline 11 & $6 \mathrm{~A}$ & 22.6 & 41.4 & $3.68 \mathrm{E}-03$ & 30 \\
\hline 11 & $6 \mathrm{~A}$ & 327.6 & 30.7 & $3.32 \mathrm{E}-03$ & 40 \\
\hline 11 & $6 \mathrm{~A}$ & 325 & 33.3 & $2.37 \mathrm{E}-03$ & 50 \\
\hline 11 & A & 10.5 & 40.8 & $2.11 \mathrm{E}-03$ & 60 \\
\hline 11 & & 312.9 & 14.2 & $1.76 \mathrm{E}-03$ & 70 \\
\hline 11 & & 13.6 & 45.6 & $1.07 \mathrm{E}-03$ & 85 \\
\hline 11 & $6 \mathrm{~A}$ & 226.1 & 3.1 & $8.98 \mathrm{E}-04$ & 100 \\
\hline 111 & & 336.9 & 9.9 & 1.04 & 115 \\
\hline 114.7 & $6 \mathrm{~B}$ & 329 & 26.4 & $2.19 \mathrm{E}-0$ & \\
\hline 114.7 & $6 \mathrm{~B}$ & 330.1 & 21 & $1.99 \mathrm{E}-02$ & \\
\hline 114.7 & $6 \mathrm{~B}$ & 329.5 & 18 & $1.79 \mathrm{E}-02$ & \\
\hline 47 & $6 \mathrm{~B}$ & 330.2 & 16.1 & $1.61 \mathrm{E}-02$ & \\
\hline . & $6 \mathrm{~B}$ & 330.6 & 12.6 & $1.44 \mathrm{E}-02$ & 12 \\
\hline 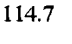 & $6 \mathrm{~B}$ & 329.1 & 15.1 & $1.32 \mathrm{E}-02$ & 15 \\
\hline & $6 \mathrm{~B}$ & 330 & 13.9 & $1.19 \mathrm{E}-02$ & 18 \\
\hline & $6 \mathrm{~B}$ & 328.5 & 16.6 & $1.09 \mathrm{E}-02$ & 2 \\
\hline & $6 \mathrm{~B}$ & & 12.2 & $9.68 \mathrm{E}-03$ & 25 \\
\hline & $6 \mathrm{~B}$ & 327.9 & 10.4 & $8.21 \mathrm{E}-03$ & 30 \\
\hline & $6 \mathrm{~B}$ & 330 & 15.7 & $5.96 \mathrm{E}-03$ & 40 \\
\hline & $6 \mathrm{~B}$ & 329.3 & 10.3 & $5.00 \mathrm{E}-03$ & \\
\hline 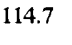 & $6 \mathrm{~B}$ & . & 20.4 & $4.46 \mathrm{E}-03$ & \\
\hline & & & & $5.06 \mathrm{E}-03$ & \\
\hline
\end{tabular}




\begin{tabular}{|c|c|c|c|c|c|}
\hline 14.7 & B & 35.4 & 10.8 & & \\
\hline 4.7 & $6 \mathrm{~B}$ & 43.9 & 17 & & \\
\hline 4.7 & $6 \mathrm{~B}$ & 31.9 & 10.1 & $3.51 \mathrm{E}$ & \\
\hline 7.7 & $7 \mathrm{~A}$ & 34.4 & 80.6 & $4.92 \mathrm{E}$ & \\
\hline 17.7 & $7 \mathrm{~A}$ & 82.6 & 30.9 & $3.81 \mathrm{E}$ & \\
\hline 17.7 & $7 \mathrm{~A}$ & 65.9 & 75.9 & $2.66 \mathrm{E}$ & \\
\hline 7.7 & $7 \mathrm{~A}$ & 67.8 & 71.8 & $1.87 \mathrm{E}$ & \\
\hline 7.7 & $7 \mathrm{~A}$ & 68.7 & 69.9 & $1.44 \mathrm{E}-02$ & \\
\hline 7.7 & $7 \mathrm{~A}$ & 79 & 66.5 & $1.17 \mathrm{E}$ & \\
\hline 7.7 & $7 \mathrm{~A}$ & 83.5 & 68.9 & $9.19 \mathrm{E}-03$ & \\
\hline 17.7 & $7 \mathrm{~A}$ & 84.8 & 63.6 & $7.96 \mathrm{E}-03$ & \\
\hline 17.7 & $7 \mathrm{~A}$ & 77.8 & 72 & $5.59 \mathrm{E}$ & \\
\hline 17.7 & $7 \mathrm{~A}$ & 71.1 & 67.5 & 5.101 & \\
\hline 7.7 & $7 \mathrm{~A}$ & 0.8 & 46.8 & 2.35 & \\
\hline 7.7 & 7A & 1.8 & 34.1 & & \\
\hline 17.7 & $7 \mathrm{~A}$ & 65.1 & 6.8 & 3.211 & \\
\hline 17.7 & $7 \mathrm{~A}$ & 10.6 & -52.3 & 2.96 & \\
\hline 17.7 & $7 \mathrm{~A}$ & 87.4 & -5.2 & 1.01 & \\
\hline 17.7 & 7A & 66.2 & -32 & 3.98 & 310 \\
\hline 17.7 & $7 \mathrm{~A}$ & 1.1 & -64.5 & 3.75 & 311 \\
\hline 20.9 & $8 \mathrm{~A}$ & 23.4 & 59.7 & 5.51 & \\
\hline 20.9 & $8 \mathrm{~A}$ & 37.5 & 53.3 & 3.12 & \\
\hline 20.9 & $8 \mathrm{~A}$ & 42.5 & 44.4 & 2.33 & \\
\hline 120.9 & 8 & 7.4 & 33.4 & & \\
\hline 20.9 & 8 & 56.4 & 16.2 & & 12 \\
\hline 120.9 & $8 \mathrm{~A}$ & 46.2 & 4.8 & -03 & 15 \\
\hline 120.9 & A & 57.4 & -3.9 & -03 & 18 \\
\hline 120.9 & $8 \mathrm{~A}$ & 64.9 & 0.8 & $1.63 \mathrm{E}$ & 21 \\
\hline 120.9 & A & 69.4 & -5.2 & $1.33 \mathrm{E}-03$ & 25 \\
\hline 120.9 & A & 82 & -14.5 & $1.27 \mathrm{E}-03$ & 30 \\
\hline 120.9 & A & 8.6 & -1.1 & 8.35E-04 & 40 \\
\hline 120.9 & 8 & 8.7 & 17.3 & $6.03 \mathrm{E}-04$ & 50 \\
\hline 120.9 & 8 & 11.8 & -32.1 & $3.81 \mathrm{E}-04$ & 60 \\
\hline 120.9 & 8 & 35.8 & -67.8 & $6.99 \mathrm{E}-04$ & 70 \\
\hline 120.9 & 8 & 143 & -8.4 & $1.19 \mathrm{E}-03$ & 85 \\
\hline 120.9 & 8 & 6.7 & -55.4 & 9.25E-04 & 100 \\
\hline 120.9 & 8 & 3.4 & -26.6 & $1.07 \mathrm{E}-03$ & 115 \\
\hline 124.2 & $500 \mathrm{~A}$ & 349.4 & 48.5 & 4.37E- 03 & \\
\hline 124.2 & A & 348.3 & 49.2 & 4.54E- 03 & \\
\hline 124.2 & $500 \mathrm{~A}$ & 350.3 & 49.2 & $4.48 \mathrm{E}-03$ & \\
\hline 124.2 & $500 \mathrm{~A}$ & 350.1 & 49.5 & 4.41E-03 & \\
\hline 124.2 & $500 \mathrm{~A}$ & 349.5 & -50 & $4.32 \mathrm{E}-03$ & 12 \\
\hline 124.2 & $500 \mathrm{~A}$ & 350.3 & -51 & $4.15 \mathrm{E}-03$ & 15 \\
\hline 124.2 & & 350.1 & -51 & $3.91 \mathrm{E}-03$ & 18 \\
\hline 124.2 & $500 \mathrm{~A}$ & 351.6 & -50.3 & $3.58 \mathrm{E}-03$ & 21 \\
\hline 124.2 & $500 \mathrm{~A}$ & 351.6 & -51.4 & $3.24 \mathrm{E}-03$ & 25 \\
\hline 124.2 & $500 \mathrm{~A}$ & 350.6 & -50.4 & $2.90 \mathrm{E}-03$ & 30 \\
\hline 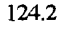 & $500 \mathrm{~A}$ & 350.3 & 48.8 & $2.21 \mathrm{E}-03$ & 40 \\
\hline 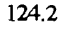 & $500 \mathrm{~A}$ & 336.4 & -52.6 & $2.09 \mathrm{E}-03$ & \\
\hline 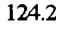 & 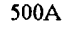 & 342.2 & -54.3 & $1.79 \mathrm{E}-03$ & 60 \\
\hline 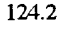 & 年 & 344.1 & 49.1 & $1.81 \mathrm{E}-03$ & \\
\hline
\end{tabular}

$\begin{array}{llllll}124.2 & 500 \mathrm{~A} & 349.5 & -53.6 & 1.73 \mathrm{E}-03 & 85\end{array}$

$\begin{array}{lllllll}124.2 & 500 \mathrm{~A} & 334 & 45 & 1.64 \mathrm{E}-03 & 100\end{array}$

$\begin{array}{lllllll}124.2 & 500 \mathrm{~A} & 343.4 & -54.3 & 1.54 \mathrm{E}-03 & 115\end{array}$

$\begin{array}{lllllll}125 & 8 \mathrm{~B} & 183 & 57.3 & 8.35 \mathrm{E}-03 & 0\end{array}$

$\begin{array}{lllllll}125 & 8 \mathrm{~B} & 179.8 & 36.7 & 6.23 \mathrm{E}-03 & 3\end{array}$

$\begin{array}{lllllll}125 & 8 \mathrm{~B} & 182.9 & 24.2 & 5.61 \mathrm{E}-03 & 6\end{array}$

$\begin{array}{llllll}125 & 8 \mathrm{~B} & 183.2 & 19.2 & 5.24 \mathrm{E}-03 & 9\end{array}$

$\begin{array}{llllll}125 & 8 \mathrm{~B} & 183.5 & 13.9 & 4.84 \mathrm{E}-03 & 12\end{array}$

$\begin{array}{llllll}125 & 8 \mathrm{~B} & 184.9 & 11.2 & 4.57 \mathrm{E}-03 & 15\end{array}$

$\begin{array}{lllllll}125 & 8 \mathrm{~B} & 184 & 9.5 & 4.30 \mathrm{E}-03 & 18\end{array}$

$\begin{array}{lllllll}125 & 8 \mathrm{~B} & 179.4 & 9.4 & 3.93 \mathrm{E}-03 & 21\end{array}$

$\begin{array}{lllllll}125 & 8 \mathrm{~B} & 179.3 & 8.3 & 3.65 \mathrm{E}-03 & 25\end{array}$

$\begin{array}{llllll}125 & 8 \mathrm{~B} & 176.5 & 2.7 & 2.96 \mathrm{E}-03 & 30\end{array}$

$\begin{array}{lllllll}125 & 8 \mathrm{~B} & 165.4 & 6.1 & 2.97 \mathrm{E}-03 & 40\end{array}$

$\begin{array}{llllll}125 & 8 \mathrm{~B} & 166.9 & 2.7 & 2.21 \mathrm{E}-03 & 50\end{array}$

$\begin{array}{llllll}125 & 8 \mathrm{~B} & 166.3 & 5.2 & 2.10 \mathrm{E}-03 & 60\end{array}$

$\begin{array}{lllllll}125 & 8 \mathrm{~B} & 160.4 & -6.2 & 2.47 \mathrm{E}-03 & 70\end{array}$

$\begin{array}{lllllll}125 & 8 \mathrm{~B} & 138.1 & -6.4 & 1.58 \mathrm{E}-03 & 85\end{array}$

$\begin{array}{lllllll}125 & 8 \mathrm{~B} & 163.4 & -8.2 & 2.05 \mathrm{E}-03 & 100\end{array}$

$\begin{array}{lllllll}125 & 8 \mathrm{~B} & 156.4 & -2 & 2.29 \mathrm{E}-03 & 115\end{array}$

$\begin{array}{lllllll}126.1 & 8 \mathrm{C} & 181.2 & 82.5 & 1.07 \mathrm{E}-02 & 0\end{array}$

$\begin{array}{lllllll}126.1 & 8 \mathrm{C} & 179 & 80.6 & 8.01 \mathrm{E}-03 & 3\end{array}$

$\begin{array}{lllllll}126.1 & 8 \mathrm{C} & 189.7 & 75.8 & 6.25 \mathrm{E}-03 & 6\end{array}$

$\begin{array}{lllllll}126.1 & 8 \mathrm{C} & 188.7 & 70.9 & 5.05 \mathrm{E}-03 & 9\end{array}$

$\begin{array}{lllllll}126.1 & 8 \mathrm{C} & 189.7 & 66.6 & 4.34 \mathrm{E}-03 & 12\end{array}$

$\begin{array}{lllllll}126.1 & 8 \mathrm{C} & 192.3 & 63.7 & 3.91 \mathrm{E}-03 & 15\end{array}$

$\begin{array}{lllllll}126.1 & 8 \mathrm{C} & 191.7 & 57.7 & 3.18 \mathrm{E}-03 & 18\end{array}$

$\begin{array}{lllllll}126.1 & 8 \mathrm{C} & 184.5 & 58.4 & 3.15 \mathrm{E}-03 & 21\end{array}$

$\begin{array}{lllllll}126.1 & 8 \mathrm{C} & 187.5 & 55.9 & 2.30 \mathrm{E}-03 & 25\end{array}$

$\begin{array}{lllllll}126.1 & 8 \mathrm{C} & 199.2 & 55.5 & 1.91 \mathrm{E}-03 & 30\end{array}$

$\begin{array}{llllll}126.1 & 8 \mathrm{C} & 182.2 & 63 & 1.37 \mathrm{E}-03 & 40\end{array}$

$\begin{array}{lllllll}126.1 & 8 \mathrm{C} & 220.2 & 53.9 & 1.26 \mathrm{E}-03 & 50\end{array}$

$\begin{array}{lllllll}126.1 & 8 \mathrm{C} & 243.3 & 49.2 & 1.39 \mathrm{E}-03 & 60\end{array}$

$\begin{array}{lllllll}126.1 & 8 \mathrm{C} & 233.1 & 48.2 & 9.65 \mathrm{E}-04 & 70\end{array}$

$\begin{array}{llllll}126.1 & 8 \mathrm{C} & 290 & 57.5 & 1.01 \mathrm{E}-03 & 85\end{array}$

$\begin{array}{llllllll}126.1 & 8 \mathrm{C} & 278.7 & 67.5 & 8.23 \mathrm{E}-04 & 100\end{array}$

$\begin{array}{lllllll}126.1 & 8 \mathrm{C} & 269.1 & 72.6 & 6.56 \mathrm{E}-04 & 115\end{array}$

$\begin{array}{llllll}128.7 & 501 \mathrm{~A} & 191.5 & 48.5 & 2.11 \mathrm{E}-02 & 0\end{array}$

$\begin{array}{lllllll}128.7 & 501 \mathrm{~A} & 189.8 & 50.3 & 1.76 \mathrm{E}-02 & 3\end{array}$

$\begin{array}{lllllll}128.7 & 501 \mathrm{~A} & 191 & 47.9 & 1.42 \mathrm{E}-02 & 6\end{array}$

$\begin{array}{llllll}128.7 & 501 \mathrm{~A} & 195.1 & 41.8 & 1.10 \mathrm{E}-02 & 9\end{array}$

$\begin{array}{llllll}128.7 & 501 \mathrm{~A} & 195 & 37.7 & 9.74 \mathrm{E}-03 & 12\end{array}$

$\begin{array}{lllllll}128.7 & 501 \mathrm{~A} & 189.5 & 37.5 & 7.65 \mathrm{E}-03 & 15\end{array}$

$\begin{array}{lllllll}128.7 & 501 \mathrm{~A} & 187.1 & 35.7 & 6.30 \mathrm{E}-03 & 18\end{array}$

$\begin{array}{lllllll}128.7 & 501 \mathrm{~A} & 191.1 & 35.3 & 5.20 \mathrm{E}-03 & 21\end{array}$

$\begin{array}{lllllll}128.7 & 501 \mathrm{~A} & 186.8 & 51.7 & 4.33 \mathrm{E}-03 & 25\end{array}$

$\begin{array}{lllllll}128.7 & 501 \mathrm{~A} & 207.2 & 35.5 & 5.07 \mathrm{E}-03 & 30\end{array}$

$\begin{array}{lllllll}128.7 & 501 \mathrm{~A} & 198.2 & 50.9 & 3.21 \mathrm{E}-03 & 40\end{array}$

$\begin{array}{llllll}128.7 & 501 \mathrm{~A} & 177.1 & 55.6 & 5.18 \mathrm{E}-03 & 50\end{array}$

$\begin{array}{llllll}128.7 & 501 \mathrm{~A} & 176 & 55 & 4.92 \mathrm{E}-03 & 60\end{array}$

$\begin{array}{llllll}128.7 & 501 \mathrm{~A} & 206.2 & 72.1 & 3.44 \mathrm{E}-03 & 70\end{array}$ $\begin{array}{llllll}128.7 & 501 \mathrm{~A} & 271.5 & 31.6 & 3.45 \mathrm{E}-03 & 85\end{array}$

$\begin{array}{lllllll}128.7 & 501 \mathrm{~A} & 253.8 & 9.7 & 3.90 \mathrm{E}-03 & 100\end{array}$

$\begin{array}{lllllll}128.7 & 501 \mathrm{~A} & 254.8 & 5 & 3.33 \mathrm{E}-03 & 115\end{array}$

$\begin{array}{llllll}130.7 & 502 \mathrm{~A} & 220.7 & 68.1 & 2.38 \mathrm{E}-02 & 0\end{array}$

$\begin{array}{llllll}130.7 & 502 \mathrm{~A} & 232.5 & 73.9 & 1.94 \mathrm{E}-02 & 3\end{array}$

$\begin{array}{llllll}130.7 & 502 \mathrm{~A} & 237.4 & 76 & 1.46 \mathrm{E}-02 & 6\end{array}$

$\begin{array}{llllll}130.7 & 502 \mathrm{~A} & 267.1 & 72.4 & 1.15 \mathrm{E}-02 & 9\end{array}$

$\begin{array}{lllllll}130.7 & 502 \mathrm{~A} & 278.9 & 72.8 & 8.83 \mathrm{E}-03 & 12\end{array}$

$\begin{array}{lllllll}130.7 & 502 \mathrm{~A} & 256.7 & 71.5 & 7.82 \mathrm{E}-03 & 15\end{array}$

$\begin{array}{llllll}130.7 & 502 \mathrm{~A} & 284.7 & 60.9 & 6.24 \mathrm{E}-03 & 18\end{array}$

$\begin{array}{lllllll}130.7 & 502 \mathrm{~A} & 275.2 & 52.7 & 4.39 \mathrm{E}-03 & 21\end{array}$

$\begin{array}{lllllll}130.7 & 502 \mathrm{~A} & 278.4 & 50.9 & 4.22 \mathrm{E}-03 & 25\end{array}$

$\begin{array}{llllll}130.7 & 502 \mathrm{~A} & 263.5 & 52.6 & 4.46 \mathrm{E}-03 & 30\end{array}$

$\begin{array}{lllllll}130.7 & 502 \mathrm{~A} & 323.1 & 77.9 & 3.39 \mathrm{E}-03 & 40\end{array}$

$\begin{array}{llllll}130.7 & 502 \mathrm{~A} & 309 & 26.7 & 4.00 \mathrm{E}-03 & 50\end{array}$

$\begin{array}{lllllll}130.7 & 502 \mathrm{~A} & 324 & -12.7 & 1.74 \mathrm{E}-03 & 60\end{array}$

$\begin{array}{lllllll}130.7 & 502 \mathrm{~A} & 348.7 & 10.5 & 1.47 \mathrm{E}-03 & 70\end{array}$

$\begin{array}{lllllll}130.7 & 502 \mathrm{~A} & 338.8 & -9.2 & 4.06 \mathrm{E}-03 & 85\end{array}$

$\begin{array}{lllllll}130.7 & 502 \mathrm{~A} & 343.6 & -38.6 & 2.69 \mathrm{E}-03 & 100\end{array}$

$\begin{array}{lllllll}130.7 & 502 \mathrm{~A} & 348.1 & -43.8 & 4.82 \mathrm{E}-03 & 115\end{array}$

$\begin{array}{lllllll}131.3 & 503 \mathrm{~A} & 177.7 & 52.6 & 2.87 \mathrm{E}-02 & 0\end{array}$

$\begin{array}{llllll}131.3 & 503 \mathrm{~A} & 175.6 & 54.2 & 2.43 \mathrm{E}-02 & 3\end{array}$

$\begin{array}{lllllll}131.3 & 503 \mathrm{~A} & 176.4 & 52.2 & 1.91 \mathrm{E}-02 & 6\end{array}$

$\begin{array}{llllll}131.3 & 503 \mathrm{~A} & 175.8 & 52.3 & 1.49 \mathrm{E}-02 & 9\end{array}$

$\begin{array}{lllllll}131.3 & 503 \mathrm{~A} & 169.7 & 48.4 & 1.16 \mathrm{E}-02 & 12\end{array}$

$\begin{array}{lllllll}131.3 & 503 \mathrm{~A} & 171.3 & 48.8 & 9.48 \mathrm{E}-03 & 15\end{array}$

$\begin{array}{llllll}131.3 & 503 \mathrm{~A} & 164.6 & 49.7 & 7.63 \mathrm{E}-03 & 18\end{array}$

$\begin{array}{lllllll}131.3 & 503 \mathrm{~A} & 163.1 & 43.2 & 7.31 \mathrm{E}-03 & 21\end{array}$

$\begin{array}{llllll}131.3 & 503 \mathrm{~A} & 152 & 40.8 & 6.91 \mathrm{E}-03 & 25\end{array}$

$\begin{array}{llllll}131.3 & 503 \mathrm{~A} & 125.7 & 55.6 & 6.28 \mathrm{E}-03 & 30\end{array}$

$\begin{array}{lllllll}131.3 & 503 \mathrm{~A} & 110.6 & 48.3 & 4.60 \mathrm{E}-03 & 40\end{array}$

$\begin{array}{llllll}131.3 & 503 \mathrm{~A} & 10.6 & 68.2 & 2.39 \mathrm{E}-03 & 50\end{array}$

$\begin{array}{lllllll}131.3 & 503 \mathrm{~A} & 15.7 & 55.4 & 2.62 \mathrm{E}-03 & 60\end{array}$

$\begin{array}{llllll}131.3 & 503 \mathrm{~A} & 2 & 20.4 & 3.02 \mathrm{E}-03 & 70\end{array}$

$\begin{array}{llllll}131.3 & 503 \mathrm{~A} & 247.2 & -18.3 & 2.10 \mathrm{E}-03 & 85\end{array}$

$\begin{array}{lllllll}131.3 & 503 \mathrm{~A} & 30.4 & 26.6 & 3.70 \mathrm{E}-03 & 100\end{array}$

$\begin{array}{llllll}131.3 & 503 \mathrm{~A} & 58.6 & 2 & 1.01 \mathrm{E}-03 & 115\end{array}$

$\begin{array}{lllllll}133.4 & 9 \mathrm{~A} & 340.2 & 72 & 6.86 \mathrm{E}-02 & 0\end{array}$

$\begin{array}{lllllll}133.4 & 9 \mathrm{~A} & 347.5 & 70 & 5.07 \mathrm{E}-02 & 3\end{array}$

$\begin{array}{lllllll}133.4 & 9 \mathrm{~A} & 349.5 & 67.2 & 3.53 \mathrm{E}-02 & 6\end{array}$

$\begin{array}{llllll}133.4 & 9 \mathrm{~A} & 350.2 & 60.6 & 2.51 \mathrm{E}-02 & 9\end{array}$

$\begin{array}{lllllll}133.4 & 9 \mathrm{~A} & 347.8 & 59.1 & 1.97 \mathrm{E}-02 & 12\end{array}$

$\begin{array}{lllllll}133.4 & 9 \mathrm{~A} & 349.3 & 58.5 & 1.64 \mathrm{E}-02 & 15\end{array}$

$\begin{array}{lllllll}133.4 & 9 \mathrm{~A} & 353.8 & 61.1 & 1.38 \mathrm{E}-02 & 18\end{array}$

$\begin{array}{llllll}133.4 & 9 \mathrm{~A} & 350.2 & 54.1 & 1.04 \mathrm{E}-02 & 21\end{array}$

$\begin{array}{lllllll}133.4 & 9 \mathrm{~A} & 339.3 & 52.6 & 9.28 \mathrm{E}-03 & 25\end{array}$

$\begin{array}{llllll}133.4 & 9 \mathrm{~A} & 353.6 & 45.3 & 7.61 \mathrm{E}-03 & 30\end{array}$

$\begin{array}{llllll}133.4 & 9 \mathrm{~A} & 345.5 & 42.9 & 5.18 \mathrm{E}-03 & 40\end{array}$

$\begin{array}{llllll}133.4 & 9 \mathrm{~A} & 355 & 24 & 2.83 \mathrm{E}-03 & 50\end{array}$

$\begin{array}{lllllll}133.4 & 9 \mathrm{~A} & 196.1 & 60.1 & 4.09 \mathrm{E}-04 & 60\end{array}$

$\begin{array}{llllll}133.4 & 9 \mathrm{~A} & 14.8 & 45.9 & 1.69 \mathrm{E}-03 & 70\end{array}$ 


\begin{tabular}{|c|c|c|c|c|c|}
\hline 133.4 & $9 \mathrm{~A}$ & 261.2 & -4.1 & $2.06 \mathrm{E}-03$ & \\
\hline 133.4 & $9 \mathrm{~A}$ & 336.7 & -37.5 & $1.84 \mathrm{E}-03$ & 100 \\
\hline 133.4 & $9 \mathrm{~A}$ & 318.4 & -32.8 & $3.40 \mathrm{E}-03$ & 11 \\
\hline 135.9 & $10 \mathrm{~A}$ & 21.1 & 52.8 & $9.31 \mathrm{E}-03$ & \\
\hline 135.9 & $10 \mathrm{~A}$ & 35.1 & 30.5 & $6.00 \mathrm{E}-03$ & \\
\hline 135.9 & $10 \mathrm{~A}$ & 42.7 & 10.8 & $5.31 \mathrm{E}-03$ & \\
\hline 135.9 & $10 \mathrm{~A}$ & 48.8 & -2.9 & $5.07 \mathrm{E}-03$ & \\
\hline 135.9 & $10 \mathrm{~A}$ & 48.4 & 4.1 & $4.93 \mathrm{E}-03$ & 1 \\
\hline 135.9 & $10 \mathrm{~A}$ & 58.5 & -5 & $4.20 \mathrm{E}-03$ & 1 \\
\hline 135.9 & $10 \mathrm{~A}$ & 63.7 & -8.9 & $4.04 \mathrm{E}-03$ & ; \\
\hline 135.9 & $10 \mathrm{~A}$ & 68.4 & -11.4 & $3.08 \mathrm{E}-03$ & 2 \\
\hline 135.9 & $10 \mathrm{~A}$ & 70.6 & -12.1 & $3.27 \mathrm{E}-03$ & 2 \\
\hline 135.9 & $10 \mathrm{~A}$ & 59.6 & -10.2 & $2.01 \mathrm{E}-03$ & 30 \\
\hline 135.9 & $10 \mathrm{~A}$ & 93.3 & -26.7 & $1.07 \mathrm{E}-03$ & 40 \\
\hline 135.9 & $10 \mathrm{~A}$ & 37.6 & -31.9 & $1.83 \mathrm{E}-03$ & 50 \\
\hline 135.9 & $10 \mathrm{~A}$ & 81.9 & -57.7 & $1.15 E-03$ & 60 \\
\hline 135.9 & $10 \mathrm{~A}$ & 61.4 & -48.1 & $1.87 \mathrm{E}-03$ & 70 \\
\hline 135.9 & $10 \mathrm{~A}$ & 54.3 & 43.4 & $1.49 \mathrm{E}-03$ & 85 \\
\hline 141 & $10 \mathrm{~B}$ & 353.4 & 62 & $2.94 \mathrm{E}-02$ & \\
\hline 141 & $10 \mathrm{~B}$ & 0 & 53.8 & $2.12 \mathrm{E}-02$ & \\
\hline 141 & $10 \mathrm{~B}$ & 9.2 & 44.2 & $1.48 \mathrm{E}-02$ & \\
\hline 141 & $10 \mathrm{~B}$ & 17.8 & 32.7 & $1.16 \mathrm{E}-02$ & \\
\hline 141 & $10 \mathrm{~B}$ & 9 & 27.8 & $1.02 \mathrm{E}-02$ & 12 \\
\hline 141 & $10 \mathrm{~B}$ & 7.7 & 17.8 & 8.01E-03 & 15 \\
\hline 141 & $10 \mathrm{~B}$ & 12.8 & 19.8 & $7.48 \mathrm{E}-03$ & 18 \\
\hline 141 & $10 \mathrm{~B}$ & 14.5 & 25.2 & $7.41 E-03$ & 21 \\
\hline 141 & $10 \mathrm{~B}$ & 359.2 & 14.7 & $6.72 \mathrm{E}-03$ & 25 \\
\hline 141 & $10 \mathrm{~B}$ & 10.4 & 9.3 & $5.56 \mathrm{E}-03$ & 30 \\
\hline 141 & $10 \mathrm{~B}$ & 58.4 & 22.1 & $5.31 \mathrm{E}-03$ & 40 \\
\hline 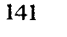 & $10 \mathrm{~B}$ & 9.5 & 5.3 & & 50 \\
\hline 141 & $10 \mathrm{~B}$ & 34.2 & -16.4 & $3.65 \mathrm{E}-03$ & 60 \\
\hline 141 & 10B & 59.1 & -2.8 & $2.24 \mathrm{E}-03$ & 70 \\
\hline 141 & $10 \mathrm{~B}$ & 20 & -56.3 & $2.30 \mathrm{E}-03$ & 85 \\
\hline 141 & $10 \mathrm{~B}$ & 85 & -50.8 & & 100 \\
\hline 141 & $10 \mathrm{~B}$ & 35.6 & -57.2 & $3.40 \mathrm{E}-03$ & 115 \\
\hline 143 & $800 \mathrm{~A}$ & 324.9 & 75.5 & $3.26 \mathrm{E}-02$ & 0 \\
\hline 143 & $800 \mathrm{~A}$ & 337.8 & 75.7 & $2.38 \mathrm{E}-02$ & . \\
\hline 143 & $800 \mathrm{~A}$ & 356.5 & 75.6 & $1.57 \mathrm{E}-02$ & 6 \\
\hline 143 & $800 \mathrm{~A}$ & 8.5 & 70.6 & $1.25 \mathrm{E}-02$ & 9 \\
\hline 143 & $800 \mathrm{~A}$ & 31 & 65.6 & $9.73 E-03$ & 12 \\
\hline 143 & $800 \mathrm{~A}$ & 36.5 & 61.9 & $8.76 \mathrm{E}-03$ & 15 \\
\hline 143 & $800 \mathrm{~A}$ & 46.2 & 56.4 & $7.52 \mathrm{E}-03$ & 18 \\
\hline 143 & $800 \mathrm{~A}$ & 35.4 & 57.7 & $6.54 \mathrm{E}-03$ & 21 \\
\hline 143 & $800 \mathrm{~A}$ & 39.6 & 67.3 & $5.09 \mathrm{E}-03$ & 25 \\
\hline 143 & $800 \mathrm{~A}$ & 30 & 31.2 & $5.30 \mathrm{E}-03$ & 30 \\
\hline 143 & $800 \mathrm{~A}$ & 44.2 & 47 & $4.38 \mathrm{E}-03$ & 40 \\
\hline 143 & $800 \mathrm{~A}$ & 38.6 & 36.6 & $4.43 \mathrm{E}-03$ & 30 \\
\hline 143 & $800 \mathrm{~A}$ & 31.7 & 21.1 & $6.60 \mathrm{E}-03$ & 60 \\
\hline 143 & $800 \mathrm{~A}$ & 50 & -7.9 & $5.37 \mathrm{E}-03$ & $\pi$ \\
\hline 1.75 & $800 \mathrm{~A}$ & 59.2 & 0.4 & $5.61 \mathrm{E}-03$ & 85 \\
\hline 143 & $800 \mathrm{~A}$ & 55 & -2.7 & $4.65 \mathrm{E}-03$ & \\
\hline
\end{tabular}

\begin{tabular}{|c|c|c|c|c|c|}
\hline 143 & $800 \mathrm{~A}$ & 43.5 & -13.3 & $3.42 \mathrm{E}-03$ & 115 \\
\hline 146.5 & $801 \mathrm{~A}$ & 40 & 60.2 & $4.05 \mathrm{E}-02$ & 0 \\
\hline 146.5 & $801 \mathrm{~A}$ & 48.7 & 47.4 & $2.92 \mathrm{E}-02$ & 3 \\
\hline 146.5 & $801 \mathrm{~A}$ & 52.2 & 26.4 & $2.10 \mathrm{E}-02$ & 6 \\
\hline 146.5 & $801 \mathrm{~A}$ & 52.5 & 10.5 & $1.77 \mathrm{E}-02$ & 9 \\
\hline 146.5 & $801 \mathrm{~A}$ & 55.4 & -1.7 & $1.62 \mathrm{E}-02$ & 12 \\
\hline 146.5 & $801 \mathrm{~A}$ & 56.8 & -5.9 & $1.54 \mathrm{E}-02$ & 15 \\
\hline 146.5 & $801 \mathrm{~A}$ & 57.9 & -11.6 & $1.50 \mathrm{E}-02$ & 18 \\
\hline 146.5 & $801 \mathrm{~A}$ & 58 & -14.5 & $1.42 \mathrm{E}-02$ & 21 \\
\hline 146.5 & $801 \mathrm{~A}$ & 57 & -12.4 & $1.34 \mathrm{E}-02$ & 25 \\
\hline 146.5 & $801 \mathrm{~A}$ & 56 & -15.1 & $1.22 \mathrm{E}-02$ & 30 \\
\hline 146.5 & $801 \mathrm{~A}$ & 65.4 & -22 & $1.15 \mathrm{E}-02$ & 40 \\
\hline 146.5 & $801 \mathrm{~A}$ & 63.4 & -17.4 & $1.05 \mathrm{E}-02$ & 50 \\
\hline 146.5 & $801 \mathrm{~A}$ & 51 & -13.6 & $8.74 \mathrm{E}-03$ & 60 \\
\hline 146.5 & $801 \mathrm{~A}$ & 59.8 & -21 & $5.28 \mathrm{E}-03$ & 70 \\
\hline 146.5 & $801 \mathrm{~A}$ & 62.6 & -23 & $5.34 \mathrm{E}-03$ & 85 \\
\hline 146.5 & $801 \mathrm{~A}$ & 77.9 & -30.8 & $5.39 \mathrm{E}-03$ & 100 \\
\hline 146.5 & $801 \mathrm{~A}$ & 79 & -22.5 & $4.91 E-03$ & 115 \\
\hline 146.6 & $802 \mathrm{~A}$ & 15.1 & 45.9 & $1.80 \mathrm{E}-02$ & 0 \\
\hline 146.6 & $802 \mathrm{~A}$ & 19.8 & 34.6 & $1.42 \mathrm{E}-02$ & 3 \\
\hline 146.6 & $802 \mathrm{~A}$ & 23.8 & 18.2 & $1.08 \mathrm{E}-02$ & 6 \\
\hline 146.6 & $802 \mathrm{~A}$ & 29.7 & 7 & $9.27 \mathrm{E}-03$ & 9 \\
\hline 146.6 & $802 \mathrm{~A}$ & 33.4 & -4.3 & $8.29 \mathrm{E}-03$ & 12 \\
\hline 146.6 & $802 \mathrm{~A}$ & 33.5 & -6.9 & $7.02 \mathrm{E}-03$ & 15 \\
\hline 146.6 & $802 \mathrm{~A}$ & 37.9 & -9.7 & 7.37E-03 & 18 \\
\hline 146.6 & $802 \mathrm{~A}$ & 36.4 & -23 & $6.38 \mathrm{E}-03$ & 21 \\
\hline 146.6 & $802 \mathrm{~A}$ & 19.2 & -21.4 & $6.18 \mathrm{E}-03$ & 25 \\
\hline 146.6 & $802 \mathrm{~A}$ & 27.4 & -23.2 & $6.60 \mathrm{E}-03$ & 30 \\
\hline 146.6 & $802 \mathrm{~A}$ & 31.6 & -33.4 & $5.82 \mathrm{E}-03$ & 40 \\
\hline 146.6 & $802 \mathrm{~A}$ & 24.9 & -34.9 & $4.33 \mathrm{E}-03$ & 50 \\
\hline 146.6 & $802 \mathrm{~A}$ & 44.9 & -23.6 & $3.40 \mathrm{E}-03$ & 60 \\
\hline 146.6 & $802 \mathrm{~A}$ & 359.1 & -40.9 & $6.68 \mathrm{E}-03$ & 70 \\
\hline 146.6 & $802 \mathrm{~A}$ & 22.7 & -14.7 & $3.51 \mathrm{E}-03$ & 85 \\
\hline 146.6 & $802 \mathrm{~A}$ & 4.8 & -26.2 & $2.82 \mathrm{E}-03$ & 100 \\
\hline 146.6 & $802 \mathrm{~A}$ & 19.1 & -45.2 & $2.51 \mathrm{E}-03$ & 115 \\
\hline 147.1 & $11 \mathrm{~A}$ & 19.3 & -5.6 & $5.13 \mathrm{E}-02$ & 0 \\
\hline 147.1 & $11 \mathrm{~A}$ & 18.7 & -11.3 & $5.02 \mathrm{E}-02$ & 3 \\
\hline 147.1 & $11 \mathrm{~A}$ & 19 & -16.7 & $4.87 \mathrm{E}-02$ & 6 \\
\hline 147.1 & $11 \mathrm{~A}$ & 19.3 & -19.5 & $4.64 \mathrm{E}-02$ & 9 \\
\hline 147.1 & $11 \mathrm{~A}$ & 18.4 & -20.5 & $4.38 \mathrm{E}-02$ & 12 \\
\hline 147.1 & $11 \mathrm{~A}$ & 18.4 & -21.6 & $4.09 \mathrm{E}-02$ & 15 \\
\hline 147.1 & $11 \mathrm{~A}$ & 18.7 & -22.6 & $3.81 \mathrm{E}-02$ & 18 \\
\hline 147.1 & $11 \mathrm{~A}$ & 18.2 & -23 & $3.51 \mathrm{E}-02$ & 21 \\
\hline 147.1 & $11 \mathrm{~A}$ & 18.9 & -22.7 & $3.12 \mathrm{E}-02$ & 25 \\
\hline 147.1 & $11 \mathrm{~A}$ & 19.1 & -22 & $2.82 \mathrm{E}-02$ & 30 \\
\hline 147.1 & $11 \mathrm{~A}$ & 19.5 & -22.4 & $2.15 \mathrm{E}-02$ & 40 \\
\hline 147.1 & $11 \mathrm{~A}$ & 17.8 & -21.2 & $1.72 \mathrm{E}-02$ & 50 \\
\hline 147.1 & $11 \mathrm{~A}$ & 17.5 & -24.2 & $1.34 \mathrm{E}-02$ & 60 \\
\hline 147.1 & $11 \mathrm{~A}$ & 16 & -24.1 & $1.18 \mathrm{E}-02$ & 70 \\
\hline 147.1 & $11 \mathrm{~A}$ & 18 & -26.6 & $1.01 \mathrm{E}-02$ & 85 \\
\hline 147.1 & $11 \mathrm{~A}$ & 13.9 & -30.1 & $8.43 \mathrm{E}-03$ & 100 \\
\hline
\end{tabular}

\begin{tabular}{|c|c|c|c|c|c|}
\hline 147.1 & $11 \mathrm{~A}$ & 12.2 & -27.1 & $8.03 E-03$ & 115 \\
\hline 147.4 & $803 \mathrm{~A}$ & 349.4 & 67.2 & $1.80 \mathrm{E}-02$ & 0 \\
\hline 147.4 & $803 \mathrm{~A}$ & 347.6 & 58.5 & $1.34 \mathrm{E}-02$ & 3 \\
\hline 147.4 & $803 \mathrm{~A}$ & 345 & 48.4 & $9.74 \mathrm{E}-03$ & 6 \\
\hline 147.4 & $803 \mathrm{~A}$ & 346.7 & 38.1 & $7.60 \mathrm{E}-03$ & 9 \\
\hline 147.4 & $803 \mathrm{~A}$ & 339.3 & 33.1 & $6.56 \mathrm{E}-03$ & 12 \\
\hline 147.4 & $803 \mathrm{~A}$ & 342.3 & 35 & $5.90 \mathrm{E}-03$ & 15 \\
\hline 147.4 & $803 \mathrm{~A}$ & 342.2 & 37 & $5.29 \mathrm{E}-03$ & 18 \\
\hline 147.4 & $803 \mathrm{~A}$ & 334 & 32 & $4.72 \mathrm{E}-03$ & 21 \\
\hline 147.4 & $803 \mathrm{~A}$ & 337.2 & 34.1 & $2.98 \mathrm{E}-03$ & 25 \\
\hline 147.4 & $803 \mathrm{~A}$ & 324.7 & 62.9 & $2.00 \mathrm{E}-03$ & 30 \\
\hline 147.4 & $803 \mathrm{~A}$ & 353 & 39.4 & $3.85 \mathrm{E}-03$ & 40 \\
\hline 147.4 & $803 \mathrm{~A}$ & 330.2 & 36.1 & $1.13 \mathrm{E}-03$ & 50 \\
\hline 147.4 & $803 \mathrm{~A}$ & 281.7 & 55.8 & $2.27 \mathrm{E}-03$ & 60 \\
\hline 147.4 & $803 \mathrm{~A}$ & 342.6 & 45.5 & $1.08 \mathrm{E}-03$ & 70 \\
\hline 147.4 & $803 \mathrm{~A}$ & 79.6 & 61.4 & $1.18 \mathrm{E}-03$ & 85 \\
\hline 147.4 & $803 \mathrm{~A}$ & 1.4 & 22.7 & $1.50 \mathrm{E}-03$ & 100 \\
\hline 147.4 & $803 \mathrm{~A}$ & 57 & 62.6 & $1.76 \mathrm{E}-03$ & 115 \\
\hline 148.3 & $804 \mathrm{~A}$ & 52.9 & -3.3 & $2.87 \mathrm{E}-02$ & 0 \\
\hline 148.3 & $804 \mathrm{~A}$ & 55.5 & -10.9 & $2.85 \mathrm{E}-02$ & 3 \\
\hline 148.3 & $804 \mathrm{~A}$ & 56.9 & -18.8 & $2.74 \mathrm{E}-02$ & 6 \\
\hline 148.3 & $804 \mathrm{~A}$ & 59.8 & -23.7 & $2.62 \mathrm{E}-02$ & 9 \\
\hline 148.3 & $804 \mathrm{~A}$ & 59.5 & -26.3 & $2.48 \mathrm{E}-02$ & 12 \\
\hline 148.3 & $804 \mathrm{~A}$ & 58 & -27.2 & $2.36 \mathrm{E}-02$ & 15 \\
\hline 148.3 & $804 \mathrm{~A}$ & 58.1 & -27.7 & $2.18 \mathrm{E}-02$ & 18 \\
\hline 148.3 & $804 \mathrm{~A}$ & 59.8 & -29 & $2.07 \mathrm{E}-02$ & 21 \\
\hline 148.3 & $804 \mathrm{~A}$ & 58.3 & -29.9 & $1.80 \mathrm{E}-02$ & 25 \\
\hline 148.3 & $804 \mathrm{~A}$ & 59.6 & -30.4 & $1.57 \mathrm{E}-02$ & 30 \\
\hline 148.3 & $804 \mathrm{~A}$ & 60 & -35.5 & $1.29 \mathrm{E}-02$ & 40 \\
\hline 148.3 & $804 \mathrm{~A}$ & 61 & -29.9 & $1.04 \mathrm{E}-02$ & 50 \\
\hline 148.3 & $804 \mathrm{~A}$ & 63.1 & -30.9 & $8.66 \mathrm{E}-03$ & 60 \\
\hline 148.3 & $804 \mathrm{~A}$ & 58.2 & -37.2 & $7.94 \mathrm{E}-03$ & 70 \\
\hline 148.3 & $804 \mathrm{~A}$ & 64.3 & -31 & $7.60 \mathrm{E}-03$ & 85 \\
\hline 148.3 & $804 \mathrm{~A}$ & 75.8 & -35.4 & $5.39 \mathrm{E}-03$ & 100 \\
\hline 148.3 & $804 \mathrm{~A}$ & 63.4 & -36.4 & $4.88 \mathrm{E}-03$ & 115 \\
\hline 149.3 & $12 \mathrm{~A}$ & 168.1 & 53.2 & $1.61 \mathrm{E}-02$ & 0 \\
\hline 149.3 & $12 \mathrm{~A}$ & 167.4 & 40.2 & $1.36 \mathrm{E}-02$ & 3 \\
\hline 149.3 & $12 \mathrm{~A}$ & 167.7 & 31.7 & $1.24 \mathrm{E}-02$ & 6 \\
\hline 149.3 & $12 \mathrm{~A}$ & 167.3 & 25.8 & $1.13 \mathrm{E}-02$ & 9 \\
\hline 149.3 & $12 \mathrm{~A}$ & 167.1 & 22.6 & $1.08 \mathrm{E}-02$ & 12 \\
\hline 149.3 & $12 \mathrm{~A}$ & 167.3 & 21.1 & $1.00 \mathrm{E}-02$ & 15 \\
\hline 149.3 & $12 \mathrm{~A}$ & 165.6 & 18.8 & $9.30 \mathrm{E}-03$ & 18 \\
\hline 149.3 & $12 \mathrm{~A}$ & 166.8 & 19.1 & $8.60 \mathrm{E}-03$ & 21 \\
\hline 149.3 & $12 \mathrm{~A}$ & 166.4 & 17.4 & $7.72 \mathrm{E}-03$ & 25 \\
\hline 149.3 & $12 \mathrm{~A}$ & 164.1 & 19.3 & $7.01 \mathrm{E}-03$ & 30 \\
\hline 149.3 & $12 \mathrm{~A}$ & 166.8 & 17.4 & $5.32 \mathrm{E}-03$ & 40 \\
\hline 149.3 & $12 \mathrm{~A}$ & 155.2 & 7.8 & $4.05 \mathrm{E}-03$ & 50 \\
\hline 149.3 & $12 \mathrm{~A}$ & 146.4 & 7.4 & $3.37 \mathrm{E}-03$ & 60 \\
\hline 149.3 & $12 \mathrm{~A}$ & 175.4 & 0.1 & $3.35 \mathrm{E}-03$ & 70 \\
\hline 149.3 & $12 \mathrm{~A}$ & 162.2 & -0.3 & $2.43 \mathrm{E}-03$ & 85 \\
\hline 149.3 & $12 \mathrm{~A}$ & 165.6 & 17.7 & $2.22 \mathrm{E}-03$ & 100 \\
\hline
\end{tabular}




\begin{tabular}{|c|c|c|c|c|c|}
\hline & $2 \mathrm{~A}$ & 71.6 & 10.4 & & \\
\hline 0.4 & $13 \mathrm{~A}$ & 32.2 & 84.7 & $69 \mathrm{E}-02$ & \\
\hline 0.4 & $13 \mathrm{~A}$ & 58.6 & 86.4 & $4.74 \mathrm{E}$ & \\
\hline 0.4 & $13 \mathrm{~A}$ & 16.2 & 85.3 & $2.89 \mathrm{E}-$ & \\
\hline 0.4 & $13 \mathrm{~A}$ & 17 & 82.9 & $1.77 \mathrm{E}-$ & \\
\hline 0.4 & $13 \mathrm{~A}$ & 19.5 & 79.8 & $1.33 \mathrm{E}-$ & \\
\hline 0.4 & $13 \mathrm{~A}$ & 24.9 & 79.4 & $1.05 \mathrm{E}-$ & \\
\hline 0.4 & $13 \mathrm{~A}$ & 22.9 & 74.4 & 8.02E-03 & \\
\hline 0.4 & $13 \mathrm{~A}$ & 16.6 & 76.1 & $6.40 \mathrm{E}-$ & \\
\hline 0.4 & $13 \mathrm{~A}$ & 3 & 69.9 & $5.26 \mathrm{E}$ & \\
\hline 0.4 & $13 \mathrm{~A}$ & 2.1 & 74.7 & 3.97E-03 & \\
\hline 0.4 & $13 \mathrm{~A}$ & 356.7 & 89.4 & 2.71E-03 & \\
\hline 0.4 & $13 \mathrm{~A}$ & 328.1 & 54.3 & $1.61 \mathrm{l}$ & \\
\hline 0.4 & $13 \mathrm{~A}$ & 348.5 & 69.2 & $1.65 \mathrm{E}$ & \\
\hline 0.4 & $13 \mathrm{~A}$ & 59.6 & 82.7 & & \\
\hline 0.4 & $13 \mathrm{~A}$ & 11.3 & 52.7 & & \\
\hline 0.4 & $13 \mathrm{~A}$ & 01.2 & 73.6 & & \\
\hline 0.4 & $13 \mathrm{~A}$ & 6.6 & 37 & & \\
\hline 9.9 & $14 \mathrm{~A}$ & 358.6 & 83.4 & & \\
\hline 9.9 & $14 \mathrm{~A}$ & 33.5 & 82.1 & & \\
\hline 9.9 & $14 \mathrm{~A}$ & 51.7 & 78.5 & & \\
\hline 9.9 & $14 \mathrm{~A}$ & 59.4 & 74.1 & & \\
\hline 9.9 & $14 \mathrm{~A}$ & 65.5 & 69.4 & & \\
\hline 9.9 & $14 \mathrm{~A}$ & 55.3 & 68.4 & & \\
\hline 9.9 & $14 \mathrm{~A}$ & 83 & 59.7 & & \\
\hline 9.9 & $14 \mathrm{~A}$ & 69.4 & 57.6 & & \\
\hline 9.9 & $14 \mathrm{~A}$ & 8.1 & 58.2 & & \\
\hline 9.9 & $14 \mathrm{~A}$ & 98 & 55.4 & & \\
\hline 9.9 & $14 \mathrm{~A}$ & 5.6 & 46.2 & & 40 \\
\hline 9.9 & 14A & 7.3 & 36.2 & & 5 \\
\hline 9.9 & $14 \mathrm{~A}$ & 70.9 & 47.6 & & 6 \\
\hline 9.9 & $14 \mathrm{~A}$ & 82.9 & 17.9 & & 7 \\
\hline 59.9 & $14 \mathrm{~A}$ & 84.3 & 0.5 & E-03 & 8 \\
\hline 59.9 & $14 \mathrm{~A}$ & 81 & 16.3 & & 100 \\
\hline 159.9 & $14 \mathrm{~A}$ & 39.5 & 45.9 & $6.47 \mathrm{E}-03$ & 115 \\
\hline 166.1 & $15 \mathrm{~A}$ & 26.2 & 83.3 & $5.67 \mathrm{E}-02$ & \\
\hline 166.1 & $15 \mathrm{~A}$ & 31.1 & 83.5 & $4.11 \mathrm{E}$ & \\
\hline 166.1 & $15 \mathrm{~A}$ & 25.8 & 82.1 & $2.62 \mathrm{E}$ & \\
\hline 166.1 & $15 \mathrm{~A}$ & 76.5 & 48.5 & $1.74 \mathrm{E}-02$ & \\
\hline 166.1 & $15 \mathrm{~A}$ & 320 & 72.4 & $1.18 \mathrm{E}-02$ & 12 \\
\hline 166.1 & $15 \mathrm{~A}$ & 22.6 & 76 & $9.09 \mathrm{E}-03$ & 1 \\
\hline 166.1 & $15 \mathrm{~A}$ & 336.3 & 71.4 & $6.83 \mathrm{E}-03$ & $1 \varepsilon$ \\
\hline 166.1 & $15 \mathrm{~A}$ & 330.2 & 70.8 & & 2 \\
\hline 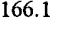 & - & 320.6 & 66.5 & & 2 \\
\hline (2) & 皮 & 347.3 & 63.7 & & $3 c$ \\
\hline 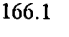 & $15 \mathrm{~A}$ & 16.6 & 30.5 & $1.78 \mathrm{E}-03$ & 40 \\
\hline - & $15 \mathrm{~A}$ & 340.2 & 34.9 & & 50 \\
\hline - & tos & 313.1 & 16.9 & $3.24 \mathrm{E}-03$ & - \\
\hline 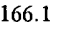 & - & 1 & 32.5 & $2.68 \mathrm{E}-03$ & F \\
\hline - & son & 353.3 & -4.4 & 5.2800 & 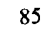 \\
\hline & & 340.3 & -18.7 & & \\
\hline
\end{tabular}

$\begin{array}{llllll}166.1 & 15 \mathrm{~A} & 293.5 & -39.8 & 3.17 \mathrm{E}-03 & 115\end{array}$ $\begin{array}{llllll}167.2 & 805 \mathrm{~A} & 317.1 & 66 & 2.28 \mathrm{E}-02 & 0\end{array}$ $\begin{array}{lllllll}167.2 & 805 \mathrm{~A} & 318.1 & 62.9 & 1.67 \mathrm{E}-02 & 3\end{array}$ $\begin{array}{lllllll}167.2 & 805 \mathrm{~A} & 317.1 & 53.8 & 1.20 \mathrm{E}-02 & 6\end{array}$ $\begin{array}{llllll}167.2 & 805 \mathrm{~A} & 318.3 & 46.8 & 9.30 \mathrm{E}-03 & 9\end{array}$ $\begin{array}{llllll}167.2 & 805 \mathrm{~A} & 320.4 & 41.1 & 7.27 \mathrm{E}-03 & 12\end{array}$ $\begin{array}{lllllll}167.2 & 805 \mathrm{~A} & 321.6 & 35.5 & 6.81 \mathrm{E}-03 & 15\end{array}$ $\begin{array}{lllllll}167.2 & 805 \mathrm{~A} & 324.6 & 31.7 & 6.41 \mathrm{E}-03 & 18\end{array}$ $\begin{array}{lllllll}167.2 & 805 \mathrm{~A} & 320.6 & 27.2 & 6.26 \mathrm{E}-03 & 21\end{array}$ $\begin{array}{lllllll}167.2 & 805 \mathrm{~A} & 323 & 23 & 5.51 \mathrm{E}-03 & 25\end{array}$ $\begin{array}{lllllll}167.2 & 805 \mathrm{~A} & 327.5 & 30.6 & 4.78 \mathrm{E}-03 & 30\end{array}$ $\begin{array}{lllllll}167.2 & 805 \mathrm{~A} & 341.5 & 24.3 & 2.59 \mathrm{E}-03 & 40\end{array}$ $\begin{array}{lllllll}167.2 & 805 \mathrm{~A} & 321.5 & -35.4 & 2.47 \mathrm{E}-03 & 50\end{array}$ $\begin{array}{lllllll}167.2 & 805 \mathrm{~A} & 336.8 & -21 & 1.43 \mathrm{E}-03 & 60\end{array}$ $\begin{array}{lllllll}167.2 & 805 \mathrm{~A} & 307.2 & -15.4 & 1.96 \mathrm{E}-03 & 70\end{array}$ $\begin{array}{lllllll}167.2 & 805 \mathrm{~A} & 241.6 & -74.7 & 8.10 \mathrm{E}-04 & 85\end{array}$ $\begin{array}{lllllll}167.2 & 805 \mathrm{~A} & 262.5 & -33 & 8.35 \mathrm{E}-04 & 100\end{array}$ $\begin{array}{lllllll}167.2 & 805 \mathrm{~A} & 36.7 & 29.2 & 6.69 \mathrm{E}-04 & 115\end{array}$ $\begin{array}{llllll}167.8 & 16 \mathrm{~A} & 295.8 & 35.6 & 2.07 \mathrm{E}-02 & 0\end{array}$ $\begin{array}{llllll}167.8 & 16 \mathrm{~A} & 296.7 & 4.1 & 1.76 \mathrm{E}-02 & 3\end{array}$ $\begin{array}{lllllll}167.8 & 16 \mathrm{~A} & 296.5 & -17 & 1.78 \mathrm{E}-02 & 6\end{array}$ $\begin{array}{lllllll}167.8 & 16 \mathrm{~A} & 296.6 & -25.1 & 1.81 \mathrm{E}-02 & 9\end{array}$ $\begin{array}{lllllll}167.8 & 16 \mathrm{~A} & 295.9 & -27.7 & 1.71 \mathrm{E}-02 & 12\end{array}$ $\begin{array}{lllllll}167.8 & 16 \mathrm{~A} & 294.9 & -31.2 & 1.58 \mathrm{E}-02 & 15\end{array}$ $\begin{array}{lllllll}167.8 & 16 \mathrm{~A} & 294.9 & -32.4 & 1.48 \mathrm{E}-02 & 18\end{array}$ $\begin{array}{lllllll}167.8 & 16 \mathrm{~A} & 296.9 & -32.7 & 1.28 \mathrm{E}-02 & 21\end{array}$ $\begin{array}{lllllll}167.8 & 16 \mathrm{~A} & 295.3 & -33.6 & 1.15 \mathrm{E}-02 & 25\end{array}$ $\begin{array}{lllllll}167.8 & 16 \mathrm{~A} & 295.4 & -33.5 & 1.06 \mathrm{E}-02 & 30\end{array}$ $\begin{array}{lllllll}167.8 & 16 \mathrm{~A} & 296.4 & -34.2 & 7.23 \mathrm{E}-03 & 40\end{array}$ $\begin{array}{lllllll}167.8 & 16 \mathrm{~A} & 307.6 & -39.4 & 5.84 \mathrm{E}-03 & 50\end{array}$ $\begin{array}{lllllll}167.8 & 16 \mathrm{~A} & 302.8 & 41.5 & 4.80 \mathrm{E}-03 & 60\end{array}$ $\begin{array}{lllllll}167.8 & 16 \mathrm{~A} & 293.9 & -44.5 & 3.94 \mathrm{E}-03 & 70\end{array}$ $\begin{array}{llllll}167.8 & 16 \mathrm{~A} & 301 & -39.5 & 4.71 \mathrm{E}-03 & 85\end{array}$ $\begin{array}{lllllll}167.8 & 16 \mathrm{~A} & 299.7 & -59.6 & 3.21 \mathrm{E}-03 & 100\end{array}$ $\begin{array}{lllllll}167.8 & 16 \mathrm{~A} & 305.5 & -36.2 & 3.47 \mathrm{E}-03 & 115\end{array}$ $\begin{array}{lllllll}168 & 806 \mathrm{~A} & 291.4 & -20.5 & 2.07 \mathrm{E}-01 & 0\end{array}$ $\begin{array}{lllllll}168 & 806 \mathrm{~A} & 290.8 & -20.4 & 2.06 \mathrm{E}-01 & 3\end{array}$ $\begin{array}{lllllll}168 & 806 \mathrm{~A} & 290.5 & -21.6 & 1.97 \mathrm{E}-01 & 6\end{array}$ $\begin{array}{lllllll}168 & 806 \mathrm{~A} & 290.4 & -22.4 & 1.80 \mathrm{E}-01 & 9\end{array}$ $\begin{array}{lllllll}168 & 806 \mathrm{~A} & 290.6 & -23 & 1.65 \mathrm{E}-01 & 12\end{array}$ $\begin{array}{lllllll}168 & 806 \mathrm{~A} & 290.6 & -23.4 & 1.50 \mathrm{E}-01 & 15\end{array}$ $\begin{array}{lllllll}168 & 806 \mathrm{~A} & 290.4 & -23 & 1.36 \mathrm{E}-01 & 18\end{array}$ $\begin{array}{lllllll}168 & 806 \mathrm{~A} & 290.4 & -22.7 & 1.23 \mathrm{E}-01 & 21\end{array}$ $\begin{array}{lllllll}168 & 806 \mathrm{~A} & 290 & -22.2 & 1.06 \mathrm{E}-01 & 25\end{array}$ $\begin{array}{lllllll}168 & 806 \mathrm{~A} & 290.3 & -22.9 & 8.88 \mathrm{E}-02 & 30\end{array}$ $\begin{array}{lllllll}168 & 806 \mathrm{~A} & 289.3 & -23.2 & 6.29 \mathrm{E}-02 & 40\end{array}$ $\begin{array}{lllllll}168 & 806 \mathrm{~A} & 289 & -25.3 & 4.58 \mathrm{E}-02 & 50\end{array}$ $\begin{array}{llllllll}168 & 806 \mathrm{~A} & 290.4 & -26.1 & 3.71 \mathrm{E}-02 & 60\end{array}$ $\begin{array}{lllllll}168 & 806 \mathrm{~A} & 290.3 & -25.1 & 3.25 \mathrm{E}-02 & 70\end{array}$ $\begin{array}{lllllll}168 & 806 \mathrm{~A} & 288.6 & -24.9 & 2.74 \mathrm{E}-02 & 85\end{array}$ $\begin{array}{lllllll}168 & 806 \mathrm{~A} & 292.2 & -25.1 & 2.53 \mathrm{E}-02 & 100\end{array}$ $\begin{array}{llllll}168 & 806 \mathrm{~A} & 287.2 & -24.8 & 2.39 \mathrm{E}-02 & 115\end{array}$ $\begin{array}{llllll}172.9 & 17 \mathrm{~A} & 14.1 & 67 & 1.32 \mathrm{E}-02 & 0\end{array}$ $\begin{array}{lllllll}172.9 & 17 \mathrm{~A} & 22.9 & 54.6 & 1.04 \mathrm{E}-02 & 3\end{array}$ $\begin{array}{lllllll}172.9 & 17 \mathrm{~A} & 30.3 & 37.8 & 7.83 \mathrm{E}-03 & 6\end{array}$ $\begin{array}{lllllll}172.9 & 17 \mathrm{~A} & 33.9 & 20.4 & 6.63 \mathrm{E}-03 & 9\end{array}$ $\begin{array}{lllllll}172.9 & 17 \mathrm{~A} & 35.1 & 14.4 & 5.65 \mathrm{E}-03 & 12\end{array}$ $\begin{array}{lllllll}172.9 & 17 \mathrm{~A} & 40.8 & 7.8 & 5.01 \mathrm{E}-0.3 & 15\end{array}$ $\begin{array}{lllllll}172.9 & 17 \mathrm{~A} & 45.9 & 4.2 & 4.53 \mathrm{E}-03 & 18\end{array}$ $\begin{array}{lllllll}172.9 & 17 \mathrm{~A} & 51.8 & -1.9 & 4.41 \mathrm{E}-03 & 21\end{array}$ $\begin{array}{llllll}172.9 & 17 \mathrm{~A} & 61.6 & 2.2 & 3.12 \mathrm{E}-03 & 25\end{array}$ $\begin{array}{llllll}172.9 & 17 \mathrm{~A} & 51.2 & 4 & 3.16 \mathrm{E}-03 & 30\end{array}$ $\begin{array}{lllllll}172.9 & 17 \mathrm{~A} & 45.8 & -10.5 & 2.80 \mathrm{E}-03 & 40\end{array}$ $\begin{array}{llllll}172.9 & 17 \mathrm{~A} & 75.9 & 1.1 & 1.74 \mathrm{E}-03 & 50\end{array}$ $\begin{array}{lllllll}172.9 & 17 \mathrm{~A} & 47.4 & -8.9 & 1.26 \mathrm{E}-03 & 60\end{array}$ $\begin{array}{lllllll}172.9 & 17 \mathrm{~A} & 46.2 & -15.7 & 2.75 \mathrm{E}-03 & 70\end{array}$ $\begin{array}{llllll}172.9 & 17 \mathrm{~A} & 56.6 & 46 & 1.71 \mathrm{E}-03 & 85\end{array}$ $\begin{array}{lllllll}172.9 & 17 \mathrm{~A} & 117.1 & 8.3 & 2.11 \mathrm{E}-03 & 100\end{array}$ $\begin{array}{lllllll}172.9 & 17 \mathrm{~A} & 96 & -53.7 & 8.21 \mathrm{E}-04 & 115\end{array}$ $\begin{array}{lllllll}173.7 & 807 \mathrm{~A} & 328.3 & 68.1 & 2.69 \mathrm{E}-02 & 0\end{array}$ $\begin{array}{lllllll}173.7 & 807 \mathrm{~A} & 334.6 & 68.5 & 2.08 \mathrm{E}-02 & 3\end{array}$ $\begin{array}{lllllll}173.7 & 807 \mathrm{~A} & 331.1 & 64.5 & 1.43 \mathrm{E}-02 & 6\end{array}$ $\begin{array}{lllllll}173.7 & 807 \mathrm{~A} & 331.2 & 60.4 & 1.05 \mathrm{E}-02 & 9\end{array}$ $\begin{array}{lllllll}173.7 & 807 \mathrm{~A} & 329.1 & 54.4 & 7.84 \mathrm{E}-03 & 12\end{array}$ $\begin{array}{lllllll}173.7 & 807 \mathrm{~A} & 325 & 59.1 & 6.20 \mathrm{E}-03 & 15\end{array}$ $\begin{array}{lllllll}173.7 & 807 \mathrm{~A} & 313.9 & 58.8 & 5.16 \mathrm{E}-03 & 18\end{array}$ $\begin{array}{lllllll}173.7 & 807 \mathrm{~A} & 327.9 & 54 & 4.98 \mathrm{E}-03 & 21\end{array}$ $\begin{array}{lllllll}173.7 & 807 \mathrm{~A} & 315.6 & 53.5 & 4.09 \mathrm{E}-03 & 25\end{array}$ $\begin{array}{lllllll}173.7 & 807 \mathrm{~A} & 317 & 62.9 & 2.53 \mathrm{E}-03 & 30\end{array}$ $\begin{array}{lllllll}173.7 & 807 \mathrm{~A} & 218 & 75.4 & 2.03 \mathrm{E}-03 & 40\end{array}$ $\begin{array}{lllllll}173.7 & 807 \mathrm{~A} & 223.3 & 62.9 & 1.03 \mathrm{E}-03 & 50\end{array}$ $\begin{array}{llllllll}173.7 & 807 \mathrm{~A} & 139.2 & 48.3 & 3.80 \mathrm{E}-04 & 60\end{array}$ $\begin{array}{lllllll}173.7 & 807 \mathrm{~A} & 247.8 & 35.4 & 1.23 \mathrm{E}-03 & 70\end{array}$ $\begin{array}{lllllll}173.7 & 807 \mathrm{~A} & 58.2 & -28.7 & 2.18 \mathrm{E}-03 & 85\end{array}$ $\begin{array}{lllllll}173.7 & 807 \mathrm{~A} & 12.8 & -33.9 & 2.05 \mathrm{E}-03 & 100\end{array}$ $\begin{array}{lllllll}173.7 & 807 \mathrm{~A} & 304.2 & 63.4 & 9.84 \mathrm{E}-04 & 115\end{array}$ $\begin{array}{lllllll}176.5 & 18 \mathrm{~A} & 333.6 & 68.9 & 1.79 \mathrm{E}-02 & 0\end{array}$ $\begin{array}{llllll}176.5 & 18 \mathrm{~A} & 355.2 & 65.9 & 1.21 \mathrm{E}-02 & 3\end{array}$ $\begin{array}{lllllll}176.5 & 18 \mathrm{~A} & 18.9 & 65.9 & 7.98 \mathrm{E}-03 & 6\end{array}$ $\begin{array}{lllllll}176.5 & 18 \mathrm{~A} & 35.6 & 60.4 & 6.35 \mathrm{E}-03 & 9\end{array}$ $\begin{array}{lllllll}176.5 & 18 \mathrm{~A} & 49.2 & 61.2 & 4.97 \mathrm{E}-03 & 12\end{array}$ $\begin{array}{lllllll}176.5 & 18 \mathrm{~A} & 62.1 & 69.4 & 3.97 \mathrm{E}-03 & 15\end{array}$ $\begin{array}{lllllll}176.5 & 18 \mathrm{~A} & 73 & 65.5 & 3.99 \mathrm{E}-03 & 18\end{array}$ $\begin{array}{lllllll}176.5 & 18 \mathrm{~A} & 72.7 & 57.5 & 3.09 \mathrm{E}-03 & 21\end{array}$ $\begin{array}{lllllll}176.5 & 18 \mathrm{~A} & 78.4 & 75.7 & 2.68 \mathrm{E}-03 & 25\end{array}$ $\begin{array}{lllllll}176.5 & 18 \mathrm{~A} & 72.9 & 60.8 & 2.73 \mathrm{E}-03 & 30\end{array}$ $\begin{array}{lllllll}176.5 & 18 \mathrm{~A} & 181.4 & 68.3 & 2.88 \mathrm{E}-03 & 40\end{array}$ $\begin{array}{lllllll}176.5 & 18 \mathrm{~A} & 224.2 & 61.2 & 1.82 \mathrm{E}-03 & 50\end{array}$ $\begin{array}{lllllll}176.5 & 18 \mathrm{~A} & 231.1 & 26.4 & 1.49 \mathrm{E}-03 & 60\end{array}$ $\begin{array}{lllllll}176.5 & 18 \mathrm{~A} & 292.9 & \mathbf{4 2 . 8} & 2.59 \mathrm{E}-03 & 70\end{array}$ $\begin{array}{lllllll}176.5 & 18 \mathrm{~A} & 205.5 & -23.5 & 3.19 \mathrm{E}-03 & 85\end{array}$ $\begin{array}{llllll}176.5 & 18 \mathrm{~A} & 230.5 & -14.4 & 2.51 \mathrm{E}-03 & 100\end{array}$ 


\begin{tabular}{|c|c|c|c|c|c|}
\hline & $18 \mathrm{~A}$ & 227 & 66.1 & & \\
\hline 9.7 & $19 \mathrm{~A}$ & 42.1 & 67.8 & $9.41 \mathrm{E}-02$ & \\
\hline 9.7 & $19 \mathrm{~A}$ & 38.8 & 68.2 & $6.90 \mathrm{E}-02$ & \\
\hline 9.7 & $19 \mathrm{~A}$ & 33.9 & 67.5 & 4.16E-02 & \\
\hline 9.7 & $19 \mathrm{~A}$ & 330.9 & 64.4 & $2.52 \mathrm{E}-02$ & \\
\hline 9.7 & $19 \mathrm{~A}$ & 324.8 & 62.9 & $1.87 \mathrm{E}-02$ & \\
\hline 9.7 & $19 \mathrm{~A}$ & 318.8 & 62.9 & $1.45 \mathrm{E}-02$ & \\
\hline 9.7 & $19 \mathrm{~A}$ & 317.4 & 59 & $1.09 \mathrm{E}-02$ & \\
\hline 9.7 & $19 \mathrm{~A}$ & 313.8 & 60.2 & $8.93 \mathrm{E}-03$ & \\
\hline 79.7 & $19 \mathrm{~A}$ & 308.4 & 53.6 & $7.22 \mathrm{E}-03$ & \\
\hline 9.7 & $19 \mathrm{~A}$ & 307.1 & 49.9 & $4.80 \mathrm{E}-03$ & \\
\hline 9.7 & $19 \mathrm{~A}$ & 289.2 & 33.3 & $3.72 \mathrm{E}-03$ & \\
\hline 9.7 & $19 \mathrm{~A}$ & 260.4 & 10.7 & 2.37E-03 & \\
\hline 9.7 & $19 \mathrm{~A}$ & 91.8 & -2 & $2.16 \mathrm{E}-03$ & \\
\hline 9.7 & $19 \mathrm{~A}$ & 78.5 & 45.8 & $1.45 \mathrm{E}-03$ & \\
\hline 79.7 & $19 \mathrm{~A}$ & 308 & 48.7 & 2.521 & \\
\hline 9.7 & 19A & 311 & 43.8 & 1.821 & 10 \\
\hline 79.7 & $19 \mathrm{~A}$ & 91.8 & -28.3 & 1.87 & . \\
\hline 30.6 & $20 \mathrm{~A}$ & 54.7 & 45.8 & & \\
\hline 30.6 & $20 \mathrm{~A}$ & 57.8 & -53.4 & & \\
\hline 30.6 & $20 \mathrm{~A}$ & 58.3 & -56 & & \\
\hline 30.6 & $20 \mathrm{~A}$ & 59.1 & -56.9 & 1.51 & \\
\hline 180.6 & $20 \mathrm{~A}$ & 159 & -57.6 & & 1 \\
\hline 180.6 & $20 \mathrm{~A}$ & 158.6 & -58.5 & & \\
\hline 180.6 & $20 \mathrm{~A}$ & 57.8 & -59.5 & & \\
\hline 180.6 & $20 \mathrm{~A}$ & 54.7 & -59.3 & & \\
\hline 180.6 & $20 \mathrm{~A}$ & 157.4 & -59.5 & 9.82 & \\
\hline 180.6 & $20 \mathrm{~A}$ & 59.2 & -62 & & \\
\hline 30.6 & $20 \mathrm{~A}$ & 162.7 & -56.6 & & \\
\hline 50.6 & $20 \mathrm{~A}$ & 52.6 & -65.9 & & \\
\hline 0.6 & $20 \mathrm{~A}$ & 158.8 & -69.6 & & \\
\hline 0.6 & $20 \mathrm{~A}$ & 80.2 & -56.3 & & \\
\hline 0.6 & $20 \mathrm{~A}$ & 61.8 & -68.7 & & \\
\hline 0.9 & 303 & 286.8 & 75.4 & & \\
\hline 0.9 & 303 & 278.2 & 66.2 & & \\
\hline 0.9 & 303 & 267 & 54.2 & & \\
\hline 0.9 & 303 & 60.7 & 39.6 & & \\
\hline 0.9 & 303 & 8.9 & 30.1 & & \\
\hline 0.9 & 303 & 4.2 & 23.5 & & 1 \\
\hline U. & $303 t$ & 36.6 & 18 & 8.37E-03 & 18 \\
\hline 20. & $303 A$ & 252.5 & 13.5 & $6.97 \mathrm{E}-03$ & 2 \\
\hline (2). & $303 \mathrm{~A}$ & 251.6 & 15 & $5.93 \mathrm{E}-03$ & 25 \\
\hline 180.9 & $303 \mathrm{~A}$ & 261.4 & 9.1 & $5.46 \mathrm{E}-03$ & 30 \\
\hline 180.9 & $303 \mathrm{~A}$ & 250.5 & -2.3 & $3.90 \mathrm{E}-03$ & 40 \\
\hline 180.9 & $303 \mathrm{~A}$ & 238.9 & 2.2 & $3.18 \mathrm{E}-03$ & \\
\hline 180.9 & $303 \mathrm{~A}$ & 267.5 & -15 & $2.91 \mathrm{E}-03$ & 60 \\
\hline 180.9 & $303 \mathrm{~A}$ & 287.2 & -0.5 & $2.61 \mathrm{E}-03$ & 70 \\
\hline 180.9 & $303 \mathrm{~A}$ & 229.7 & -28.5 & 3.32E-03 & 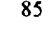 \\
\hline 180.9 & $303 \mathrm{~A}$ & 265.8 & -13.3 & $1.49 \mathrm{E}-03$ & 100 \\
\hline 50.5 & $303 \mathrm{~A}$ & 303.6 & -56.4 & $1.90 \mathrm{E}-103$ & 10 \\
\hline & $302 \mathrm{~A}$ & 219.1 & 38 & $0.00 \mathrm{~L}-\mathrm{J}$ & \\
\hline
\end{tabular}

$\begin{array}{llllll}182.5 & 302 \mathrm{~A} & 219.4 & 14.9 & 7.19 \mathrm{E}-03 & 3\end{array}$ $\begin{array}{lllllll}182.5 & 302 \mathrm{~A} & 220.3 & 2.3 & 7.07 \mathrm{E}-03 & 6\end{array}$ $\begin{array}{lllllll}182.5 & 302 \mathrm{~A} & 222.1 & -3.6 & 6.78 \mathrm{E}-03 & 9\end{array}$ $\begin{array}{lllllll}182.5 & 302 \mathrm{~A} & 221.4 & -6.8 & 6.55 \mathrm{E}-03 & 12\end{array}$ $\begin{array}{lllllll}182.5 & 302 \mathrm{~A} & 219.6 & -10.6 & 6.09 \mathrm{E}-03 & 15\end{array}$ $\begin{array}{lllllll}182.5 & 302 \mathrm{~A} & 220.7 & -9.8 & 5.77 \mathrm{E}-03 & 18\end{array}$ $\begin{array}{lllllll}182.5 & 302 \mathrm{~A} & 218.5 & -11.1 & 5.27 \mathrm{E}-03 & 21\end{array}$ $\begin{array}{lllllll}182.5 & 302 \mathrm{~A} & 216.1 & -15.1 & 4.71 \mathrm{E}-03 & 25\end{array}$ $\begin{array}{lllllll}182.5 & 302 \mathrm{~A} & 216.8 & -18.1 & 4.29 \mathrm{E}-03 & 30\end{array}$ $\begin{array}{lllllll}182.5 & 302 \mathrm{~A} & 215.2 & -22.6 & 3.16 \mathrm{E}-03 & 40\end{array}$ $\begin{array}{llllll}182.5 & 302 \mathrm{~A} & 206.5 & -18.1 & 3.15 \mathrm{E}-03 & 50\end{array}$ $\begin{array}{lllllll}182.5 & 302 \mathrm{~A} & 209.4 & -15.9 & 2.66 \mathrm{E}-03 & 60\end{array}$ $\begin{array}{llllll}182.5 & 302 \mathrm{~A} & 218.7 & -0.5 & 2.41 \mathrm{E}-03 & 70\end{array}$ $\begin{array}{lllllll}182.5 & 302 \mathrm{~A} & 202.7 & -28.6 & 2.08 \mathrm{E}-03 & 85\end{array}$ $\begin{array}{lllllll}182.5 & 302 \mathrm{~A} & 223.1 & -20.4 & 1.60 \mathrm{E}-03 & 100\end{array}$ $\begin{array}{lllllll}182.5 & 302 \mathrm{~A} & 220.9 & -21.4 & 1.72 \mathrm{E}-03 & 115\end{array}$ $\begin{array}{llllll}192.4 & 21 \mathrm{~A} & 159.5 & 83 & 1.13 \mathrm{E}-01 & 0\end{array}$ $\begin{array}{lllllll}192.4 & 21 \mathrm{~A} & 147.5 & 82.5 & 8.45 \mathrm{E}-02 & 3\end{array}$ $\begin{array}{lllllll}192.4 & 21 \mathrm{~A} & 150.5 & 81.9 & 5.35 \mathrm{E}-02 & 6\end{array}$ $\begin{array}{lllllll}192.4 & 21 \mathrm{~A} & 147.6 & 80.3 & 3.28 \mathrm{E}-02 & 9\end{array}$ $\begin{array}{llllll}192.4 & 21 \mathrm{~A} & 156 & 82.3 & 2.39 \mathrm{E}-02 & 12\end{array}$ $\begin{array}{lllllll}192.4 & 21 \mathrm{~A} & 142.1 & 81.6 & 1.87 \mathrm{E}-02 & 15\end{array}$ $\begin{array}{lllllll}192.4 & 21 \mathrm{~A} & 149.5 & 79.8 & 1.31 \mathrm{E}-02 & 18\end{array}$ $\begin{array}{llllll}192.4 & 21 \mathrm{~A} & 144.5 & 82.6 & 1.10 \mathrm{E}-02 & 21\end{array}$ $\begin{array}{lllllll}192.4 & 21 \mathrm{~A} & 251 & 80.7 & 6.65 \mathrm{E}-03 & 25\end{array}$ $\begin{array}{lllllll}192.4 & 21 \mathrm{~A} & 196.3 & 73.4 & 4.38 \mathrm{E}-03 & 30\end{array}$ $\begin{array}{llllll}192.4 & 21 \mathrm{~A} & 264.6 & 59 & 2.96 \mathrm{E}-03 & 40\end{array}$ $\begin{array}{llllll}192.4 & 21 \mathrm{~A} & 321.8 & 43 & 2.99 \mathrm{E}-03 & 50\end{array}$ $\begin{array}{llllll}192.4 & 21 \mathrm{~A} & 276.5 & 26.3 & 2.09 \mathrm{E}-03 & 60\end{array}$ $\begin{array}{lllllll}192.4 & 21 \mathrm{~A} & 274.2 & 12.4 & 2.36 \mathrm{E}-03 & 70\end{array}$ $\begin{array}{lllllll}192.4 & 21 \mathrm{~A} & 270.3 & -11.8 & 3.97 \mathrm{E}-03 & 85\end{array}$ $\begin{array}{lllllll}192.4 & 21 \mathrm{~A} & 284.7 & 1.4 & 4.06 \mathrm{E}-03 & 100\end{array}$ $\begin{array}{lllllll}192.4 & 21 \mathrm{~A} & 277.9 & 25.2 & 3.32 \mathrm{E}-03 & 115\end{array}$ $\begin{array}{lllllll}201 & 304 \mathrm{~A} & 276.4 & 69.6 & 2.20 \mathrm{E}-02 & 0\end{array}$ $\begin{array}{llllll}201 & 304 \mathrm{~A} & 275.5 & 68.7 & 1.81 \mathrm{E}-02 & 3\end{array}$ $\begin{array}{lllllll}201 & 304 \mathrm{~A} & 279 & 71.2 & 1.30 \mathrm{E}-02 & 6\end{array}$ $\begin{array}{llllll}201 & 304 \mathrm{~A} & 279.7 & 71.8 & 9.99 \mathrm{E}-03 & 9\end{array}$ $\begin{array}{llllll}201 & 304 \mathrm{~A} & 256 & 70.6 & 7.47 \mathrm{E}-03 & 12\end{array}$ $\begin{array}{llllll}201 & 304 \mathrm{~A} & 240.3 & 74.9 & 5.91 \mathrm{E}-03 & 15\end{array}$ $\begin{array}{lllllll}201 & 304 \mathrm{~A} & 230.3 & 75.6 & 5.97 \mathrm{E}-03 & 18\end{array}$ $\begin{array}{lllllll}201 & 304 \mathrm{~A} & 180.1 & 75.7 & 4.99 \mathrm{E}-03 & 21\end{array}$ $\begin{array}{lllllll}201 & 304 \mathrm{~A} & 235.3 & 70.9 & 3.48 \mathrm{E}-03 & 25\end{array}$ $\begin{array}{llllll}201 & 304 \mathrm{~A} & 225.2 & 76.5 & 3.55 \mathrm{E}-03 & 30\end{array}$ $201 \quad 304 \mathrm{~A} \quad 166.3 \quad 61.2 \quad 4.77 \mathrm{E}-03 \quad 40$ $\begin{array}{llllll}201 & 304 \mathrm{~A} & 214 & 66.4 & 3.04 \mathrm{E}-03 & 50\end{array}$ $\begin{array}{llllll}201 & 304 \mathrm{~A} & 289.5 & 55.2 & 2.94 \mathrm{E}-03 & 60\end{array}$ $\begin{array}{lllllll}201 & 304 \mathrm{~A} & 207.9 & 41 & 3.44 \mathrm{E}-03 & 70\end{array}$ $\begin{array}{llllll}201 & 304 \mathrm{~A} & 173.8 & -75.3 & 2.42 \mathrm{E}-03 & 85\end{array}$ $\begin{array}{lllllll}201 & 304 \mathrm{~A} & 135.9 & -36.4 & 1.99 \mathrm{E}-03 & 100\end{array}$ $\begin{array}{llllll}201 & 304 \mathrm{~A} & 217.2 & 41.8 & 1.92 \mathrm{E}-03 & 115\end{array}$ $\begin{array}{llllll}201.3 & 305 \mathrm{~A} & 35.2 & 78.4 & 7.74 \mathrm{E}-03 & 3\end{array}$

\begin{tabular}{|c|c|c|c|c|c|}
\hline & $305 \mathrm{~A}$ & 79 & 59.3 & $4.48 \mathrm{E}$ & \\
\hline & $305 \mathrm{~A}$ & 3.9 & 25.7 & $4.22 \mathrm{E}$ & \\
\hline & $305 \mathrm{~A}$ & 1.6 & 2.7 & $2.83 \mathrm{E}-03$ & $12+2$ \\
\hline & $305 \mathrm{~A}$ & 2.9 & -19.5 & $3.55 \mathrm{E}-03$ & . \\
\hline & $305 \mathrm{~A}$ & 5.8 & -11.3 & 4.41E-03 & \\
\hline & $305 \mathrm{~A}$ & 0.9 & -20.9 & $3.87 \mathrm{E}-03$ & 2 \\
\hline 1.3 & $305 \mathrm{~A}$ & 02.6 & -17.4 & $5.39 \mathrm{E}-03$ & 2. \\
\hline 1.3 & $305 \mathrm{~A}$ & 6.9 & -30.9 & 4.64E-03 & 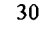 \\
\hline 1.3 & $305 \mathrm{~A}$ & 89.7 & -27.1 & $4.18 \mathrm{E}-03$ & $x^{2}+2>$ \\
\hline 1.3 & $305 \mathrm{~A}$ & 01.4 & 47.4 & $4.45 \mathrm{E}-03$ & . \\
\hline 1.3 & $305 \mathrm{~A}$ & 67 & -37.8 & $2.98 \mathrm{E}$ & \\
\hline 1.3 & $305 \mathrm{~A}$ & 76.9 & -23.5 & $4.82 \mathrm{E}-03$ & \\
\hline 1.3 & $305 \mathrm{~A}$ & 114 & -52.9 & $4.10 \mathrm{E}$ & \\
\hline 1.3 & $305 \mathrm{~A}$ & 90 & -35.4 & $8.21 \mathrm{E}$ & 100 \\
\hline 1.3 & $305 \mathrm{~A}$ & 89 & 43.2 & $6.98 \mathrm{I}$ & 115 \\
\hline 1.7 & 306 & 8.4 & 71.5 & 1.53 & \\
\hline 1.7 & 306 & 14.3 & 66.4 & 1.17 & \\
\hline 1.7 & 306 & 26.8 & 57.3 & 8.67 & \\
\hline 1.7 & 306 & 28.4 & 46.2 & 7.05 & \\
\hline 1.7 & 306 & 38.7 & 32.8 & & 12 \\
\hline 1.7 & 306 & 44.6 & 31.6 & 5.80 & 13 \\
\hline 1.7 & 306 & 46.6 & 31.5 & 5.54 & 18 \\
\hline 1.7 & 306 & 46.9 & 28.7 & 5.11 & 21 \\
\hline 1.7 & 306 & 49.4 & 22.7 & 5.37 & 23 \\
\hline 1.7 & 306 & 56.1 & 37.9 & & 30 \\
\hline & 306 & 37.5 & 20.6 & & +0 \\
\hline 1.7 & 306 & 58.4 & 32.7 & & 50 \\
\hline 1.7 & 306 & 93 & 56.7 & & 10 \\
\hline 1.7 & 306 & 48.9 & 19.8 & 3.12 & 10 \\
\hline 1.7 & 306 & 90.8 & 2 & 2.84 & 3 \\
\hline & 306 & 58.8 & 22.7 & & \\
\hline & 306 & 53.3 & 15.4 & & 15 \\
\hline & $22 \mathrm{~A}$ & 59.4 & 56.4 & & \\
\hline & $22 \mathrm{~A}$ & 60.5 & 55.3 & & \\
\hline & $2 \mathrm{~A}$ & 7.3 & 58.9 & & \\
\hline & $2 \mathrm{~A}$ & 1.4 & 11.3 & & \\
\hline & 2 & 3.7 & 14.5 & & 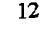 \\
\hline & 2 & 46.2 & 56.3 & & 15 \\
\hline 203.5 & $22 \mathrm{~A}$ & 63.2 & 12.9 & 1.41E-02 & 18 \\
\hline 203.5 & $22 \mathrm{~A}$ & 40 & 53.2 & $1.16 \mathrm{E}-02$ & 21 \\
\hline 203.5 & $22 \mathrm{~A}$ & 32.7 & 64.4 & $9.45 \mathrm{E}-03$ & 25 \\
\hline 203.5 & $22 \mathrm{~A}$ & 22.5 & 53.6 & $5.91 \mathrm{E}-03$ & 30 \\
\hline 203.5 & $22 \mathrm{~A}$ & 350.5 & 49.2 & 4.62E-03 & 40 \\
\hline 203.5 & $22 \mathrm{~A}$ & 91.3 & -5.1 & $3.44 \mathrm{E}-03$ & 50 \\
\hline$x^{2}$ & $22 \mathrm{~A}$ & 78.9 & -9.6 & $1.58 \mathrm{E}-03$ & \\
\hline (50) & $22 \mathrm{~A}$ & 254 & -0.5 & 4.92E-03 & 70 \\
\hline (5) & $22 \mathrm{~A}$ & 141.9 & -67.4 & $2.32 \mathrm{E}-03$ & 85 \\
\hline$x_{-1}$ & $22 \mathrm{~A}$ & 245.4 & 19.2 & $6.60 \mathrm{E}-03$ & 100 \\
\hline 200.0 & $22 \mathrm{~A}$ & 247.7 & -3.8 & $5.44 \mathrm{E}-03$ & 115 \\
\hline (n) & ( & 273.8 & 41.3 & $2.93 \mathrm{E}-02$ & 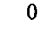 \\
\hline & (נ) & $2 / 3.8$ & 30.4 & $2.02 \mathrm{E}-02$ & \\
\hline
\end{tabular}




\begin{tabular}{|c|c|c|c|c|c|}
\hline 06 & $3 \mathrm{~A}$ & 273.9 & 37.2 & $2.39 \mathrm{D}=$ & \\
\hline 206 & $23 \mathrm{~A}$ & 274.1 & 35.9 & $2.09 \mathrm{E}-02$ & \\
\hline 06 & $23 \mathrm{~A}$ & 274 & 36.7 & $1.87 \mathrm{E}-02$ & \\
\hline 06 & $23 \mathrm{~A}$ & 275 & 38 & $1.69 \mathrm{E}-02$ & \\
\hline 06 & $3 \mathrm{~A}$ & 73.9 & 36.7 & $1.53 \mathrm{E}-02$ & \\
\hline 06 & $23 \mathrm{~A}$ & 273.5 & 38.4 & $1.33 \mathrm{E}-02$ & \\
\hline 06 & $23 \mathrm{~A}$ & 276 & 36.4 & $1.15 \mathrm{E}-02$ & \\
\hline 06 & $23 \mathrm{~A}$ & 275.7 & 37.7 & $9.85 \mathrm{E}-03$ & \\
\hline 06 & $23 \mathrm{~A}$ & 273.9 & 39.6 & $7.31 \mathrm{E}-03$ & \\
\hline 06 & $23 \mathrm{~A}$ & 273.6 & 38.6 & $7.26 \mathrm{E}-03$ & \\
\hline 206 & $23 \mathrm{~A}$ & 275 & 39.8 & $5.96 \mathrm{E}-03$ & \\
\hline 06 & $3 \mathrm{~A}$ & 81.3 & 38.5 & $4.83 \mathrm{E}-03$ & \\
\hline 06 & $23 \mathrm{~A}$ & 273 & 40.1 & $2 \mathrm{E}-03$ & \\
\hline 06 & $23 \mathrm{~A}$ & 84.2 & 40.1 & $3.74 \mathrm{E}-03$ & \\
\hline 206 & $23 \mathrm{~A}$ & 87.6 & 35.9 & E-03 & \\
\hline 06 & $23 \mathrm{~A}$ & 88.7 & 36.8 & $3.20 \mathrm{E}-03$ & \\
\hline 084 & $24 \mathrm{~A}$ & 60.9 & 41.9 & $4.66 \mathrm{E}-02$ & \\
\hline 2084 & $24 \mathrm{~A}$ & 61.8 & 36.3 & $4.39 \mathrm{E}-02$ & \\
\hline 208.4 & $24 \mathrm{~A}$ & 62.3 & 33.2 & $4.09 \mathrm{E}-02$ & \\
\hline 208.4 & $24 \mathrm{~A}$ & 263 & 31.1 & $3.81 \mathrm{E}-02$ & \\
\hline 08.4 & $24 \mathrm{~A}$ & 263.2 & 30.3 & $3.51 \mathrm{E}-02$ & \\
\hline 08.4 & $24 \mathrm{~A}$ & 63.2 & 31.4 & $3.19 \mathrm{E}-02$ & \\
\hline 08.4 & $24 \mathrm{~A}$ & 263.2 & 30.8 & & \\
\hline 08.4 & $24 \mathrm{~A}$ & 262.9 & 31.7 & & \\
\hline 08.4 & $24 \mathrm{~A}$ & 263.2 & 30.8 & & \\
\hline 08.4 & $24 \mathrm{~A}$ & 265.4 & 29.5 & $1.97 \mathrm{E}-02$ & \\
\hline 08.4 & $24 \mathrm{~A}$ & 61.4 & 27.7 & $1.44 \mathrm{E}-02$ & \\
\hline 08.4 & $24 \mathrm{~A}$ & 269.8 & 29.3 & $1.10 \mathrm{E}-02$ & \\
\hline 08.4 & $24 \mathrm{~A}$ & 72.8 & 33.1 & $8.64 \mathrm{E}-03$ & \\
\hline 08.4 & $24 \mathrm{~A}$ & 55.8 & 29.2 & $8.48 \mathrm{E}-03$ & \\
\hline 08.4 & 4 & 79.2 & 32.4 & & \\
\hline 08.4 & 14 & 54.2 & 25.7 & & 1 \\
\hline 08.4 & 4 & 51.6 & 24.7 & & 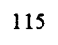 \\
\hline 12.4 & & 37.3 & 65.9 & & \\
\hline 12.4 & $25 \mathrm{~A}$ & 141 & 60.2 & & \\
\hline 12.4 & $25 \mathrm{~A}$ & 45.4 & 55.8 & & \\
\hline 12.4 & $25 \mathrm{~A}$ & 147.8 & 51.3 & $1.02 \mathrm{E}-02$ & \\
\hline 12.4 & $25 \mathrm{~A}$ & & 51.5 & & \\
\hline 12.4 & & 150.9 & 50.2 & & \\
\hline 12.4 & $25 \mathrm{~A}$ & 152 & 48.2 & & \\
\hline 012.4 & $25 \mathrm{~A}$ & 156.1 & 48.7 & & \\
\hline 12.4 & $25 \mathrm{~A}$ & 155.3 & 47.7 & & \\
\hline 112.7 & $25 \mathrm{~A}$ & 159.5 & 50.8 & & \\
\hline 12.4 & $25 \mathrm{~A}$ & 159.2 & 46 & $3.67 \mathrm{E}-03$ & \\
\hline 12.4 & $25 \mathrm{~A}$ & 104.7 & 59 & & \\
\hline 12.4 & $25 \mathrm{~A}$ & 147 & 62.4 & & \\
\hline 10.4 & $25 \mathrm{~A}$ & 157.8 & 24.3 & & $r$ \\
\hline 10,7 & $25 \mathrm{~A}$ & 343.5 & 62.4 & & 0 \\
\hline 12. & $25 \mathrm{~A}$ & 148.6 & 11.4 & & \\
\hline ( & $25 \mathrm{~A}$ & 180.3 & 33.6 & $1.86 \mathrm{E}-03$ & \\
\hline & $20 \mathrm{~A}$ & 11.3 & 13.3 & $1.17 \mathrm{E}-02$ & \\
\hline
\end{tabular}

\begin{tabular}{|c|c|c|c|c|c|c|c|c|c|c|c|}
\hline 221.5 & $26 \mathrm{~A}$ & 15.7 & 73.5 & $5.79 \mathrm{E}-02$ & 3 & 239.1 & $29 \mathrm{~A}$ & 347.8 & 82 & 2.13E-02 & 0 \\
\hline 221.5 & $26 \mathrm{~A}$ & 21.2 & 74 & $3.73 \mathrm{E}-02$ & 6 & 239.1 & $29 \mathrm{~A}$ & 354.9 & 80.7 & $1.66 \mathrm{E}-02$ & 3 \\
\hline 221.5 & $26 \mathrm{~A}$ & 28.1 & 71.6 & $2.28 \mathrm{E}-02$ & 9 & 239.1 & $29 \mathrm{~A}$ & 351.1 & 80.7 & $1.42 \mathrm{E}-02$ & 6 \\
\hline 221.5 & $26 \mathrm{~A}$ & 18.9 & 69.7 & $1.73 \mathrm{E}-02$ & 12 & 239.1 & $29 \mathrm{~A}$ & 352.9 & 78.4 & $1.20 \mathrm{E}-02$ & 9 \\
\hline 221.5 & $26 \mathrm{~A}$ & 33.8 & 67.1 & $1.30 \mathrm{E}-02$ & 15 & 239.1 & $29 \mathrm{~A}$ & 0.6 & 78.1 & $1.05 \mathrm{E}-02$ & 12 \\
\hline 221.5 & $26 \mathrm{~A}$ & 47.8 & 73.1 & $9.15 \mathrm{E}-03$ & 18 & 239.1 & $29 \mathrm{~A}$ & 359.3 & 78.1 & $9.92 \mathrm{E}-03$ & 15 \\
\hline 221.5 & $26 \mathrm{~A}$ & 84.6 & 63.1 & $8.14 \mathrm{E}-03$ & 21 & 239.1 & $29 \mathrm{~A}$ & 355.6 & 79.2 & $9.08 \mathrm{E}-03$ & 18 \\
\hline 221.5 & $26 \mathrm{~A}$ & 62.3 & 78.9 & $5.76 \mathrm{E}-03$ & 25 & 239.1 & $29 \mathrm{~A}$ & 9.7 & 75.8 & $8.00 \mathrm{E}-03$ & 21 \\
\hline 221.5 & $26 \mathrm{~A}$ & 93.1 & 80.6 & $5.69 \mathrm{E}-03$ & 30 & 239.1 & $29 \mathrm{~A}$ & 349 & 75.3 & $6.90 \mathrm{E}-03$ & 25 \\
\hline 221.5 & $26 \mathrm{~A}$ & 163.2 & 40.1 & $2.80 \mathrm{E}-03$ & 40 & 239.1 & $29 \mathrm{~A}$ & 342.9 & 76.2 & $5.27 \mathrm{E}-03$ & 30 \\
\hline 221.5 & $26 \mathrm{~A}$ & 163.7 & 47.2 & $2.94 \mathrm{E}-03$ & 50 & 239.1 & $29 \mathrm{~A}$ & 0.1 & 73.2 & $4.05 \mathrm{E}-03$ & 40 \\
\hline 221.5 & $26 \mathrm{~A}$ & 122.2 & 35.6 & $2.36 \mathrm{E}-03$ & 60 & 239.1 & $29 \mathrm{~A}$ & 338.7 & 61.3 & $3.12 \mathrm{E}-03$ & 50 \\
\hline 221.5 & $26 \mathrm{~A}$ & 120.4 & 4.4 & $1.64 \mathrm{E}-03$ & 70 & 239.1 & $29 \mathrm{~A}$ & 348.6 & 72.4 & $3.08 \mathrm{E}-03$ & 60 \\
\hline 221.5 & $26 \mathrm{~A}$ & 151.1 & -23.5 & $1.38 \mathrm{E}-03$ & 85 & 239.1 & $29 \mathrm{~A}$ & 356.9 & 73.3 & $2.84 \mathrm{E}-03$ & 70 \\
\hline 221.5 & $26 \mathrm{~A}$ & 161.2 & -19.8 & $3.26 \mathrm{E}-03$ & 100 & 245.6 & $30 \mathrm{~A}$ & 66.4 & 62.4 & $2.52 \mathrm{E}-01$ & 0 \\
\hline 221.5 & $26 \mathrm{~A}$ & 237.2 & -12.2 & $2.41 \mathrm{E}-03$ & 115 & 245.6 & $30 \mathrm{~A}$ & 65.9 & 63.2 & $2.52 \mathrm{E}-01$ & 0 \\
\hline 223.5 & $27 \mathrm{~A}$ & 157.3 & 70.2 & 4.60E-02 & 0 & 245.6 & $30 \mathrm{~A}$ & 69.7 & 60.9 & $1.88 \mathrm{E}-01$ & 3 \\
\hline 223.5 & $27 \mathrm{~A}$ & 143.9 & 69.6 & $3.24 \mathrm{E}-02$ & 3 & 245.6 & $30 \mathrm{~A}$ & 72.9 & 59.7 & $1.22 \mathrm{E}-01$ & 6 \\
\hline 223.5 & $27 \mathrm{~A}$ & 133.8 & 69.5 & $2.24 \mathrm{E}-02$ & 6 & 245.6 & $30 \mathrm{~A}$ & 76.4 & 56.8 & $7.65 \mathrm{E}-02$ & 9 \\
\hline 223.5 & $27 \mathrm{~A}$ & 121.6 & 68 & $1.64 \mathrm{E}-02$ & 9 & 245.6 & $30 \mathrm{~A}$ & 76.7 & 55.9 & $5.21 \mathrm{E}-02$ & 12 \\
\hline 223.5 & $27 \mathrm{~A}$ & 114.7 & 59.3 & $1.32 \mathrm{E}-02$ & 12 & 245.6 & $30 \mathrm{~A}$ & 79.5 & 58.9 & 3.92E-02 & 15 \\
\hline 223.5 & $27 \mathrm{~A}$ & 111.8 & 60.2 & $1.19 \mathrm{E}-02$ & 15 & 245.6 & $30 \mathrm{~A}$ & 81.1 & 59.3 & $2.85 \mathrm{E}-02$ & 18 \\
\hline 223.5 & $27 \mathrm{~A}$ & 93.4 & 67.5 & $1.14 \mathrm{E}-02$ & 18 & 245.6 & $30 \mathrm{~A}$ & 83.5 & 59.2 & $2.23 \mathrm{E}-02$ & 21 \\
\hline 223.5 & $27 \mathrm{~A}$ & 101.3 & 55.1 & $9.00 \mathrm{E}-03$ & 21 & 245.6 & $30 \mathrm{~A}$ & 85.9 & 59.3 & $1.77 \mathrm{E}-02$ & 25 \\
\hline 223.5 & $27 \mathrm{~A}$ & 92.8 & 58 & 7.95E-03 & 25 & 245.6 & $30 \mathrm{~A}$ & 96.1 & 62.9 & $1.26 \mathrm{E}-02$ & 30 \\
\hline 223.5 & $27 \mathrm{~A}$ & 89.5 & 44.3 & 7.07E-03 & 30 & 245.6 & $30 \mathrm{~A}$ & 124.2 & 64.7 & $6.70 \mathrm{E}-03$ & 40 \\
\hline 223.5 & $27 \mathrm{~A}$ & 79.3 & 50.4 & $6.14 \mathrm{E}-03$ & 30 & 245.6 & $30 \mathrm{~A}$ & 84.9 & 55 & $5.12 \mathrm{E}-03$ & 50 \\
\hline 223.5 & $27 \mathrm{~A}$ & 76.1 & 53.3 & $5.54 \mathrm{E}-03$ & 40 & 245.6 & $30 \mathrm{~A}$ & 154.9 & 77 & $5.02 \mathrm{E}-03$ & 60 \\
\hline 223.5 & $27 \mathrm{~A}$ & 93.2 & 38.6 & 4. $64 \mathrm{E}-03$ & 50 & 245.6 & $30 \mathrm{~A}$ & 182.3 & 10.4 & $1.96 \mathrm{E}-03$ & 70 \\
\hline 223.5 & $27 \mathrm{~A}$ & 29.9 & 50.2 & 2.57E-03 & 60 & 245.6 & $30 \mathrm{~A}$ & 114.2 & 39.2 & $1.97 \mathrm{E}-03$ & 85 \\
\hline 223.5 & $27 \mathrm{~A}$ & 25.2 & 14.4 & $1.01 \mathrm{E}-03$ & 70 & 245.6 & $30 \mathrm{~A}$ & 116.9 & 36.9 & $2.71 \mathrm{E}-03$ & 100 \\
\hline 223.5 & $27 \mathrm{~A}$ & 4.9 & 75.3 & $2.30 \mathrm{E}-03$ & 85 & 245.6 & $30 \mathrm{~A}$ & 145.8 & 42.3 & $4.06 \mathrm{E}-03$ & 115 \\
\hline 223.5 & $27 \mathrm{~A}$ & 69.4 & 61.2 & $1.93 \mathrm{E}-03$ & 100 & 249.8 & $31 \mathrm{~A}$ & 289.2 & 1.6 & $1.06 \mathrm{E}-02$ & 0 \\
\hline 223.5 & $27 \mathrm{~A}$ & 134.2 & -1.9 & $4.00 \mathrm{E}-03$ & 110 & 249.8 & $31 \mathrm{~A}$ & 284.5 & -5.6 & $1.03 \mathrm{E}-02$ & 3 \\
\hline 236.5 & $28 \mathrm{~A}$ & 69.7 & 61.6 & $2.61 \mathrm{E}-02$ & 0 & 249.8 & $31 \mathrm{~A}$ & 281.6 & -10.1 & $9.92 \mathrm{E}-03$ & 6 \\
\hline 236.5 & $28 \mathrm{~A}$ & 70.1 & 58.7 & $2.13 \mathrm{E}-02$ & 3 & 249.8 & $31 \mathrm{~A}$ & 279.7 & -12.6 & $9.52 \mathrm{E}-03$ & 9 \\
\hline 236.5 & $28 \mathrm{~A}$ & 69.3 & 57.5 & $1.88 \mathrm{E}-02$ & 6 & 249.8 & $31 \mathrm{~A}$ & 279 & -14.9 & $8.79 \mathrm{E}-03$ & 12 \\
\hline 236.5 & $28 \mathrm{~A}$ & 68.2 & 56.5 & $1.63 \mathrm{E}-02$ & 9 & 249.8 & $31 \mathrm{~A}$ & 278.2 & -16.5 & $7.52 \mathrm{E}-03$ & 15 \\
\hline 236.5 & $28 \mathrm{~A}$ & 68.1 & 57.6 & $1.45 \mathrm{E}-02$ & 12 & 249.8 & $31 \mathrm{~A}$ & 277.9 & -14.2 & $7.06 \mathrm{E}-03$ & 18 \\
\hline 236.5 & $28 \mathrm{~A}$ & 69.3 & 57.5 & $1.32 \mathrm{E}-02$ & 15 & 249.8 & $31 \mathrm{~A}$ & 276.2 & -15.3 & $6.44 \mathrm{E}-03$ & 21 \\
\hline 236.5 & $28 \mathrm{~A}$ & 69 & 56.6 & $1.20 \mathrm{E}-02$ & 18 & 249.8 & $31 \mathrm{~A}$ & 276.2 & -17.5 & $5.34 \mathrm{E}-03$ & 25 \\
\hline 236.5 & $28 \mathrm{~A}$ & 72.2 & 56.3 & $1.06 \mathrm{E}-02$ & 21 & 249.8 & $31 \mathrm{~A}$ & 278.2 & -22.5 & $4.68 \mathrm{E}-03$ & 30 \\
\hline 236.5 & $28 \mathrm{~A}$ & 73.1 & 56.5 & $9.13 \mathrm{E}-03$ & 25 & 249.8 & $31 \mathrm{~A}$ & 276.2 & -22.3 & $2.41 \mathrm{E}-03$ & 40 \\
\hline 236.5 & $28 \mathrm{~A}$ & 63.8 & 55.2 & $6.91 \mathrm{E}-03$ & 30 & 249.8 & $31 \mathrm{~A}$ & 279.4 & -36.1 & $2.64 \mathrm{E}-03$ & 50 \\
\hline 236.5 & $28 \mathrm{~A}$ & 73.5 & 45.1 & $5.24 \mathrm{E}-03$ & 40 & 249.8 & $31 \mathrm{~A}$ & 311.4 & -33.6 & $1.37 \mathrm{E}-03$ & 60 \\
\hline 236.5 & $28 \mathrm{~A}$ & 62.5 & 52.8 & $3.93 \mathrm{E}-03$ & 50 & 249.8 & $31 \mathrm{~A}$ & 270.2 & -31.7 & $1.29 \mathrm{E}-03$ & 70 \\
\hline 236.5 & $28 \mathrm{~A}$ & 62 & 53.1 & $2.58 \mathrm{E}-03$ & 60 & 249.8 & $31 \mathrm{~A}$ & 344.8 & -16.4 & $1.10 \mathrm{E}-03$ & 85 \\
\hline 236.5 & $28 \mathrm{~A}$ & 91.3 & 78 & 2.81E-03 & 70 & 249.8 & $31 \mathrm{~A}$ & 259.3 & -34.7 & $1.02 \mathrm{E}-03$ & 100 \\
\hline 236.5 & $28 \mathrm{~A}$ & 35.1 & 49.5 & $2.47 \mathrm{E}-03$ & 85 & 249.8 & $31 \mathrm{~A}$ & 256.4 & -23.6 & $1.62 \mathrm{E}-03$ & 115 \\
\hline 236.5 & $28 \mathrm{~A}$ & 101.9 & 62.9 & $1.90 \mathrm{E}-03$ & 100 & 251.1 & $32 \mathrm{~A}$ & 306.9 & -0.8 & $1.07 \mathrm{E}-02$ & 0 \\
\hline 236.5 & $28 \mathrm{~A}$ & 77.6 & 49.3 & 2.67E- 03 & 115 & 251.1 & $32 \mathrm{~A}$ & 306.7 & -15.4 & $1.15 \mathrm{E}-02$ & 3 \\
\hline
\end{tabular}




\begin{tabular}{|c|c|c|c|c|c|}
\hline & $2 \mathrm{~A}$ & 306.9 & -20.5 & $1.18 \mathrm{E}$ & \\
\hline & $32 \mathrm{~A}$ & 06.6 & -24 & 1.13E-02 & \\
\hline & $32 \mathrm{~A}$ & 07.5 & -26.6 & $1.04 \mathrm{E}-02$ & 12 \\
\hline & $32 \mathrm{~A}$ & 07.9 & -27.5 & $9.70 \mathrm{E}-03$ & $2^{2}$ \\
\hline & $32 \mathrm{~A}$ & 04.7 & -26.9 & $8.80 \mathrm{E}-03$ & 1 \\
\hline 1. & $32 \mathrm{~A}$ & 07.7 & -26.3 & $7.19 \mathrm{E}-03$ & 2 \\
\hline 1. & $32 \mathrm{~A}$ & 04.3 & -28.5 & $6.39 \mathrm{E}-03$ & 2 \\
\hline 1.1 & $32 \mathrm{~A}$ & 01.8 & -26 & $5.78 \mathrm{E}-03$ & 3 \\
\hline 1.1 & $32 \mathrm{~A}$ & 02.8 & -21.3 & $4.26 \mathrm{E}-03$ & 7 \\
\hline 1.1 & $32 \mathrm{~A}$ & 03.1 & -32.7 & $3.75 \mathrm{E}-03$ & 5 \\
\hline 1. & $32 \mathrm{~A}$ & 12.2 & -14.7 & $3.10 \mathrm{E}$ & \\
\hline . & $32 \mathrm{~A}$ & 89.7 & -15.6 & 3.18 & \\
\hline 1. & $32 \mathrm{~A}$ & 90.2 & -32.5 & $3.48 \mathrm{E}$ & \\
\hline 1. & $32 \mathrm{~A}$ & 75.1 & -22.3 & $2.87 \mathrm{t}$ & \\
\hline I. & $32 \mathrm{~A}$ & 82.9 & -27.3 & 2.511 & 11 \\
\hline & $33 \mathrm{~A}$ & 58.2 & 41.2 & 8.10 & \\
\hline & $33 \mathrm{~A}$ & 58.5 & 43.1 & 8.86 & \\
\hline & $33 \mathrm{~A}$ & 59.1 & 44.2 & 9.16 & \\
\hline & $33 \mathrm{~A}$ & 59.5 & 44.9 & 8.97 & \\
\hline & $3 \mathrm{~A}$ & 259 & 44.5 & 8.50 & 12 \\
\hline & $33 \mathrm{~A}$ & 58.7 & 44.6 & & 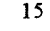 \\
\hline & $33 \mathrm{~A}$ & 01.1 & -46.8 & 7.30 & \\
\hline & , & 258 & 45.1 & & 2 \\
\hline 3 & $33 \mathrm{~A}$ & 56.4 & 44.8 & 5.61 & 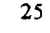 \\
\hline 3 & $33 \mathrm{~A}$ & 66.4 & 44 & 4.36 & 36 \\
\hline 3 & $33 \mathrm{~A}$ & 246 & -38 & 3.6 & $4 c-2>$ \\
\hline 3 & $33 \mathrm{~A}$ & 290.2 & -35.4 & 2.40 & 50 \\
\hline 33 & $3 \mathrm{~A}$ & 91.4 & 49.2 & 2.27 & 60 \\
\hline 3 & $3 \mathrm{~A}$ & 42.1 & -28 & 2.82 & 70 \\
\hline & $3 \mathrm{~A}$ & 06.5 & -35.5 & 1.78 & 35 \\
\hline & $3 \mathrm{~A}$ & 0.0 & -27 & & 100 \\
\hline & $3 \mathrm{~A}$ & 26.6 & -21.6 & 2.4 & 15 \\
\hline & 504 & 336.8 & -0.8 & & \\
\hline & 504 & 335.9 & -1.8 & & \\
\hline & 504 & 34.6 & -3.5 & & \\
\hline & $504 \mathrm{~A}$ & 332 & -5 & & \\
\hline & $504 \mathrm{~A}$ & 333.7 & -5.4 & & 12 \\
\hline 53.7 & $504 \mathrm{~A}$ & 334.5 & -6.5 & $9.10 \mathrm{E}-03$ & 15 \\
\hline 253.7 & $504 \mathrm{~A}$ & 333.9 & -3.5 & $8.32 \mathrm{E}-03$ & 18 \\
\hline 253.7 & $504 \mathrm{~A}$ & 335 & 4 & 7.63E-03 & 21 \\
\hline 年 & $504 \mathrm{~A}$ & 331.5 & -6.3 & $7.29 \mathrm{E}-03$ & 25 \\
\hline . & $504 \mathrm{~A}$ & 331.3 & 2.1 & $6.16 \mathrm{E}-03$ & 30 \\
\hline 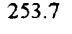 & $504 \mathrm{~A}$ & 333.8 & 0.2 & 4.87E-03 & 40 \\
\hline (20. & $504 \mathrm{~A}$ & 332.4 & 4.1 & $4.04 \mathrm{E}-03$ & 30 \\
\hline . & $504 \mathrm{~A}$ & 334.2 & -18.1 & $3.79 \mathrm{E}-03$ & 60 \\
\hline 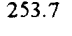 & $504 \mathrm{~A}$ & 336.2 & -2.6 & 3.23E-03 & 70 \\
\hline 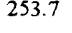 & 年 & 年 & 3.3 & $1.80 \mathrm{E}-03$ & 85 \\
\hline (1) & $504 \mathrm{~A}$ & 329.7 & -11.8 & $1.58 \mathrm{E}-03$ & 100 \\
\hline 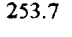 & $504 \mathrm{~A}$ & 335.9 & -9 & $2.78 \mathrm{E}-03$ & 115 \\
\hline . & $504 \mathrm{~A}$ & 331.8 & -16.6 & $1.96 \mathrm{E}-03$ & 120 \\
\hline & $504 \mathrm{E}$ & 20.7 & -32.6 & . & \\
\hline
\end{tabular}

$\begin{array}{llllll}254.6 & 504 \mathrm{~B} & 224.8 & -30.9 & 3.23 \mathrm{E}-02 & 3\end{array}$

$\begin{array}{llllll}254.6 & 504 \mathrm{~B} & 223 & -31.6 & 3.21 \mathrm{E}-02 & 6\end{array}$

$\begin{array}{lllllll}254.6 & 504 \mathrm{~B} & 221.4 & -32.9 & 3.03 \mathrm{E}-02 & 9\end{array}$

$\begin{array}{llllll}254.6 & 504 \mathrm{~B} & 221 & -32 & 2.88 \mathrm{E}-02 & 12\end{array}$

$\begin{array}{lllllll}254.6 & 504 \mathrm{~B} & 219.7 & -32.4 & 2.75 \mathrm{E}-02 & 15\end{array}$

$\begin{array}{llllll}254.6 & 504 \mathrm{~B} & 220 & -33.3 & 2.58 \mathrm{E}-02 & 18\end{array}$

$\begin{array}{lllllll}254.6 & 504 \mathrm{~B} & 218.8 & -31.9 & 2.47 \mathrm{E}-02 & 21\end{array}$

$\begin{array}{lllllll}254.6 & 504 \mathrm{~B} & 219.8 & -32.3 & 2.28 \mathrm{E}-02 & 25\end{array}$

$\begin{array}{lllllll}254.6 & 504 \mathrm{~B} & 219.9 & -32.6 & 2.02 \mathrm{E}-02 & 30\end{array}$

$\begin{array}{lllllll}254.6 & 504 \mathrm{~B} & 218.5 & -33.6 & 1.65 \mathrm{E}-02 & 40\end{array}$

$\begin{array}{lllllll}254.6 & 504 \mathrm{~B} & 217.9 & -34.1 & 1.19 \mathrm{E}-02 & 50\end{array}$

$\begin{array}{llllll}254.6 & 504 \mathrm{~B} & 219 & -33.1 & 1.07 \mathrm{E}-02 & 60\end{array}$

$\begin{array}{lllllll}254.6 & 504 \mathrm{~B} & 212.4 & -34.1 & 8.89 \mathrm{E}-03 & 70\end{array}$

$\begin{array}{lllllll}254.6 & 504 \mathrm{~B} & 224.2 & -32.3 & 7.33 \mathrm{E}-03 & 85\end{array}$

$\begin{array}{llllll}254.6 & 504 \mathrm{~B} & 212 & -39.3 & 6.10 \mathrm{E}-03 & 100\end{array}$

$\begin{array}{llllll}254.6 & 504 \mathrm{~B} & 213 & -31.3 & 5.90 \mathrm{E}-03 & 115\end{array}$

$\begin{array}{lllllll}255.1 & 504 \mathrm{C} & 187.6 & -10.7 & 3.20 \mathrm{E}-03 & 0\end{array}$

$\begin{array}{llllll}255.1 & 504 \mathrm{C} & 186.5 & -14.7 & 3.46 \mathrm{E}-03 & 3\end{array}$

$\begin{array}{llllll}255.1 & 504 \mathrm{C} & 184.5 & -19 & 3.79 \mathrm{E}-03 & 6\end{array}$

$\begin{array}{llllll}255.1 & 504 \mathrm{C} & 181.2 & -22.3 & 3.73 \mathrm{E}-03 & 9\end{array}$

$\begin{array}{lllllll}255.1 & 504 \mathrm{C} & 180.8 & -23.8 & 3.65 \mathrm{E}-03 & 12\end{array}$

$\begin{array}{lllllll}255.1 & 504 \mathrm{C} & 183.4 & -26.2 & 3.62 \mathrm{E}-03 & 15\end{array}$

$\begin{array}{lllllll}255.1 & 504 \mathrm{C} & 182 & -26.7 & 3.22 \mathrm{E}-03 & 18\end{array}$

$\begin{array}{lllllll}255.1 & 504 \mathrm{C} & 181.4 & -29.3 & 3.27 \mathrm{E}-03 & 21\end{array}$

$\begin{array}{llllll}255.1 \quad 504 \mathrm{C} & 180.4 & -30.2 & 3.11 \mathrm{E}-03 & 25\end{array}$

$\begin{array}{llllll}255.1 & 504 \mathrm{C} & 200.9 & -24.8 & 2.53 \mathrm{E}-03 & 30\end{array}$

$\begin{array}{llllll}255.1 & 504 \mathrm{C} & 169 & -32.5 & 1.97 \mathrm{E}-03 & 40\end{array}$

$\begin{array}{lllllll}255.1 & 504 \mathrm{C} & 165.8 & 41.1 & 2.20 \mathrm{E}-03 & 50\end{array}$

$\begin{array}{lllllll}255.1 & 504 \mathrm{C} & 169.2 & -32.5 & 1.04 \mathrm{E}-03 & 60\end{array}$

$\begin{array}{lllllll}255.1 & 504 \mathrm{C} & 168.8 & -19.6 & 2.06 \mathrm{E}-03 & 70\end{array}$

$\begin{array}{llllll}255.1 & 504 \mathrm{C} & 189 & -51.8 & 8.07 \mathrm{E}-04 & 85\end{array}$

$\begin{array}{llllll}255.1 & 504 \mathrm{C} & 152.8 & -31.4 & 1.33 \mathrm{E}-03 & 100\end{array}$

$\begin{array}{lllllll}255.1 & 504 \mathrm{C} & 162.4 & -66.9 & 5.61 \mathrm{E}-04 & 115\end{array}$

$\begin{array}{llllll}255.2 & 34 \mathrm{~A} & 120.6 & 29.8 & 1.08 \mathrm{E}-02 & 0\end{array}$

$\begin{array}{llllll}255.2 & 34 \mathrm{~A} & 122.9 & 24.2 & 1.05 \mathrm{E}-02 & 3\end{array}$

$\begin{array}{lllllll}255.2 & 34 \mathrm{~A} & 124.8 & 22.7 & 1.02 \mathrm{E}-02 & 6\end{array}$

$\begin{array}{llllll}255.2 & 34 \mathrm{~A} & 126.4 & 22 & 9.49 \mathrm{E}-03 & 9\end{array}$

$\begin{array}{lllllll}255.2 & 34 \mathrm{~A} & 126.2 & 19.7 & 8.69 \mathrm{E}-03 & 12\end{array}$

$\begin{array}{lllllll}255.2 & 34 \mathrm{~A} & 127.1 & 19.4 & 8.02 \mathrm{E}-03 & 15\end{array}$

$\begin{array}{lllllll}255.2 & 34 \mathrm{~A} & 126 & 19.7 & 6.99 \mathrm{E}-03 & 18\end{array}$

$\begin{array}{llllll}255.2 & 34 \mathrm{~A} & 128.2 & 19.2 & 6.25 \mathrm{E}-03 & 21\end{array}$

$\begin{array}{lllllll}255.2 & 34 \mathrm{~A} & 129.4 & 18.6 & 5.36 \mathrm{E}-03 & 25\end{array}$

$\begin{array}{lllllll}255.2 & 34 \mathrm{~A} & 120.9 & 20.7 & 4.15 \mathrm{E}-03 & 30\end{array}$

$\begin{array}{lllllll}255.2 & 34 \mathrm{~A} & 136.6 & 18 & 3.38 \mathrm{E}-03 & 40\end{array}$

$\begin{array}{llllll}255.2 & 34 \mathrm{~A} & 98.8 & 26.3 & 2.02 \mathrm{E}-03 & 50\end{array}$

$\begin{array}{llllll}255.2 & 34 \mathrm{~A} & 82 & 29.5 & 1.70 \mathrm{E}-03 & 60\end{array}$

$\begin{array}{lllllll}255.2 & 34 \mathrm{~A} & 147.7 & 16.5 & 2.44 \mathrm{E}-03 & 70\end{array}$

$\begin{array}{llllll}257.3 & 506 \mathrm{~A} & 251.7 & 17.3 & 2.48 \mathrm{E}-03 & 0\end{array}$

$\begin{array}{lllllll}257.3 & 506 \mathrm{~A} & 220.9 & 66.8 & 1.89 \mathrm{E}-03 & 3\end{array}$

$\begin{array}{llllll}257.3 & 506 \mathrm{~A} & 282.7 & 48 & 1.02 \mathrm{E}-03 & 6\end{array}$

$\begin{array}{llllll}257.3 & 506 \mathrm{~A} & 240.2 & 1.9 & 4.95 \mathrm{E}-04 & 9\end{array}$ $\begin{array}{llllll}257.3 & 506 \mathrm{~A} & 169.6 & -22.3 & 1.04 \mathrm{E}-03 & 12\end{array}$

$\begin{array}{llllll}257.3 & 506 \mathrm{~A} & 132.1 & -35.2 & 1.51 \mathrm{E}-03 \quad 15\end{array}$

$\begin{array}{lllllll}257.3 & 506 \mathrm{~A} & 137.9 & -29.5 & 1.58 \mathrm{E}-03 & 18\end{array}$

$\begin{array}{lllllll}257.3 & 506 \mathrm{~A} & 129.6 & -27.4 & 2.31 \mathrm{E}-03 & 21\end{array}$

$\begin{array}{lllllll}257.3 & 506 \mathrm{~A} & 123.9 & -22.9 & 2.30 \mathrm{E}-03 & 25\end{array}$

$\begin{array}{llllll}257.3 & 506 \mathrm{~A} & 126.8 & -16.9 & 2.45 \mathrm{E}-03 & 30\end{array}$

$\begin{array}{lllllll}257.3 & 506 \mathrm{~A} & 125.7 & -37.4 & 3.07 \mathrm{E}-03 & 40\end{array}$

$\begin{array}{lllllll}257.3 & 506 \mathrm{~A} & 169.9 & -15 & 1.83 \mathrm{E}-03 & 50\end{array}$

$\begin{array}{lllllll}257.3 & 506 \mathrm{~A} & 168.9 & -13.5 & 1.43 \mathrm{E}-03 & 60\end{array}$

$\begin{array}{lllllll}257.3 & 506 \mathrm{~A} & 132.6 & -49.3 & 2.44 \mathrm{E}-03 & 70\end{array}$

$\begin{array}{lllllll}257.3 & 506 \mathrm{~A} & 114.2 & -39.1 & 7.75 \mathrm{E}-04 & 85\end{array}$

$\begin{array}{lllllll}257.3 & 506 \mathrm{~A} & 141.1 & -66.7 & 2.30 \mathrm{E}-03 & 100\end{array}$

$\begin{array}{llllllll}257.3 & 506 \mathrm{~A} & 124.1 & -15.5 & 6.07 \mathrm{E}-04 & 115\end{array}$

$265 \quad 35 \mathrm{~B} \quad 312.3-21.6 \quad 4.75 \mathrm{E}-02 \quad 0$

$\begin{array}{llllll}265 & 35 \mathrm{~B} & 312 & -21 & 4.77 \mathrm{E}-02 & 3\end{array}$

$265 \quad 35 \mathrm{~B} \quad 312 \quad-24.4 \quad 4.53 \mathrm{E}-02 \quad 6$

$265 \quad 35 \mathrm{~B} \quad 311.4 \quad-25.1 \quad 4.23 \mathrm{E}-02 \quad 9$

$265 \quad 35 \mathrm{~B} \quad 311.1 \quad-24.9 \quad 3.91 \mathrm{E}-02 \quad 12$

$265 \quad 35 \mathrm{~B} \quad 311.3 \quad-25.6 \quad 3.63 \mathrm{E}-02 \quad 15$

$\begin{array}{lllllll}265 & 35 \mathrm{~B} & 310.8 & -25.3 & 3.26 \mathrm{E}-02 & 18\end{array}$

$\begin{array}{lllllll}265 & 35 \mathrm{~B} & 311.2 & -26.3 & 2.90 \mathrm{E}-02 & 21\end{array}$

$\begin{array}{llllll}265 & 35 \mathrm{~B} & 310.1 & -26.7 & 2.59 \mathrm{E}-02 & 25\end{array}$

$265 \quad 35 \mathrm{~B} \quad 310.1 \quad-27.2 \quad 2.23 \mathrm{E}-02 \quad 30$

$\begin{array}{llllll}265 & 35 \mathrm{~B} & 307 & -30.3 & 1.55 \mathrm{E}-02 & 40\end{array}$

$\begin{array}{llllll}265 & 35 \mathrm{~B} & 306.9 & -29 & 1.28 \mathrm{E}-02 & 50\end{array}$

$\begin{array}{llllll}265 & 35 \mathrm{~B} & 313.3 & -29 & 1.05 \mathrm{E}-02 & 60\end{array}$

$\begin{array}{llllll}265 & 35 \mathrm{~B} & 310.3 & -32.2 & 9.18 \mathrm{E}-03 & 70\end{array}$

$\begin{array}{llllll}265 & 35 \mathrm{~B} & 313 & -26.8 & 8.44 \mathrm{E}-03 & 85\end{array}$

$\begin{array}{llllll}265 & 35 \mathrm{~B} & 312.2 & -34 & 7.44 \mathrm{E}-03 & 100\end{array}$

$\begin{array}{llllll}265 & 35 \mathrm{~B} & 308 & -23.7 & 6.76 \mathrm{E}-03 & 115\end{array}$

$\begin{array}{llllll}265.3 & 35 \mathrm{C} & 264.5 & 4.7 & 8.37 \mathrm{E}-04 & 0\end{array}$

$\begin{array}{llllll}265.3 & 35 \mathrm{C} & 240.8 & -62.3 & 1.56 \mathrm{E}-03 & 3\end{array}$

$\begin{array}{llllll}265.3 & 35 \mathrm{C} & 218.4 & -72.4 & 2.13 \mathrm{E}-03 & 6\end{array}$

$\begin{array}{llllll}265.3 & 35 \mathrm{C} & 190.6 & -79.4 & 2.28 \mathrm{E}-03 & 9\end{array}$

$\begin{array}{llllll}265.3 & 35 \mathrm{C} & 187 & -78 & 2.33 \mathrm{E}-03 & 12\end{array}$

$\begin{array}{llllll}265.3 & 35 \mathrm{C} & 184.7 & -77.3 & 2.05 \mathrm{E}-03 & 15\end{array}$

$\begin{array}{lllllll}265.3 & 35 \mathrm{C} & 153.5 & -75.7 & 1.99 \mathrm{E}-03 & 18\end{array}$

$\begin{array}{lllllll}265.3 & 35 \mathrm{C} & 215.5 & -77.6 & 1.66 \mathrm{E}-03 & 21\end{array}$

$\begin{array}{lllllll}265.3 & 35 \mathrm{C} & 237.9 & -74.7 & 1.48 \mathrm{E}-03 & 25\end{array}$

$\begin{array}{lllllll}265.3 & 35 \mathrm{C} & 176.6 & -56.7 & 1.19 \mathrm{E}-03 & 30\end{array}$

$\begin{array}{lllllll}265.3 & 35 \mathrm{C} & 257.5 & -29.9 & 7.05 \mathrm{E}-04 & 40\end{array}$

$\begin{array}{llllll}265.3 & 35 \mathrm{C} & 237.9 & 2.2 & 7.09 \mathrm{E}-04 & 50\end{array}$

$\begin{array}{lllllll}265.3 & 35 \mathrm{C} & 194 & -29.2 & 9.67 \mathrm{E}-04 & 60\end{array}$

$\begin{array}{llllll}265.3 & 35 \mathrm{C} & 273.7 & -52.1 & 6.12 \mathrm{E}-04 & 70\end{array}$

$\begin{array}{lllllll}265.3 & 35 \mathrm{C} & 162.3 & -8.6 & 5.68 \mathrm{E}-04 & 85\end{array}$

$\begin{array}{lllllll}265.3 & 35 \mathrm{C} & 60.9 & -7.2 & 6.96 \mathrm{E}-04 & 100\end{array}$

$\begin{array}{lllllll}265.3 & 35 \mathrm{C} & 7.8 & -11.3 & 3.52 \mathrm{E}-04 & 115\end{array}$

$\begin{array}{llllll}270.2 & 36 \mathrm{~A} & 42.4 & 55.5 & 2.59 \mathrm{E}-03 & 0\end{array}$

$\begin{array}{llllll}270.2 & 36 \mathrm{~A} & 34.6 & -0.8 & 1.86 \mathrm{E}-03 & 3\end{array}$

$\begin{array}{llllll}270.2 & 36 \mathrm{~A} & 28.4 & -33.3 & 1.91 \mathrm{E}-03 & 6\end{array}$

$\begin{array}{llllll}270.2 & 36 \mathrm{~A} & 24.9 & -34.4 & 2.26 \mathrm{E}-03 & 9\end{array}$ 


\begin{tabular}{|c|c|c|c|c|c|}
\hline & $36 \mathrm{~A}$ & 9.1 & 42.7 & $1.91 \mathrm{E}-03$ & \\
\hline & $36 \mathrm{~A}$ & 9.7 & 48.2 & $2.01 \mathrm{E}-03$ & 15 \\
\hline & $36 \mathrm{~A}$ & 10.6 & 43.3 & $1.87 \mathrm{E}-03$ & \\
\hline & $36 \mathrm{~A}$ & 20.7 & 40.6 & $1.71 \mathrm{E}-03$ & \\
\hline & $36 \mathrm{~A}$ & 3.3 & 45.8 & $1.84 \mathrm{E}-03$ & \\
\hline 0.28 & $36 \mathrm{~A}$ & 2 & -20.4 & $9.86 \mathrm{E}-04$ & 3 \\
\hline 0.2 & $36 \mathrm{~A}$ & 49.4 & 42.7 & 7.39E-04 & 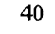 \\
\hline 0.2 & $36 \mathrm{~A}$ & 12 & -22.6 & $5.26 \mathrm{E}-04$ & 5 \\
\hline 0.2 & $36 \mathrm{~A}$ & 56.4 & 53.1 & 3.13E-04 & 60 \\
\hline 0.2 & $36 \mathrm{~A}$ & 349.5 & -8 & $6.26 \mathrm{E}-04$ & $\pi$ \\
\hline 0.2 & $36 \mathrm{~A}$ & 341.6 & 40.4 & 8.12E-04 & oj \\
\hline 4.5 & $37 \mathrm{~A}$ & 272.5 & 77.8 & 3.55E-02 & \\
\hline 4.5 & $37 \mathrm{~A}$ & 261.9 & 72.4 & $2.06 \mathrm{E}-02$ & \\
\hline 4.5 & $37 \mathrm{~A}$ & 275.1 & 53.8 & $1.17 \mathrm{E}-02$ & \\
\hline 4.5 & $37 \mathrm{~A}$ & 275.5 & 37.7 & $8.04 \mathrm{E}-03$ & \\
\hline 4.5 & $37 \mathrm{~A}$ & 285.7 & 20.4 & $4.30 \mathrm{E}-03$ & 12 \\
\hline m. & $37 \mathrm{~A}$ & 300.6 & -7.3 & $4.39 \mathrm{E}$ & 13 \\
\hline t.J & $37 \mathrm{~A}$ & 296 & -14.8 & $4.31 \mathrm{E}$ & 10 \\
\hline t.J & $37 \mathrm{~A}$ & 320.8 & -6.6 & 3.42E-03 & 21 \\
\hline $7 . .5$ & $37 \mathrm{~A}$ & 328.6 & -23.8 & $4.27 \mathrm{E}-03$ & 23 \\
\hline 4.5 & $37 \mathrm{~A}$ & 321.8 & -2.6 & $4.55 \mathrm{E}-03$ & 30 \\
\hline 4.5 & $37 \mathrm{~A}$ & 350.8 & -20.4 & $3.47 \mathrm{E}-03$ & 40 \\
\hline 4.5 & $37 \mathrm{~A}$ & 345.4 & -27.7 & $4.96 \mathrm{E}-03$ & 50 \\
\hline 4.5 & $37 \mathrm{~A}$ & 285.3 & -66.9 & $3.07 \mathrm{E}-03$ & 60 \\
\hline 4.5 & $37 \mathrm{~A}$ & 335.5 & -64.4 & $3.52 \mathrm{E}-03$ & 70 \\
\hline 4.5 & $37 \mathrm{~A}$ & 41.4 & -54.9 & $4.04 \mathrm{E}$ & 85 \\
\hline 4.5 & $37 \mathrm{~A}$ & 35.6 & 0.9 & $3.22 \mathrm{I}$ & 100 \\
\hline 4.J & $37 \mathrm{~A}$ & 135.1 & -27.4 & $5.49 \mathrm{E}$ & 115 \\
\hline 8.6 & $38 \mathrm{~A}$ & 333.7 & 33 & $7.98 \mathrm{E}$ & \\
\hline 8.6 & $38 \mathrm{~A}$ & 328.3 & 20.6 & 6.611 & \\
\hline 8.6 & $38 \mathrm{~A}$ & 325.8 & 12.6 & $6.20 \mathrm{E}$ & \\
\hline 8.6 & $38 \mathrm{~A}$ & 319.1 & 9.4 & $5.57 \mathrm{E}$ & \\
\hline 8.6 & $38 \mathrm{~A}$ & 319.1 & 7.8 & $5.17 \pi$ & 12 \\
\hline 8.6 & $38 \mathrm{~A}$ & 320.5 & 7.9 & 4.671 & 15 \\
\hline 8.6 & $38 \mathrm{~A}$ & 322.1 & 9.6 & 4.26 & 18 \\
\hline 8.6 & $38 \mathrm{~A}$ & 326.4 & 12.5 & 3.90 & 21 \\
\hline 8 & $38 \mathrm{~A}$ & 321.8 & 7.9 & 3.98 & 25 \\
\hline 8. & $38 \mathrm{~A}$ & 324.5 & 3.3 & & 30 \\
\hline & $38 \mathrm{~A}$ & 322.3 & 11.3 & & 40 \\
\hline & $38 \mathrm{~A}$ & 342.2 & 18.8 & & 50 \\
\hline & $38 \mathrm{~A}$ & 327 & 18.3 & & 00 \\
\hline & $38 \mathrm{~A}$ & 312.8 & 8.9 & & 70 \\
\hline & $38 \mathrm{~A}$ & 324.6 & -24.9 & $2.32 \mathrm{E}-03$ & 85 \\
\hline & $38 \mathrm{~A}$ & 329.5 & -1.1 & $1.68 \mathrm{E}-03$ & 100 \\
\hline 278.6 & $38 \mathrm{~A}$ & 325.3 & 3.1 & $1.91 \mathrm{E}-03$ & 115 \\
\hline 279.2 & $39 \mathrm{~A}$ & 23.1 & 68.6 & $1.54 \mathrm{E}-02$ & 0 \\
\hline 事 & $39 \mathrm{~A}$ & 23.3 & 59.4 & 9.35E-03 & 3 \\
\hline - & $39 \mathrm{~A}$ & 25.3 & 47.6 & $6.84 \mathrm{E}-03$ & 0 \\
\hline 270.2 & $39 \mathrm{~A}$ & 27.9 & 32.6 & $6.01 \mathrm{E}-03$ & 3 \\
\hline 27.2 & $39 \mathrm{~A}$ & 30.6 & 31.5 & $5.17 \mathrm{E}-03$ & 12 \\
\hline & $39 \mathrm{~A}$ & 26.8 & 25.5 & $4.57 \mathrm{E}-03$ & $1 J$ \\
\hline
\end{tabular}

$\begin{array}{llllll}279.2 & 39 \mathrm{~A} & 38.7 & 29.2 & 4.40 \mathrm{E}-03 & 18\end{array}$ $\begin{array}{lllllll}279.2 & 39 \mathrm{~A} & 29.2 & 28.7 & 3.44 \mathrm{E}-03 & 21\end{array}$ $\begin{array}{lllllll}279.2 & 39 \mathrm{~A} & 39.3 & 36.5 & 3.59 \mathrm{E}-03 & 25\end{array}$ $\begin{array}{lllllll}279.2 & 39 \mathrm{~A} & 36.1 & 26.3 & 3.64 \mathrm{E}-03 & 30\end{array}$ $\begin{array}{lllllll}279.2 & 39 \mathrm{~A} & 24.8 & 2.7 & 3.88 \mathrm{E}-03 & 40\end{array}$ $\begin{array}{lllllll}279.2 & 39 \mathrm{~A} & 35.7 & -15.7 & 2.72 \mathrm{E}-03 & 50\end{array}$ $\begin{array}{llllll}279.2 & 39 \mathrm{~A} & 23.4 & -20.3 & 3.43 \mathrm{E}-03 & 60\end{array}$ $\begin{array}{lllllll}279.2 & 39 \mathrm{~A} & 28.3 & -3.2 & 3.72 \mathrm{E}-03 & 70\end{array}$ $\begin{array}{llllll}279.2 & 39 \mathrm{~A} & 50.7 & 1.1 & 3.73 \mathrm{E}-03 & 85\end{array}$ $\begin{array}{lllllll}279.2 & 39 \mathrm{~A} & 39.3 & -7.8 & 2.66 \mathrm{E}-03 & 100\end{array}$ $\begin{array}{lllllll}279.2 & 39 \mathrm{~A} & 10.5 & 10.9 & 3.36 \mathrm{E}-03 & 115\end{array}$ $\begin{array}{llllll}283.4 & 40 \mathrm{~A} & 293 & 58.4 & 1.24 \mathrm{E}-02 & 0\end{array}$ $\begin{array}{lllllll}283.4 & 40 \mathrm{~A} & 276 & 43.1 & 9.44 \mathrm{E}-03 & 3\end{array}$ $\begin{array}{lllllll}283.4 & 40 \mathrm{~A} & 270.2 & 28.5 & 8.37 \mathrm{E}-03 & 6\end{array}$ $\begin{array}{lllllll}283.4 & 40 \mathrm{~A} & 267 & 13.1 & 6.85 \mathrm{E}-03 & 9\end{array}$ $\begin{array}{lllllll}283.4 & 40 \mathrm{~A} & 263.5 & 7.9 & 5.83 \mathrm{E}-03 & 12\end{array}$ $\begin{array}{lllllll}283.4 & 40 \mathrm{~A} & 259.9 & 4.3 & 5.44 \mathrm{E}-03 & 15\end{array}$ $\begin{array}{lllllll}283.4 & 40 \mathrm{~A} & 259.3 & 7.6 & 5.16 \mathrm{E}-03 & 18\end{array}$ $\begin{array}{lllllll}283.4 & 40 \mathrm{~A} & 257.3 & 0.2 & 4.23 \mathrm{E}-03 & 21\end{array}$ $\begin{array}{lllllll}283.4 & 40 \mathrm{~A} & 249.6 & 4.8 & 3.85 \mathrm{E}-03 & 25\end{array}$ $\begin{array}{lllllll}283.4 & 40 \mathrm{~A} & 254.4 & -12 & 2.82 \mathrm{E}-03 & 30\end{array}$ $\begin{array}{lllllll}283.4 & 40 \mathrm{~A} & 247.8 & -17.9 & 2.84 \mathrm{E}-03 & 40\end{array}$ $\begin{array}{llllll}283.4 & 40 \mathrm{~A} & 255.3 & -24.3 & 2.22 \mathrm{E}-03 & 50\end{array}$ $\begin{array}{llllll}283.4 & 40 \mathrm{~A} & 226.3 & -18.6 & 1.98 \mathrm{E}-03 & 60\end{array}$ $\begin{array}{lllllll}283.4 & 40 \mathrm{~A} & 242.9 & -26.2 & 3.12 \mathrm{E}-03 & 70\end{array}$ $\begin{array}{lllllll}283.4 & 40 \mathrm{~A} & 214.4 & -31.6 & 2.45 \mathrm{E}-03 & 85\end{array}$ $\begin{array}{llllll}283.4 & 40 \mathrm{~A} & 274.4 & -35.5 & 2.87 \mathrm{E}-03 & 100\end{array}$ $\begin{array}{lllllll}283.4 & 40 \mathrm{~A} & 284.5 & -29.9 & 1.27 \mathrm{E}-03 & 115\end{array}$ $\begin{array}{lllllll}291.3 & 41 \mathrm{~A} & 41.2 & 11.2 & 2.65 \mathrm{E}-02 & 0\end{array}$ $\begin{array}{llllll}291.3 & 41 \mathrm{~A} & 44.7 & 0.7 & 2.59 \mathrm{E}-02 & 3\end{array}$ $\begin{array}{lllllll}291.3 & 41 \mathrm{~A} & 47.1 & -5.6 & 2.52 \mathrm{E}-02 & 6\end{array}$ $\begin{array}{lllllll}291.3 & 41 \mathrm{~A} & 48.3 & -8.7 & 2.38 \mathrm{E}-02 & 9\end{array}$ $\begin{array}{lllllll}291.3 & 41 \mathrm{~A} & 48.2 & -10.3 & 2.26 \mathrm{E}-02 & 12\end{array}$ $\begin{array}{lllllll}291.3 & 41 \mathrm{~A} & 48.2 & -11.5 & 2.16 \mathrm{E}-02 & 15\end{array}$ $\begin{array}{lllllll}291.3 & 41 \mathrm{~A} & 48.2 & -12.3 & 2.08 \mathrm{E}-02 & 18\end{array}$ $\begin{array}{lllllll}291.3 & 41 \mathrm{~A} & 48.9 & -12.4 & 2.02 \mathrm{E}-02 & 21\end{array}$ $\begin{array}{lllllll}291.3 & 41 \mathrm{~A} & 49.2 & -12.9 & 1.84 \mathrm{E}-02 & 25\end{array}$ $\begin{array}{llllll}291.3 & 41 \mathrm{~A} \quad 48.9 & -11.2 & 1.68 \mathrm{E}-02 & 30\end{array}$ $\begin{array}{llllll}291.3 & 41 \mathrm{~A} \quad 47.6 & -13.7 & 1.41 \mathrm{E}-02 & 40\end{array}$ $\begin{array}{lllllll}291.3 & 41 \mathrm{~A} & 50.3 & -12.9 & 1.15 \mathrm{E}-02 & 50\end{array}$ $\begin{array}{lllllll}291.3 & 41 \mathrm{~A} & 46.8 & -10.6 & 9.82 \mathrm{E}-03 & 60\end{array}$ $\begin{array}{llllll}291.3 & 41 \mathrm{~A} & 47.5 & -13.2 & 9.61 \mathrm{E}-03 & 70\end{array}$ $\begin{array}{lllllll}291.3 & 41 \mathrm{~A} & 44.6 & -16.9 & 7.08 \mathrm{E}-03 & 85\end{array}$ $\begin{array}{lllllll}291.3 & 41 \mathrm{~A} & 38.7 & -14.3 & 7.08 \mathrm{E}-03 & 100\end{array}$ $\begin{array}{lllllll}291.3 & 41 \mathrm{~A} & 38.4 & -13.7 & 7.10 \mathrm{E}-03 & 115\end{array}$ $\begin{array}{lllllll}291.3 & 41 \mathrm{~A} & 51.5 & -24 & 6.68 \mathrm{E}-03 & 125\end{array}$ $\begin{array}{lllllll}291.3 & 41 \mathrm{~A} & 50.5 & -16.4 & 6.07 \mathrm{E}-03 & 140\end{array}$ $\begin{array}{lllllll}291.3 & 41 \mathrm{~A} & 44.8 & -12 & 5.89 \mathrm{E}-03 & 150\end{array}$ $\begin{array}{llllll}292.2 & 42 \mathrm{~A} & 215.1 & 72.4 & 1.15 \mathrm{E}-02 & 0\end{array}$ $\begin{array}{lllllll}292.2 & 42 \mathrm{~A} & 312 & 46.6 & 3.67 \mathrm{E}-06 & 3\end{array}$ $\begin{array}{lllllll}292.2 & 42 \mathrm{~A} & 253.7 & 74.3 & 6.62 \mathrm{E}-03 & 6\end{array}$ $\begin{array}{llllll}292.2 & 42 \mathrm{~A} & 269.1 & 66.6 & 5.05 \mathrm{E}-03 & 9\end{array}$ $\begin{array}{llllll}292.2 & 42 \mathrm{~A} & 281.8 & 63.6 & 4.67 \mathrm{E}-03 & 12\end{array}$ $\begin{array}{llllll}292.2 & 42 \mathrm{~A} & 271.1 & 75 & 4.46 \mathrm{E}-03 & 15\end{array}$ $\begin{array}{lllllll}292.2 & 42 \mathrm{~A} & 304.5 & 65.7 & 3.73 \mathrm{E}-03 & 18\end{array}$ $\begin{array}{lllllll}292.2 & 42 \mathrm{~A} & 323.5 & 67.4 & 3.11 \mathrm{E}-03 & 21\end{array}$ $\begin{array}{llllll}292.2 & 42 \mathrm{~A} & 286 & 43 & 3.12 \mathrm{E}-03 & 25\end{array}$ $\begin{array}{llllll}292.2 & 42 \mathrm{~A} & 285.9 & 72.4 & 4.26 \mathrm{E}-03 & 30\end{array}$ $\begin{array}{llllll}292.2 & 42 \mathrm{~A} & 280.9 & 45.9 & 1.90 \mathrm{E}-03 & 40\end{array}$ $\begin{array}{llllll}292.2 & 42 \mathrm{~A} & 275.8 & 14.2 & 2.77 \mathrm{E}-03 & 50\end{array}$ $\begin{array}{llllll}292.2 & 42 \mathrm{~A} & 238.3 & 43.9 & 1.80 \mathrm{E}-03 & 60\end{array}$ $\begin{array}{llllll}292.2 & 42 \mathrm{~A} & 269.7 & 3 & 2.16 \mathrm{E}-03 & 70\end{array}$ $\begin{array}{llllll}292.2 & 42 \mathrm{~A} & 147.8 & 44.5 & 1.93 \mathrm{E}-03 & 85\end{array}$ $\begin{array}{llllll}292.2 & 42 \mathrm{~A} & 247.3 & 1.7 & 1.59 \mathrm{E}-03 & 100\end{array}$ $\begin{array}{lllllll}292.2 & 42 \mathrm{~A} & 221.5 & 38.4 & 2.23 \mathrm{E}-03 & 115\end{array}$ $\begin{array}{llllll}304.7 \quad 51 \mathrm{~B} & 200.1 & -22.6 & 2.55 \mathrm{E}-03 & 0\end{array}$ $\begin{array}{llllll}304.7 & 51 \mathrm{~B} & 200.9 & -22.8 & 2.57 \mathrm{E}-03 & 0\end{array}$ $\begin{array}{llllll}304.7 \quad 51 \mathrm{~B} & 197.6 & -50.8 & 4.25 \mathrm{E}-03 & 3\end{array}$ $\begin{array}{lllllll}304.7 \quad 51 \mathrm{~B} & 198.2 & -56.7 & 5.17 \mathrm{E}-03 & 6\end{array}$ $\begin{array}{llllll}304.7 & 51 \mathrm{~B} & 196.7 & -57 & 5.95 \mathrm{E}-03 & 9\end{array}$ $\begin{array}{llllll}304.7 \quad 51 \mathrm{~B} & 192.2 & -59.1 & 6.16 \mathrm{E}-03 & 12\end{array}$ $\begin{array}{llllll}304.7 \quad 51 \mathrm{~B} & 190.6 & -58.9 & 6.04 \mathrm{E}-03 & 15\end{array}$ $\begin{array}{lllllll}304.7 & 51 \mathrm{~B} & 191.6 & -59.3 & 5.92 \mathrm{E}-03 & 18\end{array}$ $\begin{array}{llllll}304.7 & 51 \mathrm{~B} & 192.7 & -59 & 5.71 \mathrm{E}-03 & 21\end{array}$ $\begin{array}{lllllll}304.7 \quad 51 \mathrm{~B} & 198.1 & -59.7 & 4.97 \mathrm{E}-03 & 25\end{array}$ $\begin{array}{llllll}304.7 \quad 51 \mathrm{~B} & 191 & -60.1 & 4.46 \mathrm{E}-03 & 30\end{array}$ $\begin{array}{llllll}304.7 \quad 51 \mathrm{~B} & 196.9 & -67.8 & 3.78 \mathrm{E}-03 & 40\end{array}$ $\begin{array}{lllllll}304.7 \quad 51 \mathrm{~B} & 199.5 & -68.7 & 2.61 \mathrm{E}-03 & 50\end{array}$ $\begin{array}{llllll}304.7 \quad 51 \mathrm{~B} & 198.1 & -66.1 & 2.60 \mathrm{E}-03 & 60\end{array}$ $\begin{array}{llllll}304.7 & 51 \mathrm{~B} & 48.9 & -81.5 & 2.30 \mathrm{E}-03 & 70\end{array}$ $\begin{array}{llllll}304.7 & 51 \mathrm{~B} & 191.2 & -39.7 & 1.68 \mathrm{E}-03 & 85\end{array}$ $\begin{array}{lllllll}304.7 \quad 51 \mathrm{~B} & 211.9 & -54.3 & 1.53 \mathrm{E}-03 & 100\end{array}$ $\begin{array}{llllll}304.7 \quad 51 \mathrm{~B} & 172.9 & -57.3 & 1.71 \mathrm{E}-03 & 115\end{array}$ $\begin{array}{llllll}313.9 & 44 \mathrm{~A} & 251.4 & -6.2 & 6.68 \mathrm{E}-02 & 0\end{array}$ $\begin{array}{llllll}313.9 & 44 \mathrm{~A} & 252.3 & -6.9 & 5.74 \mathrm{E}-02 & 3\end{array}$ $\begin{array}{llllll}313.9 & 44 \mathrm{~A} & 254 & -10.2 & 3.78 \mathrm{E}-02 & 6\end{array}$ $\begin{array}{llllll}313.9 & 44 \mathrm{~A} & 256.5 & -12.1 & 2.34 \mathrm{E}-02 & 9\end{array}$ $\begin{array}{lllllll}313.9 & 44 \mathrm{~A} & 258.1 & -15.1 & 1.79 \mathrm{E}-02 & 12\end{array}$ $\begin{array}{lllllll}313.9 & 44 \mathrm{~A} & 260.6 & -19.7 & 1.47 \mathrm{E}-02 & 15\end{array}$ $\begin{array}{llllll}313.9 & 44 \mathrm{~A} & 262.1 & -17 & 1.20 \mathrm{E}-02 & 18\end{array}$ $\begin{array}{llllll}313.9 & 44 \mathrm{~A} & 260.3 & -20.2 & 1.11 \mathrm{E}-02 & 21\end{array}$ $\begin{array}{llllll}313.9 & 44 \mathrm{~A} & 261 & -18.3 & 9.62 \mathrm{E}-03 & 25\end{array}$ $\begin{array}{llllll}313.9 & 44 \mathrm{~A} & 268.5 & -21.4 & 6.08 \mathrm{E}-03 & 30\end{array}$ $\begin{array}{lllllll}313.9 & 44 \mathrm{~A} & 269.8 & -37.6 & 4.35 \mathrm{E}-03 & 40\end{array}$ $\begin{array}{llllll}313.9 & 44 \mathrm{~A} & 50.9 & -59.2 & 1.96 \mathrm{E}-03 & 50\end{array}$ $\begin{array}{lllllll}313.9 & 44 \mathrm{~A} \quad 44.3 & -30.7 & 2.97 \mathrm{E}-03 & 60\end{array}$ $\begin{array}{llllll}313.9 & 44 \mathrm{~A} & 312.9 & -57.9 & 1.19 \mathrm{E}-03 & 70\end{array}$ $\begin{array}{llllll}313.9 & 44 \mathrm{~A} \quad 53 & -33.2 & 3.61 \mathrm{E}-03 & 85\end{array}$ $\begin{array}{llllll}313.9 \quad 44 \mathrm{~A} \quad 67.6 & 40.5 & 2.35 \mathrm{E}-03 & 100\end{array}$ $\begin{array}{lllllll}313.9 & 44 \mathrm{~A} \quad 49.7 & -23.3 & 3.38 \mathrm{E}-03 & 115\end{array}$ $\begin{array}{llllll}315.3 & 504 \mathrm{D} & 116.2 & 56.6 & 6.15 \mathrm{E}-03 & 0\end{array}$ $\begin{array}{llllll}315.3 & 504 \mathrm{D} & 105.7 & 47.5 & 5.80 \mathrm{E}-03 & 3\end{array}$ 


\begin{tabular}{|c|c|c|c|c|c|}
\hline & & & 3.4 & & \\
\hline & 504D & 113.6 & 23.7 & $5.35 \mathrm{E}-03$ & \\
\hline & & & 12.7 & & \\
\hline & & & 12 & & \\
\hline & & 16.9 & 17.3 & & \\
\hline 5. & 4 & 117.6 & 6.8 & & \\
\hline 5. & 4 & 126 & 9.7 & & \\
\hline 5. & & 115.3 & 7.6 & & \\
\hline 5.3 & . & 114.3 & -1.6 & & \\
\hline 5 . & . & 124. & 10.5 & & \\
\hline 5.3 & $x_{2}$ & 102.2 & -25.4 & & \\
\hline 5.3 & 政 & 127.3 & 0.6 & & \\
\hline 5.3 & 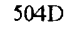 & 131.3 & -3.9 & & \\
\hline 5.3 & . & 140.2 & 4 & & \\
\hline 5.3 & & 19.7 & 1.9 & & \\
\hline 0.0 & $504 \mathrm{D}$ & 125.5 & -25.4 & & \\
\hline 5.4 & $505 \mathrm{~A}$ & 92 & -25.3 & 6085 & \\
\hline 5.4 & $505 A$ & 90.2 & -26.5 & 7.331 & \\
\hline 15.4 & $505 \mathrm{~A}$ & 92.1 & -28.1 & 7.401 & \\
\hline 15.4 & $505 \mathrm{~A}$ & 94.4 & -28.3 & & \\
\hline 15.4 & $505 \mathrm{~A}$ & 94.5 & -31.8 & 7.10 & \\
\hline 15.4 & ה & 95.7 & -32 & 0.02 & \\
\hline 5.4 & 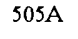 & 93 & -33 & & \\
\hline 5.4 & $05 \mathrm{~A}$ & 94.8 & -35.2 & & \\
\hline 5.4 & ה & 90.8 & 3.7 & & \\
\hline 5.4 & 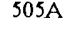 & 92.7 & 7.7 & & \\
\hline 5.4 & 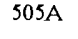 & 89.2 & -2 & 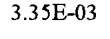 & \\
\hline 5.4 & 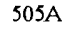 & 5.3 & -36.4 & 191 & \\
\hline 5.4 & $05 \mathrm{~A}$ & 0.1 & 42.4 & 2.87 & \\
\hline 5.4 & $5 \mathrm{~A}$ & 68.6 & 46 & $3.25 \mathrm{I}$ & \\
\hline 15.4 & $05 \mathrm{~A}$ & 98.4 & 44.3 & 2.661 & \\
\hline 15.4 & $5 \mathrm{~A}$ & 97.6 & -53.3 & 1.761 & 10 \\
\hline 15.4 & $05 \mathrm{~A}$ & 79.6 & 49.3 & $1.96 \mathrm{E}$ & 1 \\
\hline 15.6 & $6 \mathrm{~B}$ & 20.7 & 22.2 & $1.14 \mathrm{~F}$ & \\
\hline 15.6 & $6 \mathrm{~B}$ & 118 & 18.3 & 1.16 & \\
\hline 15.6 & $6 \mathrm{~B}$ & 120.2 & 14.4 & 1.161 & \\
\hline 15.6 & $6 \mathrm{~B}$ & 122.1 & 10.5 & $1.16 \mathrm{I}$ & \\
\hline 15.6 & & 1.6 & 8.6 & $1.14]$ & \\
\hline 15.6 & & 21.5 & 3.3 & $1.09 \mathrm{~F}$ & \\
\hline 15.6 & & 119.1 & 2.3 & $1.09 \mathrm{I}$ & \\
\hline . & & 118.9 & -1.7 & $1.06 \mathrm{E}$ & \\
\hline (n) & & 119.2 & -2.6 & $1.06 \mathrm{E}-02$ & \\
\hline 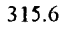 & & 116.7 & -4.3 & & \\
\hline & & 119 & -6.4 & & \\
\hline & & 118.9 & -7.5 & & \\
\hline 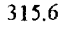 & & 117.8 & -13.6 & & \\
\hline . & & 124 & -16.3 & $6.39 \mathrm{E}-03$ & \\
\hline 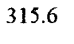 & & 131.5 & 0.2 & $6.09 \mathrm{E}-03$ & 85 \\
\hline . & (1) & 136 & -10.4 & $5.40 \mathrm{E}-03$ & 200 \\
\hline . & & 102.5 & -9 & $5.84 \mathrm{E}-03$ & Ans \\
\hline 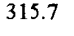 & 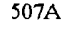 & nes & -10.1 & 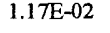 & \\
\hline
\end{tabular}

$\begin{array}{llllll}315.7 & 507 \mathrm{~A} & 103.3 & -18.2 & 1.22 \mathrm{E}-02 & 3\end{array}$

$\begin{array}{lllllll}315.7 & 507 \mathrm{~A} & 104.6 & -20.4 & 1.22 \mathrm{E}-02 & 6\end{array}$

$\begin{array}{lllllll}315.7 & 507 \mathrm{~A} & 106.1 & -20.1 & 1.19 \mathrm{E}-02 & 9\end{array}$

$\begin{array}{lllllll}315.7 & 507 \mathrm{~A} & 106 & -21.8 & 1.18 \mathrm{E}-02 & 12\end{array}$

$\begin{array}{lllllll}315.7 & 507 \mathrm{~A} & 106.7 & -24.3 & 1.10 \mathrm{E}-02 & 15\end{array}$

$\begin{array}{lllllll}315.7 & 507 \mathrm{~A} & 109 & -23.9 & 1.08 \mathrm{E}-02 & 18\end{array}$

$\begin{array}{lllllll}315.7 & 507 \mathrm{~A} & 108.6 & -26.3 & 1.04 \mathrm{E}-02 & 21\end{array}$

$\begin{array}{lllllll}315.7 & 507 \mathrm{~A} & 111.9 & -27 & 9.61 \mathrm{E}-03 & 25\end{array}$

$\begin{array}{lllllll}315.7 & 507 \mathrm{~A} & 109.2 & -29.2 & 9.07 \mathrm{E}-03 & 30\end{array}$

$\begin{array}{llllll}315.7 & 507 \mathrm{~A} & 111 & -31.3 & 7.65 \mathrm{E}-03 & 40\end{array}$

$\begin{array}{lllllll}315.7 & 507 \mathrm{~A} & 115.2 & -31.4 & 6.89 \mathrm{E}-03 & 50\end{array}$

$\begin{array}{lllllll}315.7 & 507 \mathrm{~A} & 113.1 & -34.7 & 5.88 \mathrm{E}-03 & 60\end{array}$

$\begin{array}{lllllll}315.7 & 507 \mathrm{~A} & 121.6 & -33.8 & 6.22 \mathrm{E}-03 & 70\end{array}$

$\begin{array}{llllll}315.7 & 507 \mathrm{~A} & 117.2 & -38 & 5.26 \mathrm{E}-03 & 85\end{array}$

$\begin{array}{lllllll}315.7 & 507 \mathrm{~A} & 117.9 & -34.9 & 5.97 \mathrm{E}-03 & 100\end{array}$

$\begin{array}{lllllll}315.7 & 507 \mathrm{~A} & 106.4 & -32.5 & 3.93 \mathrm{E}-03 & 115\end{array}$

$\begin{array}{llllll}315.8 & 508 \mathrm{~A} & 335.7 & 75.8 & 5.09 \mathrm{E}-03 & 0\end{array}$

$\begin{array}{lllllll}315.8 & 508 \mathrm{~A} & 356.1 & 74.9 & 4.44 \mathrm{E}-03 & 3\end{array}$

$\begin{array}{lllllll}315.8 & 508 \mathrm{~A} & 24.1 & 76.1 & 3.88 \mathrm{E}-03 & 6\end{array}$

$\begin{array}{lllllll}315.8 & 508 \mathrm{~A} & 50.4 & 73.8 & 3.57 \mathrm{E}-03 & 9\end{array}$

$\begin{array}{lllllll}315.8 & 508 \mathrm{~A} & 64.7 & 71.9 & 3.08 \mathrm{E}-03 & 12\end{array}$

$\begin{array}{lllllll}315.8 & 508 \mathrm{~A} & 63 & 70.9 & 2.28 \mathrm{E}-03 & 15\end{array}$

$\begin{array}{lllllll}315.8 & 508 \mathrm{~A} & 65.8 & 79.8 & 2.13 \mathrm{E}-03 & 18\end{array}$

$\begin{array}{lllllll}315.8 & 508 \mathrm{~A} & 60.7 & 84.5 & 1.84 \mathrm{E}-03 & 21\end{array}$

$\begin{array}{lllllll}315.8 & 508 \mathrm{~A} & 126.8 & 83.1 & 1.76 \mathrm{E}-03 & 25\end{array}$

$\begin{array}{lllllll}315.8 & 508 \mathrm{~A} & 36.2 & 82.1 & 1.25 \mathrm{E}-03 & 30\end{array}$

$\begin{array}{lllllll}315.8 & 508 \mathrm{~A} & 270.4 & 53.4 & 9.77 \mathrm{E}-04 & 40\end{array}$

$\begin{array}{llllll}315.8 & 508 \mathrm{~A} & 123.5 & 56.6 & 1.13 \mathrm{E}-03 & 50\end{array}$

$\begin{array}{lllllll}315.8 & 508 \mathrm{~A} & 271 & 40.3 & 7.74 \mathrm{E}-04 & 60\end{array}$

$\begin{array}{llllllll}315.8 & 508 \mathrm{~A} & 104.5 & -33.4 & 8.05 \mathrm{E}-04 & 70\end{array}$

$\begin{array}{lllllll}315.8 & 508 \mathrm{~A} & 110.4 & 4.1 & 9.91 \mathrm{E}-04 & 85\end{array}$

$\begin{array}{lllllll}315.8 & 508 \mathrm{~A} & 110 & 53.7 & 1.33 \mathrm{E}-03 & 100\end{array}$

$\begin{array}{lllllll}315.8 & 508 \mathrm{~A} & 77.9 & -10 & 4.93 \mathrm{E}-04 & 115\end{array}$

$\begin{array}{lllllll}315.9 & 509 \mathrm{~A} & 100.9 & 13.5 & 3.77 \mathrm{E}-03 & 0\end{array}$

$\begin{array}{llllll}315.9 & 509 \mathrm{~A} & 96.9 & 6.5 & 4.04 \mathrm{E}-03 & 3\end{array}$

$\begin{array}{lllllll}315.9 & 509 \mathrm{~A} & 100.3 & -1.4 & 4.16 \mathrm{E}-03 & 6\end{array}$

$\begin{array}{lllllll}315.9 & 509 \mathrm{~A} & 101.2 & -5.4 & 3.99 \mathrm{E}-03 & 9\end{array}$

$\begin{array}{llllll}315.9 & 509 \mathrm{~A} & 108.4 & -5.2 & 4.12 \mathrm{E}-03 & 12\end{array}$

$\begin{array}{lllllll}315.9 & 509 \mathrm{~A} & 108.4 & -9.8 & 3.66 \mathrm{E}-03 & 15\end{array}$

$\begin{array}{lllllll}315.9 & 509 \mathrm{~A} & 114.9 & 4.2 & 3.47 \mathrm{E}-03 & 18\end{array}$

$\begin{array}{lllllll}315.9 & 509 \mathrm{~A} & 112.8 & -6.2 & 3.38 \mathrm{E}-03 & 21\end{array}$

$\begin{array}{lllllll}315.9 & 509 \mathrm{~A} & 115.1 & -11.3 & 3.02 \mathrm{E}-03 & 25\end{array}$

$\begin{array}{lllllll}315.9 & 509 \mathrm{~A} & 116 & -11.9 & 2.89 \mathrm{E}-03 & 30\end{array}$

$\begin{array}{lllllll}315.9 & 509 \mathrm{~A} & 126.7 & -9.6 & 2.24 \mathrm{E}-03 & 40\end{array}$

$\begin{array}{lllllll}315.9 & 509 \mathrm{~A} & 125.5 & -16.2 & 1.55 \mathrm{E}-03 & 50\end{array}$

$\begin{array}{lllllll}315.9 & 509 \mathrm{~A} & 136.1 & -7.3 & 1.90 \mathrm{E}-03 & 60\end{array}$

$\begin{array}{lllllll}315.9 & 509 \mathrm{~A} & 124.2 & -6.4 & 1.40 \mathrm{E}-03 & 70\end{array}$

$\begin{array}{lllllll}315.9 & 509 \mathrm{~A} & 137.3 & -18.6 & 1.37 \mathrm{E}-03 & 85\end{array}$

$\begin{array}{lllllll}315.9 & 509 \mathrm{~A} & 117.1 & 9.8 & 1.42 \mathrm{E}-03 & 100\end{array}$

$\begin{array}{llllllll}315.9 & 509 \mathrm{~A} & 94.2 & -12.4 & 8.69 \mathrm{E}-04 & 115\end{array}$

$\begin{array}{llllll}316.1 & 510 \mathrm{~A} & 323.5 & 41.2 & 4.40 \mathrm{E}-03 & 0\end{array}$ $\begin{array}{llllll}316.1 & 510 \mathrm{~A} & 333.2 & 38.8 & 4.07 \mathrm{E}-03 & 3\end{array}$

$\begin{array}{lllllll}316.1 & 510 \mathrm{~A} & 337.1 & 37.9 & 3.19 \mathrm{E}-03 & 6\end{array}$

$\begin{array}{lllllll}316.1 & 510 \mathrm{~A} & 346.4 & 32.5 & 2.71 \mathrm{E}-03 & 9\end{array}$

$\begin{array}{lllllll}316.1 & 510 \mathrm{~A} & 356.2 & 35.7 & 2.26 \mathrm{E}-03 & 12\end{array}$

$\begin{array}{lllllll}316.1 & 510 \mathrm{~A} & 7.2 & 30.3 & 1.78 \mathrm{E}-03 & 15\end{array}$

$\begin{array}{llllll}316.1 & 510 \mathrm{~A} & 359.3 & 23 & 1.76 \mathrm{E}-03 & 18\end{array}$

$\begin{array}{llllll}316.1 & 510 \mathrm{~A} & 348.9 & 9.3 & 1.42 \mathrm{E}-03 & 21\end{array}$

$\begin{array}{llllll}316.1 & 510 \mathrm{~A} & 1.4 & 32.2 & 1.02 \mathrm{E}-03 & 25\end{array}$

$\begin{array}{lllllll}316.1 & 510 \mathrm{~A} & 21.2 & -15.1 & 9.76 \mathrm{E}-04 & 30\end{array}$

$\begin{array}{llllll}316.1 & 510 \mathrm{~A} & 6.5 & 4.7 & 8.17 \mathrm{E}-04 & 40\end{array}$

$\begin{array}{llllll}316.1 & 510 \mathrm{~A} & 27 & 38.9 & 1.26 \mathrm{E}-03 & 50\end{array}$

$\begin{array}{llllllll}316.1 & 510 \mathrm{~A} & 57.4 & -11.3 & 7.06 \mathrm{E}-04 & 60\end{array}$

$\begin{array}{llllllll}316.1 & 510 \mathrm{~A} & 64.4 & -31.7 & 1.21 \mathrm{E}-03 & 70\end{array}$

$\begin{array}{llllllll}316.1 & 510 \mathrm{~A} & 247.1 & -13.1 & 5.53 \mathrm{E}-04 & 85\end{array}$

$\begin{array}{lllllll}316.1 & 510 \mathrm{~A} & 70.6 & -20.8 & 1.52 \mathrm{E}-03 & 100\end{array}$

$\begin{array}{lllllll}316.1 & 510 \mathrm{~A} & 16.3 & 3.6 & 8.07 \mathrm{E}-04 & 115\end{array}$

$\begin{array}{lllllll}317.6 & 511 \mathrm{~A} & 267.1 & 78.5 & 1.00 \mathrm{E}-02 & 0\end{array}$

$\begin{array}{lllllll}317.6 & 511 \mathrm{~A} & 278.8 & 81.4 & 8.96 \mathrm{E}-03 & 3\end{array}$

$\begin{array}{lllllll}317.6 & 511 \mathrm{~A} & 239.2 & 83.2 & 7.74 \mathrm{E}-03 & 6\end{array}$

$\begin{array}{lllllll}317.6 & 511 \mathrm{~A} & 170.1 & 83.5 & 7.15 \mathrm{E}-03 & 9\end{array}$

$\begin{array}{lllllll}317.6 & 511 \mathrm{~A} & 131.7 & 80.9 & 6.27 \mathrm{E}-03 & 12\end{array}$

$\begin{array}{lllllll}317.6 & 511 \mathrm{~A} & 139.7 & 80.7 & 6.02 \mathrm{E}-03 & 15\end{array}$

$\begin{array}{lllllll}317.6 & 511 \mathrm{~A} & 135 & 72.4 & 5.96 \mathrm{E}-03 & 18\end{array}$

$\begin{array}{lllllll}317.6 & 511 \mathrm{~A} & 87.2 & 71.2 & 5.38 \mathrm{E}-03 & 21\end{array}$

$\begin{array}{lllllll}317.6 & 511 \mathrm{~A} & 76.1 & 64.2 & 5.11 \mathrm{E}-03 & 25\end{array}$

$\begin{array}{lllllll}317.6 & 511 \mathrm{~A} & 101.7 & 70.1 & 4.51 \mathrm{E}-03 & 30\end{array}$

$\begin{array}{lllllll}317.6 & 511 \mathrm{~A} & 62.3 & 67.1 & 5.43 \mathrm{E}-03 & 40\end{array}$

$\begin{array}{lllllll}317.6 & 511 \mathrm{~A} & 98.1 & 73.6 & 5.52 \mathrm{E}-03 & 50\end{array}$

$\begin{array}{lllllll}317.6 & 511 \mathrm{~A} & 110.3 & 57.8 & 4.28 \mathrm{E}-03 & 60\end{array}$

$\begin{array}{lllllll}317.6 & 511 \mathrm{~A} & 82.4 & 61.9 & 3.41 \mathrm{E}-03 & 70\end{array}$

$\begin{array}{lllllll}317.6 & 511 \mathrm{~A} & 29.8 & 72.9 & 2.75 \mathrm{E}-03 & 85\end{array}$

$\begin{array}{lllllll}317.6 & 511 \mathrm{~A} & 55.3 & 39.7 & 2.66 \mathrm{E}-03 & 100\end{array}$

$\begin{array}{lllllll}317.6 & 511 \mathrm{~A} & 49.1 & 67.4 & 2.15 \mathrm{E}-03 & 115\end{array}$

$\begin{array}{lllllll}318.4 & 512 \mathrm{~A} & 340.2 & 47.1 & 1.42 \mathrm{E}-02 & 0\end{array}$

$\begin{array}{lllllll}318.4 & 512 \mathrm{~A} & 347.9 & 44.3 & 1.36 \mathrm{E}-02 & 3\end{array}$

$\begin{array}{lllllll}318.4 & 512 \mathrm{~A} & 352.1 & 40.9 & 1.26 \mathrm{E}-02 & 6\end{array}$

$\begin{array}{lllllll}318.4 & 512 \mathrm{~A} & 355.7 & 37.7 & 1.13 \mathrm{E}-02 & 9\end{array}$

$\begin{array}{lllllll}318.4 & 512 \mathrm{~A} & 355.7 & 34.4 & 1.10 \mathrm{E}-02 & 12\end{array}$

$\begin{array}{lllllll}318.4 & 512 \mathrm{~A} & 352.8 & 32.9 & 1.03 \mathrm{E}-02 & 15\end{array}$

$\begin{array}{lllllll}318.4 & 512 \mathrm{~A} & 359 & 32.8 & 9.41 \mathrm{E}-03 & 18\end{array}$

$\begin{array}{lllllll}318.4 & 512 \mathrm{~A} & 0.3 & 23.9 & 8.91 \mathrm{E}-03 & 21\end{array}$

$\begin{array}{lllllll}318.4 & 512 \mathrm{~A} & 4.8 & 19.8 & 8.79 \mathrm{E}-03 & 25\end{array}$

$\begin{array}{lllllll}318.4 & 512 \mathrm{~A} & 9.5 & 24.1 & 7.69 \mathrm{E}-03 & 30\end{array}$

$\begin{array}{lllllll}318.4 & 512 \mathrm{~A} & 14.4 & 17.8 & 7.64 \mathrm{E}-03 & 40\end{array}$

$\begin{array}{lllllll}318.4 & 512 \mathrm{~A} & 10.5 & 16.1 & 7.16 \mathrm{E}-03 & 50\end{array}$

$\begin{array}{lllllll}318.4 & 512 \mathrm{~A} & 4.6 & 15.8 & 5.36 \mathrm{E}-03 & 60\end{array}$

$\begin{array}{llllll}318.4 & 512 \mathrm{~A} & 6.3 & 7 & 6.94 \mathrm{E}-03 & 70\end{array}$

$\begin{array}{lllllll}318.4 & 512 \mathrm{~A} & 14.9 & 0.8 & 5.70 \mathrm{E}-03 & 85\end{array}$

$\begin{array}{lllllll}318.4 & 512 \mathrm{~A} & 18.5 & 8.6 & 6.68 \mathrm{E}-03 & 100\end{array}$

$\begin{array}{lllllll}318.4 & 512 \mathrm{~A} & 359.3 & 16 & 5.80 \mathrm{E}-03 & 115\end{array}$

$\begin{array}{llllll}319.8 & 513 \mathrm{~A} & 311.2 & 68.6 & 1.21 \mathrm{E}-02 & 0\end{array}$ 


\begin{tabular}{|c|c|c|c|c|c|}
\hline & $513 \mathrm{~A}$ & 26.7 & 68.9 & $1.07 \mathrm{E}$ & \\
\hline 19.8 & $513 \mathrm{~A}$ & 333.4 & 72.6 & $9.36 \mathrm{E}-03$ & \\
\hline 9.8 & $513 \mathrm{~A}$ & 349.8 & 31.4 & $7.97 \mathrm{E}-03$ & \\
\hline 9.8 & $513 \mathrm{~A}$ & 359.2 & 68.8 & $6.80 \mathrm{E}-03$ & \\
\hline 9.8 & $513 \mathrm{~A}$ & 7.5 & 74.2 & $5.85 \mathrm{E}-03$ & 15 \\
\hline 9.8 & $513 \mathrm{~A}$ & 350.7 & 764.6 & $5.39 \mathrm{E}-03$ & 18 \\
\hline 9.8 & $513 \mathrm{~A}$ & 4.2 & 66.2 & $5.01 \mathrm{E}$ & $21+>$ \\
\hline 9.8 & $513 \mathrm{~A}$ & 18.5 & 55.8 & $3.50 \mathrm{E}-03$ & $2:$ \\
\hline 98 & $513 \mathrm{~A}$ & 8.1 & 52 & & 30 \\
\hline 198 & $513 \mathrm{~A}$ & 359.4 & 67.5 & & 40 \\
\hline 198 & $513 \mathrm{~A}$ & 334.9 & 15.4 & & 50 \\
\hline 9.8 & $513 \mathrm{~A}$ & 343.7 & 33.4 & & 60 \\
\hline 9.8 & $513 \mathrm{~A}$ & 303.7 & -7.9 & 2.47 & 7 \\
\hline 19.8 & $513 \mathrm{~A}$ & 297.9 & 32.5 & $1.14 \mathrm{E}$ & \\
\hline 9.8 & $513 \mathrm{~A}$ & 252.8 & 29.8 & $1.18 \mathrm{~F}$ & 100 \\
\hline 19.8 & $513 \mathrm{~A}$ & 301.5 & 5 & 1.931 & 11 \\
\hline & $45 \mathrm{~A}$ & 17.7 & -3.1 & $2.43 \mathrm{~F}$ & \\
\hline 25 & $45 \mathrm{~A}$ & 17.6 & -3.8 & 2.46 & \\
\hline 25 & $45 \mathrm{~A}$ & 18.3 & -6.8 & $2.46 \mathrm{E}$ & \\
\hline 25 & $45 \mathrm{~A}$ & 20.2 & -20.5 & $2.54 \mathrm{I}$ & \\
\hline 25 & $45 \mathrm{~A}$ & 21.7 & -28.6 & 2.601 & 12 \\
\hline 25 & $45 \mathrm{~A}$ & 25 & -33.8 & $2.50 \mathrm{E}$ & 15 \\
\hline 25 & $45 \mathrm{~A}$ & 26.6 & -36.5 & $2.53 \mathrm{E}$ & 18 \\
\hline 25 & $45 \mathrm{~A}$ & 26.1 & 41.3 & $2.62 \mathrm{E}$ & 18 \\
\hline 25 & $45 \mathrm{~A}$ & 26 & -40.9 & $2.66 \mathrm{E}$ & 21 \\
\hline 25 & $45 \mathrm{~A}$ & 27.3 & 42.7 & $2.38 \mathrm{E}$ & 25 \\
\hline 25 & $45 \mathrm{~A}$ & 27.3 & -38.8 & $2.18 \mathrm{E}-03$ & 30 \\
\hline 325 & $45 \mathrm{~A}$ & 19.5 & -40.7 & $1.82 \mathrm{E}-03$ & 40 \\
\hline 325 & $45 \mathrm{~A}$ & 37.2 & -39.2 & $1.24 \mathrm{E}-$ & 50 \\
\hline 325 & $45 \mathrm{~A}$ & 12.6 & -28.5 & $1.26 \mathrm{E}$ & 60 \\
\hline 25 & $45 \mathrm{~A}$ & 4 & -29.4 & $6.40 \mathrm{E}-$ & 70 \\
\hline 25 & $45 \mathrm{~A}$ & 3.9 & -34.6 & $3.85 \mathrm{E}-04$ & 85 \\
\hline 25 & $45 \mathrm{~A}$ & 358.6 & -15.5 & $1.18 \mathrm{E}-03$ & 100 \\
\hline 325 & $45 \mathrm{~A}$ & 55.9 & -51 & $4.38 \mathrm{E}-$ & 115 \\
\hline 325.8 & $46 \mathrm{~A}$ & 132.6 & -30.1 & $4.93 \mathrm{E}-03$ & 0 \\
\hline 325.8 & $46 \mathrm{~A}$ & 132 & -34.9 & $5.03 \mathrm{E}-03$ & \\
\hline 325.8 & $46 \mathrm{~A}$ & 130.3 & -39 & $5.26 \mathrm{E}-03$ & \\
\hline 325.8 & $46 \mathrm{~A}$ & 129.6 & -41.4 & $5.16 \mathrm{E}-$ & \\
\hline 325.8 & $46 \mathrm{~A}$ & 130.1 & -42.9 & $5.02 \mathrm{E}$ & 12 \\
\hline 058 & $46 \mathrm{~A}$ & 129.6 & 43.4 & $4.83 \mathrm{E}$ & 15 \\
\hline 2058 & $46 \mathrm{~A}$ & 129.8 & -43.8 & $4.48 \mathrm{E}$ & 18 \\
\hline 058 & $46 \mathrm{~A}$ & 129.4 & 44.9 & & 21 \\
\hline 3258 & $46 \mathrm{~A}$ & 127 & 44.8 & & 25 \\
\hline 3258 & $46 \mathrm{~A}$ & 128.6 & -47.2 & $3.20 \mathrm{E}-03$ & 30 \\
\hline anc & $46 \mathrm{~A}$ & 128.1 & -45.7 & $2.33 \mathrm{E}-$ & 40 \\
\hline - & $46 \mathrm{~A}$ & 134.2 & & & No \\
\hline & $46 \mathrm{~A}$ & 148.8 & -55.1 & & \\
\hline 年 & $46 \mathrm{~A}$ & 133.7 & -55.6 & & , \\
\hline 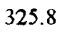 & $46 \mathrm{~A}$ & 188.6 & -56.4 & $9.17 \mathrm{E}-04$ & 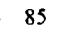 \\
\hline 0 & $46 \mathrm{~A}$ & 133.7 & 49.2 & $0.10 \mathrm{E}-0 \mathrm{t}$ & . \\
\hline & $40 \mathrm{~A}$ & 128 & 47.6 & 0.170 & \\
\hline
\end{tabular}

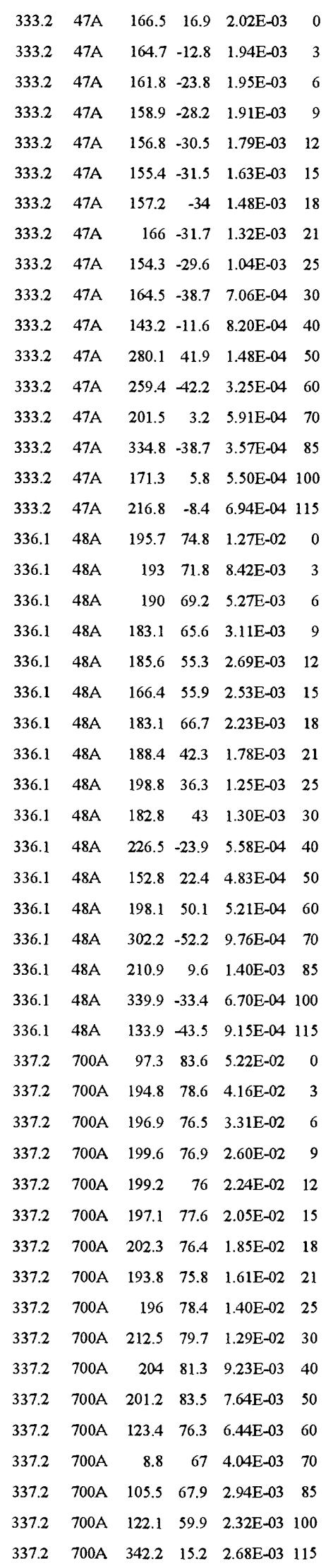

$\begin{array}{llllll}347.4 & 701 \mathrm{~A} & 294.6 & 74.4 & 5.17 \mathrm{E}-02 & 0\end{array}$ $\begin{array}{llllll}347.4 & 701 \mathrm{~A} & 297.4 & 78.8 & 4.64 \mathrm{E}-02 & 3\end{array}$ $\begin{array}{llllll}347.4 & 701 \mathrm{~A} & 81 & 51 & 4.25 \mathrm{E}-02 & 6\end{array}$ $\begin{array}{llllll}347.4 & 701 \mathrm{~A} & 297.1 & 76.7 & 3.89 \mathrm{E}-02 & 9\end{array}$ $\begin{array}{llllll}347.4 & 701 \mathrm{~A} & 78.4 & 52 & 3.53 \mathrm{E}-02 & 12\end{array}$ $\begin{array}{lllllll}347.4 & 701 \mathrm{~A} & 303.9 & 75.4 & 3.37 \mathrm{E}-02 & 15\end{array}$ $\begin{array}{llllll}347.4 & 701 \mathrm{~A} & 76 & 52.8 & 3.06 \mathrm{E}-02 & 18\end{array}$ $\begin{array}{llllll}347.4 & 701 \mathrm{~A} & 75.9 & 52.5 & 2.84 \mathrm{E}-02 & 21\end{array}$ $\begin{array}{llllll}347.4 & 701 \mathrm{~A} & 73.8 & 52.6 & 2.57 \mathrm{E}-02 & 25\end{array}$ $\begin{array}{llllll}347.4 & 701 \mathrm{~A} & 75 & 53.1 & 2.14 \mathrm{E}-02 & 30\end{array}$ $\begin{array}{llllll}347.4 & 701 \mathrm{~A} & 308.1 & 71.1 & 1.67 \mathrm{E}-02 & 40\end{array}$ $\begin{array}{lllllll}347.4 & 701 \mathrm{~A} & 314.2 & 73.7 & 1.08 \mathrm{E}-02 & 50\end{array}$ $\begin{array}{lllllll}347.4 & 701 \mathrm{~A} & 88.7 & 46.7 & 8.41 \mathrm{E}-03 & 60\end{array}$ $\begin{array}{llllll}347.4 & 701 \mathrm{~A} & 78.6 & 47 & 5.53 \mathrm{E}-03 & 70\end{array}$ $\begin{array}{lllllll}347.4 & 701 \mathrm{~A} & 168.8 & 78.6 & 3.82 \mathrm{E}-03 & 85\end{array}$ $\begin{array}{lllllll}347.4 & 701 \mathrm{~A} & 292.6 & 59.5 & 2.98 \mathrm{E}-03 & 100\end{array}$ $\begin{array}{llllll}347.4 & 701 \mathrm{~A} & 89.1 & 54.3 & 1.08 \mathrm{E}-03 & 115\end{array}$ $\begin{array}{llllll}349.1 & 702 \mathrm{~A} & 335.2 & 62.4 & 4.67 \mathrm{E}-02 & 0\end{array}$ $\begin{array}{llllll}349.1 & 702 \mathrm{~A} & 330.4 & 60.8 & 3.73 \mathrm{E}-02 & 3\end{array}$ $\begin{array}{llllll}349.1 & 702 \mathrm{~A} & 44.5 & 50 & 2.87 \mathrm{E}-02 & 6\end{array}$ $\begin{array}{llllll}349.1 & 702 \mathrm{~A} & 327.2 & 49.3 & 2.10 \mathrm{E}-02 & 9\end{array}$ $\begin{array}{llllll}349.1 & 702 \mathrm{~A} & 325.9 & 45.8 & 1.71 \mathrm{E}-02 & 12\end{array}$ $\begin{array}{lllllll}349.1 & 702 \mathrm{~A} & 17.7 & 49.5 & 1.51 \mathrm{E}-02 & 15\end{array}$ $\begin{array}{llllll}349.1 & 702 \mathrm{~A} & 9 & 46.8 & 1.32 \mathrm{E}-02 & 18\end{array}$ $\begin{array}{llllll}349.1 & 702 \mathrm{~A} & 7.6 & 48 & 1.19 \mathrm{E}-02 & 21\end{array}$ $\begin{array}{llllll}349.1 & 702 \mathrm{~A} & 0.2 & 48.3 & 1.10 \mathrm{E}-02 & 25\end{array}$ $\begin{array}{llllll}349.1 & 702 \mathrm{~A} & 354.7 & 46.7 & 9.97 \mathrm{E}-03 & 30\end{array}$ $\begin{array}{llllll}349.1 & 702 \mathrm{~A} & 349.7 & 37.5 & 8.61 \mathrm{E}-03 & 40\end{array}$ $\begin{array}{lllllll}349.1 & 702 \mathrm{~A} & 331.1 & 36.7 & 6.25 \mathrm{E}-03 & 50\end{array}$ $\begin{array}{llllll}349.1 & 702 \mathrm{~A} & 344.9 & 29.1 & 5.45 \mathrm{E}-03 & 60\end{array}$ $\begin{array}{lllllll}349.1 & 702 \mathrm{~A} & 342.2 & 36.1 & 6.37 \mathrm{E}-03 & 70\end{array}$ $\begin{array}{lllllll}349.1 & 702 \mathrm{~A} & 348.8 & 34.4 & 3.65 \mathrm{E}-03 & 85\end{array}$ $\begin{array}{lllllll}349.1 & 702 \mathrm{~A} & 328.2 & 26 & 5.43 \mathrm{E}-03 & 100\end{array}$ $\begin{array}{lllllll}349.1 & 702 \mathrm{~A} & 299 & 29.8 & 5.11 \mathrm{E}-03 & 115\end{array}$ $\begin{array}{llllll}351 & 703 \mathrm{~A} & 2.2 & 60.5 & 7.65 \mathrm{E}-02 & 0\end{array}$ $\begin{array}{llllll}351 & 703 \mathrm{~A} & 56.6 & 35.4 & 6.07 \mathrm{E}-02 & 3\end{array}$ $\begin{array}{lllllll}351 & 703 \mathrm{~A} & 55.7 & 34.8 & 4.39 \mathrm{E}-02 & 6\end{array}$ $\begin{array}{lllllll}351 & 703 \mathrm{~A} & 51.4 & 32.2 & 3.03 \mathrm{E}-02 & 9\end{array}$ $\begin{array}{llllll}351 & 703 \mathrm{~A} & 50.4 & 33.3 & 2.42 \mathrm{E}-02 & 12\end{array}$ $\begin{array}{llllll}351 & 703 \mathrm{~A} & 51.2 & 30 & 1.90 \mathrm{E}-02 & 15\end{array}$

$\begin{array}{llllll}351 & 703 \mathrm{~A} & 44.8 & 33.6 & 1.48 \mathrm{E}-02 & 18\end{array}$

$\begin{array}{llllll}351 & 703 \mathrm{~A} & 44.5 & 31.9 & 1.17 \mathrm{E}-02 & 21\end{array}$

$\begin{array}{lllllll}351 & 703 \mathrm{~A} & 38.2 & 30.2 & 9.06 \mathrm{E}-03 & 25\end{array}$

$\begin{array}{lllllll}351 & 703 \mathrm{~A} & 40.5 & 32.5 & 7.98 \mathrm{E}-03 & 30\end{array}$

$\begin{array}{llllll}351 & 703 \mathrm{~A} & 39.6 & 15.4 & 4.94 \mathrm{E}-03 & 40\end{array}$

$\begin{array}{lllllll}351 & 703 \mathrm{~A} & 29.6 & 44.1 & 3.92 \mathrm{E}-03 & 50\end{array}$

$\begin{array}{llllll}351 & 703 \mathrm{~A} & 34 & 20 & 3.28 \mathrm{E}-03 & 60\end{array}$

$\begin{array}{llllll}351 & 703 \mathrm{~A} & 23.5 & 54.8 & 2.99 \mathrm{E}-03 & 70\end{array}$

$\begin{array}{llllll}351 & 703 \mathrm{~A} & 33.4 & 3.6 & 2.14 \mathrm{E}-03 & 85\end{array}$

$\begin{array}{llllll}351 & 703 \mathrm{~A} & 121.1 & 50.9 & 1.85 \mathrm{E}-03 & 100\end{array}$

$\begin{array}{llllll}351 & 703 \mathrm{~A} & 16.2 & 48.2 & 1.21 \mathrm{E}-03 & 115\end{array}$ 


\begin{tabular}{|c|c|c|c|c|c|}
\hline & $49 \mathrm{~A}$ & 26.8 & 69.2 & 2.715 & \\
\hline 57.9 & $49 \mathrm{~A}$ & 130 & 65.8 & $2.02 E-02$ & \\
\hline 7.9 & $49 \mathrm{~A}$ & 132.1 & 62.3 & $1.70 \mathrm{E}$ & \\
\hline & $49 \mathrm{~A}$ & 38.6 & 59.6 & $1.44 \mathrm{E}$ & \\
\hline 7 & $49 \mathrm{~A}$ & 139.7 & 57.9 & $1.29 \mathrm{E}$ & \\
\hline 7.9 & $49 \mathrm{~A}$ & 136.3 & 56.8 & $1.20 \mathrm{E}$ & \\
\hline 7.9 & $49 \mathrm{~A}$ & 137.5 & 57.2 & $1.08 \mathrm{I}$ & \\
\hline 7.9 & $49 \mathrm{~A}$ & 132.6 & 51.3 & 9.381 & \\
\hline 7.9 & $49 \mathrm{~A}$ & 134.8 & 47.2 & $8.11 \mathrm{H}$ & \\
\hline 7.9 & $49 \mathrm{~A}$ & 127.3 & 48.3 & 6.65 & \\
\hline 7.9 & $49 \mathrm{~A}$ & 14.8 & 40.3 & 5.09 & \\
\hline 7.9 & $49 \mathrm{~A}$ & 14.4 & 48.1 & & \\
\hline 7.9 & $49 \mathrm{~A}$ & 99.2 & 39.8 & & \\
\hline 7.9 & $49 \mathrm{~A}$ & 95.3 & 67.9 & & \\
\hline 7.9 & $49 \mathrm{~A}$ & 03.1 & 32.1 & & \\
\hline 7.9 & $49 \mathrm{~A}$ & 89.8 & 35.4 & & 310 \\
\hline 7.9 & $49 \mathrm{~A}$ & 287 & 48.5 & & \\
\hline 9.7 & $50 \mathrm{~A}$ & 13.2 & 62.3 & & \\
\hline 9.7 & $50 \mathrm{~A}$ & 12.6 & 57.6 & & \\
\hline 9.7 & $50 \mathrm{~A}$ & 212.9 & 53.3 & $2.78 \mathrm{E}$ & \\
\hline 9.7 & $50 \mathrm{~A}$ & 212.1 & 50.1 & $2.46 \mathrm{E}$ & \\
\hline 9.7 & $50 \mathrm{~A}$ & 211.9 & 48.6 & & 2 \\
\hline 9.7 & $50 \mathrm{~A}$ & 213.4 & 48.4 & $2.08 \mathrm{E}$ & 2 \\
\hline 9.7 & $50 \mathrm{~A}$ & 212.1 & 46.4 & & \\
\hline 9.7 & $50 \mathrm{~A}$ & 214.7 & 45.3 & & \\
\hline 9.7 & $50 \mathrm{~A}$ & 214.9 & 44.7 & & \\
\hline 9.7 & $50 \mathrm{~A}$ & 210 & 44.2 & 1.42 & \\
\hline 9.7 & $50 \mathrm{~A}$ & 217.1 & 45.4 & $1.12 \mathrm{E}$ & \\
\hline 59.7 & $50 \mathrm{~A}$ & 217.3 & 45.6 & $8.65 \mathrm{E}$ & 3 \\
\hline 59.7 & $50 \mathrm{~A}$ & 215.1 & 46.2 & 6.58 & \\
\hline 59.7 & $50 \mathrm{~A}$ & 213.9 & 48.6 & 5.48 & \\
\hline 359.7 & $50 \mathrm{~A}$ & 213.5 & 53.4 & $5.45 \mathrm{E}-03$ & \\
\hline 359.7 & $50 \mathrm{~A}$ & 210.7 & 48.9 & $4.26 \mathrm{1}$ & 3 \\
\hline 359.7 & $50 \mathrm{~A}$ & 210.5 & 32.8 & $4.55 \mathrm{E}$ & $\begin{array}{lll}3 & 11\end{array}$ \\
\hline 360 & $51 \mathrm{~A}$ & 148.7 & 79.5 & $2.34 \mathrm{E}$ & \\
\hline 360 & $51 \mathrm{~A}$ & 148.1 & 76.2 & $2.02 \mathrm{E}$ & \\
\hline 360 & $51 \mathrm{~A}$ & 153.3 & 73.7 & $1.56 \mathrm{E}$ & \\
\hline 360 & $51 \mathrm{~A}$ & 156.4 & 70.5 & $1.26 \mathrm{~F}$ & \\
\hline 360 & $51 \mathrm{~A}$ & 158.2 & 67.8 & $1.05 \mathrm{E}$ & \\
\hline 360 & $51 \mathrm{~A}$ & 157.2 & 67.6 & 8.97 & \\
\hline 360 & $51 \mathrm{~A}$ & 158.9 & 64.9 & $7.83 \mathrm{E}-03$ & \\
\hline 360 & 554 & 159.6 & 63.4 & $6.90 \mathrm{E}-03$ & \\
\hline 300 & 列 & 156.7 & 62.9 & & \\
\hline 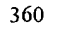 & & 158 & 62.4 & & \\
\hline 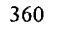 & & 169.6 & 60.7 & & \\
\hline 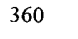 & & 168.1 & 61.1 & & \\
\hline 00 & & 175.1 & 57.5 & & \\
\hline 300 & . & 205.3 & 41.4 & & 3 \\
\hline 500 & - & 157.4 & 30 & $1.07 \mathrm{E}-03$ & $\begin{array}{ll}38 \\
8\end{array}$ \\
\hline 50 & - & 120.1 & 49.3 & $1.37 \mathrm{E}-03$ & . \\
\hline & 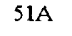 & 172.3 & 2.0 & & \\
\hline
\end{tabular}

$\begin{array}{llllll}362.7 & 704 \mathrm{~A} & 163.9 & 81.7 & 1.18 \mathrm{E}-01 & 0\end{array}$ $\begin{array}{lllllll}362.7 & 704 \mathrm{~A} & 101.3 & 34.7 & 9.88 \mathrm{E}-02 & 3\end{array}$ $\begin{array}{lllllll}362.7 & 704 \mathrm{~A} & 103.3 & 34.3 & 7.56 \mathrm{E}-02 & 6\end{array}$ $\begin{array}{lllllll}362.7 & 704 \mathrm{~A} & 152.7 & 75.6 & 5.73 \mathrm{E}-02 & 9\end{array}$ $\begin{array}{lllllll}362.7 & 704 \mathrm{~A} & 107.2 & 32.8 & 4.43 \mathrm{E}-02 & 12\end{array}$ $\begin{array}{lllllll}362.7 & 704 \mathrm{~A} & 153 & 73.3 & 3.60 \mathrm{E}-02 & 15\end{array}$ $\begin{array}{lllllll}362.7 & 704 \mathrm{~A} & 150.3 & 71.9 & 2.88 \mathrm{E}-02 & 18\end{array}$ $\begin{array}{lllllll}362.7 & 704 \mathrm{~A} & 149.6 & 71.1 & 2.50 \mathrm{E}-02 & 21\end{array}$ $\begin{array}{lllllll}362.7 & 704 \mathrm{~A} & 146.3 & 69.9 & 2.06 \mathrm{E}-02 & 25\end{array}$ $\begin{array}{lllllll}362.7 & 704 \mathrm{~A} & 152.6 & 72.4 & 1.56 \mathrm{E}-02 & 30\end{array}$ $\begin{array}{lllllll}362.7 & 704 \mathrm{~A} & 135.5 & 70.8 & 1.02 \mathrm{E}-02 & 40\end{array}$ $\begin{array}{llllll}362.7 & 704 \mathrm{~A} & 153.8 & 60.4 & 5.98 \mathrm{E}-03 & 50\end{array}$ $\begin{array}{lllllll}362.7 & 704 \mathrm{~A} & 166.2 & 63.7 & 5.09 \mathrm{E}-03 & 60\end{array}$ $\begin{array}{lllllll}362.7 & 704 \mathrm{~A} & 126.1 & 52.1 & 3.09 \mathrm{E}-03 & 70\end{array}$ $\begin{array}{lllllll}362.7 & 704 \mathrm{~A} & 95.4 & 34.5 & 2.50 \mathrm{E}-03 & 85\end{array}$ $\begin{array}{lllllll}362.7 & 704 \mathrm{~A} & 45.3 & 45.5 & 1.84 \mathrm{E}-03 & 100\end{array}$ $\begin{array}{lllllll}362.7 & 704 \mathrm{~A} & 80.2 & -22.4 & 1.91 \mathrm{E}-03 & 115\end{array}$ $\begin{array}{lllllll}371.3 & 52 \mathrm{~A} & 337.8 & 70.6 & 6.60 \mathrm{E}-02 & 0\end{array}$ $\begin{array}{lllllll}371.3 & 52 \mathrm{~A} & 346.3 & 67.3 & 5.40 \mathrm{E}-02 & 3\end{array}$ $\begin{array}{lllllll}371.3 & 52 \mathrm{~A} & 350.6 & 64.5 & 4.18 \mathrm{E}-02 & 6\end{array}$ $\begin{array}{llllll}371.3 & 52 \mathrm{~A} & 355.3 & 59.3 & 3.17 \mathrm{E}-02 & 9\end{array}$ $\begin{array}{lllllll}371.3 & 52 \mathrm{~A} & 359.9 & 56.2 & 2.66 \mathrm{E}-02 & 12\end{array}$ $\begin{array}{lllllll}371.3 & 52 \mathrm{~A} & 0 & 53.8 & 2.35 \mathrm{E}-02 & 15\end{array}$ $\begin{array}{lllllll}371.3 & 52 \mathrm{~A} & 359.8 & 51.8 & 2.04 \mathrm{E}-02 & 18\end{array}$ $\begin{array}{lllllll}371.3 & 52 \mathrm{~A} & 356.1 & 52.4 & 1.75 \mathrm{E}-02 & 21\end{array}$ $\begin{array}{lllllll}371.3 & 52 \mathrm{~A} & 356.1 & 50 & 1.42 \mathrm{E}-02 & 25\end{array}$ $\begin{array}{lllllll}371.3 & 52 \mathrm{~A} & 357.2 & 45.1 & 1.20 \mathrm{E}-02 & 30\end{array}$ $\begin{array}{lllllll}371.3 & 52 \mathrm{~A} & 343.9 & 34.7 & 9.75 \mathrm{E}-03 & 40\end{array}$ $\begin{array}{lllllll}371.3 & 52 \mathrm{~A} & 349.9 & 40.9 & 6.22 \mathrm{E}-03 & 50\end{array}$ $\begin{array}{lllllll}371.3 & 52 \mathrm{~A} & 5.1 & 36.7 & 5.18 \mathrm{E}-03 & 60\end{array}$ $\begin{array}{lllllll}371.3 & 52 \mathrm{~A} & 348.7 & 6.1 & 3.98 \mathrm{E}-03 & 70\end{array}$ $\begin{array}{lllllll}371.3 & 52 \mathrm{~A} & 15.7 & 17.8 & 2.86 \mathrm{E}-03 & 85\end{array}$ $\begin{array}{lllllll}371.3 & 52 \mathrm{~A} & 353.5 & 4.3 & 3.03 \mathrm{E}-03 & 100\end{array}$ $\begin{array}{lllllll}371.3 & 52 \mathrm{~A} & 308.5 & 41.1 & 1.69 \mathrm{E}-03 & 115\end{array}$ $\begin{array}{lllllll}375 & 300 \mathrm{~A} & 355.6 & -68.5 & 4.63 \mathrm{E}-02 & 0\end{array}$

$\begin{array}{llllll}375 & 300 \mathrm{~A} & 355 & -67 & 4.21 \mathrm{E}-02 & 3\end{array}$

$\begin{array}{lllllll}375 & 300 \mathrm{~A} & 356.2 & -66.5 & 3.75 \mathrm{E}-02 & 6\end{array}$

$\begin{array}{llllll}375 & 300 \mathrm{~A} & 356.9 & -66.2 & 3.23 \mathrm{E}-02 & 9\end{array}$

$\begin{array}{llllll}375 & 300 \mathrm{~A} & 1.3 & -66.5 & 2.86 \mathrm{E}-02 & 12\end{array}$

$\begin{array}{llllll}375 & 300 \mathrm{~A} & 1.3 & -67 & 2.59 \mathrm{E}-02 & 15\end{array}$

$\begin{array}{lllllll}375 & 300 \mathrm{~A} & 2.8 & -65.3 & 2.38 \mathrm{E}-02 & 18\end{array}$

$\begin{array}{llllll}375 \quad 300 \mathrm{~A} & 2.6 & -66.2 & 2.13 \mathrm{E}-02 & 21\end{array}$

$\begin{array}{llllll}375 & 300 \mathrm{~A} & 359 & -68.2 & 1.88 \mathrm{E}-02 & 25\end{array}$

$\begin{array}{llllll}375 & 300 \mathrm{~A} & 12.1 & -66.9 & 1.70 \mathrm{E}-02 & 30\end{array}$

$\begin{array}{lllllll}375 & 300 \mathrm{~A} & 4.9 & -67.7 & 1.31 \mathrm{E}-02 & 40\end{array}$

$\begin{array}{lllllll}375 & 300 \mathrm{~A} & 24.4 & -67.4 & 1.01 \mathrm{E}-02 & 50\end{array}$

$\begin{array}{lllllll}375 & 300 \mathrm{~A} & 67.5 & -73.7 & 6.97 \mathrm{E}-03 & 60\end{array}$

$\begin{array}{llllll}375 & 300 \mathrm{~A} & 32 & -59.7 & 6.42 \mathrm{E}-03 & 70\end{array}$

$\begin{array}{lllllll}375 & 300 \mathrm{~A} & 46.4 & -52.9 & 5.27 \mathrm{E}-03 & 85\end{array}$

$\begin{array}{lllllll}375 & 300 \mathrm{~A} & 15.6 & -66.7 & 3.77 \mathrm{E}-03 & 100\end{array}$

$\begin{array}{llllll}375 & 300 \mathrm{~A} & 64 & 45.5 & 4.03 \mathrm{E}-03 & 115\end{array}$ $\begin{array}{llllll}375.9 & 301 \mathrm{~A} & 342.1 & -65.1 & 4.55 \mathrm{E}-02 & 0\end{array}$ $\begin{array}{lllllll}375.9 & 301 \mathrm{~A} & 339.2 & -63.4 & 4.05 \mathrm{E}-02 & 3\end{array}$ $\begin{array}{lllllll}375.9 & 301 \mathrm{~A} & 338 & -63.4 & 3.53 \mathrm{E}-02 & 6\end{array}$ $\begin{array}{lllllll}375.9 & 301 \mathrm{~A} & 337.9 & -62.9 & 2.94 \mathrm{E}-02 & 9\end{array}$ $\begin{array}{lllllll}375.9 & 301 \mathrm{~A} & 341.1 & -64 & 2.54 \mathrm{E}-02 & 12\end{array}$ $\begin{array}{lllllll}375.9 & 301 \mathrm{~A} & 342.7 & -63.4 & 2.32 \mathrm{E}-02 & 15\end{array}$ $\begin{array}{lllllll}375.9 & 301 \mathrm{~A} & 347.2 & -64.1 & 2.06 \mathrm{E}-02 & 18\end{array}$ $\begin{array}{lllllll}375.9 & 301 \mathrm{~A} & 344.9 & -63.6 & 1.84 \mathrm{E}-02 & 21\end{array}$ $\begin{array}{lllllll}375.9 & 301 \mathrm{~A} & 347.4 & -62.7 & 1.60 \mathrm{E}-02 & 25\end{array}$ $\begin{array}{lllllll}375.9 & 301 \mathrm{~A} & 354.4 & -65.8 & 1.44 \mathrm{E}-02 & 30\end{array}$ $\begin{array}{lllllll}375.9 & 301 \mathrm{~A} & 343.3 & -59.9 & 1.13 \mathrm{E}-02 & 40\end{array}$ $\begin{array}{lllllll}375.9 & 301 \mathrm{~A} & 13.4 & -59.3 & 7.61 \mathrm{E}-03 & 50\end{array}$ $\begin{array}{lllllll}375.9 & 301 \mathrm{~A} & 28 & -62.6 & 5.68 \mathrm{E}-03 & 60\end{array}$ $\begin{array}{lllllll}375.9 & 301 \mathrm{~A} & 12.8 & 41.5 & 4.56 \mathrm{E}-03 & 70\end{array}$ $\begin{array}{lllllll}375.9 & 301 \mathrm{~A} & 40.8 & -36.5 & 2.60 \mathrm{E}-03 & 85\end{array}$ $\begin{array}{lllllll}375.9 & 301 \mathrm{~A} & 50.9 & -37.2 & 3.44 \mathrm{E}-03 & 100\end{array}$ $\begin{array}{lllllll}375.9 & 301 \mathrm{~A} & 60.3 & -33.8 & 6.61 \mathrm{E}-03 & 115\end{array}$ $\begin{array}{lllllll}379.6 & 53 \mathrm{~A} & 125.9 & 65.3 & 3.97 \mathrm{E}-02 & 0\end{array}$ $\begin{array}{lllllll}379.6 & 53 \mathrm{~A} & 126.5 & 65.8 & 3.98 \mathrm{E}-02 & 0\end{array}$ $\begin{array}{lllllll}379.6 & 53 \mathrm{~A} & 125.1 & 62.1 & 3.64 \mathrm{E}-02 & 3\end{array}$ $\begin{array}{lllllll}379.6 & 53 \mathrm{~A} & 125.3 & 61.2 & 3.32 \mathrm{E}-02 & 6\end{array}$ $\begin{array}{lllllll}379.6 & 53 \mathrm{~A} & 124.6 & 61.2 & 2.88 \mathrm{E}-02 & 9\end{array}$ $\begin{array}{lllllll}379.6 & 53 \mathrm{~A} & 126.9 & 62.3 & 2.65 \mathrm{E}-02 & 12\end{array}$ $\begin{array}{lllllll}379.6 & 53 \mathrm{~A} & 127.6 & 63.2 & 2.45 \mathrm{E}-02 & 15\end{array}$ $\begin{array}{lllllll}379.6 & 53 \mathrm{~A} & 126.4 & 62.4 & 2.24 \mathrm{E}-02 & 18\end{array}$ $\begin{array}{lllllll}379.6 & 53 \mathrm{~A} & 127.4 & 61.9 & 2.10 \mathrm{E}-02 & 21\end{array}$ $\begin{array}{lllllll}379.6 & 53 \mathrm{~A} & 126.6 & 60.9 & 1.99 \mathrm{E}-02 & 25\end{array}$ $\begin{array}{lllllll}379.6 & 53 \mathrm{~A} & 125.2 & 60.6 & 1.74 \mathrm{E}-02 & 30\end{array}$ $\begin{array}{lllllll}379.6 & 53 \mathrm{~A} & 125.3 & 63.7 & 1.44 \mathrm{E}-02 & 40\end{array}$ $\begin{array}{lllllll}379.6 & 53 \mathrm{~A} & 121.7 & 62.4 & 1.25 \mathrm{E}-02 & 50\end{array}$ $\begin{array}{lllllll}379.6 & 53 \mathrm{~A} & 133.5 & 60.3 & 1.00 \mathrm{E}-02 & 60\end{array}$ $\begin{array}{lllllll}379.6 & 53 \mathrm{~A} & 101.5 & 63 & 8.03 \mathrm{E}-03 & 70\end{array}$ $\begin{array}{lllllll}379.6 & 53 \mathrm{~A} & 139.1 & 50.1 & 4.96 \mathrm{E}-03 & 85\end{array}$ $\begin{array}{lllllll}379.6 & 53 \mathrm{~A} & 141.7 & 45.1 & 3.20 \mathrm{E}-03 & 100\end{array}$ $\begin{array}{lllllll}379.6 & 53 \mathrm{~A} & 113 & 61.5 & 3.85 \mathrm{E}-03 & 115\end{array}$ $\begin{array}{lllllll}394.9 & 54 \mathrm{~A} & 61.9 & 53.4 & 6.34 \mathrm{E}-02 & 0\end{array}$ $\begin{array}{lllllll}394.9 & 54 \mathrm{~A} & 62.6 & 48.6 & 5.60 \mathrm{E}-02 & 3\end{array}$ $\begin{array}{lllllll}394.9 & 54 \mathrm{~A} & 63.3 & 44.2 & 4.75 \mathrm{E}-02 & 6\end{array}$ $\begin{array}{llllll}394.9 & 54 \mathrm{~A} & 63.5 & 39.6 & 4.12 \mathrm{E}-02 & 9\end{array}$ $\begin{array}{lllllll}394.9 & 54 \mathrm{~A} & 64.2 & 36.4 & 3.64 \mathrm{E}-02 & 12\end{array}$ $\begin{array}{lllllll}394.9 & 54 \mathrm{~A} & 61.9 & 35.1 & 3.31 \mathrm{E}-02 & 15\end{array}$ $\begin{array}{lllllll}394.9 & 54 \mathrm{~A} & 60.8 & 34.3 & 2.95 \mathrm{E}-02 & 18\end{array}$ $\begin{array}{lllllll}394.9 & 54 \mathrm{~A} & 60.4 & 34.1 & 2.58 \mathrm{E}-02 & 21\end{array}$ $\begin{array}{llllll}394.9 & 54 \mathrm{~A} & 61.5 & 32 & 2.36 \mathrm{E}-02 & 25\end{array}$ $\begin{array}{llllll}394.9 & 54 \mathrm{~A} & 56 & 35.6 & 1.99 \mathrm{E}-02 & 30\end{array}$ $\begin{array}{llllll}394.9 & 54 \mathrm{~A} & 49.9 & 33 & 1.50 \mathrm{E}-02 & 40\end{array}$ $\begin{array}{lllllll}394.9 & 54 \mathrm{~A} & 55.3 & 41.6 & 1.14 \mathrm{E}-02 & 50\end{array}$ $\begin{array}{lllllll}394.9 & 54 \mathrm{~A} & 60.6 & 42.2 & 9.98 \mathrm{E}-03 & 60\end{array}$ $\begin{array}{lllllll}394.9 & 54 \mathrm{~A} & 59.3 & 36.9 & 9.73 \mathrm{E}-03 & 70\end{array}$ $\begin{array}{lllllll}394.9 & 54 \mathrm{~A} & 32.3 & 30.9 & 7.91 \mathrm{E}-03 & 85\end{array}$ $\begin{array}{lllllll}394.9 & 54 \mathrm{~A} & 71.9 & 21.3 & 5.72 \mathrm{E}-03 & 100\end{array}$ 


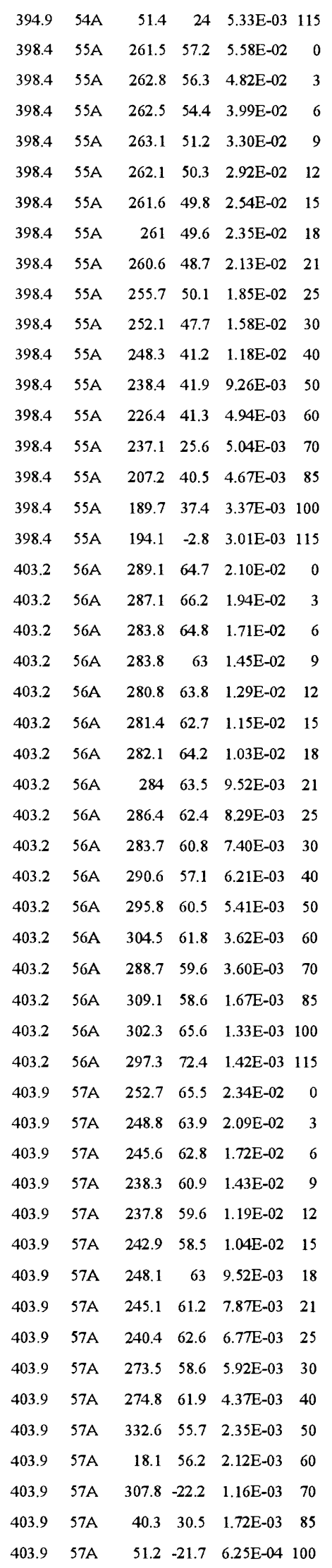

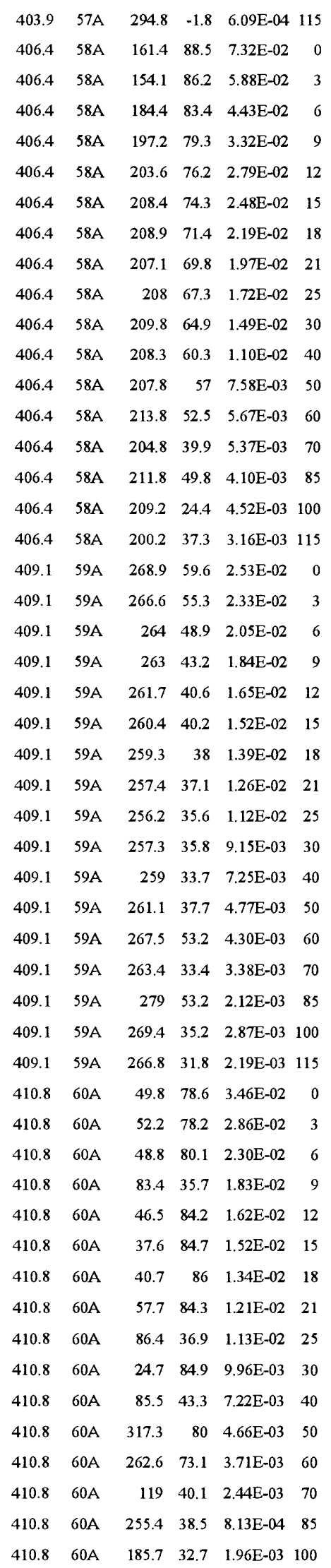

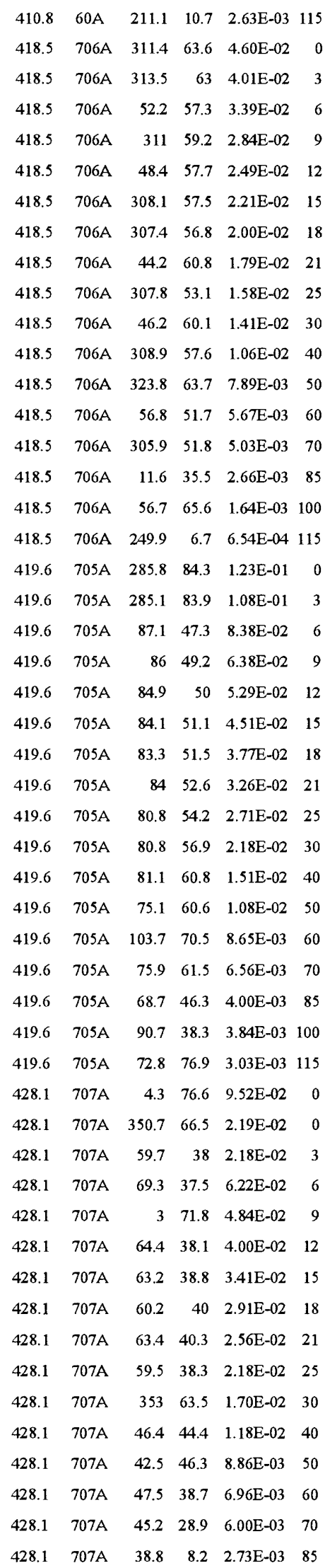




\begin{tabular}{|c|c|c|c|c|c|}
\hline & $707 \mathrm{~A}$ & 59 & -1.9 & & \\
\hline 8.1 & $707 \mathrm{~A}$ & 13.1 & 2.3 & & \\
\hline 29.7 & $708 \mathrm{~A}$ & 14.6 & 76.8 & $2.23 \mathrm{E}$ & \\
\hline 29.7 & $708 \mathrm{~A}$ & 9.1 & 77.2 & $1.97 \mathrm{E}-\mathrm{C}$ & \\
\hline 29.7 & $708 \mathrm{~A}$ & 13 & 77.2 & $1.57 \mathrm{E}-$ & \\
\hline 29.7 & $708 \mathrm{~A}$ & 73.3 & 36.1 & $1.16 \mathrm{E}-$ & \\
\hline 29.7 & $708 \mathrm{~A}$ & 8.3 & 76.2 & $9.67 \mathrm{E}-$ & 1 \\
\hline 429.7 & $708 \mathrm{~A}$ & 72.8 & 37.8 & $8.21 \mathrm{E}$ & \\
\hline 429.7 & $708 \mathrm{~A}$ & 71.4 & 39.5 & $6.79 \mathrm{E}$ & \\
\hline 29.7 & $708 \mathrm{~A}$ & 70.9 & 40.5 & $5.66 \mathrm{~F}$ & 2 \\
\hline 29.7 & $708 \mathrm{~A}$ & 71.9 & 41.5 & 4.531 & 2 \\
\hline 29.7 & $708 \mathrm{~A}$ & 71.5 & 42.2 & 3.831 & 3 \\
\hline 29.7 & $708 \mathrm{~A}$ & 25.4 & 77.5 & 2.541 & 4 \\
\hline 29.7 & $708 \mathrm{~A}$ & 77.7 & 47.4 & 1.61 & 5 \\
\hline 29.7 & $708 \mathrm{~A}$ & 79.6 & 50.5 & 1.141 & 6 \\
\hline 429.7 & $708 \mathrm{~A}$ & 88.8 & 65.3 & 8.34 & 7 \\
\hline 29.7 & $708 \mathrm{~A}$ & 92.1 & 30.8 & 5.621 & 8 \\
\hline 29.7 & $708 \mathrm{~A}$ & 68.7 & 44.8 & 3.90 & 100 \\
\hline 29.7 & $708 \mathrm{~A}$ & 164.9 & 48.9 & 4.80 & 11 \\
\hline 31.5 & $709 \mathrm{~A}$ & 26.5 & 77.2 & 8.72 & \\
\hline 31.5 & $709 \mathrm{~A}$ & 28.1 & 77.7 & 7.46 & \\
\hline 31.5 & $709 \mathrm{~A}$ & 31.3 & 78.7 & & \\
\hline 31.5 & $709 \mathrm{~A}$ & 34.8 & 78.2 & & \\
\hline 31.5 & $709 \mathrm{~A}$ & 79.3 & 32.8 & & 12 \\
\hline 1.5 & $709 \mathrm{~A}$ & 79.8 & 31.1 & & 1 \\
\hline 31.5 & $99 \mathrm{~A}$ & 45.9 & 74.9 & & 18 \\
\hline 31.5 & $709 \mathrm{~A}$ & 78.3 & 28.9 & & 2 \\
\hline 31.5 & $99 \mathrm{~A}$ & 78.9 & 29.5 & & 2 \\
\hline 31.5 & $99 \mathrm{~A}$ & 37.3 & 75 & & 30 \\
\hline 31.5 & $9 \mathrm{~A}$ & 29.2 & 71.7 & & 40 \\
\hline 31.5 & $9 \mathrm{~A}$ & 10.3 & 75.4 & & 50 \\
\hline 31.5 & $9 \mathrm{~A}$ & 81.4 & 36.1 & & 60 \\
\hline 31. & 19A & 57.1 & 36.5 & & 7 \\
\hline 31.5 & 7 & 55 & 39.9 & & 3 \\
\hline 31.5 & $9 \mathrm{~A}$ & 15.6 & 86.3 & & 100 \\
\hline 31.5 & 7 & & 66.2 & & 11 \\
\hline 33 & & & 73.4 & & \\
\hline & & & 46 & & \\
\hline & & & 71.9 & & \\
\hline & & & & & \\
\hline & & & 49.3 & & \\
\hline & & & 66.5 & & \\
\hline 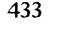 & 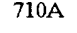 & 56.5 & 50.6 & & 10 \\
\hline . & 20 & 55.9 & 52.3 & & 21 \\
\hline 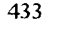 & 108 & 58 & 55.3 & & 23 \\
\hline 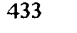 & 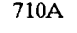 & 5.9 & 55.7 & & 30 \\
\hline 年 & 1012 & 7.1 & 62.3 & & 40 \\
\hline 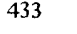 & $10 \mathrm{~m}$ & 286.5 & 57.2 & & $\pi 0$ \\
\hline & 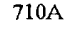 & 1.4 & 52.1 & & ( \\
\hline & s & 142.9 & 03.3 & & re \\
\hline & & & $1 . .7$ & S.Tos & \\
\hline
\end{tabular}

$\begin{array}{llllll}433 & 710 \mathrm{~A} & 192.5 & 13.1 & 2.46 \mathrm{E}-03 & 100\end{array}$

$\begin{array}{llllll}433 & 710 \mathrm{~A} & 188.9 & 7.2 & 4.19 \mathrm{E}-03 & 115\end{array}$

$\begin{array}{lllllll}436.3 & 61 \mathrm{~A} & 323.9 & 78.5 & 5.23 \mathrm{E}-03 & 0\end{array}$

$\begin{array}{lllllll}436.3 & 61 \mathrm{~A} & 299.8 & 70.9 & 3.15 \mathrm{E}-03 & 3\end{array}$

$\begin{array}{lllllll}436.3 & 61 \mathrm{~A} & 285 & 51.9 & 2.08 \mathrm{E}-03 & 6\end{array}$

$\begin{array}{llllll}436.3 & 61 \mathrm{~A} & 282.5 & 32.3 & 1.58 \mathrm{E}-03 & 9\end{array}$

$\begin{array}{lllllll}436.3 & 61 \mathrm{~A} & 276.2 & 22.5 & 1.53 \mathrm{E}-03 & 12\end{array}$

$\begin{array}{llllll}436.3 & 61 \mathrm{~A} & 279.2 & 24.7 & 1.13 \mathrm{E}-03 & 15\end{array}$

$\begin{array}{lllllll}436.3 & 61 \mathrm{~A} & 276.8 & 12.1 & 1.12 \mathrm{E}-03 & 18\end{array}$

$\begin{array}{lllllll}436.3 & 61 \mathrm{~A} & 261.1 & 9.1 & 9.23 \mathrm{E}-04 & 21\end{array}$

$\begin{array}{lllllll}436.3 & 61 \mathrm{~A} & 252.5 & 17.3 & 1.31 \mathrm{E}-03 & 25\end{array}$

$\begin{array}{lllllll}436.3 & 61 \mathrm{~A} & 280.2 & 27 & 1.15 \mathrm{E}-03 & 30\end{array}$

$\begin{array}{lllllll}436.3 & 61 \mathrm{~A} & 231 & 40.8 & 1.03 \mathrm{E}-03 & 40\end{array}$

$\begin{array}{lllllll}436.3 & 61 \mathrm{~A} & 311.3 & 38.6 & 7.61 \mathrm{E}-04 & 50\end{array}$

$\begin{array}{lllllll}436.3 & 61 \mathrm{~A} & 340.3 & 31.5 & 7.48 \mathrm{E}-04 & 60\end{array}$

$\begin{array}{lllllll}436.3 & 61 \mathrm{~A} & 198.9 & 38.1 & 1.51 \mathrm{E}-03 & 70\end{array}$

$\begin{array}{lllllll}436.3 & 61 \mathrm{~A} & 36.1 & -6.9 & 1.06 \mathrm{E}-03 & 85\end{array}$

$\begin{array}{lllllll}436.3 & 61 \mathrm{~A} & 239.6 & 37.5 & 1.26 \mathrm{E}-03 & 100\end{array}$

$\begin{array}{lllllll}436.3 & 61 \mathrm{~A} & 178.3 & 18.5 & 1.01 \mathrm{E}-03 & 115\end{array}$

$\begin{array}{lllllll}437.1 & 62 \mathrm{~A} & 82.1 & 78.8 & 3.19 \mathrm{E}-03 & 0\end{array}$

$\begin{array}{lllllll}437.1 & 62 \mathrm{~A} & 125.8 & 78.2 & 2.77 \mathrm{E}-03 & 3\end{array}$

$\begin{array}{lllllll}437.1 & 62 \mathrm{~A} & 157 & 72.6 & 2.39 \mathrm{E}-03 & 6\end{array}$

$\begin{array}{llllll}437.1 & 62 \mathrm{~A} & 175.1 & 66.2 & 2.23 \mathrm{E}-03 & 9\end{array}$

$\begin{array}{lllllll}437.1 & 62 \mathrm{~A} & 180.2 & 62.9 & 2.07 \mathrm{E}-03 & 12\end{array}$

$\begin{array}{lllllll}437.1 & 62 \mathrm{~A} & 182.7 & 56.9 & 1.93 \mathrm{E}-03 & 15\end{array}$

$\begin{array}{lllllll}437.1 & 62 \mathrm{~A} & 171.9 & 50.6 & 2.06 \mathrm{E}-03 & 18\end{array}$

$\begin{array}{lllllll}437.1 & 62 \mathrm{~A} & 180.6 & 49.1 & 1.74 \mathrm{E}-03 & 21\end{array}$

$\begin{array}{lllllll}437.1 & 62 \mathrm{~A} & 179.4 & 43.4 & 1.81 \mathrm{E}-03 & 25\end{array}$

$\begin{array}{lllllll}437.1 & 62 \mathrm{~A} & 185.6 & 49.3 & 1.38 \mathrm{E}-03 & 30\end{array}$

$\begin{array}{lllllll}437.1 & 62 \mathrm{~A} & 173.8 & 48.4 & 1.21 \mathrm{E}-03 & 40\end{array}$

$\begin{array}{lllllll}437.1 & 62 \mathrm{~A} & 164.2 & 60.9 & 6.95 \mathrm{E}-04 & 50\end{array}$

$\begin{array}{lllllll}437.1 & 62 \mathrm{~A} & 229.4 & 61 & 6.46 \mathrm{E}-04 & 60\end{array}$

$\begin{array}{lllllll}437.1 & 62 \mathrm{~A} & 224.9 & 47.3 & 8.24 \mathrm{E}-04 & 70\end{array}$

$\begin{array}{lllllll}437.1 & 62 \mathrm{~A} & 301.4 & 46.8 & 3.92 \mathrm{E}-04 & 85\end{array}$

$\begin{array}{lllllll}441.3 & 63 \mathrm{~A} & 99.9 & 61.1 & 2.66 \mathrm{E}-02 & 0\end{array}$

$\begin{array}{lllllll}441.3 & 63 \mathrm{~A} & 101.2 & 56.6 & 2.16 \mathrm{E}-02 & 3\end{array}$

$\begin{array}{llllll}441.3 & 63 \mathrm{~A} & 101.6 & 54.9 & 1.85 \mathrm{E}-02 & 6\end{array}$

$\begin{array}{lllllll}441.3 & 63 \mathrm{~A} & 102.1 & 53.1 & 1.55 \mathrm{E}-02 & 9\end{array}$

$\begin{array}{llllll}441.3 & 63 \mathrm{~A} & 103 & 51.6 & 1.35 \mathrm{E}-02 & 12\end{array}$

$\begin{array}{llllll}441.3 & 63 \mathrm{~A} & 104.5 & 53.1 & 1.18 \mathrm{E}-02 & 15\end{array}$

$\begin{array}{llllll}441.3 & 63 \mathrm{~A} & 102.6 & 52 & 1.03 \mathrm{E}-02 & 18\end{array}$

$\begin{array}{lllllll}441.3 & 63 \mathrm{~A} & 103 & 53.2 & 8.89 \mathrm{E}-03 & 21\end{array}$

$\begin{array}{lllllll}441.3 & 63 \mathrm{~A} & 102.7 & 52.8 & 7.40 \mathrm{E}-03 & 25\end{array}$

$\begin{array}{lllllll}441.3 & 63 \mathrm{~A} & 97.8 & 51.8 & 5.21 \mathrm{E}-03 & 30\end{array}$

$\begin{array}{llllll}441.3 & 63 \mathrm{~A} & 105.4 & 53.8 & 4.25 \mathrm{E}-03 & 40\end{array}$

$\begin{array}{lllllll}441.3 & 63 \mathrm{~A} & 79.7 & 50.2 & 2.92 \mathrm{E}-03 & 50\end{array}$

$\begin{array}{llllll}441.3 & 63 \mathrm{~A} & 77.2 & 41.2 & 2.47 \mathrm{E}-03 & 60\end{array}$

$\begin{array}{lllllll}441.3 & 63 \mathrm{~A} & 144.5 & 70.4 & 2.82 \mathrm{E}-03 & 70\end{array}$

$\begin{array}{lllllll}441.3 & 63 \mathrm{~A} & 76.7 & 44.1 & 2.88 \mathrm{E}-03 & 85\end{array}$

$\begin{array}{lllllll}441.3 & 63 \mathrm{~A} & 141.3 & 59.7 & 2.82 \mathrm{E}-03 & 100\end{array}$

$\begin{array}{llllll}441.3 & 63 \mathrm{~A} & 125.2 & 51.8 & 2.39 \mathrm{E}-03 & 115\end{array}$

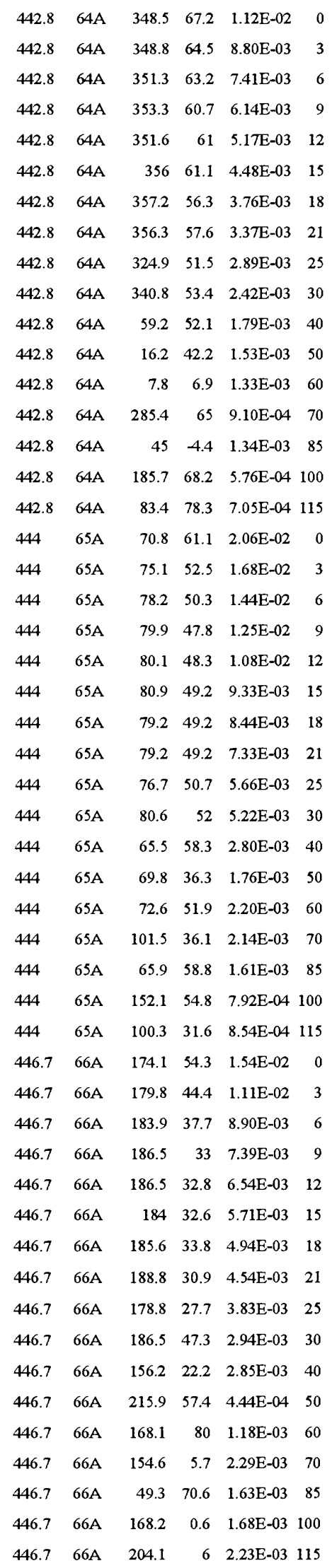




\begin{tabular}{|c|c|c|c|c|c|c|c|c|c|c|c|}
\hline 457.6 & $68 \mathrm{~A}$ & 256.8 & 75.2 & $5.20 \mathrm{E}-03$ & 0 & 463 & $71 \mathrm{~A}$ & 57.6 & 20.8 & $9.75 \mathrm{E}-03$ & 0 \\
\hline 457.6 & $68 \mathrm{~A}$ & 247.1 & 71.8 & $3.61 \mathrm{E}-03$ & 3 & 463 & $71 \mathrm{~A}$ & 58.7 & 17.2 & $9.38 \mathrm{E}-03$ & 3 \\
\hline 457.6 & $68 \mathrm{~A}$ & 243.4 & 68.6 & $2.61 \mathrm{E}-03$ & 6 & 463 & $71 \mathrm{~A}$ & 59.6 & 16.5 & $8.79 \mathrm{E}-03$ & 6 \\
\hline 457.6 & $68 \mathrm{~A}$ & 251.1 & 62.2 & $2.08 \mathrm{E}-03$ & 9 & 463 & $71 \mathrm{~A}$ & 59.5 & 16.3 & $8.05 E-03$ & 9 \\
\hline 457.6 & $68 \mathrm{~A}$ & 244.4 & 66 & $1.65 \mathrm{E}-03$ & 12 & 463 & $71 \mathrm{~A}$ & 59.3 & 16.2 & $7.40 \mathrm{E}-03$ & 12 \\
\hline 457.6 & $68 \mathrm{~A}$ & 248.2 & 67.2 & $1.45 \mathrm{E}-03$ & 15 & 463 & $71 \mathrm{~A}$ & 59.8 & 15.7 & $6.79 \mathrm{E}-03$ & 15 \\
\hline 457.6 & $68 \mathrm{~A}$ & 254.9 & 62 & $1.26 \mathrm{E}-03$ & 18 & 463 & $71 \mathrm{~A}$ & 60.4 & 15.2 & $6.27 \mathrm{E}-03$ & 18 \\
\hline 457.6 & $68 \mathrm{~A}$ & 253.3 & 60.4 & $1.12 \mathrm{E}-03$ & 21 & 463 & $71 \mathrm{~A}$ & 58.9 & 15.4 & $5.62 \mathrm{E}-03$ & 21 \\
\hline 457.6 & $68 \mathrm{~A}$ & 289 & 70.2 & $1.13 \mathrm{E}-03$ & 25 & 463 & $71 \mathrm{~A}$ & 58.9 & 15.8 & $5.10 \mathrm{E}-03$ & 25 \\
\hline 457.6 & $68 \mathrm{~A}$ & 262.8 & 47.2 & $1.09 \mathrm{E}-03$ & 30 & 463 & $71 \mathrm{~A}$ & 58.9 & 17 & $4.56 \mathrm{E}-03$ & 30 \\
\hline 457.6 & $68 \mathrm{~A}$ & 275.7 & 41 & 8.40 E-04 & 40 & 463 & $71 \mathrm{~A}$ & 59.8 & 17.1 & $3.93 E-03$ & 40 \\
\hline 457.6 & $68 \mathrm{~A}$ & 224.7 & 48.7 & $7.36 \mathrm{E}-04$ & 50 & 463 & $71 \mathrm{~A}$ & 59.6 & 20 & $3.17 \mathrm{E}-03$ & 50 \\
\hline 457.6 & $68 \mathrm{~A}$ & 249.7 & 30.6 & $3.93 \mathrm{E}-04$ & 60 & 463 & $71 \mathrm{~A}$ & 59.8 & 15.9 & $2.76 \mathrm{E}-03$ & 60 \\
\hline 457.6 & $68 \mathrm{~A}$ & 267.3 & 46.5 & $8.93 \mathrm{E}-04$ & 70 & 463 & $71 \mathrm{~A}$ & 59.2 & 17.4 & $2.81 \mathrm{E}-03$ & 70 \\
\hline 457.6 & $68 \mathrm{~A}$ & 146.5 & 48.3 & $5.92 \mathrm{E}-04$ & 85 & 463 & $71 \mathrm{~A}$ & 61.8 & 19.5 & $2.45 \mathrm{E}-03$ & 85 \\
\hline 457.6 & $68 \mathrm{~A}$ & 293 & 72.3 & $8.05 \mathrm{E}-04$ & 100 & 463 & $71 \mathrm{~A}$ & 62.2 & 18.6 & $2.03 E-03$ & 100 \\
\hline 457.6 & $68 \mathrm{~A}$ & 230.8 & 72.1 & $8.93 \mathrm{E}-04$ & 115 & 463 & $71 \mathrm{~A}$ & 63.9 & 15.6 & $2.12 \mathrm{E}-03$ & 115 \\
\hline 458.6 & $69 \mathrm{~A}$ & 64.2 & 80.3 & $9.98 \mathrm{E}-02$ & 0 & 464 & $72 \mathrm{~A}$ & 103.8 & 53.4 & $7.17 \mathrm{E}-03$ & 0 \\
\hline 458.6 & $69 \mathrm{~A}$ & 71.7 & 78.9 & $8.23 E-02$ & 3 & 464 & $72 \mathrm{~A}$ & 107.9 & 47 & $6.16 E-03$ & 3 \\
\hline 458.6 & $69 \mathrm{~A}$ & 73 & 77.8 & $5.47 \mathrm{E}-02$ & 6 & 464 & $72 \mathrm{~A}$ & 109 & 43.2 & $5.32 \mathrm{E}-03$ & 6 \\
\hline 458.6 & $69 \mathrm{~A}$ & 76.7 & 75.5 & $3.72 \mathrm{E}-02$ & 9 & 464 & $72 \mathrm{~A}$ & 113 & 42 & $4.71 \mathrm{E}-03$ & 9 \\
\hline 458.6 & $69 \mathrm{~A}$ & 80 & 74.8 & $2.89 \mathrm{E}-02$ & 12 & 464 & $72 \mathrm{~A}$ & 114 & 39.8 & $4.25 \mathrm{E}-03$ & 12 \\
\hline 458.6 & $69 \mathrm{~A}$ & 78.6 & 72.3 & $2.31 \mathrm{E}-02$ & 15 & 464 & $72 \mathrm{~A}$ & 111.6 & 41.7 & $3.88 \mathrm{E}-03$ & 15 \\
\hline 458.6 & $69 \mathrm{~A}$ & 79 & 70.9 & $1.81 \mathrm{E}-02$ & 18 & 464 & $72 \mathrm{~A}$ & 116.8 & 38.5 & $3.04 \mathrm{E}-03$ & 18 \\
\hline 458.6 & $69 \mathrm{~A}$ & 90.1 & 69.7 & $1.48 \mathrm{E}-02$ & 21 & 464 & $72 \mathrm{~A}$ & 118.9 & 41.4 & $3.17 \mathrm{E}-03$ & 21 \\
\hline 458.6 & $69 \mathrm{~A}$ & 83.3 & 71.1 & $1.16 \mathrm{E}-02$ & 25 & 464 & $72 \mathrm{~A}$ & 112.4 & 36 & $2.49 \mathrm{E}-03$ & 25 \\
\hline 458.6 & $69 \mathrm{~A}$ & 97.4 & 71.2 & $1.03 \mathrm{E}-02$ & 30 & 464 & $72 \mathrm{~A}$ & 123.4 & 46.5 & $2.53 \mathrm{E}-03$ & 30 \\
\hline 458.6 & $69 \mathrm{~A}$ & 115.2 & 63.4 & $6.04 \mathrm{E}-03$ & 40 & 464 & $72 \mathrm{~A}$ & 118.1 & 50.9 & $1.76 \mathrm{E}-03$ & 40 \\
\hline 458.6 & $69 \mathrm{~A}$ & 133.1 & 64.9 & $3.82 \mathrm{E}-03$ & 50 & 464 & $72 \mathrm{~A}$ & 111.7 & 60.5 & $9.41 \mathrm{E}-04$ & 50 \\
\hline 458.6 & $69 \mathrm{~A}$ & 132.5 & 60.1 & $2.43 \mathrm{E}-03$ & 60 & 464 & $72 \mathrm{~A}$ & 115.8 & 57.4 & $4.34 \mathrm{E}-04$ & 60 \\
\hline 458.6 & $69 \mathrm{~A}$ & 162.3 & 32.4 & $2.58 \mathrm{E}-03$ & 70 & 464 & $72 \mathrm{~A}$ & 108.9 & 68.3 & $1.06 \mathrm{E}-03$ & 70 \\
\hline 458.6 & $69 \mathrm{~A}$ & 148.9 & 25.3 & $1.89 \mathrm{E}-03$ & 85 & 464 & $72 \mathrm{~A}$ & 11.8 & 41.5 & $8.26 \mathrm{E}-04$ & 85 \\
\hline 458.6 & $69 \mathrm{~A}$ & 177.3 & 47.1 & $2.89 \mathrm{E}-03$ & 100 & 464 & $72 \mathrm{~A}$ & 107.8 & 38.6 & $1.21 \mathrm{E}-03$ & 100 \\
\hline 458.6 & $69 \mathrm{~A}$ & 166.9 & -7.7 & $2.20 \mathrm{E}-03$ & 115 & 464 & $72 \mathrm{~A}$ & 92.8 & 88.5 & $1.37 \mathrm{E}-03$ & 115 \\
\hline 461.9 & $70 \mathrm{~A}$ & 254.3 & 70.3 & $3.16 \mathrm{E}-02$ & 0 & 466.9 & $73 \mathrm{~A}$ & 338.8 & 52.7 & $4.66 \mathrm{E}-02$ & 0 \\
\hline 461.9 & $70 \mathrm{~A}$ & 247.1 & 63.7 & $2.32 \mathrm{E}-02$ & 3 & 466.9 & $73 \mathrm{~A}$ & 339.7 & 51.2 & $4.35 \mathrm{E}-02$ & 3 \\
\hline 461.9 & $70 \mathrm{~A}$ & 242.6 & 56.2 & $1.78 \mathrm{E}-02$ & 6 & 466.9 & $73 \mathrm{~A}$ & 340.1 & 50.7 & $3.95 \mathrm{E}-02$ & 6 \\
\hline 461.9 & $70 \mathrm{~A}$ & 240.4 & 47 & $1.45 \mathrm{E}-02$ & 9 & 466.9 & $73 \mathrm{~A}$ & 339.5 & 50.1 & $3.55 \mathrm{E}-02$ & 9 \\
\hline 461.9 & $70 \mathrm{~A}$ & 237.6 & 44.8 & $1.26 \mathrm{E}-02$ & 12 & 466.9 & $73 \mathrm{~A}$ & 341 & 50.2 & $3.29 \mathrm{E}-02$ & 12 \\
\hline 461.9 & $70 \mathrm{~A}$ & 235.8 & 44.1 & $1.15 \mathrm{E}-02$ & 15 & 466.9 & $73 \mathrm{~A}$ & 341 & 51.2 & $3.05 \mathrm{E}-02$ & 15 \\
\hline 461.9 & $70 \mathrm{~A}$ & 232.6 & 42.5 & $1.05 \mathrm{E}-02$ & 18 & 466.9 & $73 \mathrm{~A}$ & 341.5 & 51.9 & $2.76 \mathrm{E}-02$ & 18 \\
\hline 461.9 & $70 \mathrm{~A}$ & 233.9 & 44.8 & $9.77 \mathrm{E}-03$ & 21 & 466.9 & $73 \mathrm{~A}$ & 339.4 & 50.5 & $2.58 \mathrm{E}-02$ & 21 \\
\hline 461.9 & $70 \mathrm{~A}$ & 235.3 & 40.4 & $8.91 \mathrm{E}-03$ & 25 & 466.9 & $73 \mathrm{~A}$ & 339.8 & 50 & $2.38 \mathrm{E}-02$ & 25 \\
\hline 461.9 & $70 \mathrm{~A}$ & 233.4 & 43.5 & $8.12 \mathrm{E}-03$ & 30 & 466.9 & $73 \mathrm{~A}$ & 341.1 & 51.7 & $2.10 \mathrm{E}-02$ & 30 \\
\hline 461.9 & $70 \mathrm{~A}$ & 235.4 & 50.4 & $6.03 \mathrm{E}-03$ & 40 & 466.9 & $73 \mathrm{~A}$ & 341.9 & 51.4 & $1.46 \mathrm{E}-02$ & 40 \\
\hline 461.9 & $70 \mathrm{~A}$ & 244.1 & 40.9 & $5.30 \mathrm{E}-03$ & 50 & 466.9 & $73 \mathrm{~A}$ & 339.5 & 51.5 & $1.10 \mathrm{E}-02$ & 50 \\
\hline 461.9 & $70 \mathrm{~A}$ & 240.6 & 42.7 & $4.87 \mathrm{E}-03$ & 60 & 466.9 & $73 \mathrm{~A}$ & 340.9 & 53.8 & $8.53 \mathrm{E}-03$ & 60 \\
\hline 461.9 & $70 \mathrm{~A}$ & 245 & 35 & $5.06 \mathrm{E}-03$ & 70 & 466.9 & $73 \mathrm{~A}$ & 335.2 & 54.1 & $7.51 \mathrm{E}-03$ & 70 \\
\hline 461.9 & $70 \mathrm{~A}$ & 233.7 & 39.9 & $4.37 \mathrm{E}-03$ & 85 & 466.9 & $73 \mathrm{~A}$ & 335.6 & 48.8 & $4.55 \mathrm{E}-03$ & 85 \\
\hline 461.9 & $70 \mathrm{~A}$ & 241.6 & 43.1 & $3.75 \mathrm{E}-03$ & 100 & 466.9 & $73 \mathrm{~A}$ & 356 & 55.7 & $4.24 \mathrm{E}-03$ & 100 \\
\hline 461.9 & $70 \mathrm{~A}$ & 238 & 26.1 & $2.96 \mathrm{E}-03$ & 115 & 466.9 & $73 \mathrm{~A}$ & 326.3 & 44.8 & $4.93 \mathrm{E}-03$ & 115 \\
\hline
\end{tabular}

\begin{tabular}{|c|c|c|c|c|c|}
\hline & $4 \mathrm{~A}$ & 1.8 & 59.8 & 2.195 & \\
\hline 74.4 & $74 \mathrm{~A}$ & 10.3 & 53.2 & $1.68 \mathrm{E}-02$ & \\
\hline 4.4 & $74 \mathrm{~A}$ & 7.5 & 48.1 & $1.35 \mathrm{E}-02$ & \\
\hline 4.4 & $74 \mathrm{~A}$ & 6.6 & 45.1 & $1.14 \mathrm{E}-02$ & \\
\hline 74.4 & $74 \mathrm{~A}$ & .1 & 43.8 & $1.03 \mathrm{E}-02$ & \\
\hline 74.4 & $74 \mathrm{~A}$ & 3 & 42.1 & $9.34 \mathrm{E}-03$ & \\
\hline 74.4 & $74 \mathrm{~A}$ & 8.8 & 42 & 8.63E-03 & \\
\hline 74.4 & $74 \mathrm{~A}$ & 58.4 & 37.4 & 8.27 & \\
\hline 74.4 & $74 \mathrm{~A}$ & 5.5 & 35.5 & & \\
\hline 74.4 & $74 \mathrm{~A}$ & & 36.3 & & \\
\hline 74.4 & $74 \mathrm{~A}$ & 6.1 & 29.1 & & \\
\hline 74.4 & $74 \mathrm{~A}$ & & 32.7 & & \\
\hline 74.4 & $74 \mathrm{~A}$ & 0.4 & 33.2 & & \\
\hline 74.4 & $74 \mathrm{~A}$ & 59.5 & 30.1 & $3.42 \mathrm{E}$ & \\
\hline 74.4 & $74 \mathrm{~A}$ & 39.4 & 36.1 & & \\
\hline 74.4 & $74 \mathrm{~A}$ & 46.5 & 30.2 & & 10 \\
\hline 74.4 & $74 \mathrm{~A}$ & 34.4 & 52.1 & & 11 \\
\hline 75.2 & $75 \mathrm{~A}$ & 98.5 & 67.3 & & \\
\hline 175.2 & $75 \mathrm{~A}$ & 6.8 & 64.2 & $9.62 \mathrm{E}-03$ & \\
\hline 75.2 & $75 \mathrm{~A}$ & 15.9 & 61.1 & & \\
\hline 475.2 & $75 \mathrm{~A}$ & 93 & 59.9 & & \\
\hline 75.2 & $75 \mathrm{~A}$ & 91.1 & 59.9 & & 1 \\
\hline 75.2 & $75 \mathrm{~A}$ & 90.9 & 58.7 & & 1 \\
\hline 175.2 & $75 \mathrm{~A}$ & 92.8 & 59.9 & & 1 \\
\hline 775.2 & $75 \mathrm{~A}$ & 91.8 & 60.1 & & 2 \\
\hline 775.2 & $75 \mathrm{~A}$ & 91.7 & 57 & 4.34 & 2 \\
\hline$\$ 75.2$ & $75 \mathrm{~A}$ & 94.6 & 60 & 3.711 & 3 \\
\hline 175.2 & $75 \mathrm{~A}$ & 00.5 & 65.1 & $3.10 \mathrm{E}$ & 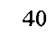 \\
\hline 175.2 & $75 \mathrm{~A}$ & 117 & 68.4 & $2.20 \mathrm{E}$ & 5 \\
\hline 75.2 & $75 \mathrm{~A}$ & 24.4 & 52.1 & $1.38 \mathrm{E}$ & 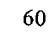 \\
\hline 75.2 & $75 \mathrm{~A}$ & 116.1 & 60.9 & $1.84 \mathrm{E}$ & 7 \\
\hline 75.2 & $75 \mathrm{~A}$ & 166 & 42.8 & $1.14 \mathrm{E}$ & 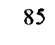 \\
\hline 475.2 & $75 \mathrm{~A}$ & 126 & 44.8 & $1.59 \mathrm{E}$ & 10 \\
\hline 775.2 & $75 \mathrm{~A}$ & 122.1 & 57.1 & $1.35 \mathrm{E}$ & 11 \\
\hline 185.2 & $76 \mathrm{~A}$ & 60.3 & 71.7 & $4.66 \mathrm{E}$ & \\
\hline 185.2 & $76 \mathrm{~A}$ & 66.1 & 67.7 & $3.72 \mathrm{E}$ & \\
\hline 185.2 & $76 \mathrm{~A}$ & 69.7 & 64.1 & $2.92 \mathrm{E}$ & \\
\hline 852 & $76 \mathrm{~A}$ & 73.8 & 60.9 & $2.33 \mathrm{E}$ & \\
\hline 852 & $76 \mathrm{~A}$ & 75.7 & 58.8 & & 1 \\
\hline $85 ?$ & $76 \mathrm{~A}$ & 76.4 & 58 & & 1 \\
\hline $85 ?$ & $76 \mathrm{~A}$ & 78.3 & 56.9 & & 1 \\
\hline $85 ?$ & $76 \mathrm{~A}$ & 772 & 56.5 & & \\
\hline 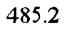 & $76 \mathrm{~A}$ & & 54.1 & & \\
\hline (1) & $76 \mathrm{~A}$ & & 54.6 & & \\
\hline 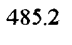 & $76 \mathrm{~A}$ & & 50.2 & & \\
\hline - & $76 \mathrm{~A}$ & & 41.2 & & \\
\hline & & & 50.4 & & \\
\hline & $76 \mathrm{~A}$ & 99.2 & 55.3 & & \\
\hline (3) & & & 44.2 & & \\
\hline 100.1 & $10 \mathrm{R}$ & 124.8 & 43.6 & & \\
\hline & & 171.9 & 49.3 & & \\
\hline
\end{tabular}




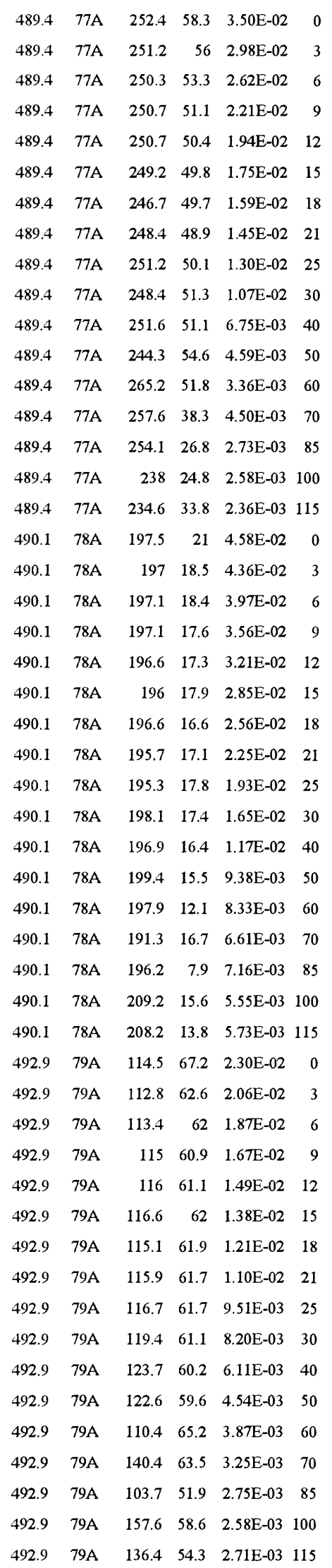

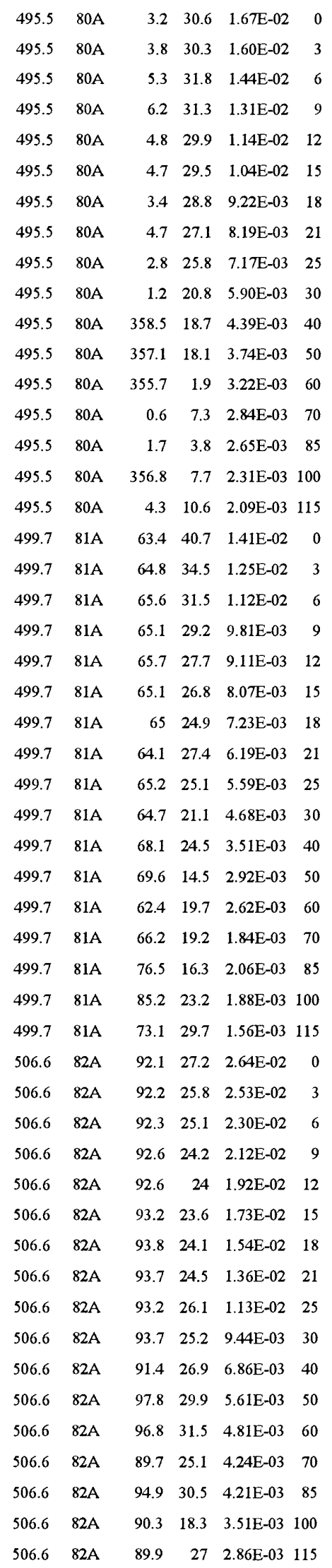

\begin{tabular}{|c|c|c|c|c|c|}
\hline 8 & $\mathrm{BA}$ & 53.9 & 50.1 & & \\
\hline 7.8 & $83 \mathrm{~A}$ & 151 & 47.2 & $2.94 \mathrm{E}$ & \\
\hline 7. & $83 \mathrm{~A}$ & 150 & 45.5 & 2.73E-02 & \\
\hline 7. & $83 \mathrm{~A}$ & 150.3 & 44.9 & $2.47 \mathrm{E}-02$ & \\
\hline 7. & $83 \mathrm{~A}$ & 150.9 & 43.8 & $2.29 \mathrm{E}$ & \\
\hline 07.8 & $83 \mathrm{~A}$ & 151.2 & 43.5 & $2.15 \mathrm{E}-02$ & \\
\hline 07.8 & $83 \mathrm{~A}$ & 151.7 & 44 & $1.91 \mathrm{E}-02$ & \\
\hline 507.8 & $83 \mathrm{~A}$ & 151.8 & 43.2 & $1.78 \mathrm{E}-02$ & \\
\hline 507.8 & $83 \mathrm{~A}$ & 151.3 & 43.5 & $1.59 \mathrm{E}-02$ & \\
\hline 07.8 & $83 \mathrm{~A}$ & 149.4 & 42.4 & $1.42 \mathrm{E}$ & \\
\hline 07.8 & $83 \mathrm{~A}$ & 150.7 & 39.6 & $1.09 \mathrm{E}$ & \\
\hline 07.8 & $83 \mathrm{~A}$ & 152.7 & 44 & $8.84 \mathrm{E}$ & \\
\hline 07.8 & $83 \mathrm{~A}$ & 157.3 & 44 & 6.82 & \\
\hline 07.8 & $83 \mathrm{~A}$ & 155.6 & 40 & 6.47 & \\
\hline 507.8 & $83 \mathrm{~A}$ & 148.7 & 36.7 & 5.00 & \\
\hline 507.8 & $83 \mathrm{~A}$ & 164.5 & 37.4 & 5.78 & 10 \\
\hline 507.8 & $83 \mathrm{~A}$ & 168.6 & 32.5 & 5.76 & 11 \\
\hline 512 & $84 \mathrm{~A}$ & 154.4 & 47.6 & 2.83 & \\
\hline 12 & $84 \mathrm{~A}$ & 154.6 & 43.3 & 2.53 & \\
\hline 512 & 34 & 153.7 & 41.4 & 2.24 & \\
\hline 512 & $4 \mathrm{~A}$ & 153.1 & 39 & & \\
\hline 512 & $4 \mathrm{~A}$ & 150.7 & 37.8 & 1.78 & \\
\hline 512 & $4 \mathrm{~A}$ & 152.6 & 38.6 & 1.5 & \\
\hline 512 & $4 \mathrm{~A}$ & 150.4 & 37.2 & 1.36 & \\
\hline 12 & $4 \mathrm{~A}$ & 51.3 & 38.3 & & \\
\hline 12 & $4 \mathrm{~A}$ & 53.9 & 37 & 1.04 & \\
\hline 12 & $4 \mathrm{~A}$ & 50.5 & 35.5 & 8.47 & \\
\hline 12 & $4 \mathrm{~A}$ & 53.5 & 31.5 & 6.33 & \\
\hline 12 & $4 \mathrm{~A}$ & 46.8 & 32.8 & & \\
\hline 12 & $4 \mathrm{~A}$ & 56.3 & 35.3 & & \\
\hline 12 & $4 \mathrm{~A}$ & 62.6 & 35.3 & & \\
\hline 12 & $4 \mathrm{~A}$ & 46.1 & 29.6 & & \\
\hline 12 & $4 \mathrm{~A}$ & 60.5 & 30.9 & & 1 \\
\hline 12 & A & 62.6 & 25.9 & & 1 \\
\hline 19. & $85 \mathrm{~A}$ & 05.3 & 60.3 & & \\
\hline 19.2 & $85 \mathrm{~A}$ & 09.9 & 54.8 & & \\
\hline . & $85 \mathrm{~A}$ & 11.5 & 52 & & \\
\hline & & 12.4 & 49.7 & & \\
\hline & $85 \mathrm{~A}$ & & 18.9 & & \\
\hline & & & 49 & & \\
\hline & $85 \mathrm{~A}$ & & 49.7 & & \\
\hline & $85 \mathrm{~A}$ & & 49.3 & & \\
\hline & $85 \mathrm{~A}$ & 18.8 & 50.7 & & \\
\hline & $85 \mathrm{~A}$ & & 49 & & \\
\hline 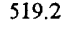 & $85 \mathrm{~A}$ & 124.9 & 52.3 & & \\
\hline & $85 \mathrm{~A}$ & 130 & 43.8 & & \\
\hline 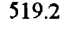 & $85 \mathrm{~A}$ & 139.6 & 54.6 & & \\
\hline 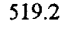 & $85 \mathrm{~A}$ & 146.7 & 41.8 & $1.66 \mathrm{t}$ & 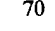 \\
\hline 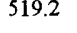 & $85 \mathrm{~A}$ & 124.8 & 70.2 & $1.49 \mathrm{E}$ & - \\
\hline & $83 \mathrm{~A}$ & 121.3 & 64.2 & 1.801 & \\
\hline & OJA & 150.1 & 34.6 & $1.11 \mathrm{E}^{-}$ & \\
\hline
\end{tabular}




\begin{tabular}{|c|c|c|c|c|c|c|c|c|c|c|c|}
\hline 520.2 & $86 \mathrm{~A}$ & 29.6 & 69.5 & $6.97 \mathrm{E}-02$ & 0 & 535.9 & $88 \mathrm{~A}$ & 322.5 & 32.5 & $1.17 \mathrm{E}-02$ & \\
\hline 520.2 & $86 \mathrm{~A}$ & 31.9 & 68.9 & $5.07 \mathrm{E}-02$ & 3 & 535.9 & $88 \mathrm{~A}$ & 323.5 & 28.3 & $1.05 \mathrm{E}-02$ & \\
\hline 520.2 & $86 \mathrm{~A}$ & 31.9 & 68.4 & $3.41 E-02$ & 6 & 535.9 & $88 \mathrm{~A}$ & 322.6 & 23.8 & $9.32 \mathrm{E}-03$ & \\
\hline 520.2 & $86 \mathrm{~A}$ & 28.7 & 64.7 & $2.18 \mathrm{E}-02$ & 9 & 535.9 & $88 \mathrm{~A}$ & 320.8 & 20.8 & $8.19 \mathrm{E}-03$ & \\
\hline 520.2 & $86 \mathrm{~A}$ & 22.7 & 65.3 & $1.74 \mathrm{E}-02$ & 12 & 535.9 & $88 \mathrm{~A}$ & 321.3 & 19.9 & $7.26 \mathrm{E}-03$ & 12 \\
\hline 520.2 & $86 \mathrm{~A}$ & 14.9 & 65.7 & $1.41 \mathrm{E}-02$ & 15 & 535.9 & $88 \mathrm{~A}$ & 318.8 & 21.8 & $6.63 \mathrm{E}-03$ & 15 \\
\hline 520.2 & $86 \mathrm{~A}$ & 14.3 & 64.8 & $1.10 \mathrm{E}-02$ & 18 & 535.9 & $88 \mathrm{~A}$ & 316.6 & 19.4 & $6.15 \mathrm{E}-03$ & 18 \\
\hline 520.2 & $86 \mathrm{~A}$ & 18.5 & 68.1 & $9.35 \mathrm{E}-03$ & 21 & 535.9 & $88 \mathrm{~A}$ & 314.6 & 19.4 & $5.48 \mathrm{E}-03$ & 21 \\
\hline 520.2 & $86 \mathrm{~A}$ & 25.3 & 74.3 & $7.47 \mathrm{E}-03$ & 25 & 535.9 & $88 \mathrm{~A}$ & 312.4 & 18.2 & $4.60 \mathrm{E}-03$ & 25 \\
\hline 520.2 & $86 \mathrm{~A}$ & 26.4 & 68.7 & $6.21 \mathrm{E}-03$ & 30 & 535.9 & $88 \mathrm{~A}$ & 307.9 & 22.2 & $4.07 \mathrm{E}-03$ & 30 \\
\hline 520.2 & $86 \mathrm{~A}$ & 11.8 & 80.9 & $3.82 \mathrm{E}-03$ & 40 & 535.9 & $88 \mathrm{~A}$ & 307.9 & 11.4 & $3.24 \mathrm{E}-03$ & 40 \\
\hline 520.2 & $86 \mathrm{~A}$ & 286.1 & 79.5 & $3.13 \mathrm{E}-03$ & 50 & 535.9 & $88 \mathrm{~A}$ & 308.5 & 13.5 & $2.20 \mathrm{E}-03$ & 50 \\
\hline 520.2 & $86 \mathrm{~A}$ & 263 & 70.4 & $2.18 \mathrm{E}-03$ & 60 & 535.9 & $88 \mathrm{~A}$ & 322.2 & -10.1 & $1.53 \mathrm{E}-03$ & 60 \\
\hline 520.2 & $86 \mathrm{~A}$ & 271.7 & 56.7 & $3.26 \mathrm{E}-03$ & 70 & 535.9 & $88 \mathrm{~A}$ & 302.9 & -5.2 & $2.24 \mathrm{E}-03$ & 70 \\
\hline 520.2 & $86 \mathrm{~A}$ & 308.4 & 33.3 & 2.83E-03 & 85 & 535.9 & $88 \mathrm{~A}$ & 296.8 & -13.3 & $1.50 \mathrm{E}-03$ & 85 \\
\hline 520.2 & $86 \mathrm{~A}$ & 285.2 & 52.5 & $4.04 \mathrm{E}-03$ & 100 & 535.9 & $88 \mathrm{~A}$ & 288 & -17.7 & $2.15 \mathrm{E}-03$ & 100 \\
\hline 520.2 & $86 \mathrm{~A}$ & 303.8 & 35.9 & $4.28 \mathrm{E}-03$ & 115 & 535.9 & $88 \mathrm{~A}$ & 289.2 & -13.6 & $1.82 \mathrm{E}-03$ & 115 \\
\hline 530.2 & $87 \mathrm{~A}$ & 87.6 & 28.4 & 1.01E-02 & 0 & 540.2 & $89 \mathrm{~A}$ & 231.9 & 70.2 & $2.77 \mathrm{E}-02$ & \\
\hline 530.2 & $87 \mathrm{~A}$ & 87 & 22.3 & $9.44 \mathrm{E}-03$ & 3 & 540.2 & $89 \mathrm{~A}$ & 231.7 & 69.5 & $2.58 \mathrm{E}-02$ & \\
\hline 530.2 & $87 \mathrm{~A}$ & 86.3 & 20.6 & 8.55E-03 & 6 & 540.2 & $89 \mathrm{~A}$ & 232.3 & 69.4 & $2.38 \mathrm{E}-02$ & \\
\hline 530.2 & $87 \mathrm{~A}$ & 86.1 & 20.1 & $7.81 \mathrm{E}-03$ & 9 & 540.2 & $89 \mathrm{~A}$ & 233.6 & 68.3 & $2.13 \mathrm{E}-02$ & \\
\hline 530.2 & $87 \mathrm{~A}$ & 87 & 18.9 & $7.00 \mathrm{E}-03$ & 12 & 540.2 & $89 \mathrm{~A}$ & 234.7 & 67.9 & $1.93 \mathrm{E}-02$ & 12 \\
\hline 530.2 & $87 \mathrm{~A}$ & 87.2 & 21.3 & $6.19 \mathrm{E}-03$ & 15 & 540.2 & $89 \mathrm{~A}$ & 231.6 & 68.7 & $1.75 \mathrm{E}-02$ & 15 \\
\hline 530.2 & $87 \mathrm{~A}$ & 86.4 & 20.2 & $5.67 \mathrm{E}-03$ & 18 & 540.2 & $89 \mathrm{~A}$ & 233.7 & 68.6 & $1.56 \mathrm{E}-02$ & 18 \\
\hline 530.2 & $87 \mathrm{~A}$ & 88.4 & 21.6 & $5.19 \mathrm{E}-03$ & 21 & 540.2 & $89 \mathrm{~A}$ & 232.2 & 69 & $1.41 \mathrm{E}-02$ & 21 \\
\hline 530.2 & $87 \mathrm{~A}$ & 90.2 & 22.8 & $4.67 \mathrm{E}-03$ & 25 & 540.2 & $89 \mathrm{~A}$ & 229.7 & 69.1 & $1.24 \mathrm{E}-02$ & 25 \\
\hline 530.2 & $87 \mathrm{~A}$ & 90.3 & 21.4 & $3.90 \mathrm{E}-03$ & 30 & 540.2 & $89 \mathrm{~A}$ & 232.8 & 68.7 & $1.06 \mathrm{E}-02$ & 30 \\
\hline 530.2 & $87 \mathrm{~A}$ & 91.2 & 23.6 & $3.16 \mathrm{E}-03$ & 40 & 540.2 & $89 \mathrm{~A}$ & 228.1 & 69.1 & 8.39E-03 & 40 \\
\hline 530.2 & $87 \mathrm{~A}$ & 93.8 & 22.3 & $2.45 \mathrm{E}-03$ & 50 & 540.2 & $89 \mathrm{~A}$ & 231 & 67 & $7.23 \mathrm{E}-03$ & 50 \\
\hline 530.2 & $87 \mathrm{~A}$ & 96.9 & 15.3 & $2.33 \mathrm{E}-03$ & 60 & 540.2 & $89 \mathrm{~A}$ & 237.3 & 71.2 & $6.38 \mathrm{E}-03$ & 60 \\
\hline 530.2 & $87 \mathrm{~A}$ & 90.1 & 27.4 & $1.89 \mathrm{E}-03$ & 70 & 540.2 & $89 \mathrm{~A}$ & 226.3 & 69.3 & $5.88 \mathrm{E}-03$ & 70 \\
\hline 530.2 & $87 \mathrm{~A}$ & 97.9 & 16.6 & $1.89 \mathrm{E}-03$ & 85 & 540.2 & $89 \mathrm{~A}$ & 237.9 & 71.1 & $5.53 \mathrm{E}-03$ & 85 \\
\hline 530.2 & $87 \mathrm{~A}$ & 94.2 & 26.1 & $1.75 \mathrm{E}-03$ & 100 & 540.2 & $89 \mathrm{~A}$ & 227.6 & 66.6 & 4.67E-03 & 100 \\
\hline 530.2 & $87 \mathrm{~A}$ & 92.7 & 24.3 & $1.59 \mathrm{E}-03$ & 115 & 540.2 & $89 \mathrm{~A}$ & 229.4 & 64.4 & $4.35 \mathrm{E}-03$ & 115 \\
\hline 535.2 & $87.5 \mathrm{~A}$ & 310.7 & 86.8 & $1.83 E-02$ & 0 & 541 & $90 \mathrm{~A}$ & 70.1 & 62.2 & $2.69 \mathrm{E}-02$ & 促 \\
\hline 535.2 & $87.5 \mathrm{~A}$ & 302.1 & 83.4 & $1.25 \mathrm{E}-02$ & 3 & 541 & $90 \mathrm{~A}$ & 80.1 & 62.2 & $1.92 \mathrm{E}-02$ & 3 \\
\hline 535.2 & $87.5 \mathrm{~A}$ & 303.5 & 78 & $8.40 \mathrm{E}-03$ & 6 & 541 & $90 \mathrm{~A}$ & 89.3 & 62 & $1.39 \mathrm{E}-02$ & 6 \\
\hline 535.2 & $87.5 \mathrm{~A}$ & 299 & 73.4 & $6.14 \mathrm{E}-03$ & 9 & 541 & $90 \mathrm{~A}$ & 92.3 & 62.8 & $1.13 \mathrm{E}-02$ & 9 \\
\hline 535.2 & $87.5 \mathrm{~A}$ & 304.5 & 69.7 & $4.99 E-03$ & 12 & 541 & $90 \mathrm{~A}$ & 94.2 & 64.6 & $9.67 \mathrm{E}-03$ & 12 \\
\hline 535.2 & $87.5 \mathrm{~A}$ & 290.2 & 71.3 & $4.83 E-03$ & 15 & 541 & $90 \mathrm{~A}$ & 96.1 & 64.9 & $8.25 E-03$ & 15 \\
\hline 535.2 & $87.5 \mathrm{~A}$ & 296.5 & 70.9 & $3.93 E-03$ & 18 & 541 & $90 \mathrm{~A}$ & 95.2 & 63.2 & $7.20 \mathrm{E}-03$ & 18 \\
\hline 535.2 & $87.5 \mathrm{~A}$ & 292.3 & 69.7 & $3.24 \mathrm{E}-03$ & 21 & 541 & $90 \mathrm{~A}$ & 101.5 & 64.3 & $6.24 \mathrm{E}-03$ & 21 \\
\hline 535.2 & $87.5 \mathrm{~A}$ & 290.7 & 58 & $2.41 E-03$ & 25 & 541 & $90 \mathrm{~A}$ & 95.1 & 64.7 & $6.04 E-03$ & 25 \\
\hline 535.2 & $87.5 \mathrm{~A}$ & 300.5 & 60.6 & $2.24 \mathrm{E}-03$ & 30 & 541 & $90 \mathrm{~A}$ & 80.6 & 68.2 & $5.10 \mathrm{E}-03$ & 30 \\
\hline 535.2 & $87.5 \mathrm{~A}$ & 259.9 & 21.4 & $1.15 \mathrm{E}-03$ & 40 & 541 & $90 \mathrm{~A}$ & 98.8 & 59.7 & $3.59 E-03$ & 40 \\
\hline 535.2 & $87.5 \mathrm{~A}$ & 274.7 & 13.1 & $1.24 \mathrm{E}-03$ & 50 & 541 & $90 \mathrm{~A}$ & 88.1 & 53.8 & $3.23 \mathrm{E}-03$ & 50 \\
\hline 535.2 & $87.5 \mathrm{~A}$ & 277 & 18.3 & $9.76 E-04$ & 60 & 541 & $90 \mathrm{~A}$ & 95.5 & 57.7 & $3.54 \mathrm{E}-03$ & 60 \\
\hline 535.2 & $87.5 \mathrm{~A}$ & 249.1 & 33.9 & $1.31 E-03$ & 70 & 541 & $90 \mathrm{~A}$ & 111.2 & 64.4 & $2.53 E-03$ & 70 \\
\hline 535.2 & $87.5 \mathrm{~A}$ & 321.8 & 50.5 & $1.39 \mathrm{E}-03$ & 85 & 541 & $90 \mathrm{~A}$ & 44.1 & 47.9 & $3.12 \mathrm{E}-03$ & 85 \\
\hline 535.2 & $87.5 \mathrm{~A}$ & 241.6 & 30.2 & $1.21 \mathrm{E}-03$ & 100 & 541 & $90 \mathrm{~A}$ & 149.1 & 71.1 & $2.63 \mathrm{E}-03$ & 100 \\
\hline 535.2 & $87.5 \mathrm{~A}$ & 232.9 & 81.1 & $6.89 \mathrm{E}-04$ & 115 & 541 & $90 \mathrm{~A}$ & 121.4 & 36.5 & $2.01 \mathrm{E}-03$ & 115 \\
\hline
\end{tabular}

\begin{tabular}{|c|c|c|c|c|c|}
\hline 4.9 & $91 \mathrm{~A}$ & 214.1 & 20.4 & $4.54 \mathrm{E}$ & \\
\hline 544.9 & $91 \mathrm{~A}$ & 213.6 & 19.7 & $4.46 \mathrm{E}-02$ & \\
\hline 544.9 & $91 \mathrm{~A}$ & 13.7 & 18.8 & $4.20 \mathrm{E}-02$ & \\
\hline 544.9 & $91 \mathrm{~A}$ & 212.9 & 17.9 & $3.85 \mathrm{E}-02$ & \\
\hline 544.9 & $91 \mathrm{~A}$ & 213.3 & 17.8 & $3.56 \mathrm{E}-02$ & \\
\hline 544.9 & $91 \mathrm{~A}$ & 213.2 & 18 & $3.25 \mathrm{E}-02$ & \\
\hline 544.9 & $91 \mathrm{~A}$ & 213.4 & 17.5 & $2.94 \mathrm{E}-02$ & \\
\hline 544.9 & $91 \mathrm{~A}$ & 212.6 & 17.2 & $2.65 \mathrm{E}-02$ & \\
\hline 544.9 & $91 \mathrm{~A}$ & 212.4 & 17.2 & $2.35 \mathrm{E}-02$ & \\
\hline 544.9 & $91 \mathrm{~A}$ & 213 & 17.6 & $2.03 \mathrm{E}-02$ & \\
\hline 544.9 & $91 \mathrm{~A}$ & 212.6 & 16.9 & $1.56 \mathrm{E}-02$ & \\
\hline 544.9 & $91 \mathrm{~A}$ & 214.5 & 15.4 & $1.26 \mathrm{E}-02$ & \\
\hline 544.9 & $91 \mathrm{~A}$ & 212.2 & 13.8 & 1.10E-02 & \\
\hline 544.9 & $91 \mathrm{~A}$ & 214.6 & 16.2 & $1.01 \mathrm{E}-02$ & \\
\hline 544.9 & $91 \mathrm{~A}$ & 214.6 & 13.1 & $8.66 \mathrm{E}-03$ & \\
\hline 544.9 & $91 \mathrm{~A}$ & 213.3 & 14.8 & $8.28 \mathrm{E}-03$ & 10 \\
\hline 544.9 & $91 \mathrm{~A}$ & 214.9 & 17.4 & $7.27 \mathrm{E}-03$ & 11 \\
\hline 545.6 & $92 \mathrm{~A}$ & 294.4 & 83.5 & $1.25 \mathrm{E}-02$ & \\
\hline 545.6 & $92 \mathrm{~A}$ & 278.6 & 82.1 & $1.01 \mathrm{E}-02$ & \\
\hline 545.6 & $92 \mathrm{~A}$ & 265.8 & 80.3 & $8.25 \mathrm{E}-03$ & \\
\hline 545.6 & $92 \mathrm{~A}$ & 260.6 & 77.7 & 7.19E-03 & \\
\hline 545.6 & $92 \mathrm{~A}$ & 257.6 & 76.7 & $6.02 \mathrm{E}-03$ & 1 \\
\hline 545.6 & $92 \mathrm{~A}$ & 270.6 & 73.6 & $5.50 \mathrm{E}-03$ & 1 \\
\hline 545.6 & $92 \mathrm{~A}$ & 274.5 & 77.3 & $4.70 \mathrm{E}-03$ & 1 \\
\hline 545.6 & $92 \mathrm{~A}$ & 253.9 & 67.7 & $4.43 \mathrm{E}-03$ & 2 \\
\hline 545.6 & $92 \mathrm{~A}$ & 272.2 & 74.8 & $3.99 \mathrm{E}-03$ & \\
\hline 545.6 & $92 \mathrm{~A}$ & 222.8 & 71.2 & $3.08 \mathrm{E}-03$ & \\
\hline 545.6 & $92 \mathrm{~A}$ & 230.1 & 48.4 & $2.94 \mathrm{E}-03$ & \\
\hline 545.6 & $92 \mathrm{~A}$ & 210.4 & 67.5 & $2.46 \mathrm{E}-03$ & \\
\hline 545.6 & $92 \mathrm{~A}$ & 13.2 & 86.5 & & \\
\hline 545.6 & $92 \mathrm{~A}$ & 253 & 27 & $2.11 \mathrm{E}-03$ & \\
\hline 545.6 & $92 \mathrm{~A}$ & 1 & 81.2 & & ( \\
\hline 040.0 & $92 \mathrm{~A}$ & 39.1 & 32.7 & $1.45 \mathrm{E}-03$ & \\
\hline 545.6 & $92 A$ & & 61.7 & $E-03$ & \\
\hline .1... & 9 & & 30.2 & 8. & \\
\hline 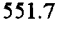 & 9 & & 30.1 & & \\
\hline 31.1 & (1) & & 30 & 7.4 & \\
\hline 551.7 & $94 \mathrm{~A}$ & 246.3 & 28.8 & & \\
\hline 551.7 & $94 \mathrm{~A}$ & 245.4 & 30 & $5.94 \mathrm{E}-03$ & 1 \\
\hline 551.7 & $94 \mathrm{~A}$ & 43.7 & 29.4 & $5.12 \mathrm{E}$ & 1 \\
\hline 551.7 & $94 \mathrm{~A}$ & 245.5 & 26.3 & & 1 \\
\hline 551.7 & $94 \mathrm{~A}$ & 241.6 & 29.5 & & 2 \\
\hline 551.7 & $94 \mathrm{~A}$ & 407 & 27 & 3.42 & 2 \\
\hline 551.7 & $94 \mathrm{~A}$ & 38.8 & 21.7 & & 30 \\
\hline 551.7 & $94 \mathrm{~A}$ & & 24.4 & & 4 \\
\hline (.) & $94 \mathrm{~A}$ & 246.5 & 21.4 & $1.79 \mathrm{E}-03$ & 50 \\
\hline 551.7 & $94 \mathrm{~A}$ & 246 & 23.1 & $1.70 E-03$ & 00 \\
\hline 551.7 & $94 \mathrm{~A}$ & 231 & 12.3 & & 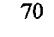 \\
\hline 年. & $94 \mathrm{~A}$ & -60 & 29 & $1.02 \mathrm{E}-03$ & 85 \\
\hline 年 & $94 \mathrm{~A}$ & 238.4 & 21.7 & $1.19 E-03$ & 100 \\
\hline 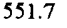 & $94 \mathrm{~A}$ & & & $\mathrm{E}-03$ & \\
\hline
\end{tabular}




\begin{tabular}{|c|c|c|c|c|c|}
\hline 55.7 & $95 \mathrm{~A}$ & 199.7 & 65.9 & $1.60 \mathrm{E}$ & \\
\hline 555.7 & $95 \mathrm{~A}$ & 201 & 63.3 & $1.48 \mathrm{E}-02$ & \\
\hline 555.7 & $95 \mathrm{~A}$ & 201.6 & 63 & $1.35 \mathrm{E}-02$ & \\
\hline 555.7 & $95 \mathrm{~A}$ & 200.9 & 62.1 & 1. $18 \mathrm{E}-02$ & \\
\hline 555.7 & $95 \mathrm{~A}$ & 98.8 & 62.8 & $1.05 \mathrm{E}-02$ & \\
\hline 555.7 & $95 \mathrm{~A}$ & 200.8 & 62.8 & $9.51 \mathrm{E}-03$ & \\
\hline 555.7 & $95 \mathrm{~A}$ & 04.8 & 60.8 & $8.32 \mathrm{E}-03$ & \\
\hline 55.7 & $95 \mathrm{~A}$ & 04.2 & 62.8 & $7.47 \mathrm{E}-03$ & \\
\hline 55.7 & $95 \mathrm{~A}$ & 06.5 & 62.8 & $6.45 \mathrm{E}-03$ & \\
\hline 555.7 & $95 \mathrm{~A}$ & 205.3 & 62.7 & $5.65 \mathrm{E}-03$ & \\
\hline 55.7 & $95 \mathrm{~A}$ & 206.1 & 62.2 & $3.87 \mathrm{E}-03$ & \\
\hline 55.7 & $95 \mathrm{~A}$ & 207.7 & 63.6 & $3.38 \mathrm{E}-03$ & \\
\hline 555.7 & $95 \mathrm{~A}$ & 203.7 & 61.4 & $3.09 \mathrm{E}-03$ & \\
\hline 555.7 & $95 \mathrm{~A}$ & 212.5 & 62.6 & $2.64 \mathrm{E}-03$ & \\
\hline 555.7 & $95 \mathrm{~A}$ & 215.8 & 62.8 & $2.44 \mathrm{E}-03$ & \\
\hline 555.7 & $95 \mathrm{~A}$ & 216.5 & 58.5 & $1.99 \mathrm{E}-03$ & 10 \\
\hline 555.7 & $95 \mathrm{~A}$ & 206.3 & 64.3 & $1.87 \mathrm{E}-03$ & 11 \\
\hline 560.6 & $96 \mathrm{~A}$ & 189.2 & 64.6 & $9.06 \mathrm{E}-03$ & \\
\hline 560.6 & $96 \mathrm{~A}$ & 185.8 & 63.3 & $8.31 E-03$ & \\
\hline 560.6 & $96 \mathrm{~A}$ & 187.3 & 63.8 & $7.34 \mathrm{E}-03$ & \\
\hline 600.6 & $96 \mathrm{~A}$ & 187.6 & 61.6 & $6.49 \mathrm{E}-03$ & \\
\hline 60.6 & $96 \mathrm{~A}$ & 193.9 & 58.9 & $5.86 \mathrm{E}-03$ & \\
\hline 60.6 & $96 \mathrm{~A}$ & 194.5 & 59.4 & $5.25 \mathrm{E}-03$ & \\
\hline 660.6 & $96 \mathrm{~A}$ & 193.4 & 58.9 & $4.57 \mathrm{E}-03$ & \\
\hline 600.6 & $96 \mathrm{~A}$ & 195.9 & 57.4 & $3.98 \mathrm{E}-03$ & \\
\hline 80.0 & $96 \mathrm{~A}$ & 201.5 & 54.6 & $3.53 \mathrm{E}-03$ & \\
\hline 00.0 & $96 \mathrm{~A}$ & 209.1 & 56.6 & $3.00 \mathrm{E}-03$ & \\
\hline 0.0 & $96 \mathrm{~A}$ & 188.1 & 48.3 & & \\
\hline 50. & $96 \mathrm{~A}$ & 22.7 & 54.9 & & \\
\hline 600.6 & $96 \mathrm{~A}$ & 195.6 & 57.3 & $1.60 \mathrm{E}-03$ & \\
\hline 60.6 & $96 \mathrm{~A}$ & 206.9 & 34.4 & $1.74 \mathrm{E}-03$ & \\
\hline 60.6 & $96 \mathrm{~A}$ & 197.1 & 57.2 & $1.10 \mathrm{E}-03$ & \\
\hline 560.6 & $96 \mathrm{~A}$ & 227.3 & 32 & $1.59 \mathrm{E}-03$ & 1 \\
\hline 560.6 & $96 \mathrm{~A}$ & 215.8 & 24.1 & $1.60 \mathrm{E}-03$ & 11 \\
\hline 61.8 & $96.5 \mathrm{~A}$ & 171 & 68.9 & $5.24 \mathrm{E}-02$ & \\
\hline 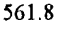 & $96.5 \mathrm{~A}$ & 173.1 & 66.8 & $4.84 \mathrm{E}-02$ & \\
\hline 4.0 & $96.5 \mathrm{~A}$ & 172.7 & 62.8 & $4.18 \mathrm{E}-02$ & \\
\hline . & $96.5 \mathrm{~A}$ & 175.1 & 59.9 & $3.71 \mathrm{E}-02$ & \\
\hline 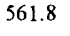 & $96.5 \mathrm{~A}$ & 179.8 & 58.2 & 3.38 & \\
\hline 01. & $96.5 \mathrm{~A}$ & 177.2 & 56.4 & 3.10 & \\
\hline 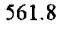 & $96.5 \mathrm{~A}$ & 180 & 54.9 & $2.85 \mathrm{E}-$ & \\
\hline 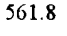 & $96.5 \mathrm{~A}$ & 176.8 & 53.1 & $2.62 \mathrm{E}-$ & \\
\hline 01.0 & $96.5 \mathrm{~A}$ & 181.8 & 51.8 & $2.37 \mathrm{E}-02$ & \\
\hline 81.0 & $96.5 \mathrm{~A}$ & 182.2 & 51 & & \\
\hline 81.8 & $96.5 \mathrm{~A}$ & 182 & 47.7 & & \\
\hline 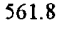 & $96.5 \mathrm{~A}$ & 186.9 & 44.2 & & \\
\hline 8.6 & $96.5 \mathrm{~A}$ & 188.6 & 44.9 & & \\
\hline 561.8 & $96.5 \mathrm{~A}$ & 184 & 38.2 & & \\
\hline & $96.5 \mathrm{~A}$ & 189.4 & 36.2 & & \\
\hline & $96.5 \mathrm{~A}$ & 192.8 & 38.2 & & \\
\hline & $96.5 \mathrm{~A}$ & & 38 & & \\
\hline
\end{tabular}

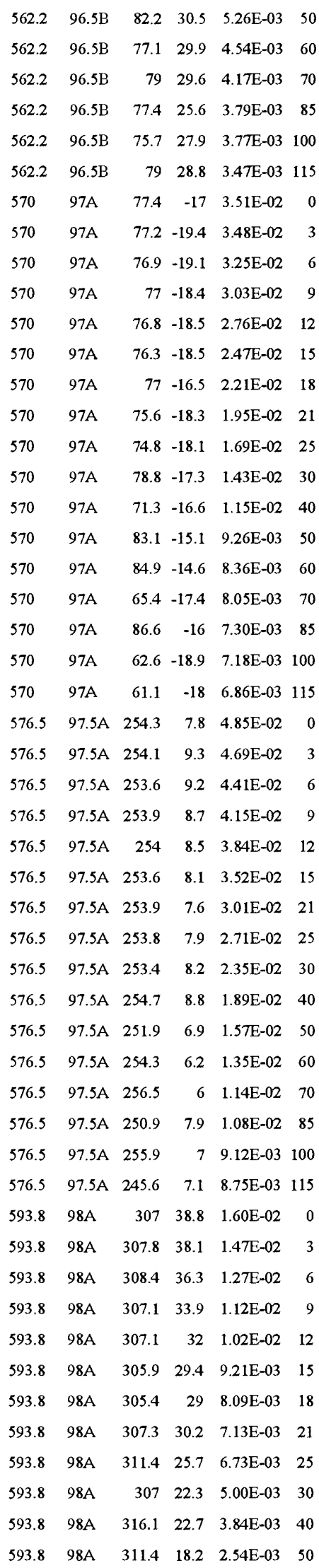

\begin{tabular}{|c|c|c|c|c|c|}
\hline 593.8 & $98 \mathrm{~A}$ & 312.3 & 27.1 & $2.30 \mathrm{E}-03$ & 60 \\
\hline 593.8 & $98 \mathrm{~A}$ & 321.6 & 5.2 & $2.20 \mathrm{E}-03$ & 70 \\
\hline 593.8 & $98 \mathrm{~A}$ & 358.4 & 24.9 & $1.36 \mathrm{E}-03$ & 85 \\
\hline 593.8 & $98 \mathrm{~A}$ & 344 & -6 & $1.36 \mathrm{E}-03$ & 100 \\
\hline 593.8 & $98 \mathrm{~A}$ & 358.1 & 4.4 & $1.29 \mathrm{E}-03$ & 115 \\
\hline 594.6 & $98 \mathrm{~A}$ & 255.9 & 20.7 & $9.95 \mathrm{E}-03$ & 0 \\
\hline 594.6 & $98 \mathrm{~A}$ & 253.9 & 18.4 & $9.65 \mathrm{E}-03$ & 3 \\
\hline 594.6 & $98 \mathrm{~A}$ & 253 & 17.1 & $9.05 \mathrm{E}-03$ & 6 \\
\hline 594.6 & $98 \mathrm{~A}$ & 252.7 & 15.9 & 8.35E-03 & 9 \\
\hline 594.6 & $98 \mathrm{~A}$ & 252.4 & 15.6 & $7.57 \mathrm{E}-03$ & 12 \\
\hline 594.6 & $98 \mathrm{~A}$ & 251.5 & 15.6 & $6.94 \mathrm{E}-03$ & 15 \\
\hline 594.6 & $98 \mathrm{~A}$ & 252.6 & 14.5 & $6.44 \mathrm{E}-03$ & 18 \\
\hline 594.6 & $98 \mathrm{~A}$ & 250.7 & 15.3 & $5.67 \mathrm{E}-03$ & 21 \\
\hline 594.6 & $98 \mathrm{~A}$ & 251.3 & 14.6 & $5.05 \mathrm{E}-03$ & 25 \\
\hline 594.6 & $98 \mathrm{~A}$ & 253.8 & 14.4 & $4.33 \mathrm{E}-03$ & 30 \\
\hline 594.6 & $98 \mathrm{~A}$ & 252.7 & 16.6 & $3.41 \mathrm{E}-03$ & 40 \\
\hline 594.6 & $98 \mathrm{~A}$ & 258.6 & 15.4 & $2.95 \mathrm{E}-03$ & 50 \\
\hline 594.6 & $98 \mathrm{~A}$ & 264.3 & 16.5 & $2.47 \mathrm{E}-03$ & 60 \\
\hline 594.6 & $98 \mathrm{~A}$ & 251.3 & 14.2 & $2.29 \mathrm{E}-03$ & 70 \\
\hline 594.6 & $98 \mathrm{~A}$ & 268.1 & 15.8 & $2.10 \mathrm{E}-03$ & 85 \\
\hline 594.6 & $98 \mathrm{~A}$ & 248.4 & 14.3 & $2.00 \mathrm{E}-03$ & 100 \\
\hline 594.6 & $98 \mathrm{~A}$ & 243.6 & 21.8 & $1.97 \mathrm{E}-03$ & 115 \\
\hline 594.6 & $98 \mathrm{~B}$ & 150.6 & 58.1 & $7.38 \mathrm{E}-03$ & 0 \\
\hline 594.6 & $98 \mathrm{~B}$ & 153.1 & 50.5 & $6.09 \mathrm{E}-03$ & 3 \\
\hline 594.6 & $98 \mathrm{~B}$ & 154.8 & 46.3 & $5.30 \mathrm{E}-03$ & 6 \\
\hline 594.6 & $98 \mathrm{~B}$ & 156.4 & 43.3 & $4.51 \mathrm{E}-03$ & 9 \\
\hline 594.6 & $98 \mathrm{~B}$ & 156.3 & 42.4 & $4.08 \mathrm{E}-03$ & 12 \\
\hline 594.6 & $98 \mathrm{~B}$ & 158.4 & 41.6 & $3.61 \mathrm{E}-03$ & 15 \\
\hline 594.6 & $98 \mathrm{~B}$ & 158.8 & 40.2 & $3.20 \mathrm{E}-03$ & 18 \\
\hline 594.6 & $98 \mathrm{~B}$ & 158 & 37.5 & $2.92 \mathrm{E}-03$ & 21 \\
\hline 594.6 & $98 \mathrm{~B}$ & 158.7 & 41.8 & $2.38 \mathrm{E}-03$ & 25 \\
\hline 594.6 & $98 \mathrm{~B}$ & 163.6 & 47.1 & $2.07 \mathrm{E}-03$ & 30 \\
\hline 594.6 & $98 \mathrm{~B}$ & 153.5 & 40.6 & $1.65 \mathrm{E}-03$ & 40 \\
\hline 594.6 & $98 \mathrm{~B}$ & 138 & 45.4 & $1.05 \mathrm{E}-03$ & 50 \\
\hline 594.6 & $98 \mathrm{~B}$ & 130.4 & 48 & $1.05 \mathrm{E}-03$ & 60 \\
\hline 594.6 & $98 \mathrm{~B}$ & 166.2 & 33.9 & $1.23 \mathrm{E}-03$ & 70 \\
\hline 594.6 & $98 \mathrm{~B}$ & 138.8 & 51.5 & $1.01 \mathrm{E}-03$ & 85 \\
\hline 594.6 & $98 \mathrm{~B}$ & 165.4 & 33.2 & $9.88 \mathrm{E}-04$ & 100 \\
\hline 594.6 & $98 \mathrm{~B}$ & 173.4 & 34.7 & $1.22 \mathrm{E}-03$ & 115 \\
\hline 595.5 & $98 \mathrm{C}$ & 194 & 24.3 & 4.19E-02 & 0 \\
\hline 595.5 & $98 \mathrm{C}$ & 194.8 & 21.3 & 4.10E-02 & 3 \\
\hline 595.5 & $98 \mathrm{C}$ & 195.1 & 21.4 & $3.78 \mathrm{E}-02$ & 6 \\
\hline 595.5 & $98 \mathrm{C}$ & 194.9 & 20.9 & $3.42 \mathrm{E}-02$ & 9 \\
\hline 595.5 & $98 \mathrm{C}$ & 194.5 & 20.3 & $3.07 \mathrm{E}-02$ & 12 \\
\hline 595.5 & $98 \mathrm{C}$ & 194.4 & 20.6 & $2.74 \mathrm{E}-02$ & 15 \\
\hline 595.5 & $98 \mathrm{C}$ & 195 & 21.2 & $2.43 \mathrm{E}-02$ & 18 \\
\hline 595.5 & $98 \mathrm{C}$ & 195.1 & 21 & $2.18 \mathrm{E}-02$ & 21 \\
\hline 595.5 & $98 \mathrm{C}$ & 195.4 & 20.9 & $1.88 \mathrm{E}-02$ & 25 \\
\hline 595.5 & $98 \mathrm{C}$ & 194.9 & 21.1 & $1.64 \mathrm{E}-02$ & 30 \\
\hline 595.5 & $98 \mathrm{C}$ & 194.9 & 20.3 & $1.29 \mathrm{E}-02$ & 40 \\
\hline 595.5 & $98 \mathrm{C}$ & 194.3 & 20.7 & $1.11 \mathrm{E}-02$ & 50 \\
\hline
\end{tabular}




\begin{tabular}{|c|c|c|c|c|c|c|c|c|c|c|c|}
\hline 595.5 & $98 \mathrm{C}$ & 191.7 & 20.5 & $9.85 \mathrm{E}-03$ & 60 & 604.1 & $101 \mathrm{~A}$ & 279.2 & 41.3 & $1.64 \mathrm{E}-03$ & 60 \\
\hline 595.5 & $98 \mathrm{C}$ & 194.5 & 20 & $9.35 \mathrm{E}-03$ & 70 & 604.1 & $101 \mathrm{~A}$ & 292.8 & 25.8 & $1.70 \mathrm{E}-03$ & 70 \\
\hline 595.5 & $98 \mathrm{C}$ & 190 & 21.5 & 8.38E-03 & 85 & 604.1 & $101 \mathrm{~A}$ & 287.6 & 29.1 & $1.03 \mathrm{E}-03$ & 85 \\
\hline 595.5 & $98 \mathrm{C}$ & 195.3 & 19.9 & $7.90 \mathrm{E}-03$ & 100 & 604.1 & $101 \mathrm{~A}$ & 303 & 28.2 & $1.20 \mathrm{E}-03$ & 100 \\
\hline 595.5 & $98 \mathrm{C}$ & 195.2 & 19.8 & $7.48 \mathrm{E}-03$ & 115 & 604.1 & $101 \mathrm{~A}$ & 297.5 & 24.1 & $1.05 \mathrm{E}-03$ & 115 \\
\hline 596.3 & $99 \mathrm{~A}$ & 291.6 & 11 & $1.65 \mathrm{E}-02$ & 0 & 604.2 & $101 \mathrm{~B}$ & 300.9 & 40.4 & $1.22 \mathrm{E}-02$ & 0 \\
\hline 596.3 & $99 \mathrm{~A}$ & 292 & 9.4 & $1.60 \mathrm{E}-02$ & 3 & 604.2 & $101 \mathrm{~B}$ & 300.8 & 39.5 & $1.09 \mathrm{E}-02$ & 3 \\
\hline 596.3 & $99 \mathrm{~A}$ & 291.6 & 8.4 & $1.48 \mathrm{E}-02$ & 6 & 604.2 & $101 \mathrm{~B}$ & 300.8 & 38.7 & $9.59 \mathrm{E}-03$ & 6 \\
\hline 596.3 & $99 \mathrm{~A}$ & 291.2 & 7.7 & 1.34E-02 & 9 & 604.2 & $101 \mathrm{~B}$ & 300.1 & 38.1 & $8.40 \mathrm{E}-03$ & 9 \\
\hline 596.3 & $99 \mathrm{~A}$ & 291.2 & 7.2 & $1.21 \mathrm{E}-02$ & 12 & 604.2 & $101 \mathrm{~B}$ & 299.9 & 38.5 & 7.36E-03 & 12 \\
\hline 596.3 & $99 \mathrm{~A}$ & 291.1 & 7.1 & $1.07 \mathrm{E}-02$ & 15 & 604.2 & $101 \mathrm{~B}$ & 302 & 40 & $6.42 \mathrm{E}-03$ & 15 \\
\hline 596.3 & $99 \mathrm{~A}$ & 291.7 & 7.8 & $9.67 \mathrm{E}-03$ & 18 & 604.2 & $101 \mathrm{~B}$ & 301.3 & 39 & $5.60 \mathrm{E}-03$ & 18 \\
\hline 596.3 & $99 \mathrm{~A}$ & 291.3 & 8.1 & $8.43 \mathrm{E}-03$ & 21 & 604.2 & $101 \mathrm{~B}$ & 301.6 & 38.3 & $4.75 \mathrm{E}-03$ & 21 \\
\hline 596.3 & $99 \mathrm{~A}$ & 291.2 & 9.2 & 7.12E-03 & 25 & 604.2 & $101 \mathrm{~B}$ & 298.8 & 37.1 & $3.96 \mathrm{E}-03$ & 25 \\
\hline 596.3 & $99 \mathrm{~A}$ & 293.2 & 6.2 & $6.30 \mathrm{E}-03$ & 30 & 604.2 & $101 \mathrm{~B}$ & 299.1 & 36.4 & $3.30 \mathrm{E}-03$ & 30 \\
\hline 596.3 & $99 \mathrm{~A}$ & 291.1 & 8.8 & $4.24 \mathrm{E}-03$ & 40 & 604.2 & $101 \mathrm{~B}$ & 296.9 & 33.6 & $1.96 \mathrm{E}-03$ & 40 \\
\hline 596.3 & $99 \mathrm{~A}$ & 297.4 & 9.5 & $4.16 \mathrm{E}-03$ & 50 & 604.2 & $101 \mathrm{~B}$ & 305.5 & 40.2 & $1.53 \mathrm{E}-03$ & 50 \\
\hline 596.3 & $99 \mathrm{~A}$ & 298.7 & 10.3 & $3.64 \mathrm{E}-03$ & 60 & 604.2 & $101 \mathrm{~B}$ & 310.6 & 40.2 & $1.15 \mathrm{E}-03$ & 60 \\
\hline 596.3 & $99 \mathrm{~A}$ & 283.5 & 6.7 & $3.16 \mathrm{E}-03$ & 70 & 604.2 & $101 \mathrm{~B}$ & 298.7 & 28.8 & $1.32 \mathrm{E}-03$ & 70 \\
\hline 596.3 & $99 \mathrm{~A}$ & 302.8 & 15.4 & $3.17 \mathrm{E}-03$ & 85 & 604.2 & $101 \mathrm{~B}$ & 296 & 23 & $9.14 \mathrm{E}-04$ & 85 \\
\hline 596.3 & $99 \mathrm{~A}$ & 280.4 & 7.4 & $2.46 \mathrm{E}-03$ & 100 & 604.2 & $101 \mathrm{~B}$ & 292.2 & 26 & 1.01E-03 & 100 \\
\hline 596.3 & $99 \mathrm{~A}$ & 281.2 & 6 & $2.27 \mathrm{E}-03$ & 115 & 604.2 & $101 \mathrm{~B}$ & 296.3 & 22.9 & $9.90 \mathrm{E}-04$ & 115 \\
\hline 600.1 & $100 \mathrm{~A}$ & 283.3 & 40.8 & $1.14 \mathrm{E}-02$ & 0 & 606 & $101 \mathrm{C}$ & 281.4 & 38.2 & $6.43 \mathrm{E}-03$ & 0 \\
\hline 600.1 & $100 \mathrm{~A}$ & 283.1 & 40.3 & $1.05 \mathrm{E}-02$ & 3 & 606 & $101 \mathrm{C}$ & 280.4 & 37.1 & $5.64 \mathrm{E}-03$ & 3 \\
\hline 600.1 & $100 \mathrm{~A}$ & 282.6 & 39.8 & $9.59 \mathrm{E}-03$ & 6 & 606 & $101 \mathrm{C}$ & 280.2 & 35.6 & $4.76 \mathrm{E}-03$ & 6 \\
\hline 600.1 & $100 \mathrm{~A}$ & 282.1 & 38.4 & $8.51 \mathrm{E}-03$ & 9 & 606 & $101 \mathrm{C}$ & 279.5 & 35.4 & $4.28 \mathrm{E}-03$ & 9 \\
\hline 600.1 & $100 \mathrm{~A}$ & 281.8 & 37.5 & $7.56 \mathrm{E}-03$ & 12 & 606 & $101 \mathrm{C}$ & 278 & 34.6 & 3.85E-03 & 12 \\
\hline 600.1 & $100 \mathrm{~A}$ & 281.5 & 37.3 & $6.77 \mathrm{E}-03$ & 15 & 606 & $101 \mathrm{C}$ & 277.5 & 34.7 & $3.20 \mathrm{E}-03$ & 15 \\
\hline 600.1 & $100 \mathrm{~A}$ & 281.8 & 36.2 & $6.03 \mathrm{E}-03$ & 18 & 606 & $101 \mathrm{C}$ & 278.1 & 34.1 & $2.97 \mathrm{E}-03$ & 18 \\
\hline 600.1 & $100 \mathrm{~A}$ & 282.6 & 38.5 & $5.26 \mathrm{E}-03$ & 21 & 606 & $101 \mathrm{C}$ & 276.4 & 35.1 & $2.31 \mathrm{E}-03$ & 21 \\
\hline 600.1 & $100 \mathrm{~A}$ & 281.4 & 35.5 & 4.77E-03 & 25 & 606 & $101 \mathrm{C}$ & 273.6 & 33.3 & $2.19 \mathrm{E}-03$ & 25 \\
\hline 600.1 & $100 \mathrm{~A}$ & 284.4 & 34 & $3.91 \mathrm{E}-03$ & 30 & 606 & $101 \mathrm{C}$ & 277.3 & 30.3 & $1.96 \mathrm{E}-03$ & 30 \\
\hline 600.1 & $100 \mathrm{~A}$ & 279.7 & 33.7 & $3.17 \mathrm{E}-03$ & 40 & 606 & $101 \mathrm{C}$ & 270.9 & 26 & $1.28 \mathrm{E}-03$ & 40 \\
\hline 600.1 & $100 \mathrm{~A}$ & 279.9 & 27.9 & $2.44 \mathrm{E}-03$ & 50 & 606 & $101 \mathrm{C}$ & 274.9 & 24.5 & $1.2 \mathrm{IE}-03$ & 50 \\
\hline 600.1 & $100 \mathrm{~A}$ & 285.3 & 39.4 & $2.47 \mathrm{E}-03$ & 60 & 606 & $101 \mathrm{C}$ & 282 & 36 & $1.10 \mathrm{E}-03$ & 60 \\
\hline 600.1 & $100 \mathrm{~A}$ & 277.7 & 32.2 & $2.06 \mathrm{E}-03$ & 70 & 606 & $101 \mathrm{C}$ & 285.6 & 22 & $9.99 \mathrm{E}-04$ & 70 \\
\hline 600.1 & $100 \mathrm{~A}$ & 285.5 & 28.4 & $1.85 \mathrm{E}-03$ & 85 & 606 & $101 \mathrm{C}$ & 296.6 & 38.8 & $6.59 \mathrm{E}-04$ & 85 \\
\hline 600.1 & $100 \mathrm{~A}$ & 280.5 & 31.7 & $1.66 \mathrm{E}-03$ & 100 & 606 & $101 \mathrm{C}$ & 274.3 & 25.3 & $7.82 \mathrm{E}-04$ & 100 \\
\hline 600.1 & $100 \mathrm{~A}$ & 277.5 & 25.6 & $1.71 \mathrm{E}-03$ & 115 & 606 & $101 \mathrm{C}$ & 276.7 & 25.1 & $9.02 \mathrm{E}-04$ & 115 \\
\hline 604.1 & $101 \mathrm{~A}$ & 289.6 & 34.4 & $1.43 \mathrm{E}-02$ & 0 & 608.3 & $102 \mathrm{~A}$ & 52.5 & 52 & $2.51 \mathrm{E}-02$ & 0 \\
\hline 604.1 & $101 \mathrm{~A}$ & 288.3 & 32.8 & $1.33 \mathrm{E}-02$ & 3 & 608.3 & $102 \mathrm{~A}$ & 55.1 & 52.1 & $2.32 \mathrm{E}-02$ & 3 \\
\hline 604.1 & $101 \mathrm{~A}$ & 287.6 & 32.1 & $1.21 \mathrm{E}-02$ & 6 & 608.3 & $102 \mathrm{~A}$ & 55.8 & 53.1 & $2.12 \mathrm{E}-02$ & 6 \\
\hline 604.1 & $101 \mathrm{~A}$ & 287.6 & 31.9 & $1.07 \mathrm{E}-02$ & 9 & 608.3 & $102 \mathrm{~A}$ & 55.9 & 53.5 & $1.88 \mathrm{E}-02$ & 9 \\
\hline 604.1 & $101 \mathrm{~A}$ & 287.7 & 31.1 & $9.43 \mathrm{E}-03$ & 12 & 608.3 & $102 \mathrm{~A}$ & 55.8 & 54 & $1.66 \mathrm{E}-02$ & 12 \\
\hline 604.1 & $101 \mathrm{~A}$ & 289.1 & 32.2 & $8.10 \mathrm{E}-03$ & 15 & 608.3 & $102 \mathrm{~A}$ & 54.8 & 55.3 & $1.50 \mathrm{E}-02$ & 15 \\
\hline 604.1 & $101 \mathrm{~A}$ & 288.2 & 31.7 & $7.32 \mathrm{E}-03$ & 18 & 608.3 & $102 \mathrm{~A}$ & 56.9 & 53.6 & $1.29 \mathrm{E}-02$ & 18 \\
\hline 604.1 & $101 \mathrm{~A}$ & 289.4 & 32 & $6.11 \mathrm{E}-03$ & 21 & 608.3 & $102 \mathrm{~A}$ & 56.3 & 54.1 & $1.14 \mathrm{E}-02$ & 21 \\
\hline 604.1 & $101 \mathrm{~A}$ & 288.9 & 30.6 & $5.10 \mathrm{E}-03$ & 25 & 608.3 & $102 \mathrm{~A}$ & 54.5 & 54.7 & $9.37 \mathrm{E}-03$ & 25 \\
\hline 604.1 & $101 \mathrm{~A}$ & 285.8 & 32.2 & $4.19 \mathrm{E}-03$ & 30 & 608.3 & $102 \mathrm{~A}$ & 56.7 & 57.1 & 7.84E-03 & 30 \\
\hline 604.1 & $101 \mathrm{~A}$ & 288.7 & 28.8 & $2.81 \mathrm{E}-03$ & 40 & 608.3 & $102 \mathrm{~A}$ & 51.1 & 54.4 & $5.34 \mathrm{E}-03$ & 40 \\
\hline 604.1 & $101 \mathrm{~A}$ & 284.7 & 25.5 & $1.81 \mathrm{E}-03$ & 50 & 608.3 & $102 \mathrm{~A}$ & 55.9 & 65.3 & 4. $12 \mathrm{E}-03$ & 50 \\
\hline
\end{tabular}

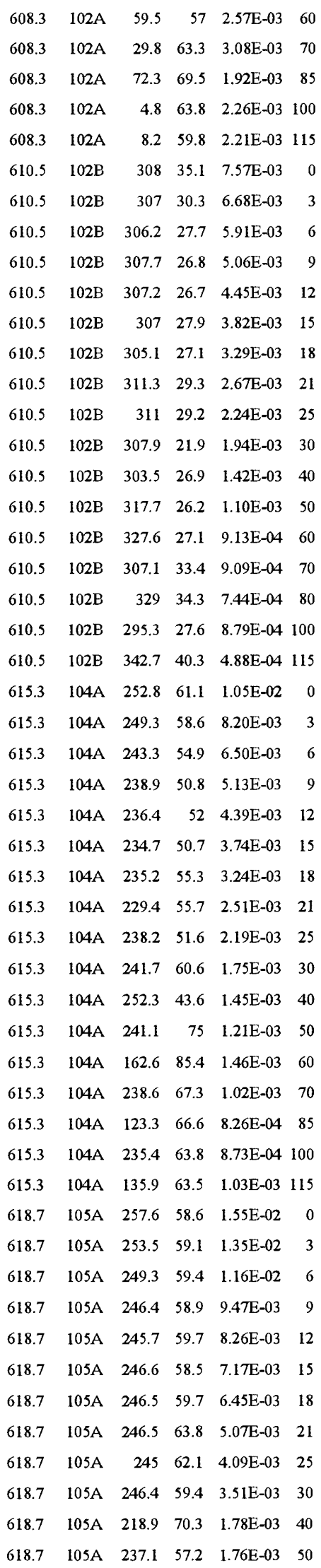


$\begin{array}{llllll}618.7 & 105 \mathrm{~A} & 241.3 & 73.6 & 2.00 \mathrm{E}-03 & 60\end{array}$

$\begin{array}{lllllll}618.7 & 105 \mathrm{~A} & 260.2 & 28.8 & 1.96 \mathrm{E}-03 & 70\end{array}$

$\begin{array}{lllllll}618.7 & 105 \mathrm{~A} & 221.2 & 71 & 1.52 \mathrm{E}-03 & 85\end{array}$

$\begin{array}{llllll}618.7 & 105 \mathrm{~A} & 250.9 & 28.2 & 1.52 \mathrm{E}-03 & 100\end{array}$

$\begin{array}{llllll}618.7 & 105 \mathrm{~A} & 250.8 & 27.2 & 1.47 \mathrm{E}-03 & 115\end{array}$

$\begin{array}{llllll}621.3 & 106 \mathrm{~A} & 18 & 71.8 & 1.29 \mathrm{E}-02 & 0\end{array}$

$\begin{array}{llllll}621.3 & 106 \mathrm{~A} & 18.9 & 71.3 & 1.13 \mathrm{E}-02 & 3\end{array}$

$\begin{array}{llllll}621.3 & 106 \mathrm{~A} & 23.5 & 71.3 & 1.01 \mathrm{E}-02 & 6\end{array}$

$\begin{array}{lllllll}621.3 & 106 \mathrm{~A} & 25.6 & 70 & 8.82 \mathrm{E}-03 & 9\end{array}$

$\begin{array}{llllll}621.3 & 106 \mathrm{~A} & 26.3 & 70.2 & 7.73 \mathrm{E}-03 & 12\end{array}$

$\begin{array}{llllll}621.3 & 106 \mathrm{~A} & 28.8 & 71.9 & 6.83 \mathrm{E}-03 & 15\end{array}$

$\begin{array}{lllllll}621.3 & 106 \mathrm{~A} & 28.7 & 71.9 & 5.95 \mathrm{E}-03 & 18\end{array}$

$\begin{array}{llllll}621.3 & 106 \mathrm{~A} & 33.1 & 73.7 & 5.34 \mathrm{E}-03 & 21\end{array}$

$\begin{array}{lllllll}621.3 & 106 \mathrm{~A} & 27.9 & 73.8 & 4.46 \mathrm{E}-03 & 25\end{array}$

$\begin{array}{llllll}621.3 & 106 \mathrm{~A} & 39.7 & 73 & 3.32 \mathrm{E}-03 & 30\end{array}$

$\begin{array}{llllll}621.3 & 106 \mathrm{~A} & 52.6 & 72.2 & 2.71 \mathrm{E}-03 & 40\end{array}$

$\begin{array}{llllll}621.3 & 106 \mathrm{~A} & 60.4 & 81 & 2.58 \mathrm{E}-03 & 50\end{array}$

$\begin{array}{llllll}621.3 & 106 \mathrm{~A} & 78.5 & 71.3 & 2.07 \mathrm{E}-03 & 60\end{array}$

$\begin{array}{llllll}621.3 & 106 \mathrm{~A} & 88.2 & 85.9 & 1.61 \mathrm{E}-03 & 70\end{array}$

$\begin{array}{llllll}621.3 & 106 \mathrm{~A} & 61.6 & 68.5 & 1.66 \mathrm{E}-03 & 85\end{array}$

$\begin{array}{lllllll}621.3 & 106 \mathrm{~A} & 221.7 & 87.4 & 1.28 \mathrm{E}-03 & 100\end{array}$

$\begin{array}{lllllll}621.3 & 106 \mathrm{~A} & 298.5 & 63.6 & 1.09 \mathrm{E}-03 & 115\end{array}$

$\begin{array}{llllll}622.8 & 107 \mathrm{~A} & 320.3 & 53.7 & 2.84 \mathrm{E}-02 & 0\end{array}$

$\begin{array}{llllll}622.8 & 107 \mathrm{~A} & 319.6 & 50 & 2.35 \mathrm{E}-02 & 3\end{array}$

$\begin{array}{lllllll}622.8 & 107 \mathrm{~A} & 319.9 & 48 & 1.97 \mathrm{E}-02 & 6\end{array}$

$\begin{array}{llllll}622.8 & 107 \mathrm{~A} & 319.3 & 47.2 & 1.64 \mathrm{E}-02 & 9\end{array}$

$\begin{array}{lllllll}622.8 & 107 \mathrm{~A} & 319.6 & 46.6 & 1.43 \mathrm{E}-02 & 12\end{array}$

$\begin{array}{lllllll}622.8 & 107 \mathrm{~A} & 322.1 & 45.1 & 1.26 \mathrm{E}-02 & 15\end{array}$

$\begin{array}{llllll}622.8 & 107 \mathrm{~A} & 320.2 & 43.9 & 1.14 \mathrm{E}-02 & 18\end{array}$

$\begin{array}{lllllll}622.8 & 107 \mathrm{~A} & 323.7 & 43.4 & 9.56 \mathrm{E}-03 & 21\end{array}$

$\begin{array}{lllllll}622.8 & 107 \mathrm{~A} & 322.9 & 46.1 & 8.18 \mathrm{E}-03 & 25\end{array}$

$\begin{array}{llllll}622.8 & 107 \mathrm{~A} & 324.4 & 44.4 & 6.97 \mathrm{E}-03 & 30\end{array}$

$\begin{array}{lllllll}622.8 & 107 \mathrm{~A} & 350.8 & 45.4 & 3.44 \mathrm{E}-03 & 40\end{array}$

$\begin{array}{lllllll}622.8 & 107 \mathrm{~A} & 332.4 & 4.1 & 2.76 \mathrm{E}-03 & 50\end{array}$

$\begin{array}{lllllll}622.8 & 107 \mathrm{~A} & 337.5 & 15.9 & 2.05 \mathrm{E}-03 & 60\end{array}$

$\begin{array}{llllll}622.8 & 107 \mathrm{~A} & 302.3 & 19.2 & 2.67 \mathrm{E}-03 & 70\end{array}$

$\begin{array}{lllllll}622.8 & 107 \mathrm{~A} & 359.6 & 43.5 & 1.90 \mathrm{E}-03 & 85\end{array}$

$\begin{array}{lllllll}622.8 \quad 107 \mathrm{~A} & 341 & 36.3 & 1.81 \mathrm{E}-03 & 100\end{array}$

$\begin{array}{llllll}622.8 & 107 \mathrm{~A} & 341.7 & 49 & 1.79 \mathrm{E}-03 & 115\end{array}$

$\begin{array}{lllllll}625.9 & 109 \mathrm{~A} & 331.6 & 53.9 & 5.59 \mathrm{E}-02 & 0\end{array}$

$\begin{array}{lllllll}625.9 & 109 \mathrm{~A} & 333.1 & 53.7 & 5.38 \mathrm{E}-02 & 3\end{array}$

$\begin{array}{llllll}625.9 & 109 \mathrm{~A} & 333 & 53.5 & 4.99 \mathrm{E}-02 & 6\end{array}$

$\begin{array}{llllll}625.9 & 109 \mathrm{~A} & 332.9 & 52.7 & 4.50 \mathrm{E}-02 & 9\end{array}$

$\begin{array}{llllll}625.9 & 109 \mathrm{~A} & 332.6 & 53.4 & 4.17 \mathrm{E}-02 & 12\end{array}$

$\begin{array}{llllll}625.9 & 109 \mathrm{~A} & 333.4 & 53.8 & 3.83 \mathrm{E}-02 & 15\end{array}$

$\begin{array}{lllllll}625.9 & 109 \mathrm{~A} & 333.1 & 53.1 & 3.46 \mathrm{E}-02 & 18\end{array}$

$\begin{array}{lllllll}625.9 & 109 \mathrm{~A} & 333.4 & 53.4 & 3.14 \mathrm{E}-02 & 21\end{array}$

$\begin{array}{llllll}625.9 & 109 \mathrm{~A} & 332.6 & 51.8 & 2.73 \mathrm{E}-02 & 25\end{array}$

$\begin{array}{lllllll}625.9 & 109 \mathrm{~A} & 330.4 & 51.2 & 2.38 \mathrm{E}-02 & 30\end{array}$

$\begin{array}{lllllll}625.9 & 109 \mathrm{~A} & 327.2 & 52.9 & 1.86 \mathrm{E}-02 & 40\end{array}$

$\begin{array}{llllll}625.9 & 109 \mathrm{~A} & 328 & 54.9 & 1.30 \mathrm{E}-02 & 50\end{array}$ $\begin{array}{llllll}625.9 & 109 \mathrm{~A} & 341 & 52.6 & 1.10 \mathrm{E}-02 & 60\end{array}$ $\begin{array}{lllllll}625.9 & 109 \mathrm{~A} & 343.2 & 47.2 & 1.05 \mathrm{E}-02 & 70\end{array}$ $\begin{array}{lllllll}625.9 & 109 \mathrm{~A} & 332.2 & 53.2 & 8.36 \mathrm{E}-03 & 85\end{array}$ $\begin{array}{llllll}625.9 & 109 \mathrm{~A} & 334.2 & 46.5 & 5.79 \mathrm{E}-03 & 100\end{array}$ $\begin{array}{lllllll}625.9 & 109 \mathrm{~A} & 332.1 & 43.5 & 6.83 \mathrm{E}-03 & 115\end{array}$ $\begin{array}{llllll}628.2 & 111 \mathrm{~A} & 345.4 & 73 & 3.41 \mathrm{E}-02 & 0\end{array}$ $\begin{array}{llllll}628.2 & 111 \mathrm{~A} & 347.4 & 74 & 2.98 \mathrm{E}-02 & 3\end{array}$ $\begin{array}{lllllll}628.2 & 111 \mathrm{~A} & 348.8 & 73.7 & 2.27 \mathrm{E}-02 & 6\end{array}$ $\begin{array}{llllll}628.2 & 111 \mathrm{~A} & 357.2 & 70.2 & 1.56 \mathrm{E}-02 & 9\end{array}$ $\begin{array}{lllllll}628.2 & 111 \mathrm{~A} & 9.5 & 69.3 & 1.24 \mathrm{E}-02 & 12\end{array}$ $\begin{array}{llllll}628.2 & 111 \mathrm{~A} & 13 & 65.6 & 1.01 \mathrm{E}-02 & 15\end{array}$ $\begin{array}{lllllll}628.2 & 111 \mathrm{~A} & 16.6 & 60.1 & 7.97 \mathrm{E}-03 & 18\end{array}$ $\begin{array}{llllll}628.2 & 111 \mathrm{~A} & 26 & 56.4 & 7.25 \mathrm{E}-03 & 21\end{array}$ $\begin{array}{llllll}628.2 & 111 \mathrm{~A} & 26.8 & 56.2 & 5.83 \mathrm{E}-03 & 25\end{array}$ $\begin{array}{llllll}628.2 & 111 \mathrm{~A} & 27.6 & 57.7 & 4.41 \mathrm{E}-03 & 30\end{array}$ $\begin{array}{lllllll}628.2 & 111 \mathrm{~A} & 11.7 & 49.5 & 2.41 \mathrm{E}-03 & 40\end{array}$ $\begin{array}{lllllll}628.2 & 111 \mathrm{~A} & 181.2 & 67.9 & 1.85 \mathrm{E}-03 & 50\end{array}$ $\begin{array}{lllllll}628.2 & 111 \mathrm{~A} & 135.8 & 55 & 2.24 \mathrm{E}-03 & 60\end{array}$ $\begin{array}{llllll}628.2 & 111 \mathrm{~A} & 352.6 & 8.1 & 1.41 \mathrm{E}-03 & 70\end{array}$ $\begin{array}{lllllll}628.2 & 111 \mathrm{~A} & 127.5 & 12.1 & 2.64 \mathrm{E}-03 & 85\end{array}$ $\begin{array}{llllll}628.2 & 111 \mathrm{~A} & 5 & 14.8 & 1.33 \mathrm{E}-03 & 100\end{array}$ $\begin{array}{lllllll}628.2 & 111 \mathrm{~A} & 9.3 & 6 & 1.90 \mathrm{E}-03 & 115\end{array}$ $\begin{array}{llllll}636.8 & 111 \mathrm{~B} & 349.2 & 77.2 & 6.85 \mathrm{E}-02 & 0\end{array}$ $\begin{array}{lllllll}636.8 & 111 \mathrm{~B} & 343.4 & 75.1 & 5.34 \mathrm{E}-02 & 3\end{array}$ $\begin{array}{lllllll}636.8 & 111 \mathrm{~B} & 337.2 & 70.9 & 3.74 \mathrm{E}-02 & 6\end{array}$ $\begin{array}{lllllll}636.8 & 111 \mathrm{~B} & 331.7 & 66.1 & 2.57 \mathrm{E}-02 & 9\end{array}$ $\begin{array}{llllll}636.8 & 111 \mathrm{~B} & 333.1 & 61.3 & 2.07 \mathrm{E}-02 & 12\end{array}$ $\begin{array}{lllllll}636.8 & 111 \mathrm{~B} & 330.2 & 59.8 & 1.69 \mathrm{E}-02 & 15\end{array}$ $\begin{array}{lllllll}636.8 & 111 \mathrm{~B} & 329.4 & 58.1 & 1.42 \mathrm{E}-02 & 18\end{array}$ $\begin{array}{llllll}636.8 & 111 \mathrm{~B} & 341.5 & 53.6 & 1.09 \mathrm{E}-02 & 21\end{array}$ $\begin{array}{lllllll}636.8 & 111 \mathrm{~B} & 329.6 & 51.5 & 8.38 \mathrm{E}-03 & 25\end{array}$ $\begin{array}{lllllll}636.8 & 111 \mathrm{~B} & 330.5 & 54.7 & 7.05 \mathrm{E}-03 & 30\end{array}$ $\begin{array}{lllllll}636.8 & 111 \mathrm{~B} & 324.4 & 49.7 & 4.58 \mathrm{E}-03 & 40\end{array}$ $\begin{array}{lllllll}636.8 & 111 \mathrm{~B} & 346.8 & 52.2 & 1.34 \mathrm{E}-03 & 50\end{array}$ $\begin{array}{llllll}636.8 & 111 \mathrm{~B} & 12 & 34.9 & 9.49 \mathrm{E}-04 & 60\end{array}$ $\begin{array}{llllll}636.8 & 111 \mathrm{~B} & 346.3 & 19.1 & 1.48 \mathrm{E}-03 & 70\end{array}$ $\begin{array}{lllllll}636.8 & 111 \mathrm{~B} & 354.9 & 64.5 & 1.17 \mathrm{E}-03 & 85\end{array}$ $\begin{array}{lllllll}636.8 & 111 \mathrm{~B} & 8.4 & 1.3 & 2.65 \mathrm{E}-03 & 100\end{array}$ $\begin{array}{llllll}636.8 & 111 \mathrm{~B} & 357.6 & 4 & 1.89 \mathrm{E}-03 & 115\end{array}$ $\begin{array}{lllllll}637.8 & 112 \mathrm{~A} & 62.6 & 72.6 & 5.86 \mathrm{E}-02 & 0\end{array}$ $\begin{array}{llllll}637.8 & 112 \mathrm{~A} & 72.2 & 69.5 & 4.77 \mathrm{E}-02 & 3\end{array}$ $\begin{array}{lllllll}637.8 & 112 \mathrm{~A} & 80.7 & 66.9 & 3.44 \mathrm{E}-02 & 6\end{array}$ $\begin{array}{llllll}637.8 & 112 \mathrm{~A} & 87.3 & 63.5 & 2.37 \mathrm{E}-02 & 9\end{array}$ $\begin{array}{llllll}637.8 & 112 \mathrm{~A} & 90 & 59.6 & 1.92 \mathrm{E}-02 & 12\end{array}$ $\begin{array}{llllll}637.8 & 112 \mathrm{~A} & 93.1 & 57.3 & 1.58 \mathrm{E}-02 & 15\end{array}$ $\begin{array}{lllllll}637.8 & 112 \mathrm{~A} & 95.4 & 55.4 & 1.26 \mathrm{E}-02 & 18\end{array}$ $\begin{array}{lllllll}637.8 & 112 \mathrm{~A} & 98.9 & 52.3 & 1.02 \mathrm{E}-02 & 21\end{array}$ $\begin{array}{lllllll}637.8 & 112 \mathrm{~A} & 104.3 & 48.6 & 7.75 \mathrm{E}-03 & 25\end{array}$ $\begin{array}{lllllll}637.8 & 112 \mathrm{~A} & 109 & 49.4 & 6.22 \mathrm{E}-03 & 30\end{array}$ $\begin{array}{llllll}637.8 & 112 \mathrm{~A} & 105.2 & 47.5 & 3.85 \mathrm{E}-03 & 40\end{array}$ $\begin{array}{lllllll}637.8 & 112 \mathrm{~A} & 94 & 51.4 & 3.00 \mathrm{E}-03 & 50\end{array}$ $\begin{array}{llllll}637.8 & 112 \mathrm{~A} & 112 & 60.3 & 2.74 \mathrm{E}-03 & 60\end{array}$ $\begin{array}{lllllll}637.8 & 112 \mathrm{~A} & 161.8 & 39.5 & 1.91 \mathrm{E}-03 & 70\end{array}$ $\begin{array}{lllllll}637.8 & 112 \mathrm{~A} & 100.5 & 41.3 & 1.51 \mathrm{E}-03 & 85\end{array}$ $\begin{array}{lllllll}637.8 & 112 \mathrm{~A} & 187.1 & 47.1 & 1.64 \mathrm{E}-03 & 100\end{array}$ $\begin{array}{lllllll}637.8 & 112 \mathrm{~A} & 162.2 & 37.1 & 1.31 \mathrm{E}-03 & 115\end{array}$ $\begin{array}{llllll}639.2 & 113 \mathrm{~A} & 68.1 & 42.9 & 2.27 \mathrm{E}-02 & 3\end{array}$ $\begin{array}{lllllll}639.2 & 113 \mathrm{~A} & 70.3 & 39.5 & 1.86 \mathrm{E}-02 & 6\end{array}$ $\begin{array}{llllll}639.2 & 113 \mathrm{~A} & 72.2 & 38.3 & 1.49 \mathrm{E}-02 & 9\end{array}$ $\begin{array}{llllll}639.2 & 113 \mathrm{~A} & 74.6 & 36.2 & 1.30 \mathrm{E}-02 & 12\end{array}$ $\begin{array}{lllllll}639.2 & 113 \mathrm{~A} & 77.1 & 33.7 & 1.16 \mathrm{E}-02 & 15\end{array}$ $\begin{array}{llllll}639.2 & 113 \mathrm{~A} & 77.4 & 34 & 1.03 \mathrm{E}-02 & 18\end{array}$ $\begin{array}{lllllll}639.2 & 113 \mathrm{~A} & 78.4 & 34.3 & 8.88 \mathrm{E}-03 & 21\end{array}$ $\begin{array}{lllllll}639.2 & 113 \mathrm{~A} & 83.1 & 35.1 & 7.33 \mathrm{E}-03 & 25\end{array}$ $\begin{array}{lllllll}639.2 & 113 \mathrm{~A} & 83.1 & 36.5 & 5.96 \mathrm{E}-03 & 30\end{array}$ $\begin{array}{llllll}639.2 & 113 \mathrm{~A} & 96.4 & 35.9 & 4.03 \mathrm{E}-03 & 40\end{array}$ $\begin{array}{lllllll}639.2 & 113 \mathrm{~A} & 85.8 & 34.6 & 2.37 \mathrm{E}-03 & 50\end{array}$ $\begin{array}{lllllll}639.2 & 113 \mathrm{~A} & 104.5 & 12.7 & 1.79 \mathrm{E}-03 & 60\end{array}$ $\begin{array}{llllll}639.2 & 113 \mathrm{~A} & 120.6 & 27.3 & 1.42 \mathrm{E}-03 & 70\end{array}$ $\begin{array}{lllllll}639.2 & 113 \mathrm{~A} & 102.6 & 20.5 & 1.15 \mathrm{E}-03 & 85\end{array}$ $\begin{array}{lllllll}639.2 & 113 \mathrm{~A} & 132.6 & 45.4 & 2.07 \mathrm{E}-03 & 100\end{array}$ $\begin{array}{lllllll}639.2 & 113 \mathrm{~A} & 91.7 & -18.5 & 6.22 \mathrm{E}-04 & 115\end{array}$ $\begin{array}{llllll}642 & 114 \mathrm{~A} & 261.8 & 38 & 2.26 \mathrm{E}-02 & 0\end{array}$ $\begin{array}{llllll}642 & 114 \mathrm{~A} & 261.2 & 37.1 & 2.16 \mathrm{E}-02 & 3\end{array}$ $\begin{array}{lllllll}642 & 114 \mathrm{~A} & 261.1 & 36.7 & 2.03 \mathrm{E}-02 & 6\end{array}$ $\begin{array}{llllll}642 & 114 \mathrm{~A} & 261 & 36.2 & 1.86 \mathrm{E}-02 & 9\end{array}$ $\begin{array}{lllllll}642 & 114 \mathrm{~A} & 261.3 & 33.7 & 1.74 \mathrm{E}-02 & 12\end{array}$ $\begin{array}{lllllll}642 & 114 \mathrm{~A} & 261.3 & 32 & 1.62 \mathrm{E}-02 & 15\end{array}$ $\begin{array}{llllll}642 & 114 \mathrm{~A} & 262.9 & 32.5 & 1.47 \mathrm{E}-02 & 18\end{array}$ $\begin{array}{llllll}642 & 114 \mathrm{~A} & 261.4 & 29.1 & 1.38 \mathrm{E}-02 & 21\end{array}$ $\begin{array}{llllll}642 & 114 \mathrm{~A} & 261.6 & 30.9 & 1.22 \mathrm{E}-02 & 25\end{array}$ $\begin{array}{llllll}642 & 114 \mathrm{~A} & 261.5 & 30.2 & 1.14 \mathrm{E}-02 & 30\end{array}$ $\begin{array}{lllllll}642 & 114 \mathrm{~A} & 261.3 & 30.3 & 8.78 \mathrm{E}-03 & 40\end{array}$ $\begin{array}{llllll}642 & 114 \mathrm{~A} & 257.5 & 26.1 & 7.41 \mathrm{E}-03 & 50\end{array}$ $\begin{array}{llllll}642 & 114 \mathrm{~A} & 268.2 & 21 & 6.37 \mathrm{E}-03 & 60\end{array}$ $\begin{array}{llllll}642 & 114 \mathrm{~A} & 265 & 28.2 & 5.46 \mathrm{E}-03 & 70\end{array}$ $\begin{array}{llllll}642 & 114 \mathrm{~A} & 254.5 & 23.6 & 3.49 \mathrm{E}-03 & 85\end{array}$ $\begin{array}{lllllll}642 & 114 \mathrm{~A} & 259.6 & 14.6 & 3.67 \mathrm{E}-03 & 100\end{array}$ $\begin{array}{llllll}642 & 114 \mathrm{~A} & 244.1 & 34.1 & 3.26 \mathrm{E}-03 & 115\end{array}$ $\begin{array}{llllll}642.4 & 115 \mathrm{~A} & 150.9 & 76.2 & 5.46 \mathrm{E}-03 & 0\end{array}$ $\begin{array}{llllll}642.4 & 115 \mathrm{~A} & 171.8 & 65.8 & 2.32 \mathrm{E}-03 & 3\end{array}$ $\begin{array}{lllllll}642.4 & 115 \mathrm{~A} & 226.6 & 24.1 & 5.16 \mathrm{E}-04 & 6\end{array}$ $\begin{array}{llllll}642.4 & 115 \mathrm{~A} & 302.7 & -59.5 & 1.35 \mathrm{E}-03 & 9\end{array}$ $\begin{array}{lllllll}642.4 & 115 \mathrm{~A} & 318.4 & -59.1 & 2.39 \mathrm{E}-03 & 12\end{array}$ $\begin{array}{lllllll}642.4 & 115 \mathrm{~A} & 326.1 & -53.7 & 2.85 \mathrm{E}-03 & 15\end{array}$ $\begin{array}{llllll}642.4 & 115 \mathrm{~A} & 329.3 & -55.5 & 3.73 \mathrm{E}-03 & 18\end{array}$ $\begin{array}{lllllll}642.4 & 115 \mathrm{~A} & 334.8 & -53.3 & 4.04 \mathrm{E}-03 & 21\end{array}$ $\begin{array}{lllllll}642.4 & 115 \mathrm{~A} & 337.3 & -53.1 & 4.55 \mathrm{E}-03 & 25\end{array}$ $\begin{array}{llllll}642.4 & 115 \mathrm{~A} & 340.2 & -54.6 & 4.73 \mathrm{E}-03 & 30\end{array}$ $\begin{array}{lllllll}642.4 & 115 \mathrm{~A} & 332.8 & -62.1 & 3.96 \mathrm{E}-03 & 40\end{array}$ $\begin{array}{llllll}642.4 & 115 \mathrm{~A} & 330.1 & -58.2 & 3.08 \mathrm{E}-03 & 50\end{array}$ $\begin{array}{llllll}642.4 & 115 \mathrm{~A} & 333.6 & 43.4 & 2.23 \mathrm{E}-03 & 60\end{array}$ 
$\begin{array}{llllll}642.4 & 115 \mathrm{~A} & 121.8 & -81.5 & 1.12 \mathrm{E}-03 & 70\end{array}$ $\begin{array}{lllllll}642.4 & 115 \mathrm{~A} & 309.4 & 40.1 & 3.41 \mathrm{E}-04 & 85\end{array}$ $\begin{array}{lllllll}642.4 & 115 \mathrm{~A} & 111.6 & -37.6 & 8.57 \mathrm{E}-04 & 100\end{array}$ $\begin{array}{lllllll}642.4 & 115 \mathrm{~A} & 138.6 & 10.9 & 8.34 \mathrm{E}-04 & 115\end{array}$ $\begin{array}{lllllll}649.7 & 117 \mathrm{~A} & 322.8 & 60.6 & 3.31 \mathrm{E}-02 & 0\end{array}$ $\begin{array}{llllll}649.7 & 117 \mathrm{~A} & 323.4 & 59.7 & 2.72 \mathrm{E}-02 & 3\end{array}$ $\begin{array}{llllll}649.7 & 117 \mathrm{~A} & 328.6 & 58.1 & 2.04 \mathrm{E}-02 & 6\end{array}$ $\begin{array}{llllll}649.7 & 117 \mathrm{~A} & 333 & 56.4 & 1.59 \mathrm{E}-02 & 9\end{array}$ $\begin{array}{llllll}649.7 & 117 \mathrm{~A} & 338.9 & 54.2 & 1.33 \mathrm{E}-02 & 12\end{array}$ $\begin{array}{lllllll}649.7 & 117 \mathrm{~A} & 339 & 54.5 & 1.14 \mathrm{E}-02 & 15\end{array}$ $\begin{array}{llllll}649.7 & 117 \mathrm{~A} & 342 & 52.8 & 9.84 \mathrm{E}-03 & 18\end{array}$ $\begin{array}{lllllll}649.7 & 117 \mathrm{~A} & 341.3 & 53.3 & 8.24 \mathrm{E}-03 & 21\end{array}$ $\begin{array}{lllllll}649.7 & 117 \mathrm{~A} & 342.9 & 51.5 & 6.81 \mathrm{E}-03 & 25\end{array}$ $\begin{array}{lllllll}649.7 & 117 \mathrm{~A} & 346.4 & 50.2 & 5.58 \mathrm{E}-03 & 30\end{array}$ $\begin{array}{llllll}649.7 & 117 \mathrm{~A} & 332.6 & 46.6 & 3.01 \mathrm{E}-03 & 40\end{array}$ $\begin{array}{llllll}649.7 & 117 \mathrm{~A} & 345.4 & 36 & 2.64 \mathrm{E}-03 & 50\end{array}$ $\begin{array}{llllll}649.7 & 117 \mathrm{~A} & 358.6 & 22 & 2.02 \mathrm{E}-03 & 60\end{array}$ $\begin{array}{lllllll}649.7 & 117 \mathrm{~A} & 308.8 & 40.7 & 4.60 \mathrm{E}-04 & 70\end{array}$ $\begin{array}{lllllll}649.7 & 117 \mathrm{~A} & 22.3 & 22.3 & 1.70 \mathrm{E}-03 & 85\end{array}$ $\begin{array}{lllllll}649.7 & 117 \mathrm{~A} & 263.9 & 47.9 & 1.12 \mathrm{E}-03 & 100\end{array}$ $\begin{array}{lllllll}649.7 & 117 \mathrm{~A} & 231.2 & 56.8 & 1.64 \mathrm{E}-03 & 115\end{array}$ $\begin{array}{lllllll}656.9 & 119 \mathrm{~A} & 181.8 & 50.6 & 2.77 \mathrm{E}-02 & 0\end{array}$ $\begin{array}{lllllll}656.9 & 119 \mathrm{~A} & 179.4 & 45.6 & 2.47 \mathrm{E}-02 & 3\end{array}$ $\begin{array}{lllllll}656.9 & 119 \mathrm{~A} & 178.8 & 41.9 & 2.19 \mathrm{E}-02 & 6\end{array}$ $\begin{array}{lllllll}656.9 & 119 \mathrm{~A} & 178 & 39.8 & 1.95 \mathrm{E}-02 & 9\end{array}$ $\begin{array}{lllllll}656.9 & 119 \mathrm{~A} & 177.1 & 39.2 & 1.74 \mathrm{E}-02 & 12\end{array}$ $\begin{array}{lllllll}656.9 & 119 \mathrm{~A} & 176.7 & 38.9 & 1.56 \mathrm{E}-02 & 15\end{array}$ $\begin{array}{lllllll}656.9 & 119 \mathrm{~A} & 178.2 & 38.7 & 1.40 \mathrm{E}-02 & 18\end{array}$ $\begin{array}{lllllll}656.9 & 119 \mathrm{~A} & 179.7 & 39.1 & 1.24 \mathrm{E}-02 & 21\end{array}$ $\begin{array}{llllll}656.9 & 119 \mathrm{~A} & 177.6 & 39.2 & 1.09 \mathrm{E}-02 & 25\end{array}$ $\begin{array}{lllllll}656.9 & 119 \mathrm{~A} & 179.2 & 40.5 & 8.87 \mathrm{E}-03 & 30\end{array}$ $\begin{array}{lllllll}656.9 & 119 \mathrm{~A} & 180.3 & 37.3 & 6.22 \mathrm{E}-03 & 40\end{array}$ $\begin{array}{lllllll}656.9 & 119 \mathrm{~A} & 179.9 & 39.3 & 4.39 \mathrm{E}-03 & 50\end{array}$ $\begin{array}{lllllll}656.9 & 119 \mathrm{~A} & 174 & 36.4 & 3.58 \mathrm{E}-03 & 60\end{array}$ $\begin{array}{lllllll}656.9 & 119 \mathrm{~A} & 186 & 36.5 & 3.57 \mathrm{E}-03 & 70\end{array}$ $\begin{array}{lllllll}656.9 & 119 \mathrm{~A} & 172.7 & 34.2 & 3.29 \mathrm{E}-03 & 85\end{array}$ $\begin{array}{lllllll}656.9 & 119 \mathrm{~A} & 186.7 & 42.8 & 2.94 \mathrm{E}-03 & 100\end{array}$ $\begin{array}{lllllll}656.9 & 119 \mathrm{~A} & 178.5 & 46.6 & 2.61 \mathrm{E}-03 & 115\end{array}$ $\begin{array}{lllllll}659.4 & 120 \mathrm{~A} & 243.2 & 37 & 3.35 \mathrm{E}-02 & 0\end{array}$ $\begin{array}{lllllll}659.4 & 120 \mathrm{~A} & 242.8 & 37.1 & 3.27 \mathrm{E}-02 & 3\end{array}$ $\begin{array}{lllllll}659.4 & 120 \mathrm{~A} & 243 & 36.7 & 3.12 \mathrm{E}-02 & 6\end{array}$ $\begin{array}{lllllll}659.4 & 120 \mathrm{~A} & 242.6 & 36.5 & 2.92 \mathrm{E}-02 & 9\end{array}$ $\begin{array}{lllllll}659.4 & 120 \mathrm{~A} & 242.8 & 36.7 & 2.75 \mathrm{E}-02 & 12\end{array}$ $\begin{array}{lllllll}659.4 & 120 \mathrm{~A} & 242.7 & 36.7 & 2.56 \mathrm{E}-02 & 15\end{array}$ $\begin{array}{lllllll}659.4 & 120 \mathrm{~A} & 242.9 & 36.3 & 2.40 \mathrm{E}-02 & 18\end{array}$ $\begin{array}{lllllll}659.4 & 120 \mathrm{~A} & 242.8 & 35.9 & 2.17 \mathrm{E}-02 & 21\end{array}$ $\begin{array}{llllll}659.4 & 120 \mathrm{~A} & 243.2 & 36 & 1.97 \mathrm{E}-02 & 25\end{array}$ $\begin{array}{lllllll}659.4 & 120 \mathrm{~A} & 243.3 & 36.2 & 1.74 \mathrm{E}-02 & 30\end{array}$ $\begin{array}{lllllll}659.4 & 120 \mathrm{~A} & 243.4 & 35.8 & 1.37 \mathrm{E}-02 & 40\end{array}$ $\begin{array}{llllll}659.4 & 120 \mathrm{~A} & 241.8 & 35.6 & 1.10 \mathrm{E}-02 & 50\end{array}$ $\begin{array}{lllllll}659.4 & 120 \mathrm{~A} & 244 & 38.5 & 8.95 \mathrm{E}-03 & 60\end{array}$ $\begin{array}{lllllll}659.4 & 120 \mathrm{~A} & 241 & 36.6 & 8.26 \mathrm{E}-03 & 70\end{array}$ $\begin{array}{lllllll}659.4 & 120 \mathrm{~A} & 243.4 & 38.7 & 6.93 \mathrm{E}-03 & 85\end{array}$ $\begin{array}{lllllll}659.4 & 120 \mathrm{~A} & 241.6 & 37.9 & 5.99 \mathrm{E}-03 & 100\end{array}$ $\begin{array}{lllllll}659.4 & 120 \mathrm{~A} & 240.2 & 33.2 & 5.31 \mathrm{E}-03 & 115\end{array}$ $\begin{array}{lllllll}661.7 & 121 \mathrm{~A} & 4.6 & 78.1 & 1.43 \mathrm{E}-01 & 0\end{array}$ $\begin{array}{lllllll}661.7 & 121 \mathrm{~A} & 15.7 & 77.3 & 1.19 \mathrm{E}-01 & 3\end{array}$ $\begin{array}{lllllll}661.7 & 121 \mathrm{~A} & 15.3 & 75.8 & 8.44 \mathrm{E}-02 & 6\end{array}$ $\begin{array}{lllllll}661.7 & 121 \mathrm{~A} & 15.3 & 73.3 & 5.84 \mathrm{E}-02 & 9\end{array}$ $\begin{array}{lllllll}661.7 & 121 \mathrm{~A} & 14.9 & 70.4 & 4.60 \mathrm{E}-02 & 12\end{array}$ $\begin{array}{lllllll}661.7 & 121 \mathrm{~A} & 18.3 & 69.1 & 3.60 \mathrm{E}-02 & 15\end{array}$ $\begin{array}{lllllll}661.7 & 121 \mathrm{~A} & 20.5 & 65.4 & 2.78 \mathrm{E}-02 & 18\end{array}$ $\begin{array}{lllllll}661.7 & 121 \mathrm{~A} & 20.9 & 64.1 & 2.26 \mathrm{E}-02 & 21\end{array}$ $\begin{array}{lllllll}661.7 & 121 \mathrm{~A} & 16.7 & 61.5 & 1.72 \mathrm{E}-02 & 25\end{array}$ $\begin{array}{lllllll}661.7 & 121 \mathrm{~A} & 21.5 & 54.9 & 1.36 \mathrm{E}-02 & 30\end{array}$ $\begin{array}{lllllll}661.7 & 121 \mathrm{~A} & 10.2 & 48.6 & 8.53 \mathrm{E}-03 & 40\end{array}$ $\begin{array}{llllll}661.7 & 121 \mathrm{~A} & 29.4 & 49 & 4.74 \mathrm{E}-03 & 50\end{array}$ $\begin{array}{lllllll}661.7 & 121 \mathrm{~A} & 27.5 & 38.3 & 4.03 \mathrm{E}-03 & 60\end{array}$ $\begin{array}{lllllll}661.7 & 121 \mathrm{~A} & 48.7 & 37.6 & 4.76 \mathrm{E}-03 & 70\end{array}$ $\begin{array}{lllllll}661.7 & 121 \mathrm{~A} & 51.2 & 26.1 & 2.38 \mathrm{E}-03 & 85\end{array}$ $\begin{array}{llllll}661.7 & 121 \mathrm{~A} & 49.5 & 26 & 3.15 \mathrm{E}-03 & 100\end{array}$ $\begin{array}{lllllll}661.7 & 121 \mathrm{~A} & 154.8 & 2.3 & 1.18 \mathrm{E}-03 & 115\end{array}$ $\begin{array}{lllllll}661.9 & 121 \mathrm{~B} & 18.6 & 80.6 & 1.04 \mathrm{E}-01 & 0\end{array}$ $\begin{array}{lllllll}661.9 & 121 \mathrm{~B} & 29.1 & 79.3 & 8.43 \mathrm{E}-02 & 3\end{array}$ $\begin{array}{lllllll}661.9 & 121 \mathrm{~B} & 30.6 & 77.7 & 5.96 \mathrm{E}-02 & 6\end{array}$ $\begin{array}{llllll}661.9 & 121 \mathrm{~B} & 31.3 & 75.9 & 4.02 \mathrm{E}-02 & 9\end{array}$ $\begin{array}{llllll}661.9 & 121 \mathrm{~B} & 27.5 & 74.6 & 3.17 \mathrm{E}-02 & 12\end{array}$ $\begin{array}{llllll}661.9 & 121 \mathrm{~B} & 27.8 & 74.2 & 2.54 \mathrm{E}-02 & 15\end{array}$ $\begin{array}{lllllll}661.9 & 121 \mathrm{~B} & 24.1 & 74.9 & 2.06 \mathrm{E}-02 & 18\end{array}$ $\begin{array}{lllllll}661.9 & 121 \mathrm{~B} & 24.3 & 70.8 & 1.65 \mathrm{E}-02 & 21\end{array}$ $\begin{array}{lllllll}661.9 & 121 \mathrm{~B} & 19.7 & 67.9 & 1.39 \mathrm{E}-02 & 25\end{array}$ $\begin{array}{lllllll}661.9 & 121 \mathrm{~B} & 25.1 & 68.5 & 1.06 \mathrm{E}-02 & 30\end{array}$ $\begin{array}{lllllll}661.9 & 121 \mathrm{~B} & 9.9 & 59.5 & 7.80 \mathrm{E}-03 & 40\end{array}$ $\begin{array}{lllllll}661.9 & 121 \mathrm{~B} & 309.7 & 69.4 & 5.62 \mathrm{E}-03 & 50\end{array}$ $\begin{array}{lllllll}661.9 & 121 \mathrm{~B} & 323.7 & 71.1 & 2.92 \mathrm{E}-03 & 60\end{array}$ $\begin{array}{lllllll}661.9 & 121 \mathrm{~B} & 331.1 & 81.1 & 2.88 \mathrm{E}-03 & 70\end{array}$ $\begin{array}{lllllll}661.9 & 121 \mathrm{~B} & 322 & 47.1 & 3.26 \mathrm{E}-03 & 85\end{array}$ $\begin{array}{lllllll}661.9 & 121 \mathrm{~B} & 209.6 & 52.3 & 1.77 \mathrm{E}-03 & 100\end{array}$ $\begin{array}{lllllll}661.9 & 121 \mathrm{~B} & 246.9 & 40.6 & 1.93 \mathrm{E}-03 & 115\end{array}$ $\begin{array}{lllllll}663.3 & 122 \mathrm{~A} & 105.6 & 39.3 & 2.98 \mathrm{E}-02 & 0\end{array}$ $\begin{array}{lllllll}663.3 & 122 \mathrm{~A} & 105.2 & 36.4 & 2.91 \mathrm{E}-02 & 3\end{array}$ $\begin{array}{lllllll}663.3 & 122 \mathrm{~A} & 105.8 & 35.5 & 2.76 \mathrm{E}-02 & 6\end{array}$ $\begin{array}{lllllll}663.3 & 122 \mathrm{~A} & 106.1 & 34.3 & 2.60 \mathrm{E}-02 & 9\end{array}$ $\begin{array}{lllllll}663.3 & 122 \mathrm{~A} & 106 & 33.4 & 2.42 \mathrm{E}-02 & 12\end{array}$ $\begin{array}{lllllll}663.3 & 122 \mathrm{~A} & 106.3 & 33.5 & 2.27 \mathrm{E}-02 & 15\end{array}$ $\begin{array}{lllllll}663.3 & 122 \mathrm{~A} & 106.2 & 33.2 & 2.06 \mathrm{E}-02 & 18\end{array}$ $\begin{array}{lllllll}663.3 & 122 \mathrm{~A} & 105.3 & 32.6 & 1.91 \mathrm{E}-02 & 21\end{array}$ $\begin{array}{lllllll}663.3 & 122 \mathrm{~A} & 105.7 & 33.2 & 1.72 \mathrm{E}-02 & 25\end{array}$ $\begin{array}{lllllll}663.3 & 122 \mathrm{~A} & 105.7 & 32.7 & 1.44 \mathrm{E}-02 & 30\end{array}$ $\begin{array}{lllllll}663.3 & 122 \mathrm{~A} & 105 & 33.4 & 1.11 \mathrm{E}-02 & 40\end{array}$ $\begin{array}{lllllll}663.3 & 122 \mathrm{~A} & 106.7 & 31.4 & 8.99 \mathrm{E}-03 & 50\end{array}$ $\begin{array}{lllllll}663.3 & 122 \mathrm{~A} & 107 & 30.8 & 7.50 \mathrm{E}-03 & 60\end{array}$ $\begin{array}{llllll}663.3 & 122 \mathrm{~A} & 102.8 & 30.4 & 6.46 \mathrm{E}-03 & 70\end{array}$ $\begin{array}{lllllll}663.3 & 122 \mathrm{~A} & 108.3 & 30 & 5.47 \mathrm{E}-03 & 85\end{array}$ $\begin{array}{lllllll}663.3 & 122 \mathrm{~A} & 98.3 & 31.4 & 4.53 \mathrm{E}-03 & 100\end{array}$ $\begin{array}{lllllll}663.3 & 122 \mathrm{~A} & 99.5 & 37.2 & 4.07 \mathrm{E}-03 & 115\end{array}$ $\begin{array}{lllllll}668.1 & 123 \mathrm{~A} & 288.8 & 65.6 & 3.56 \mathrm{E}-02 & 0\end{array}$ $\begin{array}{lllllll}668.1 & 123 \mathrm{~A} & 283.4 & 65.3 & 3.06 \mathrm{E}-02 & 3\end{array}$ $\begin{array}{lllllll}668.1 & 123 \mathrm{~A} & 279.1 & 64.2 & 2.49 \mathrm{E}-02 & 6\end{array}$ $\begin{array}{lllllll}668.1 & 123 \mathrm{~A} & 276.7 & 63.4 & 2.01 \mathrm{E}-02 & 9\end{array}$ $\begin{array}{llllll}668.1 & 123 \mathrm{~A} & 272.5 & 61 & 1.78 \mathrm{E}-02 & 12\end{array}$ $\begin{array}{lllllll}668.1 & 123 \mathrm{~A} & 273 & 59.2 & 1.55 \mathrm{E}-02 & 15\end{array}$ $\begin{array}{lllllll}668.1 & 123 \mathrm{~A} & 273 & 59.8 & 1.37 \mathrm{E}-02 & 18\end{array}$ $\begin{array}{lllllll}668.1 & 123 \mathrm{~A} & 268.5 & 58.4 & 1.15 \mathrm{E}-02 & 21\end{array}$ $\begin{array}{lllllll}668.1 & 123 \mathrm{~A} & 272.8 & 59.4 & 9.63 \mathrm{E}-03 & 25\end{array}$ $\begin{array}{lllllll}668.1 & 123 \mathrm{~A} & 269.2 & 59.4 & 8.39 \mathrm{E}-03 & 30\end{array}$ $\begin{array}{lllllll}668.1 & 123 \mathrm{~A} & 268.4 & 59 & 5.40 \mathrm{E}-03 & 40\end{array}$ $\begin{array}{lllllll}668.1 & 123 \mathrm{~A} & 275.8 & 47.7 & 4.09 \mathrm{E}-03 & 50\end{array}$ $\begin{array}{lllllll}668.1 & 123 \mathrm{~A} & 291.8 & 52.3 & 2.39 \mathrm{E}-03 & 60\end{array}$ $\begin{array}{lllllll}668.1 & 123 \mathrm{~A} & 221.1 & 34.2 & 1.49 \mathrm{E}-03 & 70\end{array}$ $\begin{array}{lllllll}668.1 & 123 \mathrm{~A} & 327.7 & 44.9 & 1.49 \mathrm{E}-03 & 85\end{array}$ $\begin{array}{lllllll}668.1 & 123 \mathrm{~A} & 287.4 & 41.7 & 1.53 \mathrm{E}-03 & 100\end{array}$ $\begin{array}{lllllll}668.1 & 123 \mathrm{~A} & 23.2 & 22.5 & 1.03 \mathrm{E}-03 & 115\end{array}$ $\begin{array}{lllllll}669.8 & 124 \mathrm{~A} & 267.6 & 56.8 & 2.62 \mathrm{E}-02 & 0\end{array}$ $\begin{array}{lllllll}669.8 & 124 \mathrm{~A} & 274.2 & 55.9 & 2.03 \mathrm{E}-02 & 3\end{array}$ $\begin{array}{lllllll}669.8 & 124 \mathrm{~A} & 271.4 & 51.6 & 1.50 \mathrm{E}-02 & 6\end{array}$ $\begin{array}{lllllll}669.8 & 124 \mathrm{~A} & 269.1 & 50.5 & 1.22 \mathrm{E}-02 & 9\end{array}$ $\begin{array}{lllllll}669.8 & 124 \mathrm{~A} & 265.9 & 50.2 & 1.02 \mathrm{E}-02 & 12\end{array}$ $\begin{array}{lllllll}669.8 & 124 \mathrm{~A} & 265.7 & 48 & 8.75 \mathrm{E}-03 & 15\end{array}$ $\begin{array}{lllllll}669.8 & 124 \mathrm{~A} & 261.9 & 46.7 & 7.69 \mathrm{E}-03 & 18\end{array}$ $\begin{array}{lllllll}669.8 & 124 \mathrm{~A} & 267.4 & 45.2 & 6.44 \mathrm{E}-03 & 21\end{array}$ $\begin{array}{lllllll}669.8 & 124 \mathrm{~A} & 265.5 & 37.1 & 5.30 \mathrm{E}-03 & 25\end{array}$ $\begin{array}{lllllll}669.8 & 124 \mathrm{~A} & 260 & 38.4 & 5.26 \mathrm{E}-03 & 30\end{array}$ $\begin{array}{lllllll}669.8 & 124 \mathrm{~A} & 258.5 & 13.1 & 4.26 \mathrm{E}-03 & 40\end{array}$ $\begin{array}{lllllll}669.8 & 124 \mathrm{~A} & 260.8 & 22.2 & 3.27 \mathrm{E}-03 & 50\end{array}$ $\begin{array}{lllllll}669.8 & 124 \mathrm{~A} & 259.5 & 15.3 & 2.82 \mathrm{E}-03 & 60\end{array}$ $\begin{array}{lllllll}669.8 & 124 \mathrm{~A} & 265.6 & 0.6 & 2.17 \mathrm{E}-03 & 70\end{array}$ $\begin{array}{lllllll}669.8 & 124 \mathrm{~A} & 237.7 & -29.7 & 1.87 \mathrm{E}-03 & 85\end{array}$ $\begin{array}{lllllll}669.8 & 124 \mathrm{~A} & 235.5 & -8.6 & 1.71 \mathrm{E}-03 & 100\end{array}$ $\begin{array}{llllll}669.8 & 124 \mathrm{~A} & 275.2 & -15.8 & 1.30 \mathrm{E}-03 & 115\end{array}$ $\begin{array}{lllllll}671.7 & 125 \mathrm{~A} & 50.5 & 67.4 & 1.34 \mathrm{E}-02 & 0\end{array}$ $\begin{array}{llllll}671.7 & 125 \mathrm{~A} & 53.1 & 64 & 1.14 \mathrm{E}-02 & 3\end{array}$ $\begin{array}{lllllll}671.7 & 125 \mathrm{~A} & 52.3 & 60.4 & 8.67 \mathrm{E}-03 & 6\end{array}$ $\begin{array}{lllllll}671.7 & 125 \mathrm{~A} & 50.7 & 58.2 & 6.65 \mathrm{E}-03 & 9\end{array}$ $\begin{array}{lllllll}671.7 & 125 \mathrm{~A} & 50.2 & 55.4 & 5.62 \mathrm{E}-03 & 12\end{array}$ $\begin{array}{lllllll}671.7 & 125 \mathrm{~A} & 45.2 & 54 & 4.71 \mathrm{E}-03 & 15\end{array}$ $\begin{array}{lllllll}671.7 & 125 \mathrm{~A} & 45.9 & 51.9 & 3.81 \mathrm{E}-03 & 18\end{array}$ $\begin{array}{lllllll}671.7 & 125 \mathrm{~A} & 40.4 & 51 & 3.24 \mathrm{E}-03 & 21\end{array}$ $\begin{array}{lllllll}671.7 & 125 \mathrm{~A} & 42.1 & 51.4 & 2.61 \mathrm{E}-03 & 25\end{array}$ $\begin{array}{lllllll}671.7 & 125 \mathrm{~A} & 43.4 & 58.1 & 1.82 \mathrm{E}-03 & 30\end{array}$ $\begin{array}{lllllll}671.7 & 125 \mathrm{~A} & 26.9 & 65.1 & 9.83 \mathrm{E}-04 & 40\end{array}$ $\begin{array}{lllllll}671.7 & 125 \mathrm{~A} & 351.7 & 22.4 & 9.31 \mathrm{E}-04 & 50\end{array}$ $\begin{array}{lllllll}671.7 & 125 \mathrm{~A} & 41.5 & 46.3 & 8.77 \mathrm{E}-04 & 60\end{array}$ 
$\begin{array}{llllll}671.7 & 125 \mathrm{~A} & 344.8 & 66.7 & 7.21 \mathrm{E}-04 & 70\end{array}$

$\begin{array}{lllllll}671.7 & 125 \mathrm{~A} & 316.1 & 57.9 & 6.00 \mathrm{E}-04 & 85\end{array}$

$\begin{array}{lllllll}671.7 & 125 \mathrm{~A} & 57.5 & 50.7 & 5.43 \mathrm{E}-04 & 100\end{array}$

$\begin{array}{lllllll}671.7 & 125 \mathrm{~A} & 287 & 48.8 & 5.19 \mathrm{E}-04 & 115\end{array}$

$\begin{array}{llllll}671.7 & 130 \mathrm{~A} & 272.7 & 42.1 & 8.79 \mathrm{E}-03 & 0\end{array}$

$\begin{array}{llllll}671.7 & 130 \mathrm{~A} & 271.3 & 40.2 & 8.16 \mathrm{E}-03 & 3\end{array}$

$\begin{array}{llllll}671.7 & 130 \mathrm{~A} & 268.8 & 37.6 & 7.25 \mathrm{E}-03 & 6\end{array}$

$\begin{array}{llllll}671.7 & 130 \mathrm{~A} & 268.4 & 34 & 6.20 \mathrm{E}-03 & 9\end{array}$

$\begin{array}{llllll}671.7 & 130 \mathrm{~A} & 266.7 & 32 & 5.51 \mathrm{E}-03 & 12\end{array}$

$\begin{array}{llllll}671.7 & 130 \mathrm{~A} & 264.2 & 31.6 & 4.85 \mathrm{E}-03 & 15\end{array}$

$\begin{array}{llllll}671.7 & 130 \mathrm{~A} & 264.3 & 29.7 & 4.19 \mathrm{E}-03 & 18\end{array}$

$\begin{array}{lllllll}671.7 & 130 \mathrm{~A} & 263.7 & 28.3 & 3.83 \mathrm{E}-03 & 21\end{array}$

$\begin{array}{llllll}671.7 & 130 \mathrm{~A} & 258.6 & 31 & 3.42 \mathrm{E}-03 & 25\end{array}$

$\begin{array}{llllll}671.7 & 130 \mathrm{~A} & 261.3 & 26.7 & 3.00 \mathrm{E}-03 & 30\end{array}$

$\begin{array}{lllllll}671.7 & 130 \mathrm{~A} & 261.3 & 32.6 & 2.37 \mathrm{E}-03 & 40\end{array}$

$\begin{array}{lllllll}671.7 & 130 \mathrm{~A} & 253.7 & 34.6 & 1.89 \mathrm{E}-03 & 50\end{array}$

$\begin{array}{lllllll}671.7 & 130 \mathrm{~A} & 264.1 & 31.4 & 1.83 \mathrm{E}-03 & 60\end{array}$

$\begin{array}{llllll}671.7 & 130 \mathrm{~A} & 253.5 & 25.9 & 1.32 \mathrm{E}-03 & 70\end{array}$

$\begin{array}{llllll}671.7 & 130 \mathrm{~A} & 255.7 & 31.6 & 1.06 \mathrm{E}-03 & 85\end{array}$

$\begin{array}{lllllll}671.7 & 130 \mathrm{~A} & 249.8 & 36.1 & 1.08 \mathrm{E}-03 & 100\end{array}$

$\begin{array}{lllllll}671.7 & 130 \mathrm{~A} & 255.3 & 33.1 & 8.97 \mathrm{E}-04 & 115\end{array}$

$\begin{array}{llllll}673.8 & 126 \mathrm{~A} & 240.8 & 45.9 & 9.37 \mathrm{E}-03 & 0\end{array}$

$\begin{array}{llllll}673.8 & 126 \mathrm{~A} & 239.1 & 45.6 & 8.65 \mathrm{E}-03 & 3\end{array}$

$\begin{array}{lllllll}673.8 & 126 \mathrm{~A} & 239.5 & 45.1 & 7.59 \mathrm{E}-03 & 6\end{array}$

$\begin{array}{llllll}673.8 & 126 \mathrm{~A} & 237.4 & 42.6 & 6.58 \mathrm{E}-03 & 9\end{array}$

$\begin{array}{llllll}673.8 & 126 \mathrm{~A} & 238.5 & 39.5 & 5.84 \mathrm{E}-03 & 12\end{array}$

$\begin{array}{llllll}673.8 & 126 \mathrm{~A} & 238.2 & 35.7 & 5.36 \mathrm{E}-03 & 15\end{array}$

$\begin{array}{lllllll}673.8 & 126 \mathrm{~A} & 237.2 & 35.8 & 4.99 \mathrm{E}-03 & 18\end{array}$

$\begin{array}{llllll}673.8 & 126 \mathrm{~A} & 237 & 33.6 & 4.30 \mathrm{E}-03 & 21\end{array}$

$\begin{array}{llllll}673.8 & 126 \mathrm{~A} & 239.2 & 29 & 3.84 \mathrm{E}-03 & 25\end{array}$

$\begin{array}{lllllll}673.8 & 126 \mathrm{~A} & 233.1 & 28.9 & 3.84 \mathrm{E}-03 & 30\end{array}$

$\begin{array}{lllllll}673.8 & 126 \mathrm{~A} & 243.9 & 22.5 & 2.96 \mathrm{E}-03 & 40\end{array}$

$\begin{array}{llllll}673.8 & 126 \mathrm{~A} & 236.6 & 4.9 & 2.48 \mathrm{E}-03 & 50\end{array}$

$\begin{array}{lllllll}673.8 & 126 \mathrm{~A} & 239.8 & -8.6 & 2.30 \mathrm{E}-03 & 60\end{array}$

$\begin{array}{llllll}673.8 & 126 \mathrm{~A} & 259.5 & 0.5 & 1.23 \mathrm{E}-03 & 70\end{array}$

$\begin{array}{llllll}673.8 & 126 \mathrm{~A} & 213.6 & -2.6 & 1.34 \mathrm{E}-03 & 85\end{array}$

$\begin{array}{lllllll}673.8 & 126 \mathrm{~A} & 278.4 & 14.4 & 9.57 \mathrm{E}-04 & 100\end{array}$

$\begin{array}{lllllll}673.8 & 126 \mathrm{~A} & 247.4 & 36.3 & 7.90 \mathrm{E}-04 & 115\end{array}$

$\begin{array}{llllll}676.5 & 131 \mathrm{~A} & 172 & -35 & 1.20 \mathrm{E}-02 & 0\end{array}$

$\begin{array}{llllll}676.5 & 131 \mathrm{~A} & 172.8 & -34.2 & 1.17 \mathrm{E}-02 & 3\end{array}$

$\begin{array}{lllllll}676.5 & 131 \mathrm{~A} & 172.8 & -33.2 & 1.08 \mathrm{E}-02 & 6\end{array}$

$\begin{array}{lllllll}676.5 & 131 \mathrm{~A} & 173.1 & -31.1 & 9.68 \mathrm{E}-03 & 9\end{array}$

$\begin{array}{llllll}676.5 & 131 \mathrm{~A} & 174 & -31.5 & 8.80 \mathrm{E}-03 & 12\end{array}$

$\begin{array}{lllllll}676.5 & 131 \mathrm{~A} & 173.5 & -30.6 & 7.72 \mathrm{E}-03 & 15\end{array}$

$\begin{array}{lllllll}676.5 & 131 \mathrm{~A} & 173.6 & -31.1 & 6.87 \mathrm{E}-03 & 18\end{array}$

$\begin{array}{lllllll}676.5 & 131 \mathrm{~A} & 173.2 & -29.8 & 5.94 \mathrm{E}-03 & 21\end{array}$

$\begin{array}{lllllll}676.5 & 131 \mathrm{~A} & 172.6 & -30.3 & 5.06 \mathrm{E}-03 & 25\end{array}$

$\begin{array}{llllll}676.5 & 13 \mathrm{IA} & \mathrm{I} 74.2 & -31.5 & 4.22 \mathrm{E}-03 & 30\end{array}$

$\begin{array}{llllll}676.5 & \text { I3IA } \quad 174 & -31.9 & 3.08 \mathrm{E}-03 & 40\end{array}$

$\begin{array}{llllll}676.5 & \text { I3IA } & 169.6 & -33 & 2.36 \mathrm{E}-03 & 50\end{array}$

$\begin{array}{llllll}676.5 & 13 \mathrm{IA} & 161.5 & -30.1 & \text { I.9IE-03 } & 60\end{array}$ $\begin{array}{llllll}676.5 & 131 \mathrm{~A} & 181.4 & -32.1 & 1.63 \mathrm{E}-03 & 70\end{array}$

$\begin{array}{lllllll}676.5 & 131 \mathrm{~A} & 159.8 & -33.9 & 1.52 \mathrm{E}-03 & 85\end{array}$

$\begin{array}{lllllll}676.5 & 131 \mathrm{~A} & 168.5 & -29.2 & 1.19 \mathrm{E}-03 & 100\end{array}$

$\begin{array}{llllll}676.5 & 131 \mathrm{~A} & 172.4 & -28.1 & 1.11 \mathrm{E}-03 & 115\end{array}$

$\begin{array}{llllll}679.3 & 132 \mathrm{~A} & 53.3 & 14.2 & 2.05 \mathrm{E}-02 & 0\end{array}$

$\begin{array}{llllll}679.3 & 132 \mathrm{~A} & 55 & 12 & 1.91 \mathrm{E}-02 & 3\end{array}$

$\begin{array}{llllll}679.3 & 132 \mathrm{~A} & 56.3 & 10.2 & 1.73 \mathrm{E}-02 & 6\end{array}$

$\begin{array}{lllllll}679.3 & 132 \mathrm{~A} & 57.5 & 8.3 & 1.59 \mathrm{E}-02 & 9\end{array}$

$\begin{array}{lllllll}679.3 & 132 \mathrm{~A} & 58.5 & 6.8 & 1.47 \mathrm{E}-02 & 12\end{array}$

$\begin{array}{lllllll}679.3 & 132 \mathrm{~A} & 58.5 & 6 & 1.40 \mathrm{E}-02 & 15\end{array}$

$\begin{array}{lllllll}679.3 & 132 \mathrm{~A} & 58.8 & 5.5 & 1.28 \mathrm{E}-02 & 18\end{array}$

$\begin{array}{lllllll}679.3 & 132 \mathrm{~A} & 58.3 & 3.9 & 1.17 \mathrm{E}-02 & 21\end{array}$

$\begin{array}{lllllll}679.3 & 132 \mathrm{~A} & 59.1 & 2.4 & 1.07 \mathrm{E}-02 & 25\end{array}$

$\begin{array}{lllllll}679.3 & 132 \mathrm{~A} & 58.9 & 2.8 & 9.27 \mathrm{E}-03 & 30\end{array}$

$\begin{array}{lllllll}679.3 & 132 \mathrm{~A} & 58.3 & 5.5 & 6.54 \mathrm{E}-03 & 40\end{array}$

$\begin{array}{llllll}679.3 & 132 \mathrm{~A} & 58.8 & 4.9 & 4.46 \mathrm{E}-03 & 50\end{array}$

$\begin{array}{lllllll}679.3 & 132 \mathrm{~A} & 62.2 & 11.4 & 3.05 \mathrm{E}-03 & 60\end{array}$

$\begin{array}{lllllll}679.3 & 132 \mathrm{~A} & 67.9 & 20.1 & 1.98 \mathrm{E}-03 & 70\end{array}$

$\begin{array}{lllllll}679.3 & 132 \mathrm{~A} & 84.8 & 1.7 & 1.66 \mathrm{E}-03 & 85\end{array}$

$\begin{array}{lllllll}679.3 & 132 \mathrm{~A} & 119.6 & 1.3 & 6.18 \mathrm{E}-04 & 100\end{array}$

$\begin{array}{lllllll}679.3 & 132 \mathrm{~A} & 114.7 & 41.8 & 7.74 \mathrm{E}-04 & 115\end{array}$

$\begin{array}{lllllll}693.2 & 133 \mathrm{~A} & 121.9 & 68.7 & 1.26 \mathrm{E}-02 & 0\end{array}$

$\begin{array}{llllll}693.2 & 133 \mathrm{~A} & 120.1 & 66 & 1.19 \mathrm{E}-02 & 3\end{array}$

$\begin{array}{llllll}693.2 & 133 \mathrm{~A} & 119.7 & 65.2 & 1.10 \mathrm{E}-02 & 6\end{array}$

$\begin{array}{lllllll}693.2 & 133 \mathrm{~A} & 119.3 & 64.6 & 9.99 \mathrm{E}-03 & 9\end{array}$

$\begin{array}{llllll}693.2 & 133 \mathrm{~A} & 120.9 & 63.6 & 9.12 \mathrm{E}-03 & 12\end{array}$

$\begin{array}{lllllll}693.2 & 133 \mathrm{~A} & 121.2 & 62.7 & 8.67 \mathrm{E}-03 & 15\end{array}$

$\begin{array}{llllll}693.2 & 133 \mathrm{~A} & 117.7 & 62 & 8.10 \mathrm{E}-03 & 18\end{array}$

$\begin{array}{lllllll}693.2 & 133 \mathrm{~A} & 123 & 62.5 & 7.35 \mathrm{E}-03 & 21\end{array}$

$\begin{array}{lllllll}693.2 & 133 \mathrm{~A} & 121.4 & 62.7 & 7.04 \mathrm{E}-03 & 25\end{array}$

$\begin{array}{llllll}693.2 & 133 \mathrm{~A} & 122 & 62.9 & 6.23 \mathrm{E}-03 & 30\end{array}$

$\begin{array}{lllllll}693.2 & 133 \mathrm{~A} & 117.6 & 62.4 & 5.34 \mathrm{E}-03 & 40\end{array}$

$\begin{array}{llllll}693.2 & 133 \mathrm{~A} & 113.6 & 59 & 4.20 \mathrm{E}-03 & 50\end{array}$

$\begin{array}{lllllll}693.2 & 133 \mathrm{~A} & 117.4 & 62.4 & 3.66 \mathrm{E}-03 & 60\end{array}$

$\begin{array}{lllllll}693.2 & 133 \mathrm{~A} & 122.4 & 59.7 & 3.11 \mathrm{E}-03 & 70\end{array}$

$\begin{array}{lllllll}693.2 & 133 \mathrm{~A} & 126.8 & 62.2 & 2.24 \mathrm{E}-03 & 85\end{array}$

$\begin{array}{lllllll}693.2 & 133 \mathrm{~A} & 139.2 & 53.5 & 1.88 \mathrm{E}-03 & 100\end{array}$

$\begin{array}{lllllll}693.2 & 133 \mathrm{~A} & 130.2 & 56.8 & 1.66 \mathrm{E}-03 & 115\end{array}$

$\begin{array}{llllll}696.4 & 134 \mathrm{~A} & 22.5 & 71.5 & 1.98 \mathrm{E}-02 & 0\end{array}$

$\begin{array}{llllll}696.4 & 134 \mathrm{~A} & 23.5 & 69.9 & 1.76 \mathrm{E}-02 & 3\end{array}$

$\begin{array}{llllll}696.4 & 134 \mathrm{~A} & 20.2 & 69.1 & 1.41 \mathrm{E}-02 & 6\end{array}$

$\begin{array}{lllllll}696.4 & 134 \mathrm{~A} & 13.7 & 67.8 & 1.16 \mathrm{E}-02 & 9\end{array}$

$\begin{array}{lllllll}696.4 & 134 \mathrm{~A} & 13.5 & 68.6 & 9.83 \mathrm{E}-03 & 12\end{array}$

$\begin{array}{llllll}696.4 & 134 \mathrm{~A} & 8.4 & 69.2 & 8.67 \mathrm{E}-03 & 15\end{array}$

$\begin{array}{lllllll}696.4 & 134 \mathrm{~A} & 3.9 & 67.4 & 7.52 \mathrm{E}-03 & 18\end{array}$

$\begin{array}{llllll}696.4 & 134 \mathrm{~A} & 5.8 & 68.1 & 6.46 \mathrm{E}-03 & 21\end{array}$

$\begin{array}{lllllll}696.4 & 134 \mathrm{~A} & 3.2 & 64.9 & 5.44 \mathrm{E}-03 & 25\end{array}$

$\begin{array}{llllll}696.4 & 134 \mathrm{~A} & 359.3 & 60.9 & 4.17 \mathrm{E}-03 & 30\end{array}$

$\begin{array}{llllll}696.4 & 134 \mathrm{~A} & 27.3 & 51.3 & 2.74 \mathrm{E}-03 & 40\end{array}$

$\begin{array}{llllll}696.4 & 134 \mathrm{~A} & 10.1 & 51.7 & 2.24 \mathrm{E}-03 & 50\end{array}$

$\begin{array}{llllll}696.4 \quad 134 \mathrm{~A} & 10 & 43.6 & 1.87 \mathrm{E}-03 & 60\end{array}$ $\begin{array}{llllll}696.4 & 134 \mathrm{~A} & 339.7 & 55.7 & 1.51 \mathrm{E}-03 & 70\end{array}$

$\begin{array}{lllllll}696.4 & 134 \mathrm{~A} & 52.8 & 68.8 & 1.08 \mathrm{E}-03 & 85\end{array}$

$\begin{array}{lllllll}696.4 & 134 \mathrm{~A} & 352.7 & 76.9 & 6.42 \mathrm{E}-04 & 100\end{array}$

$\begin{array}{lllllll}696.4 & 134 \mathrm{~A} & 342.5 & 68.8 & 7.44 \mathrm{E}-04 & 115\end{array}$

$\begin{array}{llllll}697.9 & 135 \mathrm{~A} & 81.9 & -36.1 & 1.24 \mathrm{E}-02 & 0\end{array}$

$\begin{array}{lllllll}697.9 & 135 \mathrm{~A} & 290.7 & 58.9 & 4.14 \mathrm{E}-02 & 0\end{array}$

$\begin{array}{llllll}697.9 & 135 \mathrm{~A} & 82.1 & -35.3 & 1.15 \mathrm{E}-02 & 3\end{array}$

$\begin{array}{lllllll}697.9 & 135 \mathrm{~A} & 289.3 & 58.6 & 3.63 \mathrm{E}-02 & 3\end{array}$

$\begin{array}{llllll}697.9 & 135 \mathrm{~A} & 83 & -33.3 & 1.02 \mathrm{E}-02 & 6\end{array}$

$\begin{array}{lllllll}697.9 & 135 \mathrm{~A} & 287.8 & 57.8 & 3.12 \mathrm{E}-02 & 6\end{array}$

$\begin{array}{llllll}697.9 & 135 \mathrm{~A} & 83.1 & -31.6 & 8.84 \mathrm{E}-03 & 9\end{array}$

$\begin{array}{llllll}697.9 & 135 \mathrm{~A} & 285.9 & 58.3 & 2.58 \mathrm{E}-02 & 9\end{array}$

$\begin{array}{llllll}697.9 & 135 \mathrm{~A} & 83 & -31.8 & 7.86 \mathrm{E}-03 & 12\end{array}$

$\begin{array}{lllllll}697.9 & 135 \mathrm{~A} & 286.5 & 57.8 & 2.28 \mathrm{E}-02 & 12\end{array}$

$\begin{array}{lllllll}697.9 & 135 \mathrm{~A} & 84.9 & -30.4 & 6.96 \mathrm{E}-03 & 15\end{array}$

$\begin{array}{llllll}697.9 & 135 \mathrm{~A} & 284.9 & 57.3 & 2.04 \mathrm{E}-02 & 15\end{array}$

$\begin{array}{llllll}697.9 & 135 \mathrm{~A} & 87.5 & -29.6 & 6.07 \mathrm{E}-03 & 18\end{array}$

$\begin{array}{lllllll}697.9 & 135 \mathrm{~A} & 286.1 & 60.9 & 1.80 \mathrm{E}-02 & 18\end{array}$

$\begin{array}{llllll}697.9 & 135 \mathrm{~A} & 86.5 & -31.2 & 5.05 \mathrm{E}-03 & 21\end{array}$

$\begin{array}{lllllll}697.9 & 135 \mathrm{~A} & 286.6 & 58.4 & 1.58 \mathrm{E}-02 & 21\end{array}$

$\begin{array}{llllll}697.9 & 135 \mathrm{~A} & 87.7 & -32.1 & 4.12 \mathrm{E}-03 & 25\end{array}$

$\begin{array}{lllllll}697.9 & 135 \mathrm{~A} & 284.1 & 58.8 & 1.39 \mathrm{E}-02 & 25\end{array}$

$\begin{array}{lllllll}697.9 & 135 \mathrm{~A} & 82.8 & -29.9 & 3.43 \mathrm{E}-03 & 30\end{array}$

$\begin{array}{llllll}697.9 & 135 \mathrm{~A} & 279.8 & 60.9 & 1.22 \mathrm{E}-02 & 30\end{array}$

$\begin{array}{llllll}697.9 & 135 \mathrm{~A} & 90.5 & -34 & 2.60 \mathrm{E}-03 & 40\end{array}$

$\begin{array}{llllll}697.9 & 135 \mathrm{~A} & 281.1 & 60.7 & 8.62 \mathrm{E}-03 & 40\end{array}$

$\begin{array}{lllllll}697.9 & 135 \mathrm{~A} & 104.7 & -35.4 & 1.70 \mathrm{E}-03 & 50\end{array}$

$\begin{array}{llllll}697.9 & 135 \mathrm{~A} & 275.6 & 67.3 & 5.76 \mathrm{E}-03 & 50\end{array}$

$\begin{array}{llllll}697.9 & 135 \mathrm{~A} & 103 & -35.8 & 1.84 \mathrm{E}-03 & 60\end{array}$

$\begin{array}{llllll}697.9 & 135 \mathrm{~A} & 202.8 & 75.9 & 5.02 \mathrm{E}-03 & 60\end{array}$

$\begin{array}{llllll}697.9 & 135 \mathrm{~A} & 91.9 & -21.9 & 1.59 \mathrm{E}-03 & 70\end{array}$

$\begin{array}{llllll}697.9 & 135 \mathrm{~A} & 75.1 & 88.5 & 4.37 \mathrm{E}-03 & 70\end{array}$

$\begin{array}{lllllll}697.9 & 135 \mathrm{~A} & 107.9 & -10.7 & 1.38 \mathrm{E}-03 & 85\end{array}$

$\begin{array}{llllll}697.9 & 135 \mathrm{~A} & 251.5 & 50 & 3.54 \mathrm{E}-03 & 85\end{array}$

$\begin{array}{lllllll}697.9 & 135 \mathrm{~A} & 94.2 & -28.6 & 1.46 \mathrm{E}-03 & 100\end{array}$

$\begin{array}{lllllll}697.9 & 135 \mathrm{~A} & 208.8 & 62.2 & 3.92 \mathrm{E}-03 & 100\end{array}$

$\begin{array}{llllllll}697.9 & 135 \mathrm{~A} & 94.2 & -55.7 & 9.71 \mathrm{E}-04 & 115\end{array}$

$\begin{array}{llllll}697.9 & 135 \mathrm{~A} & 216.1 & 58.1 & 4.14 \mathrm{E}-03 & 115\end{array}$

$\begin{array}{lllllll}702.9 & 136 \mathrm{~A} & 197.3 & 51.7 & 6.60 \mathrm{E}-03 & 0\end{array}$

$\begin{array}{lllllll}702.9 & 136 \mathrm{~A} & 196.3 & 46.5 & 5.69 \mathrm{E}-03 & 3\end{array}$

$\begin{array}{lllllll}702.9 & 136 \mathrm{~A} & 196.3 & 44.6 & 4.80 \mathrm{E}-03 & 6\end{array}$

$\begin{array}{llllll}702.9 & 136 \mathrm{~A} & 196.4 & 42 & 4.11 \mathrm{E}-03 & 9\end{array}$

$\begin{array}{llllll}702.9 & 136 \mathrm{~A} & 196.4 & 39.6 & 3.51 \mathrm{E}-03 & 12\end{array}$

$\begin{array}{lllllll}702.9 & 136 \mathrm{~A} & 195.7 & 37.4 & 2.96 \mathrm{E}-03 & 15\end{array}$

$\begin{array}{llllll}702.9 & 136 \mathrm{~A} & 192 & 41.2 & 2.61 \mathrm{E}-03 & 18\end{array}$

$\begin{array}{llllll}702.9 & 136 \mathrm{~A} & 189.2 & 38.7 & 2.26 \mathrm{E}-03 & 21\end{array}$

$\begin{array}{lllllll}702.9 & 136 \mathrm{~A} & 191.5 & 40.6 & 1.86 \mathrm{E}-03 & 25\end{array}$

$\begin{array}{lllllll}702.9 & 136 \mathrm{~A} & 193.8 & 42.3 & 1.49 \mathrm{E}-03 & 30\end{array}$

$\begin{array}{lllllll}702.9 & 136 \mathrm{~A} & 189.5 & 30.4 & 9.70 \mathrm{E}-04 & 40\end{array}$

$\begin{array}{llllll}702.9 & 136 \mathrm{~A} & 187.2 & 32.9 & 8.95 \mathrm{E}-04 & 50\end{array}$

$\begin{array}{llllll}702.9 & 136 \mathrm{~A} & 187 & 42.9 & 6.49 \mathrm{E}-04 & 60\end{array}$ 
$\begin{array}{llllll}702.9 & 136 \mathrm{~A} & 170.7 & 62.9 & 6.66 \mathrm{E}-04 & 70\end{array}$

$\begin{array}{llllll}702.9 & 136 \mathrm{~A} & 174.9 & 50 & 5.07 \mathrm{E}-04 & 85\end{array}$

$\begin{array}{lllllll}702.9 & 136 \mathrm{~A} & 129.6 & 33.6 & 4.32 \mathrm{E}-04 & 100\end{array}$

$\begin{array}{lllllll}702.9 & 136 \mathrm{~A} & 211.5 & 30.2 & 3.80 \mathrm{E}-04 & 115\end{array}$

$\begin{array}{llllll}704.5 & 136 \mathrm{~A} & 117.1 & 74.1 & 2.10 \mathrm{E}-02 & 0\end{array}$

$\begin{array}{llllll}704.5 & 136 \mathrm{~A} & 119 & 68 & 1.65 \mathrm{E}-02 & 3\end{array}$

$\begin{array}{llllll}704.5 & 136 \mathrm{~A} & 123.2 & 64.1 & 1.32 \mathrm{E}-02 & 6\end{array}$

$\begin{array}{llllll}704.5 & 136 \mathrm{~A} & 123.5 & 58.9 & 1.07 \mathrm{E}-02 & 9\end{array}$

$\begin{array}{llllll}704.5 & 136 \mathrm{~A} & 127.7 & 57.8 & 9.41 \mathrm{E}-03 & 12\end{array}$

$\begin{array}{llllll}704.5 & 136 \mathrm{~A} & 129.3 & 57.6 & 8.36 \mathrm{E}-03 & 15\end{array}$

$\begin{array}{llllll}704.5 & 136 \mathrm{~A} & 131.7 & 55.6 & 7.72 \mathrm{E}-03 & 18\end{array}$

$\begin{array}{llllll}704.5 & 136 \mathrm{~A} & 137.6 & 55 & 6.74 \mathrm{E}-03 & 21\end{array}$

$\begin{array}{llllll}704.5 & 136 \mathrm{~A} & 138.7 & 52.9 & 6.01 \mathrm{E}-03 & 25\end{array}$

$\begin{array}{llllll}704.5 & 136 \mathrm{~A} & 132.2 & 56.5 & 4.92 \mathrm{E}-03 & 30\end{array}$

$\begin{array}{llllll}704.5 & 136 \mathrm{~A} & 142.1 & 56.4 & 3.70 \mathrm{E}-03 & 40\end{array}$

$\begin{array}{llllll}704.5 & 136 \mathrm{~A} & 128 & 55.2 & 2.64 \mathrm{E}-03 & 50\end{array}$

$\begin{array}{llllll}704.5 & 136 \mathrm{~A} & 147.8 & 73.2 & 2.13 \mathrm{E}-03 & 60\end{array}$

$\begin{array}{llllll}704.5 & 136 \mathrm{~A} & 190.3 & 46.8 & 1.99 \mathrm{E}-03 & 70\end{array}$

$\begin{array}{llllll}704.5 & 136 \mathrm{~A} & 133.4 & 80.7 & 1.75 \mathrm{E}-03 & 85\end{array}$

$\begin{array}{llllll}704.5 & 136 \mathrm{~A} & 142 & 12.2 & 1.23 \mathrm{E}-03 & 100\end{array}$

$\begin{array}{lllllll}704.5 & 136 \mathrm{~A} & 167.7 & 1.4 & 1.33 \mathrm{E}-03 & 115\end{array}$

$\begin{array}{lllllll}704.6 & 136 \mathrm{~B} & 354.6 & 78.5 & 2.59 \mathrm{E}-02 & 0\end{array}$

$\begin{array}{llllll}704.6 & 136 \mathrm{~B} & 322.3 & 79.3 & 1.72 \mathrm{E}-02 & 3\end{array}$

$\begin{array}{llllll}704.6 & 136 \mathrm{~B} & 304 & 77.8 & 1.25 \mathrm{E}-02 & 6\end{array}$

$\begin{array}{llllll}704.6 & 136 \mathrm{~B} & 293.8 & 75.9 & 9.91 \mathrm{E}-03 & 9\end{array}$

$\begin{array}{llllll}704.6 & 136 \mathrm{~B} & 286.1 & 74.3 & 8.75 \mathrm{E}-03 & 12\end{array}$

$\begin{array}{llllll}704.6 & 136 \mathrm{~B} & 276.1 & 72.1 & 7.28 \mathrm{E}-03 & 15\end{array}$

$\begin{array}{llllll}704.6 & 136 \mathrm{~B} & 276.9 & 71.8 & 6.80 \mathrm{E}-03 & 18\end{array}$

$\begin{array}{llllll}704.6 & 136 \mathrm{~B} & 263.4 & 68.8 & 6.02 \mathrm{E}-03 & 21\end{array}$

$\begin{array}{llllll}704.6 & 136 \mathrm{~B} & 261.9 & 68.3 & 5.23 \mathrm{E}-03 & 25\end{array}$

$\begin{array}{llllll}704.6 & 136 \mathrm{~B} & 288.2 & 69.2 & 4.66 \mathrm{E}-03 & 30\end{array}$

$\begin{array}{llllll}704.6 & 136 \mathrm{~B} & 263.4 & 68.9 & 3.37 \mathrm{E}-03 & 40\end{array}$

$\begin{array}{llllll}704.6 & 136 \mathrm{~B} & 294.8 & 53.8 & 2.50 \mathrm{E}-03 & 50\end{array}$

$\begin{array}{llllll}704.6 & 136 \mathrm{~B} & 299.5 & 55.2 & 2.48 \mathrm{E}-03 & 60\end{array}$

$\begin{array}{llllll}704.6 & 136 \mathrm{~B} & 275.7 & 57.2 & 1.98 \mathrm{E}-03 & 70\end{array}$

$\begin{array}{llllll}704.6 & 136 \mathrm{~B} & 306.6 & 48.6 & 3.02 \mathrm{E}-03 & 85\end{array}$

$\begin{array}{lllllll}704.6 & 136 \mathrm{~B} & 288 & 80.2 & 1.53 \mathrm{E}-03 & 100\end{array}$

$\begin{array}{llllll}704.6 & 136 \mathrm{~B} & 285.4 & 51 & 1.15 \mathrm{E}-03 & 115\end{array}$

$\begin{array}{llllll}706.5 & 136 \mathrm{C} & 42.5 & 62.3 & 6.29 \mathrm{E}-02 & 0\end{array}$

$\begin{array}{llllll}706.5 & 136 \mathrm{C} & 45.2 & 62.3 & 4.22 \mathrm{E}-02 & 3\end{array}$

$\begin{array}{llllll}706.5 & 136 \mathrm{C} & 41.6 & 62 & 2.63 \mathrm{E}-02 & 6\end{array}$

$\begin{array}{llllll}706.5 & 136 \mathrm{C} & 35.6 & 60.5 & 1.73 \mathrm{E}-02 & 9\end{array}$

$\begin{array}{llllll}706.5 & 136 \mathrm{C} & 28.3 & 65.3 & 1.41 \mathrm{E}-02 & 12\end{array}$

$\begin{array}{llllll}706.5 & 136 \mathrm{C} & 31 & 64.4 & 1.04 \mathrm{E}-02 & 15\end{array}$

$\begin{array}{llllll}706.5 & 136 \mathrm{C} & 18.2 & 59.1 & 8.68 \mathrm{E}-03 & 18\end{array}$

$\begin{array}{llllll}706.5 & 136 \mathrm{C} & 25 & 59.6 & 7.37 \mathrm{E}-03 & 21\end{array}$

$\begin{array}{llllll}706.5 & 136 \mathrm{C} & 11.2 & 56 & 6.06 \mathrm{E}-03 & 25\end{array}$

$\begin{array}{lllllll}706.5 & 136 \mathrm{C} & 16.3 & 58 & 4.51 \mathrm{E}-03 & 30\end{array}$

$\begin{array}{llllll}706.5 & 136 \mathrm{C} & 6 & 68.8 & 4.17 \mathrm{E}-03 & 40\end{array}$

$\begin{array}{llllll}706.5 & 136 \mathrm{C} & 344.9 & 60 & 3.68 \mathrm{E}-03 & 50\end{array}$

$\begin{array}{llllll}706.5 & 136 \mathrm{C} & 22.4 & 73.6 & 2.04 \mathrm{E}-03 & 60\end{array}$ $\begin{array}{llllll}706.5 & 136 \mathrm{C} & 352.8 & 59.7 & 2.67 \mathrm{E}-03 & 70\end{array}$

$\begin{array}{llllll}706.5 & 136 \mathrm{C} & 12.1 & 72.2 & 3.34 \mathrm{E}-03 & 85\end{array}$

$\begin{array}{lllllll}706.5 & 136 \mathrm{C} & 199.4 & 37.3 & 1.79 \mathrm{E}-03 & 100\end{array}$

$\begin{array}{llllll}706.5 & 136 \mathrm{C} & 285.2 & -3.5 & 7.19 \mathrm{E}-04 & 115\end{array}$

$\begin{array}{llllll}708.5 & 137 \mathrm{~A} & 200.8 & 20.9 & 3.45 \mathrm{E}-03 & 0\end{array}$

$\begin{array}{llllll}708.5 & 137 \mathrm{~A} \quad 196 & -11.1 & 4.16 \mathrm{E}-03 \quad 3\end{array}$

$\begin{array}{llllll}708.5 & 137 \mathrm{~A} & 194.4 & -16.4 & 4.47 \mathrm{E}-03 \quad 6\end{array}$

$\begin{array}{llllll}708.5 & 137 \mathrm{~A} \quad 194.8 & -19.3 \quad 4.48 \mathrm{E}-03 \quad 9\end{array}$

$\begin{array}{llllll}708.5 & 137 \mathrm{~A} & 192.7 & -19.3 & 4.24 \mathrm{E}-03 & 12\end{array}$

$\begin{array}{llllll}708.5 & 137 \mathrm{~A} & 194.4 & -23.1 & 3.94 \mathrm{E}-03 & 15\end{array}$

$\begin{array}{llllll}708.5 & 137 \mathrm{~A} & 193.6 & -20.9 & 3.16 \mathrm{E}-03 & 21\end{array}$

$\begin{array}{llllll}708.5 & 137 \mathrm{~A} & 190.6 & -21.8 & 2.71 \mathrm{E}-03 & 25\end{array}$

$\begin{array}{llllll}708.5 & 137 \mathrm{~A} & 191.2 & -17.1 & 2.24 \mathrm{E}-03 & 30\end{array}$

$\begin{array}{llllll}708.5 & 137 \mathrm{~A} & 197.9 & -19.7 & 1.56 \mathrm{E}-03 & 40\end{array}$

$\begin{array}{llllll}708.5 & 137 \mathrm{~A} & 193.4 & -17.3 & 1.32 \mathrm{E}-03 & 50\end{array}$

$\begin{array}{llllll}708.5 & 137 \mathrm{~A} \quad 192 & -21.6 & 8.51 \mathrm{E}-04 & 60\end{array}$

$\begin{array}{llllll}708.5 & 137 \mathrm{~A} & 162.3 & 43.6 & 5.41 \mathrm{E}-04 & 70\end{array}$

$\begin{array}{llllll}708.5 & 137 \mathrm{~A} & 199.6 & -22.6 & 1.17 \mathrm{E}-03 & 85\end{array}$

$\begin{array}{lllllll}708.5 & 137 \mathrm{~A} & 168.2 & -33.4 & 7.23 \mathrm{E}-04 & 100\end{array}$

$\begin{array}{lllllll}708.5 & 137 \mathrm{~A} & 192.7 & -17.8 & 6.78 \mathrm{E}-04 & 115\end{array}$

$\begin{array}{llllll}709.9 & 138 \mathrm{~A} & 163 & 46.8 & 1.71 \mathrm{E}-03 & 0\end{array}$

$\begin{array}{llllll}709.9 & 138 \mathrm{~A} & 156 & 32.5 & 1.34 \mathrm{E}-03 & 3\end{array}$

$\begin{array}{lllllll}709.9 & 138 \mathrm{~A} & 153.2 & 17.4 & 1.20 \mathrm{E}-03 & 6\end{array}$

$\begin{array}{llllll}709.9 & 138 \mathrm{~A} & 148.9 & 9.2 & 1.18 \mathrm{E}-03 & 9\end{array}$

$\begin{array}{llllll}709.9 & 138 \mathrm{~A} & 145.5 & 4.8 & 1.15 \mathrm{E}-03 & 12\end{array}$

$\begin{array}{llllll}709.9 & 138 \mathrm{~A} & 146.5 & 0 & 1.12 \mathrm{E}-03 & 15\end{array}$

$\begin{array}{llllll}709.9 & 138 \mathrm{~A} & 142 & -5.4 & 1.06 \mathrm{E}-03 & 18\end{array}$

$\begin{array}{llllll}709.9 & 138 \mathrm{~A} & 140.4 & -3.7 & 9.92 \mathrm{E}-04 & 21\end{array}$

$\begin{array}{llllll}709.9 & 138 \mathrm{~A} & 145.7 & -0.8 & 9.13 \mathrm{E}-04 & 25\end{array}$

$\begin{array}{llllll}709.9 & 138 \mathrm{~A} & 143.9 & -7.1 & 9.09 \mathrm{E}-04 & 30\end{array}$

$\begin{array}{llllll}709.9 & 138 \mathrm{~A} & 139.1 & -5 & 8.43 \mathrm{E}-04 & 40\end{array}$

$\begin{array}{llllll}709.9 & 138 \mathrm{~A} & 144.5 & -3.3 & 5.23 \mathrm{E}-04 & 50\end{array}$

$\begin{array}{lllllll}709.9 & 138 \mathrm{~A} & 126.1 & 23.7 & 3.46 \mathrm{E}-04 & 60\end{array}$

$\begin{array}{llllll}709.9 & 138 \mathrm{~A} & 132.9 & 2.4 & 6.13 \mathrm{E}-04 & 70\end{array}$

$\begin{array}{lllllll}709.9 & 138 \mathrm{~A} & 129 & 24.8 & 3.04 \mathrm{E}-04 & 85\end{array}$

$713 \quad 140 \mathrm{~A} \quad 190.5 \quad-15.9 \quad 5.61 \mathrm{E}-03 \quad 0$

$713 \quad 140 \mathrm{~A} \quad 191.6 \quad-16.3 \quad 5.71 \mathrm{E}-03 \quad 3$

$\begin{array}{llllll}713 & 140 \mathrm{~A} & 192.8 & -18.2 & 5.90 \mathrm{E}-03 & 6\end{array}$

$713 \quad 140 \mathrm{~A} \quad 192.6 \quad-18.9 \quad 5.93 \mathrm{E}-03 \quad 9$

$\begin{array}{llllll}713 & 140 \mathrm{~A} & 193.3 & -19.9 & 5.80 \mathrm{E}-03 & 12\end{array}$

$\begin{array}{llllll}713 & 140 \mathrm{~A} & 194 & -21.2 & 5.56 \mathrm{E}-03 & 15\end{array}$

$\begin{array}{llllll}713 & 140 \mathrm{~A} & 194.4 & -21.1 & 5.27 \mathrm{E}-03 & 18\end{array}$

$\begin{array}{llllll}713 & 140 \mathrm{~A} & 194 & -22.3 & 4.79 \mathrm{E}-03 & 21\end{array}$

$\begin{array}{llllll}713 & 140 \mathrm{~A} & 192.3 & -23.7 & 4.18 \mathrm{E}-03 & 25\end{array}$

$\begin{array}{llllll}713 & 140 \mathrm{~A} & 192.6 & -26.1 & 3.53 \mathrm{E}-03 & 30\end{array}$

$\begin{array}{llllll}713 & 140 \mathrm{~A} & 192.7 & -25.2 & 2.48 \mathrm{E}-03 & 40\end{array}$

$\begin{array}{lllllll}713 & 140 \mathrm{~A} & 186.9 & -28.9 & 1.66 \mathrm{E}-03 & 50\end{array}$

$\begin{array}{lllllll}713 & 140 \mathrm{~A} & 167.9 & -25.9 & 1.30 \mathrm{E}-03 & 60\end{array}$

$\begin{array}{llllll}713 & 140 \mathrm{~A} & 192.3 & -29 & 1.04 \mathrm{E}-03 & 70\end{array}$

$\begin{array}{lllllll}713 & 140 \mathrm{~A} & 136.2 & -22.8 & 9.56 \mathrm{E}-04 & 85\end{array}$

$\begin{array}{lllllll}713 & 140 \mathrm{~A} & 192 & -26.5 & 7.54 \mathrm{E}-04 & 100\end{array}$ $\begin{array}{llllll}713 & 140 \mathrm{~A} & 193.2 & -20 & 6.47 \mathrm{E}-04 & 115\end{array}$

$\begin{array}{llllll}714.7 & 141 \mathrm{~A} & 145 & -8.5 & 5.12 \mathrm{E}-03 & 0\end{array}$

$\begin{array}{llllll}714.7 & 141 \mathrm{~A} & 146.2 & -11 & 5.16 \mathrm{E}-03 & 3\end{array}$

$\begin{array}{llllll}714.7 & 141 \mathrm{~A} \quad 146.7 & -14.2 & 4.99 \mathrm{E}-03 & 6\end{array}$

$\begin{array}{llllll}714.7 \quad 141 \mathrm{~A} \quad 145.6 & -15.1 & 4.74 \mathrm{E}-03 \quad 9\end{array}$

$\begin{array}{llllll}714.7 & 141 \mathrm{~A} \quad 146.7 & -14.5 & 4.27 \mathrm{E}-03 & 12\end{array}$

$\begin{array}{lllllll}714.7 & 141 \mathrm{~A} & 146.5 & -14.6 & 3.69 \mathrm{E}-03 & 15\end{array}$

$\begin{array}{llllll}714.7 & 141 \mathrm{~A} & 145.2 & -16.1 & 3.24 \mathrm{E}-03 & 18\end{array}$

$\begin{array}{lllllll}714.7 & 141 \mathrm{~A} & 147.5 & -16.8 & 2.71 \mathrm{E}-03 & 21\end{array}$

$\begin{array}{llllll}714.7 & 141 \mathrm{~A} & 145.9 & -16.6 & 2.21 \mathrm{E}-03 & 25\end{array}$

$\begin{array}{llllll}714.7 & 141 \mathrm{~A} & 145.7 & -15.1 & 1.89 \mathrm{E}-03 & 30\end{array}$

$\begin{array}{llllll}714.7 \quad 141 \mathrm{~A} \quad 144.4 & -16.8 \quad 1.21 \mathrm{E}-03 \quad 40\end{array}$

$\begin{array}{llllll}714.7 & 141 \mathrm{~A} & 144.5 & -14.1 & 1.07 \mathrm{E}-03 & 50\end{array}$

$\begin{array}{llllll}714.7 & 141 \mathrm{~A} & 134.5 & -7.8 & 1.16 \mathrm{E}-03 & 60\end{array}$

$\begin{array}{llllll}714.7 & 141 \mathrm{~A} & 141.2 & -4.2 & 5.11 \mathrm{E}-04 & 70\end{array}$

$\begin{array}{llllll}714.7 & 141 \mathrm{~A} & 118.6 & -6.1 & 1.15 \mathrm{E}-03 & 85\end{array}$

$\begin{array}{llllll}714.7 & 141 \mathrm{~A} & 124.3 & 2.3 & 4.35 \mathrm{E}-04 & 100\end{array}$

$\begin{array}{lllllll}714.7 & 141 \mathrm{~A} & 137.3 & 11.3 & 3.72 \mathrm{E}-04 & 115\end{array}$

$\begin{array}{llllll}714.8 & 141 \mathrm{~A} & 143.5 & -27.2 & 3.97 \mathrm{E}-03 & 0\end{array}$

$\begin{array}{llllll}714.8 & 141 \mathrm{~A} & 145.1 & -30.7 & 4.16 \mathrm{E}-03 & 3\end{array}$

$\begin{array}{lllllll}714.8 & 141 \mathrm{~A} & 144.4 & -33.1 & 4.20 \mathrm{E}-03 & 6\end{array}$

$\begin{array}{lllllll}714.8 & 141 \mathrm{~A} & 144.8 & -32.3 & 3.77 \mathrm{E}-03 & 9\end{array}$

$\begin{array}{lllllll}714.8 & 141 \mathrm{~A} & 144.9 & -32.8 & 3.35 \mathrm{E}-03 & 12\end{array}$

$\begin{array}{lllllll}714.8 & 141 \mathrm{~A} & 145.2 & -34 & 3.02 \mathrm{E}-03 & 15\end{array}$

$\begin{array}{lllllll}714.8 & 141 \mathrm{~A} & 145.6 & -33.2 & 2.60 \mathrm{E}-03 & 18\end{array}$

$\begin{array}{llllll}714.8 & 141 \mathrm{~A} & 147.6 & -33.8 & 2.28 \mathrm{E}-03 & 21\end{array}$

$\begin{array}{llllll}714.8 & 141 \mathrm{~A} & 144.3 & -33.4 & 1.92 \mathrm{E}-03 & 25\end{array}$

$\begin{array}{llllll}714.8 & 141 \mathrm{~A} & 144.5 & -33.5 & 1.44 \mathrm{E}-03 & 30\end{array}$

$\begin{array}{llllll}714.8 & 141 \mathrm{~A} & 146.4 & -30.6 & 1.10 \mathrm{E}-03 & 40\end{array}$

$\begin{array}{llllll}714.8 & 141 \mathrm{~A} & 156.2 & -34.9 & 7.12 \mathrm{E}-04 & 50\end{array}$

$\begin{array}{llllll}714.8 & 141 \mathrm{~A} & 149.9 & -26.6 & 5.78 \mathrm{E}-04 & 60\end{array}$

$\begin{array}{llllll}714.8 & 141 \mathrm{~A} & 155 & -29.2 & 6.20 \mathrm{E}-04 & 70\end{array}$

$\begin{array}{llllll}714.8 & 141 \mathrm{~A} & 151.3 & -21.3 & 4.12 \mathrm{E}-04 & 85\end{array}$

$\begin{array}{lllllll}714.8 & 141 \mathrm{~A} & 152.3 & -22.3 & 5.18 \mathrm{E}-04 & 100\end{array}$

$\begin{array}{lllllll}714.8 & 141 \mathrm{~A} & 175.4 & -28.7 & 3.67 \mathrm{E}-04 & 115\end{array}$

$\begin{array}{llllll}718.7 & 142 \mathrm{~B} & 232.9 & -6.6 & 4.44 \mathrm{E}-03 & 0\end{array}$

$\begin{array}{lllllll}718.7 & 142 \mathrm{~B} & 229.6 & -16.4 & 5.05 \mathrm{E}-03 & 3\end{array}$

$\begin{array}{llllll}718.7 & 142 \mathrm{~B} & 227.7 & -22.9 & 5.31 \mathrm{E}-03 & 6\end{array}$

$\begin{array}{llllll}718.7 & 142 \mathrm{~B} & 228.2 & -25 & 5.12 \mathrm{E}-03 & 9\end{array}$

$\begin{array}{lllllll}718.7 & 142 \mathrm{~B} & 227.9 & -25.7 & 4.62 \mathrm{E}-03 & 12\end{array}$

$\begin{array}{llllll}718.7 & 142 \mathrm{~B} & 226.4 & -26.8 & 3.93 \mathrm{E}-03 & 15\end{array}$

$\begin{array}{llllll}718.7 & 142 \mathrm{~B} & 229 & -25.3 & 3.41 \mathrm{E}-03 & 18\end{array}$

$\begin{array}{llllll}718.7 & 142 \mathrm{~B} & 229.3 & -25.2 & 2.83 \mathrm{E}-03 & 21\end{array}$

$\begin{array}{llllll}718.7 & 142 \mathrm{~B} & 231.5 & -24.5 & 2.31 \mathrm{E}-03 & 25\end{array}$

$\begin{array}{llllll}718.7 & 142 \mathrm{~B} & 237.7 & -25 & 1.74 \mathrm{E}-03 & 30\end{array}$

$\begin{array}{llllll}718.7 & 142 \mathrm{~B} & 234.4 & -22.9 & 1.04 \mathrm{E}-03 & 40\end{array}$

$\begin{array}{llllll}718.7 & 142 \mathrm{~B} & 241 & -19.1 & 7.80 \mathrm{E}-04 & 50\end{array}$

$\begin{array}{llllll}718.7 & 142 \mathrm{~B} & 237.2 & -25.5 & 3.58 \mathrm{E}-04 & 60\end{array}$

$\begin{array}{llllll}718.7 & 142 \mathrm{~B} & 237 & -13.6 & 4.45 \mathrm{E}-04 & 70\end{array}$

$\begin{array}{llllll}718.7 & 142 \mathrm{~B} & 230.7 & -31.1 & 2.65 \mathrm{E}-04 & 85\end{array}$

$\begin{array}{llllll}718.7 & 142 \mathrm{~B} & 247.9 & -13.9 & 5.47 \mathrm{E}-04 & 100\end{array}$ 
$\begin{array}{llllll}718.7 & 142 \mathrm{~B} & 248.2 & -20.1 & 3.45 \mathrm{E}-04 & 115\end{array}$ $\begin{array}{llllll}718.8 & 142 \mathrm{~A} & 203.8 & -5.8 & 4.05 \mathrm{E}-03 & 0\end{array}$ $\begin{array}{lllllll}718.8 & 142 \mathrm{~A} & 204.6 & -17.5 & 4.51 \mathrm{E}-03 & 3\end{array}$ $\begin{array}{lllllll}718.8 & 142 \mathrm{~A} & 205.8 & -24.2 & 4.78 \mathrm{E}-03 & 6\end{array}$ $\begin{array}{lllllll}718.8 & 142 \mathrm{~A} & 205.3 & -26.6 & 4.47 \mathrm{E}-03 & 9\end{array}$ $\begin{array}{lllllll}718.8 & 142 \mathrm{~A} & 205.7 & -28.6 & 4.22 \mathrm{E}-03 & 12\end{array}$ $\begin{array}{llllll}718.8 & 142 \mathrm{~A} & 205.6 & -28.5 & 3.60 \mathrm{E}-03 & 15\end{array}$ $\begin{array}{llllll}718.8 & 142 \mathrm{~A} & 205.2 & -29.2 & 3.11 \mathrm{E}-03 & 18\end{array}$ $\begin{array}{llllll}718.8 & 142 \mathrm{~A} & 204 & -28.7 & 2.48 \mathrm{E}-03 & 21\end{array}$ $\begin{array}{lllllll}718.8 & 142 \mathrm{~A} & 201.7 & -29.2 & 2.06 \mathrm{E}-03 & 25\end{array}$ $\begin{array}{lllllll}718.8 & 142 \mathrm{~A} & 208.6 & -31.8 & 1.70 \mathrm{E}-03 & 30\end{array}$ $\begin{array}{lllllll}718.8 & 142 \mathrm{~A} & 208.9 & -32.7 & 9.13 \mathrm{E}-04 & 40\end{array}$ $\begin{array}{lllllll}718.8 & 142 \mathrm{~A} & 219.9 & -20.3 & 8.25 \mathrm{E}-04 & 50\end{array}$ $\begin{array}{llllll}718.8 & 142 \mathrm{~A} & 202.2 & -13.6 & 6.45 \mathrm{E}-04 & 60\end{array}$ $\begin{array}{lllllll}718.8 & 142 \mathrm{~A} & 220.2 & -22.3 & 5.32 \mathrm{E}-04 & 70\end{array}$ $\begin{array}{llllll}718.8 & 142 \mathrm{~A} & 202.3 & -4.1 & 3.84 \mathrm{E}-04 & 85\end{array}$ $\begin{array}{llllllll}718.8 & 142 \mathrm{~A} & 220.3 & -26.1 & 2.16 \mathrm{E}-04 & 100\end{array}$ $\begin{array}{lllllll}718.8 & 142 \mathrm{~A} & 259.4 & 53 & 5.57 \mathrm{E}-04 & 115\end{array}$ $\begin{array}{llllll}718.9 & 142 \mathrm{~A} & 290.2 & -0.4 & 5.39 \mathrm{E}-03 & 0\end{array}$ $\begin{array}{llllll}718.9 & 142 \mathrm{~A} & 288.9 & -7.7 & 5.59 \mathrm{E}-03 & 3\end{array}$ $\begin{array}{lllllll}718.9 & 142 \mathrm{~A} & 288.8 & -12.9 & 5.59 \mathrm{E}-03 & 6\end{array}$ $\begin{array}{llllll}718.9 & 142 \mathrm{~A} & 288.5 & -13.7 & 5.30 \mathrm{E}-03 & 9\end{array}$ $\begin{array}{llllll}718.9 & 142 \mathrm{~A} & 287.3 & -15 & 4.76 \mathrm{E}-03 & 12\end{array}$ $\begin{array}{lllllll}718.9 & 142 \mathrm{~A} & 290.7 & -17.5 & 4.00 \mathrm{E}-03 & 15\end{array}$ $\begin{array}{lllllll}718.9 & 142 \mathrm{~A} & 288.3 & -17.4 & 3.54 \mathrm{E}-03 & 18\end{array}$ $\begin{array}{llllll}718.9 & 142 \mathrm{~A} & 291.3 & -17.8 & 3.03 \mathrm{E}-03 & 21\end{array}$ $\begin{array}{llllll}718.9 & 142 \mathrm{~A} & 290.8 & -14 & 2.46 \mathrm{E}-03 & 25\end{array}$ $\begin{array}{llllll}718.9 & 142 \mathrm{~A} & 285 & -16 & 2.01 \mathrm{E}-03 & 30\end{array}$ $\begin{array}{llllll}718.9 & 142 \mathrm{~A} & 290.6 & -17.2 & 1.20 \mathrm{E}-03 & 40\end{array}$ $\begin{array}{llllll}718.9 & 142 \mathrm{~A} & 272 & -6.9 & 1.05 \mathrm{E}-03 & 50\end{array}$ $\begin{array}{lllllll}718.9 & 142 \mathrm{~A} & 261.7 & -7.9 & 8.37 \mathrm{E}-04 & 60\end{array}$ $\begin{array}{lllllll}718.9 & 142 \mathrm{~A} & 304.7 & 1.8 & 8.28 \mathrm{E}-04 & 70\end{array}$ $\begin{array}{lllllll}718.9 & 142 \mathrm{~A} & 258.1 & -13.8 & 7.22 \mathrm{E}-04 & 85\end{array}$ $\begin{array}{lllllll}718.9 & 142 \mathrm{~A} & 313.5 & -9.3 & 6.20 \mathrm{E}-04 & 100\end{array}$ $\begin{array}{lllllll}718.9 & 142 \mathrm{~A} & 312.6 & 9.3 & 5.41 \mathrm{E}-04 & 115\end{array}$ $\begin{array}{llllll}720.9 & 143 \mathrm{~A} & 61.2 & 35.1 & 2.71 \mathrm{E}-03 & 0\end{array}$ $\begin{array}{llllll}720.9 & 143 \mathrm{~A} & 60.5 & 21.3 & 2.27 \mathrm{E}-03 & 3\end{array}$ $\begin{array}{llllll}720.9 & 143 \mathrm{~A} & 58.9 & 10.2 & 1.94 \mathrm{E}-03 & 6\end{array}$ $\begin{array}{llllll}720.9 & 143 \mathrm{~A} & 59.2 & 3.1 & 1.75 \mathrm{E}-03 & 9\end{array}$ $\begin{array}{llllll}720.9 & 143 \mathrm{~A} & 58.4 & -1.4 & 1.66 \mathrm{E}-03 & 12\end{array}$ $\begin{array}{lllllll}720.9 & 143 \mathrm{~A} & 58.3 & -2.7 & 1.52 \mathrm{E}-03 & 15\end{array}$ $\begin{array}{lllllll}720.9 & 143 \mathrm{~A} & 58 & -4.7 & 1.27 \mathrm{E}-03 & 18\end{array}$ $\begin{array}{llllll}720.9 & 143 \mathrm{~A} & 55.7 & 4.2 & 1.16 \mathrm{E}-03 & 21\end{array}$ $\begin{array}{lllllll}720.9 & 143 \mathrm{~A} & 56.8 & -5.1 & 7.97 \mathrm{E}-04 & 25\end{array}$ $\begin{array}{llllll}720.9 & 143 \mathrm{~A} & 56.9 & -9.4 & 6.66 \mathrm{E}-04 & 30\end{array}$ $\begin{array}{llllll}720.9 & 143 \mathrm{~A} & 45.9 & 1.4 & 5.90 \mathrm{E}-04 & 40\end{array}$ $\begin{array}{lllllll}720.9 & 143 \mathrm{~A} & 85.4 & -0.5 & 3.89 \mathrm{E}-04 & 50\end{array}$ $\begin{array}{llllll}720.9 & 143 \mathrm{~A} & 22.8 & -2 & 3.43 \mathrm{E}-04 & 60\end{array}$ $\begin{array}{llllll}720.9 & 143 \mathrm{~A} & 44.1 & 12.9 & 5.10 \mathrm{E}-04 & 70\end{array}$ $\begin{array}{lllllll}720.9 & 143 \mathrm{~A} & 110.9 & 7.9 & 1.40 \mathrm{E}-04 & 85\end{array}$ $\begin{array}{lllllll}720.9 & 143 \mathrm{~A} & 24.6 & -3.5 & 4.09 \mathrm{E}-04 & 100\end{array}$

\begin{tabular}{|c|c|c|c|c|c|c|c|c|c|c|c|}
\hline 720.9 & $143 A$ & 104.5 & 6.2 & $2.39 \mathrm{E}-04$ & 115 & 743.9 & $146 \mathrm{~A}$ & 162.8 & 11.8 & $6.16 \mathrm{E}-04$ & 115 \\
\hline 723.4 & $144 A$ & 205.5 & 67.2 & $2.07 \mathrm{E}-03$ & 0 & 745.2 & $147 \mathrm{~A}$ & 239.4 & 60.6 & $2.08 \mathrm{E}-02$ & 0 \\
\hline 723.4 & $144 \mathrm{~A}$ & 198.1 & 67.6 & $1.89 \mathrm{E}-03$ & 3 & 745.2 & $147 \mathrm{~A}$ & 238.6 & 59 & $1.67 \mathrm{E}-02$ & 3 \\
\hline 723.4 & $144 \mathrm{~A}$ & 186.2 & 70 & $1.71 \mathrm{E}-03$ & 6 & 745.2 & $147 \mathrm{~A}$ & 240.8 & 57 & $1.25 \mathrm{E}-02$ & 6 \\
\hline 723.4 & $144 \mathrm{~A}$ & 177.5 & 70.1 & $1.45 \mathrm{E}-03$ & 9 & 745.2 & $147 \mathrm{~A}$ & 242.3 & 55.9 & $8.74 E-03$ & 9 \\
\hline 723.4 & $144 A$ & 165.2 & 71.9 & $1.31 \mathrm{E}-03$ & 12 & 745.2 & $147 \mathrm{~A}$ & 245.4 & 50.6 & $6.81 E-03$ & 12 \\
\hline 723.4 & $144 A$ & 153.1 & 74.2 & $1.16 \mathrm{E}-03$ & 15 & 745.2 & $147 \mathrm{~A}$ & 253.8 & 47.4 & $5.09 \mathrm{E}-03$ & 15 \\
\hline 723.4 & $144 A$ & 141.5 & 70.1 & $9.76 \mathrm{E}-04$ & 18 & 745.2 & $147 \mathrm{~A}$ & 251.9 & 45.3 & $4.16 \mathrm{E}-03$ & 18 \\
\hline 723.4 & $144 A$ & 127.3 & 71.9 & $8.65 \mathrm{E}-04$ & 21 & 745.2 & $147 \mathrm{~A}$ & 256.3 & 42.2 & $3.47 \mathrm{E}-03$ & 21 \\
\hline 723.4 & $144 \mathrm{~A}$ & 125.4 & 75.6 & $8.50 \mathrm{E}-04$ & 25 & 745.2 & $147 \mathrm{~A}$ & 260.7 & 42.8 & $2.45 \mathrm{E}-03$ & 25 \\
\hline 723.4 & $144 A$ & 128.1 & 68.5 & $7.17 \mathrm{E}-04$ & 30 & 745.2 & $147 \mathrm{~A}$ & 262.7 & 41.1 & $1.98 \mathrm{E}-03$ & 30 \\
\hline 723.4 & $144 \mathrm{~A}$ & 155.3 & 76.8 & $5.02 \mathrm{E}-04$ & 40 & 745.2 & $147 \mathrm{~A}$ & 247.1 & 52.9 & $7.05 \mathrm{E}-04$ & 40 \\
\hline 723.4 & $144 \mathrm{~A}$ & 102.9 & 69 & $4.92 \mathrm{E}-04$ & 50 & 745.2 & $147 \mathrm{~A}$ & 245.2 & 38.2 & $1.01 \mathrm{E}-03$ & 50 \\
\hline 723.4 & $144 \mathrm{~A}$ & 59.5 & 74 & $3.95 \mathrm{E}-04$ & 60 & 745.2 & $147 \mathrm{~A}$ & 96.2 & 34.6 & $6.08 \mathrm{E}-04$ & 60 \\
\hline 723.4 & $144 \mathrm{~A}$ & 144.6 & 50.1 & $5.38 \mathrm{E}-04$ & 70 & 745.2 & $147 \mathrm{~A}$ & 335.3 & -24.2 & $1.15 \mathrm{E}-03$ & 70 \\
\hline 723.4 & $144 \mathrm{~A}$ & 229.6 & 84.6 & $3.55 \mathrm{E}-04$ & 85 & 745.2 & $147 \mathrm{~A}$ & 109.7 & 8.8 & 6.61E-04 & 85 \\
\hline 723.4 & $144 \mathrm{~A}$ & 172 & 35.8 & $3.89 \mathrm{E}-04$ & 100 & 745.2 & $147 \mathrm{~A}$ & 203 & -7.8 & $4.00 \mathrm{E}-04$ & 100 \\
\hline 723.4 & $144 \mathrm{~A}$ & 226.2 & 70.7 & $2.81 \mathrm{E}-04$ & 115 & 745.2 & $147 \mathrm{~A}$ & 180.7 & 7.1 & 4.75E-04 & 115 \\
\hline 742.4 & $145 \mathrm{~A}$ & 13.1 & 65.1 & $3.24 \mathrm{E}-02$ & 0 & 747.7 & $147 \mathrm{~B}$ & 38.5 & 80 & $2.99 \mathrm{E}-03$ & 0 \\
\hline 742.4 & $145 \mathrm{~A}$ & 23.7 & 65.4 & $2.69 \mathrm{E}-02$ & 3 & 747.7 & $147 B$ & 32.7 & 77.5 & $2.33 \mathrm{E}-03$ & 3 \\
\hline 742.4 & $145 \mathrm{~A}$ & 25.7 & 65.6 & $2.10 \mathrm{E}-02$ & 6 & 747.7 & $147 \mathrm{~B}$ & 32.3 & 68.7 & $1.83 \mathrm{E}-03$ & 6 \\
\hline 742.4 & $145 \mathrm{~A}$ & 27.3 & 65.6 & $1.60 \mathrm{E}-02$ & 9 & 747.7 & $147 B$ & 33.6 & 58.5 & $1.36 \mathrm{E}-03$ & 9 \\
\hline 742.4 & $145 \mathrm{~A}$ & 31.2 & 66.8 & $1.29 \mathrm{E}-02$ & 12 & 747.7 & $147 \mathrm{~B}$ & 35.8 & 45.8 & $1.12 \mathrm{E}-03$ & 12 \\
\hline 742.4 & $145 \mathrm{~A}$ & 31.3 & 67.5 & $1.12 \mathrm{E}-02$ & 15 & 747.7 & $147 B$ & 36.2 & 33.7 & $1.14 \mathrm{E}-03$ & 15 \\
\hline 742.4 & $145 \mathrm{~A}$ & 34.1 & 65.9 & $9.26 \mathrm{E}-03$ & 18 & 747.7 & $147 \mathrm{~B}$ & 32.2 & 25 & $9.13 \mathrm{E}-04$ & 18 \\
\hline 742.4 & $145 \mathrm{~A}$ & 39 & 67.2 & $7.79 \mathrm{E}-03$ & 21 & 747.7 & $147 \mathrm{~B}$ & 39.8 & 22.6 & $9.00 \mathrm{E}-04$ & 21 \\
\hline 742.4 & $145 \mathrm{~A}$ & 36.6 & 66.8 & $6.85 \mathrm{E}-03$ & 25 & 747.7 & $147 B$ & 35.2 & 16.5 & $7.16 \mathrm{E}-04$ & 25 \\
\hline 742.4 & $145 \mathrm{~A}$ & 32.1 & 65.7 & $5.30 \mathrm{E}-03$ & 30 & 747.7 & $147 B$ & 41.9 & 9.5 & $5.64 \mathrm{E}-04$ & 30 \\
\hline 742.4 & $145 \mathrm{~A}$ & 51.5 & 63.2 & $3.71 E-03$ & 40 & 747.7 & $147 \mathrm{~B}$ & 28.4 & 6.8 & $4.88 \mathrm{E}-04$ & 40 \\
\hline 742.4 & $145 \mathrm{~A}$ & 65.6 & 61.7 & $2.74 \mathrm{E}-03$ & 50 & 747.7 & $147 \mathrm{~B}$ & 19.4 & 6.9 & $4.05 \mathrm{E}-04$ & 50 \\
\hline 742.4 & $145 \mathrm{~A}$ & 121.2 & 54.9 & $2.05 \mathrm{E}-03$ & 60 & 747.7 & $147 \mathrm{~B}$ & 32.2 & 13.2 & $5.49 \mathrm{E}-04$ & 60 \\
\hline 742.4 & $145 \mathrm{~A}$ & 105.1 & 18.9 & $1.01 \mathrm{E}-03$ & 70 & 747.7 & $147 \mathrm{~B}$ & 345.1 & 58.8 & $7.46 \mathrm{E}-05$ & 70 \\
\hline 742.4 & $145 \mathrm{~A}$ & 108.5 & 40.9 & $7.61 \mathrm{E}-04$ & 85 & 747.7 & $147 \mathrm{~B}$ & 12.8 & 51.2 & $3.83 \mathrm{E}-04$ & 85 \\
\hline 742.4 & $145 \mathrm{~A}$ & 112.9 & 43.1 & $1.06 \mathrm{E}-03$ & 100 & 747.7 & $147 \mathrm{~B}$ & 201.8 & 38.1 & $1.28 \mathrm{E}-04$ & 100 \\
\hline 742.4 & $145 \mathrm{~A}$ & 76.7 & 22 & $7.56 \mathrm{E}-04$ & 115 & 747.7 & $147 \mathrm{~B}$ & 131.9 & 45 & $1.71 E-04$ & 115 \\
\hline 743.9 & $146 \mathrm{~A}$ & 360 & 64.3 & $2.78 \mathrm{E}-02$ & 0 & 748.8 & $148 \mathrm{~A}$ & 115.7 & 54.5 & $4.31 \mathrm{E}-03$ & 3 \\
\hline 743.9 & $146 \mathrm{~A}$ & 2.7 & 65.1 & $2.33 \mathrm{E}-02$ & 3 & 748.8 & $148 \mathrm{~A}$ & 114.6 & 48.3 & $3.79 \mathrm{E}-03$ & 6 \\
\hline 743.9 & $146 \mathrm{~A}$ & 3.7 & 65.5 & $1.89 \mathrm{E}-02$ & 6 & 748.8 & $148 \mathrm{~A}$ & 110.3 & 50.5 & $3.19 \mathrm{E}-03$ & 9 \\
\hline 743.9 & $146 \mathrm{~A}$ & 2.3 & 67 & $1.49 \mathrm{E}-02$ & 9 & 748.8 & $148 \mathrm{~A}$ & 107.7 & 49.4 & $2.93 \mathrm{E}-03$ & 12 \\
\hline 743.9 & $146 \mathrm{~A}$ & 2.9 & 68.6 & $1.24 \mathrm{E}-02$ & 12 & 748.8 & $148 \mathrm{~A}$ & 107.7 & 47.9 & 2.31E-03 & 15 \\
\hline 743.9 & $146 \mathrm{~A}$ & 4.8 & 70.2 & $1.12 \mathrm{E}-02$ & 15 & 748.8 & $148 \mathrm{~A}$ & 108.8 & 45.6 & $2.21 \mathrm{E}-03$ & 18 \\
\hline 743.9 & $146 \mathrm{~A}$ & 13.4 & 72.6 & $9.77 \mathrm{E}-03$ & 18 & 748.8 & $148 \mathrm{~A}$ & 105.6 & 46 & $2.08 \mathrm{E}-03$ & 21 \\
\hline 743.9 & $146 \mathrm{~A}$ & 23.7 & 70 & $8.37 \mathrm{E}-03$ & 21 & 748.8 & $148 \mathrm{~A}$ & 107.5 & 51.3 & $1.90 \mathrm{E}-03$ & 25 \\
\hline 743.9 & $146 \mathrm{~A}$ & 19.5 & 73.3 & $6.93 \mathrm{E}-03$ & 25 & 748.8 & $148 \mathrm{~A}$ & 104.6 & 41.5 & $1.65 \mathrm{E}-03$ & 30 \\
\hline 743.9 & $146 \mathrm{~A}$ & 15 & 71.5 & $5.70 \mathrm{E}-03$ & 30 & 748.8 & $148 \mathrm{~A}$ & 117.4 & 40.5 & $1.23 \mathrm{E}-03$ & 40 \\
\hline 743.9 & $146 \mathrm{~A}$ & 63.5 & 80.9 & $3.65 \mathrm{E}-03$ & 40 & 748.8 & $148 \mathrm{~A}$ & 107.6 & 44.4 & $9.53 \mathrm{E}-04$ & 50 \\
\hline 743.9 & $146 \mathrm{~A}$ & 32.4 & 75.2 & $2.20 \mathrm{E}-03$ & 50 & 748.8 & $148 \mathrm{~A}$ & 105.6 & 31.3 & $8.56 \mathrm{E}-04$ & 60 \\
\hline 743.9 & $146 \mathrm{~A}$ & 319.9 & 80.8 & $2.09 \mathrm{E}-03$ & 60 & 748.8 & $148 \mathrm{~A}$ & 111.1 & 25.1 & $8.29 \mathrm{E}-04$ & 70 \\
\hline 743.9 & $146 \mathrm{~A}$ & 187.5 & 77.6 & $1.99 \mathrm{E}-03$ & 70 & 748.8 & $148 \mathrm{~A}$ & 73.4 & 64.9 & $4.35 \mathrm{E}-04$ & 85 \\
\hline 743.9 & $146 \mathrm{~A}$ & 10.6 & 64.7 & 7.38E-04 & 85 & 748.8 & $148 \mathrm{~A}$ & 156.2 & 52.3 & $5.46 \mathrm{E}-04$ & 100 \\
\hline 743.9 & $146 \mathrm{~A}$ & 178.9 & 49.5 & $1.09 \mathrm{E}-03$ & 100 & 748.8 & $148 \mathrm{~A}$ & 126.6 & 55.3 & $5.67 \mathrm{E}-04$ & 115 \\
\hline
\end{tabular}


$\begin{array}{llllll}750.6 & 149 \mathrm{~A} & 102.3 & 65.8 & 3.52 \mathrm{E}-03 & 0\end{array}$ $\begin{array}{llllll}750.6 & 149 \mathrm{~A} & 97.1 & 65 & 3.16 \mathrm{E}-03 & 3\end{array}$ $\begin{array}{llllll}750.6 & 149 \mathrm{~A} & 91.8 & 68.7 & 2.65 \mathrm{E}-03 & 6\end{array}$ $\begin{array}{llllll}750.6 & 149 \mathrm{~A} & 89.6 & 71.1 & 2.23 \mathrm{E}-03 & 9\end{array}$ $\begin{array}{llllll}750.6 & 149 \mathrm{~A} & 80.2 & 72.2 & 1.96 \mathrm{E}-03 & 12\end{array}$ $\begin{array}{llllll}750.6 & 149 \mathrm{~A} & 74.6 & 73.6 & 1.72 \mathrm{E}-03 & 15\end{array}$ $\begin{array}{lllllll}750.6 & 149 \mathrm{~A} & 72.8 & 73.1 & 1.47 \mathrm{E}-03 & 18\end{array}$ $\begin{array}{lllllll}750.6 & 149 \mathrm{~A} & 59.7 & 72.5 & 1.28 \mathrm{E}-03 & 21\end{array}$ $\begin{array}{llllll}750.6 & 149 \mathrm{~A} & 22 & 75.1 & 1.07 \mathrm{E}-03 & 25\end{array}$ $\begin{array}{lllllll}750.6 & 149 \mathrm{~A} & 31.8 & 75.6 & 9.01 \mathrm{E}-04 & 30\end{array}$ $\begin{array}{lllllll}750.6 & 149 \mathrm{~A} & 279.4 & 87.4 & 5.20 \mathrm{E}-04 & 40\end{array}$ $\begin{array}{lllllll}750.6 & 149 \mathrm{~A} & 333.7 & 76.9 & 4.58 \mathrm{E}-04 & 50\end{array}$ $\begin{array}{lllllll}750.6 & 149 \mathrm{~A} & 148.2 & 48.6 & 4.88 \mathrm{E}-04 & 60\end{array}$ $\begin{array}{lllllll}750.6 & 149 \mathrm{~A} & 5.6 & 84.6 & 3.45 \mathrm{E}-04 & 70\end{array}$ $\begin{array}{lllllll}750.6 & 149 \mathrm{~A} & 217.8 & 16.1 & 3.42 \mathrm{E}-04 & 85\end{array}$ $\begin{array}{llllll}750.6 & 149 \mathrm{~A} & 289.2 & 20.4 & 2.99 \mathrm{E}-04 & 100\end{array}$ $\begin{array}{lllllll}750.6 & 149 \mathrm{~A} \quad 312 & -19.2 & 3.95 \mathrm{E}-04 & 115\end{array}$ $\begin{array}{llllll}752.4 & 150 \mathrm{~A} & 69.6 & 77.9 & 5.11 \mathrm{E}-03 & 0\end{array}$ $\begin{array}{llllll}752.4 & 150 \mathrm{~A} & 69.4 & 78 & 5.09 \mathrm{E}-03 & 0\end{array}$ $\begin{array}{llllll}752.4 & 150 \mathrm{~A} & 68.6 & 77.4 & 4.42 \mathrm{E}-03 & 3\end{array}$ $\begin{array}{llllll}752.4 & 150 \mathrm{~A} & 79.2 & 80.3 & 4.00 \mathrm{E}-03 & 6\end{array}$ $\begin{array}{llllll}752.4 & 150 \mathrm{~A} & 108.2 & 79 & 3.35 \mathrm{E}-03 & 9\end{array}$ $\begin{array}{llllll}752.4 & 150 \mathrm{~A} & 134.1 & 84.9 & 2.86 \mathrm{E}-03 & 12\end{array}$ $\begin{array}{llllll}752.4 & 150 \mathrm{~A} & 92.9 & 80.8 & 2.80 \mathrm{E}-03 & 15\end{array}$ $\begin{array}{lllllll}752.4 & 150 \mathrm{~A} & 135.1 & 76.7 & 2.54 \mathrm{E}-03 & 18\end{array}$ $\begin{array}{llllll}752.4 & 150 \mathrm{~A} & 107.6 & 83 & 2.43 \mathrm{E}-03 & 21\end{array}$ $\begin{array}{llllll}752.4 & 150 \mathrm{~A} & 76.9 & 81.6 & 2.31 \mathrm{E}-03 & 25\end{array}$ $\begin{array}{llllll}752.4 & 150 \mathrm{~A} & 79.1 & 75.4 & 2.05 \mathrm{E}-03 & 30\end{array}$ $\begin{array}{llllll}752.4 & 150 \mathrm{~A} & 82.1 & 61.3 & 1.34 \mathrm{E}-03 & 40\end{array}$ $\begin{array}{lllllll}752.4 & 150 \mathrm{~A} & 83.9 & 47.9 & 1.56 \mathrm{E}-03 & 50\end{array}$ $\begin{array}{lllllll}752.4 & 150 \mathrm{~A} & 68.8 & 50.3 & 1.38 \mathrm{E}-03 & 60\end{array}$ $\begin{array}{lllllll}752.4 & 150 \mathrm{~A} & 78.6 & 49.9 & 1.38 \mathrm{E}-03 & 70\end{array}$ $\begin{array}{lllllll}752.4 & 150 \mathrm{~A} & 41.3 & 22.7 & 7.98 \mathrm{E}-04 & 85\end{array}$ $\begin{array}{lllllll}752.4 & 150 \mathrm{~A} & 88.3 & 37.6 & 7.10 \mathrm{E}-04 & 100\end{array}$ $\begin{array}{lllllll}752.4 & 150 \mathrm{~A} & 75.7 & 42.8 & 1.24 \mathrm{E}-03 & 115\end{array}$ $\begin{array}{lllllll}756.3 & 151 \mathrm{~A} & 186.5 & 37.9 & 8.66 \mathrm{E}-03 & 0\end{array}$ $\begin{array}{llllll}756.3 & 151 \mathrm{~A} & 187.5 & 12.4 & 7.66 \mathrm{E}-03 & 3\end{array}$ $\begin{array}{lllllll}756.3 & 151 \mathrm{~A} & 189.6 & -1.4 & 7.62 \mathrm{E}-03 & 6\end{array}$ $\begin{array}{llllll}756.3 & 151 \mathrm{~A} & 191.7 & -6.8 & 8.03 \mathrm{E}-03 & 9\end{array}$ $\begin{array}{lllllll}756.3 & 151 \mathrm{~A} & 190.6 & -7.2 & 6.99 \mathrm{E}-03 & 12\end{array}$ $\begin{array}{lllllll}756.3 & 151 \mathrm{~A} & 192.9 & -8.4 & 6.80 \mathrm{E}-03 & 15\end{array}$ $\begin{array}{lllllll}756.3 & 151 \mathrm{~A} & 191.5 & -2.3 & 5.93 \mathrm{E}-03 & 18\end{array}$ $\begin{array}{lllllll}756.3 & 151 \mathrm{~A} & 185.6 & -2.3 & 4.59 \mathrm{E}-03 & 21\end{array}$ $\begin{array}{llllll}756.3 & 151 \mathrm{~A} & 175.2 & -3.6 & 3.72 \mathrm{E}-03 & 25\end{array}$ $\begin{array}{lllllll}756.3 & 151 \mathrm{~A} & 187.7 & 5.5 & 3.36 \mathrm{E}-03 & 30\end{array}$ $\begin{array}{lllllll}756.3 & 151 \mathrm{~A} & 188.9 & 10.7 & 2.47 \mathrm{E}-03 & 40\end{array}$ $\begin{array}{lllllll}756.3 & 151 \mathrm{~A} & 207 & 34.3 & 2.03 \mathrm{E}-03 & 50\end{array}$ $\begin{array}{llllll}756.3 & 151 \mathrm{~A} & 177 & 6 & 1.27 \mathrm{E}-03 & 60\end{array}$ $\begin{array}{lllllll}756.3 & 151 \mathrm{~A} & 173.5 & 15.6 & 1.86 \mathrm{E}-03 & 70\end{array}$ $\begin{array}{lllllll}756.3 & 151 \mathrm{~A} & 149 & -18.5 & 1.21 \mathrm{E}-03 & 85\end{array}$ $\begin{array}{lllllll}756.3 & 151 \mathrm{~A} & 142.1 & 39.6 & 1.44 \mathrm{E}-03 & 100\end{array}$ $\begin{array}{llllll}756.3 & 151 \mathrm{~A} & 200.2 & 27 & 2.87 \mathrm{E}-03 & 115\end{array}$ $\begin{array}{lllllll}758.4 & 152 \mathrm{~A} & 225.6 & 85.2 & 1.97 \mathrm{E}-02 & 0\end{array}$ $\begin{array}{lllllll}758.4 & 152 \mathrm{~A} & 269.8 & 86 & 1.10 \mathrm{E}-02 & 3\end{array}$ $\begin{array}{lllllll}758.4 & 152 \mathrm{~A} & 318 & 80.3 & 6.63 \mathrm{E}-03 & 6\end{array}$ $\begin{array}{llllll}758.4 & 152 \mathrm{~A} & 327.3 & 75.5 & 4.30 \mathrm{E}-03 & 9\end{array}$ $\begin{array}{lllllll}758.4 & 152 \mathrm{~A} & 326.2 & 62.3 & 3.38 \mathrm{E}-03 & 12\end{array}$ $\begin{array}{lllllll}758.4 & 152 \mathrm{~A} & 341.4 & 49.9 & 2.92 \mathrm{E}-03 & 15\end{array}$ $\begin{array}{lllllll}758.4 & 152 \mathrm{~A} & 353.2 & 61.8 & 2.32 \mathrm{E}-03 & 18\end{array}$ $\begin{array}{lllllll}758.4 & 152 \mathrm{~A} & 337.8 & 54.9 & 1.64 \mathrm{E}-03 & 21\end{array}$ $\begin{array}{lllllll}758.4 & 152 \mathrm{~A} & 339 & 73.4 & 1.68 \mathrm{E}-03 & 25\end{array}$ $\begin{array}{lllllll}758.4 & 152 \mathrm{~A} & 290.5 & 61.5 & 1.80 \mathrm{E}-03 & 30\end{array}$ $\begin{array}{lllllll}758.4 & 152 \mathrm{~A} & 49.3 & 43.3 & 1.33 \mathrm{E}-03 & 40\end{array}$ $\begin{array}{lllllll}758.4 & 152 \mathrm{~A} & 358.2 & 43.9 & 1.74 \mathrm{E}-03 & 50\end{array}$ $\begin{array}{lllllll}758.4 & 152 \mathrm{~A} & 17.6 & 25.1 & 1.45 \mathrm{E}-03 & 60\end{array}$ $\begin{array}{lllllll}758.4 & 152 \mathrm{~A} & 11.7 & 32 & 1.73 \mathrm{E}-03 & 70\end{array}$ $\begin{array}{lllllll}758.4 & 152 \mathrm{~A} & 32.9 & 44.5 & 1.83 \mathrm{E}-03 & 85\end{array}$ $\begin{array}{llllll}758.4 & 152 \mathrm{~A} & 51.2 & 33.3 & 1.07 \mathrm{E}-03 & 100\end{array}$ $\begin{array}{lllllll}758.4 & 152 \mathrm{~A} & 281 & 31.8 & 1.43 \mathrm{E}-03 & 115\end{array}$ $\begin{array}{llllll}760.3 & 153 \mathrm{~A} & 103.2 & 60 & 1.33 \mathrm{E}-02 & 0\end{array}$ $\begin{array}{llllll}760.3 & 153 \mathrm{~A} & 104.3 & 56.9 & 1.16 \mathrm{E}-02 & 3\end{array}$ $\begin{array}{llllll}760.3 & 153 \mathrm{~A} & 105.5 & 54.7 & 9.72 \mathrm{E}-03 & 6\end{array}$ $\begin{array}{llllll}760.3 & 153 \mathrm{~A} & 108 & 51.9 & 8.23 \mathrm{E}-03 & 9\end{array}$ $\begin{array}{llllll}760.3 & 153 \mathrm{~A} & 108.9 & 50.4 & 7.20 \mathrm{E}-03 & 12\end{array}$ $\begin{array}{llllll}760.3 & 153 \mathrm{~A} & 109.4 & 48 & 6.38 \mathrm{E}-03 & 15\end{array}$ $\begin{array}{lllllll}760.3 & 153 \mathrm{~A} & 112.6 & 48.7 & 5.86 \mathrm{E}-03 & 18\end{array}$ $\begin{array}{lllllll}760.3 & 153 \mathrm{~A} & 109.8 & 45.8 & 5.47 \mathrm{E}-03 & 21\end{array}$ $\begin{array}{lllllll}760.3 & 153 \mathrm{~A} & 118.5 & 46.8 & 4.87 \mathrm{E}-03 & 25\end{array}$ $\begin{array}{lllllll}760.3 & 153 \mathrm{~A} & 111.7 & 51.8 & 4.57 \mathrm{E}-03 & 30\end{array}$ $\begin{array}{lllllll}760.3 & 153 \mathrm{~A} & 110.7 & 53.2 & 3.72 \mathrm{E}-03 & 40\end{array}$ $\begin{array}{llllll}760.3 & 153 \mathrm{~A} & 113.9 & 51 & 2.62 \mathrm{E}-03 & 50\end{array}$ $\begin{array}{lllllll}760.3 & 153 \mathrm{~A} & 86 & 61.1 & 2.58 \mathrm{E}-03 & 60\end{array}$ $\begin{array}{lllllll}760.3 & 153 \mathrm{~A} & 121.4 & 51.5 & 1.96 \mathrm{E}-03 & 70\end{array}$ $\begin{array}{lllllll}760.3 & 153 \mathrm{~A} & 97.5 & 74.5 & 1.51 \mathrm{E}-03 & 85\end{array}$ $\begin{array}{lllllll}760.3 & 153 \mathrm{~A} & 173.5 & 73.6 & 1.99 \mathrm{E}-03 & 100\end{array}$ $\begin{array}{lllllll}760.3 & 153 \mathrm{~A} & 124.7 & 76.4 & 8.72 \mathrm{E}-04 & 115\end{array}$ $\begin{array}{llllll}770.2 & 153 \mathrm{~B} & 71.4 & 76.7 & 5.54 \mathrm{E}-02 & 0\end{array}$ $\begin{array}{llllll}770.2 & 153 \mathrm{~B} & 75 & 74.1 & 5.08 \mathrm{E}-02 & 3\end{array}$ $\begin{array}{llllll}770.2 & 153 \mathrm{~B} & 74.5 & 74.8 & 4.48 \mathrm{E}-02 & 6\end{array}$ $\begin{array}{llllll}770.2 & 153 \mathrm{~B} & 73 & 75.6 & 3.84 \mathrm{E}-02 & 9\end{array}$ $\begin{array}{llllll}770.2 & 153 \mathrm{~B} & 73.4 & 75.9 & 3.46 \mathrm{E}-02 & 12\end{array}$ $\begin{array}{llllll}770.2 & 153 \mathrm{~B} & 71.7 & 76.6 & 3.07 \mathrm{E}-02 & 15\end{array}$ $\begin{array}{llllll}770.2 & 153 \mathrm{~B} & 70.6 & 77.6 & 2.67 \mathrm{E}-02 & 18\end{array}$ $\begin{array}{llllll}770.2 & 153 \mathrm{~B} & 82.3 & 77 & 2.40 \mathrm{E}-02 & 21\end{array}$ $\begin{array}{lllllll}770.2 & 153 \mathrm{~B} & 88.7 & 76.1 & 2.08 \mathrm{E}-02 & 25\end{array}$ $\begin{array}{llllll}770.2 & 153 \mathrm{~B} & 90.6 & 77.4 & 1.66 \mathrm{E}-02 & 30\end{array}$ $\begin{array}{llllll}770.2 & 153 \mathrm{~B} & 103.2 & 76.4 & 1.08 \mathrm{E}-02 & 40\end{array}$ $\begin{array}{lllllll}770.2 & 153 \mathrm{~B} & 153 & 76.6 & 6.54 \mathrm{E}-03 & 50\end{array}$ $\begin{array}{llllll}770.2 & 153 \mathrm{~B} & 122.3 & 56.1 & 4.13 \mathrm{E}-03 & 60\end{array}$ $\begin{array}{lllllll}770.2 & 153 \mathrm{~B} & 173.4 & 62.3 & 3.01 \mathrm{E}-03 & 70\end{array}$ $\begin{array}{lllllll}770.2 & 153 \mathrm{~B} & 181.2 & 58.4 & 1.59 \mathrm{E}-03 & 85\end{array}$ $\begin{array}{lllllll}770.2 & 153 \mathrm{~B} & 192.5 & 61.8 & 3.15 \mathrm{E}-03 & 100\end{array}$ $\begin{array}{llllll}770.2 & 153 \mathrm{~B} & 219.4 & 50.2 & 2.57 \mathrm{E}-03 & 115\end{array}$ $\begin{array}{llllll}773 & 153 \mathrm{C} & 71.3 & 62.8 & 7.45 \mathrm{E}-02 & 0\end{array}$ $\begin{array}{llllll}773 & 153 \mathrm{C} & 73.7 & 60.4 & 6.66 \mathrm{E}-02 & 3\end{array}$ $\begin{array}{llllll}773 & 153 \mathrm{C} & 74.9 & 60.9 & 5.61 \mathrm{E}-02 & 6\end{array}$ $\begin{array}{llllll}773 & 153 \mathrm{C} & 74.8 & 60.4 & 4.60 \mathrm{E}-02 & 9\end{array}$ $\begin{array}{llllll}773 & 153 \mathrm{C} & 73.7 & 61.9 & 3.92 \mathrm{E}-02 & 12\end{array}$ $\begin{array}{llllll}773 & 153 \mathrm{C} & 75.3 & 63.5 & 3.35 \mathrm{E}-02 & 15\end{array}$ $\begin{array}{llllll}773 & 153 \mathrm{C} & 71.9 & 65.4 & 2.95 \mathrm{E}-02 & 18\end{array}$ $\begin{array}{llllll}773 & 153 \mathrm{C} & 77.9 & 65.3 & 2.49 \mathrm{E}-02 & 21\end{array}$ $\begin{array}{llllll}773 & 153 \mathrm{C} & 76.1 & 62.8 & 2.08 \mathrm{E}-02 & 25\end{array}$ $\begin{array}{llllll}773 & 153 \mathrm{C} & 73.7 & 70.6 & 1.70 \mathrm{E}-02 & 30\end{array}$ $\begin{array}{llllll}773 & 153 \mathrm{C} & 91.4 & 65 & 1.07 \mathrm{E}-02 & 40\end{array}$ $\begin{array}{lllllll}773 & 153 \mathrm{C} & 57.8 & 84.8 & 5.70 \mathrm{E}-03 & 50\end{array}$ $\begin{array}{llllll}773 & 153 \mathrm{C} & 244.8 & 68 & 2.24 \mathrm{E}-03 & 60\end{array}$ $\begin{array}{llllll}773 & 153 \mathrm{C} & 255.6 & 3.8 & 3.60 \mathrm{E}-03 & 70\end{array}$ $\begin{array}{llllll}773 & 153 \mathrm{C} & 274.4 & -13.6 & 3.90 \mathrm{E}-03 & 85\end{array}$ $\begin{array}{llllll}773 & 153 \mathrm{C} & 248.2 & -26.4 & 2.86 \mathrm{E}-03 & 100\end{array}$ $\begin{array}{llllll}773 & 153 \mathrm{C} & 247.2 & -35.3 & 3.70 \mathrm{E}-03 & 115\end{array}$ $\begin{array}{llllll}775.1 & 153 \mathrm{E} & 341.9 & 64.2 & 5.46 \mathrm{E}-02 & 0\end{array}$ $\begin{array}{llllll}775.1 & 153 \mathrm{E} & 343.8 & 63.6 & 4.74 \mathrm{E}-02 & 3\end{array}$ $\begin{array}{llllll}775.1 & 153 \mathrm{E} & 344.2 & 63.5 & 3.88 \mathrm{E}-02 & 6\end{array}$ $\begin{array}{llllll}775.1 & 153 \mathrm{E} & 345.9 & 63 & 3.07 \mathrm{E}-02 & 9\end{array}$ $\begin{array}{llllll}775.1 & 153 \mathrm{E} & 348.4 & 63.3 & 2.67 \mathrm{E}-02 & 12\end{array}$ $\begin{array}{llllll}775.1 & 153 \mathrm{E} & 353.4 & 62.9 & 2.31 \mathrm{E}-02 & 15\end{array}$ $\begin{array}{llllll}775.1 & 153 \mathrm{E} & 351.2 & 63.8 & 2.02 \mathrm{E}-02 & 18\end{array}$ $\begin{array}{lllllll}775.1 & 153 \mathrm{E} & 353.5 & 61.8 & 1.72 \mathrm{E}-02 & 21\end{array}$ $\begin{array}{llllll}775.1 & 153 \mathrm{E} & 354.3 & 62.3 & 1.40 \mathrm{E}-02 & 25\end{array}$ $\begin{array}{llllll}775.1 & 153 \mathrm{E} & 351.9 & 65.9 & 1.20 \mathrm{E}-02 & 30\end{array}$ $\begin{array}{llllll}775.1 & 153 \mathrm{E} & 335 & 53.8 & 7.37 \mathrm{E}-03 & 40\end{array}$ $\begin{array}{llllll}775.1 & 153 \mathrm{E} & 329.6 & 68.4 & 4.29 \mathrm{E}-03 & 50\end{array}$ $\begin{array}{lllllll}775.1 & 153 \mathrm{E} & 352.8 & 76 & 2.63 \mathrm{E}-03 & 60\end{array}$ $\begin{array}{llllll}775.1 & 153 \mathrm{E} & 209.9 & 77.8 & 1.97 \mathrm{E}-03 & 70\end{array}$ $\begin{array}{lllllll}775.1 & 153 \mathrm{E} & 182.8 & 20.8 & 6.12 \mathrm{E}-04 & 85\end{array}$ $\begin{array}{lllllll}775.1 & 153 \mathrm{E} & 234.4 & 79.6 & 8.25 \mathrm{E}-04 & 100\end{array}$ $\begin{array}{llllll}775.1 & 153 \mathrm{E} & 249.3 & 57.2 & 2.14 \mathrm{E}-03 & 115\end{array}$ $\begin{array}{llllll}777.6 & 153 \mathrm{D} & 204 & -8.7 & 9.16 \mathrm{E}-02 & 0\end{array}$ $\begin{array}{lllllll}777.6 & 153 \mathrm{D} & 203.4 & -11.8 & 9.32 \mathrm{E}-02 & 3\end{array}$ $\begin{array}{lllllll}777.6 & 153 \mathrm{D} & 203.1 & -13.4 & 8.91 \mathrm{E}-02 & 6\end{array}$ $\begin{array}{llllll}777.6 & 153 \mathrm{D} & 203.6 & -13.2 & 8.22 \mathrm{E}-02 & 9\end{array}$ $\begin{array}{lllllll}777.6 & 153 \mathrm{D} & 202.9 & -13.4 & 7.46 \mathrm{E}-02 & 12\end{array}$ $\begin{array}{llllll}777.6 & 153 \mathrm{D} & 202.6 & -13.8 & 6.68 \mathrm{E}-02 & 15\end{array}$ $\begin{array}{lllllll}777.6 & 153 \mathrm{D} & 202.4 & -12.7 & 5.92 \mathrm{E}-02 & 18\end{array}$ $\begin{array}{lllllll}777.6 & 153 \mathrm{D} & 203.4 & -12.7 & 5.25 \mathrm{E}-02 & 21\end{array}$ $\begin{array}{lllllll}777.6 & 153 \mathrm{D} & 201.1 & -11.4 & 4.50 \mathrm{E}-02 & 25\end{array}$ $\begin{array}{llllll}777.6 & 153 \mathrm{D} & 201.8 & -12.1 & 3.70 \mathrm{E}-02 & 30\end{array}$ $\begin{array}{llllll}777.6 & 153 \mathrm{D} & 203.3 & -12.5 & 2.68 \mathrm{E}-02 & 40\end{array}$ $\begin{array}{llllll}777.6 & 153 \mathrm{D} & 198.7 & -15.7 & 1.87 \mathrm{E}-02 & 50\end{array}$ $\begin{array}{llllll}777.6 \quad 153 \mathrm{D} & 192.1 & -11.2 & 1.57 \mathrm{E}-02 & 60\end{array}$ $\begin{array}{llllll}777.6 & 153 \mathrm{D} & 199.3 & -17.8 & 1.52 \mathrm{E}-02 & 70\end{array}$ $\begin{array}{llllll}777.6 & 153 \mathrm{D} & 188.3 & -17 & 1.25 \mathrm{E}-02 & 85\end{array}$ $\begin{array}{llllll}777.6 & 153 \mathrm{D} & 203.1 & -15.4 & 1.21 \mathrm{E}-02 & 100\end{array}$ 
$\begin{array}{llllll}777.6 & 153 \mathrm{D} & 205.9 & -18.6 & 1.14 \mathrm{E}-02 & 115\end{array}$ $\begin{array}{llllll}782.5 & 154 \mathrm{~A} & 317.3 & 19.3 & 1.01 \mathrm{E}-02 & 0\end{array}$ $\begin{array}{llllll}782.5 & 154 \mathrm{~A} & 347.8 & -22 & 1.05 \mathrm{E}-02 & 3\end{array}$ $\begin{array}{llllll}782.5 & 154 \mathrm{~A} & 348.2 & -32.4 & 1.21 \mathrm{E}-02 & 6\end{array}$ $\begin{array}{llllll}782.5 & 154 \mathrm{~A} & 349.8 & -32.7 & 1.25 \mathrm{E}-02 & 9\end{array}$ $\begin{array}{llllll}782.5 & 154 \mathrm{~A} & 348.5 & -34.8 & 1.24 \mathrm{E}-02 & 12\end{array}$ $\begin{array}{llllll}782.5 & 154 \mathrm{~A} & 349.2 & -38.1 & 1.15 \mathrm{E}-02 & 15\end{array}$ $\begin{array}{llllll}782.5 & 154 \mathrm{~A} & 350.8 & -36.9 & 1.04 \mathrm{E}-02 & 18\end{array}$ $\begin{array}{lllllll}782.5 & 154 \mathrm{~A} & 350.7 & -38.3 & 9.32 \mathrm{E}-03 & 21\end{array}$ $\begin{array}{lllllll}782.5 & 154 \mathrm{~A} & 351.5 & \mathbf{3} 8.8 & 8.03 \mathrm{E}-03 & 25\end{array}$ $\begin{array}{lllllll}782.5 & 154 \mathrm{~A} & 352.9 & -33.2 & 7.00 \mathrm{E}-03 & 30\end{array}$ $\begin{array}{llllll}782.5 & 154 \mathrm{~A} & 354.8 & -33.3 & 4.83 \mathrm{E}-03 & 40\end{array}$ $\begin{array}{lllllll}782.5 & 154 \mathrm{~A} & 345.9 & -24.8 & 4.46 \mathrm{E}-03 & 50\end{array}$ $\begin{array}{lllllll}782.5 & 154 \mathrm{~A} & 345.5 & -24 & 4.21 \mathrm{E}-03 & 60\end{array}$ $\begin{array}{llllll}782.5 & 154 \mathrm{~A} & 2.6 & -52.2 & 2.85 \mathrm{E}-03 & 70\end{array}$ $\begin{array}{lllllll}782.5 & 154 \mathrm{~A} & 340.8 & -26.5 & 3.02 \mathrm{E}-03 & 85\end{array}$ $\begin{array}{lllllll}782.5 & 154 \mathrm{~A} & 349.8 & 40.8 & 2.10 \mathrm{E}-03 & 100\end{array}$ $\begin{array}{lllllll}782.5 & 154 \mathrm{~A} & 339.4 & -43.4 & 2.49 \mathrm{E}-03 & 115\end{array}$ $\begin{array}{llllll}783.5 & 155 \mathrm{~A} & 356.4 & -30.7 & 9.05 \mathrm{E}-03 & 0\end{array}$ $\begin{array}{llllll}783.5 & 155 \mathrm{~A} & 358.1 & -33.6 & 9.48 \mathrm{E}-03 & 3\end{array}$ $\begin{array}{llllll}783.5 & 155 \mathrm{~A} & 0.8 & -35.9 & 9.85 \mathrm{E}-03 & 6\end{array}$ $\begin{array}{llllll}783.5 & 155 \mathrm{~A} & 2.4 & -36 & 9.79 \mathrm{E}-03 & 9\end{array}$ $\begin{array}{llllll}783.5 & 155 \mathrm{~A} & 3.6 & -36.4 & 9.46 \mathrm{E}-03 & 12\end{array}$ $\begin{array}{lllllll}783.5 & 155 \mathrm{~A} & 4.2 & -37.3 & 8.80 \mathrm{E}-03 & 15\end{array}$ $\begin{array}{llllll}783.5 & 155 \mathrm{~A} & 2.7 & -36.3 & 7.66 \mathrm{E}-03 & 21\end{array}$ $\begin{array}{llllll}783.5 & 155 \mathrm{~A} & 5 & 40.4 & 6.37 \mathrm{E}-03 & 25\end{array}$ $\begin{array}{lllllll}783.5 & 155 \mathrm{~A} & 3.3 & 43.1 & 5.33 \mathrm{E}-03 & 30\end{array}$ $\begin{array}{lllllll}783.5 & 155 \mathrm{~A} & 355.6 & -31.5 & 5.07 \mathrm{E}-03 & 40\end{array}$ $\begin{array}{llllll}783.5 & 155 \mathrm{~A} & 1.8 & -59.2 & 2.55 \mathrm{E}-03 & 50\end{array}$ $\begin{array}{llllll}783.5 & 155 \mathrm{~A} & 347.9 & -21.1 & 4.43 \mathrm{E}-03 & 60\end{array}$ $\begin{array}{llllll}783.5 & 155 \mathrm{~A} & 346.4 & -17.6 & 4.37 \mathrm{E}-03 & 70\end{array}$ $\begin{array}{llllll}783.5 & 155 \mathrm{~A} & 265 & -81.9 & 1.56 \mathrm{E}-03 & 85\end{array}$ $\begin{array}{lllllll}783.5 & 155 \mathrm{~A} & 344 & -13.7 & 4.44 \mathrm{E}-03 & 100\end{array}$ $\begin{array}{lllllll}783.5 & 155 \mathrm{~A} & 233.4 & -75.6 & 1.44 \mathrm{E}-03 & 115\end{array}$ $\begin{array}{llllll}783.5 & 155 \mathrm{~B} & 31.1 & 66.4 & 3.29 \mathrm{E}-02 & 0\end{array}$ $\begin{array}{llllll}783.5 & 155 \mathrm{~B} & 35 & 65.5 & 2.80 \mathrm{E}-02 & 3\end{array}$ $\begin{array}{llllll}783.5 & 155 \mathrm{~B} & 33.6 & 68.1 & 2.37 \mathrm{E}-02 & 6\end{array}$ $\begin{array}{llllll}783.5 & 155 \mathrm{~B} & 33.2 & 69.7 & 2.02 \mathrm{E}-02 & 9\end{array}$ $\begin{array}{llllll}783.5 & 155 \mathrm{~B} & 31 & 71.2 & 1.76 \mathrm{E}-02 & 12\end{array}$ $\begin{array}{llllll}783.5 & 155 \mathrm{~B} & 27.1 & 74.7 & 1.58 \mathrm{E}-02 & 15\end{array}$ $\begin{array}{lllllll}783.5 & 155 \mathrm{~B} & 28.9 & 76.9 & 1.40 \mathrm{E}-02 & 18\end{array}$ $\begin{array}{llllll}783.5 & 155 \mathrm{~B} & 14.9 & 81.7 & 1.19 \mathrm{E}-02 & 21\end{array}$ $\begin{array}{llllll}783.5 & 155 \mathrm{~B} & 32.3 & 81.5 & 1.08 \mathrm{E}-02 & 25\end{array}$ $\begin{array}{llllll}783.5 & 155 \mathrm{~B} & 24.2 & 73.9 & 9.27 \mathrm{E}-03 & 30\end{array}$ $\begin{array}{llllll}783.5 & 155 \mathrm{~B} & 356.8 & 86.9 & 6.68 \mathrm{E}-03 & 40\end{array}$ $\begin{array}{llllll}783.5 & 155 \mathrm{~B} & 23.1 & 82.2 & 5.76 \mathrm{E}-03 & 50\end{array}$ $\begin{array}{lllllll}783.5 & 155 \mathrm{~B} & 111 & 73.4 & 4.07 \mathrm{E}-03 & 60\end{array}$ $\begin{array}{llllll}783.5 & 155 \mathrm{~B} & 100.3 & 76.2 & 3.64 \mathrm{E}-03 & 70\end{array}$ $\begin{array}{llllll}783.5 & 155 \mathrm{~B} & 184.2 & 74.9 & 3.26 \mathrm{E}-03 & 85\end{array}$ $\begin{array}{lllllll}783.5 & 155 \mathrm{~B} & 138.8 & 59.6 & 2.48 \mathrm{E}-03 & 100\end{array}$ $\begin{array}{llllll}783.5 & 155 \mathrm{~B} & 188.3 & 67.1 & 3.05 \mathrm{E}-03 & 115\end{array}$ $\begin{array}{llllll}791.7 & 307 \mathrm{~A} & 343.6 & 60.8 & 6.56 \mathrm{E}-02 & 0\end{array}$ $\begin{array}{llllll}791.7 & 307 \mathrm{~A} \quad 346 & 60.5 & 5.95 \mathrm{E}-02 & 3\end{array}$ $\begin{array}{llllll}791.7 & 307 \mathrm{~A} & 345.5 & 61 & 5.31 \mathrm{E}-02 & 6\end{array}$ $\begin{array}{llllll}791.7 & 307 \mathrm{~A} & 345.8 & 60.8 & 4.56 \mathrm{E}-02 & 9\end{array}$ $\begin{array}{llllll}791.7 & 307 \mathrm{~A} & 346.2 & 61.8 & 4.04 \mathrm{E}-02 & 12\end{array}$ $\begin{array}{lllllll}791.7 & 307 \mathrm{~A} & 347.3 & 62.6 & 3.66 \mathrm{E}-02 & 15\end{array}$ $\begin{array}{lllllll}791.7 & 307 \mathrm{~A} & 349.1 & 62.7 & 3.18 \mathrm{E}-02 & 18\end{array}$ $\begin{array}{llllll}791.7 & 307 \mathrm{~A} & 352.5 & 64.2 & 2.82 \mathrm{E}-02 & 21\end{array}$ $\begin{array}{llllll}791.7 & 307 \mathrm{~A} & 348.7 & 63.1 & 2.40 \mathrm{E}-02 & 25\end{array}$ $\begin{array}{llllll}791.7 & 307 \mathrm{~A} & 351.5 & 65.3 & 2.03 \mathrm{E}-02 & 30\end{array}$ $\begin{array}{llllll}791.7 & 307 \mathrm{~A} & 354.1 & 69.5 & 1.34 \mathrm{E}-02 & 40\end{array}$ $\begin{array}{lllllll}791.7 & 307 \mathrm{~A} & 345.7 & 72.2 & 8.34 \mathrm{E}-03 & 50\end{array}$ $\begin{array}{llllll}791.7 & 307 \mathrm{~A} & 340.3 & 77.9 & 5.19 \mathrm{E}-03 & 60\end{array}$ $\begin{array}{llllll}791.7 & 307 \mathrm{~A} & 75 & 77.9 & 2.65 \mathrm{E}-03 & 70\end{array}$ $\begin{array}{llllll}791.7 & 307 \mathrm{~A} & 196.2 & 33 & 1.70 \mathrm{E}-03 & 85\end{array}$ $\begin{array}{lllllll}791.7 & 307 \mathrm{~A} & 173.5 & 8.6 & 1.82 \mathrm{E}-03 & 100\end{array}$ $\begin{array}{lllllll}791.7 & 307 \mathrm{~A} & 150.5 & 3.1 & 9.83 \mathrm{E}-04 & 115\end{array}$ $\begin{array}{llllll}792.7 & 308 \mathrm{~A} & 345.3 & 58.6 & 5.00 \mathrm{E}-02 & 0\end{array}$ $\begin{array}{lllllll}792.7 & 308 \mathrm{~A} & 345.1 & 57.8 & 4.32 \mathrm{E}-02 & 3\end{array}$ $\begin{array}{llllll}792.7 & 308 \mathrm{~A} & 344.8 & 57.3 & 3.57 \mathrm{E}-02 & 6\end{array}$ $\begin{array}{llllll}792.7 & 308 \mathrm{~A} & 348.1 & 55.1 & 2.86 \mathrm{E}-02 & 9\end{array}$ $\begin{array}{llllll}792.7 & 308 \mathrm{~A} & 350.4 & 56.2 & 2.41 \mathrm{E}-02 & 12\end{array}$ $\begin{array}{lllllll}792.7 & 308 \mathrm{~A} & 347.2 & 55.6 & 2.09 \mathrm{E}-02 & 15\end{array}$ $\begin{array}{lllllll}792.7 & 308 \mathrm{~A} & 350.6 & 55.1 & 1.82 \mathrm{E}-02 & 18\end{array}$ $\begin{array}{lllllll}792.7 & 308 \mathrm{~A} & 355.4 & 55.8 & 1.57 \mathrm{E}-02 & 21\end{array}$ $\begin{array}{lllllll}792.7 & 308 \mathrm{~A} & 355.5 & 56.1 & 1.27 \mathrm{E}-02 & 25\end{array}$ $\begin{array}{lllllll}792.7 & 308 \mathrm{~A} & 357.3 & 59.6 & 1.10 \mathrm{E}-02 & 30\end{array}$ $\begin{array}{lllllll}792.7 & 308 \mathrm{~A} & 355.4 & 61.8 & 7.04 \mathrm{E}-03 & 40\end{array}$ $\begin{array}{llllll}792.7 & 308 \mathrm{~A} & 7.7 & 57 & 4.89 \mathrm{E}-03 & 50\end{array}$ $\begin{array}{llllll}792.7 & 308 \mathrm{~A} & 44.2 & 45.2 & 2.37 \mathrm{E}-03 & 60\end{array}$ $\begin{array}{llllll}792.7 & 308 \mathrm{~A} & 62.9 & 24.1 & 2.50 \mathrm{E}-03 & 70\end{array}$ $\begin{array}{llllll}792.7 & 308 \mathrm{~A} & 58.7 & 34.1 & 2.21 \mathrm{E}-03 & 85\end{array}$ $\begin{array}{lllllll}792.7 & 308 \mathrm{~A} & 102.5 & -1.1 & 2.01 \mathrm{E}-03 & 100\end{array}$ $\begin{array}{llllll}792.7 & 308 \mathrm{~A} & 60 & -13.6 & 2.43 \mathrm{E}-03 & 115\end{array}$ $\begin{array}{llllll}794.8 & 309 \mathrm{~A} & 349.1 & 49 & 3.34 \mathrm{E}-02 & 0\end{array}$ $\begin{array}{llllll}794.8 & 309 \mathrm{~A} & 351 & 48.3 & 2.99 \mathrm{E}-02 & 3\end{array}$ $\begin{array}{llllll}794.8 & 309 \mathrm{~A} & 351.2 & 47.5 & 2.59 \mathrm{E}-02 & 6\end{array}$ $\begin{array}{llllll}794.8 & 309 \mathrm{~A} & 351 & 45.7 & 2.17 \mathrm{E}-02 & 9\end{array}$ $\begin{array}{llllll}794.8 & 309 \mathrm{~A} & 350.8 & 45 & 1.87 \mathrm{E}-02 & 12\end{array}$ $\begin{array}{lllllll}794.8 & 309 \mathrm{~A} & 351.4 & 45.5 & 1.65 \mathrm{E}-02 & 15\end{array}$ $\begin{array}{lllllll}794.8 & 309 \mathrm{~A} & 352.8 & 45 & 1.43 \mathrm{E}-02 & 18\end{array}$ $\begin{array}{llllll}794.8 & 309 \mathrm{~A} & 351 & 45.3 & 1.26 \mathrm{E}-02 & 21\end{array}$ $\begin{array}{lllllll}794.8 & 309 \mathrm{~A} & 353.1 & 47.1 & 1.08 \mathrm{E}-02 & 25\end{array}$ $\begin{array}{lllllll}794.8 & 309 \mathrm{~A} & 354.3 & 48.6 & 8.88 \mathrm{E}-03 & 30\end{array}$ $\begin{array}{llllll}794.8 & 309 \mathrm{~A} & 9.1 & 49 & 5.65 \mathrm{E}-03 & 40\end{array}$ $\begin{array}{llllll}794.8 & 309 \mathrm{~A} & 2.9 & 59.2 & 4.26 \mathrm{E}-03 & 50\end{array}$ $\begin{array}{llllll}794.8 & 309 \mathrm{~A} & 31.1 & 64.2 & 2.99 \mathrm{E}-03 & 60\end{array}$ $\begin{array}{lllllll}794.8 & 309 \mathrm{~A} & 359.9 & 48.6 & 2.61 \mathrm{E}-03 & 70\end{array}$ $\begin{array}{llllll}794.8 & 309 \mathrm{~A} & 109.8 & 66.6 & 1.40 \mathrm{E}-03 & 85\end{array}$ $\begin{array}{lllllll}794.8 & 309 \mathrm{~A} & 30.5 & 40.1 & 2.18 \mathrm{E}-03 & 100\end{array}$ $\begin{array}{lllllll}794.8 & 309 \mathrm{~A} & 16.7 & 47.8 & 1.89 \mathrm{E}-03 & 115\end{array}$ $\begin{array}{llllll}795.4 & 310 \mathrm{~A} & 287.5 & 39.2 & 3.60 \mathrm{E}-02 & 0\end{array}$ $\begin{array}{lllllll}795.4 & 310 \mathrm{~A} & 284.7 & 35.6 & 3.27 \mathrm{E}-02 & 3\end{array}$ $\begin{array}{lllllll}795.4 & 310 \mathrm{~A} & 280.2 & 30.5 & 2.90 \mathrm{E}-02 & 6\end{array}$ $\begin{array}{llllll}795.4 & 310 \mathrm{~A} & 274.4 & 23 & 2.58 \mathrm{E}-02 & 9\end{array}$ $\begin{array}{lllllll}795.4 & 310 \mathrm{~A} & 269.5 & 17.4 & 2.37 \mathrm{E}-02 & 12\end{array}$ $\begin{array}{llllll}795.4 & 310 \mathrm{~A} & 265.9 & 13.1 & 2.19 \mathrm{E}-02 & 15\end{array}$ $\begin{array}{llllll}795.4 & 310 \mathrm{~A} & 261.2 & 5.4 & 2.08 \mathrm{E}-02 & 18\end{array}$ $\begin{array}{lllllll}795.4 & 310 \mathrm{~A} & 258.6 & 0.4 & 1.92 \mathrm{E}-02 & 21\end{array}$ $\begin{array}{llllll}795.4 & 310 \mathrm{~A} & 254.6 & -6 & 1.89 \mathrm{E}-02 & 25\end{array}$ $\begin{array}{lllllll}795.4 & 310 \mathrm{~A} & 253.3 & -12.1 & 1.80 \mathrm{E}-02 & 30\end{array}$ $\begin{array}{lllllll}795.4 & 310 \mathrm{~A} & 248.7 & -20.6 & 1.53 \mathrm{E}-02 & 40\end{array}$ $\begin{array}{lllllll}795.4 & 310 \mathrm{~A} & 245.2 & -21.6 & 1.25 \mathrm{E}-02 & 50\end{array}$ $\begin{array}{lllllll}795.4 & 310 \mathrm{~A} & 243.8 & -27.3 & 8.78 \mathrm{E}-03 & 60\end{array}$ $\begin{array}{llllll}795.4 & 310 \mathrm{~A} & 243.3 & -24.6 & 5.51 \mathrm{E}-03 & 70\end{array}$ $\begin{array}{llllll}795.4 & 310 \mathrm{~A} & 255 & -15.6 & 2.38 \mathrm{E}-03 & 85\end{array}$ $\begin{array}{lllllll}795.4 & 310 \mathrm{~A} & 245.6 & 21.8 & 1.39 \mathrm{E}-03 & 100\end{array}$ $\begin{array}{lllllll}795.4 & 310 \mathrm{~A} & 31 & 54.3 & 9.87 \mathrm{E}-04 & 115\end{array}$ $\begin{array}{llllll}797.1 & 311 \mathrm{~A} & 23.2 & 63.4 & 3.70 \mathrm{E}-02 & 0\end{array}$ $\begin{array}{lllllll}797.1 & 311 \mathrm{~A} & 23.1 & 62.6 & 3.35 \mathrm{E}-02 & 3\end{array}$ $\begin{array}{llllll}797.1 & 311 \mathrm{~A} & 22.5 & 62.9 & 2.95 \mathrm{E}-02 & 6\end{array}$ $\begin{array}{llllll}797.1 & 311 \mathrm{~A} & 21 & 62.9 & 2.48 \mathrm{E}-02 & 9\end{array}$ $\begin{array}{lllllll}797.1 & 311 \mathrm{~A} & 19.4 & 63.8 & 2.19 \mathrm{E}-02 & 12\end{array}$ $\begin{array}{lllllll}797.1 & 311 \mathrm{~A} & 17.4 & 65.3 & 1.98 \mathrm{E}-02 & 15\end{array}$ $\begin{array}{lllllll}797.1 & 311 \mathrm{~A} & 18 & 66.2 & 1.74 \mathrm{E}-02 & 18\end{array}$ $\begin{array}{lllllll}797.1 & 311 \mathrm{~A} & 16.3 & 67.3 & 1.53 \mathrm{E}-02 & 21\end{array}$ $\begin{array}{lllllll}797.1 & 311 \mathrm{~A} & 19.2 & 70.9 & 1.32 \mathrm{E}-02 & 25\end{array}$ $\begin{array}{lllllll}797.1 & 311 \mathrm{~A} & 22.9 & 72.1 & 1.14 \mathrm{E}-02 & 30\end{array}$ $\begin{array}{lllllll}797.1 & 311 \mathrm{~A} & 21.5 & 69.9 & 7.60 \mathrm{E}-03 & 40\end{array}$ $\begin{array}{llllll}797.1 & 311 \mathrm{~A} & 55.2 & 71.9 & 4.64 \mathrm{E}-03 & 50\end{array}$ $\begin{array}{llllll}797.1 & 311 \mathrm{~A} & 44 & 62.5 & 3.37 \mathrm{E}-03 & 60\end{array}$ $\begin{array}{lllllll}797.1 & 311 \mathrm{~A} & 112.7 & 82.8 & 2.42 \mathrm{E}-03 & 70\end{array}$ $\begin{array}{lllllll}797.1 & 311 \mathrm{~A} & 177.1 & 30 & 1.32 \mathrm{E}-03 & 85\end{array}$ $\begin{array}{llllll}797.1 & 311 \mathrm{~A} & 81.2 & 26.9 & 1.11 \mathrm{E}-03 & 100\end{array}$ $\begin{array}{lllllll}797.1 & 311 \mathrm{~A} & 327.4 & 24.6 & 1.97 \mathrm{E}-04 & 115\end{array}$ $\begin{array}{llllll}799.4 & 312 \mathrm{~A} & 9.1 & 83.7 & 5.54 \mathrm{E}-02 & 0\end{array}$ $\begin{array}{lllllll}799.4 & 312 \mathrm{~A} & 10 & 83.7 & 4.99 \mathrm{E}-02 & 3\end{array}$ $\begin{array}{lllllll}799.4 & 312 \mathrm{~A} & 6.7 & 84.3 & 4.47 \mathrm{E}-02 & 6\end{array}$ $\begin{array}{llllll}799.4 & 312 \mathrm{~A} & 2.6 & 84.1 & 3.83 \mathrm{E}-02 & 9\end{array}$ $\begin{array}{llllll}799.4 & 312 \mathrm{~A} & 4.6 & 84.2 & 3.37 \mathrm{E}-02 & 12\end{array}$ $\begin{array}{llllll}799.4 & 312 \mathrm{~A} & 4.9 & 83.9 & 3.06 \mathrm{E}-02 & 15\end{array}$ $\begin{array}{llllll}799.4 & 312 \mathrm{~A} & 4.2 & 84.6 & 2.71 \mathrm{E}-02 & 18\end{array}$ $\begin{array}{llllll}799.4 & 312 \mathrm{~A} & 6.4 & 84.8 & 2.41 \mathrm{E}-02 & 21\end{array}$ $\begin{array}{lllllll}799.4 & 312 \mathrm{~A} & 6 & 84.7 & 2.07 \mathrm{E}-02 & 25\end{array}$ $\begin{array}{lllllll}799.4 & 312 \mathrm{~A} & 328.8 & 85.4 & 1.77 \mathrm{E}-02 & 30\end{array}$ $\begin{array}{llllll}799.4 & 312 \mathrm{~A} & 344.6 & 83 & 1.19 \mathrm{E}-02 & 40\end{array}$ $\begin{array}{lllllll}799.4 & 312 \mathrm{~A} & 299.7 & 85.7 & 8.82 \mathrm{E}-03 & 50\end{array}$ $\begin{array}{llllll}799.4 & 312 \mathrm{~A} & 11.2 & 82.9 & 5.33 \mathrm{E}-03 & 60\end{array}$ $\begin{array}{llllll}799.4 & 312 \mathrm{~A} & 30.1 & 56.3 & 3.64 \mathrm{E}-03 & 70\end{array}$ $\begin{array}{llllll}799.4 & 312 \mathrm{~A} & 2.6 & 50.8 & 2.53 \mathrm{E}-03 & 85\end{array}$ $\begin{array}{lllllll}799.4 & 312 \mathrm{~A} & 40.9 & 30 & 1.67 \mathrm{E}-03 & 100\end{array}$ $\begin{array}{llllll}799.4 & 312 \mathrm{~A} & 324.4 & 3.3 & 1.99 \mathrm{E}-03 & 115\end{array}$ 


\begin{tabular}{|c|c|c|c|c|c|}
\hline & & & 71.5 & & \\
\hline & & 80.1 & 70.2 & & \\
\hline 0.4 & $3 \mathrm{~A}$ & 87.2 & 68 & $3.62 \mathrm{E}$ & \\
\hline 0.4 & $3 \mathrm{~A}$ & 89.5 & 65.6 & $2.88 \mathrm{E}-\mathrm{C}$ & \\
\hline 0.4 & $3 \mathrm{~A}$ & 87.8 & 63.9 & $2.45 \mathrm{E}-$ & \\
\hline 0.4 & $13 \mathrm{~A}$ & 87.3 & 63.7 & $2.19 \mathrm{E}$ & \\
\hline 0.4 & $313 \mathrm{~A}$ & 90.2 & 63.7 & $1.91 \mathrm{E}$ & \\
\hline 0.4 & $313 \mathrm{~A}$ & 85.1 & 63.1 & $1.65 \mathrm{E}$ & \\
\hline 0.4 & $313 \mathrm{~A}$ & 87.2 & 60.5 & $1.39 \mathrm{E}$ & \\
\hline 0.4 & $313 \mathrm{~A}$ & 91.8 & 63.3 & $1.19 \mathrm{E}$ & \\
\hline 0.4 & $313 \mathrm{~A}$ & 89.9 & 60.7 & $7.93 \mathrm{E}$ & \\
\hline 0.4 & $313 \mathrm{~A}$ & 117.2 & 61.9 & 6.281 & \\
\hline 0.4 & $313 \mathrm{~A}$ & 124.1 & 68.7 & $3.86 \mathrm{H}$ & \\
\hline 0.4 & $313 \mathrm{~A}$ & 132.1 & 76.9 & $2.88 \mathrm{I}$ & \\
\hline 0.4 & $313 \mathrm{~A}$ & 202.1 & 21.3 & $1.94 \mathrm{E}$ & \\
\hline 00.4 & $313 \mathrm{~A}$ & 145.2 & 64.5 & $2.08 \mathrm{I}$ & 10 \\
\hline 00.4 & $313 \mathrm{~A}$ & 177.9 & 40 & 1.72 & 115 \\
\hline 1.3 & $314 A$ & 57 & 72.8 & & \\
\hline 1. & $314 A$ & 57.4 & 72.5 & & \\
\hline 01. & $314 \mathrm{~A}$ & 59.3 & 72.9 & & \\
\hline 1. & $314 \mathrm{~A}$ & 59.6 & 72.3 & & \\
\hline $1 .{ }^{2}$ & $14 \mathrm{~A}$ & 59.2 & 72.8 & & \\
\hline 1. & $14 \mathrm{~A}$ & 59.8 & 72.8 & & \\
\hline 1. & $14 \mathrm{~A}$ & 62.9 & 73.5 & & \\
\hline 1. & $14 \mathrm{~A}$ & 58.6 & 73.3 & & \\
\hline 1. & $4 \mathrm{~A}$ & 62.8 & 73.4 & & \\
\hline 1. & $14 \mathrm{~A}$ & 58.5 & 78.6 & & \\
\hline 1. & $4 \mathrm{~A}$ & 1.8 & 80.4 & & \\
\hline 1. & $4 \mathrm{~A}$ & 9.9 & 84.1 & & \\
\hline 1 & $14 \mathrm{~A}$ & 130.8 & 79.7 & & \\
\hline 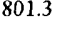 & $314 \mathrm{~A}$ & 231.7 & 64.7 & & \\
\hline & $314 \mathrm{~A}$ & 173.6 & 64.7 & & \\
\hline & $4 \mathrm{~A}$ & 190.8 & 25.2 & & 100 \\
\hline & $4 \mathrm{~A}$ & 190.4 & 17.1 & & \\
\hline & & & 71.9 & & \\
\hline & & & 71.6 & & \\
\hline & & & .6 & & \\
\hline & & & 4 & & \\
\hline 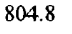 & & & & & \\
\hline & & & 70.9 & & \\
\hline 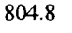 & & & 71 & & \\
\hline 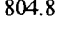 & & & 72.2 & & \\
\hline 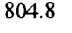 & & 31.4 & 12 & & \\
\hline 0 & & 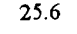 & 73 & & \\
\hline 47.0 & & .3 & 73.4 & & \\
\hline 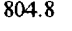 & $5 \mathrm{~A}$ & 4.1 & 73.3 & & \\
\hline 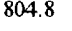 & $5 \mathrm{~A}$ & 322.9 & 70.2 & 7.67 & \\
\hline & & 320.1 & 68.5 & & r \\
\hline & $315 \mathrm{~A}$ & 329.9 & 62.8 & $4.77 \mathrm{E}-03$ & O- \\
\hline & $315 \mathrm{~A}$ & 277.8 & 61.4 & $4.45 \mathrm{E}-03$ & 10 \\
\hline & & 8.3 & 8 & $4.80 \mathrm{E}-$ & \\
\hline
\end{tabular}

\begin{tabular}{|c|c|c|c|c|c|}
\hline & & 27.6 & 62.6 & & \\
\hline & $16 \mathrm{~A}$ & 29.8 & 61.4 & $4.24 \mathrm{I}$ & \\
\hline 4.9 & $16 \mathrm{~A}$ & 30.1 & 61.7 & $3.73 \mathrm{E}$ & \\
\hline 4.9 & $316 \mathrm{~A}$ & 0.7 & 60.8 & $3.15 \mathrm{E}$ & \\
\hline 4.9 & $316 \mathrm{~A}$ & 31 & 61.4 & $2.78 \mathrm{I}$ & \\
\hline 4.9 & $316 \mathrm{~A}$ & 31.3 & 62.4 & $2.53 \mathrm{E}$ & \\
\hline 4.9 & $316 \mathrm{~A}$ & 32 & 63.3 & $2.22 \mathrm{E}$ & \\
\hline 4.9 & $316 f$ & 32.3 & 63.1 & $1.99 \mathrm{E}-02$ & \\
\hline 4.9 & $316 \mathrm{~A}$ & 31.5 & 63.4 & $1.71 \mathrm{H}$ & \\
\hline 4.9 & $316 \mathrm{~A}$ & 28.8 & 65.4 & 1.56 & \\
\hline 4.9 & $316 \mathrm{~A}$ & 43.9 & 63.5 & 1.00 & \\
\hline 4.9 & $316 \mathrm{~A}$ & 40.5 & 67 & 7.38 & \\
\hline 4.9 & $316 \mathrm{~A}$ & 24 & 67.5 & 4.06 & \\
\hline 4.9 & $316 \mathrm{~A}$ & 117.7 & 46.4 & 2.16 & \\
\hline 4.9 & $316 \mathrm{~A}$ & 141.8 & 70 & 2.05 & \\
\hline 4.9 & $316 \mathrm{~A}$ & 128.4 & 25.2 & -03 & 10 \\
\hline 4.9 & $316 \mathrm{~A}$ & 109.8 & 45.4 & & 11 \\
\hline 12.8 & $156 \mathrm{~A}$ & 232.3 & 70.7 & & \\
\hline 12.8 & $156 \mathrm{~A}$ & 223.5 & 70.3 & & \\
\hline 12.8 & $156 \mathrm{~A}$ & 216.5 & 68.2 & & \\
\hline 12.8 & $156 \mathrm{~A}$ & 214.4 & 64.8 & & \\
\hline 2.8 & $156 \mathrm{~A}$ & 212.5 & 63.1 & 5.45 & \\
\hline 12.8 & $156 \mathrm{~A}$ & 209.4 & 62.1 & & \\
\hline 12.8 & $156 \mathrm{~A}$ & 208.3 & 61 & & \\
\hline 12.8 & $156 \mathrm{~A}$ & 205. & 59 & & \\
\hline 12.8 & $156 \mathrm{~A}$ & 207.2 & 57.6 & & \\
\hline 12.8 & $156 \mathrm{~A}$ & 204.2 & 57.6 & & \\
\hline 2.8 & $156 \mathrm{~A}$ & 204.3 & 55.8 & & \\
\hline 2. & $156 \mathrm{~A}$ & 20 & 53.5 & & \\
\hline 12.8 & $156 \mathrm{~A}$ & 203.4 & 48.2 & & \\
\hline 12.8 & $156 \mathrm{~A}$ & 205.4 & 49.8 & & \\
\hline 2. & $156 \mathrm{~A}$ & 217.5 & 38 & & \\
\hline & $156 \mathrm{~A}$ & 194.7 & 49.7 & & \\
\hline & $156 \mathrm{~A}$ & 208.5 & 21.4 & & \\
\hline & & & 71.9 & & \\
\hline & & & 70.7 & & \\
\hline & & & & & \\
\hline & & & 9 & & \\
\hline & & & & & \\
\hline & & & 2.5 & & \\
\hline & & & 0.9 & & \\
\hline . & & 210.3 & 59.7 & & \\
\hline . & & & 57 & & \\
\hline . & & 6.7 & 58.8 & & \\
\hline 020 & & 03.2 & 53.8 & 1.49 & 4 \\
\hline 20. & $7 \mathrm{~A}$ & 186.8 & 36.7 & & 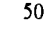 \\
\hline 020 & $7 \mathrm{~A}$ & 160.8 & 15.7 & 6.58 & o \\
\hline & $7 \mathrm{~A}$ & 135.7 & 50.5 & 4.30E-03 & . \\
\hline & $157 \mathrm{~A}$ & 151.9 & 24.6 & 4.11E-03 & 0. \\
\hline & & 159.9 & 59.6 & 4.581 & \\
\hline & & 1.2 & 41.2 & & \\
\hline
\end{tabular}

$\begin{array}{llllll}825.7 & 158 \mathrm{~A} & 58.4 & 81.2 & 1.41 \mathrm{E}-01 & 0\end{array}$ $\begin{array}{lllllll}825.7 & 158 \mathrm{~A} & 65.1 & 79.1 & 1.28 \mathrm{E}-01 & 3\end{array}$ $\begin{array}{llllll}825.7 & 158 \mathrm{~A} & 62.7 & 79.8 & 1.09 \mathrm{E}-01 & 6\end{array}$ $\begin{array}{lllllll}825.7 & 158 \mathrm{~A} & 53.9 & 80.7 & 8.77 \mathrm{E}-02 & 9\end{array}$ $\begin{array}{lllllll}825.7 & 158 \mathrm{~A} & 47 & 81.5 & 7.59 \mathrm{E}-02 & 12\end{array}$ $\begin{array}{lllllll}825.7 & 158 \mathrm{~A} & 32.4 & 82.1 & 6.57 \mathrm{E}-02 & 15\end{array}$ $\begin{array}{lllllll}825.7 & 158 \mathrm{~A} & 22.9 & 83.3 & 5.78 \mathrm{E}-02 & 18\end{array}$ $\begin{array}{llllll}825.7 & 158 \mathrm{~A} & 20.1 & 82 & 5.06 \mathrm{E}-02 & 21\end{array}$ $\begin{array}{lllllll}825.7 & 158 \mathrm{~A} & 7.5 & 82.4 & 4.39 \mathrm{E}-02 & 25\end{array}$ $\begin{array}{lllllll}825.7 & 158 \mathrm{~A} & 351 & 84 & 3.79 \mathrm{E}-02 & 30\end{array}$ $\begin{array}{lllllll}825.7 & 158 \mathrm{~A} & 341.2 & 80.5 & 2.44 \mathrm{E}-02 & 40\end{array}$ $\begin{array}{lllllll}825.7 & 158 \mathrm{~A} & 303.9 & 77.8 & 1.57 \mathrm{E}-02 & 50\end{array}$ $\begin{array}{lllllll}825.7 & 158 \mathrm{~A} & 312.6 & 72.1 & 1.14 \mathrm{E}-02 & 60\end{array}$ $\begin{array}{lllllll}825.7 & 158 \mathrm{~A} & 295.9 & 35.5 & 6.69 \mathrm{E}-03 & 70\end{array}$ $\begin{array}{lllllll}825.7 & 158 \mathrm{~A} & 271.9 & 46.3 & 5.51 \mathrm{E}-03 & 85\end{array}$ $\begin{array}{lllllll}825.7 & 158 \mathrm{~A} & 276.4 & 35.6 & 2.17 \mathrm{E}-03 & 100\end{array}$ $\begin{array}{lllllll}825.7 & 158 \mathrm{~A} & 248.3 & 30 & 5.11 \mathrm{E}-03 & 115\end{array}$ $\begin{array}{lllllll}842.4 & 711 \mathrm{~A} & 325.5 & 56.9 & 9.89 \mathrm{E}-02 & 0\end{array}$ $\begin{array}{lllllll}842.4 & 711 \mathrm{~A} & 326.3 & 55.8 & 8.43 \mathrm{E}-02 & 3\end{array}$ $\begin{array}{lllllll}842.4 & 711 \mathrm{~A} & 324.1 & 54.6 & 6.93 \mathrm{E}-02 & 6\end{array}$ $\begin{array}{lllllll}842.4 & 711 \mathrm{~A} & 38.7 & 50.9 & 5.41 \mathrm{E}-02 & 9\end{array}$ $\begin{array}{lllllll}842.4 & 711 \mathrm{~A} & 325.2 & 54.3 & 4.63 \mathrm{E}-02 & 12\end{array}$ $\begin{array}{lllllll}842.4 & 711 \mathrm{~A} & 38.7 & 52.3 & 3.82 \mathrm{E}-02 & 15\end{array}$ $\begin{array}{lllllll}842.4 & 711 \mathrm{~A} & 39.5 & 54.4 & 3.28 \mathrm{E}-02 & 18\end{array}$ $\begin{array}{llllll}842.4 & 711 \mathrm{~A} & 40.4 & 54.5 & 2.89 \mathrm{E}-02 & 21\end{array}$ $\begin{array}{lllllll}842.4 & 711 \mathrm{~A} & 38.9 & 56.3 & 2.46 \mathrm{E}-02 & 25\end{array}$ $\begin{array}{lllllll}842.4 & 711 \mathrm{~A} & 43.8 & 56.1 & 1.99 \mathrm{E}-02 & 30\end{array}$ $\begin{array}{lllllll}842.4 & 711 \mathrm{~A} & 30.7 & 63.6 & 1.36 \mathrm{E}-02 & 40\end{array}$ $\begin{array}{lllllll}842.4 & 711 \mathrm{~A} & 38 & 62.3 & 8.99 \mathrm{E}-03 & 50\end{array}$ $\begin{array}{lllllll}842.4 & 711 \mathrm{~A} & 54.9 & 48.8 & 5.96 \mathrm{E}-03 & 60\end{array}$ $\begin{array}{lllllll}842.4 & 711 \mathrm{~A} & 11 & 45.3 & 3.89 \mathrm{E}-03 & 70\end{array}$ $\begin{array}{lllllll}842.4 & 711 \mathrm{~A} & 52.1 & -16.1 & 3.19 \mathrm{E}-03 & 85\end{array}$ $\begin{array}{lllllll}842.4 & 711 \mathrm{~A} & 29.2 & -9.6 & 4.05 \mathrm{E}-03 & 100\end{array}$ $\begin{array}{lllllll}842.4 & 711 \mathrm{~A} & 337.4 & -15.7 & 2.66 \mathrm{E}-03 & 115\end{array}$ $\begin{array}{lllllll}850.1 & 712 \mathrm{~A} & 322.5 & 67.1 & 1.18 \mathrm{E}-01 & 0\end{array}$ $\begin{array}{lllllll}850.1 & 712 \mathrm{~A} & 324.5 & 66.4 & 1.07 \mathrm{E}-01 & 3\end{array}$ $\begin{array}{lllllll}850.1 & 712 \mathrm{~A} & 328.1 & 66.8 & 9.41 \mathrm{E}-02 & 6\end{array}$ $\begin{array}{lllllll}850.1 & 712 \mathrm{~A} & 328.7 & 66.8 & 8.15 \mathrm{E}-02 & 9\end{array}$ $\begin{array}{lllllll}850.1 & 712 \mathrm{~A} & 330.1 & 67.3 & 7.15 \mathrm{E}-02 & 12\end{array}$ $\begin{array}{lllllll}850.1 & 712 \mathrm{~A} & 329.6 & 67.3 & 6.24 \mathrm{E}-02 & 15\end{array}$ $\begin{array}{lllllll}850.1 & 712 \mathrm{~A} & 331.4 & 69 & 5.53 \mathrm{E}-02 & 18\end{array}$ $\begin{array}{lllllll}850.1 & 712 \mathrm{~A} & 337.4 & 68.3 & 4.79 \mathrm{E}-02 & 21\end{array}$ $\begin{array}{lllllll}850.1 & 712 \mathrm{~A} & 339.5 & 68.5 & 4.14 \mathrm{E}-02 & 25\end{array}$ $\begin{array}{lllllll}850.1 & 712 \mathrm{~A} & 342.5 & 68.7 & 3.68 \mathrm{E}-02 & 30\end{array}$ $\begin{array}{lllllll}850.1 & 712 \mathrm{~A} & 346.7 & 68.6 & 2.28 \mathrm{E}-02 & 40\end{array}$ $\begin{array}{lllllll}850.1 & 712 \mathrm{~A} & 348.6 & 78 & 1.52 \mathrm{E}-02 & 50\end{array}$ $\begin{array}{llllll}850.1 & 712 \mathrm{~A} & 323 & 75.5 & 9.95 \mathrm{E}-03 & 60\end{array}$ $\begin{array}{lllllll}850.1 & 712 \mathrm{~A} & 244.1 & 76.7 & 5.58 \mathrm{E}-03 & 70\end{array}$ $\begin{array}{lllllll}850.1 & 712 \mathrm{~A} & 121.8 & 63.8 & 5.07 \mathrm{E}-03 & 85\end{array}$ $\begin{array}{lllllll}850.1 & 712 \mathrm{~A} & 169.8 & 39.8 & 2.09 \mathrm{E}-03 & 100\end{array}$ $\begin{array}{lllllll}850.1 & 712 \mathrm{~A} & 173.1 & 46.9 & 2.18 \mathrm{E}-03 & 115\end{array}$ 
$\begin{array}{llllll}869.3 & 712 \mathrm{~B} & 316 & 58.8 & 2.46 \mathrm{E}-01 & 0\end{array}$ $\begin{array}{llllll}869.3 & 712 \mathrm{~B} & 319.3 & 60 & 2.21 \mathrm{E}-01 & 3\end{array}$ $\begin{array}{llllll}869.3 & 712 \mathrm{~B} & 51.1 & 54.7 & 1.86 \mathrm{E}-01 & 6\end{array}$ $\begin{array}{lllllll}869.3 & 712 \mathrm{~B} & 53.1 & 53.7 & 1.50 \mathrm{E}-01 & 9\end{array}$ $\begin{array}{llllll}869.3 & 712 \mathrm{~B} & 55.5 & 53.2 & 1.28 \mathrm{E}-01 & 12\end{array}$ $\begin{array}{lllllll}869.3 & 712 \mathrm{~B} & 318.8 & 65.1 & 1.09 \mathrm{E}-01 & 15\end{array}$ $\begin{array}{llllll}869.3 & 712 \mathrm{~B} & 60.7 & 52.2 & 9.52 \mathrm{E}-02 & 18\end{array}$ $\begin{array}{llllll}869.3 & 712 \mathrm{~B} & 319.8 & 68.9 & 8.20 \mathrm{E}-02 & 21\end{array}$ $\begin{array}{llllll}869.3 & 712 \mathrm{~B} & 64.6 & 52.6 & 6.92 \mathrm{E}-02 & 25\end{array}$ $\begin{array}{llllll}869.3 & 712 \mathrm{~B} & 310 & 68.5 & 5.88 \mathrm{E}-02 & 30\end{array}$ $\begin{array}{llllll}869.3 & 712 \mathrm{~B} & 323.5 & 72.5 & 3.77 \mathrm{E}-02 & 40\end{array}$ $\begin{array}{llllll}869.3 & 712 \mathrm{~B} & 318.6 & 75.1 & 2.48 \mathrm{E}-02 & 50\end{array}$ $\begin{array}{lllllll}869.3 & 712 \mathrm{~B} & 85.8 & 35.7 & 1.60 \mathrm{E}-02 & 60\end{array}$ $\begin{array}{llllll}869.3 & 712 \mathrm{~B} & 117.8 & 84 & 1.03 \mathrm{E}-02 & 70\end{array}$ $\begin{array}{lllllll}869.3 & 712 \mathrm{~B} & 113.9 & 23.8 & 4.95 \mathrm{E}-03 & 85\end{array}$ $\begin{array}{lllllll}869.3 & 712 \mathrm{~B} & 109.6 & 4.7 & 3.04 \mathrm{E}-03 & 100\end{array}$ $\begin{array}{lllllll}869.3 & 712 \mathrm{~B} & 61 & 4.4 & 3.54 \mathrm{E}-03 & 115\end{array}$ $\begin{array}{llllll}879.5 & 712 \mathrm{C} & 282 & -16.7 & 3.26 \mathrm{E}-02 & 0\end{array}$ $\begin{array}{llllll}879.5 & 712 \mathrm{C} & 283.5 & -22.7 & 3.50 \mathrm{E}-02 & 3\end{array}$ $\begin{array}{llllll}879.5 & 712 \mathrm{C} & 284.1 & -25.7 & 3.61 \mathrm{E}-02 & 6\end{array}$ $\begin{array}{llllll}879.5 & 712 \mathrm{C} & 284.4 & -26.9 & 3.53 \mathrm{E}-02 & 9\end{array}$ $\begin{array}{llllll}879.5 & 712 \mathrm{C} & 284.2 & -26.8 & 3.42 \mathrm{E}-02 & 12\end{array}$ $\begin{array}{llllll}879.5 & 712 \mathrm{C} & 283.7 & -27.4 & 3.24 \mathrm{E}-02 & 15\end{array}$ $\begin{array}{lllllll}879.5 & 712 \mathrm{C} & 283.7 & -26.3 & 3.04 \mathrm{E}-02 & 18\end{array}$ $\begin{array}{llllll}879.5 & 712 \mathrm{C} & 284.2 & -27.2 & 2.78 \mathrm{E}-02 & 21\end{array}$ $\begin{array}{llllll}879.5 & 712 \mathrm{C} & 284 & -27.5 & 2.53 \mathrm{E}-02 & 25\end{array}$ $\begin{array}{llllll}879.5 & 712 \mathrm{C} & 282.7 & -27.3 & 2.28 \mathrm{E}-02 & 30\end{array}$ $\begin{array}{llllll}879.5 & 712 \mathrm{C} & 283.5 & -28.6 & 1.81 \mathrm{E}-02 & 40\end{array}$ $\begin{array}{llllll}879.5 & 712 \mathrm{C} & 286 & -33.9 & 1.60 \mathrm{E}-02 & 50\end{array}$ $\begin{array}{llllll}879.5 & 712 \mathrm{C} & 293.5 & -40 & 1.48 \mathrm{E}-02 & 60\end{array}$ $\begin{array}{llllll}879.5 & 712 \mathrm{C} & 290.5 & 40.6 & 1.25 \mathrm{E}-02 & 70\end{array}$ $\begin{array}{llllll}879.5 & 712 \mathrm{C} & 292.8 & -38.3 & 1.22 \mathrm{E}-02 & 85\end{array}$ $\begin{array}{lllllll}879.5 & 712 \mathrm{C} & 286.5 & 40.7 & 1.22 \mathrm{E}-02 & 100\end{array}$ $\begin{array}{llllll}879.5 & 712 \mathrm{C} \quad 291.1 & 41.6 & 1.10 \mathrm{E}-02 & 115\end{array}$ $\begin{array}{llllll}889.4 & 713 \mathrm{~A} & 36.4 & 66.8 & 4.44 \mathrm{E}-02 & 0\end{array}$ $\begin{array}{llllll}889.4 & 713 \mathrm{~A} & 34.1 & 67.4 & 4.15 \mathrm{E}-02 & 3\end{array}$ $\begin{array}{lllllll}889.4 & 713 \mathrm{~A} & 38.1 & 67.3 & 3.80 \mathrm{E}-02 & 6\end{array}$ $\begin{array}{llllll}889.4 & 713 \mathrm{~A} & 36.9 & 68.6 & 3.33 \mathrm{E}-02 & 9\end{array}$ $\begin{array}{lllllll}889.4 & 713 \mathrm{~A} & 37.9 & 69.4 & 3.06 \mathrm{E}-02 & 12\end{array}$ $\begin{array}{llllll}889.4 & 713 \mathrm{~A} & 72.5 & 26.9 & 2.74 \mathrm{E}-02 & 15\end{array}$ $\begin{array}{llllll}889.4 & 713 \mathrm{~A} & 73.3 & 27.4 & 2.47 \mathrm{E}-02 & 18\end{array}$ $\begin{array}{llllll}889.4 & 713 \mathrm{~A} & 74.5 & 27.7 & 2.19 \mathrm{E}-02 & 21\end{array}$ $\begin{array}{llllll}889.4 & 713 \mathrm{~A} & 76.7 & 27.1 & 1.90 \mathrm{E}-02 & 25\end{array}$ $\begin{array}{lllllll}889.4 & 713 \mathrm{~A} & 76.1 & 26.1 & 1.65 \mathrm{E}-02 & 30\end{array}$ $\begin{array}{llllll}889.4 & 713 \mathrm{~A} & 54.9 & 72.3 & 1.12 \mathrm{E}-02 & 40\end{array}$ $\begin{array}{lllllll}889.4 & 713 \mathrm{~A} & 74.7 & 18 & 8.48 \mathrm{E}-03 & 50\end{array}$ $\begin{array}{llllll}889.4 & 713 \mathrm{~A} & 54.5 & 66.9 & 5.13 \mathrm{E}-03 & 60\end{array}$ $\begin{array}{lllllll}889.4 & 713 \mathrm{~A} & 75.3 & 25.7 & 3.65 \mathrm{E}-03 & 70\end{array}$ $\begin{array}{lllllll}889.4 & 713 \mathrm{~A} & 53.4 & 25.7 & 2.53 \mathrm{E}-03 & 85\end{array}$ $\begin{array}{lllllll}889.4 & 713 \mathrm{~A} & 55.5 & 45.4 & 1.98 \mathrm{E}-03 & 100\end{array}$ $\begin{array}{lllllll}889.4 & 713 \mathrm{~A} & 93.5 & 3.9 & 1.41 \mathrm{E}-03 & 115\end{array}$ $\begin{array}{llllll}893.4 & 714 \mathrm{~A} & 204.9 & 75.1 & 8.70 \mathrm{E}-02 & 0\end{array}$ $\begin{array}{llllll}893.4 & 714 \mathrm{~A} & 199.6 & 76 & 8.05 \mathrm{E}-02 & 3\end{array}$ $\begin{array}{lllllll}893.4 & 714 \mathrm{~A} & 107.6 & 43.2 & 7.44 \mathrm{E}-02 & 6\end{array}$ $\begin{array}{lllllll}893.4 & 714 \mathrm{~A} & 204.3 & 76.7 & 6.64 \mathrm{E}-02 & 9\end{array}$ $\begin{array}{llllll}893.4 & 714 \mathrm{~A} & 192.9 & 77.9 & 6.07 \mathrm{E}-02 & 12\end{array}$ $\begin{array}{llllll}893.4 & 714 \mathrm{~A} & 190 & 78.8 & 5.69 \mathrm{E}-02 & 15\end{array}$ $\begin{array}{llllll}893.4 & 714 \mathrm{~A} & 104.5 & 42 & 5.08 \mathrm{E}-02 & 18\end{array}$ $\begin{array}{llllll}893.4 & 714 \mathrm{~A} & 191.4 & 79.1 & 4.67 \mathrm{E}-02 & 21\end{array}$ $\begin{array}{llllll}893.4 & 714 \mathrm{~A} & 103.4 & 42 & 4.07 \mathrm{E}-02 & 25\end{array}$ $\begin{array}{llllll}893.4 & 714 \mathrm{~A} & 195.2 & 80.7 & 3.53 \mathrm{E}-02 & 30\end{array}$ $\begin{array}{llllll}893.4 & 714 \mathrm{~A} & 102 & 45.1 & 2.52 \mathrm{E}-02 & 40\end{array}$ $\begin{array}{lllllll}893.4 & 714 \mathrm{~A} & 214.5 & 80.1 & 1.82 \mathrm{E}-02 & 50\end{array}$ $\begin{array}{lllllll}893.4 & 714 \mathrm{~A} & 103.4 & 50.2 & 1.22 \mathrm{E}-02 & 60\end{array}$ $\begin{array}{lllllll}893.4 & 714 \mathrm{~A} & 235.6 & 78.5 & 8.49 \mathrm{E}-03 & 70\end{array}$ $\begin{array}{lllllll}893.4 & 714 \mathrm{~A} & 254.3 & 70.8 & 5.78 \mathrm{E}-03 & 85\end{array}$ $\begin{array}{lllllll}893.4 & 714 \mathrm{~A} & 98.8 & 47.1 & 3.25 \mathrm{E}-03 & 100\end{array}$ $\begin{array}{lllllll}893.4 & 714 \mathrm{~A} & 107.4 & 41.8 & 3.17 \mathrm{E}-03 & 115\end{array}$ $\begin{array}{lllllll}909.8 & 715 \mathrm{~A} & 6.4 & -58.5 & 9.04 \mathrm{E}-02 & 0\end{array}$ $\begin{array}{llllll}909.8 & 715 \mathrm{~A} & 312 & -34.7 & 7.61 \mathrm{E}-02 & 3\end{array}$ $\begin{array}{lllllll}909.8 & 715 \mathrm{~A} & 6 & -55.7 & 6.28 \mathrm{E}-02 & 6\end{array}$ $\begin{array}{llllll}909.8 & 715 \mathrm{~A} & 9.8 & -53.3 & 4.82 \mathrm{E}-02 & 9\end{array}$ $\begin{array}{llllll}909.8 & 715 \mathrm{~A} & 11.7 & -53.3 & 4.11 \mathrm{E}-02 & 12\end{array}$ $\begin{array}{llllll}909.8 & 715 \mathrm{~A} & 318.9 & -36.9 & 3.38 \mathrm{E}-02 & 15\end{array}$ $\begin{array}{lllllll}909.8 & 715 \mathrm{~A} & 318.4 & -38.4 & 2.93 \mathrm{E}-02 & 18\end{array}$ $\begin{array}{lllllll}909.8 & 715 \mathrm{~A} & 319.9 & -40.8 & 2.51 \mathrm{E}-02 & 21\end{array}$ $\begin{array}{llllll}909.8 & 715 \mathrm{~A} & 17.5 & -51.7 & 2.10 \mathrm{E}-02 & 25\end{array}$ $\begin{array}{llllll}909.8 & 715 \mathrm{~A} & 19 & -52.6 & 1.82 \mathrm{E}-02 & 30\end{array}$ $\begin{array}{lllllll}909.8 & 715 \mathrm{~A} & 33.4 & -50.8 & 1.03 \mathrm{E}-02 & 40\end{array}$ $\begin{array}{llllll}909.8 & 715 \mathrm{~A} & 25.5 & 47 & 6.62 \mathrm{E}-03 & 50\end{array}$ $\begin{array}{lllllll}909.8 & 715 \mathrm{~A} & 334.9 & -43.2 & 3.14 \mathrm{E}-03 & 60\end{array}$ $\begin{array}{llllll}909.8 & 715 \mathrm{~A} & 165.2 & -68 & 2.93 \mathrm{E}-03 & 70\end{array}$ $\begin{array}{llllll}909.8 & 715 \mathrm{~A} & 91 & -14.2 & 9.24 \mathrm{E}-04 & 85\end{array}$ $\begin{array}{lllllll}909.8 & 715 \mathrm{~A} & 113.9 & -37.1 & 2.89 \mathrm{E}-03 & 100\end{array}$ $\begin{array}{lllllll}909.8 & 715 \mathrm{~A} & 169 & -10.5 & 8.96 \mathrm{E}-04 & 115\end{array}$ $\begin{array}{llllll}915.3 & 159 \mathrm{~A} & 341.8 & 53.2 & 2.09 \mathrm{E}-01 & 0\end{array}$ $\begin{array}{llllll}915.3 & 159 \mathrm{~A} & 345.3 & 54 & 1.73 \mathrm{E}-01 & 3\end{array}$ $\begin{array}{llllll}915.3 & 159 \mathrm{~A} & 346.2 & 53.7 & 1.39 \mathrm{E}-01 & 6\end{array}$ $\begin{array}{llllll}915.3 & 159 \mathrm{~A} & 346.6 & 52.1 & 1.08 \mathrm{E}-01 & 9\end{array}$ $\begin{array}{llllll}915.3 & 159 \mathrm{~A} & 346.4 & 51.6 & 9.10 \mathrm{E}-02 & 12\end{array}$ $\begin{array}{lllllll}915.3 & 159 \mathrm{~A} & 347.3 & 51.7 & 7.71 \mathrm{E}-02 & 15\end{array}$ $\begin{array}{lllllll}915.3 & 159 \mathrm{~A} & 345.7 & 51.2 & 6.67 \mathrm{E}-02 & 18\end{array}$ $\begin{array}{llllll}915.3 & 159 \mathrm{~A} & 343.9 & 52.5 & 5.70 \mathrm{E}-02 & 21\end{array}$ $\begin{array}{lllllll}915.3 & 159 \mathrm{~A} & 342.5 & 53.4 & 4.88 \mathrm{E}-02 & 25\end{array}$ $\begin{array}{llllll}915.3 & 159 \mathrm{~A} & 344.1 & 55 & 4.08 \mathrm{E}-02 & 30\end{array}$ $\begin{array}{llllll}915.3 & 159 \mathrm{~A} & 336.9 & 55 & 2.63 \mathrm{E}-02 & 40\end{array}$ $\begin{array}{lllllll}915.3 & 159 \mathrm{~A} & 329.8 & 63.9 & 1.69 \mathrm{E}-02 & 50\end{array}$ $\begin{array}{lllllll}915.3 & 159 \mathrm{~A} & 308.3 & 73.9 & 1.27 \mathrm{E}-02 & 60\end{array}$ $\begin{array}{lllllll}915.3 & 159 \mathrm{~A} & 271.5 & 69.5 & 8.67 \mathrm{E}-03 & 70\end{array}$ $\begin{array}{llllll}915.3 & 159 \mathrm{~A} & 215.8 & 54 & 7.45 \mathrm{E}-03 & 85\end{array}$ $\begin{array}{lllllll}915.3 & 159 \mathrm{~A} & 256.8 & 66.7 & 5.70 \mathrm{E}-03 & 100\end{array}$ $\begin{array}{lllllll}915.3 & 159 \mathrm{~A} & 155.2 & 49.8 & 5.90 \mathrm{E}-03 & 115\end{array}$

\begin{tabular}{|c|c|c|c|c|c|}
\hline 919.8 & $160 \mathrm{~A}$ & 225.5 & 75 & $7.06 \mathrm{E}-02$ & 0 \\
\hline 919.8 & $160 \mathrm{~A}$ & 226.1 & 74.8 & $7.07 \mathrm{E}-02$ & 3 \\
\hline 919.8 & $160 \mathrm{~A}$ & 224.4 & 75.4 & $7.08 \mathrm{E}-02$ & 6 \\
\hline 919.8 & $160 \mathrm{~A}$ & 225.9 & 75.1 & $7.01 \mathrm{E}-02$ & 6 \\
\hline 919.8 & $160 \mathrm{~A}$ & 224.2 & 76.6 & $6.24 \mathrm{E}-02$ & 0 \\
\hline 919.8 & $160 \mathrm{~A}$ & 223.7 & 78.9 & $5.62 \mathrm{E}-02$ & 12 \\
\hline 919.8 & $160 A$ & 227 & 80.5 & $5.13 \mathrm{E}-02$ & 15 \\
\hline 919.8 & $160 A$ & 228.1 & 81 & $4.59 \mathrm{E}-02$ & 18 \\
\hline 919.8 & $160 \mathrm{~A}$ & 232.6 & 82.3 & $4.14 \mathrm{E}-02$ & 21 \\
\hline 919.8 & $160 \mathrm{~A}$ & 237.7 & 82 & $3.59 \mathrm{E}-02$ & 25 \\
\hline 919.8 & $160 \mathrm{~A}$ & 237.1 & 83.3 & $3.08 \mathrm{E}-02$ & 30 \\
\hline 919.8 & $160 \mathrm{~A}$ & 241.9 & 86.5 & $2.14 \mathrm{E}-02$ & 40 \\
\hline 919.8 & $160 \mathrm{~A}$ & 253.2 & 84.1 & $1.52 \mathrm{E}-02$ & 50 \\
\hline 919.8 & $160 \mathrm{~A}$ & 359.3 & 84.6 & $1.06 \mathrm{E}-02$ & 60 \\
\hline 919.8 & $160 \mathrm{~A}$ & 8.2 & 73.9 & $7.60 \mathrm{E}-03$ & 70 \\
\hline 919.8 & $160 \mathrm{~A}$ & 29.1 & 69 & $4.82 \mathrm{E}-03$ & 85 \\
\hline 919.8 & $160 \mathrm{~A}$ & 352.4 & 51.3 & $5.18 \mathrm{E}-03$ & 100 \\
\hline 919.8 & $160 \mathrm{~A}$ & 1.4 & 42.4 & $3.20 \mathrm{E}-03$ & 115 \\
\hline 929.6 & $716 \mathrm{~A}$ & 327.9 & 70.9 & $1.02 \mathrm{E}-01$ & 0 \\
\hline 929.6 & $716 \mathrm{~A}$ & 65.4 & 47.7 & $9.33 \mathrm{E}-02$ & 3 \\
\hline 929.6 & $716 \mathrm{~A}$ & 67 & 47.3 & $8.39 \mathrm{E}-02$ & 6 \\
\hline 929.6 & $716 \mathrm{~A}$ & 69.2 & 46.1 & $7.25 \mathrm{E}-02$ & 9 \\
\hline 929.6 & $716 \mathrm{~A}$ & 71.1 & 45.1 & $6.54 \mathrm{E}-02$ & 12 \\
\hline 929.6 & $716 \mathrm{~A}$ & 71.8 & 44.9 & $5.92 \mathrm{E}-02$ & 15 \\
\hline 929.6 & $716 \mathrm{~A}$ & 73.3 & 43.6 & $5.22 \mathrm{E}-02$ & 18 \\
\hline 929.6 & $716 \mathrm{~A}$ & 73.6 & 43.7 & 4.71E-02 & 21 \\
\hline 929.6 & $716 \mathrm{~A}$ & 74.5 & 43.6 & $4.10 \mathrm{E}-02$ & 25 \\
\hline 929.6 & $716 \mathrm{~A}$ & 341.7 & 78.8 & $3.41 \mathrm{E}-02$ & 30 \\
\hline 929.6 & $716 \mathrm{~A}$ & 77.7 & 43.5 & $2.37 \mathrm{E}-02$ & 40 \\
\hline 929.6 & $716 \mathrm{~A}$ & 80 & 45.3 & $1.58 \mathrm{E}-02$ & 50 \\
\hline 929.6 & $716 \mathrm{~A}$ & 81.7 & 49.9 & $8.97 \mathrm{E}-03$ & 60 \\
\hline 929.6 & $716 \mathrm{~A}$ & 82.7 & 47.4 & $5.54 \mathrm{E}-03$ & 70 \\
\hline 929.6 & $716 \mathrm{~A}$ & 89.2 & 33.5 & $2.71 \mathrm{E}-03$ & 85 \\
\hline 929.6 & $716 \mathrm{~A}$ & 61.9 & 66.6 & $2.09 \mathrm{E}-03$ & 100 \\
\hline 929.6 & $716 \mathrm{~A}$ & 39.3 & -10.2 & $1.84 \mathrm{E}-03$ & 115 \\
\hline 931.1 & $717 \mathrm{~A}$ & 186.5 & 71 & $8.38 \mathrm{E}-02$ & 0 \\
\hline 931.1 & $717 \mathrm{~A}$ & 186.6 & 67.5 & $7.16 \mathrm{E}-02$ & 3 \\
\hline 931.1 & $717 \mathrm{~A}$ & 187.9 & 65.3 & $5.98 \mathrm{E}-02$ & 6 \\
\hline 931.1 & $717 \mathrm{~A}$ & 189.4 & 63.8 & $4.82 \mathrm{E}-02$ & 9 \\
\hline 931.1 & $717 \mathrm{~A}$ & 191.7 & 62.8 & $4.05 E-02$ & 12 \\
\hline 931.1 & $717 \mathrm{~A}$ & 192.7 & 62.4 & $3.50 \mathrm{E}-02$ & 15 \\
\hline 931.1 & $717 \mathrm{~A}$ & 192.1 & 62.9 & $2.97 \mathrm{E}-02$ & 18 \\
\hline 931.1 & $717 \mathrm{~A}$ & 187.2 & 59.9 & $2.60 \mathrm{E}-02$ & 21 \\
\hline 931.1 & $717 \mathrm{~A}$ & 186.9 & 60.3 & $2.15 \mathrm{E}-02$ & 25 \\
\hline 931.1 & $717 \mathrm{~A}$ & 196.5 & 61.9 & $1.89 \mathrm{E}-02$ & 30 \\
\hline 931.1 & $717 \mathrm{~A}$ & 197.3 & 57.8 & $1.28 \mathrm{E}-02$ & 40 \\
\hline 931.1 & $717 \mathrm{~A}$ & 228.4 & 59.5 & $9.30 \mathrm{E}-03$ & 50 \\
\hline 931.1 & $717 \mathrm{~A}$ & 246.8 & 50.8 & $4.96 \mathrm{E}-03$ & 60 \\
\hline 931.1 & $717 \mathrm{~A}$ & 233.9 & 39.5 & $3.28 \mathrm{E}-03$ & 70 \\
\hline 931.1 & $717 \mathrm{~A}$ & 263.5 & -0.3 & $2.27 \mathrm{E}-03$ & 85 \\
\hline 931.1 & $717 \mathrm{~A}$ & 3.8 & -17.7 & $1.69 \mathrm{E}-03$ & 100 \\
\hline
\end{tabular}


$\begin{array}{llllll}931.1 & 717 \mathrm{~A} & 244.9 & 31.4 & 3.12 \mathrm{E}-03 & 115\end{array}$ $\begin{array}{llllll}942.9 & 161 \mathrm{~A} & 341.4 & 79.6 & 6.36 \mathrm{E}-02 & 0\end{array}$ $\begin{array}{lllllll}942.9 & 161 \mathrm{~A} & 354.2 & 81 & 5.85 \mathrm{E}-02 & 3\end{array}$ $\begin{array}{lllllll}942.9 & 161 \mathrm{~A} & 357 & 82.3 & 5.37 \mathrm{E}-02 & 6\end{array}$ $\begin{array}{llllll}942.9 & 161 \mathrm{~A} & 357.5 & 82.9 & 4.78 \mathrm{E}-02 & 9\end{array}$ $\begin{array}{lllllll}942.9 & 161 \mathrm{~A} & 358 & 83.3 & 4.31 \mathrm{E}-02 & 12\end{array}$ $\begin{array}{lllllll}942.9 & 161 \mathrm{~A} & 358 & 83.4 & 3.96 \mathrm{E}-02 & 15\end{array}$ $\begin{array}{lllllll}942.9 & 161 \mathrm{~A} & 7.2 & 83.8 & 3.51 \mathrm{E}-02 & 18\end{array}$ $\begin{array}{lllllll}942.9 & 161 \mathrm{~A} & 10 & 84.7 & 3.09 \mathrm{E}-02 & 21\end{array}$ $\begin{array}{llllll}942.9 & 161 \mathrm{~A} & 10 & 85.1 & 2.59 \mathrm{E}-02 & 25\end{array}$ $\begin{array}{llllll}942.9 & 161 \mathrm{~A} & 24.6 & 85.3 & 2.09 \mathrm{E}-02 & 30\end{array}$ $\begin{array}{llllll}942.9 & 161 \mathrm{~A} & 27 & 85 & 1.37 \mathrm{E}-02 & 40\end{array}$ $\begin{array}{lllllll}942.9 & 161 \mathrm{~A} & 332.8 & 84.6 & 8.57 \mathrm{E}-03 & 50\end{array}$ $\begin{array}{lllllll}942.9 & 161 \mathrm{~A} & 120 & 81.5 & 5.24 \mathrm{E}-03 & 60\end{array}$ $\begin{array}{lllllll}942.9 & 161 \mathrm{~A} & 278.9 & 82.7 & 4.11 \mathrm{E}-03 & 70\end{array}$ $\begin{array}{lllllll}942.9 & 161 \mathrm{~A} & 152.5 & 79.3 & 2.65 \mathrm{E}-03 & 85\end{array}$ $\begin{array}{lllllll}942.9 & 161 \mathrm{~A} & 136.7 & 71.8 & 3.48 \mathrm{E}-03 & 100\end{array}$ $\begin{array}{lllllll}942.9 & 161 \mathrm{~A} & 276.8 & 75.4 & 2.54 \mathrm{E}-03 & 115\end{array}$ $\begin{array}{llllll}944 & 162 \mathrm{~A} & 51.3 & 69.3 & 3.79 \mathrm{E}-02 & 0\end{array}$ $\begin{array}{llllll}944 & 162 \mathrm{~A} & 60.1 & 67.6 & 3.37 \mathrm{E}-02 & 3\end{array}$ $\begin{array}{llllll}944 & 162 \mathrm{~A} & 61 & 68.1 & 2.88 \mathrm{E}-02 & 6\end{array}$ $\begin{array}{llllll}944 & 162 \mathrm{~A} & 59.1 & 67.4 & 2.27 \mathrm{E}-02 & 9\end{array}$ $\begin{array}{llllll}944 & 162 \mathrm{~A} & 59.7 & 67.6 & 1.87 \mathrm{E}-02 & 12\end{array}$ $\begin{array}{llllll}944 & 162 \mathrm{~A} & 54.9 & 69.1 & 1.60 \mathrm{E}-02 & 15\end{array}$ $\begin{array}{lllllll}944 & 162 \mathrm{~A} & 55.3 & 71.4 & 1.29 \mathrm{E}-02 & 18\end{array}$ $\begin{array}{llllll}944 & 162 \mathrm{~A} & 50.1 & 70.9 & 1.04 \mathrm{E}-02 & 21\end{array}$ $\begin{array}{lllllll}944 & 162 \mathrm{~A} & 49.1 & 69.2 & 8.16 \mathrm{E}-03 & 25\end{array}$ $\begin{array}{lllllll}944 & 162 \mathrm{~A} & 36.3 & 80.7 & 7.29 \mathrm{E}-03 & 30\end{array}$ $\begin{array}{lllllll}944 & 162 \mathrm{~A} & 339.7 & 78.1 & 3.40 \mathrm{E}-03 & 40\end{array}$ $\begin{array}{llllll}944 & 162 \mathrm{~A} & 283.6 & 67.3 & 2.66 \mathrm{E}-03 & 50\end{array}$ $\begin{array}{lllllll}944 & 162 \mathrm{~A} & 295.2 & -4.8 & 2.39 \mathrm{E}-03 & 60\end{array}$ $\begin{array}{lllllll}944 & 162 \mathrm{~A} & 228.9 & -70.4 & 1.89 \mathrm{E}-03 & 70\end{array}$ $\begin{array}{lllllll}944 & 162 \mathrm{~A} & 317.8 & -57.2 & 2.17 \mathrm{E}-03 & 85\end{array}$ $\begin{array}{lllllll}944 & 162 \mathrm{~A} & 258.8 & -82.1 & 2.40 \mathrm{E}-03 & 100\end{array}$ $\begin{array}{lllllll}944 & 162 \mathrm{~A} & 193.1 & -52.6 & 1.87 \mathrm{E}-03 & 115\end{array}$ $\begin{array}{llllll}1037.3 & 165 \mathrm{~A} & 135.8 & 39.5 & 3.88 \mathrm{E}-02 & 0\end{array}$ $\begin{array}{llllll}1037.3 & 165 \mathrm{~A} & 129.2 & 32 & 3.51 \mathrm{E}-02 & 3\end{array}$ $\begin{array}{lllllll}1037.3 & 165 \mathrm{~A} & 129.7 & 22.4 & 3.57 \mathrm{E}-02 & 6\end{array}$ $\begin{array}{llllll}1037.3 & 165 \mathrm{~A} & 130.1 & 13.4 & 3.60 \mathrm{E}-02 & 9\end{array}$ $\begin{array}{llllll}1037.3 & 165 \mathrm{~A} & 130.6 & 8.8 & 3.59 \mathrm{E}-02 & 12\end{array}$ $\begin{array}{llllll}1037.3 & 165 \mathrm{~A} & 131.4 & 6.2 & 3.48 \mathrm{E}-02 & 15\end{array}$ $\begin{array}{lllllll}1037.3 & 165 \mathrm{~A} & 131 & 4.5 & 3.33 \mathrm{E}-02 & 18\end{array}$ $\begin{array}{llllll}1037.3 & 165 \mathrm{~A} & 132.3 & 3.1 & 3.05 \mathrm{E}-02 & 21\end{array}$ $\begin{array}{lllllll}1037.3 & 165 \mathrm{~A} & 133 & 2.6 & 2.75 \mathrm{E}-02 & 25\end{array}$ $\begin{array}{llllll}1037.3 & 165 \mathrm{~A} & 129.7 & 3 & 2.39 \mathrm{E}-02 & 30\end{array}$ $\begin{array}{llllll}1037.3 & 165 \mathrm{~A} & 133.3 & -3.3 & 1.97 \mathrm{E}-02 & 40\end{array}$ $\begin{array}{llllll}1037.3 & 165 \mathrm{~A} & 128.3 & -5 & 1.45 \mathrm{E}-02 & 50\end{array}$ $\begin{array}{lllllll}1037.3 & 165 \mathrm{~A} & 126.2 & -10.8 & 1.24 \mathrm{E}-02 & 60\end{array}$ $\begin{array}{lllllll}1037.3 & 165 \mathrm{~A} & 138.7 & -12.2 & 1.26 \mathrm{E}-02 & 70\end{array}$ $\begin{array}{lllllll}1037.3 & 165 \mathrm{~A} & 120.6 & -20.5 & 1.06 \mathrm{E}-02 & 85\end{array}$ $\begin{array}{lllllll}1037.3 & 165 \mathrm{~A} & 132.6 & -16 & 1.22 \mathrm{E}-02 & 100\end{array}$ $\begin{array}{llllll}1037.3 & 165 \mathrm{~A} & 136.2 & -14.9 & 1.11 \mathrm{E}-02 & 115\end{array}$ $\begin{array}{lllllll}1037.5 & 166 \mathrm{~A} & 321.1 & 59.7 & 7.46 \mathrm{E}-02 & 0\end{array}$ $\begin{array}{llllll}1037.5 & 166 \mathrm{~A} & 327.6 & 57.4 & 6.81 \mathrm{E}-02 & 3\end{array}$ $\begin{array}{lllllll}1037.5 & 166 \mathrm{~A} & 328.5 & 56.7 & 5.88 \mathrm{E}-02 & 6\end{array}$ $\begin{array}{llllll}1037.5 & 166 \mathrm{~A} & 328.9 & 55.6 & 4.96 \mathrm{E}-02 & 9\end{array}$ $\begin{array}{llllll}1037.5 & 166 \mathrm{~A} & 329.8 & 55.4 & 4.29 \mathrm{E}-02 & 12\end{array}$ $\begin{array}{llllll}1037.5 & 166 \mathrm{~A} & 330.5 & 55.4 & 3.76 \mathrm{E}-02 & 15\end{array}$ $\begin{array}{llllll}1037.5 & 166 \mathrm{~A} & 330.5 & 54.6 & 2.96 \mathrm{E}-02 & 21\end{array}$ $\begin{array}{llllll}1037.5 & 166 \mathrm{~A} & 329.2 & 53.6 & 2.50 \mathrm{E}-02 & 25\end{array}$ $\begin{array}{llllll}1037.5 & 166 \mathrm{~A} & 332 & 55 & 2.05 \mathrm{E}-02 & 30\end{array}$ $\begin{array}{llllll}1037.5 & 166 \mathrm{~A} & 339.3 & 58 & 1.53 \mathrm{E}-02 & 40\end{array}$ $\begin{array}{lllllll}1037.5 & 166 \mathrm{~A} & 332.1 & 59.1 & 8.69 \mathrm{E}-03 & 50\end{array}$ $\begin{array}{lllllll}1037.5 & 166 \mathrm{~A} & 349.5 & 50.1 & 5.63 \mathrm{E}-03 & 60\end{array}$ $\begin{array}{lllllll}1037.5 & 166 \mathrm{~A} & 13.1 & 58.3 & 4.63 \mathrm{E}-03 & 70\end{array}$ $\begin{array}{lllllll}1037.5 & 166 \mathrm{~A} & 61.8 & 7.9 & 1.32 \mathrm{E}-03 & 85\end{array}$ $\begin{array}{lllllll}1037.5 & 166 \mathrm{~A} & 32.9 & 10.8 & 3.03 \mathrm{E}-03 & 100\end{array}$ $\begin{array}{lllllll}1037.5 & 166 \mathrm{~A} & 90.2 & -26.6 & 1.31 \mathrm{E}-03 & 115\end{array}$ $\begin{array}{llllll}1037.6 & 167 \mathrm{~A} & 310.6 & 68.8 & 6.40 \mathrm{E}-02 & 0\end{array}$ $\begin{array}{llllll}1037.6 & 167 \mathrm{~A} & 319.7 & 65.2 & 5.79 \mathrm{E}-02 & 3\end{array}$ $\begin{array}{lllllll}1037.6 & 167 \mathrm{~A} & 320.7 & 64.7 & 5.00 \mathrm{E}-02 & 6\end{array}$ $\begin{array}{lllllll}1037.6 & 167 \mathrm{~A} & 321 & 64.4 & 4.12 \mathrm{E}-02 & 9\end{array}$ $\begin{array}{lllllll}1037.6 & 167 \mathrm{~A} & 322.9 & 64.6 & 3.57 \mathrm{E}-02 & 12\end{array}$ $\begin{array}{llllll}1037.6 & 167 \mathrm{~A} & 321.5 & 64.6 & 3.10 \mathrm{E}-02 & 15\end{array}$ $\begin{array}{llllll}1037.6 & 167 \mathrm{~A} & 325.9 & 65.3 & 2.73 \mathrm{E}-02 & 18\end{array}$ $\begin{array}{lllllll}1037.6 & 167 \mathrm{~A} & 323.5 & 65.6 & 2.33 \mathrm{E}-02 & 21\end{array}$ $\begin{array}{llllll}1037.6 & 167 \mathrm{~A} & 325.5 & 66.6 & 2.03 \mathrm{E}-02 & 25\end{array}$ $\begin{array}{lllllll}1037.6 & 167 \mathrm{~A} & 326.6 & 67.6 & 1.81 \mathrm{E}-02 & 30\end{array}$ $\begin{array}{lllllll}1037.6 & 167 \mathrm{~A} & 327.8 & 67.1 & 1.09 \mathrm{E}-02 & 40\end{array}$ $\begin{array}{lllllll}1037.6 & 167 \mathrm{~A} & 358.3 & 75.9 & 7.17 \mathrm{E}-03 & 50\end{array}$ $\begin{array}{llllll}1037.6 & 167 \mathrm{~A} & 141.3 & 71.7 & 2.96 \mathrm{E}-03 & 60\end{array}$ $\begin{array}{llllll}1037.6 & 167 \mathrm{~A} & 153 & 24.4 & 2.32 \mathrm{E}-03 & 70\end{array}$ $\begin{array}{llllll}1037.6 & 167 \mathrm{~A} & 163.6 & 45.1 & 2.77 \mathrm{E}-03 & 85\end{array}$ $\begin{array}{lllllll}1037.6 & 167 \mathrm{~A} & 165.2 & 18 & 2.56 \mathrm{E}-03 & 100\end{array}$ $\begin{array}{lllllll}1037.6 & 167 \mathrm{~A} & 164.6 & -2.4 & 3.08 \mathrm{E}-03 & 115\end{array}$ $\begin{array}{llllll}1043.2 & 168 \mathrm{~A} & 144.1 & 61.3 & 9.55 \mathrm{E}-02 & 0\end{array}$ $\begin{array}{llllll}1043.2 & 168 \mathrm{~A} & 142.4 & 58 & 7.89 \mathrm{E}-02 & 3\end{array}$ $\begin{array}{llllll}1043.2 & 168 \mathrm{~A} & 142 & 56.7 & 5.95 \mathrm{E}-02 & 6\end{array}$ $\begin{array}{llllll}1043.2 & 168 \mathrm{~A} & 142 & 55.7 & 4.47 \mathrm{E}-02 & 9\end{array}$ $\begin{array}{lllllll}1043.2 & 168 \mathrm{~A} & 143.3 & 56.1 & 3.59 \mathrm{E}-02 & 12\end{array}$ $\begin{array}{llllll}1043.2 & 168 \mathrm{~A} & 143.7 & 56 & 2.94 \mathrm{E}-02 & 15\end{array}$ $\begin{array}{llllll}1043.2 & 168 \mathrm{~A} & 142.1 & 57.4 & 2.40 \mathrm{E}-02 & 18\end{array}$ $\begin{array}{llllll}1043.2 & 168 \mathrm{~A} & 141 & 55.4 & 1.89 \mathrm{E}-02 & 21\end{array}$ $\begin{array}{lllllll}1043.2 & 168 \mathrm{~A} & 136 & 60.9 & 1.46 \mathrm{E}-02 & 25\end{array}$ $\begin{array}{llllll}1043.2 & 168 \mathrm{~A} & 139.9 & 64.8 & 1.17 \mathrm{E}-02 & 30\end{array}$ $\begin{array}{lllllll}1043.2 & 168 \mathrm{~A} & 109.8 & 63.2 & 4.56 \mathrm{E}-03 & 40\end{array}$ $\begin{array}{llllll}1043.2 & 168 \mathrm{~A} & 76.7 & 55.3 & 2.13 \mathrm{E}-03 & 50\end{array}$ $\begin{array}{llllll}1043.2 & 168 \mathrm{~A} & 305.8 & 66.6 & 2.01 \mathrm{E}-03 & 60\end{array}$ $\begin{array}{llllll}1043.2 & 168 \mathrm{~A} & 332.1 & 35.6 & 1.41 \mathrm{E}-03 & 70\end{array}$ $\begin{array}{lllllll}1043.2 & 168 \mathrm{~A} & 19.8 & -59.3 & 1.58 \mathrm{E}-03 & 85\end{array}$ $\begin{array}{llllll}1043.2 & 168 \mathrm{~A} & 3.3 & 80 & 2.21 \mathrm{E}-03 & 100\end{array}$ $\begin{array}{lllllll}1043.2 & 168 \mathrm{~A} & 4.7 & 31.3 & 2.39 \mathrm{E}-03 & 115\end{array}$ $\begin{array}{llllll}1047.8 & 169 \mathrm{~A} & 215.1 & 61.5 & 4.20 \mathrm{E}-02 & 0\end{array}$ $\begin{array}{lllllll}1047.8 & 169 \mathrm{~A} & 219.4 & 62.6 & 3.39 \mathrm{E}-02 & 3\end{array}$ $\begin{array}{llllll}1047.8 & 169 \mathrm{~A} & 222.6 & 61.3 & 2.66 \mathrm{E}-02 & 6\end{array}$ $\begin{array}{llllll}1047.8 & 169 \mathrm{~A} & 219.6 & 60.4 & 2.10 \mathrm{E}-02 & 9\end{array}$ $\begin{array}{llllll}1047.8 & 169 \mathrm{~A} & 219.5 & 60.4 & 1.80 \mathrm{E}-02 & 12\end{array}$ $\begin{array}{lllllll}1047.8 & 169 \mathrm{~A} & 220.6 & 59.7 & 1.56 \mathrm{E}-02 & 15\end{array}$ $\begin{array}{llllll}1047.8 & 169 \mathrm{~A} & 219.3 & 60.3 & 1.28 \mathrm{E}-02 & 18\end{array}$ $\begin{array}{lllllll}1047.8 & 169 \mathrm{~A} & 225.5 & 59.1 & 1.11 \mathrm{E}-02 & 21\end{array}$ $\begin{array}{lllllll}1047.8 & 169 \mathrm{~A} & 223.6 & 60.1 & 9.66 \mathrm{E}-03 & 25\end{array}$ $\begin{array}{lllllll}1047.8 & 169 \mathrm{~A} & 221.3 & 63.9 & 8.92 \mathrm{E}-03 & 30\end{array}$ $\begin{array}{lllllll}1047.8 & 169 \mathrm{~A} & 213.1 & 61.8 & 5.27 \mathrm{E}-03 & 40\end{array}$ $\begin{array}{lllllll}1047.8 & 169 \mathrm{~A} & 274.8 & 64.8 & 2.43 \mathrm{E}-03 & 50\end{array}$ $\begin{array}{lllllll}1047.8 & 169 \mathrm{~A} & 45.5 & 49.3 & 1.22 \mathrm{E}-03 & 60\end{array}$ $\begin{array}{lllllll}1047.8 & 169 \mathrm{~A} & 242.5 & 44.1 & 1.65 \mathrm{E}-03 & 70\end{array}$ $\begin{array}{llllll}1047.8 & 169 \mathrm{~A} & 29.5 & 17.1 & 1.38 \mathrm{E}-03 & 85\end{array}$ $\begin{array}{lllllll}1047.8 & 169 \mathrm{~A} & 347.1 & 32.6 & 4.38 \mathrm{E}-04 & 100\end{array}$ $\begin{array}{lllllll}1047.8 & 169 \mathrm{~A} & 325.4 & 10.8 & 1.39 \mathrm{E}-03 & 115\end{array}$ $\begin{array}{llllll}1054.1 & 170 \mathrm{~A} & 342.9 & 60.8 & 3.75 \mathrm{E}-02 & 0\end{array}$ $\begin{array}{llllll}1054.1 & 170 \mathrm{~A} & 345.3 & 61.8 & 3.33 \mathrm{E}-02 & 3\end{array}$ $\begin{array}{llllll}1054.1 & 170 \mathrm{~A} & 345.1 & 62 & 2.84 \mathrm{E}-02 & 6\end{array}$ $\begin{array}{llllll}1054.1 & 170 \mathrm{~A} & 344.7 & 61.6 & 2.43 \mathrm{E}-02 & 9\end{array}$ $\begin{array}{llllll}1054.1 & 170 \mathrm{~A} & 343.7 & 61.7 & 2.12 \mathrm{E}-02 & 12\end{array}$ $\begin{array}{llllll}1054.1 & 170 \mathrm{~A} & 346.2 & 61.8 & 1.84 \mathrm{E}-02 & 15\end{array}$ $\begin{array}{lllllll}1054.1 & 170 \mathrm{~A} & 345.4 & 63 & 1.67 \mathrm{E}-02 & 18\end{array}$ $\begin{array}{lllllll}1054.1 & 170 \mathrm{~A} & 348.2 & 61.1 & 1.44 \mathrm{E}-02 & 21\end{array}$ $\begin{array}{lllllll}1054.1 & 170 \mathrm{~A} & 342.6 & 60.9 & 1.26 \mathrm{E}-02 & 25\end{array}$ $\begin{array}{lllllll}1054.1 & 170 \mathrm{~A} & 350.4 & 62.6 & 1.07 \mathrm{E}-02 & 30\end{array}$ $\begin{array}{lllllll}1054.1 & 170 \mathrm{~A} & 337 & 66.4 & 6.75 \mathrm{E}-03 & 40\end{array}$ $\begin{array}{lllllll}1054.1 & 170 \mathrm{~A} & 295 & 74.6 & 3.57 \mathrm{E}-03 & 50\end{array}$ $\begin{array}{llllll}1054.1 & 170 \mathrm{~A} & 49.9 & 69.4 & 1.60 \mathrm{E}-03 & 60\end{array}$ $\begin{array}{llllll}1054.1 & 170 \mathrm{~A} & 170.4 & 23.2 & 1.03 \mathrm{E}-03 & 70\end{array}$ $\begin{array}{lllllll}1054.1 & 170 \mathrm{~A} & 222.6 & -45.3 & 4.63 \mathrm{E}-04 & 85\end{array}$ $\begin{array}{llllll}1054.1 & 170 \mathrm{~A} & 77.8 & 60 & 1.69 \mathrm{E}-03 & 100\end{array}$ $\begin{array}{lllllll}1054.1 & 170 \mathrm{~A} & 82.6 & 36.3 & 1.18 \mathrm{E}-03 & 115\end{array}$ $\begin{array}{lllllll}1056.1 & 171 \mathrm{~A} & 333.3 & 67.4 & 4.57 \mathrm{E}-02 & 0\end{array}$ $\begin{array}{llllll}1056.1 & 171 \mathrm{~A} & 340 & 66 & 3.82 \mathrm{E}-02 & 3\end{array}$ $\begin{array}{llllll}1056.1 & 171 \mathrm{~A} & 342 & 63.6 & 2.99 \mathrm{E}-02 & 6\end{array}$ $\begin{array}{llllll}1056.1 & 171 \mathrm{~A} & 343.8 & 61.1 & 2.30 \mathrm{E}-02 & 9\end{array}$ $\begin{array}{llllll}1056.1 & 171 \mathrm{~A} & 345.7 & 59 & 2.00 \mathrm{E}-02 & 12\end{array}$ $\begin{array}{lllllll}1056.1 & 171 \mathrm{~A} & 347 & 57.6 & 1.66 \mathrm{E}-02 & 15\end{array}$ $\begin{array}{llllll}1056.1 & 171 \mathrm{~A} & 347.1 & 57.1 & 1.49 \mathrm{E}-02 & 18\end{array}$ $\begin{array}{lllllll}1056.1 & 171 \mathrm{~A} & 350.3 & 54.4 & 1.27 \mathrm{E}-02 & 21\end{array}$ $\begin{array}{lllllll}1056.1 & 171 \mathrm{~A} & 348.8 & 53.4 & 1.09 \mathrm{E}-02 & 25\end{array}$ $\begin{array}{llllll}1056.1 & 171 \mathrm{~A} & 352.2 & 51 & 9.21 \mathrm{E}-03 & 30\end{array}$ $\begin{array}{llllll}1056.1 & 171 \mathrm{~A} & 356.6 & 50.8 & 5.59 \mathrm{E}-03 & 40\end{array}$ $\begin{array}{llllll}1056.1 & 171 \mathrm{~A} & 17.1 & 24.9 & 2.85 \mathrm{E}-03 & 50\end{array}$ $\begin{array}{llllll}1056.1 & 171 \mathrm{~A} & 48.9 & 12.2 & 2.58 \mathrm{E}-03 & 60\end{array}$ $\begin{array}{lllllll}1056.1 & 171 \mathrm{~A} & 24.4 & 16.1 & 2.40 \mathrm{E}-03 & 70\end{array}$ $\begin{array}{lllllll}1056.1 & 171 \mathrm{~A} & 47.1 & 7.1 & 2.55 \mathrm{E}-03 & 85\end{array}$ $\begin{array}{lllllll}1056.1 & 171 \mathrm{~A} & 1.8 & 8.3 & 1.67 \mathrm{E}-03 & 100\end{array}$ $\begin{array}{lllllll}1056.1 & 171 \mathrm{~A} & 110.4 & 16 & 1.17 \mathrm{E}-03 & 115\end{array}$ 
$\begin{array}{llllll}1058.1 & 172 \mathrm{~A} & 317.5 & 56.5 & 3.32 \mathrm{E}-02 & 0\end{array}$ $\begin{array}{lllllll}1058.1 & 172 \mathrm{~A} & 317.8 & 55.5 & 2.90 \mathrm{E}-02 & 3\end{array}$ $\begin{array}{lllllll}1058.1 & 172 \mathrm{~A} & 317.4 & 55.2 & 2.52 \mathrm{E}-02 & 6\end{array}$ $\begin{array}{lllllll}1058.1 & 172 \mathrm{~A} & 316 & 54.3 & 2.10 \mathrm{E}-02 & 9\end{array}$ $\begin{array}{lllllll}1058.1 & 172 \mathrm{~A} & 317.7 & 54.5 & 1.80 \mathrm{E}-02 & 12\end{array}$ $\begin{array}{lllllll}1058.1 & 172 \mathrm{~A} & 320.6 & 55.5 & 1.59 \mathrm{E}-02 & 15\end{array}$ $\begin{array}{llllll}1058.1 & 172 \mathrm{~A} & 318.6 & 55.3 & 1.37 \mathrm{E}-02 & 18\end{array}$ $\begin{array}{lllllll}1058.1 & 172 \mathrm{~A} & 318.4 & 54.9 & 1.23 \mathrm{E}-02 & 21\end{array}$ $\begin{array}{llllll}1058.1 & 172 \mathrm{~A} & 320.7 & 54.2 & 1.05 \mathrm{E}-02 & 25\end{array}$ $\begin{array}{lllllll}1058.1 & 172 \mathrm{~A} & 324.3 & 56.3 & 8.69 \mathrm{E}-03 & 30\end{array}$ $\begin{array}{llllll}1058.1 & 172 \mathrm{~A} & 322.4 & 55.4 & 5.89 \mathrm{E}-03 & 40\end{array}$ $\begin{array}{lllllll}1058.1 & 172 \mathrm{~A} & 358 & 63.2 & 3.90 \mathrm{E}-03 & 50\end{array}$ $\begin{array}{llllll}1058.1 & 172 \mathrm{~A} & 7.4 & 77 & 2.49 \mathrm{E}-03 & 60\end{array}$ $\begin{array}{llllll}1058.1 & 172 \mathrm{~A} & 111.4 & 53.2 & 1.85 \mathrm{E}-03 & 70\end{array}$ $\begin{array}{llllll}1058.1 & 172 \mathrm{~A} & 104.2 & 32.1 & 1.66 \mathrm{E}-03 & 85\end{array}$ $\begin{array}{lllllll}1058.1 & 172 \mathrm{~A} & 91.5 & 23.6 & 9.68 \mathrm{E}-04 & 100\end{array}$ $\begin{array}{llllll}1058.1 & 172 \mathrm{~A} & 131.7 & -76.2 & 2.87 \mathrm{E}-04 & 115\end{array}$ $\begin{array}{llllll}1062.8 & 173 \mathrm{~A} & 20.9 & 64.2 & 2.53 \mathrm{E}-02 & 0\end{array}$ $\begin{array}{lllllll}1062.8 & 173 \mathrm{~A} & 31.7 & 63.8 & 2.08 \mathrm{E}-02 & 3\end{array}$ $\begin{array}{lllllll}1062.8 & 173 \mathrm{~A} & 45.4 & 61.2 & 1.62 \mathrm{E}-02 & 6\end{array}$ $\begin{array}{lllllll}1062.8 & 173 \mathrm{~A} & 54.2 & 55.2 & 1.32 \mathrm{E}-02 & 9\end{array}$ $\begin{array}{llllll}1062.8 & 173 \mathrm{~A} & 55.5 & 52.9 & 1.18 \mathrm{E}-02 & 12\end{array}$ $\begin{array}{llllll}1062.8 & 173 \mathrm{~A} & 53 & 51.6 & 1.04 \mathrm{E}-02 & 15\end{array}$ $\begin{array}{llllll}1062.8 & 173 \mathrm{~A} & 56.6 & 46.8 & 9.01 \mathrm{E}-03 & 18\end{array}$ $\begin{array}{lllllll}1062.8 & 173 \mathrm{~A} & 51.4 & 44 & 7.78 \mathrm{E}-03 & 21\end{array}$ $\begin{array}{llllll}1062.8 & 173 \mathrm{~A} & 43.6 & 39.2 & 6.81 \mathrm{E}-03 & 25\end{array}$ $\begin{array}{lllllll}1062.8 & 173 \mathrm{~A} & 43.5 & 32.7 & 4.93 \mathrm{E}-03 & 30\end{array}$ $\begin{array}{lllllll}1062.8 & 173 \mathrm{~A} & 34 & 15.5 & 4.80 \mathrm{E}-03 & 40\end{array}$ $\begin{array}{lllllll}1062.8 & 173 \mathrm{~A} & 25.3 & 8 & 3.01 \mathrm{E}-03 & 50\end{array}$ $\begin{array}{lllllll}1062.8 & 173 \mathrm{~A} & 25.3 & -6.4 & 2.89 \mathrm{E}-03 & 60\end{array}$ $\begin{array}{lllllll}1062.8 & 173 \mathrm{~A} & 16.3 & 21.2 & 3.60 \mathrm{E}-03 & 70\end{array}$ $\begin{array}{llllll}1062.8 & 173 \mathrm{~A} & 32.4 & 3.3 & 3.09 \mathrm{E}-03 & 85\end{array}$ $\begin{array}{lllllll}1062.8 & 173 \mathrm{~A} & 12.1 & 18.9 & 2.60 \mathrm{E}-03 & 100\end{array}$ $\begin{array}{llllll}1062.8 & 173 \mathrm{~A} & 10.1 & 11.2 & 3.12 \mathrm{E}-03 & 115\end{array}$ $\begin{array}{lllllll}1064.7 & 174 \mathrm{~A} & 272.5 & 56.3 & 8.64 \mathrm{E}-02 & 0\end{array}$ $\begin{array}{lllllll}1064.7 & 174 \mathrm{~A} & 270.8 & 54.9 & 7.53 \mathrm{E}-02 & 3\end{array}$ $\begin{array}{llllll}1064.7 & 174 \mathrm{~A} & 269.8 & 54.4 & 6.34 \mathrm{E}-02 & 6\end{array}$ $\begin{array}{llllll}1064.7 & 174 \mathrm{~A} & 270.1 & 53.7 & 5.15 \mathrm{E}-02 & 9\end{array}$ $\begin{array}{llllll}1064.7 & 174 \mathrm{~A} & 271.1 & 54.8 & 4.40 \mathrm{E}-02 & 12\end{array}$ $\begin{array}{llllll}1064.7 & 174 \mathrm{~A} & 270.4 & 55.2 & 3.83 \mathrm{E}-02 & 15\end{array}$ $\begin{array}{llllll}1064.7 & 174 \mathrm{~A} & 270.9 & 55.1 & 3.35 \mathrm{E}-02 & 18\end{array}$ $\begin{array}{llllll}1064.7 & 174 \mathrm{~A} & 265.9 & 53.1 & 2.49 \mathrm{E}-02 & 25\end{array}$ $\begin{array}{llllll}1064.7 & 174 \mathrm{~A} & 266.1 & 50.7 & 2.10 \mathrm{E}-02 & 30\end{array}$ $\begin{array}{llllll}1064.7 & 174 \mathrm{~A} & 265.5 & 58.2 & 1.34 \mathrm{E}-02 & 40\end{array}$ $\begin{array}{lllllll}1064.7 & 174 \mathrm{~A} & 246.4 & 63.3 & 6.99 \mathrm{E}-03 & 50\end{array}$ $\begin{array}{lllllll}1064.7 & 174 \mathrm{~A} & 349.1 & 82.1 & 4.17 \mathrm{E}-03 & 60\end{array}$ $\begin{array}{lllllll}1064.7 & 174 \mathrm{~A} & 101.2 & 61.6 & 3.14 \mathrm{E}-03 & 70\end{array}$ $\begin{array}{llllll}1064.7 & 174 \mathrm{~A} & 328.2 & 42.5 & 1.45 \mathrm{E}-03 & 85\end{array}$ $\begin{array}{lllllll}1064.7 & 174 \mathrm{~A} & 120.6 & 34.1 & 2.16 \mathrm{E}-03 & 100\end{array}$ $\begin{array}{lllllll}1064.7 & 174 \mathrm{~A} & 120.9 & -37.9 & 2.24 \mathrm{E}-03 & 115\end{array}$ $\begin{array}{llllll}1181.3 & 175 \mathrm{~A} & 214.8 & 67.2 & 6.18 \mathrm{E}-03 & 0\end{array}$ $\begin{array}{llllll}1181.3 & 175 \mathrm{~A} & 208.6 & 65 & 5.76 \mathrm{E}-03 & 3\end{array}$ $\begin{array}{llllll}1181.3 & 175 \mathrm{~A} & 202.3 & 64.5 & 5.07 \mathrm{E}-03 & 6\end{array}$ $\begin{array}{llllll}1181.3 & 175 \mathrm{~A} & 205.3 & 64 & 4.28 \mathrm{E}-03 & 9\end{array}$ $\begin{array}{lllllll}1181.3 & 175 \mathrm{~A} & 196.3 & 61.2 & 3.55 \mathrm{E}-03 & 12\end{array}$ $\begin{array}{lllllll}1181.3 & 175 \mathrm{~A} & 191 & 62.5 & 3.11 \mathrm{E}-03 & 15\end{array}$ $\begin{array}{llllll}1181.3 & 175 \mathrm{~A} & 196.3 & 63.3 & 2.58 \mathrm{E}-03 & 18\end{array}$ $\begin{array}{llllll}1181.3 & 175 \mathrm{~A} & 193.6 & 66.3 & 1.99 \mathrm{E}-03 & 25\end{array}$ $\begin{array}{lllllll}1181.3 & 175 \mathrm{~A} & 174.7 & 57.1 & 1.67 \mathrm{E}-03 & 30\end{array}$ $\begin{array}{lllllll}1181.3 & 175 \mathrm{~A} & 171.3 & 50.4 & 1.33 \mathrm{E}-03 & 40\end{array}$ $\begin{array}{lllllll}1181.3 & 175 \mathrm{~A} & 329.3 & 83.6 & 6.97 \mathrm{E}-04 & 50\end{array}$ $\begin{array}{lllllll}1181.3 & 175 \mathrm{~A} & 214.7 & 81.3 & 6.33 \mathrm{E}-04 & 60\end{array}$ $\begin{array}{lllllll}1181.3 & 175 \mathrm{~A} & 179.6 & 87 & 6.97 \mathrm{E}-04 & 70\end{array}$ $\begin{array}{llllll}1181.3 & 175 \mathrm{~A} & 49.8 & 70.2 & 5.85 \mathrm{E}-04 & 85\end{array}$ $\begin{array}{lllllll}1181.3 & 175 \mathrm{~A} & 211 & 51.7 & 8.50 \mathrm{E}-04 & 100\end{array}$ $\begin{array}{lllllll}1181.3 & 175 \mathrm{~A} & 59.5 & 43.6 & 7.07 \mathrm{E}-04 & 115\end{array}$ $\begin{array}{lllllll}1205 & 177 \mathrm{~A} & 335.5 & 67.4 & 4.54 \mathrm{E}-02 & 0\end{array}$ $\begin{array}{lllllll}1205 & 177 \mathrm{~A} & 341.4 & 67.4 & 4.32 \mathrm{E}-02 & 3\end{array}$ $\begin{array}{lllllll}1205 & 177 \mathrm{~A} & 342.7 & 66.8 & 4.18 \mathrm{E}-02 & 6\end{array}$ $\begin{array}{lllllll}1205 & 177 \mathrm{~A} & 343.1 & 65.4 & 3.92 \mathrm{E}-02 & 9\end{array}$ $\begin{array}{lllllll}1205 & 177 \mathrm{~A} & 342.7 & 63.9 & 3.71 \mathrm{E}-02 & 12\end{array}$ $\begin{array}{lllllll}1205 & 177 \mathrm{~A} & 343.1 & 63.2 & 3.59 \mathrm{E}-02 & 15\end{array}$ $\begin{array}{lllllll}1205 & 177 \mathrm{~A} & 343.1 & 62.2 & 3.34 \mathrm{E}-02 & 18\end{array}$ $\begin{array}{llllll}1205 & 177 \mathrm{~A} & 345.8 & 62.3 & 3.19 \mathrm{E}-02 & 21\end{array}$ $\begin{array}{lllllll}1205 & 177 \mathrm{~A} & 348.3 & 61.5 & 2.95 \mathrm{E}-02 & 25\end{array}$ $\begin{array}{lllllll}1205 & 177 \mathrm{~A} & 347.5 & 59.4 & 2.55 \mathrm{E}-02 & 30\end{array}$ $\begin{array}{llllll}1205 & 177 \mathrm{~A} & 351.4 & 59 & 2.00 \mathrm{E}-02 & 40\end{array}$ $\begin{array}{llllll}1205 & 177 \mathrm{~A} & 357.2 & 56.4 & 1.45 \mathrm{E}-02 & 50\end{array}$ $\begin{array}{lllllll}1205 & 177 \mathrm{~A} & 356.7 & 58.5 & 1.17 \mathrm{E}-02 & 60\end{array}$ $\begin{array}{llllll}1205 & 177 \mathrm{~A} & 1.2 & 62.2 & 9.90 \mathrm{E}-03 & 70\end{array}$ $\begin{array}{llllll}1205 & 177 \mathrm{~A} & 10 & 47.1 & 6.40 \mathrm{E}-03 & 85\end{array}$ $\begin{array}{lllllll}1205 & 177 \mathrm{~A} & 13.5 & 56.1 & 5.55 \mathrm{E}-03 & 100\end{array}$ $\begin{array}{llllll}1205 & 177 \mathrm{~A} & 12.4 & 51.6 & 5.10 \mathrm{E}-03 & 115\end{array}$ $\begin{array}{lllllll}1207 & 178 \mathrm{~A} & 309.3 & 77.1 & 3.90 \mathrm{E}-02 & 0\end{array}$ $\begin{array}{lllllll}1207 & 178 \mathrm{~A} & 313.4 & 77.1 & 3.61 \mathrm{E}-02 & 3\end{array}$ $\begin{array}{lllllll}1207 & 178 \mathrm{~A} & 318.3 & 74.9 & 3.25 \mathrm{E}-02 & 6\end{array}$ $\begin{array}{llllll}1207 & 178 \mathrm{~A} & 319.4 & 73.5 & 2.80 \mathrm{E}-02 & 9\end{array}$ $\begin{array}{llllll}1207 & 178 \mathrm{~A} & 319.3 & 72.3 & 2.46 \mathrm{E}-02 & 12\end{array}$ $\begin{array}{llllll}1207 & 178 \mathrm{~A} & 322.1 & 71.6 & 2.28 \mathrm{E}-02 & 15\end{array}$ $\begin{array}{llllll}1207 & 178 \mathrm{~A} & 320.1 & 70.1 & 1.95 \mathrm{E}-02 & 18\end{array}$ $\begin{array}{llllll}1207 & 178 \mathrm{~A} & 323 & 69.6 & 1.74 \mathrm{E}-02 & 21\end{array}$ $\begin{array}{lllllll}1207 & 178 \mathrm{~A} & 321.7 & 70.1 & 1.39 \mathrm{E}-02 & 25\end{array}$ $\begin{array}{lllllll}1207 & 178 \mathrm{~A} & 316.2 & 73.5 & 9.79 \mathrm{E}-03 & 30\end{array}$ $\begin{array}{llllll}1207 & 178 \mathrm{~A} & 298.4 & 75.4 & 5.99 \mathrm{E}-03 & 40\end{array}$ $\begin{array}{lllllll}1207 & 178 \mathrm{~A} & 255.7 & 77.4 & 3.86 \mathrm{E}-03 & 50\end{array}$ $\begin{array}{lllllll}1207 & 178 \mathrm{~A} & 211.7 & 81.6 & 2.94 \mathrm{E}-03 & 60\end{array}$ $\begin{array}{lllllll}1207 & 178 \mathrm{~A} & 177.5 & 68.9 & 2.69 \mathrm{E}-03 & 70\end{array}$ $\begin{array}{lllllll}1207 & 178 \mathrm{~A} & 158.1 & 66.1 & 2.57 \mathrm{E}-03 & 85\end{array}$ $\begin{array}{llllll}1207 & 178 \mathrm{~A} & 150.5 & 43 & 2.34 \mathrm{E}-03 & 100\end{array}$ $\begin{array}{llllll}1207 & 178 \mathrm{~A} & 171.2 & 42.6 & 2.40 \mathrm{E}-03 & 115\end{array}$ $\begin{array}{lllllll}1209 & 179 \mathrm{~A} & 64.3 & 83 & 3.26 \mathrm{E}-02 & 0\end{array}$ $\begin{array}{llllll}1209 & 179 \mathrm{~A} & 60 & 81.5 & 3.01 \mathrm{E}-02 & 3\end{array}$

\begin{tabular}{|c|c|c|c|c|c|}
\hline & & 6.9 & 80.3 & & \\
\hline & $179 \mathrm{~A}$ & 34 & 77.9 & 2.30E-02 & \\
\hline & 179A & 33.9 & 76.2 & 2.03E-02 & 12 \\
\hline & $179 \mathrm{~A}$ & 26.7 & 75.1 & $1.87 \mathrm{E}-02$ & \\
\hline & 179 & 24.7 & 76.1 & $1.60 \mathrm{E}-02$ & 18 \\
\hline & $179 \mathrm{~A}$ & 18.9 & 73.9 & $1.41 \mathrm{E}-02$ & 21 \\
\hline 209 & $179 \mathrm{~A}$ & 16.1 & 72.9 & $1.12 \mathrm{E}-02$ & 28 \\
\hline 209 & $179 \mathrm{~A}$ & 18.3 & 71.6 & $8.85 \mathrm{E}-03$ & 30 \\
\hline 09 & $179 \mathrm{~A}$ & 19.3 & 60.3 & $5.97 \mathrm{E}-03$ & 40 \\
\hline 09 & $179 \mathrm{~A}$ & 22.4 & 36.5 & 3.33E-03 & 50 \\
\hline 09 & $179 \mathrm{~A}$ & 72.7 & 63.4 & $1.64 \mathrm{E}-03$ & 60 \\
\hline 09 & $179 \mathrm{~A}$ & 356.7 & 37.3 & 2.18E-03 & 70 \\
\hline 209 & $179 \mathrm{~A}$ & 61.6 & 33.1 & $1.85 \mathrm{E}-03$ & \\
\hline 209 & $179 \mathrm{~A}$ & 22.7 & -3.6 & $1.30 \mathrm{E}-03$ & 100 \\
\hline 09 & $179 \mathrm{~A}$ & 341.6 & 14.9 & $1.70 \mathrm{E}-03$ & 115 \\
\hline 211 & $180 \mathrm{~A}$ & 305.3 & 79.4 & $1.61 \mathrm{E}-02$ & \\
\hline 11 & $180 \mathrm{~A}$ & 319.9 & 80.3 & $1.46 \mathrm{E}-02$ & \\
\hline 211 & $180 \mathrm{~A}$ & 326.3 & 77.2 & $1.26 \mathrm{E}-02$ & \\
\hline 211 & $180 \mathrm{~A}$ & 336.4 & 73.4 & $1.08 \mathrm{E}-02$ & \\
\hline 211 & $180 \mathrm{~A}$ & 339 & 68.3 & & 12 \\
\hline 211 & $180 \mathrm{~A}$ & 345 & 63.2 & & 10 \\
\hline 211 & $180 \mathrm{~A}$ & 342.8 & 63.4 & & 18 \\
\hline 211 & $180 \mathrm{~A}$ & 349 & 60.7 & & 21 \\
\hline 211 & $180 \mathrm{~A}$ & 352.5 & 57.4 & & 20 \\
\hline 211 & $180 \mathrm{~A}$ & 344.9 & 66.5 & & 30 \\
\hline 211 & $180 \mathrm{~A}$ & 35.3 & 42.6 & $2.81 \mathrm{E}$ & 40 \\
\hline 211 & $180 \mathrm{~A}$ & 16.6 & 42.8 & & 50 \\
\hline 211 & $180 \mathrm{~A}$ & 7.7 & 13.9 & $1.45 \mathrm{E}$ & 00 \\
\hline 211 & $180 \mathrm{~A}$ & 358.9 & 33.7 & $3.06 \mathrm{E}$ & 70 \\
\hline 211 & $180 \mathrm{~A}$ & 30.8 & 38.3 & $1.85 \mathrm{E}$ & 85 \\
\hline 211 & $180 \mathrm{~A}$ & 47.4 & 49.1 & $1.09 \mathrm{E}-03$ & 100 \\
\hline 211 & $180 \mathrm{~A}$ & 53 & 1.5 & $1.59 \mathrm{E}-03$ & 115 \\
\hline 215.4 & $181 \mathrm{~A}$ & 67.8 & 56.3 & $3.64 \mathrm{E}-$ & 0 \\
\hline 215.4 & $181 \mathrm{~A}$ & 64.1 & 54.2 & $3.28 \mathrm{E}$ & \\
\hline 215.4 & $181 \mathrm{~A}$ & 61.6 & 53.3 & $2.84 \mathrm{E}$ & \\
\hline 215.4 & $181 \mathrm{~A}$ & 57.1 & 52.4 & $2.48 \mathrm{E}-$ & \\
\hline 215.4 & $181 \mathrm{~A}$ & 53.6 & 52.1 & $2.13 \mathrm{E}$ & 12 \\
\hline 215.4 & $181 \mathrm{~A}$ & 54.8 & 52.9 & $1.93 \mathrm{E}$ & 15 \\
\hline 215.4 & $181 \mathrm{~A}$ & 47.7 & 56.3 & $1.70 \mathrm{E}-$ & 18 \\
\hline 215.4 & $181 \mathrm{~A}$ & 43.6 & 57.6 & & \\
\hline 215.4 & $181 \mathrm{~A}$ & 49.3 & 56.7 & & \\
\hline 215.4 & $181 \mathrm{~A}$ & 40 & 63.8 & $8.58 \mathrm{E}$ & \\
\hline 215.4 & $181 \mathrm{~A}$ & 74.6 & 69.9 & & \\
\hline 215.4 & $181 \mathrm{~A}$ & 91.3 & 59.3 & & \\
\hline 215.4 & $181 \mathrm{~A}$ & 28.8 & 86.2 & 2.65E-03 & \\
\hline 215.4 & $181 \mathrm{~A}$ & 185.8 & 45.4 & $2.06 \mathrm{E}-03$ & 10 \\
\hline 215.4 & $181 \mathrm{~A}$ & 165.2 & 56.3 & $1.43 \mathrm{E}-03$ & 0 \\
\hline 215.4 & $181 \mathrm{~A}$ & 159.5 & 26.9 & $2.28 \mathrm{E}-03$ & 100 \\
\hline 215.4 & $181 \mathrm{~A}$ & 196.6 & 34.9 & $2.46 \mathrm{E}-03$ & 12 \\
\hline 225 & $182 \mathrm{~A}$ & 302.6 & 60.6 & $3.52 \mathrm{E}-02$ & \\
\hline & $182 \mathrm{~A}$ & 304.3 & 61.4 & $3.30 \mathrm{OE}-02$ & \\
\hline
\end{tabular}


$\begin{array}{llllll}1225.6 & 182 \mathrm{~A} & 301.6 & 60 & 3.19 \mathrm{E}-02 & 6\end{array}$ $\begin{array}{lllllll}1225.6 & 182 \mathrm{~A} & 299.8 & 57.4 & 2.96 \mathrm{E}-02 & 9\end{array}$ $\begin{array}{lllllll}1225.6 & 182 \mathrm{~A} & 298.8 & 55.9 & 2.76 \mathrm{E}-02 & 12\end{array}$ $\begin{array}{lllllll}1225.6 & 182 \mathrm{~A} & 297.3 & 55.7 & 2.60 \mathrm{E}-02 & 15\end{array}$ $\begin{array}{lllllll}1225.6 & 182 \mathrm{~A} & 296.1 & 53.9 & 2.44 \mathrm{E}-02 & 18\end{array}$ $\begin{array}{lllllll}1225.6 & 182 \mathrm{~A} & 295.6 & 53.8 & 1.96 \mathrm{E}-02 & 25\end{array}$ $\begin{array}{llllll}1225.6 & 182 \mathrm{~A} & 297.1 & 55.3 & 1.73 \mathrm{E}-02 & 30\end{array}$ $\begin{array}{lllllll}1225.6 & 182 \mathrm{~A} & 298.3 & 52.7 & 1.32 \mathrm{E}-02 & 40\end{array}$ $\begin{array}{lllllll}1225.6 & 182 \mathrm{~A} & 297.7 & 58.3 & 9.22 \mathrm{E}-03 & 50\end{array}$ $\begin{array}{lllllll}1225.6 & 182 \mathrm{~A} & 304.1 & 47.3 & 6.01 \mathrm{E}-03 & 60\end{array}$ $\begin{array}{lllllll}1225.6 & 182 \mathrm{~A} & 309.2 & 51.6 & 4.88 \mathrm{E}-03 & 70\end{array}$ $\begin{array}{lllllll}1225.6 & 182 \mathrm{~A} & 263.4 & 67.2 & 2.94 \mathrm{E}-03 & 85\end{array}$ $\begin{array}{lllllll}1225.6 & 182 \mathrm{~A} & 298.6 & 70 & 3.33 \mathrm{E}-03 & 100\end{array}$ $\begin{array}{llllll}1225.6 & 182 \mathrm{~A} & 240.2 & 59.3 & 2.60 \mathrm{E}-03 & 115\end{array}$ $\begin{array}{lllllll}1233.5 & 183 \mathrm{~A} & 58 & 67.4 & 5.29 \mathrm{E}-02 & 0\end{array}$ $\begin{array}{lllllll}1233.5 & 183 \mathrm{~A} & 57.3 & 67.4 & 4.83 \mathrm{E}-02 & 3\end{array}$ $\begin{array}{lllllll}1233.5 & 183 \mathrm{~A} & 52.1 & 68.7 & 4.14 \mathrm{E}-02 & 6\end{array}$ $\begin{array}{lllllll}1233.5 & 183 \mathrm{~A} & 43.1 & 69.2 & 3.38 \mathrm{E}-02 & 9\end{array}$ $\begin{array}{lllllll}1233.5 & 183 \mathrm{~A} & 36.1 & 68.1 & 2.97 \mathrm{E}-02 & 12\end{array}$ $\begin{array}{lllllll}1233.5 & 183 \mathrm{~A} & 34.5 & 68.7 & 2.60 \mathrm{E}-02 & 15\end{array}$ $\begin{array}{lllllll}1233.5 & 183 \mathrm{~A} & 30.8 & 68.1 & 2.23 \mathrm{E}-02 & 18\end{array}$ $\begin{array}{lllllll}1233.5 & 183 \mathrm{~A} & 25 & 71.2 & 1.93 \mathrm{E}-02 & 21\end{array}$ $\begin{array}{llllll}1233.5 & 183 \mathrm{~A} & 26 & 69.7 & 1.61 \mathrm{E}-02 & 25\end{array}$ $\begin{array}{lllllll}1233.5 & 183 \mathrm{~A} & 20.4 & 68 & 1.29 \mathrm{E}-02 & 30\end{array}$ $\begin{array}{lllllll}1233.5 & 183 \mathrm{~A} & 23.7 & 68.4 & 7.48 \mathrm{E}-03 & 40\end{array}$ $\begin{array}{lllllll}1233.5 & 183 \mathrm{~A} & 344.1 & 67.3 & 4.45 \mathrm{E}-03 & 50\end{array}$ $\begin{array}{lllllll}1233.5 & 183 \mathrm{~A} & 21.3 & 72.8 & 4.10 \mathrm{E}-03 & 60\end{array}$ $\begin{array}{llllll}1233.5 & 183 \mathrm{~A} & 44.5 & 69.3 & 2.99 \mathrm{E}-03 & 70\end{array}$ $\begin{array}{lllllll}1233.5 & 183 \mathrm{~A} & 75.5 & 59.4 & 3.96 \mathrm{E}-03 & 85\end{array}$ $\begin{array}{lllllll}1233.5 & 183 \mathrm{~A} & 79.7 & 54.5 & 3.86 \mathrm{E}-03 & 100\end{array}$ $\begin{array}{lllllll}1233.5 & 183 \mathrm{~A} & 102.6 & 56.9 & 3.52 \mathrm{E}-03 & 115\end{array}$ $\begin{array}{lllllll}1235.2 & 184 \mathrm{~A} & 33 & 47.5 & 4.27 \mathrm{E}-02 & 0\end{array}$ $\begin{array}{llllll}1235.2 & 184 \mathrm{~A} & 31.6 & 47 & 4.09 \mathrm{E}-02 & 3\end{array}$ $\begin{array}{lllllll}1235.2 & 184 \mathrm{~A} & 29.9 & 47.1 & 3.85 \mathrm{E}-02 & 6\end{array}$ $\begin{array}{llllll}1235.2 & 184 \mathrm{~A} & 28.7 & 45.3 & 3.51 \mathrm{E}-02 & 9\end{array}$ $\begin{array}{llllll}1235.2 & 184 \mathrm{~A} & 28.8 & 45.2 & 3.27 \mathrm{E}-02 & 12\end{array}$ $\begin{array}{lllllll}1235.2 & 184 \mathrm{~A} & 28.3 & 45.9 & 2.98 \mathrm{E}-02 & 15\end{array}$ $\begin{array}{llllll}1235.2 & 184 \mathrm{~A} & 28.5 & 44.1 & 2.60 \mathrm{E}-02 & 18\end{array}$ $\begin{array}{llllll}1235.2 & 184 \mathrm{~A} & 26.8 & 45.4 & 2.31 \mathrm{E}-02 & 21\end{array}$ $\begin{array}{llllll}1235.2 & 184 \mathrm{~A} & 26 & 45.5 & 1.94 \mathrm{E}-02 & 25\end{array}$ $\begin{array}{llllll}1235.2 & 184 \mathrm{~A} & 24.8 & 47 & 1.54 \mathrm{E}-02 & 30\end{array}$ $\begin{array}{lllllll}1235.2 & 184 \mathrm{~A} & 21.1 & 49.3 & 1.03 \mathrm{E}-02 & 40\end{array}$ $\begin{array}{lllllll}1235.2 & 184 \mathrm{~A} & 14.4 & 51.4 & 6.40 \mathrm{E}-03 & 50\end{array}$ $\begin{array}{lllllll}1235.2 & 184 \mathrm{~A} & 1.7 & 55.2 & 4.28 \mathrm{E}-03 & 60\end{array}$ $\begin{array}{llllll}1235.2 & 184 \mathrm{~A} & 336 & 57.3 & 3.54 \mathrm{E}-03 & 70\end{array}$ $\begin{array}{lllllll}1235.2 & 184 \mathrm{~A} & 9.7 & 62 & 2.46 \mathrm{E}-03 & 85\end{array}$ $\begin{array}{lllllll}1235.2 & 184 \mathrm{~A} & 6.6 & 65 & 1.98 \mathrm{E}-03 & 100\end{array}$ $\begin{array}{lllllll}1235.2 & 184 \mathrm{~A} & 97.9 & 75.5 & 2.24 \mathrm{E}-03 & 115\end{array}$ $\begin{array}{lllllll}1237.2 & 185 \mathrm{~A} & 74 & 47.2 & 3.92 \mathrm{E}-02 & 0\end{array}$ $\begin{array}{llllll}1237.2 & 185 \mathrm{~A} & 73.4 & 45.3 & 3.63 \mathrm{E}-02 & 3\end{array}$ $\begin{array}{lllllll}1237.2 & 185 \mathrm{~A} & 72 & 43.6 & 3.10 \mathrm{E}-02 & 6\end{array}$ $\begin{array}{llllll}1237.2 & 185 \mathrm{~A} & 70.7 & 40.5 & 2.65 \mathrm{E}-02 & 9\end{array}$ $\begin{array}{lllllll}1237.2 & 185 \mathrm{~A} & 71.6 & 37.9 & 2.31 \mathrm{E}-02 & 12\end{array}$ $\begin{array}{lllllll}1237.2 & 185 \mathrm{~A} & 70.2 & 36.6 & 2.01 \mathrm{E}-02 & 15\end{array}$ $\begin{array}{lllllll}1237.2 & 185 \mathrm{~A} & 70.3 & 36.4 & 1.80 \mathrm{E}-02 & 18\end{array}$ $\begin{array}{lllllll}1237.2 & 185 \mathrm{~A} & 70.5 & 35.9 & 1.53 \mathrm{E}-02 & 21\end{array}$ $\begin{array}{lllllll}1237.2 & 185 \mathrm{~A} & 64 & 38.7 & 1.20 \mathrm{E}-02 & 25\end{array}$ $\begin{array}{lllllll}1237.2 & 185 \mathrm{~A} & 65.9 & 38.9 & 9.19 \mathrm{E}-03 & 30\end{array}$ $\begin{array}{lllllll}1237.2 & 185 \mathrm{~A} & 50.2 & 53.1 & 5.26 \mathrm{E}-03 & 40\end{array}$ $\begin{array}{llllll}1237.2 & 185 \mathrm{~A} & 67.5 & 65.3 & 4.31 \mathrm{E}-03 & 50\end{array}$ $\begin{array}{lllllll}1237.2 & 185 \mathrm{~A} & 29.7 & 43.9 & 1.72 \mathrm{E}-03 & 60\end{array}$ $\begin{array}{lllllll}1237.2 & 185 \mathrm{~A} & 27.6 & 55.5 & 2.77 \mathrm{E}-03 & 70\end{array}$ $\begin{array}{llllll}1237.2 & 185 \mathrm{~A} & 81.4 & 44.8 & 1.21 \mathrm{E}-03 & 85\end{array}$ $\begin{array}{lllllll}1237.2 & 185 \mathrm{~A} & 78.1 & 62.2 & 2.39 \mathrm{E}-03 & 100\end{array}$ $\begin{array}{lllllll}1237.2 & 185 \mathrm{~A} & 18 & 64.2 & 1.71 \mathrm{E}-03 & 115\end{array}$ $\begin{array}{lllllll}1239.2 & 186 \mathrm{~A} & 328 & 65.6 & 4.21 \mathrm{E}-02 & 0\end{array}$ $\begin{array}{lllllll}1239.2 & 186 \mathrm{~A} & 328.9 & 65.2 & 4.13 \mathrm{E}-02 & 3\end{array}$ $\begin{array}{lllllll}1239.2 & 186 \mathrm{~A} & 329.5 & 63.5 & 3.88 \mathrm{E}-02 & 6\end{array}$ $\begin{array}{lllllll}1239.2 & 186 \mathrm{~A} & 329.8 & 61.3 & 3.55 \mathrm{E}-02 & 9\end{array}$ $\begin{array}{lllllll}1239.2 & 186 \mathrm{~A} & 330.1 & 61.1 & 3.29 \mathrm{E}-02 & 12\end{array}$ $\begin{array}{lllllll}1239.2 & 186 \mathrm{~A} & 332.4 & 60.9 & 3.04 \mathrm{E}-02 & 15\end{array}$ $\begin{array}{llllll}1239.2 & 186 \mathrm{~A} & 332 & 60.1 & 2.69 \mathrm{E}-02 & 18\end{array}$ $\begin{array}{lllllll}1239.2 & 186 \mathrm{~A} & 334.8 & 61.1 & 2.41 \mathrm{E}-02 & 21\end{array}$ $\begin{array}{lllllll}1239.2 & 186 \mathrm{~A} & 339.6 & 62.9 & 2.05 \mathrm{E}-02 & 25\end{array}$ $\begin{array}{lllllll}1239.2 & 186 \mathrm{~A} & 335.9 & 62.9 & 1.61 \mathrm{E}-02 & 30\end{array}$ $\begin{array}{lllllll}1239.2 & 186 \mathrm{~A} & 345.7 & 65.8 & 1.09 \mathrm{E}-02 & 40\end{array}$ $\begin{array}{lllllll}1239.2 & 186 \mathrm{~A} & 337.9 & 71.8 & 7.34 \mathrm{E}-03 & 50\end{array}$ $\begin{array}{lllllll}1239.2 & 186 \mathrm{~A} & 334.9 & 77.1 & 5.53 \mathrm{E}-03 & 60\end{array}$ $\begin{array}{llllll}1239.2 & 186 \mathrm{~A} & 12.3 & 78 & 4.26 \mathrm{E}-03 & 70\end{array}$ $\begin{array}{lllllll}1239.2 & 186 \mathrm{~A} & 347.9 & 86.4 & 3.97 \mathrm{E}-03 & 85\end{array}$ $\begin{array}{lllllll}1239.2 & 186 \mathrm{~A} & 343.1 & 86.6 & 3.26 \mathrm{E}-03 & 100\end{array}$ $\begin{array}{lllllll}1239.2 & 186 \mathrm{~A} & 95.9 & 74.6 & 2.66 \mathrm{E}-03 & 115\end{array}$ $\begin{array}{lllllll}1241.2 & 187 \mathrm{~A} & 57.2 & 68.2 & 3.01 \mathrm{E}-02 & 0\end{array}$ $\begin{array}{lllllll}1241.2 & 187 \mathrm{~A} & 54.4 & 69.6 & 2.88 \mathrm{E}-02 & 3\end{array}$ $\begin{array}{lllllll}1241.2 & 187 \mathrm{~A} & 51.3 & 68.5 & 2.74 \mathrm{E}-02 & 6\end{array}$ $\begin{array}{lllllll}1241.2 & 187 \mathrm{~A} & 47.6 & 66.3 & 2.56 \mathrm{E}-02 & 9\end{array}$ $\begin{array}{lllllll}1241.2 & 187 \mathrm{~A} & 45.9 & 64.3 & 2.40 \mathrm{E}-02 & 12\end{array}$ $\begin{array}{lllllll}1241.2 & 187 \mathrm{~A} & 43.2 & 62.8 & 2.28 \mathrm{E}-02 & 15\end{array}$ $\begin{array}{lllllll}1241.2 & 187 \mathrm{~A} & 43.5 & 61.1 & 2.11 \mathrm{E}-02 & 18\end{array}$ $\begin{array}{llllll}1241.2 & 187 \mathrm{~A} & 42.5 & 60.7 & 1.98 \mathrm{E}-02 & 21\end{array}$ $\begin{array}{lllllll}1241.2 & 187 \mathrm{~A} & 42.9 & 59.6 & 1.76 \mathrm{E}-02 & 25\end{array}$ $\begin{array}{llllll}1241.2 & 187 \mathrm{~A} & 42.1 & 58.2 & 1.57 \mathrm{E}-02 & 30\end{array}$ $\begin{array}{llllll}1241.2 & 187 \mathrm{~A} & 38.9 & 53.7 & 1.19 \mathrm{E}-02 & 40\end{array}$ $\begin{array}{lllllll}1241.2 & 187 \mathrm{~A} & 42.9 & 50.6 & 9.17 \mathrm{E}-03 & 50\end{array}$ $\begin{array}{lllllll}1241.2 & 187 \mathrm{~A} & 46.3 & 46.3 & 6.46 \mathrm{E}-03 & 60\end{array}$ $\begin{array}{lllllll}1241.2 & 187 \mathrm{~A} & 45.5 & 49.8 & 4.39 \mathrm{E}-03 & 70\end{array}$ $\begin{array}{lllllll}1241.2 & 187 \mathrm{~A} & 58.9 & 45 & 3.72 \mathrm{E}-03 & 85\end{array}$ $\begin{array}{lllllll}1241.2 & 187 \mathrm{~A} & 36.7 & 44.1 & 2.48 \mathrm{E}-03 & 100\end{array}$ $\begin{array}{llllll}1241.2 & 187 \mathrm{~A} & 63 & 49.9 & 3.17 \mathrm{E}-03 & 115\end{array}$ $\begin{array}{lllllll}1245.4 & 188 \mathrm{~A} & 86.4 & 65.1 & 3.55 \mathrm{E}-02 & 0\end{array}$ $\begin{array}{lllllll}1245.4 & 188 \mathrm{~A} & 84.1 & 62.3 & 3.30 \mathrm{E}-02 & 3\end{array}$ $\begin{array}{lllllll}1245.4 & 188 \mathrm{~A} & 81.7 & 61.5 & 2.98 \mathrm{E}-02 & 6\end{array}$

\begin{tabular}{|c|c|c|c|c|c|}
\hline & & & & & \\
\hline 245.4 & $188 \mathrm{~A}$ & 4.5 & 60 & $2.28 \mathrm{E}-02$ & 12 \\
\hline 45.4 & $188 \mathrm{~A}$ & 71.4 & 60 & $2.02 \mathrm{E}-02$ & 15 \\
\hline 45.4 & $188 \mathrm{~A}$ & 72.5 & 57.8 & $1.75 \mathrm{E}-02$ & 18 \\
\hline 245.4 & $188 \mathrm{~A}$ & 70.6 & 55.5 & $1.51 \mathrm{E}-02$ & \\
\hline 245.4 & $188 \mathrm{~A}$ & 73.9 & 52.9 & $1.30 \mathrm{E}-02$ & $25+2$ \\
\hline 245.4 & $188 \mathrm{~A}$ & 72.6 & 48.4 & $1.04 \mathrm{E}-02$ & $30-2>$ \\
\hline 245.4 & $188 \mathrm{~A}$ & 69.6 & 37.1 & $8.07 \mathrm{E}-03$ & 40 \\
\hline 245.4 & $188 \mathrm{~A}$ & 70.1 & 35 & $6.73 \mathrm{E}-03$ & \\
\hline 245.4 & $188 \mathrm{~A}$ & 63.2 & 32.3 & $5.40 \mathrm{E}-03$ & $600+2$ \\
\hline 245.4 & $188 \mathrm{~A}$ & 63.8 & 41.3 & 4.31E-03 & \\
\hline 245.4 & $188 \mathrm{~A}$ & 71.7 & 36 & $4.58 \mathrm{E}$ & \\
\hline 245.4 & $188 \mathrm{~A}$ & 66.9 & 52.2 & $2.87 \mathrm{E}-03$ & 100 \\
\hline 245.4 & $188 \mathrm{~A}$ & 70.5 & 19.2 & $3.08 \mathrm{E}-03$ & 115 \\
\hline 246.4 & $189 \mathrm{~A}$ & 70.1 & 71.9 & $8.39 \mathrm{E}-03$ & \\
\hline 246.4 & $189 \mathrm{~A}$ & 81.7 & 68.1 & $7.20 \mathrm{E}-03$ & \\
\hline 246.4 & $189 \mathrm{~A}$ & 86.9 & 65.3 & $6.42 \mathrm{E}-03$ & \\
\hline 2464 & $189 \mathrm{~A}$ & 89.5 & 62.7 & $5.75 \mathrm{E}-03$ & \\
\hline 246.4 & $189 \mathrm{~A}$ & & 61.2 & $5.31 \mathrm{E}-03$ & \\
\hline 246.4 & $189 \mathrm{~A}$ & 91.7 & 61.7 & & 18 \\
\hline 246.4 & $189 \mathrm{~A}$ & 92.6 & 60.8 & & \\
\hline 246.4 & $189 \mathrm{~A}$ & & & & \\
\hline $2+0.4$ & $189 \mathrm{~A}$ & & 63.1 & & \\
\hline 246.4 & $189 \mathrm{~A}$ & & & & \\
\hline 246.4 & $189 \mathrm{~A}$ & & & & \\
\hline 246.4 & $189 \mathrm{~A}$ & 86.5 & 50.8 & & \\
\hline 246.4 & $189 \mathrm{~A}$ & 11.1 & 49 & & $\pi$ \\
\hline 246.4 & $189 \mathrm{~A}$ & 19.3 & 60.8 & $1.60 \mathrm{E}-03$ & 00 \\
\hline 246.4 & $189 \mathrm{~A}$ & 73.4 & 31.5 & $2.23 \mathrm{E}-03$ & $\pi$ \\
\hline 246.4 & $189 \mathrm{~A}$ & 132 & 63.5 & $1.35 \mathrm{E}-03$ & 83 \\
\hline 246.4 & $189 \mathrm{~A}$ & 140.5 & 52.3 & $1.37 \mathrm{E}-03$ & 100 \\
\hline 246.4 & $189 \mathrm{~A}$ & 65.2 & 15.4 & 2.371 & 1 \\
\hline 246.9 & $190 \mathrm{~A}$ & 167.2 & 44.9 & $8.03 \mathrm{E}$ & \\
\hline 246.9 & $190 \mathrm{~A}$ & 164.6 & 43.6 & $7.84 \mathrm{E}-02$ & \\
\hline 246.9 & $190 \mathrm{~A}$ & 163.5 & 43.3 & $7.09 \mathrm{E}-02$ & \\
\hline 246.9 & $190 \mathrm{~A}$ & 164 & 41.6 & 6.24 & \\
\hline 246.9 & $190 \mathrm{~A}$ & 163.9 & 42.1 & $5.53 \mathrm{E}$ & 12 \\
\hline 246.9 & $190 \mathrm{~A}$ & 165.2 & 42.3 & $4.89 \mathrm{I}$ & 15 \\
\hline 246.9 & $190 \mathrm{~A}$ & 166.3 & 42.3 & 4.31E-02 & 18 \\
\hline 246.9 & $190 \mathrm{~A}$ & 163.6 & 41.6 & $3.74 \mathrm{E}$ & 21 \\
\hline 246.9 & $190 \mathrm{~A}$ & 168 & 40.1 & $3.08 \mathrm{E}-02$ & \\
\hline 246.9 & $190 \mathrm{~A}$ & 165.3 & 45.1 & $2.66 \mathrm{E}-02$ & \\
\hline 246.9 & $190 \mathrm{~A}$ & 164.1 & 41.2 & & \\
\hline 20.9 & $190 \mathrm{~A}$ & 175.7 & 37.4 & & \\
\hline (2) & $190 \mathrm{~A}$ & 181.8 & 36.8 & & \\
\hline $2+0.7$ & $190 \mathrm{~A}$ & 176 & 29.2 & & \\
\hline 1240.9 & $190 \mathrm{~A}$ & 170.3 & 39.7 & $6.16 \mathrm{E}-03$ & 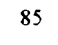 \\
\hline 1240.9 & $190 \mathrm{~A}$ & 171.4 & 21.1 & 4.33E-03 & 100 \\
\hline $10+0.9$ & $190 \mathrm{~A}$ & 170.0 & 25 & $2.3+[-60$ & 10 \\
\hline 202.9 & $191 \mathrm{~A}$ & 263.6 & 66.2 & $0.73 \mathrm{E}-0.2$ & \\
\hline (1) & $191 \mathrm{~A}$ & 202 & 60.1 & $0.015-02$ & \\
\hline
\end{tabular}


$\begin{array}{llllll}1262.9 & 191 \mathrm{~A} & 261.4 & 62.1 & 4.99 \mathrm{E}-02 & 6\end{array}$ $\begin{array}{llllll}1262.9 & 191 \mathrm{~A} \quad 262 & 58.6 & 4.10 \mathrm{E}-02 & 9\end{array}$ $\begin{array}{llllll}1262.9 \quad 191 \mathrm{~A} & 262.3 \quad 57.8 & 3.57 \mathrm{E}-02 & 12\end{array}$ $\begin{array}{llllll}1262.9 & 191 \mathrm{~A} & 261.6 & 56.4 & 3.12 \mathrm{E}-02 & 15\end{array}$ $\begin{array}{llllll}1262.9 & 191 \mathrm{~A} & 263.4 & 57.8 & 2.75 \mathrm{E}-02 & 18\end{array}$ $\begin{array}{llllll}1262.9 & 191 \mathrm{~A} & 261.6 & 53.6 & 2.32 \mathrm{E}-02 & 21\end{array}$ $\begin{array}{llllll}1262.9 & 191 \mathrm{~A} & 263.6 & 51.1 & 2.01 \mathrm{E}-02 & 25\end{array}$ $\begin{array}{llllll}1262.9 & 191 \mathrm{~A} & 264.4 & 55.7 & 1.65 \mathrm{E}-02 & 30\end{array}$ $\begin{array}{llllll}1262.9 & 191 \mathrm{~A} & 274.3 & 49.7 & 1.01 \mathrm{E}-02 & 40\end{array}$ $\begin{array}{llllll}1262.9 & 191 \mathrm{~A} & 279.9 & 49.9 & 4.09 \mathrm{E}-03 & 50\end{array}$ $\begin{array}{llllll}1262.9 & 191 \mathrm{~A} & 265.3 & 34.6 & 4.06 \mathrm{E}-03 & 60\end{array}$ $\begin{array}{llllll}1262.9 & 191 \mathrm{~A} & 279.4 & 37.4 & 2.90 \mathrm{E}-03 & 70\end{array}$ $\begin{array}{llllll}1262.9 & 191 \mathrm{~A} & 321.3 & 49.3 & 1.72 \mathrm{E}-03 & 85\end{array}$ $\begin{array}{lllllll}1262.9 & 191 \mathrm{~A} & 300.1 & 28.1 & 1.79 \mathrm{E}-03 & 100\end{array}$ $\begin{array}{llllll}1262.9 & 191 \mathrm{~A} & 275.9 & 21.8 & 1.10 \mathrm{E}-03 & 115\end{array}$ $\begin{array}{llllll}1263.6 & 192 \mathrm{~A} & 278.8 & 51.8 & 3.99 \mathrm{E}-02 & 0\end{array}$ $\begin{array}{lllllll}1263.6 & 192 \mathrm{~A} & 277 & 53.1 & 3.79 \mathrm{E}-02 & 3\end{array}$ $\begin{array}{llllll}1263.6 & 192 \mathrm{~A} & 69.9 & 79 & 3.36 \mathrm{E}-02 & 6\end{array}$ $\begin{array}{llllll}1263.6 & 192 \mathrm{~A} & 275 & 50.2 & 2.87 \mathrm{E}-02 & 9\end{array}$ $\begin{array}{llllll}1263.6 & 192 \mathrm{~A} & 275.5 & 49.4 & 2.55 \mathrm{E}-02 & 12\end{array}$ $\begin{array}{llllll}1263.6 & 192 \mathrm{~A} & 275.1 & 48.7 & 2.29 \mathrm{E}-02 & 15\end{array}$ $\begin{array}{llllll}1263.6 & 192 \mathrm{~A} & 275.4 & 50 & 2.02 \mathrm{E}-02 & 18\end{array}$ $\begin{array}{llllll}1263.6 & 192 \mathrm{~A} & 276.8 & 46.8 & 1.80 \mathrm{E}-02 & 21\end{array}$ $\begin{array}{llllll}1263.6 & 192 \mathrm{~A} & 276.3 & 45.6 & 1.59 \mathrm{E}-02 & 25\end{array}$ $\begin{array}{llllll}1263.6 & 192 \mathrm{~A} & 282 & 52.5 & 1.39 \mathrm{E}-02 & 30\end{array}$ $\begin{array}{llllll}1263.6 & 192 \mathrm{~A} & 279.6 & 45.5 & 1.01 \mathrm{E}-02 & 40\end{array}$ $\begin{array}{llllll}1263.6 & 192 \mathrm{~A} & 279.3 & 51.2 & 6.63 \mathrm{E}-03 & 50\end{array}$ $\begin{array}{lllllll}1263.6 & 192 \mathrm{~A} & 294.8 & 46.7 & 4.45 \mathrm{E}-03 & 60\end{array}$ $\begin{array}{llllll}1263.6 & 192 \mathrm{~A} & 279.7 & 36.3 & 2.50 \mathrm{E}-03 & 70\end{array}$ $\begin{array}{llllll}1263.6 & 192 \mathrm{~A} & 294.4 & 62.5 & 2.14 \mathrm{E}-03 & 85\end{array}$ $\begin{array}{lllllll}1263.6 & 192 \mathrm{~A} & 309.1 & 23.2 & 2.09 \mathrm{E}-03 & 100\end{array}$ $\begin{array}{lllllll}1263.6 & 192 \mathrm{~A} & 282.9 & -34 & 2.53 \mathrm{E}-03 & 115\end{array}$ $\begin{array}{llllll}1265.5 & 193 \mathrm{~A} & 87.6 & 80.5 & 6.01 \mathrm{E}-02 & 0\end{array}$ $\begin{array}{llllll}1265.5 & 193 \mathrm{~A} & 89.1 & 78.1 & 4.82 \mathrm{E}-02 & 3\end{array}$ $\begin{array}{lllllll}1265.5 & 193 \mathrm{~A} & 90.4 & 74.8 & 3.46 \mathrm{E}-02 & 6\end{array}$ $\begin{array}{llllll}1265.5 & 193 \mathrm{~A} & 90.6 & 70.8 & 2.41 \mathrm{E}-02 & 9\end{array}$ $\begin{array}{llllll}1265.5 & 193 \mathrm{~A} & 90 & 67.5 & 1.95 \mathrm{E}-02 & 12\end{array}$ $\begin{array}{llllll}1265.5 & 193 \mathrm{~A} & 91.9 & 65.2 & 1.60 \mathrm{E}-02 & 15\end{array}$ $\begin{array}{lllllll}1265.5 & 193 \mathrm{~A} & 95.7 & 65.9 & 1.30 \mathrm{E}-02 & 18\end{array}$ $\begin{array}{llllll}1265.5 & 193 \mathrm{~A} & 94 & 61.9 & 1.07 \mathrm{E}-02 & 21\end{array}$ $\begin{array}{llllll}1265.5 & 193 \mathrm{~A} & 96.4 & 60.8 & 9.29 \mathrm{E}-03 & 25\end{array}$ $\begin{array}{llllll}1265.5 & 193 \mathrm{~A} & 92.5 & 58.1 & 6.45 \mathrm{E}-03 & 30\end{array}$ $\begin{array}{llllll}1265.5 & 193 \mathrm{~A} & 124.8 & 58.5 & 3.29 \mathrm{E}-03 & 40\end{array}$ $\begin{array}{llllll}1265.5 & 193 \mathrm{~A} & 95.5 & 37.9 & 2.49 \mathrm{E}-03 & 50\end{array}$ $\begin{array}{lllllll}1265.5 & 193 \mathrm{~A} & 105.4 & 7.5 & 2.27 \mathrm{E}-03 & 60\end{array}$ $\begin{array}{lllllll}1265.5 & 193 \mathrm{~A} & 117.4 & -13.5 & 1.99 \mathrm{E}-03 & 70\end{array}$ $\begin{array}{llllll}1265.5 & 193 \mathrm{~A} & 105.6 & 6.9 & 2.10 \mathrm{E}-03 & 85\end{array}$ $\begin{array}{lllllll}1265.5 & 193 \mathrm{~A} & 94.4 & -20.8 & 2.13 \mathrm{E}-03 & 100\end{array}$ $\begin{array}{lllllll}1265.5 & 193 \mathrm{~A} & 109.5 & -24.7 & 1.64 \mathrm{E}-03 & 115\end{array}$ $\begin{array}{llllll}1269.3 & 194 \mathrm{~A} & 359.7 & 57.9 & 1.76 \mathrm{E}-02 & 0\end{array}$ $\begin{array}{llllll}1269.3 & 194 \mathrm{~A} & 358.9 & 57.8 & 1.74 \mathrm{E}-02 & 3\end{array}$ $\begin{array}{llllll}1269.3 & 194 \mathrm{~A} & 356.9 & 57 & 1.62 \mathrm{E}-02 & 6\end{array}$ $\begin{array}{llllll}1269.3 & 194 \mathrm{~A} & 358.3 & 56.9 & 1.53 \mathrm{E}-02 & 9\end{array}$ $\begin{array}{llllll}1269.3 & 194 \mathrm{~A} & 358 & 58.3 & 1.44 \mathrm{E}-02 & 12\end{array}$ $\begin{array}{llllll}1269.3 & 194 \mathrm{~A} & 357 & 59.4 & 1.31 \mathrm{E}-02 & 15\end{array}$ $\begin{array}{llllll}1269.3 & 194 \mathrm{~A} & 356.8 & 59.1 & 1.21 \mathrm{E}-02 & 18\end{array}$ $\begin{array}{llllll}1269.3 & 194 \mathrm{~A} & 354.6 & 58.5 & 1.10 \mathrm{E}-02 & 21\end{array}$ $\begin{array}{llllll}1269.3 & 194 \mathrm{~A} & 349.9 & 59.2 & 9.86 \mathrm{E}-03 & 25\end{array}$ $\begin{array}{llllll}1269.3 & 194 \mathrm{~A} & 354.5 & 59.5 & 8.46 \mathrm{E}-03 & 30\end{array}$ $\begin{array}{llllll}1269.3 & 194 \mathrm{~A} & 345 & 62.4 & 6.30 \mathrm{E}-03 & 40\end{array}$ $\begin{array}{lllllll}1269.3 & 194 \mathrm{~A} & 336.4 & 65.6 & 4.55 \mathrm{E}-03 & 50\end{array}$ $\begin{array}{lllllll}1269.3 & 194 \mathrm{~A} & 341.7 & 68.9 & 3.37 \mathrm{E}-03 & 60\end{array}$ $\begin{array}{llllll}1269.3 & 194 \mathrm{~A} & 346.5 & 63.9 & 2.34 \mathrm{E}-03 & 70\end{array}$ $\begin{array}{llllll}1269.3 & 194 \mathrm{~A} & 317.3 & 65.3 & 2.31 \mathrm{E}-03 & 85\end{array}$ $\begin{array}{llllll}1269.3 & 194 \mathrm{~A} & 269 & 52.4 & 1.36 \mathrm{E}-03 & 100\end{array}$ $\begin{array}{llllll}1269.3 \quad 194 \mathrm{~A} \quad 275 \quad 32 & 1.31 \mathrm{E}-03 & 115\end{array}$ $\begin{array}{llllll}1273.1 & 195 \mathrm{~A} & 277.8 & -51.3 & 1.16 \mathrm{E}-01 & 0\end{array}$ $\begin{array}{lllllll}1273.1 & 195 \mathrm{~A} & 278.1 & -50.4 & 1.14 \mathrm{E}-01 & 3\end{array}$ $\begin{array}{llllll}1273.1 & 195 \mathrm{~A} & 278.3 & -50.5 & 1.08 \mathrm{E}-01 & 6\end{array}$ $\begin{array}{lllllll}1273.1 & 195 \mathrm{~A} & 278.4 & -50.5 & 1.01 \mathrm{E}-01 & 9\end{array}$ $\begin{array}{llllll}1273.1 & 195 \mathrm{~A} & 278.9 & -50.5 & 9.40 \mathrm{E}-02 & 12\end{array}$ $\begin{array}{lllllll}1273.1 & 195 \mathrm{~A} & 278.8 & -50.4 & 8.72 \mathrm{E}-02 & 15\end{array}$ $\begin{array}{lllllll}1273.1 & 195 \mathrm{~A} & 278.9 & -50 & 8.03 \mathrm{E}-02 & 18\end{array}$ $\begin{array}{llllll}1273.1 & 195 \mathrm{~A} & 278.7 & 48.9 & 7.39 \mathrm{E}-02 & 21\end{array}$ $\begin{array}{lllllll}1273.1 & 195 \mathrm{~A} & 278.9 & -48.5 & 6.37 \mathrm{E}-02 & 25\end{array}$ $\begin{array}{lllllll}1273.1 & 195 \mathrm{~A} & 278.6 & 49.7 & 5.30 \mathrm{E}-02 & 30\end{array}$ $\begin{array}{lllllll}1273.1 & 195 \mathrm{~A} & 278.4 & 46.8 & 3.78 \mathrm{E}-02 & 40\end{array}$ $\begin{array}{llllll}1273.1 & 195 \mathrm{~A} & 280.4 & -51.6 & 2.47 \mathrm{E}-02 & 50\end{array}$ $\begin{array}{llllll}1273.1 & 195 \mathrm{~A} & 288 & -53.2 & 1.71 \mathrm{E}-02 & 60\end{array}$ $\begin{array}{lllllll}1273.1 & 195 \mathrm{~A} & 276.9 & -38.8 & 1.16 \mathrm{E}-02 & 70\end{array}$ $\begin{array}{lllllll}1273.1 & 195 \mathrm{~A} & 288.7 & -27.8 & 7.89 \mathrm{E}-03 & 85\end{array}$ $\begin{array}{lllllll}1273.1 & 195 \mathrm{~A} & 278.4 & -26.1 & 6.00 \mathrm{E}-03 & 100\end{array}$ $\begin{array}{lllllll}1273.1 & 195 \mathrm{~A} & 270.4 & -22 & 7.01 \mathrm{E}-03 & 115\end{array}$ $\begin{array}{llllll}1277.1 & 196 \mathrm{~A} & 6.8 & 71.8 & 5.15 \mathrm{E}-02 & 0\end{array}$ $\begin{array}{llllll}1277.1 & 196 \mathrm{~A} & 13.7 & 70.2 & 4.45 \mathrm{E}-02 & 3\end{array}$ $\begin{array}{llllll}1277.1 & 196 \mathrm{~A} & 16.1 & 68.7 & 3.64 \mathrm{E}-02 & 6\end{array}$ $\begin{array}{llllll}1277.1 & 196 \mathrm{~A} & 16.5 & 67.8 & 2.95 \mathrm{E}-02 & 9\end{array}$ $\begin{array}{llllll}1277.1 & 196 \mathrm{~A} & 15.2 & 67 & 2.55 \mathrm{E}-02 & 12\end{array}$ $\begin{array}{llllll}1277.1 & 196 \mathrm{~A} & 12.8 & 66.5 & 2.19 \mathrm{E}-02 & 15\end{array}$ $\begin{array}{lllllll}1277.1 & 196 \mathrm{~A} & 11.8 & 66.9 & 1.95 \mathrm{E}-02 & 18\end{array}$ $\begin{array}{llllll}1277.1 & 196 \mathrm{~A} & 12.2 & 67.5 & 1.70 \mathrm{E}-02 & 21\end{array}$ $\begin{array}{lllllll}1277.1 & 196 \mathrm{~A} & 7.5 & 68.5 & 1.39 \mathrm{E}-02 & 25\end{array}$ $\begin{array}{llllll}1277.1 & 196 \mathrm{~A} & 5.1 & 70 & 1.14 \mathrm{E}-02 & 30\end{array}$ $\begin{array}{llllll}1277.1 & 196 \mathrm{~A} & 12.9 & 72.5 & 6.13 \mathrm{E}-03 & 40\end{array}$ $\begin{array}{llllll}1277.1 & 196 \mathrm{~A} & 293.2 & 70.1 & 2.70 \mathrm{E}-03 & 50\end{array}$ $\begin{array}{llllll}1277.1 & 196 \mathrm{~A} & 327.5 & 64.7 & 1.72 \mathrm{E}-03 & 60\end{array}$ $\begin{array}{lllllll}1277.1 & 196 \mathrm{~A} & 213.1 & 68.5 & 1.79 \mathrm{E}-03 & 70\end{array}$ $\begin{array}{lllllll}1277.1 & 196 \mathrm{~A} & 237.8 & 34.9 & 1.27 \mathrm{E}-03 & 85\end{array}$ $\begin{array}{lllllll}1277.1 & 196 \mathrm{~A} & 288.5 & 25.7 & 2.13 \mathrm{E}-03 & 100\end{array}$ $\begin{array}{lllllll}1277.1 & 196 \mathrm{~A} & 332.1 & -20.5 & 1.80 \mathrm{E}-03 & 115\end{array}$ $\begin{array}{llllll}1278.2 & 197 \mathrm{~A} & 19.3 & 58 & 5.06 \mathrm{E}-02 & 0\end{array}$ $\begin{array}{llllll}1278.2 & 197 \mathrm{~A} & 20.9 & 56.1 & 4.60 \mathrm{E}-02 & 3\end{array}$

\begin{tabular}{|c|c|c|c|c|}
\hline 78. & $197 \mathrm{~A}$ & 21.6 & 54.5 & $3.90 \mathrm{E}$ \\
\hline 278.2 & $197 \mathrm{~A}$ & 22.2 & 52.8 & $3.28 \mathrm{E}-02$ \\
\hline 78.2 & $197 \mathrm{~A}$ & 22.4 & 51.1 & $89 \mathrm{E}-02$ \\
\hline 78.2 & $197 \mathrm{~A}$ & 24.2 & 51.3 & S0E-02 \\
\hline 78.2 & $197 \mathrm{~A}$ & 2.1 & 51.6 & $2.32 \mathrm{E}-02$ \\
\hline 78.2 & $197 \mathrm{~A}$ & 19 & 48.5 & $1.91 \mathrm{E}-02$ \\
\hline 78.2 & $197 \mathrm{~A}$ & 23.3 & 48.8 & $1.67 \mathrm{E}-02$ \\
\hline 78.2 & $197 \mathrm{~A}$ & 21.9 & 50.4 & $1.47 \mathrm{E}-02$ \\
\hline 278.2 & $197 \mathrm{~A}$ & 35 & 40.8 & 8.05E-03 \\
\hline 278.2 & $197 \mathrm{~A}$ & 46.9 & 43.6 & $4.54 \mathrm{E}-03$ \\
\hline 278.2 & $197 \mathrm{~A}$ & 65.8 & 31.8 & $3.75 \mathrm{E}-03$ \\
\hline 78.2 & $197 \mathrm{~A}$ & 54.7 & 13.1 & $2.04 \mathrm{E}-03$ \\
\hline 78.2 & $197 \mathrm{~A}$ & 92.2 & 36.2 & $2.92 \mathrm{E}-03$ \\
\hline 278.2 & $197 \mathrm{~A}$ & 82.3 & 41.2 & $2.61 \mathrm{E}-03$ \\
\hline 278.2 & $197 \mathrm{~A}$ & 56.9 & 53 & $5.05 \mathrm{E}-04$ \\
\hline 1281.1 & $198 \mathrm{~A}$ & 62.2 & 72.6 & $3.54 \mathrm{E}-02$ \\
\hline 81.1 & $198 \mathrm{~A}$ & 64.3 & 70.8 & $3.24 \mathrm{E}-02$ \\
\hline 81.1 & $198 \mathrm{~A}$ & 64.7 & 70.5 & $2.81 \mathrm{E}-02$ \\
\hline 81.1 & $198 \mathrm{~A}$ & 66.5 & 69.5 & $2.33 \mathrm{E}-02$ \\
\hline 81.1 & $198 \mathrm{~A}$ & 69.7 & 68.9 & $2.05 \mathrm{E}-02$ \\
\hline 1281.1 & $198 \mathrm{~A}$ & 72.4 & 68.4 & $1.83 \mathrm{E}-02$ \\
\hline 1281.1 & $198 \mathrm{~A}$ & 71.9 & 68.7 & $1.63 \mathrm{E}-02$ \\
\hline 1281.1 & $198 \mathrm{~A}$ & 77 & 66.9 & $1.44 \mathrm{E}-02$ \\
\hline 81.1 & $198 \mathrm{~A}$ & 78.6 & 67.1 & $1.25 \mathrm{E}-02$ \\
\hline 81.1 & $198 \mathrm{~A}$ & & 71.7 & \\
\hline 81.1 & $198 \mathrm{~A}$ & & 59.1 & $8.00 \mathrm{E}-03$ \\
\hline & $198 \mathrm{~A}$ & 05.1 & 66.4 & \\
\hline & $198 \mathrm{~A}$ & 27.7 & & \\
\hline & $198 \mathrm{~A}$ & 53.3 & & \\
\hline & $198 \mathrm{~A}$ & 104 & & \\
\hline & 198 & 5.4 & 37 & \\
\hline & $198 \mathrm{~A}$ & 74.6 & 43.3 & \\
\hline & $199 \mathrm{~A}$ & & 84.3 & \\
\hline 282.1 & $199 \mathrm{~A}$ & 76.6 & 83.4 & \\
\hline 282.1 & $199 \mathrm{~A}$ & & 35.1 & \\
\hline & $199 \mathrm{~A}$ & 74.7 & 83.5 & \\
\hline & $199 \mathrm{~A}$ & & & \\
\hline & $199 \mathrm{~A}$ & & 84.1 & \\
\hline & 199A & & 82.9 & \\
\hline & $199 \mathrm{~A}$ & & 34.7 & \\
\hline & $199 \mathrm{~A}$ & & 36 & \\
\hline & $199 \mathrm{~A}$ & 16.4 & 80.8 & $1.97 \mathrm{E}-02$ \\
\hline & $199 \mathrm{~A}$ & 77 & 44.3 & \\
\hline & $199 \mathrm{~A}$ & 335.6 & 62 & \\
\hline 282.1 & $199 \mathrm{~A}$ & 324.8 & 63.7 & \\
\hline 282.1 & $199 \mathrm{~A}$ & & & \\
\hline 1282.1 & $199 \mathrm{~A}$ & 7.5 & 19.8 & \\
\hline 1202.1 & $199 A$ & & 71.6 & \\
\hline 402.4 & $199 A$ & & 15.3 & $2.01 \mathrm{E}-03$ \\
\hline 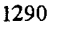 & $200 \mathrm{~A}$ & 41.5 & -56.9 & $2.85 \mathrm{E}-02$ \\
\hline & & & & \\
\hline
\end{tabular}


$\begin{array}{llllll}1290 & 200 \mathrm{~A} & 339.6 & -54.6 & 2.61 \mathrm{E}-02 & 6\end{array}$

$\begin{array}{llllll}1290 & 200 \mathrm{~A} & 340.1 & -53.3 & 2.42 \mathrm{E}-02 & 9\end{array}$

$\begin{array}{llllll}1290 & 200 \mathrm{~A} & 340.6 & -53.6 & 2.27 \mathrm{E}-02 & 12\end{array}$

$\begin{array}{llllll}1290 & 200 \mathrm{~A} & 339.6 & -53.5 & 2.12 \mathrm{E}-02 & 15\end{array}$

$\begin{array}{llllll}1290 & 200 \mathrm{~A} & 339.9 & -53 & 1.96 \mathrm{E}-02 & 18\end{array}$

$\begin{array}{llllll}1290 & 200 \mathrm{~A} & 339.9 & -52.4 & 1.84 \mathrm{E}-02 & 21\end{array}$

$1290 \quad 200 \mathrm{~A} \quad 339.7 \quad-51.5 \quad 1.63 \mathrm{E}-02 \quad 25$

$\begin{array}{llllll}1290 & 200 \mathrm{~A} & 338.3 & -52.7 & 1.41 \mathrm{E}-02 & 30\end{array}$

$\begin{array}{lllllll}1290 & 200 \mathrm{~A} & 340.6 & -53.2 & 1.04 \mathrm{E}-02 & 40\end{array}$

$\begin{array}{llllll}1290 & 200 \mathrm{~A} & 349.5 & -55.3 & 7.24 \mathrm{E}-03 & 50\end{array}$

$\begin{array}{llllll}1290 & 200 \mathrm{~A} & 342.1 & -65.2 & 4.06 \mathrm{E}-03 & 60\end{array}$

$\begin{array}{llllll}1290 & 200 \mathrm{~A} & 1.4 & -61.3 & 2.90 \mathrm{E}-03 & 70\end{array}$

$\begin{array}{llllll}1290 & 200 \mathrm{~A} & 356.8 & -61.2 & 2.24 \mathrm{E}-03 & 85\end{array}$

$\begin{array}{llllll}1290 & 200 \mathrm{~A} & 10.7 & -38 & 1.29 \mathrm{E}-03 & 100\end{array}$

$\begin{array}{lllllll}1290 & 200 \mathrm{~A} & 35.4 & 49.3 & 2.58 \mathrm{E}-03 & 115\end{array}$

$\begin{array}{llllll}1306.4 & 201 \mathrm{~A} & 348.2 & 64.1 & 4.09 \mathrm{E}-02 & 0\end{array}$

$\begin{array}{llllll}1306.4 & 201 \mathrm{~A} & 353.6 & 62.3 & 3.68 \mathrm{E}-02 & 3\end{array}$

$\begin{array}{llllll}1306.4 & 201 \mathrm{~A} & 354.5 & 60.2 & 3.10 \mathrm{E}-02 & 6\end{array}$

$\begin{array}{llllll}1306.4 & 201 \mathrm{~A} & 49.2 & 35.2 & 2.61 \mathrm{E}-02 & 9\end{array}$

$\begin{array}{llllll}1306.4 & 201 \mathrm{~A} & 47.7 & 34.2 & 2.29 \mathrm{E}-02 & 12\end{array}$

$\begin{array}{llllll}1306.4 & 201 \mathrm{~A} & 46.7 & 35.2 & 2.03 \mathrm{E}-02 & 15\end{array}$

$\begin{array}{llllll}1306.4 & 201 \mathrm{~A} & 354.7 & 55.1 & 1.85 \mathrm{E}-02 & 18\end{array}$

$\begin{array}{llllll}1306.4 & 201 \mathrm{~A} & 356.5 & 54.2 & 1.65 \mathrm{E}-02 & 21\end{array}$

$\begin{array}{llllll}1306.4 & 201 \mathrm{~A} & 355.1 & 54.8 & 1.44 \mathrm{E}-02 & 25\end{array}$

$\begin{array}{llllll}1306.4 & 201 \mathrm{~A} & 354.1 & 54.5 & 1.30 \mathrm{E}-02 & 30\end{array}$

$\begin{array}{lllllll}1306.4 & 201 \mathrm{~A} & 349.9 & 49.6 & 8.55 \mathrm{E}-03 & 40\end{array}$

$\begin{array}{llllll}1306.4 & 201 \mathrm{~A} & 352.8 & 49.2 & 6.51 \mathrm{E}-03 & 50\end{array}$

$\begin{array}{llllll}1306.4 & 201 \mathrm{~A} & 354.2 & 50.5 & 4.40 \mathrm{E}-03 & 60\end{array}$

$\begin{array}{llllll}1306.4 & 201 \mathrm{~A} & 345 & 49.3 & 3.05 \mathrm{E}-03 & 70\end{array}$

$\begin{array}{llllll}1306.4 & 201 \mathrm{~A} & 359.5 & 42.7 & 2.45 \mathrm{E}-03 & 85\end{array}$

$\begin{array}{llllll}1306.4 & 201 \mathrm{~A} & 27 & 4.4 & 7.20 \mathrm{E}-04 & 100\end{array}$

$\begin{array}{llllll}1306.4 & 201 \mathrm{~A} & 260.3 & 50.1 & 9.57 \mathrm{E}-04 & 115\end{array}$

$\begin{array}{llllll}1309.4 & 202 \mathrm{~A} & 204.3 & 78.6 & 7.61 \mathrm{E}-02 & 0\end{array}$

$\begin{array}{llllll}1309.4 & 202 \mathrm{~A} & 198.5 & 77.1 & 6.57 \mathrm{E}-02 & 3\end{array}$

$\begin{array}{llllll}1309.4 & 202 \mathrm{~A} & 191.4 & 75.6 & 5.11 \mathrm{E}-02 & 6\end{array}$

$\begin{array}{llllll}1309.4 & 202 \mathrm{~A} & 183.8 & 74.6 & 3.75 \mathrm{E}-02 & 9\end{array}$

$\begin{array}{llllll}1309.4 & 202 \mathrm{~A} & 178.1 & 73.9 & 3.06 \mathrm{E}-02 & 12\end{array}$

$\begin{array}{llllll}1309.4 & 202 \mathrm{~A} & 179 & 75 & 2.53 \mathrm{E}-02 & 15\end{array}$

$\begin{array}{llllll}1309.4 & 202 \mathrm{~A} & 176.1 & 75.1 & 2.07 \mathrm{E}-02 & 18\end{array}$

$\begin{array}{llllll}1309.4 & 202 \mathrm{~A} & 179.6 & 76.8 & 1.70 \mathrm{E}-02 & 21\end{array}$

$\begin{array}{lllllll}1309.4 & 202 \mathrm{~A} & 192.1 & 77.9 & 1.34 \mathrm{E}-02 & 25\end{array}$

$\begin{array}{llllll}1309.4 & 202 \mathrm{~A} & 193.2 & 78.4 & 1.05 \mathrm{E}-02 & 30\end{array}$

$\begin{array}{llllll}1309.4 & 202 \mathrm{~A} & 261 & 87.3 & 5.49 \mathrm{E}-03 & 40\end{array}$

$\begin{array}{llllll}1309.4 & 202 \mathrm{~A} & 341.4 & 65.1 & 2.65 \mathrm{E}-03 & 50\end{array}$

$\begin{array}{llllll}1309.4 & 202 \mathrm{~A} & 355.9 & 23 & 1.56 \mathrm{E}-03 & 60\end{array}$

$\begin{array}{llllll}1309.4 & 202 \mathrm{~A} & 11.6 & -6.2 & 3.04 \mathrm{E}-03 & 70\end{array}$

$\begin{array}{llllll}1309.4 & 202 \mathrm{~A} & 12.5 & -52.4 & 3.45 \mathrm{E}-03 & 85\end{array}$

$\begin{array}{lllllll}1309.4 & 202 \mathrm{~A} & 358.1 & -23.7 & 3.19 \mathrm{E}-03 & 100\end{array}$

$\begin{array}{lllllll}1309.4 & 202 \mathrm{~A} & 25.3 & -38.3 & 2.92 \mathrm{E}-03 & 115\end{array}$

$\begin{array}{llllll}1311.4 & 203 \mathrm{~A} & 114.2 & 76.9 & 1.15 \mathrm{E}-01 & 0\end{array}$

$\begin{array}{llllll}1311.4 & 203 \mathrm{~A} & 110.1 & 73.4 & 9.73 \mathrm{E}-02 & 3\end{array}$ $\begin{array}{llllll}1311.4 & 203 \mathrm{~A} & 106.8 & 71.2 & 7.41 \mathrm{E}-02 & 6\end{array}$

$\begin{array}{llllll}1311.4 & 203 \mathrm{~A} & 101.8 & 67.9 & 5.57 \mathrm{E}-02 & 9\end{array}$

$\begin{array}{lllllll}1311.4 & 203 \mathrm{~A} & 99.3 & 66.6 & 4.55 \mathrm{E}-02 & 12\end{array}$

$\begin{array}{lllllll}1311.4 & 203 \mathrm{~A} & 98.3 & 66.5 & 3.64 \mathrm{E}-02 & 15\end{array}$

$\begin{array}{lllllll}1311.4 & 203 \mathrm{~A} & 94.7 & 64.6 & 3.08 \mathrm{E}-02 & 18\end{array}$

$\begin{array}{lllllll}1311.4 & 203 \mathrm{~A} & 97.8 & 62.2 & 2.44 \mathrm{E}-02 & 21\end{array}$

$\begin{array}{llllll}1311.4 & 203 \mathrm{~A} & 102.4 & 64 & 1.96 \mathrm{E}-02 & 25\end{array}$

$\begin{array}{llllll}1311.4 & 203 \mathrm{~A} & 95.5 & 62.8 & 1.62 \mathrm{E}-02 & 30\end{array}$

$\begin{array}{lllllll}1311.4 & 203 \mathrm{~A} & 91.9 & 65.8 & 9.20 \mathrm{E}-03 & 40\end{array}$

$\begin{array}{lllllll}1311.4 & 203 \mathrm{~A} & 78.8 & 68.1 & 6.00 \mathrm{E}-03 & 50\end{array}$

$\begin{array}{llllll}1311.4 & 203 \mathrm{~A} & 42.3 & 50.3 & 2.97 \mathrm{E}-03 & 60\end{array}$

$\begin{array}{lllllll}1311.4 & 203 \mathrm{~A} & 85.6 & 65.9 & 2.28 \mathrm{E}-03 & 70\end{array}$

$\begin{array}{llllll}1311.4 & 203 \mathrm{~A} & 5 & 50.5 & 2.35 \mathrm{E}-03 & 85\end{array}$

$\begin{array}{llllll}1311.4 & 203 \mathrm{~A} & 61.4 & 51.9 & 3.54 \mathrm{E}-03 & 100\end{array}$

$\begin{array}{llllll}1311.4 & 203 \mathrm{~A} & 56.2 & 74.6 & 1.83 \mathrm{E}-03 & 115\end{array}$

$\begin{array}{llllll}1313.3 & 204 \mathrm{~A} & 108.9 & 73.5 & 8.80 \mathrm{E}-02 & 0\end{array}$

$\begin{array}{llllll}1313.3 & 204 \mathrm{~A} & 108.2 & 69.3 & 7.40 \mathrm{E}-02 & 3\end{array}$

$\begin{array}{llllll}1313.3 & 204 \mathrm{~A} & 105.7 & 66.2 & 5.57 \mathrm{E}-02 & 6\end{array}$

$\begin{array}{llllll}1313.3 & 204 \mathrm{~A} & 102.8 & 62.7 & 4.07 \mathrm{E}-02 & 9\end{array}$

$\begin{array}{llllll}1313.3 & 204 \mathrm{~A} & 99.5 & 61.9 & 3.17 \mathrm{E}-02 & 12\end{array}$

$\begin{array}{llllll}1313.3 & 204 \mathrm{~A} & 99.2 & 57 & 2.55 \mathrm{E}-02 & 15\end{array}$

$\begin{array}{llllll}1313.3 & 204 \mathrm{~A} & 95 & 58.7 & 2.08 \mathrm{E}-02 & 18\end{array}$

$\begin{array}{llllll}1313.3 & 204 \mathrm{~A} & 91.1 & 55.3 & 1.72 \mathrm{E}-02 & 21\end{array}$

$\begin{array}{lllllll}1313.3 & 204 \mathrm{~A} & 88.1 & 52.2 & 1.38 \mathrm{E}-02 & 25\end{array}$

$\begin{array}{llllll}1313.3 & 204 \mathrm{~A} & 92.1 & 47.6 & 1.08 \mathrm{E}-02 & 30\end{array}$

$\begin{array}{lllllll}1313.3 & 204 \mathrm{~A} & 78.9 & 28.7 & 4.29 \mathrm{E}-03 & 40\end{array}$

$\begin{array}{llllll}1313.3 & 204 \mathrm{~A} & 68.1 & -8.9 & 2.65 \mathrm{E}-03 & 50\end{array}$

$\begin{array}{llllll}1313.3 & 204 \mathrm{~A} & 76.4 & 13.4 & 2.42 \mathrm{E}-03 & 60\end{array}$

$\begin{array}{llllll}1313.3 & 204 \mathrm{~A} & 33.5 & 43.5 & 3.83 \mathrm{E}-03 & 70\end{array}$

$\begin{array}{llllll}1313.3 \quad 204 \mathrm{~A} & 34.8 & -37.8 & 2.24 \mathrm{E}-03 & 85\end{array}$

$\begin{array}{lllllll}1313.3 & 204 \mathrm{~A} & 70.9 & 17.5 & 4.35 \mathrm{E}-04 & 100\end{array}$

$\begin{array}{llllll}1313.3 & 204 \mathrm{~A} & 355.3 & -64.2 & 2.55 \mathrm{E}-03 & 115\end{array}$

$\begin{array}{llllll}1320 & 205 \mathrm{~A} & 68.5 & 69.4 & 5.68 \mathrm{E}-02 & 0\end{array}$

$\begin{array}{lllllll}1320 & 205 \mathrm{~A} & 70 & 66.7 & 4.94 \mathrm{E}-02 & 3\end{array}$

$\begin{array}{lllllll}1320 & 205 \mathrm{~A} & 69.1 & 67.7 & 3.93 \mathrm{E}-02 & 6\end{array}$

$\begin{array}{llllll}1320 & 205 \mathrm{~A} & 66.9 & 68.2 & 2.90 \mathrm{E}-02 & 9\end{array}$

$\begin{array}{llllll}1320 & 205 \mathrm{~A} & 64.9 & 70.6 & 2.34 \mathrm{E}-02 & 12\end{array}$

$\begin{array}{llllll}1320 & 205 \mathrm{~A} & 64.5 & 71.6 & 1.84 \mathrm{E}-02 & 15\end{array}$

$\begin{array}{llllll}1320 & 205 \mathrm{~A} & 62.9 & 77.3 & 1.58 \mathrm{E}-02 & 18\end{array}$

$\begin{array}{llllll}1320 & 205 \mathrm{~A} & 60.6 & 79.2 & 1.18 \mathrm{E}-02 & 21\end{array}$

$\begin{array}{llllll}1320 & 205 \mathrm{~A} & 75.9 & 76.5 & 9.06 \mathrm{E}-03 & 25\end{array}$

$\begin{array}{llllll}1320 & 205 \mathrm{~A} & 342.7 & 88.3 & 7.89 \mathrm{E}-03 & 30\end{array}$

$\begin{array}{llllll}1320 & 205 \mathrm{~A} & 250.1 & 55.8 & 4.51 \mathrm{E}-03 & 40\end{array}$

$\begin{array}{llllll}1320 & 205 \mathrm{~A} & 240.5 & 26 & 3.17 \mathrm{E}-03 & 50\end{array}$

$\begin{array}{llllll}1320 & 205 \mathrm{~A} & 199.9 & 10.9 & 1.69 \mathrm{E}-03 & 60\end{array}$

$\begin{array}{llllll}1320 & 205 \mathrm{~A} & 242.4 & 14.3 & 1.25 \mathrm{E}-03 & 70\end{array}$

$\begin{array}{lllllll}1320 & 205 \mathrm{~A} & 199.7 & 6.4 & 3.55 \mathrm{E}-03 & 85\end{array}$

$\begin{array}{lllllll}1320 & 205 \mathrm{~A} & 258.7 & 36.6 & 1.65 \mathrm{E}-03 & 100\end{array}$

$\begin{array}{llllllll}1320 & 205 \mathrm{~A} & 162.8 & 40.3 & 8.93 \mathrm{E}-04 & 115\end{array}$

$\begin{array}{llllll}1328.3 & 206 \mathrm{~A} & 198.3 & 78.3 & 1.59 \mathrm{E}-01 & 0\end{array}$

$\begin{array}{llllll}1328.3 & 206 \mathrm{~A} & 187.1 & 76.6 & 1.41 \mathrm{E}-01 & 3\end{array}$ $\begin{array}{llllll}1328.3 & 206 \mathrm{~A} & 182.8 & 74.2 & 1.16 \mathrm{E}-01 & 6\end{array}$

$\begin{array}{llllll}1328.3 & 206 \mathrm{~A} & 178.6 & 70.8 & 8.79 \mathrm{E}-02 & 9\end{array}$

$\begin{array}{llllll}1328.3 & 206 \mathrm{~A} & 173.9 & 69.2 & 7.22 \mathrm{E}-02 & 12\end{array}$

$\begin{array}{lllllll}1328.3 & 206 \mathrm{~A} & 169.8 & 68.6 & 6.21 \mathrm{E}-02 & 15\end{array}$

$\begin{array}{llllll}1328.3 & 206 \mathrm{~A} & 163.1 & 66.8 & 5.16 \mathrm{E}-02 & 18\end{array}$

$\begin{array}{llllll}1328.3 & 206 \mathrm{~A} & 160.9 & 64.5 & 4.35 \mathrm{E}-02 & 21\end{array}$

$\begin{array}{lllllll}1328.3 & 206 \mathrm{~A} & 153.5 & 62.7 & 3.53 \mathrm{E}-02 & 25\end{array}$

$\begin{array}{lllllll}1328.3 & 206 \mathrm{~A} & 148.5 & 60.9 & 2.95 \mathrm{E}-02 & 30\end{array}$

$\begin{array}{lllllll}1328.3 & 206 \mathrm{~A} & 137.9 & 60.3 & 2.05 \mathrm{E}-02 & 40\end{array}$

$\begin{array}{llllll}1328.3 & 206 \mathrm{~A} & 135.6 & 55.2 & 1.46 \mathrm{E}-02 & 50\end{array}$

$\begin{array}{lllllll}1328.3 & 206 \mathrm{~A} & 118.1 & 56.1 & 1.13 \mathrm{E}-02 & 60\end{array}$

$\begin{array}{lllllll}1328.3 & 206 \mathrm{~A} & 108.8 & 53.6 & 8.98 \mathrm{E}-03 & 70\end{array}$

$\begin{array}{lllllll}1328.3 & 206 \mathrm{~A} & 109.9 & 24.4 & 6.39 \mathrm{E}-03 & 85\end{array}$

$\begin{array}{llllll}1328.3 & 206 \mathrm{~A} & 88.8 & 41.8 & 5.19 \mathrm{E}-03 & 100\end{array}$

$\begin{array}{lllllll}1328.3 & 206 \mathrm{~A} & 77.9 & 24.8 & 2.87 \mathrm{E}-03 & 115\end{array}$

$\begin{array}{llllll}1338.2 & 207 \mathrm{~A} & 19.8 & 64.8 & 1.54 \mathrm{E}-01 & 0\end{array}$

$\begin{array}{llllll}1338.2 & 207 \mathrm{~A} & 21.9 & 64.5 & 1.38 \mathrm{E}-01 & 3\end{array}$

$\begin{array}{llllll}1338.2 & 207 \mathrm{~A} & 21.9 & 64.6 & 1.16 \mathrm{E}-01 & 6\end{array}$

$\begin{array}{llllll}1338.2 & 207 \mathrm{~A} & 27.3 & 63.9 & 9.73 \mathrm{E}-02 & 9\end{array}$

$\begin{array}{llllll}1338.2 & 207 \mathrm{~A} & 26.7 & 64.3 & 8.41 \mathrm{E}-02 & 12\end{array}$

$\begin{array}{lllllll}1338.2 & 207 \mathrm{~A} & 28.7 & 62.5 & 7.05 \mathrm{E}-02 & 15\end{array}$

$\begin{array}{llllll}1338.2 & 207 \mathrm{~A} & 27 & 66 & 6.50 \mathrm{E}-02 & 18\end{array}$

$\begin{array}{llllll}1338.2 & 207 \mathrm{~A} & 26 & 64.3 & 5.68 \mathrm{E}-02 & 21\end{array}$

$\begin{array}{lllllll}1338.2 & 207 \mathrm{~A} & 28.3 & 65.1 & 4.78 \mathrm{E}-02 & 25\end{array}$

$\begin{array}{lllllll}1338.2 & 207 \mathrm{~A} & 16.2 & 69.6 & 4.19 \mathrm{E}-02 & 30\end{array}$

$\begin{array}{lllllll}1338.2 & 207 \mathrm{~A} & 30 & 66.7 & 2.03 \mathrm{E}-02 & 40\end{array}$

$\begin{array}{lllllll}1338.2 & 207 \mathrm{~A} & 62.2 & 79.1 & 9.08 \mathrm{E}-03 & 50\end{array}$

$\begin{array}{llllll}1338.2 & 207 \mathrm{~A} & 283.7 & 68.7 & 7.49 \mathrm{E}-03 & 60\end{array}$

$\begin{array}{llllll}1338.2 & 207 \mathrm{~A} & 211 & -17.6 & 4.36 \mathrm{E}-03 & 70\end{array}$

$\begin{array}{llllll}1338.2 & 207 \mathrm{~A} & 307.5 & 11.4 & 2.31 \mathrm{E}-03 & 85\end{array}$

$\begin{array}{llllll}1338.2 & 207 \mathrm{~A} & 268 & -20.6 & 3.76 \mathrm{E}-03 & 100\end{array}$

$\begin{array}{lllllll}1338.2 & 207 \mathrm{~A} & 267.7 & -54.1 & 5.94 \mathrm{E}-03 & 115\end{array}$

$\begin{array}{llllll}1340.4 & 208 \mathrm{~A} & 22.2 & 58.8 & 1.12 \mathrm{E}-01 & 0\end{array}$

$\begin{array}{lllllll}1340.4 & 208 \mathrm{~A} & 24.8 & 57.9 & 1.06 \mathrm{E}-01 & 3\end{array}$

$\begin{array}{llllll}1340.4 & 208 \mathrm{~A} & 25 & 57.2 & 9.45 \mathrm{E}-02 & 6\end{array}$

$\begin{array}{llllll}1340.4 & 208 \mathrm{~A} & 24.8 & 56.5 & 8.52 \mathrm{E}-02 & 9\end{array}$

$\begin{array}{lllllll}1340.4 & 208 \mathrm{~A} & 25.3 & 56.4 & 7.79 \mathrm{E}-02 & 12\end{array}$

$\begin{array}{llllll}1340.4 & 208 \mathrm{~A} & 26.3 & 55.5 & 7.11 \mathrm{E}-02 & 15\end{array}$

$\begin{array}{llllll}1340.4 & 208 \mathrm{~A} & 24.4 & 57.1 & 6.70 \mathrm{E}-02 & 18\end{array}$

$\begin{array}{lllllll}1340.4 & 208 \mathrm{~A} & 27.5 & 54.1 & 5.87 \mathrm{E}-02 & 21\end{array}$

$\begin{array}{llllll}1340.4 & 208 \mathrm{~A} & 25 & 55.3 & 4.99 \mathrm{E}-02 & 25\end{array}$

$\begin{array}{llllll}1340.4 & 208 \mathrm{~A} & 20.6 & 59 & 4.45 \mathrm{E}-02 & 30\end{array}$

$\begin{array}{llllll}1340.4 & 208 \mathrm{~A} & 28.4 & 50.9 & 2.24 \mathrm{E}-02 & 40\end{array}$

$\begin{array}{llllll}1340.4 & 208 \mathrm{~A} & 19.2 & 53.2 & 1.14 \mathrm{E}-02 & 50\end{array}$

$\begin{array}{llllll}1340.4 & 208 \mathrm{~A} & 22.7 & 25.9 & 5.65 \mathrm{E}-03 & 60\end{array}$

$\begin{array}{llllll}1340.4 & 208 \mathrm{~A} & 9.1 & 24.7 & 3.17 \mathrm{E}-03 & 70\end{array}$

$\begin{array}{lllllll}1340.4 & 208 \mathrm{~A} & 9.5 & -0.1 & 2.25 \mathrm{E}-03 & 85\end{array}$

$\begin{array}{llllll}1340.4 & 208 \mathrm{~A} & 285.6 & 46.3 & 2.50 \mathrm{E}-03 & 100\end{array}$

$\begin{array}{llllll}1340.4 & 208 \mathrm{~A} & 291.2 & -38 & 2.32 \mathrm{E}-03 & 115\end{array}$

$\begin{array}{lllllll}1358.6 & 209 \mathrm{~A} & 58.5 & -19.3 & 4.24 \mathrm{E}-03 & 0\end{array}$

$\begin{array}{llllll}1358.6 & 209 \mathrm{~A} & 310.7 & 73.1 & 1.32 \mathrm{E}-01 & 3\end{array}$ 
$\begin{array}{llllll}1358.6 & 209 \mathrm{~A} & 310.6 & 72.3 & 1.04 \mathrm{E}-01 & 6\end{array}$ $\begin{array}{lllllll}1358.6 & 209 \mathrm{~A} & 312.7 & 72.5 & 8.13 \mathrm{E}-02 & 9\end{array}$ $\begin{array}{llllll}1358.6 & 209 \mathrm{~A} & 315.2 & 71.4 & 6.55 \mathrm{E}-02 & 12\end{array}$ $\begin{array}{llllll}1358.6 & 209 \mathrm{~A} & 318 & 71.2 & 5.48 \mathrm{E}-02 & 15\end{array}$ $\begin{array}{llllll}1358.6 & 209 \mathrm{~A} & 317.4 & 70.3 & 4.79 \mathrm{E}-02 & 18\end{array}$ $\begin{array}{llllll}1358.6 & 209 \mathrm{~A} & 320.2 & 71 & 4.18 \mathrm{E}-02 & 21\end{array}$ $\begin{array}{lllllll}1358.6 & 209 \mathrm{~A} & 316.3 & 68 & 3.57 \mathrm{E}-02 & 25\end{array}$ $\begin{array}{llllll}1358.6 & 209 \mathrm{~A} & 312.8 & 67.3 & 3.12 \mathrm{E}-02 & 30\end{array}$ $\begin{array}{lllllll}1358.6 & 209 \mathrm{~A} & 323.2 & 72.6 & 2.44 \mathrm{E}-02 & 40\end{array}$ $\begin{array}{llllll}1358.6 & 209 \mathrm{~A} & 326.2 & 76 & 1.52 \mathrm{E}-02 & 50\end{array}$ $\begin{array}{lllllll}1358.6 & 209 \mathrm{~A} & 354.5 & 72.6 & 1.04 \mathrm{E}-02 & 60\end{array}$ $\begin{array}{lllllll}1358.6 & 209 \mathrm{~A} & 52.7 & 51.7 & 7.95 \mathrm{E}-03 & 70\end{array}$ $\begin{array}{lllllll}1358.6 & 209 \mathrm{~A} & 59.5 & 8.1 & 4.64 \mathrm{E}-03 & 85\end{array}$ $\begin{array}{llllllll}1358.6 & 209 \mathrm{~A} & 58.4 & -25.3 & 5.68 \mathrm{E}-03 & 100\end{array}$ $\begin{array}{lllllll}1358.6 & 209 \mathrm{~A} & 56.5 & -19.5 & 4.31 \mathrm{E}-03 & 115\end{array}$ $\begin{array}{lllllll}1358.6 & 209 \mathrm{~A} & 58.1 & -35.9 & 7.17 \mathrm{E}-03 & 130\end{array}$ $\begin{array}{lllllll}1359 & 209 \mathrm{~B} & 76.9 & 73.6 & 2.64 \mathrm{E}-01 & 0\end{array}$ $\begin{array}{lllllll}1359 & 209 \mathrm{~B} & 77.6 & 71.7 & 2.15 \mathrm{E}-01 & 3\end{array}$ $\begin{array}{lllllll}1359 & 209 \mathrm{~B} & 77.4 & 69.8 & 1.45 \mathrm{E}-01 & 6\end{array}$ $\begin{array}{lllllll}1359 & 209 \mathrm{~B} & 78.4 & 69.5 & 1.02 \mathrm{E}-01 & 9\end{array}$ $\begin{array}{llllll}1359 & 209 \mathrm{~B} & 79 & 69.6 & 7.98 \mathrm{E}-02 & 12\end{array}$ $\begin{array}{llllll}1359 & 209 \mathrm{~B} & 84.1 & 65.9 & 6.17 \mathrm{E}-02 & 15\end{array}$ $\begin{array}{lllllll}1359 & 209 \mathrm{~B} & 84.2 & 69.8 & 5.14 \mathrm{E}-02 & 18\end{array}$ $\begin{array}{lllllll}1359 & 209 \mathrm{~B} & 90.6 & 62.9 & 4.16 \mathrm{E}-02 & 21\end{array}$ $\begin{array}{llllll}1359 & 209 \mathrm{~B} & 92 & 61.7 & 3.31 \mathrm{E}-02 & 25\end{array}$ $\begin{array}{llllll}1359 & 209 \mathrm{~B} & 104.5 & 73.5 & 2.69 \mathrm{E}-02 & 30\end{array}$ $\begin{array}{llllll}1359 & 209 \mathrm{~B} & 196.6 & 52.4 & 8.12 \mathrm{E}-03 & 40\end{array}$ $\begin{array}{lllllll}1359 & 209 \mathrm{~B} & 232.4 & -2.4 & 1.01 \mathrm{E}-02 & 50\end{array}$ $\begin{array}{lllllll}1359 & 209 \mathrm{~B} & 224.6 & -12.2 & 1.11 \mathrm{E}-02 & 60\end{array}$ $\begin{array}{lllllll}1359 & 209 \mathrm{~B} & 217.4 & -42.6 & 1.09 \mathrm{E}-02 & 70\end{array}$ $\begin{array}{lllllll}1359 & 209 \mathrm{~B} & 221.4 & -28.8 & 9.82 \mathrm{E}-03 & 85\end{array}$ $\begin{array}{lllllll}1359 & 209 \mathrm{~B} & 233.2 & 48.8 & 1.41 \mathrm{E}-02 & 100\end{array}$ $\begin{array}{lllllll}1359 & 209 \mathrm{~B} & 218 & 44.8 & 9.41 \mathrm{E}-03 & 115\end{array}$ $\begin{array}{lllllll}1361 & 210 \mathrm{~A} & 84.8 & 75.4 & 6.40 \mathrm{E}-02 & 0\end{array}$ $\begin{array}{lllllll}1361 & 210 \mathrm{~A} & 89.4 & 71.6 & 5.24 \mathrm{E}-02 & 3\end{array}$ $\begin{array}{lllllll}1361 & 210 \mathrm{~A} & 89.8 & 68.3 & 3.82 \mathrm{E}-02 & 6\end{array}$ $\begin{array}{lllllll}1361 & 210 \mathrm{~A} & 90.9 & 65.2 & 2.72 \mathrm{E}-02 & 9\end{array}$ $\begin{array}{lllllll}1361 & 210 \mathrm{~A} & 91.1 & 64.6 & 2.14 \mathrm{E}-02 & 12\end{array}$ $\begin{array}{llllll}1361 & 210 \mathrm{~A} & 94.6 & 63.4 & 1.74 \mathrm{E}-02 & 15\end{array}$ $\begin{array}{lllllll}1361 & 210 \mathrm{~A} & 90.7 & 64.9 & 1.49 \mathrm{E}-02 & 18\end{array}$ $\begin{array}{lllllll}1361 & 210 \mathrm{~A} & 91.6 & 57.3 & 1.17 \mathrm{E}-02 & 21\end{array}$ $\begin{array}{lllllll}1361 & 210 \mathrm{~A} & 86.9 & 61.6 & 8.97 \mathrm{E}-03 & 25\end{array}$ $\begin{array}{lllllll}1361 & 210 \mathrm{~A} & 92 & 67.9 & 7.19 \mathrm{E}-03 & 30\end{array}$ $\begin{array}{lllllll}1361 & 210 \mathrm{~A} & 71.1 & 42.4 & 4.57 \mathrm{E}-03 & 40\end{array}$ $\begin{array}{lllllll}1361 & 210 \mathrm{~A} & 112.4 & 61.7 & 2.84 \mathrm{E}-03 & 50\end{array}$ $\begin{array}{lllllll}1361 & 210 \mathrm{~A} & 35.5 & 20.9 & 2.29 \mathrm{E}-03 & 60\end{array}$ $\begin{array}{llllll}1361 & 210 \mathrm{~A} & 28.6 & 22 & 2.3 \mathrm{tE}-03 & 70\end{array}$ $\begin{array}{lllllll}1361 & 210 \mathrm{~A} & 149.5 & -25.5 & 2.08 \mathrm{E}-03 & 85\end{array}$ $\begin{array}{lllllll}1361 & 210 \mathrm{~A} & 63.4 & 43.8 & 1.16 \mathrm{E}-03 & 100\end{array}$ $\begin{array}{lllllll}1361 & 210 \mathrm{~A} & 260.2 & 46.3 & 2.04 \mathrm{E}-03 & 115\end{array}$ $\begin{array}{llllll}1362.8 & 211 \mathrm{~A} & 89.1 & 66.5 & 9.95 \mathrm{E}-02 & 0\end{array}$

\begin{tabular}{|c|c|c|c|c|c|c|c|c|c|c|c|}
\hline 1362.8 & $211 \mathrm{~A}$ & 88.2 & 63.6 & 8.83E-02 & 3 & 1377.3 & $252 \mathrm{~A}$ & 358.9 & 74.7 & $8.63 \mathrm{E}-02$ & 2 \\
\hline 1362.8 & $211 \mathrm{~A}$ & 85.4 & 61.4 & $6.94 \mathrm{E}-02$ & 6 & 1377.3 & $252 \mathrm{~A}$ & 2.2 & 74.3 & $6.55 \mathrm{E}-02$ & 2 \\
\hline 1362.8 & $211 \mathrm{~A}$ & 86.2 & 57.9 & $5.27 \mathrm{E}-02$ & 9 & 1377.3 & $252 \mathrm{~A}$ & 2.6 & 73.8 & 4.95E-02 & 2 \\
\hline 1362.8 & $211 \mathrm{~A}$ & 83.4 & 56.4 & $4.30 \mathrm{E}-02$ & 12 & 1377.3 & $252 \mathrm{~A}$ & 3.4 & 74.9 & $3.92 \mathrm{E}-02$ & 212 \\
\hline 1362.8 & $211 \mathrm{~A}$ & 83.2 & 52.4 & $3.61 \mathrm{E}-02$ & 15 & 1377.3 & $252 \mathrm{~A}$ & 10.3 & 75.3 & $3.02 \mathrm{E}-02$ & 15 \\
\hline 1362.8 & $211 \mathrm{~A}$ & 83.4 & 53.5 & $3.06 \mathrm{E}-02$ & 18 & 1377.3 & $252 \mathrm{~A}$ & 21.2 & 75.4 & $2.46 \mathrm{E}-02$ & 18 \\
\hline 1362.8 & $211 \mathrm{~A}$ & 84.5 & 46.3 & $2.46 \mathrm{E}-02$ & 21 & 1377.3 & $252 \mathrm{~A}$ & 20 & 74.3 & $2.07 \mathrm{E}-02$ & 221 \\
\hline 1362.8 & $211 \mathrm{~A}$ & 84.9 & 40.6 & $2.03 \mathrm{E}-02$ & 25 & 1377.3 & $252 \mathrm{~A}$ & 19.1 & 76.8 & $1.69 \mathrm{E}-02$ & 25 \\
\hline 1362.8 & $211 \mathrm{~A}$ & 85.4 & 45.2 & $1.65 \mathrm{E}-02$ & 30 & 1377.3 & $252 \mathrm{~A}$ & 0.8 & 74 & $1.43 \mathrm{E}-02$ & 30 \\
\hline 1362.8 & $211 \mathrm{~A}$ & 89.7 & 25.2 & 8.53E-03 & 40 & 1377.3 & $252 \mathrm{~A}$ & 31 & 76.1 & $6.47 \mathrm{E}-03$ & 40 \\
\hline 1362.8 & $211 \mathrm{~A}$ & 102.2 & 10.2 & $4.68 \mathrm{E}-03$ & 50 & 1377.3 & $252 \mathrm{~A}$ & 317.9 & 81.7 & 4.65E-03 & 50 \\
\hline 1362.8 & $211 \mathrm{~A}$ & 108.3 & -31.9 & $3.71 \mathrm{E}-03$ & 60 & 1377.3 & $252 \mathrm{~A}$ & 137 & 61.2 & $2.57 \mathrm{E}-03$ & 60 \\
\hline 1362.8 & $211 \mathrm{~A}$ & 91.3 & -36.8 & $3.53 \mathrm{E}-03$ & 70 & 1377.3 & $252 \mathrm{~A}$ & 159.8 & 66 & $1.52 \mathrm{E}-03$ & 70 \\
\hline 1362.8 & $211 \mathrm{~A}$ & 132.9 & -34.3 & $3.76 \mathrm{E}-03$ & 85 & 1377.3 & $252 \mathrm{~A}$ & 86.8 & 22.3 & 4. $68 \mathrm{E}-03$ & 85 \\
\hline 1362.8 & $211 \mathrm{~A}$ & 128.2 & -54.4 & $2.52 \mathrm{E}-03$ & 100 & 1377.3 & $252 \mathrm{~A}$ & 131.1 & 51.7 & $2.78 \mathrm{E}-03$ & 3100 \\
\hline 1362.8 & $211 \mathrm{~A}$ & 175.7 & 19.2 & $2.17 \mathrm{E}-03$ & 115 & 1377.3 & $252 \mathrm{~A}$ & 136.8 & 72.1 & $1.48 \mathrm{E}-03$ & 3115 \\
\hline 1365.4 & $250 \mathrm{~A}$ & 151.5 & 70.5 & $9.54 \mathrm{E}-02$ & 0 & 1379 & $253 \mathrm{~A}$ & 2 & 63.1 & $1.11 \mathrm{E}-01$ & 0 \\
\hline 1365.4 & $250 \mathrm{~A}$ & 147.3 & 69.2 & 8.69E-02 & 3 & 1379 & $253 \mathrm{~A}$ & 3.9 & 61.7 & $9.92 \mathrm{E}-02$ & 3 \\
\hline 1365.4 & $250 \mathrm{~A}$ & 146.7 & 70.7 & 7.45E-02 & 6 & 1379 & $253 \mathrm{~A}$ & 3.3 & 59.6 & $8.27 \mathrm{E}-02$ & 6 \\
\hline 1365.4 & $250 \mathrm{~A}$ & 145.4 & 72.1 & $6.31 \mathrm{E}-02$ & 9 & 1379 & $253 \mathrm{~A}$ & 3.3 & 58.1 & 7.10E-02 & 9 \\
\hline 1365.4 & $250 \mathrm{~A}$ & 143.9 & 73 & $5.56 \mathrm{E}-02$ & 12 & 1379 & $253 \mathrm{~A}$ & 4.6 & 57.6 & $6.44 \mathrm{E}-02$ & 12 \\
\hline 1365.4 & $250 \mathrm{~A}$ & 140.6 & 73.8 & $4.86 \mathrm{E}-02$ & 15 & 1379 & $253 \mathrm{~A}$ & 5 & 57.4 & $5.56 \mathrm{E}-02$ & 15 \\
\hline 1365.4 & $250 \mathrm{~A}$ & 141.9 & 75.7 & 4.19E-02 & 18 & 1379 & $253 \mathrm{~A}$ & 5.6 & 57.3 & $5.13 \mathrm{E}-02$ & 18 \\
\hline 1365.4 & $250 \mathrm{~A}$ & 141.2 & 75.5 & $3.61 \mathrm{E}-02$ & 21 & 1379 & $253 \mathrm{~A}$ & 6.7 & 57.3 & $4.61 \mathrm{E}-02$ & 21 \\
\hline 1365.4 & $250 \mathrm{~A}$ & 145.1 & 76 & $2.95 \mathrm{E}-02$ & 25 & 1379 & $253 \mathrm{~A}$ & 5.3 & 57.9 & 4.02E- 02 & 25 \\
\hline 1365.4 & $250 \mathrm{~A}$ & 141.6 & 77.1 & $2.34 \mathrm{E}-02$ & 30 & 1379 & $253 \mathrm{~A}$ & 4.2 & 59 & $3.70 \mathrm{E}-02$ & 30 \\
\hline 1365.4 & $250 \mathrm{~A}$ & 147.5 & 75.2 & $1.40 \mathrm{E}-02$ & 40 & 1379 & $253 \mathrm{~A}$ & 3.3 & 54.6 & $2.27 \mathrm{E}-02$ & 40 \\
\hline 1365.4 & $250 \mathrm{~A}$ & 215.2 & 84.2 & 7.95E-03 & 50 & 1379 & $253 \mathrm{~A}$ & 9.3 & 56.4 & $1.36 \mathrm{E}-02$ & 50 \\
\hline 1365.4 & $250 \mathrm{~A}$ & 88.6 & 80.8 & $5.95 \mathrm{E}-03$ & 60 & 1379 & $253 \mathrm{~A}$ & 23.7 & 56.3 & $6.67 \mathrm{E}-03$ & 60 \\
\hline 1365.4 & $250 \mathrm{~A}$ & 175.3 & 76.8 & 3.74E-03 & 70 & 1379 & $253 \mathrm{~A}$ & 355.2 & 43.4 & $4.01 \mathrm{E}-03$ & 70 \\
\hline 1365.4 & $250 \mathrm{~A}$ & 203.4 & 35.8 & $1.88 \mathrm{E}-03$ & 85 & 1379 & $253 \mathrm{~A}$ & 352.4 & 19.5 & $3.62 \mathrm{E}-03$ & 85 \\
\hline 1365.4 & $250 \mathrm{~A}$ & 184.2 & 63.3 & $1.54 \mathrm{E}-03$ & 100 & 1379 & $253 \mathrm{~A}$ & 213.7 & 0.5 & $7.52 \mathrm{E}-04$ & +100 \\
\hline 1365.4 & $250 \mathrm{~A}$ & 10.6 & 66.1 & $2.11 \mathrm{E}-03$ & 115 & 1379 & $253 \mathrm{~A}$ & 343.4 & 42.2 & $1.92 \mathrm{E}-03$ & 3115 \\
\hline 1375.1 & $251 \mathrm{~A}$ & 2.1 & 64.8 & 7.75E-02 & 0 & 1381 & $254 \mathrm{~A}$ & 332.5 & 64.6 & $1.24 \mathrm{E}-01$ & 0 \\
\hline 1375.1 & $251 \mathrm{~A}$ & 5.9 & 63.4 & 7.03E-02 & 3 & 1381 & $254 \mathrm{~A}$ & 333.3 & 62.6 & $1.08 \mathrm{E}-01$ & 3 \\
\hline 1375.1 & $251 \mathrm{~A}$ & 4.6 & 62.4 & $6.13 \mathrm{E}-02$ & 6 & 1381 & $254 \mathrm{~A}$ & 333.3 & 60 & 8.82E-02 & 6 \\
\hline 1375.1 & $251 \mathrm{~A}$ & 3.5 & 60.8 & $5.27 \mathrm{E}-02$ & 9 & 1381 & $254 \mathrm{~A}$ & 333.6 & 56.5 & $6.84 \mathrm{E}-02$ & 9 \\
\hline 1375.1 & $251 \mathrm{~A}$ & 1.4 & 60 & $4.58 \mathrm{E}-02$ & 12 & 1381 & $254 \mathrm{~A}$ & 333.8 & 55.2 & $5.67 \mathrm{E}-02$ & 12 \\
\hline 1375.1 & $251 \mathrm{~A}$ & 356.7 & 60.5 & $4.05 \mathrm{E}-02$ & 15 & 1381 & $254 \mathrm{~A}$ & 334.3 & 54.3 & $4.81 \mathrm{E}-02$ & 15 \\
\hline 1375.1 & $251 \mathrm{~A}$ & 355.9 & 59.3 & 3.44E-02 & 18 & 1381 & $254 \mathrm{~A}$ & 336 & 54.7 & $4.18 \mathrm{E}-02$ & 18 \\
\hline 1375.1 & $251 \mathrm{~A}$ & 0.9 & 59.7 & 2.94E-02 & 21 & 1381 & $254 \mathrm{~A}$ & 334.8 & 53.1 & $3.48 \mathrm{E}-02$ & 21 \\
\hline 1375.1 & $251 \mathrm{~A}$ & 355.7 & 58.7 & $2.51 \mathrm{E}-02$ & 25 & 1381 & $254 \mathrm{~A}$ & 335.7 & 52.5 & $2.90 \mathrm{E}-02$ & 25 \\
\hline 1375.1 & $251 \mathrm{~A}$ & 355.8 & 60.4 & $2.01 \mathrm{E}-02$ & 30 & 1381 & $254 \mathrm{~A}$ & 341.9 & 51.5 & $2.59 \mathrm{E}-02$ & 30 \\
\hline 1375.1 & $251 \mathrm{~A}$ & 354.2 & 62.9 & $1.47 \mathrm{E}-02$ & 40 & 1381 & $254 \mathrm{~A}$ & 340.8 & 50.5 & $1.63 \mathrm{E}-02$ & 40 \\
\hline 1375.1 & $251 \mathrm{~A}$ & 346.4 & 50.1 & $7.16 \mathrm{E}-03$ & 50 & 1381 & $254 \mathrm{~A}$ & 340.4 & 51.6 & $1.20 \mathrm{E}-02$ & 50 \\
\hline 1375.1 & $251 \mathrm{~A}$ & 340.7 & 46.6 & $4.27 \mathrm{E}-03$ & 60 & 1381 & $254 \mathrm{~A}$ & 345.2 & 42 & $6.88 \mathrm{E}-03$ & 60 \\
\hline 1375.1 & $251 \mathrm{~A}$ & 338.5 & 25.6 & 3.33E-03 & 70 & 1381 & $254 \mathrm{~A}$ & 20.6 & 37.9 & $2.66 \mathrm{E}-03$ & 70 \\
\hline 1375.1 & $251 \mathrm{~A}$ & 309.4 & 39.4 & $3.50 \mathrm{E}-03$ & 85 & 1381 & $254 \mathrm{~A}$ & 7.1 & 29.7 & $2.05 \mathrm{E}-03$ & 85 \\
\hline 1375.1 & $251 \mathrm{~A}$ & 333 & -14.6 & $2.46 \mathrm{E}-03$ & 100 & 1381 & $254 \mathrm{~A}$ & 104.9 & -10.2 & $2.22 \mathrm{E}-03$ & 100 \\
\hline 1375.1 & $251 \mathrm{~A}$ & 324.8 & -2.1 & $4.15 \mathrm{E}-03$ & 115 & 1381 & $254 \mathrm{~A}$ & 359.5 & -75.8 & 5.71E-04 & 115 \\
\hline 1377.3 & $252 \mathrm{~A}$ & 349.7 & 75.1 & $1.04 \mathrm{E}-01$ & 0 & 1383 & $255 \mathrm{~A}$ & 335.6 & 78.6 & $1.54 \mathrm{E}-01$ & 0 \\
\hline
\end{tabular}




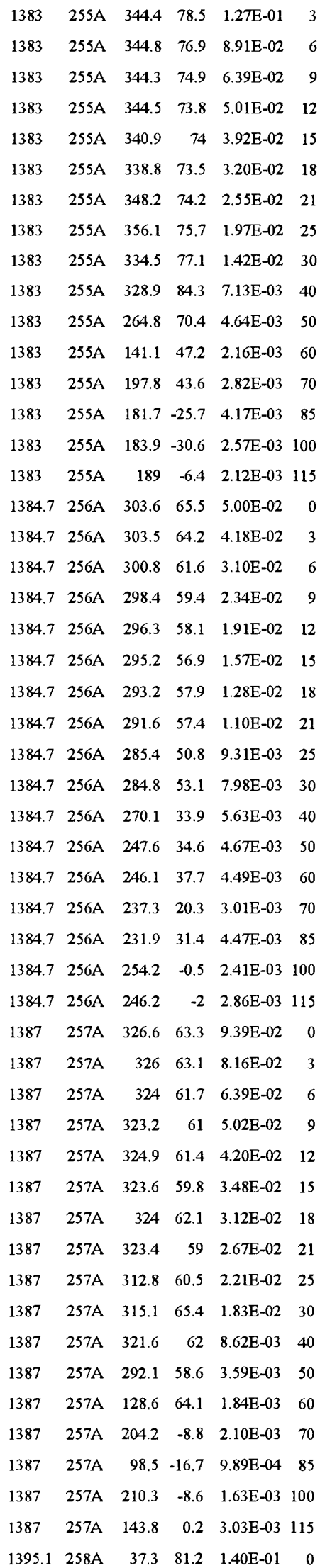

$\begin{array}{llllll}1395.1 & 258 \mathrm{~A} & 54.6 & 78.8 & 1.19 \mathrm{E}-01 & 3\end{array}$ $\begin{array}{lllllll}1395.1 & 258 \mathrm{~A} & 61 & 76.8 & 8.79 \mathrm{E}-02 & 6\end{array}$ $\begin{array}{llllll}1395.1 & 258 \mathrm{~A} & 63.2 & 75.4 & 6.43 \mathrm{E}-02 & 9\end{array}$ $\begin{array}{lllllll}1395.1 & 258 \mathrm{~A} & 68.3 & 75.3 & 5.13 \mathrm{E}-02 & 12\end{array}$ $\begin{array}{llllll}1395.1 & 258 \mathrm{~A} & 66.5 & 73.3 & 4.27 \mathrm{E}-02 & 15\end{array}$ $\begin{array}{llllll}1395.1 & 258 \mathrm{~A} & 68.6 & 73.3 & 3.60 \mathrm{E}-02 & 18\end{array}$ $\begin{array}{llllll}1395.1 & 258 \mathrm{~A} & 69.1 & 70.2 & 2.94 \mathrm{E}-02 & 21\end{array}$ $\begin{array}{llllll}1395.1 & 258 \mathrm{~A} & 72.1 & 67.6 & 2.40 \mathrm{E}-02 & 25\end{array}$ $\begin{array}{llllll}1395.1 & 258 \mathrm{~A} & 69.3 & 71 & 2.01 \mathrm{E}-02 & 30\end{array}$ $\begin{array}{llllll}1395.1 & 258 \mathrm{~A} & 83.9 & 63.7 & 1.04 \mathrm{E}-02 & 40\end{array}$ $\begin{array}{llllll}1395.1 & 258 \mathrm{~A} & 66.1 & 68.8 & 5.12 \mathrm{E}-03 & 50\end{array}$ $\begin{array}{llllll}1395.1 & 258 \mathrm{~A} & 10.7 & 74.9 & 1.61 \mathrm{E}-03 & 60\end{array}$ $\begin{array}{llllll}1395.1 & 258 \mathrm{~A} & 239.8 & 76.5 & 6.08 \mathrm{E}-04 & 70\end{array}$ $\begin{array}{llllll}1395.1 & 258 \mathrm{~A} & 219.1 & -70.8 & 1.37 \mathrm{E}-03 & 85\end{array}$ $\begin{array}{llllll}1395.1 & 258 \mathrm{~A} & 237 & -12 & 2.27 \mathrm{E}-03 & 100\end{array}$ $\begin{array}{llllll}1395.1 & 258 \mathrm{~A} & 191.9 & 47 & 1.56 \mathrm{E}-03 & 115\end{array}$ $\begin{array}{llllll}1397 & 259 \mathrm{~A} & 294 & 86.7 & 9.51 \mathrm{E}-02 & 0\end{array}$ $\begin{array}{llllll}1397 & 259 \mathrm{~A} & 354.3 & 88.9 & 7.93 \mathrm{E}-02 & 3\end{array}$ $\begin{array}{llllll}1397 & 259 \mathrm{~A} & 39.5 & 88.4 & 5.92 \mathrm{E}-02 & 6\end{array}$ $\begin{array}{llllll}1397 \quad 259 \mathrm{~A} & 49 & 87.6 & 4.22 \mathrm{E}-02 & 9\end{array}$ $\begin{array}{llllll}1397 & 259 \mathrm{~A} & 44.5 & 88 & 3.31 \mathrm{E}-02 & 12\end{array}$ $\begin{array}{llllll}1397 & 259 \mathrm{~A} & 35.2 & 88.5 & 2.72 \mathrm{E}-02 & 15\end{array}$ $\begin{array}{llllll}1397 \quad 259 \mathrm{~A} & 29.6 & 87.6 & 2.27 \mathrm{E}-02 & 18\end{array}$ $\begin{array}{llllll}1397 & 259 \mathrm{~A} & 47.5 & 87.7 & 1.83 \mathrm{E}-02 & 21\end{array}$ $\begin{array}{llllll}1397 \quad 259 \mathrm{~A} & 86 & 85.2 & 1.50 \mathrm{E}-02 & 25\end{array}$ $\begin{array}{llllll}1397 & 259 \mathrm{~A} & 5.6 & 88.1 & 1.22 \mathrm{E}-02 & 30\end{array}$ $\begin{array}{llllll}1397 & 259 \mathrm{~A} & 96.2 & 85.1 & 6.13 \mathrm{E}-03 & 40\end{array}$ $\begin{array}{llllll}1397 \quad 259 \mathrm{~A} & 107.6 & 82.1 & 3.85 \mathrm{E}-03 & 50\end{array}$ $\begin{array}{llllll}1397 & 259 \mathrm{~A} & 50.2 & 65 & 2.08 \mathrm{E}-03 & 60\end{array}$ $\begin{array}{llllll}1397 & 259 \mathrm{~A} & 282.8 & 47.4 & 1.50 \mathrm{E}-03 & 70\end{array}$ $\begin{array}{lllllll}1397 & 259 \mathrm{~A} & 255 & 8.4 & 6.33 \mathrm{E}-04 & 85\end{array}$ $\begin{array}{llllll}1397 & 259 \mathrm{~A} & 279.6 & 65.2 & 2.01 \mathrm{E}-03 & 100\end{array}$ $\begin{array}{llllll}1397 & 259 \mathrm{~A} & 153.9 & 77.5 & 2.07 \mathrm{E}-03 & 115\end{array}$ $\begin{array}{llllll}1397.7 & 260 \mathrm{~A} & 138.9 & 75.1 & 8.39 \mathrm{E}-02 & 0\end{array}$ $\begin{array}{llllll}1397.7 & 260 \mathrm{~A} & 131 & 71.7 & 7.36 \mathrm{E}-02 & 3\end{array}$ $\begin{array}{llllll}1397.7 & 260 \mathrm{~A} & 103.5 & 23.2 & 5.97 \mathrm{E}-02 & 6\end{array}$ $\begin{array}{llllll}1397.7 & 260 \mathrm{~A} & 103.7 & 21.5 & 4.91 \mathrm{E}-02 & 9\end{array}$ $\begin{array}{llllll}1397.7 & 260 \mathrm{~A} & 123.8 & 66.1 & 4.26 \mathrm{E}-02 & 12\end{array}$ $\begin{array}{llllll}1397.7 & 260 \mathrm{~A} & 122.2 & 64.5 & 3.74 \mathrm{E}-02 & 15\end{array}$ $\begin{array}{llllll}1397.7 & 260 \mathrm{~A} & 121.2 & 63.5 & 3.29 \mathrm{E}-02 & 18\end{array}$ $\begin{array}{llllll}1397.7 & 260 \mathrm{~A} & 119.1 & 63.3 & 2.94 \mathrm{E}-02 & 21\end{array}$ $\begin{array}{llllll}1397.7 & 260 \mathrm{~A} & 119.2 & 62.3 & 2.57 \mathrm{E}-02 & 25\end{array}$ $\begin{array}{llllll}1397.7 & 260 \mathrm{~A} & 116.7 & 62.9 & 2.24 \mathrm{E}-02 & 30\end{array}$ $\begin{array}{llllll}1397.7 & 260 \mathrm{~A} & 111.3 & 59.4 & 1.58 \mathrm{E}-02 & 40\end{array}$ $\begin{array}{llllll}1397.7 & 260 \mathrm{~A} & 115.8 & 63.9 & 1.03 \mathrm{E}-02 & 50\end{array}$ $\begin{array}{llllll}1397.7 & 260 \mathrm{~A} & 131 & 62 & 6.39 \mathrm{E}-03 & 60\end{array}$ $\begin{array}{lllllll}1397.7 & 260 \mathrm{~A} & 109.8 & 59.6 & 4.26 \mathrm{E}-03 & 70\end{array}$ $\begin{array}{llllll}1397.7 & 260 \mathrm{~A} & 126.9 & 44.9 & 2.84 \mathrm{E}-03 & 85\end{array}$ $\begin{array}{lllllll}1397.7 & 260 \mathrm{~A} & 213.1 & 26 & 7.46 \mathrm{E}-04 & 100\end{array}$ $\begin{array}{lllllll}1397.7 & 260 \mathrm{~A} & 142.2 & 1.2 & 8.19 \mathrm{E}-04 & 110\end{array}$ $\begin{array}{llllll}1399 & 261 \mathrm{~A} & 254.2 & 80 & 4.41 \mathrm{E}-02 & 0\end{array}$ $\begin{array}{llllll}1399 & 261 \mathrm{~A} & 250.6 & 79.7 & 3.70 \mathrm{E}-02 & 3\end{array}$

$\begin{array}{llllll}1399 & 261 \mathrm{~A} & 254.7 & 77.3 & 2.72 \mathrm{E}-02 & 6\end{array}$

$\begin{array}{llllll}1399 & 261 \mathrm{~A} & 253.9 & 74.8 & 2.02 \mathrm{E}-02 & 9\end{array}$

$\begin{array}{llllll}1399 & 261 \mathrm{~A} & 253.8 & 74.3 & 1.59 \mathrm{E}-02 & 12\end{array}$

$\begin{array}{llllll}1399 & 261 \mathrm{~A} & 256.3 & 73.9 & 1.28 \mathrm{E}-02 & 15\end{array}$

$\begin{array}{lllllll}1399 & 261 \mathrm{~A} & 267 & 73.4 & 1.06 \mathrm{E}-02 & 18\end{array}$

$\begin{array}{llllll}1399 & 261 \mathrm{~A} & 256.5 & 70.8 & 8.64 \mathrm{E}-03 & 21\end{array}$

$\begin{array}{llllll}1399 & 261 \mathrm{~A} & 269 & 65.2 & 6.93 \mathrm{E}-03 & 25\end{array}$

$\begin{array}{llllll}1399 & 261 \mathrm{~A} & 252.8 & 70 & 5.81 \mathrm{E}-03 & 30\end{array}$

$\begin{array}{llllll}1399 & 261 \mathrm{~A} & 262.7 & 68.1 & 2.95 \mathrm{E}-03 & 40\end{array}$

$\begin{array}{llllll}1399 & 261 \mathrm{~A} & 246.1 & 50.5 & 2.09 \mathrm{E}-03 & 50\end{array}$

$\begin{array}{llllll}1399 & 261 \mathrm{~A} & 279.6 & 84.8 & 2.24 \mathrm{E}-03 & 60\end{array}$

$\begin{array}{llllll}1399 & 261 \mathrm{~A} & 275.8 & 64.1 & 1.43 \mathrm{E}-03 & 70\end{array}$

$\begin{array}{llllll}1399 & 261 \mathrm{~A} & 42.6 & 22.8 & 1.46 \mathrm{E}-03 & 85\end{array}$

$\begin{array}{lllllll}1399 & 261 \mathrm{~A} & 85.5 & 23.7 & 6.82 \mathrm{E}-04 & 100\end{array}$

$\begin{array}{lllllll}1399 & 261 \mathrm{~A} & 58.3 & -2.7 & 8.02 \mathrm{E}-04 & 115\end{array}$

$\begin{array}{llllll}1401 & 262 \mathrm{~A} & 228.4 & 79.8 & 4.70 \mathrm{E}-02 & 0\end{array}$

$\begin{array}{llllll}1401 & 262 \mathrm{~A} & 216 & 80.5 & 3.95 \mathrm{E}-02 & 3\end{array}$

$\begin{array}{llllll}1401 & 262 \mathrm{~A} & 214.4 & 80.1 & 2.99 \mathrm{E}-02 & 6\end{array}$

$\begin{array}{llllll}1401 & 262 \mathrm{~A} & 216.7 & 79.8 & 2.16 \mathrm{E}-02 & 9\end{array}$

$\begin{array}{llllll}1401 & 262 \mathrm{~A} & 222 & 80.3 & 1.72 \mathrm{E}-02 & 12\end{array}$

$\begin{array}{lllllll}1401 & 262 \mathrm{~A} & 227.4 & 80.9 & 1.42 \mathrm{E}-02 & 15\end{array}$

$\begin{array}{llllll}1401 & 262 \mathrm{~A} & 234.5 & 80.8 & 1.19 \mathrm{E}-02 & 18\end{array}$

$\begin{array}{llllll}1401 & 262 \mathrm{~A} & 253.2 & 82.8 & 9.40 \mathrm{E}-03 & 21\end{array}$

$\begin{array}{llllll}1401 & 262 \mathrm{~A} & 262.6 & 86.5 & 7.16 \mathrm{E}-03 & 25\end{array}$

$\begin{array}{llllll}1401 & 262 \mathrm{~A} & 100.9 & 87.6 & 5.74 \mathrm{E}-03 & 30\end{array}$

$\begin{array}{llllll}1401 & 262 \mathrm{~A} & 340.2 & 84.2 & 2.64 \mathrm{E}-03 & 40\end{array}$

$\begin{array}{llllll}1401 & 262 \mathrm{~A} & 247.4 & 78.1 & 1.99 \mathrm{E}-03 & 50\end{array}$

$\begin{array}{llllll}1401 & 262 \mathrm{~A} & 32.4 & 32.3 & 1.21 \mathrm{E}-03 & 60\end{array}$

$\begin{array}{llllll}1401 & 262 \mathrm{~A} & 307.6 & 65.5 & 1.24 \mathrm{E}-03 & 70\end{array}$

$\begin{array}{llllll}1401 & 262 \mathrm{~A} & 29.9 & 39.9 & 1.18 \mathrm{E}-03 & 85\end{array}$

$\begin{array}{lllllll}1401 & 262 \mathrm{~A} & 349.8 & 19.4 & 6.17 \mathrm{E}-04 & 100\end{array}$

$\begin{array}{llllll}1401 & 262 \mathrm{~A} & 36.9 & 30 & 1.13 \mathrm{E}-03 & 115\end{array}$

$\begin{array}{llllll}1403 & 263 \mathrm{~A} & 98 & 61.1 & 8.17 \mathrm{E}-02 & 0\end{array}$

$\begin{array}{llllll}1403 & 263 \mathrm{~A} & 95.5 & 56.7 & 7.13 \mathrm{E}-02 & 3\end{array}$

$\begin{array}{llllll}1403 & 263 \mathrm{~A} & 92.8 & 54 & 5.62 \mathrm{E}-02 & 6\end{array}$

$\begin{array}{llllll}1403 \quad 263 \mathrm{~A} & 91.2 & 50.1 & 4.31 \mathrm{E}-02 & 9\end{array}$

$\begin{array}{llllll}1403 & 263 \mathrm{~A} & 88.5 & 49.3 & 3.47 \mathrm{E}-02 & 12\end{array}$

$\begin{array}{lllllll}1403 & 263 \mathrm{~A} & 86 & 48.1 & 2.88 \mathrm{E}-02 & 15\end{array}$

$\begin{array}{llllll}1403 & 263 \mathrm{~A} & 88.3 & 48.9 & 2.40 \mathrm{E}-02 & 18\end{array}$

$\begin{array}{llllll}1403 & 263 \mathrm{~A} & 86.5 & 46.2 & 1.90 \mathrm{E}-02 & 21\end{array}$

$\begin{array}{llllll}1403 & 263 \mathrm{~A} & 89.3 & 43.8 & 1.51 \mathrm{E}-02 & 25\end{array}$

$\begin{array}{llllll}1403 & 263 \mathrm{~A} & 85.3 & 53.1 & 1.07 \mathrm{E}-02 & 30\end{array}$

$\begin{array}{llllll}1403 & 263 \mathrm{~A} & 82.8 & 42.9 & 5.13 \mathrm{E}-03 & 40\end{array}$

$\begin{array}{llllll}1403 & 263 \mathrm{~A} & 298 & 29 & 2.01 \mathrm{E}-03 & 50\end{array}$

$\begin{array}{llllll}1403 & 263 \mathrm{~A} & 253.6 & -12.8 & 7.72 \mathrm{E}-04 & 60\end{array}$

$\begin{array}{llllll}1403 & 263 \mathrm{~A} & 263.7 & -28.9 & 3.84 \mathrm{E}-03 & 70\end{array}$

$\begin{array}{llllll}1403 & 263 \mathrm{~A} & 327.8 & -35.2 & 3.12 \mathrm{E}-03 & 85\end{array}$

$\begin{array}{llllll}1403 & 263 \mathrm{~A} & 280.2 & 49.6 & 4.45 \mathrm{E}-03 & 100\end{array}$

$\begin{array}{llllll}1403 \quad 263 \mathrm{~A} & 273.1 & -27.5 & 5.19 \mathrm{E}-03 & 115\end{array}$

$\begin{array}{llllll}1407 & 264 \mathrm{~A} & 293.5 & 78.1 & 2.72 \mathrm{E}-01 & 0\end{array}$ 


\begin{tabular}{|c|c|c|c|c|c|}
\hline 1407 & $264 \mathrm{~A}$ & 295.3 & 79.1 & $2.28 \mathrm{E}-01$ & \\
\hline 1407 & $264 A$ & 296.9 & 78.1 & $1.65 \mathrm{E}-01$ & \\
\hline 1407 & $264 \mathrm{~A}$ & 297.9 & 76.4 & $1.22 \mathrm{E}-01$ & \\
\hline 407 & $264 \mathrm{~A}$ & 298.2 & 75.1 & $9.38 \mathrm{E}-02$ & \\
\hline 1407 & $264 \mathrm{~A}$ & 295.1 & 72.7 & $7.27 \mathrm{E}-02$ & \\
\hline 1407 & $264 \mathrm{~A}$ & 296.8 & 71.5 & $5.63 \mathrm{E}-02$ & \\
\hline 1407 & $264 \mathrm{~A}$ & 302.1 & 69.8 & $4.56 \mathrm{E}-02$ & \\
\hline 407 & $264 \mathrm{~A}$ & 299 & 67.3 & $3.54 \mathrm{E}-02$ & \\
\hline 407 & $264 \mathrm{~A}$ & 303.6 & 67.6 & 2.6 & \\
\hline 407 & $264 A$ & 312.6 & 64.1 & $1.49 \mathrm{E}-02$ & \\
\hline 1407 & $264 A$ & 300.3 & 64.5 & $9.18 \mathrm{E}-03$ & \\
\hline 1407 & $264 \mathrm{~A}$ & 21.5 & 42.8 & $3.17 \mathrm{E}-03$ & \\
\hline 1407 & $264 A$ & 289.2 & 26.2 & $3.31 \mathrm{E}-03$ & \\
\hline 1407 & $264 A$ & 353.5 & 37.8 & $1.08 \mathrm{E}-03$ & \\
\hline 1407 & $264 \mathrm{~A}$ & 139.2 & 28.3 & $2.57 \mathrm{E}-03$ & 10 \\
\hline 1407 & $264 A$ & 76.4 & 56.7 & $2.02 \mathrm{E}-03$ & 11 \\
\hline 1409 & $265 A$ & 286.2 & 78.2 & $1.26 \mathrm{E}-01$ & \\
\hline 1409 & $265 \mathrm{~A}$ & 286.9 & 79 & $1.11 \mathrm{E}-01$ & \\
\hline 1409 & $265 \mathrm{~A}$ & 289 & 77.5 & $8.38 \mathrm{E}-02$ & \\
\hline 1409 & $265 A$ & 289.1 & 75.8 & $6.44 \mathrm{E}-02$ & \\
\hline 409 & $265 A$ & 291.7 & 74.7 & $5.27 \mathrm{E}-02$ & \\
\hline 409 & $265 \mathrm{~A}$ & 293.8 & 73.5 & $4.30 \mathrm{E}-02$ & 1 \\
\hline 1409 & $265 \mathrm{~A}$ & 296.7 & 72.5 & $3.43 \mathrm{E}-02$ & 1 \\
\hline 1409 & $265 \mathrm{~A}$ & 293.5 & 71.4 & $2.88 \mathrm{E}-02$ & 2 \\
\hline 409 & $265 \mathrm{~A}$ & 294 & 69.4 & $2.29 \mathrm{E}-02$ & \\
\hline 409 & $265 \mathrm{~A}$ & 298.9 & 71.5 & $1.73 \mathrm{E}-02$ & 3 \\
\hline 1409 & $265 \mathrm{~A}$ & 302.6 & 71.8 & 8.99E-03 & 4 \\
\hline 409 & $265 \mathrm{~A}$ & 305 & 74.4 & $5.27 \mathrm{E}-03$ & \\
\hline 409 & $265 \mathrm{~A}$ & 238.7 & 70.9 & $3.69 \mathrm{E}-03$ & \\
\hline 409 & $265 \mathrm{~A}$ & 222.6 & 55.7 & & \\
\hline 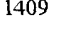 & $265 A$ & 180.8 & 34.8 & $1.30 \mathrm{E}$ & 8 \\
\hline 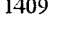 & $265 \mathrm{~A}$ & 196.2 & 20.7 & & 10 \\
\hline 09 & $265 \mathrm{~A}$ & 170 & -31.3 & 1.2 & 115 \\
\hline $5 x$ & $266 \mathrm{~A}$ & 253.5 & 75.3 & $2.06 \mathrm{E}-02$ & \\
\hline 411 & $66 \mathrm{~A}$ & 247.1 & 74.9 & 1.72 & \\
\hline 411 & $266 \mathrm{~A}$ & 247.1 & 74.6 & $1.27 \mathrm{E}-02$ & \\
\hline 2 & $66 \mathrm{~A}$ & 243.5 & 74.5 & $9.30 \mathrm{E}-03$ & \\
\hline & $66 \mathrm{~A}$ & 242.4 & 74.8 & & 1 \\
\hline & $66 \mathrm{~A}$ & 227.2 & 75.5 & & 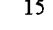 \\
\hline & $66 \mathrm{~A}$ & 215.4 & 79 & & 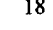 \\
\hline & $266 \mathrm{~A}$ & 238 & 75.1 & & 2 \\
\hline & $266 \mathrm{~A}$ & 236.3 & (1) & & 2 \\
\hline & $266 \mathrm{~A}$ & 165.8 & 83.8 & & 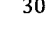 \\
\hline & 2003 & 47.2 & 64.2 & & 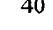 \\
\hline 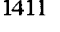 & 0018 & 29.5 & 39.4 & & 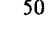 \\
\hline & 20003 & 46.8 & 50.5 & & 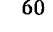 \\
\hline 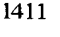 & $266 \mathrm{~A}$ & 42.6 & 71 & $1.39 \mathrm{E}-03$ & 70 \\
\hline$\theta x$ & 2000 & 54.9 & 30.8 & $1.98 \mathrm{E}-03$ & 85 \\
\hline 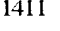 & $266 \mathrm{~A}$ & 345.7 & 36.4 & $6.89 \mathrm{E}-04$ & 100 \\
\hline $8 x$ & $266 \mathrm{~A}$ & 5.6 & 55.3 & $1.30 \mathrm{E}-03$ & 115 \\
\hline- & Un & 122.1 & 66.5 & $2.03 \mathrm{E}-02$ & \\
\hline
\end{tabular}

\begin{tabular}{|c|c|c|c|c|c|c|c|c|c|c|c|}
\hline 1413 & $267 \mathrm{~A}$ & 123.4 & 62.3 & $1.67 \mathrm{E}-02$ & 3 & 1424 & $271 \mathrm{~A}$ & 205.2 & 66.1 & $1.15 \mathrm{E}-01$ & 3 \\
\hline 1413 & $267 \mathrm{~A}$ & 118.2 & 55.2 & $1.35 \mathrm{E}-02$ & 6 & 1424 & $271 \mathrm{~A}$ & 204.4 & 66.5 & $9.72 \mathrm{E}-02$ & 6 \\
\hline 1413 & $267 \mathrm{~A}$ & 116.5 & 49.4 & $1.15 \mathrm{E}-02$ & 9 & 1424 & $271 \mathrm{~A}$ & 205.5 & 67 & $8.13 \mathrm{E}-02$ & 9 \\
\hline 1413 & $267 \mathrm{~A}$ & 110.8 & 47.9 & $9.73 \mathrm{E}-03$ & 12 & 1424 & $271 \mathrm{~A}$ & 206.7 & 68 & $7.10 \mathrm{E}-02$ & 12 \\
\hline 1413 & $267 \mathrm{~A}$ & 108.2 & 46.6 & $7.71 \mathrm{E}-03$ & 15 & 1424 & $271 \mathrm{~A}$ & 210.8 & 66.9 & $6.16 \mathrm{E}-02$ & 15 \\
\hline 1413 & $267 \mathrm{~A}$ & 110.8 & 48.4 & $6.58 \mathrm{E}-03$ & 18 & 1424 & $271 \mathrm{~A}$ & 206.7 & 68.3 & $5.27 \mathrm{E}-02$ & 18 \\
\hline 1413 & $267 \mathrm{~A}$ & 116.6 & 51 & $5.31 \mathrm{E}-03$ & 21 & 1424 & $271 \mathrm{~A}$ & 209.3 & 70.2 & $4.62 \mathrm{E}-02$ & 21 \\
\hline 1413 & $267 \mathrm{~A}$ & 126.3 & 52.2 & $3.97 \mathrm{E}-03$ & 25 & 1424 & $271 \mathrm{~A}$ & 211.2 & 70.8 & $3.93 \mathrm{E}-02$ & 25 \\
\hline 1413 & $267 \mathrm{~A}$ & 127.7 & 46.4 & $2.87 \mathrm{E}-03$ & 30 & 1424 & $271 \mathrm{~A}$ & 212.1 & 72.4 & $3.36 \mathrm{E}-02$ & 30 \\
\hline 1413 & $267 \mathrm{~A}$ & 104.8 & 74.1 & $2.05 \mathrm{E}-03$ & 40 & 1424 & $271 \mathrm{~A}$ & 197.2 & 67.5 & $1.97 \mathrm{E}-02$ & 40 \\
\hline 1413 & $267 \mathrm{~A}$ & 206.7 & 31.6 & $1.36 \mathrm{E}-03$ & 50 & 1424 & $271 \mathrm{~A}$ & 218.4 & 64.2 & $1.35 \mathrm{E}-02$ & 50 \\
\hline 1413 & $267 \mathrm{~A}$ & 194.5 & 27.7 & $1.60 \mathrm{E}-03$ & 60 & 1424 & $271 \mathrm{~A}$ & 197.5 & 63.1 & 8.39E-03 & 60 \\
\hline 1413 & $267 \mathrm{~A}$ & 156.8 & 65.2 & $1.93 \mathrm{E}-03$ & 70 & 1424 & $271 \mathrm{~A}$ & 193 & 62.4 & $8.52 \mathrm{E}-03$ & 70 \\
\hline 1413 & $267 \mathrm{~A}$ & 146.5 & 37.5 & $2.28 \mathrm{E}-03$ & 85 & 1424 & $271 \mathrm{~A}$ & 213.3 & 55.7 & $4.56 \mathrm{E}-03$ & 85 \\
\hline 1413 & $267 \mathrm{~A}$ & 357.3 & 56.7 & $1.60 \mathrm{E}-03$ & 100 & 1424 & $271 \mathrm{~A}$ & 192 & 62.3 & $5.99 \mathrm{E}-03$ & 100 \\
\hline 1413 & $267 \mathrm{~A}$ & 295.2 & 40.1 & $1.74 \mathrm{E}-03$ & 115 & 1424 & $271 \mathrm{~A}$ & 210.4 & 57.6 & $6.18 \mathrm{E}-03$ & 115 \\
\hline 1414.4 & $268 \mathrm{~A}$ & 239 & 50.9 & $2.25 \mathrm{E}-02$ & 0 & 1432.2 & $272 \mathrm{~A}$ & 246.9 & 61.3 & $1.89 \mathrm{E}-01$ & 0 \\
\hline 1414.4 & $268 \mathrm{~A}$ & 239 & 47.6 & $2.02 \mathrm{E}-02$ & 3 & 1432.2 & $272 \mathrm{~A}$ & 242.5 & 62.3 & $1.68 \mathrm{E}-01$ & 3 \\
\hline 1414.4 & $268 \mathrm{~A}$ & 239.8 & 44 & $1.69 \mathrm{E}-02$ & 6 & 1432.2 & $272 \mathrm{~A}$ & 241.8 & 63.2 & $1.43 \mathrm{E}-01$ & 6 \\
\hline 1414.4 & $268 \mathrm{~A}$ & 166.7 & 69 & $1.39 \mathrm{E}-02$ & 9 & 1432.2 & $272 \mathrm{~A}$ & 244.2 & 63.7 & $1.20 \mathrm{E}-01$ & 9 \\
\hline 1414.4 & $268 \mathrm{~A}$ & 243.3 & 40.6 & $1.22 \mathrm{E}-02$ & 12 & 1432.2 & $272 \mathrm{~A}$ & 244.6 & 64.5 & $1.05 \mathrm{E}-01$ & 12 \\
\hline 1414.4 & $268 \mathrm{~A}$ & 243.9 & 38.8 & $1.07 \mathrm{E}-02$ & 15 & 1432.2 & $272 \mathrm{~A}$ & 246.1 & 65.8 & $9.44 \mathrm{E}-02$ & 15 \\
\hline 1414.4 & $268 \mathrm{~A}$ & 178.7 & 69.8 & $9.56 \mathrm{E}-03$ & 18 & 1432.2 & $272 \mathrm{~A}$ & 245.9 & 66.3 & $8.38 \mathrm{E}-02$ & 18 \\
\hline 1414.4 & $268 \mathrm{~A}$ & 197.9 & 68.3 & $8.52 \mathrm{E}-03$ & 21 & 1432.2 & $272 \mathrm{~A}$ & 243.5 & 67.6 & $7.48 \mathrm{E}-02$ & 21 \\
\hline 1414.4 & $268 \mathrm{~A}$ & 245.2 & 32.4 & $6.97 \mathrm{E}-03$ & 25 & 1432.2 & $272 \mathrm{~A}$ & 248 & 68.8 & $6.56 \mathrm{E}-02$ & 25 \\
\hline 1414.4 & $268 \mathrm{~A}$ & 246 & 35.2 & $6.70 \mathrm{E}-03$ & 30 & 1432.2 & $272 \mathrm{~A}$ & 242.2 & 69.8 & $5.62 \mathrm{E}-02$ & 30 \\
\hline 1414.4 & $268 \mathrm{~A}$ & 254.4 & 28.5 & $3.88 \mathrm{E}-03$ & 40 & 1432.2 & $272 \mathrm{~A}$ & 246.3 & 68.6 & 4.03E-02 & 40 \\
\hline 1414.4 & $268 \mathrm{~A}$ & 254.4 & 34.2 & 4.41E-03 & 50 & 1432.2 & $272 \mathrm{~A}$ & 246.2 & 73.8 & $2.71 \mathrm{E}-02$ & 50 \\
\hline 1414.4 & $268 \mathrm{~A}$ & 210.1 & 70.2 & $2.42 \mathrm{E}-03$ & 60 & 1432.2 & $272 \mathrm{~A}$ & 221.6 & 71.5 & $2.08 \mathrm{E}-02$ & 60 \\
\hline 1414.4 & $268 \mathrm{~A}$ & 255.6 & 6 & 2.34E-03 & 70 & 1432.2 & $272 \mathrm{~A}$ & 172.9 & 71.9 & $1.36 \mathrm{E}-02$ & 70 \\
\hline 1414.4 & $268 \mathrm{~A}$ & 222 & 57.7 & $2.98 \mathrm{E}-03$ & 85 & 1432.2 & $272 \mathrm{~A}$ & 246.6 & 74.3 & $9.07 \mathrm{E}-03$ & 85 \\
\hline 1414.4 & $268 \mathrm{~A}$ & 277.3 & 72.8 & $1.29 \mathrm{E}-03$ & 100 & 1432.2 & $272 \mathrm{~A}$ & 90.5 & 32.7 & $6.98 \mathrm{E}-03$ & 100 \\
\hline 1414.4 & $268 \mathrm{~A}$ & 283.6 & 5.5 & $1.65 \mathrm{E}-03$ & 115 & 1432.2 & $272 \mathrm{~A}$ & 184.1 & 58.4 & $9.00 \mathrm{E}-03$ & 115 \\
\hline 1422.4 & $270 \mathrm{~A}$ & 297.8 & 65 & $2.34 \mathrm{E}-02$ & 0 & 1434 & $273 \mathrm{~A}$ & 248.1 & 52.8 & $1.08 \mathrm{E}-01$ & 0 \\
\hline 1422.4 & $270 \mathrm{~A}$ & 299.6 & 65.4 & $2.18 \mathrm{E}-02$ & 3 & 1434 & $273 \mathrm{~A}$ & 248.6 & 53.7 & $9.86 \mathrm{E}-02$ & 3 \\
\hline 1422.4 & $270 \mathrm{~A}$ & 299.1 & 63.6 & $2.01 \mathrm{E}-02$ & 6 & 1434 & $273 \mathrm{~A}$ & 251.1 & 53.6 & $8.16 \mathrm{E}-02$ & 6 \\
\hline 1422.4 & $270 \mathrm{~A}$ & 298.2 & 62.7 & $1.85 \mathrm{E}-02$ & 9 & 1434 & $273 \mathrm{~A}$ & 255 & 51.9 & $6.77 \mathrm{E}-02$ & 9 \\
\hline 1422.4 & $270 \mathrm{~A}$ & 298.4 & 61.5 & $1.73 \mathrm{E}-02$ & 12 & 1434 & $273 \mathrm{~A}$ & 257 & 51.2 & $5.85 \mathrm{E}-02$ & 12 \\
\hline 1422.4 & $270 \mathrm{~A}$ & 298.4 & 61.4 & $1.58 \mathrm{E}-02$ & 15 & 1434 & $273 \mathrm{~A}$ & 259.3 & 50.4 & $4.94 \mathrm{E}-02$ & 15 \\
\hline 1422.4 & $270 \mathrm{~A}$ & 298.8 & 58.4 & $1.40 \mathrm{E}-02$ & 18 & 1434 & $273 \mathrm{~A}$ & 263.2 & 49.6 & $4.37 \mathrm{E}-02$ & 18 \\
\hline 1422.4 & $270 \mathrm{~A}$ & 299.2 & 61 & $1.26 \mathrm{E}-02$ & 21 & 1434 & $273 \mathrm{~A}$ & 265.3 & 47.9 & $3.81 \mathrm{E}-02$ & 21 \\
\hline 1422.4 & $270 \mathrm{~A}$ & 297.5 & 60.5 & $1.10 \mathrm{E}-02$ & 25 & 1434 & $273 \mathrm{~A}$ & 266.1 & 48.2 & $3.22 \mathrm{E}-02$ & 25 \\
\hline 1422.4 & $270 \mathrm{~A}$ & 296.3 & 54.2 & $8.66 \mathrm{E}-03$ & 30 & 1434 & $273 \mathrm{~A}$ & 271.4 & 48.9 & $2.65 \mathrm{E}-02$ & 30 \\
\hline 1422.4 & $270 \mathrm{~A}$ & 301.7 & 57.7 & $6.16 \mathrm{E}-03$ & 40 & 1434 & $273 \mathrm{~A}$ & 280.5 & 51.3 & $1.51 \mathrm{E}-02$ & 40 \\
\hline 1422.4 & $270 \mathrm{~A}$ & 293.3 & 53.5 & $3.92 \mathrm{E}-03$ & 50 & 1434 & $273 \mathrm{~A}$ & 278.1 & 59.1 & $8.79 \mathrm{E}-03$ & 50 \\
\hline 1422.4 & $270 \mathrm{~A}$ & 312.8 & 46.7 & $3.02 \mathrm{E}-03$ & 60 & 1434 & $273 \mathrm{~A}$ & 304.5 & 63.4 & $6.41 \mathrm{E}-03$ & 60 \\
\hline 1422.4 & $270 \mathrm{~A}$ & 311.4 & 49.8 & $3.09 \mathrm{E}-03$ & 70 & 1434 & $273 \mathrm{~A}$ & 305.5 & 67.8 & $5.85 \mathrm{E}-03$ & 70 \\
\hline 1422.4 & $270 \mathrm{~A}$ & 279.6 & 50.5 & $1.57 \mathrm{E}-03$ & 85 & 1434 & $273 \mathrm{~A}$ & 328.8 & 43.5 & $4.00 \mathrm{E}-03$ & 85 \\
\hline 1422.4 & $270 \mathrm{~A}$ & 274.4 & 81.7 & $1.31 \mathrm{E}-03$ & 100 & 1434 & $273 \mathrm{~A}$ & 357 & 45.4 & $1.09 \mathrm{E}-03$ & 100 \\
\hline 1422.4 & $270 \mathrm{~A}$ & 256.7 & 35.3 & $1.50 \mathrm{E}-03$ & 115 & 1434 & $273 \mathrm{~A}$ & 293 & 60.2 & $3.10 \mathrm{E}-03$ & 115 \\
\hline 1424 & $271 \mathrm{~A}$ & 208.8 & 65.6 & $1.26 \mathrm{E}-01$ & 0 & 1436 & $274 \mathrm{~A}$ & 232.7 & 52.3 & $1.03 \mathrm{E}-01$ & 0 \\
\hline
\end{tabular}




\begin{tabular}{|c|c|c|c|c|c|}
\hline 1436 & $274 \mathrm{~A}$ & 230.7 & 51.6 & $9.04 \mathrm{E}-02$ & 3 \\
\hline 1436 & $274 \mathrm{~A}$ & 229.7 & 49.5 & $7.13 \mathrm{E}-02$ & 6 \\
\hline 1436 & $274 \mathrm{~A}$ & 230.6 & 47.9 & $5.75 \mathrm{E}-02$ & 9 \\
\hline 1436 & $274 \mathrm{~A}$ & 229.8 & 47.8 & $4.81 \mathrm{E}-02$ & 12 \\
\hline 1436 & $274 \mathrm{~A}$ & 231.1 & 46.6 & $4.02 \mathrm{E}-02$ & 15 \\
\hline 1436 & $274 \mathrm{~A}$ & 231.2 & 46.6 & $3.44 \mathrm{E}-02$ & 18 \\
\hline 1436 & $274 \mathrm{~A}$ & 229 & 45.8 & $3.02 E-02$ & 21 \\
\hline 1436 & $274 \mathrm{~A}$ & 232.3 & 46.1 & $2.46 \mathrm{E}-02$ & 25 \\
\hline 1436 & $274 \mathrm{~A}$ & 229.4 & 49.4 & $1.91 \mathrm{E}-02$ & 30 \\
\hline 1436 & $274 \mathrm{~A}$ & 235.1 & 47.6 & $1.08 \mathrm{E}-02$ & 40 \\
\hline 1436 & $274 \mathrm{~A}$ & 220.6 & 53.7 & $3.93 \mathrm{E}-03$ & 50 \\
\hline 1436 & $274 \mathrm{~A}$ & 240.2 & 67.1 & $2.40 \mathrm{E}-03$ & 60 \\
\hline 1436 & $274 \mathrm{~A}$ & 271.3 & 38.1 & $1.49 \mathrm{E}-03$ & 70 \\
\hline 1436 & $274 \mathrm{~A}$ & 111.6 & 9.4 & $1.24 \mathrm{E}-03$ & 85 \\
\hline 1436 & $274 \mathrm{~A}$ & 192.6 & 5.9 & $2.44 \mathrm{E}-03$ & 100 \\
\hline 1436 & $274 \mathrm{~A}$ & 180.1 & 1.3 & $3.78 \mathrm{E}-03$ & 115 \\
\hline 1440 & $275 \mathrm{~A}$ & 208.9 & 76.7 & $1.65 \mathrm{E}-01$ & 0 \\
\hline 1440 & $275 \mathrm{~A}$ & 197.8 & 75.1 & $1.33 \mathrm{E}-01$ & 3 \\
\hline 1440 & $275 \mathrm{~A}$ & 191.5 & 72.4 & $9.52 \mathrm{E}-02$ & 6 \\
\hline 1440 & $275 \mathrm{~A}$ & 187.3 & 69.1 & $6.76 \mathrm{E}-02$ & 9 \\
\hline 1440 & $275 \mathrm{~A}$ & 188.2 & 68 & $5.39 \mathrm{E}-02$ & 12 \\
\hline 1440 & $275 \mathrm{~A}$ & 189.9 & 65.6 & $4.43 \mathrm{E}-02$ & 15 \\
\hline 1440 & $275 \mathrm{~A}$ & 188.6 & 63.3 & $3.51 \mathrm{E}-02$ & 18 \\
\hline 1440 & $275 \mathrm{~A}$ & 182.6 & 63.5 & $2.87 \mathrm{E}-02$ & 21 \\
\hline 1440 & $275 \mathrm{~A}$ & 192.7 & 65.2 & $2.27 \mathrm{E}-02$ & 25 \\
\hline 1440 & $275 \mathrm{~A}$ & 185.9 & 62.1 & $1.70 \mathrm{E}-02$ & 30 \\
\hline 1440 & $275 \mathrm{~A}$ & 192.6 & 63.4 & $1.01 \mathrm{E}-02$ & 40 \\
\hline 1440 & $275 \mathrm{~A}$ & 207.8 & 61.5 & $6.44 \mathrm{E}-03$ & 50 \\
\hline 1440 & $275 \mathrm{~A}$ & 199.8 & 34 & $4.32 \mathrm{E}-03$ & 60 \\
\hline 1440 & $275 \mathrm{~A}$ & 158.1 & 65.4 & $3.17 \mathrm{E}-03$ & 70 \\
\hline 1440 & $275 \mathrm{~A}$ & 147.1 & 54.5 & $2.01 \mathrm{E}-03$ & 85 \\
\hline 1440 & $275 \mathrm{~A}$ & 155.5 & 12.1 & $2.25 \mathrm{E}-03$ & 100 \\
\hline 1440 & $275 \mathrm{~A}$ & 149.5 & 39.8 & $3.89 \mathrm{E}-03$ & 115 \\
\hline 1444 & $276 \mathrm{~A}$ & 196.7 & 56.9 & $8.18 \mathrm{E}-02$ & 0 \\
\hline 1444 & $276 \mathrm{~A}$ & 194.6 & 56 & $7.44 \mathrm{E}-02$ & 3 \\
\hline 1444 & $276 \mathrm{~A}$ & 194.7 & 55.2 & $6.11 \mathrm{E}-02$ & 6 \\
\hline 1444 & $276 \mathrm{~A}$ & 194.1 & 53.7 & $4.78 \mathrm{E}-02$ & 9 \\
\hline 1444 & $276 \mathrm{~A}$ & 194.6 & 53.1 & $3.95 \mathrm{E}-02$ & 12 \\
\hline 1444 & $276 \mathrm{~A}$ & 195.1 & 53 & $3.31 \mathrm{E}-02$ & 15 \\
\hline 1444 & $276 \mathrm{~A}$ & 195 & 53.8 & $2.70 \mathrm{E}-02$ & 18 \\
\hline 1444 & $276 \mathrm{~A}$ & 194.7 & 54 & $2.21 \mathrm{E}-02$ & 21 \\
\hline 1444 & $276 \mathrm{~A}$ & 199 & 53.2 & $1.77 \mathrm{E}-02$ & 25 \\
\hline 1444 & $276 \mathrm{~A}$ & 200.1 & 53.1 & $1.45 \mathrm{E}-02$ & 30 \\
\hline 1444 & $276 \mathrm{~A}$ & 190.6 & 58.8 & $7.11 \mathrm{E}-03$ & 40 \\
\hline 1444 & $276 \mathrm{~A}$ & 194.7 & 48.5 & $5.39 \mathrm{E}-03$ & 50 \\
\hline 1444 & $276 \mathrm{~A}$ & 208.3 & 62.9 & $2.87 \mathrm{E}-03$ & 60 \\
\hline 1444 & $276 \mathrm{~A}$ & 181.7 & 43.2 & $1.63 \mathrm{E}-03$ & 70 \\
\hline 1444 & $276 \mathrm{~A}$ & 126.2 & 44.5 & $2.26 \mathrm{E}-03$ & 85 \\
\hline 1444 & $276 \mathrm{~A}$ & 165.3 & 59.1 & $1.59 \mathrm{E}-03$ & 100 \\
\hline 1444 & $276 \mathrm{~A}$ & 91.7 & 42.1 & $1.26 \mathrm{E}-03$ & 115 \\
\hline 1446 & $277 \mathrm{~A}$ & 198.1 & 58.4 & $8.42 E-02$ & 0 \\
\hline
\end{tabular}

$\begin{array}{llllll}1446 & 277 \mathrm{~A} & 195.9 & 58.6 & 7.74 \mathrm{E}-02 & 3\end{array}$

$\begin{array}{llllll}1446 & 277 \mathrm{~A} & 197.8 & 58.2 & 6.43 \mathrm{E}-02 & 6\end{array}$

$\begin{array}{llllll}1446 & 277 \mathrm{~A} & 194.3 & 58.4 & 5.38 \mathrm{E}-02 & 9\end{array}$

$\begin{array}{llllll}1446 & 277 \mathrm{~A} & 193.9 & 58.9 & 4.38 \mathrm{E}-02 & 12\end{array}$

$\begin{array}{llllll}1446 & 277 \mathrm{~A} & 194.4 & 58.5 & 3.73 \mathrm{E}-02 & 15\end{array}$

$\begin{array}{llllll}1446 & 277 \mathrm{~A} & 193.5 & 58.3 & 3.23 \mathrm{E}-02 & 18\end{array}$

$\begin{array}{llllll}1446 & 277 \mathrm{~A} & 197.8 & 58.3 & 2.60 \mathrm{E}-02 & 21\end{array}$

$\begin{array}{llllll}1446 & 277 \mathrm{~A} & 197.2 & 55.5 & 2.28 \mathrm{E}-02 & 25\end{array}$

$\begin{array}{llllll}1446 & 277 \mathrm{~A} & 198.1 & 60.8 & 1.96 \mathrm{E}-02 & 30\end{array}$

$1446 \quad 277 \mathrm{~A} \quad 204.5 \quad 66 \quad 1.02 \mathrm{E}-02 \quad 40$

$\begin{array}{lllllll}1446 & 277 \mathrm{~A} & 236.6 & 58 & 7.56 \mathrm{E}-03 & 50\end{array}$

$\begin{array}{lllllll}1446 & 277 \mathrm{~A} & 199.4 & 58 & 6.23 \mathrm{E}-03 & 60\end{array}$

$\begin{array}{llllll}1446 & 277 \mathrm{~A} & 49.8 & 36 & 2.20 \mathrm{E}-03 & 70\end{array}$

$\begin{array}{llllll}1446 & 277 \mathrm{~A} & 145.9 & 65.4 & 3.00 \mathrm{E}-03 & 85\end{array}$

$\begin{array}{llllll}1446 & 277 \mathrm{~A} & 1.9 & 60.9 & 2.15 \mathrm{E}-03 & 100\end{array}$

$\begin{array}{llllll}1446 & 277 \mathrm{~A} & 355.8 & 64.3 & 3.20 \mathrm{E}-03 & 115\end{array}$

$\begin{array}{lllllll}1448 & 278 \mathrm{~A} & 214.6 & 62.6 & 1.58 \mathrm{E}-01 & 0\end{array}$

$\begin{array}{llllll}1448 & 278 \mathrm{~A} & 209.1 & 61 & 1.41 \mathrm{E}-01 & 3\end{array}$

$\begin{array}{llllll}1448 & 278 \mathrm{~A} & 207.7 & 59 & 1.17 \mathrm{E}-01 & 6\end{array}$

$\begin{array}{llllll}1448 & 278 \mathrm{~A} & 207.7 & 56.7 & 9.57 \mathrm{E}-02 & 9\end{array}$

$\begin{array}{lllllll}1448 & 278 \mathrm{~A} & 207.1 & 55.9 & 8.14 \mathrm{E}-02 & 12\end{array}$

$\begin{array}{llllll}1448 & 278 \mathrm{~A} & 205.8 & 55.5 & 7.01 \mathrm{E}-02 & 15\end{array}$

$\begin{array}{llllll}1448 & 278 \mathrm{~A} & 203.4 & 55.6 & 6.06 \mathrm{E}-02 & 18\end{array}$

$\begin{array}{llllll}1448 & 278 \mathrm{~A} & 205.2 & 54.9 & 5.03 \mathrm{E}-02 & 21\end{array}$

$\begin{array}{llllll}1448 & 278 \mathrm{~A} & 205 & 54.7 & 4.21 \mathrm{E}-02 & 25\end{array}$

$\begin{array}{llllll}1448 & 278 \mathrm{~A} & 203 & 54.4 & 3.59 \mathrm{E}-02 & 30\end{array}$

$\begin{array}{llllll}1448 & 278 \mathrm{~A} & 202.9 & 50.7 & 2.40 \mathrm{E}-02 & 40\end{array}$

$\begin{array}{llllll}1448 & 278 \mathrm{~A} & 202.9 & 56.3 & 1.59 \mathrm{E}-02 & 50\end{array}$

$\begin{array}{llllll}1448 & 278 \mathrm{~A} & 196 & 54.2 & 9.65 \mathrm{E}-03 & 60\end{array}$

$\begin{array}{lllllll}1448 & 278 \mathrm{~A} & 196.4 & 42.7 & 5.44 \mathrm{E}-03 & 70\end{array}$

$\begin{array}{llllll}1448 & 278 \mathrm{~A} & 141.3 & 26.2 & 3.56 \mathrm{E}-03 & 85\end{array}$

$\begin{array}{llllll}1448 & 278 \mathrm{~A} & 178.3 & -31.3 & 2.94 \mathrm{E}-03 & 100\end{array}$

$\begin{array}{lllllll}1448 & 278 \mathrm{~A} & 174.6 & -9.3 & 2.90 \mathrm{E}-03 & 115\end{array}$

$\begin{array}{lllllll}1449.5 & 279 \mathrm{~A} & 191.4 & 57.4 & 1.14 \mathrm{E}-01 & 0\end{array}$

$\begin{array}{llllll}1449.5 & 279 \mathrm{~A} & 190.6 & 55.3 & 9.93 \mathrm{E}-02 & 3\end{array}$

$\begin{array}{lllllll}1449.5 & 279 \mathrm{~A} & 191.5 & 52.7 & 7.64 \mathrm{E}-02 & 6\end{array}$

$\begin{array}{llllll}1449.5 & 279 \mathrm{~A} & 191.6 & 50.1 & 6.09 \mathrm{E}-02 & 9\end{array}$

$\begin{array}{llllll}1449.5 & 279 \mathrm{~A} & 190.6 & 50.9 & 4.93 \mathrm{E}-02 & 12\end{array}$

$\begin{array}{lllllll}1449.5 & 279 \mathrm{~A} & 190.9 & 50.7 & 4.01 \mathrm{E}-02 & 15\end{array}$

$\begin{array}{lllllll}1449.5 & 279 \mathrm{~A} & 190.7 & 51.4 & 3.54 \mathrm{E}-02 & 18\end{array}$

$\begin{array}{llllll}1449.5 & 279 \mathrm{~A} & 192.7 & 50.8 & 2.80 \mathrm{E}-02 & 21\end{array}$

$\begin{array}{llllll}1449.5 & 279 \mathrm{~A} & 199.3 & 51.5 & 2.33 \mathrm{E}-02 & 25\end{array}$

$\begin{array}{llllll}1449.5 & 279 \mathrm{~A} & 188.4 & 52.6 & 1.73 \mathrm{E}-02 & 30\end{array}$

$\begin{array}{lllllll}1449.5 & 279 \mathrm{~A} & 203.6 & 43.7 & 8.73 \mathrm{E}-03 & 40\end{array}$

$\begin{array}{llllll}1449.5 & 279 \mathrm{~A} & 211.9 & 56.8 & 6.34 \mathrm{E}-03 & 50\end{array}$

$\begin{array}{lllllll}1449.5 & 279 \mathrm{~A} & 220.8 & 12.3 & 6.97 \mathrm{E}-03 & 60\end{array}$

$\begin{array}{llllll}1449.5 & 279 \mathrm{~A} & 260.1 & 27.1 & 4.56 \mathrm{E}-03 & 70\end{array}$

$\begin{array}{llllll}1449.5 & 279 \mathrm{~A} & 245.2 & 26.7 & 5.95 \mathrm{E}-03 & 85\end{array}$

$\begin{array}{llllll}1449.5 & 279 \mathrm{~A} & 258.7 & 26.9 & 3.94 \mathrm{E}-03 & 100\end{array}$

$\begin{array}{llllll}1449.5 & 279 \mathrm{~A} & 263.2 & 13.8 & 3.37 \mathrm{E}-03 & 115\end{array}$

$\begin{array}{llllll}1474 & 281 \mathrm{~A} & 9.4 & 52.3 & 1.63 \mathrm{E}-01 & 0\end{array}$ \begin{tabular}{rrrrrr}
1474 & $281 \mathrm{~A}$ & 10.5 & 51.4 & $1.44 \mathrm{E}-01$ & 3 \\
1474 & $281 \mathrm{~A}$ & 10.3 & 50.9 & $1.15 \mathrm{E}-01$ & 6 \\
1474 & $281 \mathrm{~A}$ & 10 & 49.2 & $9.24 \mathrm{E}-02$ & 9 \\
1474 & $281 \mathrm{~A}$ & 10.1 & 49 & $7.81 \mathrm{E}-02$ & 12 \\
1474 & $281 \mathrm{~A}$ & 8.8 & 48.6 & $6.70 \mathrm{E}-02$ & 15 \\
1474 & $281 \mathrm{~A}$ & 11.3 & 50.1 & $5.68 \mathrm{E}-02$ & 18 \\
1474 & $281 \mathrm{~A}$ & 10.5 & 48.8 & $4.77 \mathrm{E}-02$ & 21 \\
1474 & $281 \mathrm{~A}$ & 8.5 & 50.3 & $3.94 \mathrm{E}-02$ & 25 \\
1474 & $281 \mathrm{~A}$ & 11.1 & 53.4 & $3.28 \mathrm{E}-02$ & 30 \\
1474 & $281 \mathrm{~A}$ & 15.3 & 52.3 & $1.83 \mathrm{E}-02$ & 40 \\
1474 & $281 \mathrm{~A}$ & 12.3 & 56.1 & $1.04 \mathrm{E}-02$ & 50 \\
1474 & $281 \mathrm{~A}$ & 0.8 & 30.1 & $6.57 \mathrm{E}-03$ & 60 \\
1474 & $281 \mathrm{~A}$ & 9.7 & 70.8 & $5.42 \mathrm{E}-03$ & 70 \\
1474 & $281 \mathrm{~A}$ & 74.3 & 52.1 & $5.25 \mathrm{E}-03$ & 85 \\
1477 & $282 \mathrm{~A}$ & 6.6 & 81.7 & $5.81 \mathrm{E}-03$ & 85 \\
1474 & $281 \mathrm{~A}$ & 34 & 28.6 & $3.99 \mathrm{E}-03$ & 100 \\
1477 & $282 \mathrm{~A}$ & 78.4 & 46 & $4.15 \mathrm{E}-03$ & 100 \\
1474 & $281 \mathrm{~A}$ & 34.6 & 49.2 & $4.44 \mathrm{E}-03$ & 115 \\
1477 & $282 \mathrm{~A}$ & 292.9 & 57.3 & $1.51 \mathrm{E}-01$ & 0 \\
1477 & $282 \mathrm{~A}$ & 295.4 & 55.9 & $1.38 \mathrm{E}-01$ & 3 \\
1477 & $282 \mathrm{~A}$ & 296.7 & 55.4 & $1.16 \mathrm{E}-01$ & 6 \\
1477 & $282 \mathrm{~A}$ & 296.9 & 55.3 & $9.24 \mathrm{E}-02$ & 9 \\
1477 & $282 \mathrm{~A}$ & 297.9 & 57.1 & $7.83 \mathrm{E}-02$ & 12 \\
\hline 1477 & $282 \mathrm{~A}$ & 296.5 & 57.4 & $6.71 \mathrm{E}-02$ & 15 \\
\hline 147 & $282 \mathrm{~A}$ & 297 & 61.7 & $5.82 \mathrm{E}-02$ & 18 \\
\hline 147 & $282 \mathrm{~A}$ & 298.2 & 58.5 & $5.06 \mathrm{E}-02$ & 21 \\
147 & 60.4 & 64.4 & $4.16 \mathrm{E}-02$ & 25 \\
147.3 & 299.5 & 62.8 & $2.27 \mathrm{E}-02$ & 40 \\
1403 & 115
\end{tabular}

$\begin{array}{lllllll}1482.3 & 283 \mathrm{~A} & 278.3 & 61.4 & 7.06 \mathrm{E}-02 & 0\end{array}$

$\begin{array}{llllll}1482.3 & 283 \mathrm{~A} & 279.6 & 63.1 & 6.76 \mathrm{E}-02 & 3\end{array}$

$\begin{array}{llllll}1482.3 & 283 \mathrm{~A} & 280.4 & 63.4 & 6.00 \mathrm{E}-02 & 6\end{array}$

$\begin{array}{llllll}1482.3 & 283 \mathrm{~A} & 279.3 & 63.3 & 5.17 \mathrm{E}-02 & 9\end{array}$

$\begin{array}{llllll}1482.3 & 283 \mathrm{~A} & 279.9 & 64.3 & 4.69 \mathrm{E}-02 & 12\end{array}$

$\begin{array}{lllllll}1482.3 & 283 \mathrm{~A} & 283 & 65.5 & 4.19 \mathrm{E}-02 & 15\end{array}$

$\begin{array}{lllllll}1482.3 & 283 \mathrm{~A} & 281.4 & 66.9 & 3.70 \mathrm{E}-02 & 18\end{array}$

$\begin{array}{lllllll}1482.3 & 283 \mathrm{~A} & 285 & 68.8 & 3.20 \mathrm{E}-02 & 21\end{array}$

$\begin{array}{llllll}1482.3 & 283 \mathrm{~A} & 285 & 67.2 & 2.75 \mathrm{E}-02 & 25\end{array}$

$\begin{array}{llllll}1482.3 & 283 \mathrm{~A} & 285 & 68.1 & 2.23 \mathrm{E}-02 & 30\end{array}$

$\begin{array}{llllll}1482.3 & 283 \mathrm{~A} & 285.7 & 69.1 & 1.42 \mathrm{E}-02 & 40\end{array}$

$\begin{array}{llllll}1482.3 & 283 \mathrm{~A} & 291.2 & 69.9 & 1.01 \mathrm{E}-02 & 50\end{array}$

$\begin{array}{llllll}1482.3 & 283 \mathrm{~A} & 297.6 & 71.1 & 6.65 \mathrm{E}-03 & 60\end{array}$

$\begin{array}{llllll}1482.3 & 283 \mathrm{~A} & 279.2 & 67.1 & 4.93 \mathrm{E}-03 & 70\end{array}$

$\begin{array}{llllll}1482.3 & 283 \mathrm{~A} & 297.5 & 61 & 4.15 \mathrm{E}-03 & 85\end{array}$

$\begin{array}{lllllll}1482.3 & 283 \mathrm{~A} & 262.1 & 41.9 & 2.59 \mathrm{E}-03 & 100\end{array}$

$\begin{array}{llllll}1482.3 & 283 \mathrm{~A} & 270.3 & 43.7 & 3.11 \mathrm{E}-03 & 115\end{array}$

$\begin{array}{llllll}1484.1 & 284 \mathrm{~A} & 29.2 & 53.3 & 5.73 \mathrm{E}-02 & 0\end{array}$ 


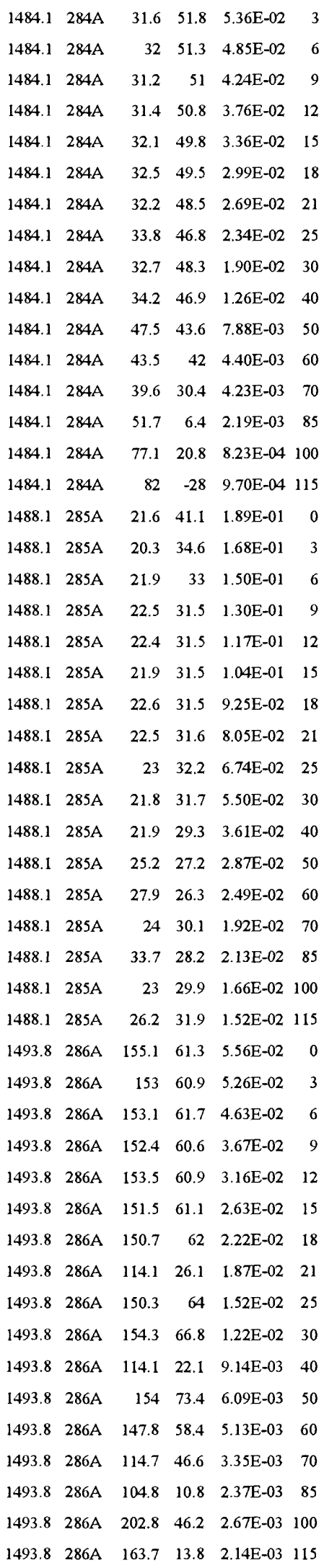




\section{Appendix B, Whole-core magnetic susceptibility measurements}

\begin{tabular}{|c|c|c|c|c|c|c|c|c|}
\hline \multirow[t]{2}{*}{ Drive \# } & Depth & MS (SI & & 50.3 & $5.63 \mathrm{E}-03$ & & 108.16 & $1.66 \mathrm{E}-03$ \\
\hline & $(\mathbf{f t})$ & volume) & & 50.5 & $3.90 \mathrm{E}-03$ & & 108.27 & $1.98 \mathrm{E}-03$ \\
\hline Drive 1 & 26.5 & $2.07 \mathrm{E}-03$ & & 50.73 & $4.48 \mathrm{E}-03$ & & 108.35 & $2.58 \mathrm{E}-03$ \\
\hline \multirow{9}{*}{ Drive 3} & 33.5 & $3.19 \mathrm{E}-03$ & Drive 9 & 57.5 & $1.69 \mathrm{E}-03$ & & 108.37 & $2.84 \mathrm{E}-03$ \\
\hline & 34.25 & 3.53E-03 & & 58.5 & $2.45 \mathrm{E}-03$ & & 108.39 & $3.04 \mathrm{E}-03$ \\
\hline & 34.5 & $3.53 \mathrm{E}-03$ & Drive 10 & 81.2 & $1.19 \mathrm{E}-03$ & & 108.43 & $4.48 \mathrm{E}-03$ \\
\hline & 33.1 & 2.64E-03 & & 81.66 & $1.53 \mathrm{E}-03$ & & 108.96 & 4. $64 \mathrm{E}-03$ \\
\hline & 35.2 & $4.20 \mathrm{E}-03$ & & 82 & $1.24 \mathrm{E}-03$ & & 109.1 & 7.93E-03 \\
\hline & 35.5 & $3.64 \mathrm{E}-03$ & Drive 12 & 87.5 & $1.49 \mathrm{E}-03$ & Drive 17 & 110.3 & $3.28 \mathrm{E}-03$ \\
\hline & 36.5 & $3.70 \mathrm{E}-03$ & & 87.6 & $1.89 \mathrm{E}-03$ & & 110.81 & 2.93E-03 \\
\hline & 36.5 & $5.00 \mathrm{E}-03$ & & 87.87 & $1.81 \mathrm{E}-03$ & & 110.88 & $2.33 \mathrm{E}-03$ \\
\hline & 36.8 & $3.49 \mathrm{E}-03$ & & 88.06 & $1.65 \mathrm{E}-03$ & & 110.88 & $1.25 \mathrm{E}-03$ \\
\hline \multirow[t]{13}{*}{ Drive 4} & 37.3 & $1.69 \mathrm{E}-03$ & & 88.3 & $1.05 \mathrm{E}-03$ & & 111.17 & 7.77E-04 \\
\hline & 37.3 & $1.69 \mathrm{E}-03$ & Drive $12 b$ & 88.63 & $1.60 \mathrm{E}-03$ & & 111.46 & 8.85E-04 \\
\hline & 37.5 & $2.42 \mathrm{E}-03$ & & 88.7 & $2.11 \mathrm{E}-03$ & & 111.76 & $1.82 \mathrm{E}-03$ \\
\hline & 37.5 & $3.42 \mathrm{E}-03$ & & 88.8 & 4.01E-03 & Drive $17 b$ & 112.3 & $1.41 \mathrm{E}-03$ \\
\hline & 37.73 & 4.09E-03 & & 88.88 & $5.32 \mathrm{E}-03$ & & 112.47 & $1.50 \mathrm{E}-03$ \\
\hline & 38.18 & 4. $13 \mathrm{E}-03$ & & 88.91 & $5.32 \mathrm{E}-03$ & & 113 & $1.53 \mathrm{E}-03$ \\
\hline & 38.22 & 4. $20 \mathrm{E}-03$ & & 89.5 & $2.68 \mathrm{E}-03$ & Drive $17 \mathrm{c}$ & 114.2 & $1.21 \mathrm{E}-03$ \\
\hline & 37.3 & $1.69 \mathrm{E}-03$ & & 90.2 & $3.75 \mathrm{E}-03$ & & 114.45 & $1.48 \mathrm{E}-03$ \\
\hline & 37.5 & $2.38 \mathrm{E}-03$ & Drive 13 & 92.1 & $1.69 \mathrm{E}-03$ & & 114.77 & $9.96 \mathrm{E}-04$ \\
\hline & 37.75 & $3.40 \mathrm{E}-03$ & & 92.26 & 2.64E-03 & & 114.85 & $1.26 \mathrm{E}-03$ \\
\hline & 38.18 & $4.09 \mathrm{E}-03$ & & 92.4 & $2.33 \mathrm{E}-03$ & & 115 & $1.46 \mathrm{E}-03$ \\
\hline & 38.22 & 4. $13 \mathrm{E}-03$ & & 92.47 & $2.04 \mathrm{E}-03$ & & 115.72 & $2.27 \mathrm{E}-03$ \\
\hline & 38.5 & 4.24E-03 & & 92.5 & $1.96 \mathrm{E}-03$ & Drive $17 d$ & 116.2 & $1.48 \mathrm{E}-03$ \\
\hline \multirow[t]{4}{*}{ Drive 5} & 40.6 & $4.48 \mathrm{E}-03$ & & 93.18 & $1.73 \mathrm{E}-03$ & & 116.6 & $1.53 \mathrm{E}-03$ \\
\hline & 40.68 & $4.92 \mathrm{E}-03$ & Drive $13 b$ & 93.7 & $1.69 \mathrm{E}-03$ & & 117 & $1.44 \mathrm{E}-03$ \\
\hline & 41.16 & 4.92E-03 & Drive 14 & 95.5 & $2.71 \mathrm{E}-03$ & Drive 18 & 120.32 & $1.27 \mathrm{E}-03$ \\
\hline & 41.5 & $4.24 \mathrm{E}-03$ & & 96.5 & $1.66 \mathrm{E}-03$ & & 120.43 & $1.02 \mathrm{E}-03$ \\
\hline \multirow[t]{21}{*}{ Drive 6} & 45.1 & $2.19 \mathrm{E}-03$ & & 96.72 & $1.11 \mathrm{E}-03$ & & 120.65 & $5.91 \mathrm{E}-04$ \\
\hline & 45.2 & $4.28 \mathrm{E}-03$ & & 97 & $1.52 \mathrm{E}-03$ & & 120.8 & $6.98 \mathrm{E}-04$ \\
\hline & 45.3 & $4.24 \mathrm{E}-03$ & Drive $14 \mathrm{~b}$ & 97.5 & $1.96 \mathrm{E}-03$ & & 121.5 & $1.67 \mathrm{E}-03$ \\
\hline & 45.4 & 4. $24 \mathrm{E}-03$ & & 98.2 & $1.79 \mathrm{E}-03$ & Drive 19 & 123.5 & $1.54 \mathrm{E}-03$ \\
\hline & 45.5 & 4.24E-03 & Drive 15 & 99.5 & $2.13 \mathrm{E}-03$ & Drive 20 & 124.49 & $2.76 \mathrm{E}-04$ \\
\hline & 45.6 & 4.24E-03 & & 99.62 & $1.95 \mathrm{E}-03$ & & 124.65 & $2.40 \mathrm{E}-04$ \\
\hline & 45.7 & $4.24 \mathrm{E}-03$ & & 99.75 & $1.44 \mathrm{E}-03$ & & 125.12 & $1.90 \mathrm{E}-04$ \\
\hline & 45.8 & $3.65 \mathrm{E}-03$ & & 100 & $1.13 \mathrm{E}-03$ & & 125.25 & $1.72 \mathrm{E}-04$ \\
\hline & 45.9 & $3.42 \mathrm{E}-03$ & & 100.4 & $1.18 \mathrm{E}-03$ & & 125.4 & $1.67 \mathrm{E}-04$ \\
\hline & 46 & $3.34 \mathrm{E}-03$ & & 100.69 & 1.15E-03 & & 125.9 & 2.73E-04 \\
\hline & 46.1 & 3.36E-03 & & 100.82 & $1.25 \mathrm{E}-03$ & Drive 21 & 134 & $2.34 \mathrm{E}-03$ \\
\hline & 46.2 & $3.59 \mathrm{E}-03$ & Drive $15 b$ & 102 & $1.11 \mathrm{E}-03$ & & 134.25 & $2.32 \mathrm{E}-03$ \\
\hline & 46.3 & $3.92 \mathrm{E}-03$ & Drive $15 \mathrm{c}$ & 104.5 & $1.55 \mathrm{E}-03$ & & 134.55 & $1.97 \mathrm{E}-03$ \\
\hline & 46.4 & 3. $92 \mathrm{E}-03$ & & 104.81 & $1.52 \mathrm{E}-03$ & & 134.76 & $2.54 \mathrm{E}-03$ \\
\hline & 46.5 & $4.09 \mathrm{E}-03$ & Drive 16 & 105.78 & $1.15 \mathrm{E}-03$ & Drive $21 \mathrm{~b}$ & 135.25 & 2.03E-03 \\
\hline & 46.6 & $3.83 \mathrm{E}-03$ & & 105.88 & $1.35 \mathrm{E}-03$ & & 135.45 & $1.89 \mathrm{E}-03$ \\
\hline & 46.7 & $3.21 \mathrm{E}-03$ & & 105.98 & $1.29 \mathrm{E}-03$ & & 135.5 & $1.65 \mathrm{E}-03$ \\
\hline & 46.8 & $3.24 \mathrm{E}-03$ & & 106.06 & $1.21 \mathrm{E}-03$ & & 135.55 & $1.39 \mathrm{E}-03$ \\
\hline & 46.9 & $3.67 \mathrm{E}-03$ & & 106.25 & $1.31 \mathrm{E}-03$ & & 135.6 & $1.14 \mathrm{E}-03$ \\
\hline & 47 & $3.42 \mathrm{E}-03$ & & 106.8 & $2.26 \mathrm{E}-03$ & & 135.85 & $1.02 \mathrm{E}-03$ \\
\hline & 47.1 & $2.32 \mathrm{E}-03$ & & 107.32 & $2.90 \mathrm{E}-03$ & & 135.9 & $1.17 \mathrm{E}-03$ \\
\hline \multirow[t]{4}{*}{ Drive 7} & 47.5 & 2.83E-03 & Drive $16 b$ & 107.7 & $1.48 \mathrm{E}-03$ & & 136 & $1.69 \mathrm{E}-03$ \\
\hline & 47.85 & 4.17E-03 & & 107.8 & $1.74 \mathrm{E}-03$ & & 136.7 & $2.01 \mathrm{E}-03$ \\
\hline & 48.5 & $4.48 \mathrm{E}-03$ & & 107.85 & $1.60 \mathrm{E}-03$ & Drive $21 \mathrm{c}$ & 138 & $1.51 \mathrm{E}-03$ \\
\hline & 48.9 & $2.95 \mathrm{E}-03$ & & 107.9 & $1.69 \mathrm{E}-03$ & Drive 22 & 141.2 & $1.85 \mathrm{E}-03$ \\
\hline Drive $7 \mathrm{~b}$ & 49.5 & $2.89 \mathrm{E}-03$ & & 108.01 & $1.55 \mathrm{E}-03$ & & 142 & $1.75 \mathrm{E}-03$ \\
\hline
\end{tabular}




\begin{tabular}{|c|c|c|c|c|c|c|c|c|}
\hline Drive $22 b$ & 143.5 & $1.44 \mathrm{E}-03$ & & 194 & $2.53 \mathrm{E}-03$ & & 263 & $1.48 \mathrm{E}-02$ \\
\hline \multirow[t]{7}{*}{ Drive 23} & 145.97 & $9.92 \mathrm{E}-04$ & Drive 32 & 201.02 & $2.37 \mathrm{E}-03$ & & 263.2 & 8.33E-02 \\
\hline & 146.03 & $1.28 \mathrm{E}-03$ & & 202 & $1.30 \mathrm{E}-03$ & & 263.6 & $1.97 \mathrm{E}-03$ \\
\hline & 146.21 & $1.35 \mathrm{E}-03$ & Drive 33 & 204 & $2.44 \mathrm{E}-03$ & Drive 43 & 265.25 & $1.13 \mathrm{E}-03$ \\
\hline & 146.28 & $1.35 \mathrm{E}-03$ & & 204.6 & $5.71 \mathrm{E}-04$ & & 265.6 & $1.30 \mathrm{E}-03$ \\
\hline & 147.18 & $9.28 \mathrm{E}-04$ & Drive $33 b$ & 205.8 & $2.94 \mathrm{E}-04$ & & 265.67 & $1.27 \mathrm{E}-03$ \\
\hline & 147.51 & $1.36 \mathrm{E}-03$ & & 206.3 & 8.57E-04 & & 265.8 & $1.17 \mathrm{E}-03$ \\
\hline & 147.56 & $1.36 \mathrm{E}-03$ & & 206.4 & $9.68 \mathrm{E}-04$ & & 265.25 & $1.35 \mathrm{E}-03$ \\
\hline \multirow[t]{6}{*}{ Drive 24} & 149.08 & 4.01E-04 & & 206.5 & $1.04 \mathrm{E}-03$ & Drive $43 b$ & 266.95 & $1.16 \mathrm{E}-03$ \\
\hline & 149.21 & $5.47 \mathrm{E}-04$ & Drive $33 c$ & 207.5 & $1.05 \mathrm{E}-03$ & & 267.3 & $1.42 \mathrm{E}-03$ \\
\hline & 149.42 & $1.18 \mathrm{E}-03$ & & 208 & 7.74E-04 & Drive 45 & 270.55 & $4.09 \mathrm{E}-04$ \\
\hline & 149.52 & 1. $13 \mathrm{E}-03$ & & 208.84 & $3.30 \mathrm{E}-04$ & & 270.8 & $1.09 \mathrm{E}-03$ \\
\hline & 149.9 & $1.02 \mathrm{E}-03$ & Drive $33 d$ & 210 & $5.04 \mathrm{E}-04$ & & 271.4 & $4.09 \mathrm{E}-04$ \\
\hline & 150.6 & 7.34E-04 & & 210.32 & 8.93E-04 & Drive 46 & 273.4 & $1.07 \mathrm{E}-03$ \\
\hline \multirow[t]{2}{*}{ Drive $24 b$} & 151.08 & $6.66 \mathrm{E}-04$ & & 210.7 & $8.81 \mathrm{E}-04$ & & 273.9 & $1.45 \mathrm{E}-03$ \\
\hline & 151.5 & $2.90 \mathrm{E}-03$ & & 210.8 & $1.05 \mathrm{E}-03$ & & 274.2 & $1.60 \mathrm{E}-03$ \\
\hline \multirow[t]{5}{*}{ Drive 25} & 159.2 & 2. $10 \mathrm{E}-03$ & & 210.86 & $1.09 \mathrm{E}-03$ & & 274.5 & $1.85 \mathrm{E}-03$ \\
\hline & 159.42 & $1.65 \mathrm{E}-03$ & Drive 34 & 211.3 & 8.49E-04 & Drive $46 b$ & 275.7 & 2.27E-03 \\
\hline & 159.65 & $1.56 \mathrm{E}-03$ & & 212.35 & $1.42 \mathrm{E}-03$ & & 276.1 & $3.03 \mathrm{E}-03$ \\
\hline & 160.1 & $3.42 \mathrm{E}-03$ & Drive 35 & 221.8 & $2.09 \mathrm{E}-03$ & & 276.4 & $2.59 \mathrm{E}-03$ \\
\hline & 160.6 & $2.28 \mathrm{E}-03$ & & 222 & $2.00 \mathrm{E}-03$ & Drive 47 & 278.4 & 7.22E-04 \\
\hline Drive $25 b$ & 162 & $1.67 \mathrm{E}-03$ & & 222.2 & $1.91 \mathrm{E}-03$ & & 278.95 & $5.59 \mathrm{E}-04$ \\
\hline \multirow[t]{2}{*}{ Drive $25 c$} & 163.1 & $2.11 \mathrm{E}-03$ & Drive $35 b$ & 223.25 & $1.80 \mathrm{E}-03$ & & 279.5 & $8.05 \mathrm{E}-04$ \\
\hline & 163.8 & $2.42 \mathrm{E}-03$ & & 223.55 & $2.07 \mathrm{E}-03$ & & 279.8 & $8.01 \mathrm{E}-04$ \\
\hline \multirow[t]{7}{*}{ Drive 26} & 164.75 & 2.13E-03 & Drive 37 & 236.55 & $2.21 \mathrm{E}-03$ & Drive $47 \mathrm{~b}$ & 281.1 & $6.58 \mathrm{E}-04$ \\
\hline & 164.91 & $1.68 \mathrm{E}-03$ & & 237.03 & 2.17E-03 & Drive 48 & 282.75 & $6.66 \mathrm{E}-04$ \\
\hline & 165.3 & 1.73E-03 & & 237.15 & $2.13 \mathrm{E}-03$ & & 283 & $6.58 \mathrm{E}-04$ \\
\hline & 165.46 & $3.38 \mathrm{E}-03$ & & 237.2 & $1.80 \mathrm{E}-03$ & Drive $48 b$ & 285.1 & $1.43 \mathrm{E}-03$ \\
\hline & 165.5 & $3.83 \mathrm{E}-03$ & & 237.25 & $1.39 \mathrm{E}-03$ & & 285.3 & $1.53 \mathrm{E}-03$ \\
\hline & 165.57 & $4.01 \mathrm{E}-03$ & & 237.3 & $1.03 \mathrm{E}-03$ & & 285.85 & $1.61 \mathrm{E}-03$ \\
\hline & 166.3 & 2. $10 \mathrm{E}-03$ & & 237.35 & $7.58 \mathrm{E}-04$ & Drive 49 & 291.1 & $5.67 \mathrm{E}-04$ \\
\hline \multirow[t]{3}{*}{ Drive $26 \mathrm{~b}$} & 167.1 & $1.42 \mathrm{E}-03$ & & 237.4 & $5.83 \mathrm{E}-04$ & Drive 50 & 291.93 & $1.09 \mathrm{E}-03$ \\
\hline & 167.92 & $1.18 \mathrm{E}-03$ & & 237.5 & 4.17E-04 & & 292.4 & $9.88 \mathrm{E}-04$ \\
\hline & 168.3 & $1.82 \mathrm{E}-03$ & & 237.8 & $5.91 \mathrm{E}-04$ & & 292.7 & 1. $10 \mathrm{E}-03$ \\
\hline \multirow[t]{3}{*}{ Drive $26 c$} & 168.85 & $2.60 \mathrm{E}-03$ & Drive $37 b$ & 239.3 & 8.33E-04 & & 293.1 & $1.09 \mathrm{E}-03$ \\
\hline & 169.2 & $3.28 \mathrm{E}-03$ & Drive 38 & 234.45 & $1.51 \mathrm{E}-03$ & & 293.4 & $1.22 \mathrm{E}-03$ \\
\hline & 169.6 & $2.23 \mathrm{E}-03$ & & 243.64 & $1.14 \mathrm{E}-03$ & Drive $50 \mathrm{~b}$ & 293.9 & $1.26 \mathrm{E}-03$ \\
\hline \multirow[t]{2}{*}{ Drive 27} & 171 & $2.59 \mathrm{E}-03$ & & 243.92 & $1.37 \mathrm{E}-03$ & & 294.1 & $1.59 \mathrm{E}-03$ \\
\hline & 172 & $1.84 \mathrm{E}-03$ & & 244 & $1.45 \mathrm{E}-03$ & & 295.45 & $1.43 \mathrm{E}-03$ \\
\hline \multirow[t]{3}{*}{ Drive $27 b$} & 172.8 & $1.93 \mathrm{E}-03$ & Drive $38 b$ & 245.25 & $1.57 \mathrm{E}-03$ & Drive $50 c$ & 296.18 & $1.38 \mathrm{E}-03$ \\
\hline & 173.7 & $2.59 \mathrm{E}-03$ & & 245.55 & $1.62 \mathrm{E}-03$ & Drive 51 & 303.65 & $1.54 \mathrm{E}-03$ \\
\hline & 174 & 2.03E-03 & Drive 39 & 249.02 & $1.22 \mathrm{E}-03$ & Drive $51 \mathrm{~b}$ & 304.3 & $1.56 \mathrm{E}-03$ \\
\hline \multirow[t]{3}{*}{ Drive 28} & 175.7 & $1.39 \mathrm{E}-03$ & & 249.33 & $7.81 \mathrm{E}-04$ & & 304.7 & $5.08 \mathrm{E}-04$ \\
\hline & 176 & $1.96 \mathrm{E}-03$ & & 249.43 & 6.07E-04 & & 305.65 & 7.97E-04 \\
\hline & 177 & $1.79 \mathrm{E}-03$ & & 249.49 & $5.28 \mathrm{E}-04$ & Drive 51c & 306.3 & $7.50 \mathrm{E}-04$ \\
\hline \multirow[t]{4}{*}{ Drive 29} & 179.1 & $4.88 \mathrm{E}-04$ & Drive 40 & 250.1 & 7.77E-04 & & 306.7 & 6.70E-04 \\
\hline & 179.4 & $7.38 \mathrm{E}-04$ & & 251 & $3.41 \mathrm{E}-04$ & & 307 & $6.66 \mathrm{E}-04$ \\
\hline & 179.7 & $9.96 \mathrm{E}-04$ & & 251.1 & $3.13 \mathrm{E}-04$ & & 307.3 & 4.92E-04 \\
\hline & 180.1 & $9.04 \mathrm{E}-04$ & & 251.2 & 3.07E-04 & & 307.5 & $6.51 \mathrm{E}-04$ \\
\hline \multirow[t]{2}{*}{ Drive $29 b$} & 181.3 & $6.19 \mathrm{E}-04$ & & 251.3 & $3.22 \mathrm{E}-04$ & Drive 52 & 312.2 & $6.98 \mathrm{E}-04$ \\
\hline & 182.2 & $6.58 \mathrm{E}-04$ & & 251.4 & $3.82 \mathrm{E}-04$ & & 312.5 & $6.90 \mathrm{E}-04$ \\
\hline \multirow[t]{8}{*}{ Drive 31} & 190.66 & 2.53E-03 & & 251.44 & 4.28E-04 & & 312.8 & $6.55 \mathrm{E}-04$ \\
\hline & 190.78 & $2.49 \mathrm{E}-03$ & & 251.76 & $1.36 \mathrm{E}-03$ & & 313.3 & $5.12 \mathrm{E}-04$ \\
\hline & 190.9 & $1.95 \mathrm{E}-03$ & Drive $40 \mathrm{~b}$ & 253 & $3.04 \mathrm{E}-04$ & & 313.6 & $5.16 \mathrm{E}-04$ \\
\hline & 190.98 & $1.78 \mathrm{E}-03$ & Drive $40 c$ & 254.5 & $2.83 \mathrm{E}-04$ & Drive 53 & 321.9 & 8.81E-04 \\
\hline & 191.1 & 2.69E-03 & Drive 42 & 260.3 & $1.07 \mathrm{E}-08$ & & 322.3 & 8.25E-04 \\
\hline & 191.82 & $2.89 \mathrm{E}-03$ & & 261.5 & $1.24 \mathrm{E}-03$ & & 322.5 & $8.45 \mathrm{E}-04$ \\
\hline & 192.06 & $2.31 \mathrm{E}-03$ & Drive $42 b$ & 262.3 & $6.03 \mathrm{E}-04$ & & 322.8 & 8.81E-04 \\
\hline & 192.35 & $1.44 \mathrm{E}-03$ & & 262.7 & $9.08 \mathrm{E}-04$ & & 323.2 & 8.77E-04 \\
\hline Drive $31 b$ & 193 & $1.85 \mathrm{E}-03$ & & 262.95 & 7.93E-03 & Drive $53 b$ & 324.1 & $6.98 \mathrm{E}-04$ \\
\hline
\end{tabular}




\begin{tabular}{|c|c|c|c|c|c|c|c|c|}
\hline & 324.9 & $5.67 \mathrm{E}-04$ & & 411.75 & $6.70 \mathrm{E}-04$ & & 474.1 & 4.64E- 04 \\
\hline & 325.2 & 7.26E-04 & & 411.9 & $6.39 \mathrm{E}-04$ & & 474.7 & $8.96 \mathrm{E}-04$ \\
\hline \multirow[t]{3}{*}{ Drive $53 c$} & 325.9 & 4.68E-04 & Drive 74 & 416.9 & $1.51 \mathrm{E}-03$ & Drive 86 & 484.1 & $1.79 \mathrm{E}-03$ \\
\hline & 326.1 & 1.04E-03 & & 417.4 & $1.66 \mathrm{E}-03$ & & 484.67 & $9.68 \mathrm{E}-04$ \\
\hline & 326.4 & 7.85E-04 & & 417.9 & $1.76 \mathrm{E}-03$ & & 484.96 & 8.96E-04 \\
\hline Drive 55 & 333.45 & 4.68E-04 & Drive $74 b$ & 418.8 & $1.70 \mathrm{E}-03$ & & 485.35 & 8.09E-04 \\
\hline \multirow{3}{*}{ Drive $55 \mathrm{~b}$} & 334.25 & $9.76 \mathrm{E}-04$ & & 419.2 & $1.65 \mathrm{E}-03$ & Drive 86b & 486.05 & $1.19 \mathrm{E}-03$ \\
\hline & 335.17 & $1.82 \mathrm{E}-03$ & & 419.4 & $1.95 \mathrm{E}-03$ & & 486.7 & 7.14E-04 \\
\hline & 335.35 & $1.84 \mathrm{E}-03$ & Drive 77aa & 428.3 & $1.23 \mathrm{E}-03$ & & 486.85 & $6.94 \mathrm{E}-04$ \\
\hline \multirow[t]{2}{*}{ Drive 56} & 337.3 & $1.32 \mathrm{E}-03$ & & 428.8 & $1.13 \mathrm{E}-03$ & Drive 87 & 489.2 & $9.56 \mathrm{E}-04$ \\
\hline & 337.6 & $1.07 \mathrm{E}-03$ & & 429.3 & $1.46 \mathrm{E}-03$ & & 489.7 & 9.04E-04 \\
\hline \multirow[t]{3}{*}{ Drive 58} & 347.4 & $8.57 \mathrm{E}-04$ & Drive $77 \mathrm{ab}$ & 430.3 & $1.58 \mathrm{E}-03$ & & 490.4 & $9.08 \mathrm{E}-04$ \\
\hline & 347.9 & $1.06 \mathrm{E}-03$ & & 430.8 & $1.94 \mathrm{E}-03$ & & 490.55 & 8.96E-04 \\
\hline & 348.6 & $1.15 \mathrm{E}-03$ & & 431.3 & $1.54 \mathrm{E}-03$ & Drive 87b & 491.1 & $1.25 \mathrm{E}-03$ \\
\hline \multirow[t]{3}{*}{ Drive $58 b$} & 349.4 & $1.27 \mathrm{E}-03$ & Drive 77 ac & 432.15 & $1.17 \mathrm{E}-03$ & & 491.48 & $9.00 \mathrm{E}-04$ \\
\hline & 350.12 & $1.70 \mathrm{E}-03$ & Drive $77 \mathrm{ba}$ & 433.2 & $1.71 \mathrm{E}-03$ & & 491.7 & 8.41E-04 \\
\hline & 350.74 & $1.03 \mathrm{E}-03$ & & 433.6 & $1.66 \mathrm{E}-03$ & & 491.9 & 8.41E-04 \\
\hline Drive $58 \mathrm{c}$ & 351.15 & $1.20 \mathrm{E}-03$ & & 433.9 & $1.45 \mathrm{E}-03$ & Drive 88 & 493.2 & 5.71E-04 \\
\hline Drive 59 & 353.17 & $1.07 \mathrm{E}-03$ & & 434.2 & 1.61E-03 & & 493.7 & $1.13 \mathrm{E}-03$ \\
\hline \multirow[t]{3}{*}{ Drive 61} & 357.94 & $1.02 \mathrm{E}-03$ & & 434.5 & $1.75 \mathrm{E}-03$ & & 494 & $1.30 \mathrm{E}-03$ \\
\hline & 358.4 & 7.34E-04 & Drive $77 \mathrm{bb}$ & 435.3 & $1.95 \mathrm{E}-03$ & & 494.4 & $1.15 \mathrm{E}-03$ \\
\hline & 358.9 & $4.32 \mathrm{E}-04$ & & 435.7 & $2.00 \mathrm{E}-03$ & Drive $88 b$ & 495.05 & $9.12 \mathrm{E}-04$ \\
\hline Drive $61 \mathrm{~b}$ & 360.7 & $1.48 \mathrm{E}-03$ & & 436 & $1.87 \mathrm{E}-03$ & & 495.2 & $6.51 \mathrm{E}-04$ \\
\hline \multirow{2}{*}{ Drive 61c } & 361.52 & $8.37 \mathrm{E}-04$ & & 436.55 & $1.39 \mathrm{E}-03$ & & 495.7 & 3.65E-04 \\
\hline & 362.67 & $1.35 \mathrm{E}-03$ & Drive $77 b c$ & 437.25 & $2.13 \mathrm{E}-03$ & & 494.95 & 8.45E-04 \\
\hline \multirow[t]{3}{*}{ Drive 63} & 369.8 & $1.61 \mathrm{E}-03$ & Drive 78 & 438.8 & $2.59 \mathrm{E}-03$ & Drive 89 & 498.3 & $5.20 \mathrm{E}-04$ \\
\hline & 370.4 & $2.02 \mathrm{E}-03$ & & 438.9 & $3.20 \mathrm{E}-03$ & & 498.8 & 8.25E-04 \\
\hline & 371 & $1.60 \mathrm{E}-03$ & & 439 & $2.92 \mathrm{E}-03$ & & 499.3 & $6.78 \mathrm{E}-04$ \\
\hline \multirow[t]{3}{*}{ Drive $63 b$} & 371.7 & $1.61 \mathrm{E}-03$ & & 439.4 & $1.56 \mathrm{E}-03$ & Drive $89 b$ & 500.1 & $1.36 \mathrm{E}-03$ \\
\hline & 372.1 & $1.97 \mathrm{E}-03$ & & 440 & $2.01 \mathrm{E}-03$ & & 500.5 & $1.47 \mathrm{E}-03$ \\
\hline & 373.1 & $1.77 \mathrm{E}-03$ & Drive $78 b$ & 440.8 & $1.52 \mathrm{E}-03$ & & 500.8 & $1.38 \mathrm{E}-03$ \\
\hline \multirow[t]{3}{*}{ Drive 64} & 375.45 & $1.50 \mathrm{E}-03$ & & 441.1 & $7.02 \mathrm{E}-04$ & & 501.1 & $1.58 \mathrm{E}-03$ \\
\hline & 375.7 & $1.43 \mathrm{E}-03$ & & 441.5 & 7. $62 \mathrm{E}-04$ & & 501.3 & $1.70 \mathrm{E}-03$ \\
\hline & 375.8 & $1.38 \mathrm{E}-03$ & & 441.8 & $6.86 \mathrm{E}-04$ & Drive 90 & 502.15 & $1.57 \mathrm{E}-03$ \\
\hline Drive 65 & 378.87 & $1.73 \mathrm{E}-03$ & & 442.1 & 8.77E-04 & & 502.45 & $1.27 \mathrm{E}-03$ \\
\hline \multirow{2}{*}{ Drive 69} & 395.25 & $1.40 \mathrm{E}-03$ & Drive $78 c$ & 442.58 & $9.12 \mathrm{E}-04$ & & 502.85 & $1.38 \mathrm{E}-03$ \\
\hline & 396.1 & $1.30 \mathrm{E}-03$ & & 442.97 & $4.52 \mathrm{E}-04$ & & 503.15 & $1.20 \mathrm{E}-03$ \\
\hline \multirow[t]{3}{*}{ Drive $69 \mathrm{~b}$} & 396.9 & $9.56 \mathrm{E}-04$ & Drive 79 & 443.75 & $5.59 \mathrm{E}-04$ & & 503.65 & $1.49 \mathrm{E}-03$ \\
\hline & 397.4 & $1.15 \mathrm{E}-03$ & & 444.3 & 4.09E-04 & Drive 90b & 504.15 & $1.57 \mathrm{E}-03$ \\
\hline & 397.9 & $1.40 \mathrm{E}-03$ & & 444.6 & $3.30 \mathrm{E}-04$ & & 504.35 & $1.60 \mathrm{E}-03$ \\
\hline \multirow[t]{3}{*}{ Drive $69 c$} & 398.7 & $1.24 \mathrm{E}-03$ & & 445 & $3.64 \mathrm{E}-04$ & & 504.85 & $1.44 \mathrm{E}-03$ \\
\hline & 399.14 & $8.45 \mathrm{E}-04$ & Drive $79 b$ & 445.8 & $3.82 \mathrm{E}-04$ & & 505.15 & $1.42 \mathrm{E}-03$ \\
\hline & 399.9 & $2.24 \mathrm{E}-03$ & & 446.4 & $4.72 \mathrm{E}-04$ & & 505.55 & $9.24 \mathrm{E}-04$ \\
\hline \multirow[t]{6}{*}{ Drive 70} & 401.45 & $2.51 \mathrm{E}-03$ & & 447.1 & $8.29 \mathrm{E}-04$ & Drive $90 \mathrm{c}$ & 506.15 & $9.64 \mathrm{E}-04$ \\
\hline & 401.93 & $3.09 \mathrm{E}-03$ & Drive $79 c$ & 447.55 & $1.23 \mathrm{E}-03$ & & 506.35 & $1.01 \mathrm{E}-03$ \\
\hline & 402 & $3.14 \mathrm{E}-03$ & Drive 80 & 457.4 & $3.39 \mathrm{E}-04$ & & 507.55 & $1.59 \mathrm{E}-03$ \\
\hline & 402.26 & $2.54 \mathrm{E}-03$ & Drive 80b & 458.4 & $1.35 \mathrm{E}-03$ & Drive 91 & 508.4 & $1.23 \mathrm{E}-03$ \\
\hline & 402.45 & $1.31 \mathrm{E}-03$ & & 458.85 & $1.88 \mathrm{E}-03$ & & 508.7 & $1.36 \mathrm{E}-03$ \\
\hline & 402.7 & $8.73 \mathrm{E}-04$ & Drive 81 & 461.6 & $3.46 \mathrm{E}-04$ & & 509.1 & $1.28 \mathrm{E}-03$ \\
\hline \multirow[t]{3}{*}{ Drive $70 \mathrm{~b}$} & 403.5 & 7.97E-04 & & 462.4 & $3.12 \mathrm{E}-04$ & & 509.4 & $1.42 \mathrm{E}-03$ \\
\hline & 403.7 & $5.71 \mathrm{E}-04$ & & 462.8 & $2.20 \mathrm{E}-04$ & Drive $91 b$ & 510 & $9.00 \mathrm{E}-04$ \\
\hline & 404.16 & $1.65 \mathrm{E}-03$ & Drive $81 b$ & 463.4 & $2.76 \mathrm{E}-04$ & & 510.3 & $8.89 \mathrm{E}-04$ \\
\hline Drive 71 & 404.95 & $8.13 \mathrm{E}-04$ & & 463.8 & $5.75 \mathrm{E}-04$ & & 510.85 & $1.20 \mathrm{E}-03$ \\
\hline Drive 72 & 406.6 & $1.03 \mathrm{E}-03$ & Drive 82 & 466.4 & $1.10 \mathrm{E}-03$ & & 511 & $1.34 \mathrm{E}-03$ \\
\hline & 406.9 & $1.00 \mathrm{E}-03$ & & 467.2 & $6.62 \mathrm{E}-04$ & & 511.5 & $1.56 \mathrm{E}-03$ \\
\hline & 407.1 & $1.12 \mathrm{E}-03$ & Drive $82 b$ & 468.65 & 2.84E-04 & Drive 9lc & 512.15 & $6.07 \mathrm{E}-04$ \\
\hline Drive $72 b$ & 407.65 & 8.37E-04 & Drive 83 & 471.6 & $1.23 \mathrm{E}-03$ & & 512.3 & $7.85 \mathrm{E}-04$ \\
\hline & 408.7 & $1.18 \mathrm{E}-03$ & & 472.1 & $1.28 \mathrm{E}-03$ & Drive 92 & 514.7 & $6.19 \mathrm{E}-04$ \\
\hline Drive 73 & 411.05 & 7.42E-04 & & 472.6 & $1.19 \mathrm{E}-03$ & & 515.15 & $6.62 \mathrm{E}-04$ \\
\hline & 411.2 & 8.09E-04 & Drive 83b & 473.6 & $6.58 \mathrm{E}-04$ & & 515.4 & $6.55 \mathrm{E}-04$ \\
\hline
\end{tabular}




\begin{tabular}{|c|c|c|c|c|c|c|c|c|}
\hline \multirow[t]{4}{*}{ Drive $92 b$} & 516 & 7.50E-04 & Drive 101 & 559.35 & $8.41 \mathrm{E}-04$ & Drive 119 & 641.65 & 7.97E-04 \\
\hline & 516.3 & 8. $13 \mathrm{E}-04$ & & 559.6 & $3.05 \mathrm{E}-04$ & & 642.14 & $4.28 \mathrm{E}-04$ \\
\hline & 516.75 & $1.08 \mathrm{E}-03$ & & 560.35 & $3.97 \mathrm{E}-04$ & Drive 120 & 650.4 & $1.43 \mathrm{E}-03$ \\
\hline & 516.9 & 7.62E-04 & Drive $101 \mathrm{~b}$ & 560.8 & $3.67 \mathrm{E}-04$ & & 650.6 & $9.76 \mathrm{E}-04$ \\
\hline \multirow[t]{4}{*}{ Drive 93} & 519.55 & $1.12 \mathrm{E}-03$ & & 560.95 & $3.65 \mathrm{E}-04$ & Drive $120 b$ & 651.15 & $3.04 \mathrm{E}-02$ \\
\hline & 519.8 & $1.17 \mathrm{E}-03$ & Drive 103 & 569.5 & $7.81 \mathrm{E}-04$ & & 651.4 & $1.49 \mathrm{E}-03$ \\
\hline & 519.9 & $1.21 \mathrm{E}-03$ & & 569.7 & $5.71 \mathrm{E}-04$ & & 651.5 & $1.40 \mathrm{E}-03$ \\
\hline & 520.4 & $1.25 \mathrm{E}-03$ & Drive 105 & 573.6 & $2.39 \mathrm{E}-04$ & Drive 122 & 662.6 & $6.62 \mathrm{E}-04$ \\
\hline \multirow[t]{6}{*}{ Drive $93 b$} & 520.9 & $1.50 \mathrm{E}-03$ & Drive 106 & 576.25 & $3.65 \mathrm{E}-04$ & & 662.9 & $7.30 \mathrm{E}-04$ \\
\hline & 521.1 & $1.34 \mathrm{E}-03$ & & 576.8 & $9.28 \mathrm{E}-04$ & & 663.5 & $3.28 \mathrm{E}-04$ \\
\hline & 531.35 & $1.43 \mathrm{E}-03$ & & 577 & $5.95 \mathrm{E}-04$ & & 663.7 & $3.88 \mathrm{E}-04$ \\
\hline & 521.65 & $1.50 \mathrm{E}-03$ & & 577.1 & $4.44 \mathrm{E}-04$ & & 664.1 & 7.93E-04 \\
\hline & 521.9 & $1.42 \mathrm{E}-03$ & Drive 109 & 593.5 & $3.81 \mathrm{E}-04$ & Drive $122 b$ & 664.5 & 8.21E-04 \\
\hline & 522.2 & $1.35 \mathrm{E}-03$ & & 593.6 & $4.24 \mathrm{E}-04$ & & 664.7 & 8.37E-04 \\
\hline \multirow[t]{5}{*}{ Drive 94} & 524 & $1.12 \mathrm{E}-03$ & Drive 111 & 598.6 & $5.59 \mathrm{E}-04$ & Drive 123 & 668.5 & $1.02 \mathrm{E}-03$ \\
\hline & 524.3 & $1.03 \mathrm{E}-03$ & & 599.1 & $3.02 \mathrm{E}-04$ & & 669 & 8.53E-04 \\
\hline & 524.5 & $1.01 \mathrm{E}-03$ & & 599.45 & $2.86 \mathrm{E}-04$ & & 669.5 & 7.74E-04 \\
\hline & 524.95 & $1.04 \mathrm{E}-03$ & Drive $111 b$ & 600.5 & $3.16 \mathrm{E}-04$ & Drive 124 & 670.8 & $1.40 \mathrm{E}-03$ \\
\hline & 525.4 & $8.65 \mathrm{E}-04$ & Drive 114 & 612.3 & $4.24 \mathrm{E}-04$ & & 671.3 & $4.01 \mathrm{E}-04$ \\
\hline Drive $94 b$ & 525.85 & $5.32 \mathrm{E}-04$ & & 612.6 & $2.81 \mathrm{E}-04$ & & 672 & $6.98 \mathrm{E}-04$ \\
\hline \multirow[t]{4}{*}{ Drive 95} & 529 & $9.76 \mathrm{E}-04$ & & 613.15 & $2.91 \mathrm{E}-04$ & Drive $124 b$ & 672.63 & $2.61 \mathrm{E}-04$ \\
\hline & 529.07 & $1.01 \mathrm{E}-03$ & & 613.65 & $6.66 \mathrm{E}-04$ & & 672.75 & $2.52 \mathrm{E}-04$ \\
\hline & 529.2 & $9.16 \mathrm{E}-04$ & Drive $114 b$ & 614.3 & $3.68 \mathrm{E}-04$ & & 673.15 & $1.85 \mathrm{E}-04$ \\
\hline & 529.7 & $9.08 \mathrm{E}-04$ & & 615.35 & $5.75 \mathrm{E}-04$ & & 673.55 & $2.35 \mathrm{E}-04$ \\
\hline \multirow[t]{6}{*}{ Drive 96} & 534.35 & $6.78 \mathrm{E}-04$ & Drive $114 \mathrm{c}$ & 616.2 & $2.45 \mathrm{E}-04$ & Drive 125 & 676.7 & 3.39E-04 \\
\hline & 534.5 & $9.28 \mathrm{E}-04$ & & 616.35 & $3.36 \mathrm{E}-04$ & Drive 126 & 679.5 & $2.83 \mathrm{E}-04$ \\
\hline & 534.7 & 8.49E-04 & & 616.8 & $5.79 \mathrm{E}-04$ & & 679.8 & 3.32E-04 \\
\hline & 534.9 & 8.01E-04 & & 617.05 & $2.38 \mathrm{E}-04$ & & 680.3 & $6.35 \mathrm{E}-04$ \\
\hline & 535.3 & $5.28 \mathrm{E}-04$ & Drive 115 & 617.45 & $1.96 \mathrm{E}-04$ & Drive 130 & 692.8 & $9.72 \mathrm{E}-04$ \\
\hline & 535.5 & $6.70 \mathrm{E}-04$ & & 617.75 & $2.28 \mathrm{E}-04$ & & 693.1 & 7.14E-04 \\
\hline \multirow[t]{4}{*}{ Drive $96 \mathrm{~b}$} & 536.25 & $1.59 \mathrm{E}-03$ & & 618.05 & $2.25 \mathrm{E}-04$ & & 694.4 & $7.18 \mathrm{E}-04$ \\
\hline & 536.6 & $7.62 \mathrm{E}-04$ & & 618.2 & $2.26 \mathrm{E}-04$ & Drive $130 b$ & 694.9 & $1.02 \mathrm{E}-03$ \\
\hline & 536.8 & $5.28 \mathrm{E}-04$ & & 618.6 & $2.22 \mathrm{E}-04$ & & 695.4 & $1.21 \mathrm{E}-03$ \\
\hline & 537 & $4.60 \mathrm{E}-04$ & Drive $115 b$ & 618.8 & $5.36 \mathrm{E}-04$ & & 695.7 & $1.27 \mathrm{E}-03$ \\
\hline \multirow[t]{6}{*}{ Drive 97} & 539.5 & $5.12 \mathrm{E}-04$ & & 619 & $2.91 \mathrm{E}-04$ & Drive 131 & 697.52 & $6.70 \mathrm{E}-04$ \\
\hline & 539.7 & $4.40 \mathrm{E}-04$ & & 619.2 & $2.85 \mathrm{E}-04$ & & 697.62 & 8.41E-04 \\
\hline & 539.9 & 4. 13E-04 & Drive $115 c$ & 619.45 & $3.40 \mathrm{E}-04$ & & 698.12 & $8.49 \mathrm{E}-04$ \\
\hline & 540.35 & $2.83 \mathrm{E}-04$ & & 619.75 & $4.60 \mathrm{E}-04$ & Drive 133 & 703.3 & $3.36 \mathrm{E}-04$ \\
\hline & 540.6 & $3.44 \mathrm{E}-04$ & & 620.05 & $2.93 \mathrm{E}-04$ & & 703.5 & $2.09 \mathrm{E}-04$ \\
\hline & 540.7 & $3.89 \mathrm{E}-04$ & & 620.35 & $3.16 \mathrm{E}-04$ & & 703.8 & $2.02 \mathrm{E}-04$ \\
\hline Drive $97 b$ & 541.42 & $5.12 \mathrm{E}-04$ & & 620.55 & $2.65 \mathrm{E}-04$ & & 703.9 & $1.94 \mathrm{E}-04$ \\
\hline \multirow[t]{5}{*}{ Drive 98} & 544.5 & $5.99 \mathrm{E}-04$ & Drive 116 & 621.7 & $2.80 \mathrm{E}-04$ & & 704.3 & $1.31 \mathrm{E}-04$ \\
\hline & 544.65 & $5.08 \mathrm{E}-04$ & & 622.2 & $2.95 \mathrm{E}-04$ & Drive $133 b$ & 705 & 4.01E-04 \\
\hline & 545.1 & $6.35 \mathrm{E}-04$ & & 623.22 & $5.71 \mathrm{E}-04$ & & 705.3 & $5.67 \mathrm{E}-04$ \\
\hline & 545.3 & $6.15 \mathrm{E}-04$ & Drive $116 b$ & 623.8 & $8.21 \mathrm{E}-04$ & & 706.05 & $7.14 \mathrm{E}-04$ \\
\hline & 545.75 & $5.16 \mathrm{E}-04$ & & 624.4 & $2.22 \mathrm{E}-03$ & Drive $133 c$ & 706.8 & $6.74 \mathrm{E}-04$ \\
\hline \multirow[t]{4}{*}{ Drive $98 b$} & 546.25 & 4.44E-04 & Drive 117 & 626.2 & $1.20 \mathrm{E}-03$ & & 707.1 & 8.17E-04 \\
\hline & 546.4 & $4.40 \mathrm{E}-04$ & & 626.25 & $1.11 \mathrm{E}-03$ & & 707.5 & $2.47 \mathrm{E}-04$ \\
\hline & 547.1 & 8.77E-04 & Drive $117 b$ & 627.65 & $3.71 \mathrm{E}-04$ & & 707.95 & 1.73E-04 \\
\hline & 547.4 & $9.04 \mathrm{E}-04$ & & 627.7 & 4.01E-04 & Drive 134 & 709.4 & $1.11 \mathrm{E}-04$ \\
\hline \multirow[t]{6}{*}{ Drive 99} & 549.13 & $5.36 \mathrm{E}-04$ & Drive 118 & 634.9 & $1.15 \mathrm{E}-03$ & & 710.4 & $2.27 \mathrm{E}-04$ \\
\hline & 549.6 & $2.54 \mathrm{E}-04$ & & 635.1 & $9.16 \mathrm{E}-04$ & Drive $134 b$ & 711.05 & $2.51 \mathrm{E}-04$ \\
\hline & 550.01 & $1.78 \mathrm{E}-04$ & & 635.6 & $1.40 \mathrm{E}-03$ & & 711.3 & $2.49 \mathrm{E}-04$ \\
\hline & 550.15 & $2.17 \mathrm{E}-04$ & & 636 & $9.32 \mathrm{E}-04$ & & 711.7 & $4.36 \mathrm{E}-04$ \\
\hline & 550.65 & $5.51 \mathrm{E}-04$ & Drive $118 b$ & 637 & $9.36 \mathrm{E}-04$ & Drive 135 & 713.3 & $1.81 \mathrm{E}-04$ \\
\hline & 550.75 & $6.15 \mathrm{E}-04$ & & 637.5 & $1.46 \mathrm{E}-03$ & & 713.65 & $1.28 \mathrm{E}-04$ \\
\hline \multirow[t]{2}{*}{ Drive $99 b$} & 551.15 & $5.71 \mathrm{E}-04$ & & 638.15 & $1.04 \mathrm{E}-03$ & & 714.1 & $1.64 \mathrm{E}-04$ \\
\hline & 551.25 & $5.67 \mathrm{E}-04$ & Drive $118 \mathrm{c}$ & 638.65 & $1.28 \mathrm{E}-03$ & & 714.5 & $1.98 \mathrm{E}-04$ \\
\hline \multirow[t]{2}{*}{ Drive 100} & 554.9 & $4.60 \mathrm{E}-04$ & & 638.9 & $1.25 \mathrm{E}-03$ & Drive $135 b$ & 715.05 & 1.75E-04 \\
\hline & 555.85 & $3.18 \mathrm{E}-02$ & & 639.07 & $1.10 \mathrm{E}-03$ & & 715.25 & $1.99 \mathrm{E}-04$ \\
\hline
\end{tabular}




\begin{tabular}{|c|c|c|c|c|c|c|c|c|}
\hline \multirow[t]{4}{*}{ Drive 136} & 717.1 & 1.29E-04 & Drive $147 \mathrm{c}$ & 793.3 & $7.14 \mathrm{E}-04$ & & 916.85 & $1.18 \mathrm{E}-03$ \\
\hline & 717.4 & $1.42 \mathrm{E}-04$ & & 793.8 & $5.95 \mathrm{E}-04$ & Drive $166 \mathrm{~b}$ & 920.8 & $2.50 \mathrm{E}-03$ \\
\hline & 717.9 & $1.47 \mathrm{E}-04$ & & 794 & $6.03 \mathrm{E}-04$ & & 920.9 & $2.26 \mathrm{E}-03$ \\
\hline & 718.4 & $1.48 \mathrm{E}-04$ & & 794.3 & $5.91 \mathrm{E}-04$ & & 921.4 & $9.88 \mathrm{E}-04$ \\
\hline \multirow[t]{3}{*}{ Drive $136 b$} & 719.55 & $1.57 \mathrm{E}-04$ & & 794.85 & $6.11 \mathrm{E}-04$ & & 921.65 & 8.21E-04 \\
\hline & 719.9 & $1.69 \mathrm{E}-04$ & Drive $147 \mathrm{~d}$ & 795.4 & $7.81 \mathrm{E}-04$ & Drive 167 & 929.85 & $1.02 \mathrm{E}-03$ \\
\hline & 719.6 & $1.23 \mathrm{E}-04$ & & 796.7 & $7.14 \mathrm{E}-04$ & & 929.9 & $9.76 \mathrm{E}-04$ \\
\hline \multirow[t]{3}{*}{ Drive $136 \mathrm{c}$} & 721.3 & $1.57 \mathrm{E}-04$ & Drive 150 & 813 & $1.69 \mathrm{E}-03$ & & 930 & 8.73E-04 \\
\hline & 721.4 & $1.60 \mathrm{E}-04$ & & 813.3 & $1.69 \mathrm{E}-03$ & & 930.5 & $1.81 \mathrm{E}-03$ \\
\hline & 722.2 & $1.40 \mathrm{E}-04$ & & 813.5 & $1.75 \mathrm{E}-03$ & Drive 168 & 931.25 & $1.96 \mathrm{E}-03$ \\
\hline Drive 137 & 723 & $1.90 \mathrm{E}-04$ & & 813.7 & 1.79E-03 & & 931.65 & $1.82 \mathrm{E}-03$ \\
\hline Drive 138 & 732.8 & $6.90 \mathrm{E}-04$ & & 814 & $1.81 \mathrm{E}-03$ & Drive 170 & 948.85 & $1.25 \mathrm{E}-03$ \\
\hline \multirow[t]{3}{*}{ Drive 140} & 742.6 & $6.78 \mathrm{E}-04$ & & 814.3 & $1.66 \mathrm{E}-03$ & & 948.95 & 8.05E-04 \\
\hline & 742.7 & $7.46 \mathrm{E}-04$ & Drive $150 \mathrm{~b}$ & 814.9 & $1.67 \mathrm{E}-03$ & Drive $170 \mathrm{~b}$ & 950.15 & $1.42 \mathrm{E}-03$ \\
\hline & 743 & $7.18 \mathrm{E}-04$ & & 815.2 & $1.73 \mathrm{E}-03$ & & 950.55 & 1.15E-03 \\
\hline Drive $140 \mathrm{~b}$ & 744.44 & $6.15 \mathrm{E}-04$ & & 815.5 & $1.61 \mathrm{E}-03$ & & 950.85 & $1.07 \mathrm{E}-03$ \\
\hline \multirow[t]{4}{*}{ Drive $141 \mathrm{c}$} & 749.17 & $1.85 \mathrm{E}-04$ & & 815.9 & $1.71 \mathrm{E}-03$ & & 951.25 & $6.23 \mathrm{E}-04$ \\
\hline & 749.5 & $1.71 \mathrm{E}-04$ & & 816.2 & $1.81 \mathrm{E}-03$ & Drive 171 & 977.25 & $7.54 \mathrm{E}-03$ \\
\hline & 749.9 & $1.87 \mathrm{E}-04$ & Drive $150 c$ & 816.9 & $2.30 \mathrm{E}-03$ & & 977.6 & 4.05E-02 \\
\hline & 750.3 & $2.55 \mathrm{E}-04$ & & 817.9 & $1.79 \mathrm{E}-03$ & & 977.8 & 4.52E-03 \\
\hline \multirow[t]{4}{*}{ Drive 142} & 752.75 & 2.17E-04 & & 818.2 & $1.59 \mathrm{E}-03$ & & 978.25 & 2.74E-03 \\
\hline & 753.35 & $2.30 \mathrm{E}-04$ & Drive $150 \mathrm{~d}$ & 818.8 & 2.99E-03 & & 978.55 & $2.76 \mathrm{E}-03$ \\
\hline & 753.75 & $2.50 \mathrm{E}-04$ & & 819.1 & 2.12E-03 & Drive $171 b$ & 980.4 & $2.51 \mathrm{E}-03$ \\
\hline & 753.95 & $2.26 \mathrm{E}-04$ & & 819.5 & $1.49 \mathrm{E}-03$ & & 980.8 & $1.32 \mathrm{E}-03$ \\
\hline \multirow[t]{5}{*}{ Drive $142 b$} & 754.55 & $2.83 \mathrm{E}-04$ & & 819.8 & $2.40 \mathrm{E}-03$ & Drive 173 & 1002.7 & $1.57 \mathrm{E}-03$ \\
\hline & 754.85 & 8.37E-04 & & 820 & $1.79 \mathrm{E}-03$ & & 1002.9 & $1.56 \mathrm{E}-03$ \\
\hline & 755.15 & 1. $10 \mathrm{E}-03$ & Drive 151 & 822.6 & $2.35 \mathrm{E}-03$ & Drive $173 b$ & 1004.45 & 2.33E-03 \\
\hline & 755.35 & $1.25 \mathrm{E}-03$ & & 823.2 & $1.94 \mathrm{E}-03$ & & 1004.55 & $2.28 \mathrm{E}-03$ \\
\hline & 755.85 & 1.17E-03 & & 823.6 & $2.25 \mathrm{E}-03$ & Drive 174 & 1017.7 & $4.28 \mathrm{E}-03$ \\
\hline \multirow[t]{6}{*}{ Drive $142 c$} & 756.45 & $1.38 \mathrm{E}-03$ & Drive $151 b$ & 824.25 & $1.73 \mathrm{E}-03$ & & 1018 & $3.90 \mathrm{E}-03$ \\
\hline & 756.65 & $1.32 \mathrm{E}-03$ & & 824.39 & $2.11 \mathrm{E}-03$ & & 1018.4 & $3.41 \mathrm{E}-03$ \\
\hline & 757.05 & $1.11 \mathrm{E}-03$ & & 825.4 & 2. $67 \mathrm{E}-03$ & & 1018.75 & $2.71 \mathrm{E}-03$ \\
\hline & 757.25 & $1.02 \mathrm{E}-03$ & Drive 153 & 842.4 & $1.97 \mathrm{E}-03$ & Drive 175 & 1022.37 & 1.19E-03 \\
\hline & 757.65 & $9.20 \mathrm{E}-04$ & & 842.5 & $2.09 \mathrm{E}-03$ & Drive 176 & 1032 & $2.88 \mathrm{E}-03$ \\
\hline & 757.95 & 8.05E-04 & & 843.6 & $2.20 \mathrm{E}-03$ & & 1032.3 & $3.59 \mathrm{E}-03$ \\
\hline \multirow[t]{3}{*}{ Drive $142 d$} & 758.45 & $7.46 \mathrm{E}-04$ & & 843.7 & $1.98 \mathrm{E}-03$ & & 1032.6 & $2.71 \mathrm{E}-03$ \\
\hline & 758.85 & $6.15 \mathrm{E}-04$ & Drive 155 & 853 & $3.21 \mathrm{E}-03$ & Drive $176 b$ & 1033.5 & $1.84 \mathrm{E}-03$ \\
\hline & 759.65 & $5.87 \mathrm{E}-04$ & & 853.3 & $3.13 \mathrm{E}-03$ & & 1033.85 & $2.04 \mathrm{E}-03$ \\
\hline \multirow[t]{2}{*}{ Drive 143} & 761.35 & 7.97E-04 & & 853.75 & 4.01E-03 & Drive 177 & 1035.7 & $1.44 \mathrm{E}-03$ \\
\hline & 761.75 & 5.51E-04 & & 854.05 & $3.72 \mathrm{E}-03$ & & 1036.5 & $1.82 \mathrm{E}-03$ \\
\hline \multirow[t]{2}{*}{ Drive 144} & 770.5 & $1.13 \mathrm{E}-03$ & Drive $155 b$ & 855.1 & $4.96 \mathrm{E}-03$ & & 1036.55 & $2.25 \mathrm{E}-03$ \\
\hline & 770.8 & $1.02 \mathrm{E}-03$ & & 855.2 & 4. $60 \mathrm{E}-03$ & Drive 178 & 1042.55 & $2.67 \mathrm{E}-03$ \\
\hline Drive 145 & 777.25 & $8.53 \mathrm{E}-04$ & & 855.3 & $4.68 \mathrm{E}-03$ & & 1042.67 & $2.51 \mathrm{E}-03$ \\
\hline \multirow[t]{3}{*}{ Drive $145 b$} & 778.4 & $2.67 \mathrm{E}-03$ & Drive 157 & 869.35 & $8.77 \mathrm{E}-03$ & & 1042.9 & 2. 12E-03 \\
\hline & 778.7 & $2.96 \mathrm{E}-03$ & & 869.5 & 8.57E-03 & & 1043.3 & $3.34 \mathrm{E}-03$ \\
\hline & 778.8 & $3.03 \mathrm{E}-03$ & Drive 158 & 879.4 & $1.92 \mathrm{E}-03$ & & 1043.5 & $4.01 \mathrm{E}-03$ \\
\hline \multirow[t]{4}{*}{ Drive 146} & 782.45 & $1.65 \mathrm{E}-03$ & & 879.7 & $2.41 \mathrm{E}-03$ & & 1043.7 & $2.87 \mathrm{E}-03$ \\
\hline & 782.8 & $1.98 \mathrm{E}-03$ & & 880.6 & $1.62 \mathrm{E}-03$ & Drive $178 b$ & 1044.7 & $9.12 \mathrm{E}-04$ \\
\hline & 783 & $2.00 \mathrm{E}-03$ & Drive 159 & 889.45 & $1.01 \mathrm{E}-03$ & & 1045.3 & $1.50 \mathrm{E}-03$ \\
\hline & 783.2 & $1.52 \mathrm{E}-03$ & Drive 160 & 893.76 & $1.54 \mathrm{E}-03$ & & 1045.7 & $1.69 \mathrm{E}-03$ \\
\hline \multirow[t]{2}{*}{ Drive $146 b$} & 784.3 & $1.60 \mathrm{E}-03$ & & 894.1 & $1.28 \mathrm{E}-03$ & Drive $178 c$ & 1046.25 & $1.97 \mathrm{E}-03$ \\
\hline & 784.6 & $1.83 \mathrm{E}-03$ & & 984.25 & 1. $19 \mathrm{E}-03$ & & 1046.55 & $1.92 \mathrm{E}-03$ \\
\hline \multirow[t]{4}{*}{ Drive 147} & 789.25 & $7.74 \mathrm{E}-04$ & Drive 163 & 902.6 & $2.02 \mathrm{E}-03$ & Drive 179 & 1053.5 & $1.44 \mathrm{E}-03$ \\
\hline & 789.9 & $9.60 \mathrm{E}-04$ & Drive 164 & 910.2 & $3.70 \mathrm{E}-03$ & & 1053.8 & $1.20 \mathrm{E}-03$ \\
\hline & 790.3 & $1.23 \mathrm{E}-03$ & Drive 165 & 914.05 & $4.32 \mathrm{E}-03$ & Drive $179 b$ & 1054.3 & $1.03 \mathrm{E}-03$ \\
\hline & 790.6 & 8.77E-04 & & 914.4 & $6.74 \mathrm{E}-03$ & & 1054.7 & $1.01 \mathrm{E}-03$ \\
\hline Drive $147 \mathrm{~b}$ & 791.25 & $1.12 \mathrm{E}-03$ & & 914.9 & $3.39 \mathrm{E}-03$ & & 1055.1 & $1.00 \mathrm{E}-03$ \\
\hline & 791.7 & $9.40 \mathrm{E}-04$ & & 915.05 & $2.76 \mathrm{E}-03$ & & 1055.5 & $9.32 \mathrm{E}-04$ \\
\hline & 792.3 & $1.26 \mathrm{E}-03$ & Drive $165 b$ & 916.4 & $1.16 \mathrm{E}-03$ & & 1055.8 & 8.69E-04 \\
\hline & 792.6 & $1.25 \mathrm{E}-03$ & & 916.7 & $1.09 \mathrm{E}-03$ & Drive $179 c$ & 1056.3 & $9.76 \mathrm{E}-04$ \\
\hline
\end{tabular}




\begin{tabular}{|c|c|c|c|c|c|c|c|c|}
\hline & 1056.5 & $9.56 \mathrm{E}-04$ & & 1169.5 & $2.66 \mathrm{E}-03$ & & 1244.82 & $1.25 \mathrm{E}-03$ \\
\hline & 1056.9 & $1.00 \mathrm{E}-03$ & Drive 193 & 1171.1 & 7.93E-04 & Drive 203b & 1247.22 & $3.42 \mathrm{E}-03$ \\
\hline & 1057.3 & $1.09 \mathrm{E}-03$ & Drive 194 & 1181.09 & $3.81 \mathrm{E}-04$ & Drive 204 & 1254.82 & $8.09 \mathrm{E}-0$ \\
\hline & 1057.7 & $1.01 \mathrm{E}-03$ & & 1181.12 & $3.76 \mathrm{E}-04$ & & 1255.32 & $9.76 \mathrm{E}-0$ \\
\hline \multirow[t]{3}{*}{ Drive 180} & 1062.37 & 7.06E-04 & & 1181.5 & $1.05 \mathrm{E}-03$ & Drive 205 & 1260.35 & $2.97 \mathrm{E}-0$ \\
\hline & 1063.6 & $1.98 \mathrm{E}-03$ & & 1182 & $1.17 \mathrm{E}-03$ & & 1260.45 & $3.67 \mathrm{E}-0$ \\
\hline & 1063.8 & $2.10 \mathrm{E}-03$ & & 1182.35 & $1.21 \mathrm{E}-03$ & & 1260.94 & 3.63E-03 \\
\hline \multirow[t]{4}{*}{ Drive $180 \mathrm{~b}$} & 1064.5 & 2.39E-03 & & 1182.65 & $1.47 \mathrm{E}-03$ & & 1261.1 & $2.51 \mathrm{E}-03$ \\
\hline & 1064.86 & $4.20 \mathrm{E}-03$ & Drive $194 b$ & 1183.4 & 8.81E-04 & & 1261.25 & $2.30 \mathrm{E}-03$ \\
\hline & 1065.2 & $1.94 \mathrm{E}-03$ & & 1183.73 & $8.29 \mathrm{E}-04$ & Drive $205 b$ & 1261.7 & $1.65 \mathrm{E}-0$ \\
\hline & 1065.58 & $2.59 \mathrm{E}-03$ & & 1184.1 & $9.04 \mathrm{E}-04$ & & 1262 & $1.82 \mathrm{E}-03$ \\
\hline \multirow[t]{3}{*}{ Drive 182} & 1076.5 & $1.25 \mathrm{E}-03$ & & 1184.25 & 1.10E-03 & & 1262.1 & $1.89 \mathrm{E}-03$ \\
\hline & 1076.9 & $1.92 \mathrm{E}-03$ & Drive $194 c$ & 1184.88 & $1.67 \mathrm{E}-03$ & & 1363.15 & $2.13 \mathrm{E}-0$ \\
\hline & 1077.73 & $1.82 \mathrm{E}-03$ & & 1184.97 & $2.07 \mathrm{E}-03$ & & 1263.25 & $2.04 \mathrm{E}-03$ \\
\hline \multirow[t]{3}{*}{ Drive 183} & 1092.7 & $1.63 \mathrm{E}-03$ & & 1185.05 & 2.19E-03 & Drive 205c & 1263.8 & $1.72 \mathrm{E}-03$ \\
\hline & 1093 & 1.35E-03 & Drive 195 & 1189.82 & $9.64 \mathrm{E}-04$ & & 1264 & $1.77 \mathrm{E}-03$ \\
\hline & 1094.35 & $3.15 \mathrm{E}-03$ & & 1190.3 & $1.22 \mathrm{E}-03$ & & 1264.3 & $1.83 \mathrm{E}-03$ \\
\hline \multirow[t]{2}{*}{ Drive $183 b$} & 1094.75 & $3.91 \mathrm{E}-03$ & Drive $195 b$ & 1192 & 7.34E-04 & & 1264.7 & $1.58 \mathrm{E}-03$ \\
\hline & 1095.05 & 4.20E-03 & & 1192.3 & 7.14E-04 & & 1265 & $1.71 \mathrm{E}-03$ \\
\hline \multirow[t]{5}{*}{ Drive 184} & 1096.48 & 1.13E-03 & & 1192.7 & 8.41E-04 & & 1265.25 & $1.60 \mathrm{E}-03$ \\
\hline & 1097.3 & $1.40 \mathrm{E}-03$ & Drive 196 & 1196.1 & $7.85 \mathrm{E}-04$ & Drive $205 d$ & 1265.73 & $1.50 \mathrm{E}-03$ \\
\hline & 1097.4 & $1.41 \mathrm{E}-03$ & & 1196.53 & $5.28 \mathrm{E}-04$ & & 1266 & $1.54 \mathrm{E}-03$ \\
\hline & 1097.8 & 1.69E-03 & Drive 197 & 1204.75 & 7.18E-04 & & 1266.3 & $1.52 \mathrm{E}-03$ \\
\hline & 1097.9 & $1.73 \mathrm{E}-03$ & Drive $197 b$ & 1205.42 & $7.14 \mathrm{E}-04$ & & 1266.64 & $1.31 \mathrm{E}-03$ \\
\hline \multirow[t]{4}{*}{ Drive 185} & 1102.1 & $1.48 \mathrm{E}-03$ & & 1205.67 & 4.20E-04 & & 1266.8 & $1.38 \mathrm{E}-03$ \\
\hline & 1102.15 & $1.58 \mathrm{E}-03$ & & 1206.1 & $5.12 \mathrm{E}-04$ & Drive 205e & 1267.82 & $1.44 \mathrm{E}-03$ \\
\hline & 1102.25 & $1.61 \mathrm{E}-03$ & & 1206.2 & 7.77E-04 & Drive 206 & 1269.53 & $1.55 \mathrm{E}-03$ \\
\hline & 1102.35 & $1.55 \mathrm{E}-03$ & & 1206.6 & $9.88 \mathrm{E}-04$ & & 1269.72 & $1.70 \mathrm{E}-03$ \\
\hline Drive 186 & 1112.8 & $1.53 \mathrm{E}-03$ & & 1206.9 & $9.96 \mathrm{E}-04$ & & 1270.2 & $2.61 \mathrm{E}-03$ \\
\hline \multirow[t]{4}{*}{ Drive 187} & 1116.1 & $1.63 \mathrm{E}-03$ & Drive 197c & 1207.5 & $1.01 \mathrm{E}-03$ & & 1270.5 & $3.47 \mathrm{E}-03$ \\
\hline & 1116.5 & 2.72E-03 & & 1208 & $1.01 \mathrm{E}-03$ & & 1270.8 & $8.53 \mathrm{E}-03$ \\
\hline & 1116.6 & $3.00 \mathrm{E}-03$ & & 1208.4 & $1.22 \mathrm{E}-03$ & Drive $206 \mathrm{~b}$ & 1271.3 & $1.53 \mathrm{E}-03$ \\
\hline & 1117.2 & $3.93 \mathrm{E}-03$ & & 1208.8 & $1.14 \mathrm{E}-03$ & & 1271.5 & $1.34 \mathrm{E}-03$ \\
\hline \multirow[t]{6}{*}{ Drive $187 \mathrm{~b}$} & 1117.93 & 7.30E-03 & Drive 197d & 1209.5 & $1.08 \mathrm{E}-03$ & & 1271.7 & $1.10 \mathrm{E}-03$ \\
\hline & 1118.1 & $5.28 \mathrm{E}-03$ & & 1209.9 & $9.12 \mathrm{E}-04$ & & 1271.9 & $1.11 \mathrm{E}-03$ \\
\hline & 1118.6 & $6.82 \mathrm{E}-03$ & & 1210 & $6.47 \mathrm{E}-04$ & & 1272.1 & $1.35 \mathrm{E}-03$ \\
\hline & 1118.85 & 7.42E-03 & & 1210.5 & $7.34 \mathrm{E}-04$ & & 1272.62 & $2.19 \mathrm{E}-03$ \\
\hline & 1119.1 & $8.01 \mathrm{E}-03$ & & 1210.9 & 7.97E-04 & Drive 206c & 1273.35 & $1.67 \mathrm{E}-03$ \\
\hline & 1119.3 & 8.13E-03 & Drive $197 \mathrm{e}$ & 1211.92 & 7.34E-04 & & 1273.7 & $1.61 \mathrm{E}-03$ \\
\hline Drive $187 \mathrm{c}$ & 1119.9 & $1.90 \mathrm{E}-03$ & Drive 198 & 1213.33 & 7.54E-04 & & 1274 & $1.49 \mathrm{E}-03$ \\
\hline \multirow[t]{4}{*}{ Drive 188} & 1122.6 & $2.05 \mathrm{E}-03$ & & 1213.5 & 7.62E-04 & & 1274.2 & $1.52 \mathrm{E}-03$ \\
\hline & 1123 & $1.52 \mathrm{E}-03$ & & 1214.75 & 4.92E-04 & & 1274.5 & $1.44 \mathrm{E}-03$ \\
\hline & 1123.35 & $1.20 \mathrm{E}-03$ & Drive $198 b$ & 1215.75 & $9.80 \mathrm{E}-04$ & & 1274.8 & $1.19 \mathrm{E}-03$ \\
\hline & 1123.8 & $1.42 \mathrm{E}-03$ & Drive 200 & 1223.93 & 7.89E-04 & Drive 206d & 1275.45 & $9.12 \mathrm{E}-04$ \\
\hline \multirow[t]{3}{*}{ Drive $188 b$} & 1124.25 & $9.08 \mathrm{E}-04$ & & 1224.93 & $6.15 \mathrm{E}-04$ & & 1275.9 & $9.52 \mathrm{E}-04$ \\
\hline & 1125 & $1.04 \mathrm{E}-03$ & Drive $200 \mathrm{~b}$ & 1225.77 & $7.10 \mathrm{E}-04$ & & 1276.5 & $9.16 \mathrm{E}-04$ \\
\hline & 1125.5 & $1.04 \mathrm{E}-03$ & & 1226.93 & $1.42 \mathrm{E}-03$ & & 1276.7 & $9.68 \mathrm{E}-04$ \\
\hline \multirow[t]{2}{*}{ Drive 189} & 1133.05 & $9.96 \mathrm{E}-04$ & & 1227.06 & $1.29 \mathrm{E}-03$ & Drive 206e & 1277.3 & $1.89 \mathrm{E}-03$ \\
\hline & 1133.7 & $9.80 \mathrm{E}-04$ & Drive 201 & 1233.87 & 7.42E-04 & & 1277.5 & 2.03E-03 \\
\hline \multirow[t]{3}{*}{ Drive 192} & 1162.5 & $5.24 \mathrm{E}-03$ & Drive $201 \mathrm{~b}$ & 1235.87 & $6.15 \mathrm{E}-04$ & & 1277.7 & $2.07 \mathrm{E}-03$ \\
\hline & 1163 & $3.74 \mathrm{E}-03$ & & 1236.2 & $6.90 \mathrm{E}-04$ & Drive 207 & 1279.6 & $2.36 \mathrm{E}-03$ \\
\hline & 1163.5 & $2.61 \mathrm{E}-03$ & & 1236.37 & $7.62 \mathrm{E}-04$ & & 1279.85 & $1.71 \mathrm{E}-03$ \\
\hline \multirow[t]{3}{*}{ Drive $192 b$} & 1164.5 & 2.93E-03 & Drive 201c & 1237.87 & $6.70 \mathrm{E}-04$ & & 1280.35 & $1.76 \mathrm{E}-03$ \\
\hline & 1165 & $2.43 \mathrm{E}-03$ & & 1238.28 & $6.51 \mathrm{E}-04$ & & 1280.8 & $1.71 \mathrm{E}-03$ \\
\hline & 1165.5 & $2.81 \mathrm{E}-03$ & & 1238.62 & $6.03 \mathrm{E}-04$ & Drive $207 \mathrm{~b}$ & 1281.65 & $1.40 \mathrm{E}-03$ \\
\hline \multirow[t]{3}{*}{ Drive 192c } & 1166.5 & $2.82 \mathrm{E}-03$ & Drive 201d & 1239.62 & $5.59 \mathrm{E}-04$ & & 1281.75 & $1.42 \mathrm{E}-03$ \\
\hline & 1167 & 2.30E-03 & & 1239.95 & 4.96E-04 & Drive $207 \mathrm{c}$ & 1284.05 & $1.23 \mathrm{E}-03$ \\
\hline & 1167.5 & $1.87 \mathrm{E}-03$ & & 1240.37 & $6.58 \mathrm{E}-04$ & Drive 208 & 1289.52 & $1.56 \mathrm{E}-03$ \\
\hline Drive $192 d$ & 1168.5 & 3.36E-03 & & 1240.62 & $4.52 \mathrm{E}-04$ & Drive 209 & 1299.3 & $1.58 \mathrm{E}-03$ \\
\hline & 1169 & $3.17 \mathrm{E}-03$ & Drive 203 & 1244.44 & $9.84 \mathrm{E}-04$ & & 1299.5 & $1.60 \mathrm{E}-03$ \\
\hline
\end{tabular}




\begin{tabular}{|c|c|c|c|c|c|c|c|c|}
\hline & 1299.7 & $1.60 \mathrm{E}-03$ & & 1329.3 & 3.75E-03 & & 1395.47 & 2.02E-03 \\
\hline \multirow[t]{8}{*}{ Drive 210} & 1305.7 & $1.70 \mathrm{E}-03$ & & 1329.6 & $3.76 \mathrm{E}-03$ & & 1396.1 & 4.28E-03 \\
\hline & 1305.93 & $2.01 \mathrm{E}-03$ & & 1329.8 & $4.52 \mathrm{E}-03$ & & 1396.15 & $4.05 \mathrm{E}-03$ \\
\hline & 1306 & $2.05 \mathrm{E}-03$ & & 1329.95 & 5.39E-03 & & 1396.2 & $3.11 \mathrm{E}-03$ \\
\hline & 1306.15 & $2.00 \mathrm{E}-03$ & Drive 213 & 1337.05 & 4.92E-03 & & 1396.3 & $2.96 \mathrm{E}-03$ \\
\hline & 1306.55 & $1.77 \mathrm{E}-03$ & & 1337.2 & $5.63 \mathrm{E}-03$ & & 1396.4 & $3.30 \mathrm{E}-03$ \\
\hline & 1306.7 & $2.21 \mathrm{E}-03$ & & 1337.6 & $5.08 \mathrm{E}-03$ & & 1396.47 & $3.49 \mathrm{E}-03$ \\
\hline & 1306.9 & $2.01 \mathrm{E}-03$ & Drive $213 b$ & 1338.6 & $4.48 \mathrm{E}-03$ & & 1396.6 & $2.53 \mathrm{E}-03$ \\
\hline & 1307.1 & $1.92 \mathrm{E}-03$ & & 1338.9 & $5.00 \mathrm{E}-03$ & & 1396.7 & $2.43 \mathrm{E}-03$ \\
\hline \multirow[t]{2}{*}{ Drive $210 b$} & 1307.95 & $1.80 \mathrm{E}-03$ & & 1339.2 & $4.17 \mathrm{E}-03$ & Drive $219 b$ & 1397.3 & $1.26 \mathrm{E}-03$ \\
\hline & 1308.96 & $2.60 \mathrm{E}-03$ & & 1339.7 & $3.51 \mathrm{E}-03$ & & 1397.4 & $1.41 \mathrm{E}-03$ \\
\hline \multirow{7}{*}{ Drive $210 c$} & 1309.9 & $3.70 \mathrm{E}-03$ & Drive $213 c$ & 1340.25 & $4.28 \mathrm{E}-03$ & & 1398.1 & $1.65 \mathrm{E}-03$ \\
\hline & 1310.2 & $4.52 \mathrm{E}-03$ & & 1340.9 & $2.00 \mathrm{E}-03$ & & 1398.69 & $1.38 \mathrm{E}-03$ \\
\hline & 1310.3 & $4.92 \mathrm{E}-03$ & & 1341.1 & $2.04 \mathrm{E}-03$ & & 1398.8 & $9.04 \mathrm{E}-04$ \\
\hline & 1310.5 & 4.01E-03 & & 1341.6 & $1.90 \mathrm{E}-03$ & Drive $219 c$ & 1399.5 & $1.12 \mathrm{E}-03$ \\
\hline & 1310.7 & $3.24 \mathrm{E}-03$ & Drive 215 & 1355.6 & $2.19 \mathrm{E}-03$ & & 1399.7 & $1.66 \mathrm{E}-03$ \\
\hline & 1310.9 & 2.71E-03 & & 1356 & 3.19E-03 & & 1400 & $1.25 \mathrm{E}-03$ \\
\hline & 1311.1 & $2.38 \mathrm{E}-03$ & Drive $215 b$ & 1358 & $2.45 \mathrm{E}-03$ & & 1400.3 & $1.28 \mathrm{E}-03$ \\
\hline \multirow[t]{5}{*}{ Drive $210 \mathrm{~d}$} & 1311.8 & $3.72 \mathrm{E}-03$ & & 1358.2 & $2.12 \mathrm{E}-03$ & Drive $219 \mathrm{~d}$ & 1401.3 & $9.44 \mathrm{E}-04$ \\
\hline & 1312.1 & $3.59 \mathrm{E}-03$ & & 1358.4 & 2.04E-03 & & 1401.5 & $1.01 \mathrm{E}-03$ \\
\hline & 1312.4 & $6.27 \mathrm{E}-03$ & & 1358.6 & $1.73 \mathrm{E}-03$ & & 1401.7 & $1.11 \mathrm{E}-03$ \\
\hline & 1312.7 & $4.01 \mathrm{E}-03$ & Drive $215 c$ & 1359.3 & $5.79 \mathrm{E}-03$ & & 1401.9 & $1.23 \mathrm{E}-03$ \\
\hline & 1312.9 & $3.11 \mathrm{E}-03$ & & 1359.65 & $3.41 \mathrm{E}-03$ & & 1402.1 & $1.30 \mathrm{E}-03$ \\
\hline \multirow[t]{7}{*}{ Drive 211} & 1316.3 & $2.58 \mathrm{E}-03$ & & 1360.5 & $3.57 \mathrm{E}-03$ & & 1402.3 & $1.31 \mathrm{E}-03$ \\
\hline & 1316.5 & $3.15 \mathrm{E}-03$ & Drive $216 b$ & 1365.65 & $2.08 \mathrm{E}-03$ & & 1402.5 & $1.39 \mathrm{E}-03$ \\
\hline & 1316.7 & $3.46 \mathrm{E}-03$ & Drive 217 & 1375.3 & 2.26E-03 & & 1402.7 & $1.38 \mathrm{E}-03$ \\
\hline & 1316.9 & $3.89 \mathrm{E}-03$ & & 1375.8 & $2.77 \mathrm{E}-03$ & Drive $219 \mathrm{e}$ & 1403.5 & $3.65 \mathrm{E}-03$ \\
\hline & 1317.1 & $5.04 \mathrm{E}-03$ & & 1376.2 & $2.92 \mathrm{E}-03$ & & 1403.7 & $4.36 \mathrm{E}-03$ \\
\hline & 1317.3 & 4.60E-03 & & 1376.4 & $3.10 \mathrm{E}-03$ & Drive 220 & 1405.3 & $4.20 \mathrm{E}-03$ \\
\hline & 1317.5 & 4.64E-03 & & 1376.6 & $3.46 \mathrm{E}-03$ & & 1405.5 & $4.24 \mathrm{E}-03$ \\
\hline \multirow[t]{8}{*}{ Drive $211 \mathrm{~b}$} & 1318.3 & $2.59 \mathrm{E}-03$ & Drive $217 b$ & 1377.6 & $3.88 \mathrm{E}-03$ & & 1405.7 & $5.00 \mathrm{E}-03$ \\
\hline & 1318.5 & $3.15 \mathrm{E}-03$ & & 1377.9 & 4.13E-03 & & 1405.9 & $4.88 \mathrm{E}-03$ \\
\hline & 1318.7 & $3.45 \mathrm{E}-03$ & & 1378.2 & $3.04 \mathrm{E}-03$ & & 1406.1 & $4.60 \mathrm{E}-03$ \\
\hline & 1318.9 & $3.89 \mathrm{E}-03$ & & 1378.25 & 2.99E-03 & & 1406.3 & 4.24E-03 \\
\hline & 1319.1 & $5.04 \mathrm{E}-03$ & & 1378.45 & $5.04 \mathrm{E}-03$ & & 1406.5 & $3.37 \mathrm{E}-03$ \\
\hline & 1319.3 & $4.88 \mathrm{E}-03$ & & 1378.55 & $6.82 \mathrm{E}-03$ & & 1406.7 & $3.10 \mathrm{E}-03$ \\
\hline & 1319.5 & $4.60 \mathrm{E}-03$ & & 1378.8 & $4.96 \mathrm{E}-03$ & Drive $220 b$ & 1407.7 & $2.24 \mathrm{E}-03$ \\
\hline & 1319.7 & $4.64 \mathrm{E}-03$ & Drive $217 \mathrm{c}$ & 1379.3 & $3.53 \mathrm{E}-03$ & & 1407.9 & $2.26 \mathrm{E}-03$ \\
\hline \multirow[t]{6}{*}{ Drive $211 \mathrm{c}$} & 1320.3 & 2.67E-03 & & 1379.5 & $3.25 \mathrm{E}-03$ & & 1408.1 & 2.47E-03 \\
\hline & 1320.5 & $2.84 \mathrm{E}-03$ & & 1379.7 & $2.64 \mathrm{E}-03$ & & 1408.3 & $2.42 \mathrm{E}-03$ \\
\hline & 1320.7 & 2.84E-03 & & 1379.9 & $1.74 \mathrm{E}-03$ & & 1408.5 & $2.20 \mathrm{E}-03$ \\
\hline & 1320.9 & $3.05 \mathrm{E}-03$ & & 1380.4 & $3.13 \mathrm{E}-03$ & & 1408.7 & $2.11 \mathrm{E}-03$ \\
\hline & 1321.1 & $3.81 \mathrm{E}-03$ & & 1380.6 & $3.71 \mathrm{E}-03$ & Drive 220c & 1409.3 & $2.11 \mathrm{E}-03$ \\
\hline & 1321.3 & $4.76 \mathrm{E}-03$ & Drive $217 \mathrm{~d}$ & 1381.4 & $3.84 \mathrm{E}-03$ & & 1409.5 & $2.19 \mathrm{E}-03$ \\
\hline \multirow[t]{5}{*}{ Drive 211d } & 1322.3 & 2.49E-03 & & 1381.6 & $3.76 \mathrm{E}-03$ & & 1409.7 & $2.11 \mathrm{E}-03$ \\
\hline & 1322.5 & $2.20 \mathrm{E}-03$ & & 1381.8 & $3.71 \mathrm{E}-03$ & & 1409.9 & $2.41 \mathrm{E}-03$ \\
\hline & 1322.9 & $1.87 \mathrm{E}-03$ & & 1382 & $3.61 \mathrm{E}-03$ & & 1410.1 & $2.30 \mathrm{E}-03$ \\
\hline & 1323.1 & 2.17E-03 & & 1382.2 & $3.51 \mathrm{E}-03$ & & 1410.7 & $1.14 \mathrm{E}-03$ \\
\hline & 1323.3 & $2.16 \mathrm{E}-03$ & & 1382.4 & $3.44 \mathrm{E}-03$ & Drive $220 \mathrm{~d}$ & 1411.5 & $1.11 \mathrm{E}-03$ \\
\hline Drive 211 e & 1324.45 & 2.01E-03 & & 1382.6 & $3.24 \mathrm{E}-03$ & & 1411.9 & $1.06 \mathrm{E}-03$ \\
\hline \multirow[t]{2}{*}{ Drive 212} & 1325.54 & $1.65 \mathrm{E}-03$ & Drive $217 \mathrm{e}$ & 1383.3 & $3.75 \mathrm{E}-03$ & & 1412.1 & $1.22 \mathrm{E}-03$ \\
\hline & 1326.08 & $6.66 \mathrm{E}-04$ & & 1383.7 & $2.50 \mathrm{E}-03$ & & 1412.3 & $9.88 \mathrm{E}-04$ \\
\hline \multirow[t]{5}{*}{ Drive $212 b$} & 1326.65 & $3.97 \mathrm{E}-04$ & & 1384.25 & $1.63 \mathrm{E}-03$ & & 1412.5 & $8.69 \mathrm{E}-04$ \\
\hline & 1327.1 & $9.44 \mathrm{E}-04$ & Drive 218 & 1385.3 & $7.58 \mathrm{E}-04$ & & 1412.7 & $8.13 \mathrm{E}-04$ \\
\hline & 1327.4 & $2.14 \mathrm{E}-03$ & & 1385.5 & $9.76 \mathrm{E}-04$ & Drive 221 & 1415.6 & 3.32E-04 \\
\hline & 1327.7 & $3.02 \mathrm{E}-03$ & & 1385.85 & $2.66 \mathrm{E}-03$ & Drive & 1423 & $4.36 \mathrm{E}-04$ \\
\hline & 1328.1 & $3.75 \mathrm{E}-03$ & & 1386.3 & $6.43 \mathrm{E}-03$ & $221 a b$ & & \\
\hline \multirow[t]{3}{*}{ Drive $212 \mathrm{c}$} & 1328.6 & $4.88 \mathrm{E}-03$ & & 1383.5 & $6.23 \mathrm{E}-03$ & Drive & 1429.3 & 4.48E-03 \\
\hline & 1328.85 & $5.95 \mathrm{E}-03$ & & 1386.36 & 4. $32 \mathrm{E}-03$ & $221 b b$ & & \\
\hline & 1329.2 & $5.24 \mathrm{E}-03$ & Drive 219 & 1395.27 & $2.64 \mathrm{E}-03$ & & 1429.5 & 4.72E-03 \\
\hline
\end{tabular}




\begin{tabular}{|c|c|c|c|c|c|}
\hline & 1429.7 & 4.24E-03 & Drive $223 e$ & 1450.3 & $7.54 \mathrm{E}-03$ \\
\hline & 1430.1 & $6.19 \mathrm{E}-03$ & & 1450.59 & $6.03 \mathrm{E}-03$ \\
\hline \multirow[t]{8}{*}{ Drive 222} & 1432.35 & $5.95 \mathrm{E}-03$ & Drive 225 & 1461.75 & $8.53 \mathrm{E}-04$ \\
\hline & 1432.5 & $5.43 \mathrm{E}-03$ & Drive 226 & 1462.45 & $1.05 \mathrm{E}-03$ \\
\hline & 1432.7 & $6.55 \mathrm{E}-03$ & & 1462.72 & $1.16 \mathrm{E}-03$ \\
\hline & 1432.9 & $8.89 \mathrm{E}-03$ & & 1463.1 & $6.51 \mathrm{E}-04$ \\
\hline & 1433.1 & $1.10 \mathrm{E}-02$ & & 1463.2 & $6.70 \mathrm{E}-04$ \\
\hline & 1433.3 & $7.02 \mathrm{E}-03$ & Drive $226 b$ & 1464.5 & $5.99 \mathrm{E}-03$ \\
\hline & 1433.5 & $6.74 \mathrm{E}-03$ & & 1464.7 & $4.56 \mathrm{E}-03$ \\
\hline & 1433.7 & 7.54E-03 & & 1464.9 & $3.68 \mathrm{E}-03$ \\
\hline \multirow[t]{6}{*}{ Drive $222 b$} & 1434.5 & 4.24E-03 & & 1465.1 & 3.19E-03 \\
\hline & 1434.9 & $6.58 \mathrm{E}-03$ & & 1465.3 & $3.17 \mathrm{E}-03$ \\
\hline & 1435.1 & $5.08 \mathrm{E}-03$ & & 1465.5 & 3.83E-03 \\
\hline & 1435.3 & $5.16 \mathrm{E}-03$ & & 1465.7 & $3.90 \mathrm{E}-03$ \\
\hline & 1435.5 & $5.08 \mathrm{E}-03$ & Drive $226 c$ & 1466.3 & $5.24 \mathrm{E}-03$ \\
\hline & 1435.7 & 4.52E-03 & & 1466.5 & $6.82 \mathrm{E}-03$ \\
\hline \multirow[t]{8}{*}{ Drive $222 \mathrm{c}$} & 1436.3 & $4.48 \mathrm{E}-03$ & & 1466.6 & $9.32 \mathrm{E}-03$ \\
\hline & 1436.5 & $5.12 \mathrm{E}-03$ & & 1466.8 & $1.58 \mathrm{E}-02$ \\
\hline & 1436.7 & $5.75 \mathrm{E}-03$ & & 1466.95 & $1.49 \mathrm{E}-02$ \\
\hline & 1436.9 & $7.50 \mathrm{E}-03$ & & 1467.2 & $1.54 \mathrm{E}-02$ \\
\hline & 1437.1 & $6.47 \mathrm{E}-03$ & & 1467.4 & $1.09 \mathrm{E}-02$ \\
\hline & 1437.3 & $5.51 \mathrm{E}-03$ & Drive 227 & 1472.3 & $3.74 \mathrm{E}-03$ \\
\hline & 1437.5 & $4.40 \mathrm{E}-03$ & & 1473.07 & 4.96E-03 \\
\hline & 1437.7 & $4.05 \mathrm{E}-03$ & & 1473.4 & $5.63 \mathrm{E}-03$ \\
\hline \multirow[t]{7}{*}{ Drive $222 \mathrm{~d}$} & 1438.3 & $7.85 \mathrm{E}-03$ & & 1473.7 & $4.05 \mathrm{E}-03$ \\
\hline & 1438.5 & $1.27 \mathrm{E}-02$ & Drive $227 b$ & 1474.3 & $3.74 \mathrm{E}-03$ \\
\hline & 1438.7 & $1.08 \mathrm{E}-02$ & & 1474.5 & 4.24E-03 \\
\hline & 1438.9 & 1.19E-02 & & 1474.9 & 4.44E-03 \\
\hline & 1439.1 & $7.02 \mathrm{E}-03$ & & 1475.1 & 4.40E-03 \\
\hline & 1439.3 & 4.48E-03 & & 1475.65 & 3.67E-03 \\
\hline & 1439.6 & $5.67 \mathrm{E}-03$ & Drive $227 \mathrm{c}$ & 1476.9 & 4.48E-03 \\
\hline \multirow[t]{4}{*}{ Drive $222 \mathrm{e}$} & 1440.4 & 3.37E-03 & & 1477.5 & 4.32E-03 \\
\hline & 1440.8 & $6.35 \mathrm{E}-03$ & & 1478.1 & $2.65 \mathrm{E}-03$ \\
\hline & 1441.18 & $6.07 \mathrm{E}-03$ & Drive 228 & 1482.5 & $1.36 \mathrm{E}-03$ \\
\hline & 1448.5 & 4.72E-03 & & 1482.83 & $1.69 \mathrm{E}-03$ \\
\hline \multirow[t]{7}{*}{ Drive 223} & 1442.3 & $2.93 \mathrm{E}-03$ & Drive $228 b$ & 1484.45 & $1.49 \mathrm{E}-03$ \\
\hline & 1442.5 & $2.92 \mathrm{E}-03$ & & 1484.55 & $1.93 \mathrm{E}-03$ \\
\hline & 1442.7 & $3.27 \mathrm{E}-03$ & & 1485.65 & 2.05E-03 \\
\hline & 1443.1 & $3.53 \mathrm{E}-03$ & & 1485.75 & $2.21 \mathrm{E}-03$ \\
\hline & 1443.3 & $3.56 \mathrm{E}-03$ & Drive $228 c$ & 1486.25 & $2.87 \mathrm{E}-03$ \\
\hline & 1443.5 & $2.75 \mathrm{E}-03$ & & 1486.7 & $2.46 \mathrm{E}-03$ \\
\hline & 1443.7 & $2.63 \mathrm{E}-03$ & Drive 229 & 1493.2 & $1.32 \mathrm{E}-03$ \\
\hline \multirow[t]{7}{*}{ Drive 223b } & 1444.3 & $3.83 \mathrm{E}-03$ & & 1493.4 & $1.36 \mathrm{E}-03$ \\
\hline & 1444.5 & $4.84 \mathrm{E}-03$ & Drive $229 b$ & 1494.6 & $1.57 \mathrm{E}-03$ \\
\hline & 1444.7 & $4.52 \mathrm{E}-03$ & & 1496.22 & $1.61 \mathrm{E}-03$ \\
\hline & 1445.1 & $7.50 \mathrm{E}-03$ & & & \\
\hline & 1445.3 & $1.55 \mathrm{E}-02$ & & & \\
\hline & 1445.5 & 7.02E-03 & & & \\
\hline & 1445.7 & 4.24E-03 & & & \\
\hline \multirow[t]{6}{*}{ Drive $223 c$} & 1446.3 & $3.86 \mathrm{E}-03$ & & & \\
\hline & 1446.7 & $3.78 \mathrm{E}-03$ & & & \\
\hline & 1446.9 & 3.84E-03 & & & \\
\hline & 1447.3 & $4.24 \mathrm{E}-03$ & & & \\
\hline & 1447.5 & 4.01E-03 & & & \\
\hline & 1447.7 & 4.24E-03 & & & \\
\hline \multirow[t]{5}{*}{ Drive $223 d$} & 1448.3 & $5.43 \mathrm{E}-03$ & & & \\
\hline & 1448.5 & 3.67E-03 & & & \\
\hline & 1448.7 & $3.34 \mathrm{E}-03$ & & & \\
\hline & 1448.9 & 4.64E-03 & & & \\
\hline & 1449.8 & $5.91 \mathrm{E}-03$ & & & \\
\hline
\end{tabular}




\section{Appendix C, Magnetic property data for 98th Street core samples}

Note: Depth, in $\mathrm{ft}$; MS, magnetic susceptibility (SI volume); NRM, natural remanent magnetization (A/m); Q, Koenigsberger ratio; Total mag., calculated total magnetization $(\mathrm{A} / \mathrm{m})$; Polarity, magnetic polarity where $\mathrm{N}, \mathrm{R}$, and I are normal, reversed, and intermediate, respectively, having A (best), B, or C (worst) quality; Size, estimated sediment grain size for most samples where 2 is coarse sand, 3 is medium sand, 4 is fine sand, 5 is silty, fine sand, 6 is clayey, silty, fine sand, 7 is silt, 8 is silty clay, and 9 is clay.

\begin{tabular}{|c|c|c|c|c|c|c|c|}
\hline Depth & Sample & MS & NRM & $\mathbf{Q}$ & Total mag. & Polarity & Size \\
\hline 25.7 & $13 \mathrm{~B}$ & & $3.30 \mathrm{E}-02$ & & & N-B & \\
\hline 26.8 & $14 \mathrm{~B}$ & $6.14 \mathrm{E}-03$ & $3.14 \mathrm{E}-02$ & 0.13 & $2.74 \mathrm{E}-01$ & N-B & 4 \\
\hline 38.1 & $15 B$ & $1.02 \mathrm{E}-02$ & $6.34 \mathrm{E}-02$ & 0.16 & 4.67E-01 & N-B & 4 \\
\hline 44.8 & $16 \mathrm{~B}$ & $1.46 \mathrm{E}-02$ & $1.21 \mathrm{E}-01$ & 0.21 & $6.98 \mathrm{E}-01$ & $\mathrm{~N}-\mathrm{A}$ & 3.7 \\
\hline 46.5 & $17 \mathrm{~B}$ & $9.68 \mathrm{E}-03$ & $1.11 \mathrm{E}-01$ & 0.29 & $4.94 \mathrm{E}-01$ & N-A & 4 \\
\hline 47.8 & $18 \mathrm{~B}$ & 1. $15 \mathrm{E}-02$ & $1.56 \mathrm{E}-01$ & 0.34 & $6.11 \mathrm{E}-01$ & $\mathrm{~N}-\mathrm{A}$ & 3.7 \\
\hline 49.8 & $19 \mathrm{~B}$ & $5.59 \mathrm{E}-03$ & $6.02 \mathrm{E}-02$ & 0.27 & $2.81 \mathrm{E}-01$ & N-A & 3 \\
\hline 81.1 & $20 \mathrm{~B}$ & 4.64E-03 & $1.94 \mathrm{E}-02$ & 0.11 & $2.03 \mathrm{E}-01$ & N-B & 5 \\
\hline 87.8 & $1 \mathrm{~A}$ & 4.73E-04 & $6.28 \mathrm{E}-02$ & 3.36 & $8.15 \mathrm{E}-02$ & $\mathrm{~N}-\mathrm{C}$ & 4 \\
\hline 93.1 & $2 \mathrm{C}$ & $3.68 \mathrm{E}-04$ & $3.37 \mathrm{E}-02$ & 2.32 & $4.82 \mathrm{E}-02$ & N-B & 8 \\
\hline 96.7 & $2 \mathrm{~A}$ & $1.95 \mathrm{E}-04$ & $3.66 \mathrm{E}-02$ & 4.75 & $4.43 \mathrm{E}-02$ & $\mathrm{~N}-\mathrm{A}$ & 4 \\
\hline 99.3 & $2 \mathrm{~B}$ & $2.50 \mathrm{E}-04$ & $3.40 \mathrm{E}-02$ & 3.44 & 4.39E-02 & $\mathrm{N}-\mathrm{A}$ & 8 \\
\hline 103.4 & $3 \mathrm{~A}$ & $1.26 \mathrm{E}-04$ & $2.15 \mathrm{E}-02$ & 4.31 & $2.65 \mathrm{E}-02$ & $\mathrm{~N}-\mathrm{A}$ & 8 \\
\hline 104.6 & $4 \mathrm{~A}$ & & $3.49 \mathrm{E}-02$ & & & $\mathrm{~N}-\mathrm{A}$ & \\
\hline 106.2 & $5 \mathrm{~A}$ & $4.23 \mathrm{E}-04$ & $6.59 \mathrm{E}-02$ & 3.94 & $8.26 \mathrm{E}-02$ & N-A & 6 \\
\hline 111 & $6 \mathrm{~A}$ & $1.11 \mathrm{E}-04$ & $1.09 \mathrm{E}-02$ & 2.49 & $1.53 \mathrm{E}-02$ & N-B & 6 \\
\hline 114.7 & $6 \mathrm{~B}$ & $1.36 \mathrm{E}-03$ & $2.19 \mathrm{E}-02$ & 0.41 & $7.56 \mathrm{E}-02$ & N-B & 7.5 \\
\hline 117.7 & $7 \mathrm{~A}$ & 3.77E-04 & $4.92 \mathrm{E}-02$ & 3.30 & $6.41 \mathrm{E}-02$ & $\mathrm{~N}-\mathrm{C}$ & 4 \\
\hline 120.9 & $8 \mathrm{~A}$ & $1.19 \mathrm{E}-04$ & $5.51 \mathrm{E}-03$ & 1.17 & $-8.18 \mathrm{E}-04$ & $\mathrm{R}-\mathrm{C}$ & 8 \\
\hline 124.2 & $500 \mathrm{~A}$ & & 4.37E-03 & & & R-A & \\
\hline 125 & $8 \mathrm{~B}$ & & $8.35 \mathrm{E}-03$ & & & $\mathrm{R}-\mathrm{C}$ & \\
\hline 126.1 & $8 \mathrm{C}$ & $6.16 \mathrm{E}-05$ & $1.07 \mathrm{E}-02$ & 4.39 & $1.31 \mathrm{E}-02$ & $\mathrm{~N}-\mathrm{B}$ & 9 \\
\hline 128.7 & $501 \mathrm{~A}$ & & $2.11 \mathrm{E}-02$ & & & $\mathrm{~N}-\mathrm{C}$ & \\
\hline 130.7 & $502 \mathrm{~A}$ & & $2.38 \mathrm{E}-02$ & & & $\mathrm{~N}-\mathrm{B}$ & \\
\hline 131.3 & $503 \mathrm{~A}$ & & $2.87 \mathrm{E}-02$ & & & $\mathrm{~N}-\mathrm{B}$ & \\
\hline 133.4 & $9 \mathrm{~A}$ & 5.37E-04 & $6.86 \mathrm{E}-02$ & 3.23 & 8.99E-02 & $\mathrm{N}-\mathrm{A}$ & 4 \\
\hline 135.9 & $10 \mathrm{~A}$ & $2.26 \mathrm{E}-03$ & $9.31 \mathrm{E}-03$ & 0.10 & $8.02 \mathrm{E}-02$ & $\mathrm{R}-\mathrm{C}$ & 6.5 \\
\hline 141 & $10 \mathrm{~B}$ & & 2.94E-02 & & & N-B & \\
\hline 143 & $800 \mathrm{~A}$ & $3.94 \mathrm{E}-04$ & $3.26 \mathrm{E}-02$ & 2.09 & $4.82 \mathrm{E}-02$ & $\mathrm{~N}-\mathrm{C}$ & 3 \\
\hline 146.5 & $801 \mathrm{~A}$ & $5.04 \mathrm{E}-04$ & $4.05 \mathrm{E}-02$ & 2.03 & $-2.06 \mathrm{E}-02$ & $\mathrm{R}-\mathrm{C}$ & 4 \\
\hline 146.6 & $802 \mathrm{~A}$ & $3.60 \mathrm{E}-04$ & $1.80 \mathrm{E}-02$ & 1.26 & $-3.77 \mathrm{E}-03$ & R-B & 4 \\
\hline 147.1 & $11 \mathrm{~A}$ & $1.67 \mathrm{E}-04$ & $5.13 \mathrm{E}-02$ & 7.79 & $-4.47 \mathrm{E}-02$ & $\mathrm{R}-\mathrm{A}$ & 8 \\
\hline 147.4 & $803 \mathrm{~A}$ & $2.61 \mathrm{E}-04$ & $1.80 \mathrm{E}-02$ & 1.74 & $2.83 \mathrm{E}-02$ & $\mathrm{~N}-\mathrm{B}$ & 5 \\
\hline 148.3 & $804 \mathrm{~A}$ & $2.42 \mathrm{E}-04$ & $2.87 \mathrm{E}-02$ & 3.00 & $-1.91 \mathrm{E}-02$ & R-A & 5 \\
\hline 149.3 & $12 \mathrm{~A}$ & $1.22 \mathrm{E}-04$ & $1.61 \mathrm{E}-02$ & 3.33 & $2.09 \mathrm{E}-02$ & $\mathrm{~N}-\mathrm{B}$ & 8 \\
\hline 150.4 & $13 \mathrm{~A}$ & $1.76 \mathrm{E}-03$ & $6.69 \mathrm{E}-02$ & 0.96 & $1.37 \mathrm{E}-01$ & $\mathrm{~N}-\mathrm{C}$ & 5 \\
\hline 159.9 & $14 \mathrm{~A}$ & $5.28 \mathrm{E}-04$ & $8.03 \mathrm{E}-02$ & 3.85 & $1.01 \mathrm{E}-01$ & $\mathrm{~N}-\mathrm{C}$ & 3.5 \\
\hline 166.1 & $15 \mathrm{~A}$ & 4.16E-04 & $5.67 \mathrm{E}-02$ & 3.44 & 7.32E-02 & $\mathrm{N}-\mathrm{C}$ & 5 \\
\hline 167.2 & $805 \mathrm{~A}$ & $2.45 \mathrm{E}-04$ & $2.28 \mathrm{E}-02$ & 2.35 & $3.25 \mathrm{E}-02$ & $\mathrm{~N}-\mathrm{A}$ & 5 \\
\hline 167.8 & $16 \mathrm{~A}$ & & $207 \mathrm{E}-02$ & & & $\mathrm{R}-\mathrm{A}$ & \\
\hline
\end{tabular}




\begin{tabular}{|c|c|c|c|c|c|c|c|}
\hline 168 & $806 \mathrm{~A}$ & 2.29E-04 & $2.07 \mathrm{E}-01$ & 22.82 & $-1.98 \mathrm{E}-01$ & R-A & 6 \\
\hline 172.9 & $17 \mathrm{~A}$ & $2.72 \mathrm{E}-04$ & $1.32 \mathrm{E}-02$ & 1.23 & $-2.45 \mathrm{E}-03$ & $\mathrm{R}-\mathrm{C}$ & 5 \\
\hline 173.7 & $807 \mathrm{~A}$ & 4.10E-04 & $2.69 \mathrm{E}-02$ & 1.66 & $4.31 \mathrm{E}-02$ & N-B & 4 \\
\hline 176.5 & $18 \mathrm{~A}$ & $3.94 \mathrm{E}-03$ & $1.79 \mathrm{E}-02$ & 0.11 & $1.74 \mathrm{E}-01$ & $\mathrm{~N}-\mathrm{C}$ & 4 \\
\hline 179.7 & $19 \mathrm{~A}$ & $2.56 \mathrm{E}-04$ & $9.41 \mathrm{E}-02$ & 9.29 & $1.04 \mathrm{E}-01$ & $\mathrm{~N}-\mathrm{A}$ & 6 \\
\hline 180.6 & $20 \mathrm{~A}$ & $9.11 \mathrm{E}-04$ & $1.33 \mathrm{E}-02$ & 0.37 & 2.27E-02 & $\mathrm{R}-\mathrm{A}$ & 9 \\
\hline 180.9 & $303 \mathrm{~A}$ & & $3.81 \mathrm{E}-02$ & & & N-B & \\
\hline 182.5 & $302 \mathrm{~A}$ & 1.17E-03 & $8.00 \mathrm{E}-03$ & 0.17 & $5.41 \mathrm{E}-02$ & $\mathrm{R}-\mathrm{C}$ & 8.5 \\
\hline 192.4 & $21 \mathrm{~A}$ & & 1.13E-01 & & & $\mathrm{N}-\mathrm{C}$ & \\
\hline 201 & $304 \mathrm{~A}$ & $5.78 \mathrm{E}-03$ & $2.20 \mathrm{E}-02$ & 0.10 & $2.51 \mathrm{E}-01$ & $\mathrm{~N}-\mathrm{C}$ & 4 \\
\hline 201.3 & $305 \mathrm{~A}$ & $4.46 \mathrm{E}-03$ & 7.74E-03 & 0.04 & $1.84 \mathrm{E}-01$ & $\mathrm{~N}-\mathrm{C}$ & 4 \\
\hline 201.7 & $306 \mathrm{~A}$ & $3.28 \mathrm{E}-03$ & $1.53 \mathrm{E}-02$ & 0.12 & $1.45 \mathrm{E}-01$ & $\mathrm{~N}-\mathrm{C}$ & 6 \\
\hline 203.5 & $22 \mathrm{~A}$ & 6.74E-04 & $1.00 \mathrm{E}-01$ & 3.75 & $1.27 \mathrm{E}-01$ & $\mathrm{~N}-\mathrm{C}$ & 4 \\
\hline 206 & $23 \mathrm{~A}$ & & $2.93 \mathrm{E}-02$ & & & N-A & \\
\hline 208.4 & $24 \mathrm{~A}$ & $1.18 \mathrm{E}-04$ & $4.66 \mathrm{E}-02$ & 10.01 & $5.13 \mathrm{E}-02$ & N-A & 9 \\
\hline 212.4 & $25 \mathrm{~A}$ & $1.43 \mathrm{E}-03$ & $1.90 \mathrm{E}-02$ & 0.34 & $7.57 \mathrm{E}-02$ & N-A & 8 \\
\hline 221.5 & $26 \mathrm{~A}$ & 4.76E-03 & 7.77E-02 & 0.41 & $2.66 \mathrm{E}-01$ & $\mathrm{~N}-\mathrm{C}$ & 4 \\
\hline 223.5 & $27 \mathrm{~A}$ & & $4.60 \mathrm{E}-02$ & & & N-B & \\
\hline 236.5 & $28 \mathrm{~A}$ & $1.28 \mathrm{E}-03$ & 2.61E-02 & 0.52 & 7.67E-02 & $\mathrm{N}-\mathrm{C}$ & 9 \\
\hline 239.1 & $29 \mathrm{~A}$ & & $2.13 \mathrm{E}-02$ & & & $\mathrm{~N}-\mathrm{C}$ & \\
\hline 245.6 & $30 \mathrm{~A}$ & 4.49E-03 & $2.52 \mathrm{E}-01$ & 1.42 & $4.30 \mathrm{E}-01$ & $\mathrm{~N}-\mathrm{C}$ & 4 \\
\hline 249.8 & $31 \mathrm{~A}$ & 1.39E-03 & $1.06 \mathrm{E}-02$ & 0.19 & $4.45 \mathrm{E}-02$ & R-B & 9 \\
\hline 251.1 & $32 \mathrm{~A}$ & 1.12E-03 & $1.07 \mathrm{E}-02$ & 0.24 & 3.37E-02 & R-A & 9 \\
\hline 253 & $33 \mathrm{~A}$ & $5.28 \mathrm{E}-04$ & 8. $10 \mathrm{E}-03$ & 0.39 & $1.28 \mathrm{E}-02$ & R-A & 9 \\
\hline 253.7 & $504 \mathrm{~A}$ & & $1.08 \mathrm{E}-02$ & & & I-A & \\
\hline 254.6 & $504 \mathrm{~B}$ & & $3.23 \mathrm{E}-02$ & & & R-A & \\
\hline 255.1 & $504 C$ & & $3.20 \mathrm{E}-03$ & & & R-B & \\
\hline 255.2 & $34 \mathrm{~A}$ & $6.10 \mathrm{E}-04$ & $1.08 \mathrm{E}-02$ & 0.45 & $3.49 \mathrm{E}-02$ & $\mathrm{~N}-\mathrm{A}$ & 9 \\
\hline 257.3 & $506 \mathrm{~A}$ & & $2.48 \mathrm{E}-03$ & & & $\mathrm{R}-\mathrm{C}$ & \\
\hline 265 & $35 \mathrm{~B}$ & & $4.75 \mathrm{E}-02$ & & & R-A & \\
\hline 265.3 & $35 \mathrm{C}$ & $1.05 \mathrm{E}-03$ & 8.37E-04 & 0.02 & $4.08 \mathrm{E}-02$ & $R-B$ & 8 \\
\hline 270.2 & $36 \mathrm{~A}$ & $9.92 \mathrm{E}-04$ & $2.59 \mathrm{E}-03$ & 0.07 & $3.66 \mathrm{E}-02$ & R-B & 8.5 \\
\hline 274.5 & $37 \mathrm{~A}$ & & $3.55 \mathrm{E}-02$ & & & $\mathrm{~N}-\mathrm{C}$ & \\
\hline 278.6 & $38 \mathrm{~A}$ & 1.37E-03 & 7.98E-03 & 0.15 & $6.21 \mathrm{E}-02$ & $\mathrm{~N}-\mathrm{C}$ & 8.5 \\
\hline 279.2 & $39 \mathrm{~A}$ & $2.37 \mathrm{E}-03$ & $1.54 \mathrm{E}-02$ & 0.16 & $1.09 \mathrm{E}-01$ & N-B & 8 \\
\hline 283.4 & $40 \mathrm{~A}$ & $1.82 \mathrm{E}-03$ & $1.24 \mathrm{E}-02$ & 0.17 & $5.97 \mathrm{E}-02$ & $\mathrm{R}-\mathrm{C}$ & 5 \\
\hline 291.3 & $41 \mathrm{~A}$ & $2.15 \mathrm{E}-03$ & $2.65 \mathrm{E}-02$ & 0.31 & $5.86 \mathrm{E}-02$ & $\mathrm{R}-\mathrm{B}$ & 2.4 \\
\hline 292.2 & $42 \mathrm{~A}$ & $2.67 \mathrm{E}-03$ & $1.15 \mathrm{E}-02$ & 0.11 & 1.17E-01 & $\mathrm{N}-\mathrm{C}$ & 4 \\
\hline 304.7 & $51 \mathrm{~B}$ & & $2.55 \mathrm{E}-03$ & & & R-B & \\
\hline 313.9 & $44 \mathrm{~A}$ & $1.95 \mathrm{E}-03$ & $6.68 \mathrm{E}-02$ & 0.87 & $1.03 \mathrm{E}-02$ & $\mathrm{R}-\mathrm{C}$ & 5 \\
\hline 315.3 & $504 \mathrm{D}$ & & $6.15 \mathrm{E}-03$ & & & $\mathrm{~N}-\mathrm{C}$ & \\
\hline 315.4 & $505 \mathrm{~A}$ & & $6.98 \mathrm{E}-03$ & & & R-B & \\
\hline 315.6 & $506 \mathrm{~B}$ & & 1. $14 \mathrm{E}-02$ & & & $\mathrm{R}-\mathrm{C}$ & \\
\hline 315.7 & $507 \mathrm{~A}$ & & $1.17 \mathrm{E}-02$ & & & $R-B$ & \\
\hline 315.8 & $508 \mathrm{~A}$ & & $5.09 \mathrm{E}-03$ & & & N-B & \\
\hline 315.9 & $509 A$ & & $3.77 \mathrm{E}-03$ & & & $\mathrm{R}-\mathrm{C}$ & \\
\hline 316.1 & $510 \mathrm{~A}$ & & $4.40 \mathrm{E}-03$ & & & N-B & \\
\hline 317.6 & $511 \mathrm{~A}$ & & $1.00 \mathrm{E}-02$ & & & N-B & \\
\hline 318.4 & $512 \mathrm{~A}$ & & $1.42 \mathrm{E}-02$ & & & N-B & \\
\hline 319.8 & $513 \mathrm{~A}$ & & $1.21 \mathrm{E}-02$ & & & N-B & \\
\hline 325 & $45 \mathrm{~A}$ & & $3.54 \mathrm{E}-03$ & & & R-B & \\
\hline 325.8 & $46 \mathrm{~A}$ & $6.51 \mathrm{E}-04$ & 4.93E-03 & 0.19 & $2.08 \mathrm{E}-02$ & $R-A$ & 8 \\
\hline 333.2 & $47 \mathrm{~A}$ & $5.25 \mathrm{E}-04$ & $2.02 \mathrm{E}-03$ & 0.10 & $1.87 \mathrm{E}-02$ & $R-B$ & 9 \\
\hline 336.1 & $48 \mathrm{~A}$ & $1.51 \mathrm{E}-03$ & $1.27 \mathrm{E}-02$ & 0.21 & 7.24E-02 & $\mathrm{N}-\mathrm{C}$ & 9 \\
\hline
\end{tabular}




\begin{tabular}{|c|c|c|c|c|c|c|c|}
\hline 337.2 & $700 \mathrm{~A}$ & & $5.22 \mathrm{E}-02$ & & & N-B & \\
\hline 347.4 & $701 \mathrm{~A}$ & $1.93 \mathrm{E}-04$ & $5.17 \mathrm{E}-02$ & 6.76 & $5.93 \mathrm{E}-02$ & N-B & 4 \\
\hline 349.1 & $702 \mathrm{~A}$ & $1.74 \mathrm{E}-04$ & $4.67 \mathrm{E}-02$ & 6.77 & $5.36 \mathrm{E}-02$ & N-B & 4 \\
\hline 351 & $703 \mathrm{~A}$ & $3.72 \mathrm{E}-04$ & 7.65E-02 & 5.19 & $9.12 \mathrm{E}-02$ & N-A & 4 \\
\hline 357.9 & 49A & & $2.41 \mathrm{E}-02$ & & & N-B & \\
\hline 359.7 & $50 \mathrm{~A}$ & 1.19E-03 & $3.50 \mathrm{E}-02$ & 0.74 & $8.21 \mathrm{E}-02$ & $\mathrm{~N}-\mathrm{A}$ & 6 \\
\hline 360 & $51 \mathrm{~A}$ & 8.88E-04 & 2.34E-02 & 0.67 & 5.85E-02 & N-A & 4 \\
\hline 362.7 & $704 \mathrm{~A}$ & 2.39E-04 & 1.18E-01 & 12.49 & $1.27 \mathrm{E}-01$ & $\mathrm{~N}-\mathrm{A}$ & 4 \\
\hline 371.3 & $52 \mathrm{~A}$ & $3.98 \mathrm{E}-03$ & $6.60 \mathrm{E}-02$ & 0.42 & $2.23 \mathrm{E}-01$ & N-A & 4 \\
\hline 375 & $300 \mathrm{~A}$ & $3.83 \mathrm{E}-03$ & $4.63 \mathrm{E}-02$ & 0.31 & $1.05 \mathrm{E}-01$ & $\mathrm{R}-\mathrm{A}$ & 5 \\
\hline 375.9 & $301 \mathrm{~A}$ & $3.71 \mathrm{E}-03$ & $4.55 \mathrm{E}-02$ & 0.31 & $1.01 \mathrm{E}-01$ & $\mathrm{R}-\mathrm{A}$ & 4.5 \\
\hline 379.6 & $53 \mathrm{~A}$ & & $3.97 \mathrm{E}-02$ & & & N-A & \\
\hline 394.9 & $54 \mathrm{~A}$ & & $6.34 \mathrm{E}-02$ & & & $\mathrm{~N}-\mathrm{A}$ & \\
\hline 398.4 & $55 \mathrm{~A}$ & & $5.58 \mathrm{E}-02$ & & & N-A & \\
\hline 403.2 & $56 \mathrm{~A}$ & $1.54 \mathrm{E}-03$ & 2. $10 \mathrm{E}-02$ & 0.34 & $8.21 \mathrm{E}-02$ & $\mathrm{~N}-\mathrm{A}$ & 6 \\
\hline 403.9 & $57 \mathrm{~A}$ & $2.06 \mathrm{E}-03$ & $2.34 \mathrm{E}-02$ & 0.29 & $1.05 \mathrm{E}-01$ & N-A & 6 \\
\hline 406.4 & $58 \mathrm{~A}$ & & 7.32E-02 & & & N-B & \\
\hline 409.1 & $59 \mathrm{~A}$ & $1.76 \mathrm{E}-03$ & $2.53 \mathrm{E}-02$ & 0.36 & $9.49 \mathrm{E}-02$ & N-A & 6 \\
\hline 410.8 & $60 \mathrm{~A}$ & & $3.46 \mathrm{E}-02$ & & & $\mathrm{~N}-\mathrm{C}$ & \\
\hline 418.5 & $706 \mathrm{~A}$ & $3.10 \mathrm{E}-04$ & $4.60 \mathrm{E}-02$ & 3.76 & $5.82 \mathrm{E}-02$ & N-B & 3.5 \\
\hline 419.5 & $705 \mathrm{~A}$ & & $1.23 \mathrm{E}-01$ & & & N-A & \\
\hline 428.1 & $707 \mathrm{~A}$ & $3.21 \mathrm{E}-04$ & $9.52 \mathrm{E}-02$ & 7.51 & $1.08 \mathrm{E}-01$ & N-B & 3.5 \\
\hline 429.7 & $708 \mathrm{~A}$ & $3.41 \mathrm{E}-04$ & $2.23 \mathrm{E}-01$ & 16.54 & $2.36 \mathrm{E}-01$ & N-B & 4 \\
\hline 431.5 & $709 \mathrm{~A}$ & $2.51 \mathrm{E}-04$ & $8.72 \mathrm{E}-02$ & 8.77 & $9.71 \mathrm{E}-02$ & N-B & 4 \\
\hline 433 & $710 \mathrm{~A}$ & $3.13 \mathrm{E}-04$ & $1.09 \mathrm{E}-01$ & 8.81 & $1.21 \mathrm{E}-01$ & $\mathrm{~N}-\mathrm{C}$ & 5 \\
\hline 436.3 & $61 \mathrm{~A}$ & $1.06 \mathrm{E}-04$ & $5.23 \mathrm{E}-03$ & 1.24 & $9.44 \mathrm{E}-03$ & $\mathrm{~N}-\mathrm{A}$ & 9 \\
\hline 437.1 & $62 \mathrm{~A}$ & $6.77 \mathrm{E}-04$ & 3.19E-03 & 0.12 & $3.00 \mathrm{E}-02$ & N-B & 8 \\
\hline 441.3 & $63 \mathrm{~A}$ & $1.50 \mathrm{E}-03$ & $2.66 \mathrm{E}-02$ & 0.45 & 8.58E-02 & $\mathrm{N}-\mathrm{A}$ & 9 \\
\hline 442.8 & $64 \mathrm{~A}$ & $9.68 \mathrm{E}-05$ & $1.12 \mathrm{E}-02$ & 2.93 & $1.50 \mathrm{E}-02$ & N-B & 9 \\
\hline 444 & $65 \mathrm{~A}$ & $1.10 \mathrm{E}-04$ & $2.06 \mathrm{E}-02$ & 4.72 & $2.50 \mathrm{E}-02$ & N-A & 9 \\
\hline 446.7 & $66 \mathrm{~A}$ & $9.54 \mathrm{E}-05$ & $1.54 \mathrm{E}-02$ & 4.08 & $1.92 \mathrm{E}-02$ & N-B & 9 \\
\hline 457 & $67 \mathrm{~A}$ & $6.25 \mathrm{E}-04$ & & & & & 9 \\
\hline 457.6 & $68 \mathrm{~A}$ & 7.62E-04 & $5.20 \mathrm{E}-03$ & 0.17 & $3.53 \mathrm{E}-02$ & N-B & 9 \\
\hline 458.6 & $69 \mathrm{~A}$ & $2.67 \mathrm{E}-04$ & $9.98 \mathrm{E}-02$ & 9.45 & 1. $10 \mathrm{E}-01$ & $\mathrm{~N}-\mathrm{A}$ & 5 \\
\hline 461.9 & $70 \mathrm{~A}$ & $8.96 \mathrm{E}-05$ & $3.16 \mathrm{E}-02$ & 8.92 & $3.51 \mathrm{E}-02$ & $\mathrm{~N}-\mathrm{A}$ & 5 \\
\hline 463 & $71 \mathrm{~A}$ & $3.27 \mathrm{E}-05$ & $9.75 \mathrm{E}-03$ & 7.54 & $1.10 \mathrm{E}-02$ & $\mathrm{~N}-\mathrm{A}$ & 9 \\
\hline 464 & $72 \mathrm{~A}$ & $7.31 \mathrm{E}-05$ & $7.71 \mathrm{E}-03$ & 2.67 & $1.06 \mathrm{E}-02$ & N-B & 9 \\
\hline 466.9 & $73 \mathrm{~A}$ & $2.07 \mathrm{E}-04$ & $4.66 \mathrm{E}-02$ & 5.68 & $5.48 \mathrm{E}-02$ & $\mathrm{~N}-\mathrm{A}$ & 5 \\
\hline 474.4 & $74 \mathrm{~A}$ & $9.68 \mathrm{E}-05$ & $2.19 \mathrm{E}-02$ & 5.72 & $2.57 \mathrm{E}-02$ & $\mathrm{~N}-\mathrm{A}$ & 4 \\
\hline 475.2 & $75 \mathrm{~A}$ & & $1.21 \mathrm{E}-02$ & & & N-B & \\
\hline 485.2 & $76 \mathrm{~A}$ & $1.52 \mathrm{E}-04$ & $4.66 \mathrm{E}-02$ & 7.76 & $5.26 \mathrm{E}-02$ & N-B & 4 \\
\hline 489.4 & $77 \mathrm{~A}$ & $2.00 \mathrm{E}-04$ & $3.50 \mathrm{E}-02$ & 4.43 & 4.29E-02 & N-A & 5 \\
\hline 490.1 & $78 \mathrm{~A}$ & $1.35 \mathrm{E}-04$ & $4.58 \mathrm{E}-02$ & 8.58 & $5.11 \mathrm{E}-02$ & $\mathrm{~N}-\mathrm{A}$ & 9 \\
\hline 495.5 & $80 \mathrm{~A}$ & $5.23 \mathrm{E}-05$ & $1.67 \mathrm{E}-02$ & 8.07 & $1.88 \mathrm{E}-02$ & $\mathrm{~N}-\mathrm{A}$ & 9 \\
\hline 499.7 & $81 \mathrm{~A}$ & $6.87 \mathrm{E}-05$ & $1.41 \mathrm{E}-02$ & 5.19 & $1.68 \mathrm{E}-02$ & $\mathrm{~N}-\mathrm{A}$ & 8 \\
\hline 506.6 & $82 \mathrm{~A}$ & $7.73 \mathrm{E}-05$ & $2.64 \mathrm{E}-02$ & 8.63 & $2.95 \mathrm{E}-02$ & $\mathrm{~N}-\mathrm{A}$ & 8 \\
\hline 507.8 & $83 \mathrm{~A}$ & $1.71 \mathrm{E}-03$ & $3.16 \mathrm{E}-02$ & 0.47 & $9.93 \mathrm{E}-02$ & $\mathrm{~N}-\mathrm{A}$ & 9 \\
\hline 512 & $84 \mathrm{~A}$ & $1.46 \mathrm{E}-03$ & $2.83 \mathrm{E}-02$ & 0.49 & $8.58 \mathrm{E}-02$ & N-A & 9 \\
\hline 519.2 & $85 \mathrm{~A}$ & & $1.97 \mathrm{E}-02$ & & & $\mathrm{~N}-\mathrm{A}$ & \\
\hline 520.2 & $86 \mathrm{~A}$ & $3.61 \mathrm{E}-03$ & $6.97 \mathrm{E}-02$ & 0.49 & 2. $13 \mathrm{E}-01$ & N-B & 5 \\
\hline 530.2 & $87 \mathrm{~A}$ & $4.76 \mathrm{E}-05$ & 1.01E-02 & 5.36 & $1.20 \mathrm{E}-02$ & N-A & 8.5 \\
\hline 535.2 & $87.5 \mathrm{~A}$ & $8.78 \mathrm{E}-05$ & $1.83 \mathrm{E}-02$ & 5.27 & 2. $18 \mathrm{E}-02$ & N-B & 6 \\
\hline 535.9 & $88 \mathrm{~A}$ & $9.22 \mathrm{E}-04$ & $1.17 \mathrm{E}-02$ & 0.32 & 4. $82 \mathrm{E}-02$ & N-B & 6 \\
\hline 540.2 & $89 \mathrm{~A}$ & $5.58 \mathrm{E}-05$ & $2.77 \mathrm{E}-02$ & 12.56 & $2.99 \mathrm{E}-02$ & N-A & 8.5 \\
\hline
\end{tabular}




\begin{tabular}{|c|c|c|c|c|c|c|c|}
\hline 541 & $90 \mathrm{~A}$ & $1.82 \mathrm{E}-03$ & $2.69 \mathrm{E}-02$ & 0.37 & $9.90 \mathrm{E}-02$ & N-B & 7.5 \\
\hline 544.9 & $91 \mathrm{~A}$ & $9.30 \mathrm{E}-05$ & $4.54 \mathrm{E}-02$ & 12.34 & $4.91 \mathrm{E}-02$ & $\mathrm{~N}-\mathrm{A}$ & 7.5 \\
\hline 545.6 & $92 \mathrm{~A}$ & 1.19E-04 & $1.25 \mathrm{E}-02$ & 2.66 & $1.72 \mathrm{E}-02$ & N-B & 6.5 \\
\hline 550.5 & $93 \mathrm{~A}$ & $4.87 \mathrm{E}-04$ & & & & & 9 \\
\hline 551.7 & $94 \mathrm{~A}$ & $4.13 \mathrm{E}-05$ & $8.77 \mathrm{E}-03$ & 5.37 & $1.04 \mathrm{E}-02$ & $\mathrm{~N}-\mathrm{A}$ & 8 \\
\hline 555.7 & $95 \mathrm{~A}$ & 4.71E-05 & $1.60 \mathrm{E}-02$ & 8.58 & $1.79 \mathrm{E}-02$ & N-A & 9 \\
\hline 560.6 & $96 \mathrm{~A}$ & $5.64 \mathrm{E}-05$ & $9.06 \mathrm{E}-03$ & 4.06 & 1. $13 \mathrm{E}-02$ & $\mathrm{~N}-\mathrm{A}$ & 8.5 \\
\hline 561.8 & $96.5 \mathrm{~A}$ & $1.50 \mathrm{E}-04$ & $5.24 \mathrm{E}-02$ & 8.86 & $5.83 \mathrm{E}-02$ & $\mathrm{~N}-\mathrm{A}$ & 5 \\
\hline 562.2 & $96.5 \mathrm{~B}$ & $6.08 \mathrm{E}-05$ & $5.26 \mathrm{E}-03$ & 2.19 & $7.66 \mathrm{E}-03$ & $\mathrm{~N}-\mathrm{C}$ & 8.5 \\
\hline 570 & $97 \mathrm{~A}$ & $5.22 \mathrm{E}-04$ & $3.51 \mathrm{E}-02$ & 1.70 & $-1.45 \mathrm{E}-02$ & R-A & 8.5 \\
\hline 576.5 & $97.5 \mathrm{~A}$ & $1.16 \mathrm{E}-03$ & $4.85 \mathrm{E}-02$ & 1.06 & $9.44 \mathrm{E}-02$ & $\mathrm{~N}-\mathrm{C}$ & 6.5 \\
\hline 593.8 & $98 \mathrm{~A}$ & $9.76 \mathrm{E}-05$ & $1.60 \mathrm{E}-02$ & 4.15 & $1.99 \mathrm{E}-02$ & $\mathrm{~N}-\mathrm{A}$ & 4 \\
\hline 594.6 & $98 \mathrm{~B}$ & $5.39 \mathrm{E}-05$ & 7.38E-03 & 3.46 & $9.51 \mathrm{E}-03$ & N-A & 8 \\
\hline 594.6 & 98 & $5.56 \mathrm{E}-04$ & $9.95 \mathrm{E}-03$ & 0.45 & $3.19 \mathrm{E}-02$ & $\mathrm{~N}-\mathrm{A}$ & 9 \\
\hline 595.5 & $98 \mathrm{C}$ & $6.00 \mathrm{E}-05$ & 4. $19 \mathrm{E}-02$ & 17.65 & $4.43 \mathrm{E}-02$ & $\mathrm{~N}-\mathrm{A}$ & 9 \\
\hline 596.3 & $99 \mathrm{~A}$ & $4.20 \mathrm{E}-05$ & $1.65 \mathrm{E}-02$ & 9.94 & $1.82 \mathrm{E}-02$ & N-B & 9 \\
\hline 600.1 & $100 \mathrm{~A}$ & $5.44 \mathrm{E}-04$ & $1.14 \mathrm{E}-02$ & 0.53 & $3.29 \mathrm{E}-02$ & N-A & 9 \\
\hline 604.1 & $101 \mathrm{~A}$ & 4.07E-05 & $1.43 \mathrm{E}-02$ & 8.88 & $1.59 \mathrm{E}-02$ & $\mathrm{~N}-\mathrm{A}$ & 8.5 \\
\hline 604.2 & $101 B$ & 4.78E-05 & $1.22 \mathrm{E}-02$ & 6.46 & $1.41 \mathrm{E}-02$ & $\mathrm{~N}-\mathrm{A}$ & 8 \\
\hline 606 & 101B & $4.01 \mathrm{E}-05$ & $6.43 \mathrm{E}-03$ & 4.06 & 8.01E-03 & $\mathrm{N}-\mathrm{A}$ & 9 \\
\hline 606 & 101D & $4.40 \mathrm{E}-05$ & & & & & 8.5 \\
\hline 608.3 & $102 \mathrm{~A}$ & $6.25 \mathrm{E}-05$ & $2.51 \mathrm{E}-02$ & 10.15 & $2.76 \mathrm{E}-02$ & N-A & 8 \\
\hline 610.5 & $102 B$ & $4.02 \mathrm{E}-04$ & 7.57E-03 & 0.48 & $2.35 \mathrm{E}-02$ & $\mathrm{~N}-\mathrm{A}$ & 8 \\
\hline 615.3 & $104 \mathrm{~A}$ & $9.26 \mathrm{E}-04$ & $1.05 \mathrm{E}-02$ & 0.29 & 4.71E-02 & $\mathrm{N}-\mathrm{B}$ & 9 \\
\hline 618.7 & $105 \mathrm{~A}$ & $1.04 \mathrm{E}-03$ & $1.55 \mathrm{E}-02$ & 0.38 & $5.67 \mathrm{E}-02$ & N-A & 9 \\
\hline 621.3 & $106 \mathrm{~A}$ & $5.72 \mathrm{E}-05$ & $1.29 \mathrm{E}-02$ & 5.70 & $1.52 \mathrm{E}-02$ & N-A & 9 \\
\hline 622.8 & $107 \mathrm{~A}$ & $1.95 \mathrm{E}-03$ & $2.84 \mathrm{E}-02$ & 0.37 & $1.05 \mathrm{E}-01$ & N-A & 8 \\
\hline 625.9 & $109 A$ & $2.67 \mathrm{E}-03$ & $5.59 \mathrm{E}-02$ & 0.53 & $1.62 \mathrm{E}-01$ & N-A & 7 \\
\hline 628.2 & $111 \mathrm{~A}$ & 3. $32 \mathrm{E}-03$ & $3.41 \mathrm{E}-02$ & 0.26 & $1.65 \mathrm{E}-01$ & N-B & 4 \\
\hline 636.8 & $111 \mathrm{~B}$ & $2.50 \mathrm{E}-03$ & $6.85 \mathrm{E}-02$ & 0.69 & $1.67 \mathrm{E}-01$ & N-B & 5 \\
\hline 637.8 & $112 \mathrm{~A}$ & $1.82 \mathrm{E}-03$ & $5.86 \mathrm{E}-02$ & 0.81 & $1.31 \mathrm{E}-01$ & N-B & 5 \\
\hline 639.2 & $113 \mathrm{~A}$ & $1.27 \mathrm{E}-04$ & $2.27 \mathrm{E}-02$ & 4.53 & $2.77 \mathrm{E}-02$ & $\mathrm{~N}-\mathrm{A}$ & 7 \\
\hline 642 & $114 \mathrm{~A}$ & $1.18 \mathrm{E}-03$ & $2.26 \mathrm{E}-02$ & 0.48 & $6.92 \mathrm{E}-02$ & $\mathrm{~N}-\mathrm{A}$ & 5 \\
\hline 642.4 & 115 & $9.87 \mathrm{E}-04$ & $5.46 \mathrm{E}-03$ & 0.14 & $3.36 \mathrm{E}-02$ & R-B & 6 \\
\hline 643.6 & $116 \mathrm{~A}$ & $2.17 \mathrm{E}-03$ & & & & & 7 \\
\hline 649.7 & $117 \mathrm{~A}$ & $1.95 \mathrm{E}-03$ & $3.31 \mathrm{E}-02$ & 0.43 & $1.10 \mathrm{E}-01$ & $\mathrm{~N}-\mathrm{A}$ & 7 \\
\hline 650.9 & $118 \mathrm{~A}$ & $1.54 \mathrm{E}-03$ & & & & & 7.5 \\
\hline 656.9 & $119 \mathrm{~A}$ & $8.47 \mathrm{E}-05$ & $2.77 \mathrm{E}-02$ & 8.27 & $3.10 \mathrm{E}-02$ & $\mathrm{~N}-\mathrm{A}$ & 7.5 \\
\hline 659.4 & $120 \mathrm{~A}$ & 4.92E-05 & $3.35 \mathrm{E}-02$ & 17.22 & $3.54 \mathrm{E}-02$ & N-A & 8 \\
\hline 661.7 & $121 \mathrm{~A}$ & $3.19 \mathrm{E}-04$ & $1.43 \mathrm{E}-01$ & 11.34 & $1.56 \mathrm{E}-01$ & N-A & 3.5 \\
\hline 661.9 & $121 \mathrm{~B}$ & 2.67E-04 & $1.04 \mathrm{E}-01$ & 9.84 & $1.15 \mathrm{E}-01$ & $\mathrm{~N}-\mathrm{A}$ & 3.5 \\
\hline 663.3 & $122 \mathrm{~A}$ & $6.27 \mathrm{E}-05$ & $2.98 \mathrm{E}-02$ & 12.02 & $3.23 \mathrm{E}-02$ & $\mathrm{~N}-\mathrm{A}$ & 8 \\
\hline 668.1 & $123 \mathrm{~A}$ & $1.49 \mathrm{E}-04$ & $3.56 \mathrm{E}-02$ & 6.03 & $4.15 \mathrm{E}-02$ & $\mathrm{~N}-\mathrm{A}$ & 4 \\
\hline 669.8 & $124 \mathrm{~A}$ & $1.65 \mathrm{E}-04$ & $2.62 \mathrm{E}-02$ & 4.02 & $3.27 \mathrm{E}-02$ & N-A & 4 \\
\hline 671.7 & $130 \mathrm{~A}$ & $4.81 \mathrm{E}-05$ & 8.79E-03 & 4.62 & $1.07 \mathrm{E}-02$ & N-A & 9 \\
\hline 671.7 & $125 \mathrm{~A}$ & $6.54 \mathrm{E}-05$ & $1.34 \mathrm{E}-02$ & 5.18 & $1.60 \mathrm{E}-02$ & N-A & 7.5 \\
\hline 673.8 & $126 \mathrm{~A}$ & & $9.37 \mathrm{E}-03$ & 39.47 & $9.61 \mathrm{E}-03$ & N-B & 8 \\
\hline 676.5 & $131 \mathrm{~A}$ & 4.79E-04 & $1.20 \mathrm{E}-02$ & 0.63 & $3.10 \mathrm{E}-02$ & $\mathrm{R}-\mathrm{A}$ & 8 \\
\hline 679.3 & $132 \mathrm{~A}$ & & $2.05 \mathrm{E}-02$ & & & $\mathrm{I}-\mathrm{A}$ & \\
\hline 693.2 & $133 \mathrm{~A}$ & $5.30 \mathrm{E}-05$ & $1.26 \mathrm{E}-02$ & 6.02 & $1.47 \mathrm{E}-02$ & $\mathrm{~N}-\mathrm{A}$ & 8.5 \\
\hline 696.4 & $134 \mathrm{~A}$ & $1.27 \mathrm{E}-03$ & $1.98 \mathrm{E}-02$ & 0.40 & $6.99 \mathrm{E}-02$ & N-B & 8 \\
\hline 697.9 & $135 \mathrm{~A}$ & $2.36 \mathrm{E}-04$ & $1.24 \mathrm{E}-02$ & 1.33 & $-3.08 \mathrm{E}-03$ & $\mathrm{R}-\mathrm{A}$ & 8 \\
\hline 697.9 & $135 \mathrm{~A}$ & $2.36 \mathrm{E}-04$ & $4.41 \mathrm{E}-02$ & 4.73 & $5.34 \mathrm{E}-02$ & $\mathrm{~N}-\mathrm{A}$ & 8 \\
\hline 702.9 & $136 \mathrm{~A}$ & 4.13E-05 & $6.60 \mathrm{E}-03$ & 4.04 & $8.23 \mathrm{E}-03$ & N-A & 6 \\
\hline
\end{tabular}




\begin{tabular}{|c|c|c|c|c|c|c|c|}
\hline 704.5 & $136 \mathrm{~A}$ & $1.05 \mathrm{E}-04$ & $2.10 \mathrm{E}-02$ & 5.07 & $2.51 \mathrm{E}-02$ & N-A & 8.5 \\
\hline 704.6 & $136 \mathrm{~B}$ & $1.24 \mathrm{E}-04$ & $2.59 \mathrm{E}-02$ & 5.27 & $3.08 \mathrm{E}-02$ & $\mathrm{~N}-\mathrm{C}$ & 8 \\
\hline 706.5 & $136 \mathrm{C}$ & $2.34 \mathrm{E}-04$ & 6.29E-02 & 6.79 & $7.22 \mathrm{E}-02$ & N-B & 5 \\
\hline 708.5 & $137 \mathrm{~A}$ & 4.37E-05 & $3.45 \mathrm{E}-03$ & 2.00 & $-1.72 \mathrm{E}-03$ & R-B & 9 \\
\hline 709.9 & $138 \mathrm{~A}$ & $3.05 \mathrm{E}-05$ & $1.71 \mathrm{E}-03$ & 1.42 & $-5.04 \mathrm{E}-04$ & R-B & 9 \\
\hline 713 & $140 \mathrm{~A}$ & $2.75 \mathrm{E}-05$ & $5.61 \mathrm{E}-03$ & 5.16 & $-4.52 \mathrm{E}-03$ & R-A & 9 \\
\hline 714.7 & $141 \mathrm{~A}$ & $2.42 \mathrm{E}-05$ & $5.12 \mathrm{E}-03$ & 5.35 & $-4.16 \mathrm{E}-03$ & R-A & 9 \\
\hline 714.8 & $141 \mathrm{~A}$ & $3.30 \mathrm{E}-04$ & 3.97E-03 & 0.30 & $9.08 \mathrm{E}-03$ & $\mathrm{R}-\mathrm{A}$ & 9 \\
\hline 718.7 & $142 B$ & $3.28 \mathrm{E}-05$ & 4.44E-03 & 3.42 & $-3.14 \mathrm{E}-03$ & R-A & 9 \\
\hline 718.8 & $142 \mathrm{~A}$ & $3.82 \mathrm{E}-05$ & $4.05 \mathrm{E}-03$ & 2.68 & $-2.54 \mathrm{E}-03$ & R-A & 9 \\
\hline 718.9 & $142 \mathrm{~A}$ & 4.64E-04 & 5.39E-03 & 0.29 & $1.29 \mathrm{E}-02$ & R-A & 9 \\
\hline 720.9 & $143 \mathrm{~A}$ & 3.99E-04 & $2.71 \mathrm{E}-03$ & 0.17 & $1.31 \mathrm{E}-02$ & $\mathrm{R}-\mathrm{C}$ & 9 \\
\hline 723.4 & $144 \mathrm{~A}$ & $2.53 \mathrm{E}-05$ & $2.07 \mathrm{E}-03$ & 2.07 & 3.07E-03 & N-B & 9 \\
\hline 742.4 & $145 \mathrm{~A}$ & & $3.24 \mathrm{E}-02$ & & & N-A & \\
\hline 743.9 & $146 \mathrm{~A}$ & $1.93 \mathrm{E}-03$ & $2.78 \mathrm{E}-02$ & 0.36 & $1.04 \mathrm{E}-01$ & $\mathrm{~N}-\mathrm{A}$ & 5 \\
\hline 745.2 & $147 \mathrm{~A}$ & $2.09 \mathrm{E}-03$ & $2.08 \mathrm{E}-02$ & 0.25 & $1.03 \mathrm{E}-01$ & N-A & 5 \\
\hline 747.7 & 147B & & $2.99 \mathrm{E}-03$ & & & $\mathrm{~N}-\mathrm{C}$ & \\
\hline 748.8 & $148 \mathrm{~A}$ & & $4.31 \mathrm{E}-03$ & & & N-B & \\
\hline 750.6 & $149 \mathrm{~A}$ & $4.04 \mathrm{E}-04$ & $3.52 \mathrm{E}-03$ & 0.22 & $1.95 \mathrm{E}-02$ & N-B & 8.5 \\
\hline 752.4 & $150 \mathrm{~A}$ & $6.19 \mathrm{E}-04$ & $5.11 \mathrm{E}-03$ & 0.21 & $2.96 \mathrm{E}-02$ & $\mathrm{~N}-\mathrm{A}$ & 8 \\
\hline 756.3 & $151 \mathrm{~A}$ & $3.00 \mathrm{E}-03$ & $8.66 \mathrm{E}-03$ & 0.07 & $1.10 \mathrm{E}-01$ & $\mathrm{R}-\mathrm{B}$ & 6 \\
\hline 758.4 & $152 \mathrm{~A}$ & $2.11 \mathrm{E}-03$ & $1.97 \mathrm{E}-02$ & 0.24 & $1.03 \mathrm{E}-01$ & $\mathrm{~N}-\mathrm{C}$ & 7 \\
\hline 760.3 & $153 \mathrm{~A}$ & $8.12 \mathrm{E}-05$ & 1.33E-02 & 4.14 & $1.65 \mathrm{E}-02$ & N-A & 8 \\
\hline 770.2 & $153 \mathrm{~B}$ & $1.98 \mathrm{E}-04$ & $5.54 \mathrm{E}-02$ & 7.08 & $6.32 \mathrm{E}-02$ & $\mathrm{~N}-\mathrm{A}$ & 4 \\
\hline 773 & $153 \mathrm{C}$ & 4.07E-04 & $7.45 \mathrm{E}-02$ & 4.63 & $9.06 \mathrm{E}-02$ & $\mathrm{~N}-\mathrm{A}$ & 4 \\
\hline 775.1 & $153 \mathrm{E}$ & $2.73 \mathrm{E}-04$ & $5.46 \mathrm{E}-02$ & 5.05 & $6.54 \mathrm{E}-02$ & N-A & 4 \\
\hline 777.6 & $153 \mathrm{D}$ & $6.84 \mathrm{E}-04$ & $9.16 \mathrm{E}-02$ & 3.39 & $-6.46 \mathrm{E}-02$ & $\mathrm{R}-\mathrm{B}$ & 7 \\
\hline 782.5 & $154 \mathrm{~A}$ & & $1.01 \mathrm{E}-02$ & & & R-A & \\
\hline 783.5 & $155 \mathrm{~A}$ & $8.12 \mathrm{E}-05$ & $9.06 \mathrm{E}-03$ & 2.82 & $-5.85 \mathrm{E}-03$ & $\mathrm{R}-\mathrm{B}$ & 8 \\
\hline 783.6 & $155 \mathrm{~B}$ & $1.93 \mathrm{E}-04$ & $3.29 \mathrm{E}-02$ & 4.30 & 4.05E-02 & N-A & 5 \\
\hline 791.7 & $307 \mathrm{~A}$ & & $6.56 \mathrm{E}-02$ & & & $\mathrm{~N}-\mathrm{A}$ & \\
\hline 792.7 & $308 \mathrm{~A}$ & $3.27 \mathrm{E}-03$ & $5.00 \mathrm{E}-02$ & 0.39 & $1.79 \mathrm{E}-01$ & $\mathrm{~N}-\mathrm{A}$ & 4 \\
\hline 794.8 & $309 \mathrm{~A}$ & $1.81 \mathrm{E}-03$ & 3.34E-02 & 0.47 & $1.05 \mathrm{E}-01$ & $\mathrm{~N}-\mathrm{A}$ & 3.5 \\
\hline 795.4 & $310 \mathrm{~A}$ & $2.34 \mathrm{E}-03$ & $3.60 \mathrm{E}-02$ & 0.39 & & R-B & 3.5 \\
\hline 797.1 & $311 \mathrm{~A}$ & 2.03E-03 & $3.70 \mathrm{E}-02$ & 0.46 & $1.17 \mathrm{E}-01$ & N-A & 3.5 \\
\hline 799.4 & $312 \mathrm{~A}$ & $2.95 \mathrm{E}-03$ & $5.54 \mathrm{E}-02$ & 0.47 & $1.72 \mathrm{E}-01$ & $\mathrm{~N}-\mathrm{A}$ & 3.5 \\
\hline 800.4 & $313 \mathrm{~A}$ & 3.11E-03 & $4.97 \mathrm{E}-02$ & 0.40 & $1.73 \mathrm{E}-01$ & N-A & 3.5 \\
\hline 801.3 & $314 \mathrm{~A}$ & $2.58 \mathrm{E}-03$ & 4.93E-02 & 0.48 & $1.51 \mathrm{E}-01$ & $\mathrm{~N}-\mathrm{A}$ & 3.5 \\
\hline 804.8 & $315 \mathrm{~A}$ & 3.17E-03 & $7.45 \mathrm{E}-02$ & 0.59 & $2.00 \mathrm{E}-01$ & $\mathrm{~N}-\mathrm{A}$ & 4 \\
\hline 804.9 & $316 \mathrm{~A}$ & $2.58 \mathrm{E}-03$ & 4. $66 \mathrm{E}-02$ & 0.46 & $1.49 \mathrm{E}-01$ & $\mathrm{~N}-\mathrm{A}$ & 4 \\
\hline 812.8 & $156 \mathrm{~A}$ & & $1.16 \mathrm{E}-01$ & & & N-A & \\
\hline 820.8 & $157 \mathrm{~A}$ & 8.27E-03 & $1.61 \mathrm{E}-01$ & 0.49 & $4.88 \mathrm{E}-01$ & $\mathrm{~N}-\mathrm{A}$ & 3 \\
\hline 825.7 & $158 \mathrm{~A}$ & 8.36E-04 & $1.41 \mathrm{E}-01$ & 4.26 & $1.74 \mathrm{E}-01$ & $\mathrm{~N}-\mathrm{A}$ & 3.5 \\
\hline 842.4 & $711 \mathrm{~A}$ & $4.53 \mathrm{E}-04$ & $9.89 \mathrm{E}-02$ & 5.53 & $1.17 \mathrm{E}-01$ & $\mathrm{~N}-\mathrm{A}$ & 3 \\
\hline 850.1 & $712 \mathrm{~A}$ & $7.89 \mathrm{E}-04$ & $1.18 \mathrm{E}-01$ & 3.78 & $1.49 \mathrm{E}-01$ & $\mathrm{~N}-\mathrm{A}$ & 3.5 \\
\hline 869.3 & $712 \mathrm{~B}$ & $1.33 \mathrm{E}-03$ & $2.46 \mathrm{E}-01$ & 4.66 & $2.99 \mathrm{E}-01$ & $\mathrm{~N}-\mathrm{B}$ & 5 \\
\hline 879.5 & $712 \mathrm{C}$ & 4.38E-04 & $3.26 \mathrm{E}-02$ & 1.88 & $-1.53 \mathrm{E}-02$ & $\mathrm{R}-\mathrm{A}$ & 7 \\
\hline 889.4 & $713 \mathrm{~A}$ & $1.90 \mathrm{E}-04$ & 4.44E-02 & 5.90 & $5.19 \mathrm{E}-02$ & $\mathrm{~N}-\mathrm{C}$ & 3.5 \\
\hline 893.4 & $714 \mathrm{~A}$ & & $8.70 \mathrm{E}-02$ & & & $\mathrm{~N}-\mathrm{C}$ & \\
\hline 909.8 & $715 \mathrm{~A}$ & $6.52 \mathrm{E}-04$ & 9.04E-02 & 3.51 & $-6.46 \mathrm{E}-02$ & $\mathrm{R}-\mathrm{A}$ & 4 \\
\hline 915.3 & $159 A$ & $8.30 \mathrm{E}-04$ & $2.09 \mathrm{E}-01$ & 6.37 & $2.42 \mathrm{E}-01$ & $\mathrm{~N}-\mathrm{A}$ & 3.5 \\
\hline 919.8 & $160 \mathrm{~A}$ & $3.52 \mathrm{E}-04$ & 7.08E-02 & 5.09 & 8.47E-02 & $\mathrm{N}-\mathrm{A}$ & 4 \\
\hline 929.6 & $716 \mathrm{~A}$ & $2.26 \mathrm{E}-02$ & $1.02 \mathrm{E}-01$ & 0.11 & $9.97 \mathrm{E}-01$ & $\mathrm{~N}-\mathrm{A}$ & 3 \\
\hline 931.1 & $717 \mathrm{~A}$ & $5.28 \mathrm{E}-04$ & $8.38 \mathrm{E}-02$ & 4.01 & $1.05 \mathrm{E}-01$ & $\mathrm{~N}-\mathrm{A}$ & 5 \\
\hline
\end{tabular}




\begin{tabular}{|c|c|c|c|c|c|c|c|}
\hline 942.9 & $161 \mathrm{~A}$ & $1.42 \mathrm{E}-04$ & $6.36 \mathrm{E}-02$ & 11.35 & $6.92 \mathrm{E}-02$ & $\mathrm{~N}-\mathrm{A}$ & 3.5 \\
\hline 944 & $162 \mathrm{~A}$ & & $3.79 \mathrm{E}-02$ & & & N-A & \\
\hline 1037.3 & $165 \mathrm{~A}$ & & $3.88 \mathrm{E}-02$ & & & $\mathrm{~N}-\mathrm{C}$ & \\
\hline 1037.5 & $166 \mathrm{~A}$ & $6.51 \mathrm{E}-03$ & $7.46 \mathrm{E}-02$ & 0.29 & $3.32 \mathrm{E}-01$ & $\mathrm{~N}-\mathrm{A}$ & 5 \\
\hline 1037.6 & $167 \mathrm{~A}$ & $5.28 \mathrm{E}-03$ & $6.40 \mathrm{E}-02$ & 0.31 & 2.73E-01 & N-A & 5 \\
\hline 1043.2 & $168 \mathrm{~A}$ & 6.27E-03 & $9.55 \mathrm{E}-02$ & 0.39 & $3.43 \mathrm{E}-01$ & $\mathrm{~N}-\mathrm{A}$ & 5 \\
\hline 1047.8 & $169 A$ & 3.17E-03 & 4.20E-02 & 0.33 & $1.68 \mathrm{E}-01$ & $\mathrm{~N}-\mathrm{A}$ & 7.5 \\
\hline 1054.1 & $170 \mathrm{~A}$ & 2.77E-03 & $3.75 \mathrm{E}-02$ & 0.34 & $1.47 \mathrm{E}-01$ & $\mathrm{~N}-\mathrm{A}$ & 4 \\
\hline 1056.1 & $171 \mathrm{~A}$ & $2.56 \mathrm{E}-03$ & $4.57 \mathrm{E}-02$ & 0.45 & $1.47 \mathrm{E}-01$ & N-A & 4 \\
\hline 1058.1 & $172 \mathrm{~A}$ & $2.58 \mathrm{E}-03$ & $3.32 \mathrm{E}-02$ & 0.33 & $1.35 \mathrm{E}-01$ & N-A & 5 \\
\hline 1062.8 & $173 \mathrm{~A}$ & $2.18 \mathrm{E}-03$ & $2.53 \mathrm{E}-02$ & 0.29 & $1.12 \mathrm{E}-01$ & N-B & 3 \\
\hline 1064.7 & $174 \mathrm{~A}$ & 7.67E-03 & 8.64E-02 & 0.28 & $3.90 \mathrm{E}-01$ & N-A & 6 \\
\hline 1181.3 & $175 \mathrm{~A}$ & $5.15 \mathrm{E}-04$ & $6.18 \mathrm{E}-03$ & 0.30 & $2.66 \mathrm{E}-02$ & N-A & 7 \\
\hline 1205 & $177 \mathrm{~A}$ & 2.23E-03 & $4.54 \mathrm{E}-02$ & 0.51 & $1.34 \mathrm{E}-01$ & N-A & 3.5 \\
\hline 1207 & $178 \mathrm{~A}$ & $2.83 \mathrm{E}-03$ & $3.90 \mathrm{E}-02$ & 0.35 & $1.51 \mathrm{E}-01$ & N-A & 4 \\
\hline 1209 & $179 \mathrm{~A}$ & $2.80 \mathrm{E}-03$ & $3.26 \mathrm{E}-02$ & 0.29 & $1.43 \mathrm{E}-01$ & N-A & 4 \\
\hline 1211 & $180 \mathrm{~A}$ & 2.01E-03 & $1.61 \mathrm{E}-02$ & 0.20 & $9.56 \mathrm{E}-02$ & N-B & 4 \\
\hline 1215.4 & $181 \mathrm{~A}$ & $3.24 \mathrm{E}-03$ & $3.64 \mathrm{E}-02$ & 0.28 & $1.64 \mathrm{E}-01$ & N-A & 4 \\
\hline 1225.6 & $182 \mathrm{~A}$ & $2.06 \mathrm{E}-03$ & $3.52 \mathrm{E}-02$ & 0.43 & $1.17 \mathrm{E}-01$ & N-A & 3 \\
\hline 1233.5 & $183 \mathrm{~A}$ & $4.68 \mathrm{E}-03$ & $5.29 \mathrm{E}-02$ & 0.29 & 2.38E-01 & $\mathrm{N}-\mathrm{A}$ & 4 \\
\hline 1235.2 & $184 \mathrm{~A}$ & $1.73 \mathrm{E}-03$ & 4.27E-02 & 0.62 & $1.11 \mathrm{E}-01$ & N-A & 4 \\
\hline 1237.2 & $185 \mathrm{~A}$ & $3.38 \mathrm{E}-03$ & $3.92 \mathrm{E}-02$ & 0.29 & $1.73 \mathrm{E}-01$ & N-A & 4 \\
\hline 1239.2 & $186 \mathrm{~A}$ & $1.60 \mathrm{E}-03$ & $4.21 \mathrm{E}-02$ & 0.66 & $1.05 \mathrm{E}-01$ & N-A & 4 \\
\hline 1241.2 & $187 \mathrm{~A}$ & 2.12E-03 & $3.01 \mathrm{E}-02$ & 0.36 & $1.14 \mathrm{E}-01$ & N-A & 3 \\
\hline 1245.4 & $188 \mathrm{~A}$ & $3.00 \mathrm{E}-03$ & $3.55 \mathrm{E}-02$ & 0.30 & $1.54 \mathrm{E}-01$ & N-A & 4 \\
\hline 1246.4 & $189 \mathrm{~A}$ & & 8.39E-03 & & & N-B & \\
\hline 1246.9 & $190 \mathrm{~A}$ & 7.75E-03 & 8.03E-02 & 0.26 & $3.87 \mathrm{E}-01$ & N-A & 4 \\
\hline 1262.9 & $191 \mathrm{~A}$ & 4.78E-03 & $6.73 \mathrm{E}-02$ & 0.36 & $2.56 \mathrm{E}-01$ & N-A & 5 \\
\hline 1263.6 & $192 \mathrm{~A}$ & & $3.99 \mathrm{E}-02$ & & & $\mathrm{~N}-\mathrm{A}$ & \\
\hline 1265.5 & $193 \mathrm{~A}$ & 3.39E-03 & $6.01 \mathrm{E}-02$ & 0.45 & $1.94 \mathrm{E}-01$ & $\mathrm{~N}-\mathrm{A}$ & 4 \\
\hline 1269.3 & $194 \mathrm{~A}$ & 8.45E-04 & $1.76 \mathrm{E}-02$ & 0.53 & $5.10 \mathrm{E}-02$ & N-A & 8.5 \\
\hline 1273.1 & $195 \mathrm{~A}$ & $6.47 \mathrm{E}-03$ & $1.16 \mathrm{E}-01$ & 0.45 & $1.40 \mathrm{E}-01$ & $\mathrm{R}-\mathrm{A}$ & 6 \\
\hline 1277.1 & $196 \mathrm{~A}$ & 3.36E-03 & $5.15 \mathrm{E}-02$ & 0.39 & $1.84 \mathrm{E}-01$ & N-A & 4 \\
\hline 1278.2 & $197 \mathrm{~A}$ & $5.23 \mathrm{E}-03$ & $5.06 \mathrm{E}-02$ & 0.24 & $2.58 \mathrm{E}-01$ & $\mathrm{~N}-\mathrm{A}$ & 4 \\
\hline 1281.1 & $198 \mathrm{~A}$ & $3.10 \mathrm{E}-03$ & $3.54 \mathrm{E}-02$ & 0.29 & $1.58 \mathrm{E}-01$ & N-A & 3 \\
\hline 1282.1 & $199 \mathrm{~A}$ & $4.56 \mathrm{E}-04$ & $2.39 \mathrm{E}-02$ & 1.33 & 4.19E-02 & $\mathrm{N}-\mathrm{C}$ & 4 \\
\hline 1290 & $200 \mathrm{~A}$ & $1.42 \mathrm{E}-03$ & $2.85 \mathrm{E}-02$ & 0.51 & $2.77 \mathrm{E}-02$ & $\mathrm{R}-\mathrm{A}$ & 3 \\
\hline 1306.4 & $201 \mathrm{~A}$ & 2.09E-04 & $4.09 \mathrm{E}-02$ & 4.95 & 4.92E-02 & $\mathrm{N}-\mathrm{A}$ & 5 \\
\hline 1309.4 & $202 \mathrm{~A}$ & $5.25 \mathrm{E}-03$ & 7.61E-02 & 0.37 & 2.84E-01 & N-B & 4 \\
\hline 1311.4 & $203 \mathrm{~A}$ & 7.64E-03 & $1.15 \mathrm{E}-01$ & 0.38 & 4.17E-01 & $\mathrm{N}-\mathrm{A}$ & 3.7 \\
\hline 1313.3 & $204 \mathrm{~A}$ & $6.95 \mathrm{E}-03$ & 8.80E-02 & 0.32 & 3.63E-01 & $\mathrm{N}-\mathrm{A}$ & 3.7 \\
\hline 1320 & $205 \mathrm{~A}$ & $6.00 \mathrm{E}-03$ & $5.68 \mathrm{E}-02$ & 0.24 & $2.94 \mathrm{E}-01$ & N-B & 3.7 \\
\hline 1328.3 & $206 \mathrm{~A}$ & & $1.59 \mathrm{E}-01$ & & & N-A & \\
\hline 1338.2 & $207 \mathrm{~A}$ & $1.79 \mathrm{E}-02$ & $1.54 \mathrm{E}-01$ & 0.22 & $8.62 \mathrm{E}-01$ & $\mathrm{~N}-\mathrm{A}$ & 4 \\
\hline 1340.4 & $208 \mathrm{~A}$ & $1.10 \mathrm{E}-02$ & 1. $12 \mathrm{E}-01$ & 0.26 & $5.47 \mathrm{E}-01$ & $\mathrm{~N}-\mathrm{A}$ & 4 \\
\hline 1358.6 & $209 \mathrm{~A}$ & & $1.32 \mathrm{E}-01$ & & & N-A & \\
\hline 1359 & 209B & $2.36 \mathrm{E}-02$ & $2.64 \mathrm{E}-01$ & 0.28 & $1.20 \mathrm{E}+00$ & N-A & 4 \\
\hline 1361 & $210 \mathrm{~A}$ & $4.43 E-03$ & $6.40 \mathrm{E}-02$ & 0.37 & $2.39 \mathrm{E}-01$ & N-A & 4 \\
\hline 1362.8 & $211 \mathrm{~A}$ & $1.04 \mathrm{E}-02$ & $9.95 \mathrm{E}-02$ & 0.24 & 5.12E-01 & N-A & 4 \\
\hline 1365.4 & $250 \mathrm{~A}$ & $5.78 \mathrm{E}-04$ & $9.54 \mathrm{E}-02$ & 4.17 & $1.18 \mathrm{E}-01$ & N-A & 4 \\
\hline 1375.1 & $251 \mathrm{~A}$ & $6.60 \mathrm{E}-03$ & $7.75 \mathrm{E}-02$ & 0.30 & $3.39 \mathrm{E}-01$ & N-A & 3 \\
\hline 1377.3 & $252 \mathrm{~A}$ & $6.11 \mathrm{E}-04$ & $1.04 \mathrm{E}-01$ & 4.30 & $1.28 \mathrm{E}-01$ & N-A & 3.5 \\
\hline 1379 & $253 \mathrm{~A}$ & $6.62 \mathrm{E}-04$ & $1.11 \mathrm{E}-01$ & 4.24 & $1.37 \mathrm{E}-01$ & N-A & 4.5 \\
\hline 1381 & $254 \mathrm{~A}$ & $7.23 \mathrm{E}-04$ & $1.24 \mathrm{E}-02$ & 0.43 & $4.10 \mathrm{E}-02$ & N-A & 4 \\
\hline
\end{tabular}




$\begin{array}{llllllll}1383 & 255 \mathrm{~A} & 5.26 \mathrm{E}-04 & 1.54 \mathrm{E}-01 & 7.40 & 1.75 \mathrm{E}-01 & \text { N-A } & 4 \\ 1384.7 & 256 \mathrm{~A} & 3.16 \mathrm{E}-04 & 5.00 \mathrm{E}-02 & 4.00 & 6.25 \mathrm{E}-02 & \text { N-A } & 4 \\ 1387 & 257 \mathrm{~A} & 6.65 \mathrm{E}-04 & 9.39 \mathrm{E}-02 & 3.57 & 1.20 \mathrm{E}-01 & \text { N-A } & 5 \\ 1395.1 & 258 \mathrm{~A} & 5.97 \mathrm{E}-04 & 1.40 \mathrm{E}-01 & 5.93 & 1.64 \mathrm{E}-01 & \text { N-A } & 4 \\ 1397 & 259 \mathrm{~A} & 2.80 \mathrm{E}-04 & 9.51 \mathrm{E}-02 & 8.60 & 1.06 \mathrm{E}-01 & \text { N-A } & 4 \\ 1397.7 & 260 \mathrm{~A} & 4.97 \mathrm{E}-03 & 8.39 \mathrm{E}-02 & 0.43 & 2.80 \mathrm{E}-01 & \text { N-A } & 6 \\ 1399 & 261 \mathrm{~A} & 1.92 \mathrm{E}-04 & 4.41 \mathrm{E}-02 & 5.82 & 5.17 \mathrm{E}-02 & \text { N-A } & 4 \\ 1401 & 262 \mathrm{~A} & 1.84 \mathrm{E}-04 & 4.70 \mathrm{E}-02 & 6.46 & 5.43 \mathrm{E}-02 & \text { N-A } & 4 \\ 1403 & 263 \mathrm{~A} & 5.63 \mathrm{E}-04 & 8.17 \mathrm{E}-02 & 3.67 & 1.04 \mathrm{E}-01 & \text { N-A } & 5 \\ 1407 & 264 \mathrm{~A} & 6.99 \mathrm{E}-04 & 2.72 \mathrm{E}-01 & 9.84 & 3.00 \mathrm{E}-01 & \text { N-A } & 4 \\ 1409 & 265 \mathrm{~A} & 4.13 \mathrm{E}-03 & 1.26 \mathrm{E}-01 & 0.77 & 2.89 \mathrm{E}-01 & \text { N-A } & 4 \\ 1411 & 266 \mathrm{~A} & 1.28 \mathrm{E}-04 & 2.06 \mathrm{E}-02 & 4.06 & 2.57 \mathrm{E}-02 & \text { N-B } & 4 \\ 1413 & 267 \mathrm{~A} & 1.48 \mathrm{E}-04 & 2.03 \mathrm{E}-02 & 3.48 & 2.61 \mathrm{E}-02 & \text { N-A } & 4 \\ 1414.4 & 268 \mathrm{~A} & 2.11 \mathrm{E}-03 & 2.25 \mathrm{E}-02 & 0.27 & 1.06 \mathrm{E}-01 & \text { N-B } & 4 \\ 1422.4 & 270 \mathrm{~A} & 8.52 \mathrm{E}-05 & 2.34 \mathrm{E}-02 & 6.95 & 2.68 \mathrm{E}-02 & \text { N-A } & 4 \\ 1424 & 271 \mathrm{~A} & 6.93 \mathrm{E}-04 & 1.26 \mathrm{E}-01 & 4.60 & 1.53 \mathrm{E}-01 & \text { N-A } & 4 \\ 1432.2 & 272 \mathrm{~A} & 1.34 \mathrm{E}-02 & 1.89 \mathrm{E}-01 & 0.36 & 7.19 \mathrm{E}-01 & \text { N-A } & 3 \\ 1434 & 273 \mathrm{~A} & 6.66 \mathrm{E}-04 & 1.08 \mathrm{E}-01 & 4.10 & 1.34 \mathrm{E}-01 & \text { N-A } & 4 \\ 1436 & 274 \mathrm{~A} & 6.33 \mathrm{E}-04 & 1.03 \mathrm{E}-01 & 4.11 & 1.28 \mathrm{E}-01 & \text { N-A } & 4 \\ 1440 & 275 \mathrm{~A} & 7.53 \mathrm{E}-03 & 1.65 \mathrm{E}-01 & 0.55 & 4.63 \mathrm{E}-01 & \text { N-A } & 4 \\ 1444 & 276 \mathrm{~A} & 5.23 \mathrm{E}-04 & 8.18 \mathrm{E}-02 & 3.95 & 1.02 \mathrm{E}-01 & \text { N-A } & 4 \\ 1446 & 277 \mathrm{~A} & 6.69 \mathrm{E}-04 & 8.42 \mathrm{E}-02 & 3.18 & 1.11 \mathrm{E}-01 & \text { N-A } & 4 \\ 1448 & 278 \mathrm{~A} & 1.13 \mathrm{E}-02 & 1.58 \mathrm{E}-01 & 0.35 & 6.04 \mathrm{E}-01 & \text { N-A } & 3 \\ 1449.5 & 279 \mathrm{~A} & 6.95 \mathrm{E}-04 & 1.14 \mathrm{E}-01 & 4.15 & 1.41 \mathrm{E}-01 & \text { N-A } & 3.7 \\ 1474 & 281 \mathrm{~A} & 9.33 \mathrm{E}-04 & 1.63 \mathrm{E}-01 & 4.42 & 2.00 \mathrm{E}-01 & \text { N-A } & 4 \\ 1477 & 282 \mathrm{~A} & & 1.51 \mathrm{E}-01 & & & \text { N-A } & 2 \\ 1482.3 & 283 \mathrm{~A} & 2.64 \mathrm{E}-04 & 7.06 \mathrm{E}-02 & 6.76 & 8.10 \mathrm{E}-02 & \text { N-A } & 4 \\ 1484.1 & 284 \mathrm{~A} & 3.08 \mathrm{E}-04 & 5.73 \mathrm{E}-02 & 4.70 & 6.95 \mathrm{E}-02 & \text { N-A } & 5 \\ 1488.1 & 285 \mathrm{~A} & 6.58 \mathrm{E}-04 & 1.89 \mathrm{E}-01 & 7.26 & 2.15 \mathrm{E}-01 & \text { N-A } & 9 \\ 1493.8 & 286 \mathrm{~A} & & 5.56 \mathrm{E}-02 & & & \text { N-B } & \end{array}$

